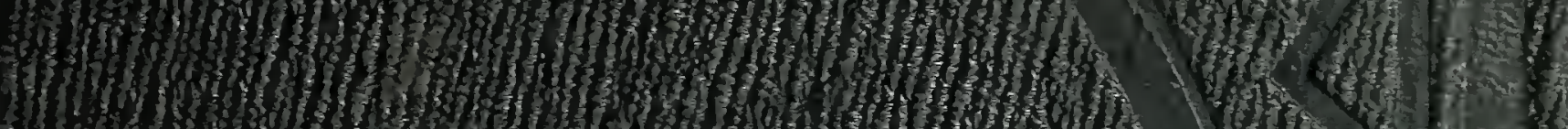

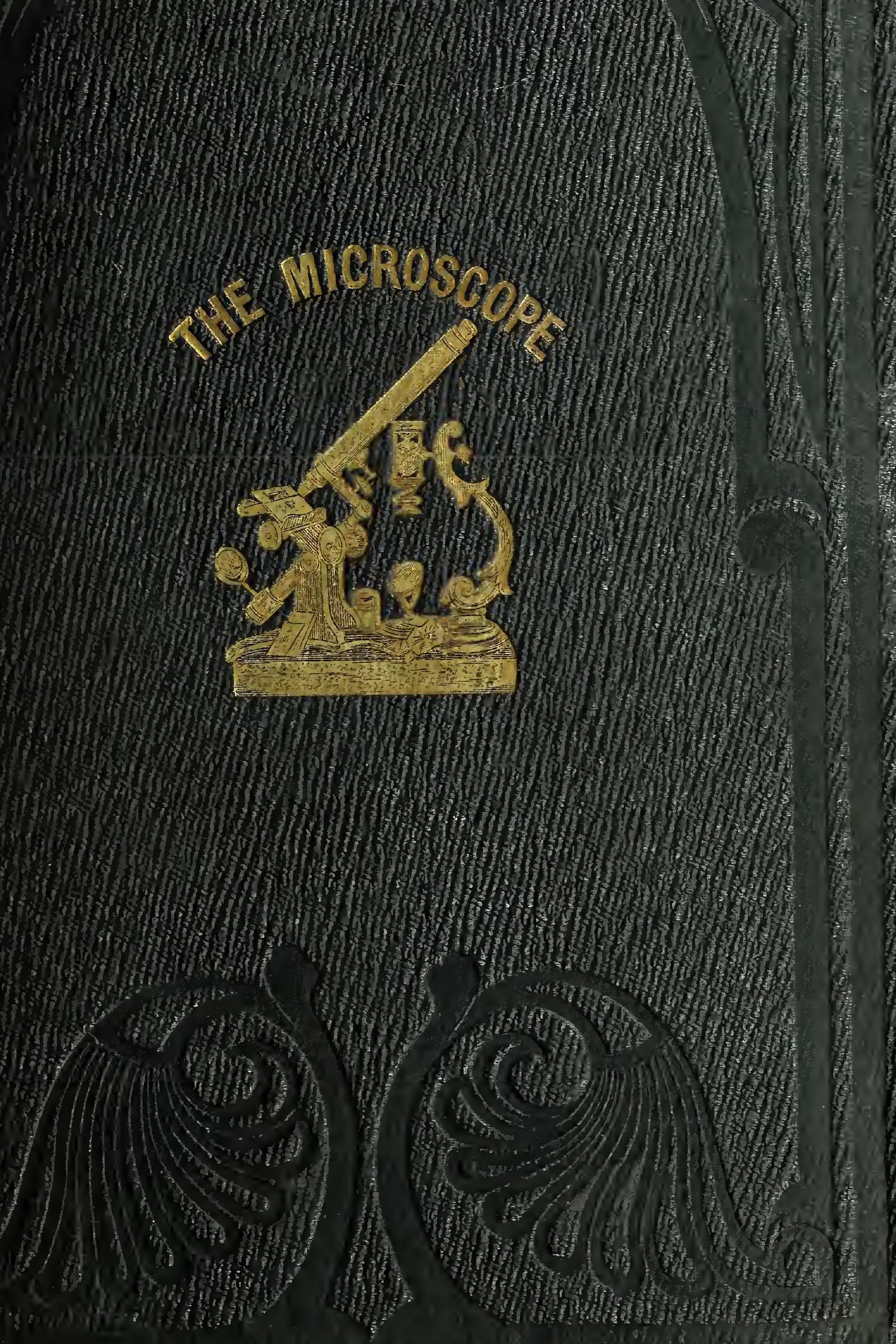



1854

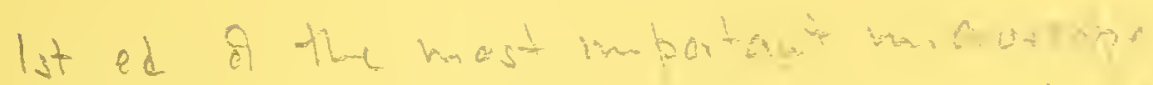
beck of the 19a. continuiber in tormt to l5ed.18as and ad iall. 



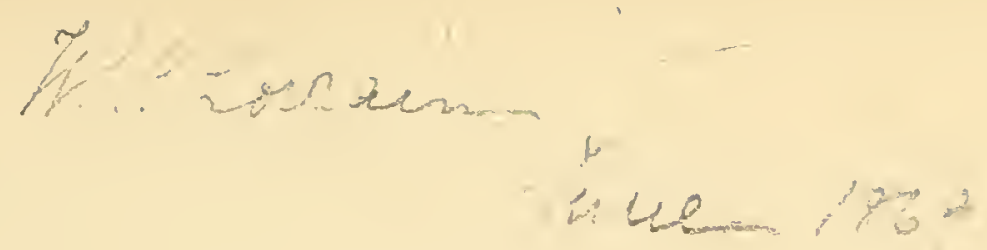

THE MICROSCOPE. 

Digitized by the Internet Archive in 2018 with funding from University of Toronto 


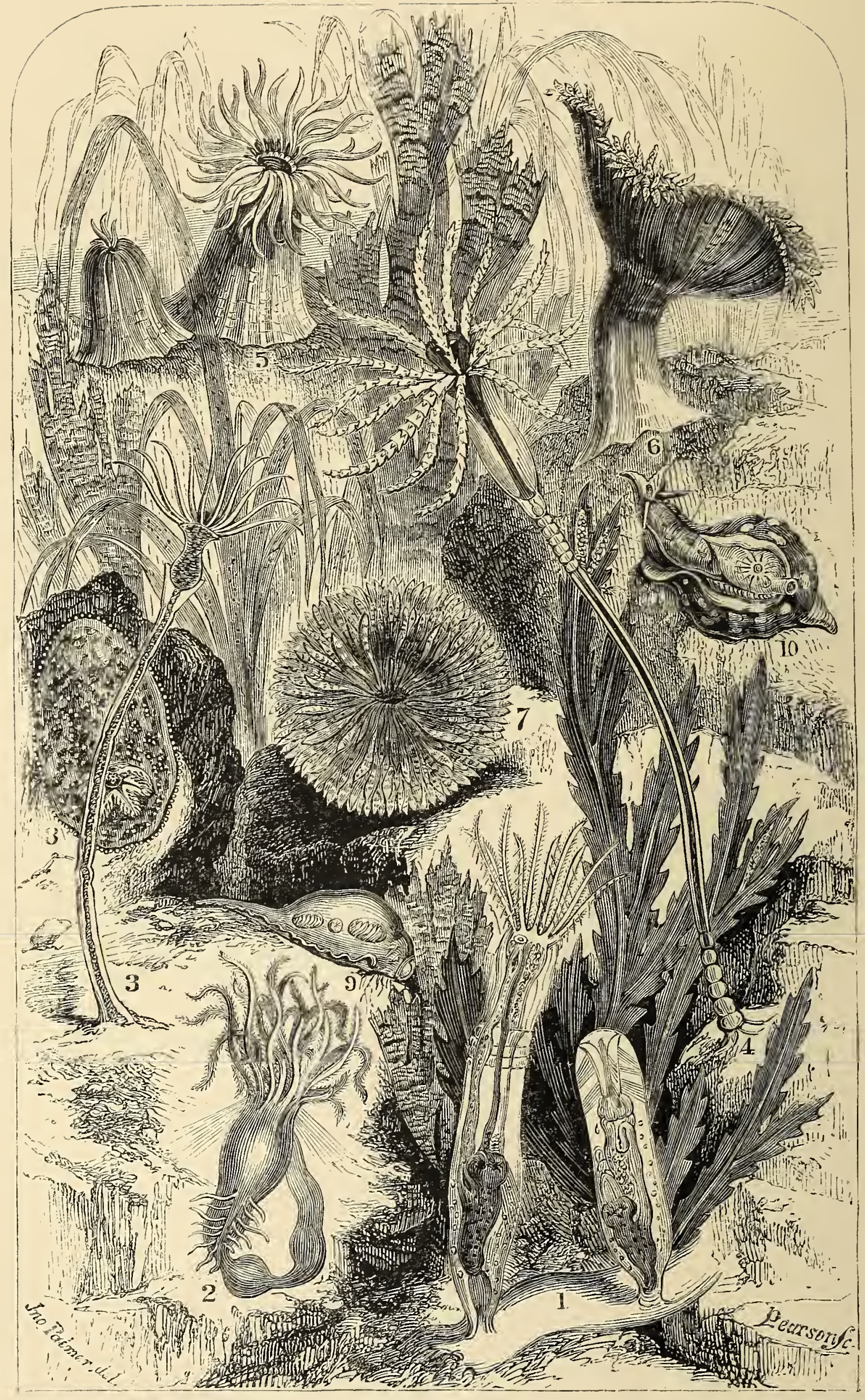




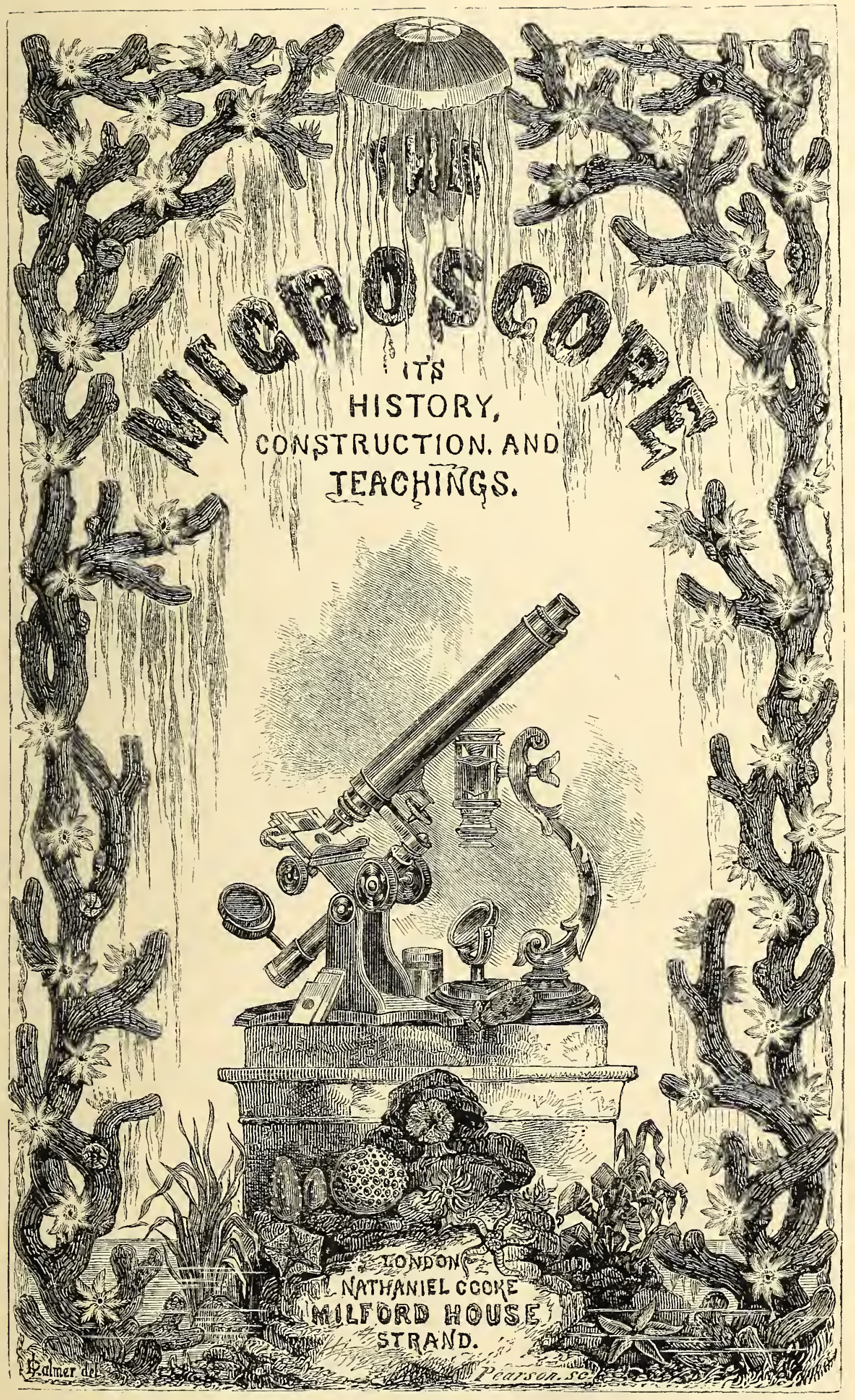





\section{MICROSCOPE:}

ITS

\section{HISTORY, CONSTRUCTION, AND APPLICATIONS.}

EEING A

FAMILIAR INTRODUCTION TO THE USE OF THE INSTRUMENT

A $\times \mathbf{D}$

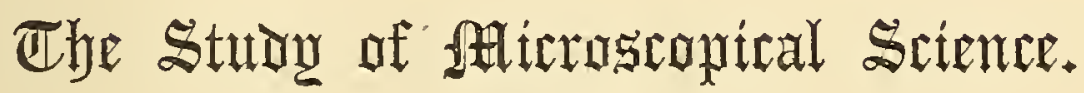

BY JABEZ HOGG, M.R.C.S.

FELLOTF OF THE MEDICAL SOCIETT OF LONDON,

MEMBER OF THE MICROSCOPICAL AND PATHOLOGICAL SOCIETIES OF LONDON, AUTHOR OF "ELEMENTS OF NATURAL PHILOSOPHY," ETC.

Hllustrated with upwards of ffibe

$$
\text { IONDON: }
$$

PUBLISHED AT THE OFFICE OF

THE ILLUSTRATED LONDON LIBRARI, MILFORD HOUSE, MILFORD LANE, STRAND;

AND W. S. ORR AND CO., AMEN CORNER, PATERNOSTER ROW. 


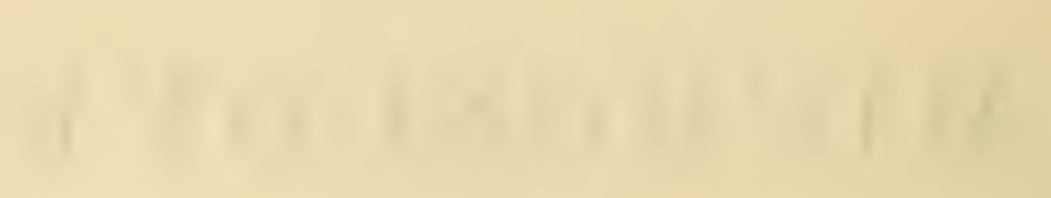




\section{Mำ}

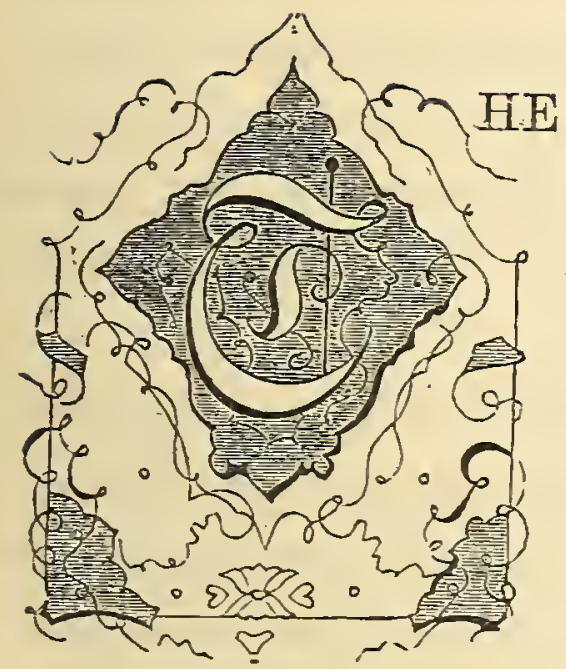

author of the present publication entered upon his task with some hesitation and diffidence: but the reasons which influenced him to undertake it may be briefly told; and they at once explain his motives, and plead his justification, for the work which he now ventures to submit to the indulgent consideration of his readers.

It had:been to him for some time a subject of regret, that one of the most useful and fascinating of studies-the study that belongs to the domain of microscopic observation - should be, if not wholly neglected, at best but coldly and indifferently appreciated, by the great mass of the general public; and he formed a strong opinion that this apathy and inattention were mainly attributable to the want of some concise, yet sufficiently comprehensive, popular account of the Microscope, both as regards the management and manipulation of the instrument, and the varied wonders and hidden realms of beauty that are disclosed and developed by its aid. He saw around him valuable, erudite, and splendid volumes; which, however, being chiefly destined for circulation amongst a special class of readers, were necessarily, from the nature of their contents and the style of their production, published at a price that renders them practically unattainable by the great bulk of the public. They constitute careful and beautiful contributions to the purposes of science; but they cannot adequately serve to bring the value and charm of microscopic studies home, so to speak, to the firesides of the people. Repeatedly, day after day, new and interesting discoveries, 
and further amplifications of truths already discerned, have been made; but they have been either scattered in serials, or, more usually, devoted to the pages of class publications. Thus this most important and attractive study has been, in a great measure, the province of the few only, who have derived from it a rich store of enlightenment and gratification; the many not having, however, participated, to any general extent, in the instruction and entertainment which follow in its train.

The manifold and varied uses and advantages of the Microscope crowd upon us in such profusion, that we can only attempt to enumerate them in the briefest and most rapid manner in these few passages of introduction. It is not many years since this invaluable instrument was regarded in the light of a costly toy; it is now the inseparable companion of the man of science.

In the medical world its utility and necessity are fully appreciated, even by those who formerly were slow to see its benefits. Knowledge which could not be obtained by the minutest dissection is acquired by the aid of the Microscope, which has become as essential to the anatomist and pathologist as the scalpel to the one and bedside observation to the other. The smallest portion of a diseased structure, placed under a Microscope, will tell more in one minute to the experienced eye than could be ascertained by many days' examination of the gross masses of disease in the ordinary method. Microscopic agency, in thus assisting the medical man, materially contributes to the alleviation of those multiplied "ills which flesh is heir to."

So fully impressed were the Council of the Royal College of Surgeons with the importance of the facts brought to light in a short space of time, that in 1841 they determined to establish a Professorship of Histology, and to form a collection of preparations of the elementary tissues of both animals and vegetables, healthy and morbid, adapted to illustrate the uses and results of microscopical investigations. From that time histological anatomy deservedly became an important branch of the education of the medical student.

The Microscope, as an ally of Chemistry, enables us to discover very minutely and completely the changes of form and colour effected by test-fluids upon solids; it dissects for us, so to speak, the most 
multiplex compounds; it opens to the mind an extended and rast panorama, opulent in wonders, rich in beauties, and boundless in extent.

In prosecuting the study of Vegetable Physiology, the Microscope is an indispensable instrument; it empowers the student to trace the earliest forms of regetable life, and the functions of the different tissues and ressels in plants. Valuable assistance is derived from its agency in detecting the adulteration of our articles of food, as has been verified by the exposures - which must have done great good - that have from time to time appeared in the Lancet. In the examination of suspected flour, an article of so much importance to all persons, the Microscope enables us to judge of the size and shape of the starch-grains, the markings of them, and their isolation and agglomeration, and thus to distinguish the starch-grains of one meal from those of another. In the necessarily limited space of this work, the author has only been able to glance at the subject of the minute structure of regetables; but the remarks and reflections which he has deroted to it will, he hopes, lead the microscopist to a full investigation of one of the most beautiful departments of nature.

By "conducting the eye to the confines of the visible form," the Microscope proves an effective auxiliary in defining the geometric properties of bodies. Its influence as an instrument of research upon the structure of bodies has been compared to that of the galvanic battery, in the hands of Dary, upon Chemistry. It has enabled us to detect the smallest structural difference, heretofore inappreciable; and in our analysis to define positively the structure of tissues, beyond the capability of the greatest magnifying power to change or modify.

The Zoologist finds in the Microscope a necessary co-operator. To the Geologist it reveals, among a multiplicity of other facts, "that our large coal-beds are the ruins of a gigantic vegetation; and the vast limestone rocks, which are so abundant on the earth's surface, are the catacombs of myriads of animal tribes, that are too minute to be perceived by the unaided vision."

In medico-legal investigations the Microscope has been frequently called into use; and in some cases human life has been pending upon its accuracy of decision. 
The Microscope not only assists studies, and develops objects of profound interest, but it also opens up innumerable sources of entertainment and amusement, in the ordinary conventional acceptation of these terms. It discloses to us peculiarities and attractions in abundance. It impresses us with the wonderful and beautifully-skilful adaptation of all parts of creation, and fills our minds with additional reverence and admiration for the beneficent and Almighty Creator. It enables us to bring to light latent beauties which the eye could never trace. "Nature," it has been well observed, "is not loquacious, although filled with inexhaustible stores; she presents enough at first sight to attract the thoughtful ; but mankind must interrogate and study her, in order to come at all, perhaps to a thousandth part, of that which she has to communicate."

It is not necessary, in this place, to recapitulate further the various ways in which the Microscope is of paramount importance to the seeker after truth, the student, and the man of scientific acquirements; and, it may be added, of infinite charm and interest to all.

The author has briefly explained the considerations which prompted him, and the objects which he hoped to advance, in undertaking the present work. He begs now to conclude these preliminary observations with a few words in explanation of his arrangements, and by way of acknowledgment to those to whom he is indebted. He has sought, in the volume that he now lays before the public, to point out and elucidate, at once in a practical manner and in a popular style, the vast fund of utility and amusement which the Microscope affords; and has endeavoured to touch upon most of the interesting subjects for microscopic observation as fully as the restrictions of a limited space, and the nature of a succinct summary, would permit. To have dwelt upon each in complete detail would have necessitated volume upon volume,--expensive books must have resulted,-and this would have entirely frustrated the aim which the writer had in view; he has, therefore, contented himself with the humble, but, he trusts, not useless task, of setting up a finger-post, so to say, to direct the inquirer into the wider road. In the sections of the work devoted to the very minute portion of creation, he has ventured to dwell somewhat more upon the subject, in the belief that that department is more espe- 
cially the province of the microscopist than many others. He has arranged his topics under special headings, and in separate chapters, for the sake of greater clearness and precision; and has brought the everwelcome aid of illustrations to convey his explanatory remarks more vividly to the minds of his readers. To his friend, Mr. J. G. Kelly, he has to express his thanks for valuable assistance afforded in making. drawings of the objects from the Microscope and camera lucida; as also to $\mathrm{Mr}$. George Pearson, for the admirable manner in which he has engraved them. The author has minutely described the use and manipulations of the Microscope, so as to render its management, he ventures to hope, intelligible to all; and his marked and warm acknowledgments are due to the eminent maker and improver of the Microscope, Mr. Ross, for his revision of this portion of the work. He is peculiarly indebted to Professor John Quekett, whose very valuable lectures, delivered in the Royal College of Surgeons, and other multifarious and successful researches, have pre-eminently distinguished him as the microscopist of the day. From notes made at the lectures spoken of, and from the many admirable papers which this gentleman has published, much sound information has been gleaned; and the author has to thank him, in the most sincere and cordial manner, for the ready acquiescence that he gave to the writer's wish to cull from the choicest of the mass of contributions with which he has enriched the microscopical science of the day. The author has also freely arailed himself of the researches of other scientific investigators, - Ehrenberg; Carpenter, Johnston, Ralfs, Busk, Gosse, Huxley, and the members of the Microscopical Society of London, to the foundation of which excellent society we may ascribe the rise and progress of many improvements in the instrument; and it has tended, moreover, to stimulate discoveries, and induce greater accuracy of observation. In the extracts which he has made from these various sources, the author's object has been to give his readers, in addition to the results of his own original investigations, some adequate notion of the leading theories, the most characteristic views, and the most useful instructions, of these distinguished authorities, whose names are intimately identified with the studies of the Microscope.

Finally, it is the author's hope that, by the instrumentality of this 
volume, he may possibly assist in bringing the Microscope, and its most valuable and delightful studies, before the general public in a more familiar, compendious, and economical form than has hitherto been attempted; and that he may thus, in these days of a diffused taste for reading and the spread of cheap publications, submit some further food for the exercise of the mental and intellectual faculties,contribute to the additional amusement and instruction of the family circle around the domestic hearth,-and aid the student of nature in investigating the wonderful and exquisite works of the Almighty Hand. If it shall be the good fortune of this work, which is now confided with great diffidence to the consideration of the public, to succeed, in however slight a degree, in furthering this design, the author will feel sincerely happy; and will be fully repaid for the attention, time, and labour, that he has expended in writing, arranging, and compiling it.

6 Gower Street, Bedford Square, May 1854. 


\section{DIRECTIONS FOR PLACING THE PLATES.}

Frontispiece and Engraved Title to face each other, and precede the printed title-page.

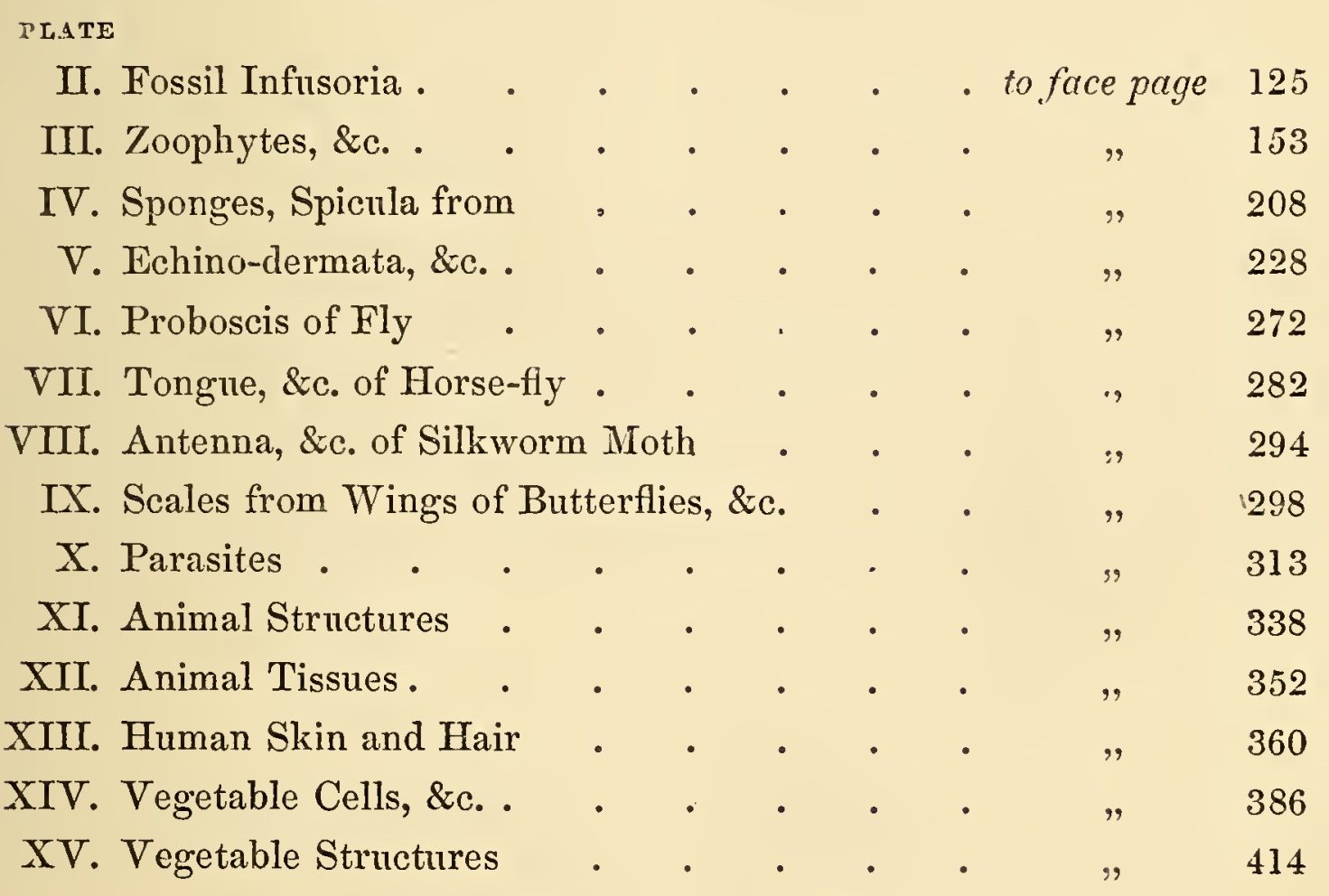





\section{CONTENTS.}

\section{PART I.}

HISTORT OF THE INTENTION AND IMPROFEMENTS OF THE MICROSCOPE.

\section{CHAPTER. I.}

Derivation, and period of invention. Known before Christ. Single microscopes of Leeuwenhoek, Swammerdam, Lyonet, and Ellis. Lieberkuhn's reflecting instruments in the Royal College of 'Surgeons. Leeuwenhoek's microscopes; Dr. Hooke's. Stephen Gray's glass globules. Dr. Hooke's compound instrument. Dirini's object-glasses. Bonnani's, Martin's, Sir Isaac Newton's, Dr. Barker's, and Dr. Smith's discoreries. Lieberkuhn's solar microscope. Baker's, Dr. Hill's, M. Delabarre"s, and Mr. G. Adams" published accounts of the microscope. Frauenhofer's, Pelligne's, Cheralier's, and Amici's experiments. Tulley's, Dr. Goring's, and Lister's experiments. Amici's achromatic glasses. Sir John Herschel's, Airy's, Barlow's, Coddington's, and Lister's combinations. Mr. Ross's improrements. Dr. Goring's, Solly's, Bowerbank's, and Valentine's improrements and assistance. Reports of the Juries of the Great Exhibition on the instruments manufactured by the London makers

\section{CHAPTER II.}

Mechanical and optical principles inrolved in the construction of the modern microscope: Mode of estimating the magnifying power. Rays of light passing through transparent media. Forms of lenses. Refraction of light through lenses, and method of tracing the progress of rays. Spherical aberration of lenses. Sir John Herschel's and Coddington's lenses. Chromatic and achromatic aberration of lenses. Limit of distinct vision through lenses. Dr. Wollaston's doublet. Holland's eye-piece. Wollaston and Coddington's lenses. Stanhope lens. Ross's simple microscope. Valentine's simple microscope. The compound microscope, Mr. Ross's remarks upon. Lister's, Powell's, Tülley's, and Ross's improvements. Mr. Ross's correction of object-glasses. Mr. Varley's corrections of the eye-piece. Huyghenian and micrometer eye-pieces. Mr. Lister's stage-micrometer. Mr. Jackson's eye-piece micrometer. Dr. Hannorer's table of comparatire measures. M. Nobert's glass micrometers. Sets of eye-pieces, their value. 
Magnifying power of Ross's object-glasses. Mechanical arrangements. Mr. Ross's smaller instrument. Ross's large compound microscope. Baker's student's microscope. Useful Remarks in Juries' report on the microscopes at the Great Exhibition

Page 12

\section{CHAPTER III.}

Directions for using the instrument. Sir David Brewster's directions for viewing objects. Mr. J. Smith on the management of light in viewing objects. Mr. Ross upon illumination. The diaphragm. Varley's white cloud. Wollaston's condenser. The achromatic illuminator. Wenham's parabolic reflector. Mr. Shadbolt's improvement. Condensing lenses. Mr. Brooke's double re.. flector. Lieberkuhn's condenser. Mr. Highley's achromatic lamp. Forceps. Dipping tubes. Varley's forceps. Collecting net. Animalcule box. Mr. Williamson's collecting-stick. Varley's animalculæ cages. Smith and Beck's chara troughs. Dissecting knives and needles; mode of using the same. Dissections under the microscope. Valentin's knife, improved. Dissecting scissors. Cutting glass covers. Mounting and preserving objects. Mr. Gorham's "cementing pencil." Object-holder. Air-pump. Mr. Boys' directions for mounting. Mr. Deane's mounting fluid. Mr. Warrington's fluid, and directions for using it. Mr. Goadby's solutions. Mode of injecting. Ruysch's and Breschet's method. Goadby's process. Dr. Schacht's reagents and preservative fluids. Dr. Parkes' caution. Mr. Ralfs' directions for preserving algæ, \&c. Mr. Topping's fluid. Mr. Shadbolt on collecting: objects. Localities of microscopic plants, \&c. The camera-lucida. Quekett's directions for using the camera-lucida. Polarised light. Nicol's prisms. On using polarising prisms; Mr. Woodward's explanations; Mr. J. King's remarks on. Dr. Herepath's new salt of quinine. Mr. Legg's phenomena of polarised light. Crystals of urinary salts. Crystals of ice. Starch grains under polarised light. Binocularity applied to the microscope. Application of photography to the microscope . . . . Page 50

\section{PART II.}

\section{CHAPTER I.}

A glance at the microscopic world. History of animalculæ. Professor Quekett on viewing ciliary movement. Infusorial animalculæ. Baker's account of wheel-animalculæ. The marine animalculæ. Phosphorescent appearance of the sea, described by Darwin, Captain Scoresby, and Mr. Gosse. Polygastric animalculæ. Monads. Ehrenberg's description of monads. The Breast-plate monad. The Proteus. Enchelis. Vibrio. Astatia. Bacillaria. Navicula. Vorticella. Stentors. Rotifera. Brachiona. Notommata aurita; described by Mr. Gosse. Floscularia. Melicerta ringens; described by Mr. Gosse. Bergmehl. Professor Owen on the uses of animalculæ. Polypifera. Dr. John- 
ston on British zoophytes. Hydra ; first described by Leeuwenhoek. Trembley's description of hydra. Marine animal plants. Alcyonidæ; Professor Grant's description of their ora. Asteroidæ. Corallidæ. Jukes' and Captain Basil Hall's descriptions of coral-reefs. Gorgoniæ. Crisiadæ. Mr. Busk's description of the Shepherd's Purse and Snake Corallines. Celleporidæ. Madreporidæ. Tubiporidæ. Pennatulidæ. Sertulariadæ. Actiniadæ. Lucernariæ. Bryozoæ. Flustræ. Escharæ. Vesiculariadæ. Zoological Society's marine vivaria, Mr. Gosse on. Porifera. Sponges ; described by Professor Dr. Grant. Dr. Lankaster's definition of regetable and animal life. Alcyonellæ. Limniades. Clionæ. Skeletons of zoophytes. Spiculæ of sponges. Mr. Carter on the seed-like bodies in sponges. Dr. Golding Bird on preserving the polypidoms of zoophytes. Fossil infusoria. Professor Quekett on mounting infusoria. Professor Redfern on isolating Nariculæ, \&c. Diatomaceæ. Professor Bailey's contributions to the Museum of the College of Surgeons. The value of studying fossil infusoria to artists. Mr. Shadbolt on the Arachnoidiscus. Ker. W. Smith on collecting and preserving Diatomaceæ. Santhidiæ. Rev. J. B. Read on animals found in chalk. Foraminiferæ. Echinidæ. Acalephæ. Annelidæ. Crustaceæ. Mollusca. Pearls. Gasteropoda. Lymnæa stagnalis, derelopment of. Mode of showing the structure of shell; Dr. Harrey on collecting salt-water specimens. List of interesting specimens

Page 120

\section{CHAPTER II.}

INSECTS.

Their beauty of organisation. 'The Crane-fly. The Wasp's tongue, feelers, \&c. Eye of fly. Sucker on foot. Breathing-aperture. Feet and legs. Tongue and piercing-apparatus of fly. Mr. G. Newport on the tongue of the fly. The wings of insects. The Dragon-fly. The Gnat. The Water-beetle. The Cricket. The common beetle. The Death-watch. The Bacon-beetle. The Cockchafer. Buttertlies and moths; Mr. G. Newport on the structure of their tongues; scales from their wings. Mrr. Topping's test-objects. Eggs of insects. Saws of Saw-fly. The Froth-fly. Aphides. The Ant. The Ant-lion. Stings of insects. The Honey-bee. The Spider. Parasites. The Flea. The Louse. The Itch-insect. Entozoa folliculorum. The Guinea-rorm. Echinococci. Dr. J. Leidy on regetable parasites. Dr. Burnett on parasites, and their relation to other animals;--found on the sparrow and snail, by Dr. Robins. On the rater-snail. Swammerdam's dissections of insects. Parts of insects for examination. Insect changes ; their distribution, their commercial value, and general uses

\section{CHAPTER III.}

ANIMAL STRUCTURE.

Histological history of animal life; the value of the microscope in the study of. Dr. Carpenter's definition of a cell. The metamorphoses of the cell. Mr. 
Huxley's review of the unicellular theory. Mr. Huxley's sketch of a general theory of the structure of animals and plants. The vital or molecular forces. The elementary tissues ; Professor Quekett's classification of. Simple membrane. Blood-cells. Crystallisation of blood. Capillaries. Fibrous tissue, elastic and non-elastic. Muscular fibre. Structure of the skin. Structure of hair. Nerves. Cartilage. Consolidated tissues; teeth. Bone. Professor Quekett on the structure of bone. Dr. Bennett on mode of investigating animal structures. To view the circulation of the blood in the frog. The newt. Structure of fishes . . . . . . . . . . Page 334

\section{CHAPTER IV. \\ VEGETABLE STRUCTURE.}

Vital and chemical characteristics of plants. Mülder's description of the vegetable cell. The Yeast-plant. Fungoid growths. The red snow of the northern regions. Scotch Siller-cups. Confervæ. Algæ. Mosses. The Volvox globata. Desmidieæ. Ralfs on the British Desmidieæ. The cell-transformation. Cellular tissue. Woody fibre. Lactiferous tissue. Crystals in plants. Raphides. Siliceous cuticle of grasses. Starch. Adulterations of wheat-flour. Potato disease. Hairs on the stinging-nettle. Chlorophyl. Nitella. Vallisneria. Chara. Mr. Varley on the cultivation of Chara. Vascular tissue ; Mr. Edwin Quekett upon the development of. Preparation of vegetable tissues. Machine for cutting sections. Method of making sections. Concluding remarks . . . . . . 。 Page 386 


\title{
THE MICROSCOPE.
}

\section{PART I.}

\section{HISTORY OF THE INVENTION AND IMPROVEMENTS OF THE MICROSCOPE.}

\begin{abstract}
CHAPTER I.

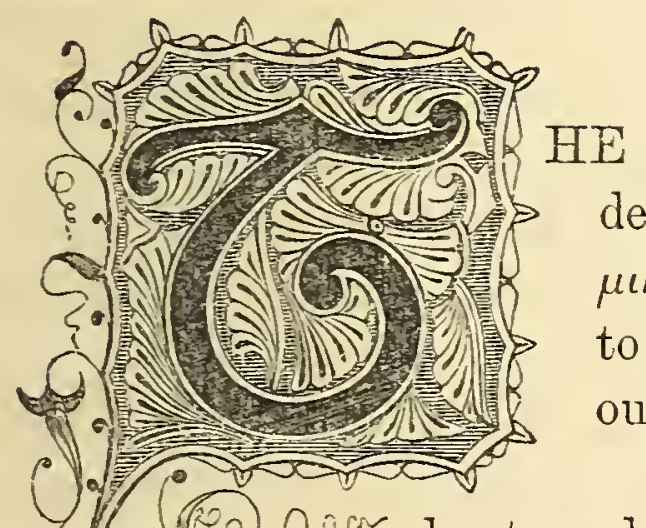

HISTORY OF THE MIICROSCOPE.

HE instrument known as the Microscope derives its name from two Greek words,

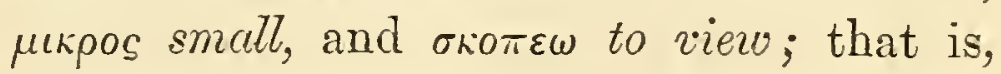
to see or view such minute objects as without its aid would be invisible.

The invention of the microscope cannot for be traced with any degree of certainty before the year 1660 , a period fruitful in discoveries. The honour of the invention is claimed by the Italians and the Dutch; the name of the inventor, however, is lost. Probably the discorery did not at first appear sufficiently important to engage the attention of those men who, ef by their reputation in science, were able to establish an hand down the name of its inventor to succeeding ages.

It is not difficult to fix the period when the microscope first began to be generally known, and to be used for the purpose of examining minute objects; for though we are ignorant of the name of the first inventor, we are acquainted with the names of those who introduced it into public view. Zacharias Jansens and his son are said to have made microscopes before the year 1590:
\end{abstract}


about that time the ingenious Cornelius Drebell brought one made by them with him to England, and showed it to William Borrell and others. It is possible this instrument of Drebell's was not strictly what is now called a microscope, but was rather a kind of microscopic telescope, something similar in principle to that lately described by M. Aepinus in a letter to the Academy of Sciences at St. Petersburg. It was formed of a copper tube six feet long and one inch in diameter, supported by three brass pillars in the shape of dolphins; these were fixed to a base of ebony, on which the objects to be viewed by the microscope were placed. Fontana, in a work which he published in 1646 , says that he had made microscopes in the year 1618 : this may be perfectly true, without derogating from the merit of the Jansens; for we have many instances in our own times of more than one person having made the same invention nearly simultaneously, without any communication from one to the other. In 1685 Stelluti published a description of the parts of a bee, which he had examined with a microscope.

If we consider the microscope as an instrument consisting of one lens only, it is not at all improbable that it was known at a very early period, nay even in a degree to the Greeks and Romans; at any rate, it is tolerably certain that spectacles were used as early as the thirteenth century. Now as the glasses of these were made of different convexities, and consequently of different magnifying powers, it is natural to suppose that smaller and more convex lenses were made, and applied to the examination of minute objects.

Aristophanes, who lived five centuries before Christ, speaks of a "burning-sphere." 'Seneca, who wrote in the first half-century of the Christian era, says that small and indistinct objects become larger and more distinct in form when scen through a globe of glass filled with water. Pliny also mentions the burning property of lenses made of glass.

The history of the microscope, like that of nations and arts, has had its brilliant periods, in which it shone with uncommon splendour, and was cultivated with extraordinary ardour; and these have been succeeded by intervals marked with no discovery, and in which the science seemed to fade away, or at least to lie dormant, till some favourable circumstance - the discovery of a new object, or some new improvement in the instruments of observation-awakened the attention of the curious, and reanimated their researches. Thus, soon after the invention of the microscope, the field it presented to observation was cultivated by men of the first rank in science, who enriched almost every branch of natural history by the discoveries they made by means of this instrument. 
We shall first speak of the single microscope, that, as we have already observed, having been invented and used long before the double or compound microscope. When the lenses of the single microscope are very convex, and consequently the magnifying porver very great, the field of view is small; and it is so difficult to adjust with accuracy their focal distance, that it requires some practice to render the use of them familiar. It was with an instrument of this kind that Leeuwenhoek and Swammerdam, Lyonet and Ellis, examined the invisible forms of nature, laid open some of her hidden recesses, and by their example stimulated others to the same pursuit.

The construction of the single microscope is so simple, that it is susceptible of but little improvement, and has therefore undergone few alterations; and these have been chiefly confined to the mode of mounting it, or to additions to its apparatus. The greatest improvement this instrument has received was made by Dr. Lieberkuhn,* about the year 1740 : it consists in placing: the small lens in the centre of a highly-polished concave speculum of silver, by which means a strong light is reflected upon the upper surface of an object, which is thus examined with great ease and pleasure. Before this contrivance, it was almost impossible to examine small opaque objects with any degree of exactness and satisfaction; for the dark side of the object being next the eye, and also overshadowed by the proximity of the instrument, its appearance was necessarily obscure and indistinct.

Lieberkuhn adapted a separate microscope to every object: but all this labour was not bestowed on trifling objects; his were generally the most curious anatomical preparations, twelve of which, with their microscopes, are deposited in the Museum of the Royal College of Surgeons.

Lieberkuhn's instrument, fig. 1, is thus described by Professor Quekett: $\uparrow a b$ represents a piece of brass tube, about an inch long and an inch in diameter, which is provided with a cap at each extremity; the one at $a$ carries a small double-convex lens of half an inch in focal length, whilst the one at $b$ carries a condensing lens three-quarters of an inch in diameter.

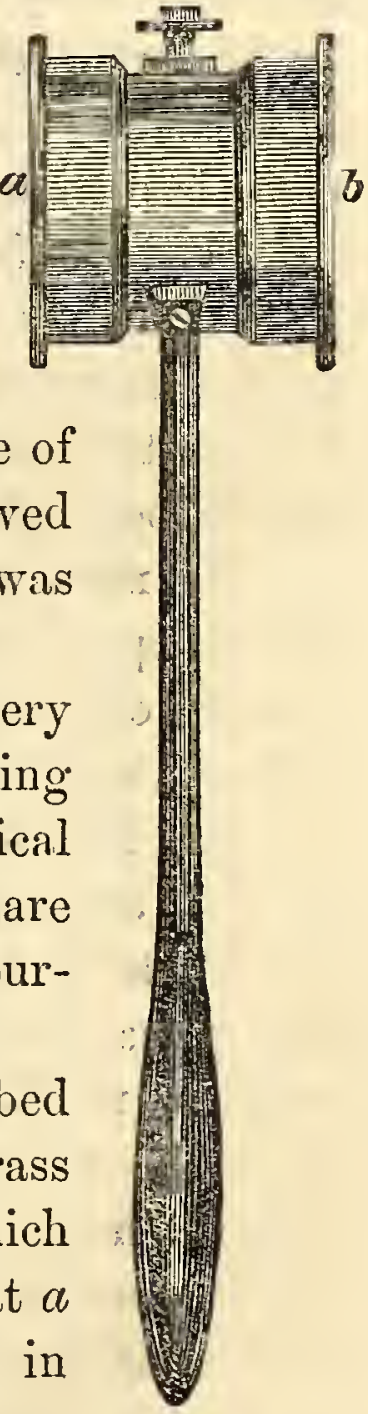

fig. 1 .

* Dr. Nathaniel Lieberkuhn of Berlin.

+ Practical Treatise on the Microscope, p. 16. 
A vertical section of one of these instruments is seen in fig. 2: a represents the magnifier, which is lodged in a cavity formed partly by

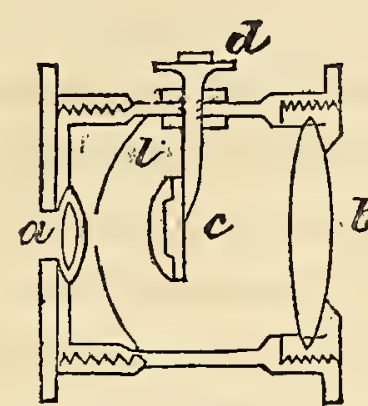

fig. 2. the cap $a$ and by the silver cup or speculum $l$. In front of the lens is the speculum $l$, which is a quarter of an inch thick at its edge, and whose focus is about half an inch; in front of this again there is a disc of metal $c$, three-eighths of an inch in diameter, connected by a wire with the small knob $d$; upon this disc the injected object is fastened, and is covered over with some kind of varnish which has

LIEBERKUHN'S MICROSCOPE. dried of a hemispherical figure. Between this knob and the inside and outside of the tube there are two slips of thin brass, which act as springs to keep the wire and disc steady. When the knob is moved, the injected object is carried to or from the lens, so as to be in its focus, and to be seen distinctly, whilst the condensing lens $b$ serves to concentrate the light on the speculum. To the lower part of the tube a handle of ebony, about three inches in length, is attached by a brass ferrule and two screws. The use of this instrument is obvious: it is held in the hand in such a position that the rays of light from a lamp or white cloud may fall on the condenser $b$, by which they are concentrated on the speculum $l$; this, again, further condenses them on the object and the disc $c$, which object, when so illuminated, can readily be adjusted by the little knob $d$, so as to be in the focus of the small magnifier at $a$.

We must not omit in this place some account of Leeuwenhoek's microscopes, which were rendered famous throughout all Europe, on account of the numerous discoveries he had made with them, as well as from his afterwards bequeathing a part of them to the Royal Society.

The microscopes he used were all single, and fitted up in a convenient and simple manner: each consisted of a very small doubleconvex lens, let into a socket between two plates riveted together, and pierced with a small hole; the object was placed on a silver point or needle, which, by means of screws adapted for that purpose, might be turned about, raised or depressed at pleasure, and thus be brought nearer to, or be removed farther from, the glass, as the eye of the observer, the nature of the object, and the convenient examination of its parts required.

Leeuwenhoek fixed his objects, if they were solid, to these points with glue; if they were fluid, he fitted them on a little plate of talc, or exceedingly thin-blown glass, which he afterwards glued to the needle in the same manner as his other objects. The glasses 
were all exceedingly clear, and of different magnifying powers, proportioned to the nature of the object and the parts designed to be examined.

He observed, in his letter to the Royal Society, that, from upwards of forty years' experience, he had found the most considerable discoveries were to be made with glasses of moderate magnifying power, which exhibited the object with the most perfect brightness and distinctness. Each instrument was devoted to one or two objects; hence he had always some hundreds by him.

About the year 1665, small glass globules began to be occasionally applied to the single microscope, instead of convex lenses. By these globules an immense magnifying power was obtained. Their invention has been generally attributed to M. Hartsoeker; though it appears that we are really indebted to the celebrated Dr. Hooke for this discovery, for he described the manner of making them in the preface to his Micrographia Illustrata, published in the year 1656 .

Mr. Stephen Gray* having observed some irregular particles within a glass globule, and finding that they appeared distinct and prodigiously magnified when held close to his eye, concluded, that if he placed a globule of water in which there were any particles more opaque than the water near his eye, he should see those particles distinctly and highly magnified. The result of this idea far exceeded his expectation. His method was, to take on a pin a small portion of water which he knew contained some minute animalculæ; this he laid on the end of a small piece of brass wire, till there was formed somewhat more than a hemisphere of water; on applying it then to the eye, he found the animalculæ most enormously magnified;: for those which were scarcely discernible with his glass globules, with this appeared as large as ordinary-sized peas. Montucla observes, that when any objects are enclosed within this transparent globule, the hinder part of the globule acts like a concave mirror, provided the objects be situated between that surface and the focus; and that by this means they are magnified three times and a half more than they would be in the usual way.

An extempore microscope may be formed by taking up a small drop of water on the point of a pin, and placing it over a fine hole made in a piece of metal; but as the refractive power of water is less than that of glass, these globules do not magnify so much as glass ones of the same size: this was also one of Mr. Gray's magnifiers. The same ingenious author invented another water-microscope, con-

* Philosophical Transactions, 1696. 
sisting of two drops of water separated in part by a thin brass plate, but touching near the centre, which were thus rendered equivalent to a double-convex lens of unequal convexities.

Dr. Hooke described the method of using this single microscope: "If you are desirous," he says, "of obtaining a microscope with one single refraction, and consequently capable of procuring. the greatest clearness and brightness any one kind of microscope is susceptible of, spread a: little of the fluid you intend to examine on a glass plate; bring this under one of your microscopic globules, then move it gently upwards till the fluid touches the globule, to which it will soon adhere, and that so firmly as to bear being moved a little backwards or forwards. By looking through the globule, you will then. have a perfect view of the animalculæ in the drop."

The three first compound microscopes that attract our notice are those of Dr. Hooke, Eustachio Divini, and Philip Bonnani. Dr. Hooke gives an account of his in the preface to his Micrographia, published in the year 1667 : it was about three inches in diameter, seven inches long, and furnished. with four draw-out tubes, by which it might be lengthened as occasion. required; it had three glasses - a small object-glass, a middle glass, and a deep eye-glass: Dr. Hooke used all the glasses when he wanted to take in a considerable part of an object at once, as by the middle glass a number of radiating pencils were conveyed to the eye which would otherwise have been lost; but when he wanted to examine with accuracy the small parts of any substance, he took out the middle glass, and only made use of the eye and object lenses; for the fewer the refractions are, the clearer and brighter the object appears.

Dr. Hooke also gave us the first and most simple method of finding how much any compound microscope magnifies an object. $\mathrm{He}$ placed an accurate scale, divided into very minute parts of an inch, on the stage of the microscope; adjusted the microscope till the divisions appeared distinct; and then observed with the other eye how many divisions of a rule similarly divided and laid on the stage were included in one of the magnified divisions ; "for if one division, as seen with one eye through the microscope, extends to thirty divisions on the rule, which is seen by the naked eye, it is evident that the diameter of the object is increased or magnified thirty times."

An account of Eustachio Divini's microscope was read at the Royal Society in 1668. "It consisted of an object-lens, a middle glass, and two eye-glasses, which were plano-convex lenses, and were placed so that they touched each other in the centre of their convex surfaces; 
by which means the glass takes in more of an object, the field is larger, the extremities of it less curved, and the magnifying power greater. The tube in which the glasses were enclosed was as large as a man's leg, and the eye-glasses as broad as the palm; of the hand. It: had four several lengths : when shut up was 16 inches long, and magnified the diameter of an object 41 times, at the second length 90 , at the third length 111, and at the fourth length 143 . times." It does not appear that Divini, varied the object-glasses.

Philip Bonnani published an account of his two microscopes in 1698: Both were compound. The first was similar to that which Mr. Martin published as new, in his Micrographica. Nova, in 1712, His second was like the former, composed of three glasses, one for the eye, a middle glass, and an object lens; they were mounted in a cylindrical tube, which was placed in a horizontal position; behind the stage was a small tube with a conver lens at each end; beyond this was a lamp; the whole capable of various adjustments, and regulated by a pinion and rack. The small tube was used to condense the light on to the object, and spread it uniformly over, according to its nature, and the magnifying power that was used.

A short time before this, Sir Isaac Newton having discovered his celebrated theory of light and colours, was led to improve the telescope; and in 1672 he is said to have applied his principles most successfully to the construction of a compound reflecting microscope. He also pointed out the proper mode of illuminating objects by artificial light, as he describes it, "of any convenient colour not too much compounded, mono-chromatic." We find other two plans of this kind; the first that of Dr. Robert Barker, and the second that of Dr. Smith. In the latter there were two reflecting mirrors, one concave, and the other convex : the image: was viewed by a lens. This microscope, though far from being executed in the best manuer, performed, says Dr. Smith, very well, so that he did not doubt it would have excelled others, had it been properly: finished.

In. 17.38, Lieberkuhn's inyention of the solar microscope was communicated to the public. The vast magnifying power obtained by this instrument, the colossal grandeur witli which it exhibited the "minutiæ of nature," the pleasure which arose from being able to display the same object to a number of observers at the same time, by affording a new source of rational amusement, increased the number of microscopic observers, who were further stimulated to the same pursuits. by Mr. Trembley's, famous discovery of the polype. The discovery of the wonderful properties of this little animal, together with the works 
of Mr. Trembley, Mr. Baker, and Mr. Adams, combined to spread the reputation of the instrument.

In 1742, Mr. Henry Baker, F.R.S., published an admirable treatise on the microscope. He also read several papers before the Royal Society on the subject of his mieroscopic discoveries. In our titlepage we have represented an elegant scroll "pocket microscope with a speculum," described by him as a new invention.

In 1770 , Dr. Hill published a treatise, in which he endeavours by means of the microscope to explain the construction of timber, and to show the number, the nature, and office of its several parts, their various arrangements and proportions in the different kinds; and he points out a way of judging, from the structure of trees, the uses they will best serve in the affairs of life.

M. L. F. Delabarre published an account of his microscope in 1777. It does not appear that it was superior in any respect to those that were then made in England. It was inferior to some; for those made by Mr. Adams, in 1771, possessed all the advantages of Delabarre's in a higher degree, except that of changing the eye-glasses.

In 1774, Mr. George Adams, the son of the above, improved his father's invention, and rendered it useful for viewing opaque as weil as transparent objects. This instrument, made and described by him,* continued in use up to the time of the invention of the achromatic improvement, proposed and made in 1815 for Amici, who subsequently gave so much time to the investigation of polarised light, and the adaptation of a polarising apparatus to the microscope.

In the year 1816, Frauenhofer, a celebrated optician of Munich, constructed object-glasses for the microscope of a single achromatic lens, in which the two glasses, although in juxtaposition, were not cemented together : these glasses were very thick, and of long focus. Although such considerable improvements had taken place in the making of achromatic object-glasses since their first discovery by Euler in 1776 , we find even at so late a period as 1821 , M. Biot writing, "that opticians regarded as impossible the construction of a good achromatic microscope." Dr. Wollaston, too, was of the same opinion, " that the compound instrument would never rival the single."

In 1823, experiments were commenced in France by M. Selligues, which were followed up by Frauenhofer in Munich, by Amici in Modena, by M. Chevalier in Paris, and by the late Dr. Goring and Mr. Tulley in London. To M. Selligues we are indebted for the first plan

* Microscopical Essays, 1787. 
of making an object-glass composed of four achromatic compound lenses, each consisting of two lenses. The focal length of each objectglass was eighteen lines, its diameter six lines, and its thickness in the centre six lines, the aperture only one line. They could be used combined or separated.

A microscope constructed on this principle, by M. Chevalier, was presented by M. Selligues to the Academie des Sciences on the 5th of April, 1824. In the same year, and without a knowledge of what had been done on the Continent, the late Mr. Tulley, at the suggestion of Dr. Goring, constructed an achromatic object-glass for a compound microscope of nine-tenths of an inch focal length, composed of three lenses, and transmitting a pencil of eighteen degrees; this was the first that had been made in England.

It was at one time hoped, as precious stones are more refractive than glass, and as the increased refractive power is unaccompanied by a corresponding increase in chromatic dispersion, that they would furnish valuable materials for lenses, inasmuch as the refractions would be accomplished by shallower curves, and consequently with diminished spherical aberration.

But these hopes were disappointed. Every thing that ingenuity and perseverance could accomplish was tried by Mr. Varley and Mr. Pritchard, under the patronage of Dr. Goring. It appeared, however, that the great reflective power, the doubly-refracting property, the colour, and the heterogeneous structure of the jewels which were tried, much more than counterbalanced the benefits arising from their greater refractive powers, and left no doubt of the superiority of skilfully-made glass doublets and triplets.

The idea is now, in fact, abandoned; and the same remark is applicable to the attempts at constructing fluid lenses, and to the projects for giving to glass other than spherical surfaces; none of which have come into extensive use.

Mr. Lister, who was engaged with Mr. Tulley in the perfecting the achromatic object-glass, finding that none of the microscope-stands hitherto made were sufficiently steady for the use of high powers, directed his attention to the improvement of this part of the instrument; and in order to carry out his views, he employed Mr. James Smith, now one of our first opticians, to execute a stand from his own drawings, which he completed early in 1826.

In March 1825 M. Chevalier presented to the Society for the Encouragement of the Sciences an achromatic lens of four lines focus, two lines in diameter, and one line in thickness in the centre. This lens 
was greatly superior to the one before noticed, which had been made by him for M. Selligues.

In 1826, Professor Amici, who from the year 1815 to 1824 had abandoned his experiments on the achromatic object-glass, was: induced, after the report of Fresnel to the Academy of Science, to resume them; and in 1827 he brought to this country and to: Paris a horizontal microscope, in which the object-glass was composed of three lenses superposed, each having a focus of six lines and a large aperture. This microscope had also extra eye-pieces, by which the magnifying power could be increased. A microscope constructed on Amici's plan by Chevalier, during the stay of that physician in Paris, was exhibited at the Louvre, and a silver medal was awarded to its maker.

"While these practical investigations were in progress," says Mr. Ross, "the subject of achromatism engaged the attention of some of the most profound mathematicians in England. Sir John Herschel, Professors Airy and Barlow, Mr. Coddington, and others, contributed largely to the theoretical examination of the subject; and though the results of their labours were not immediately applicable to the microscope, they essentially promoted its improvement.

"Mr. Jackson Lister, in 1829, succeeded in forming a combination of lenses upon the theory propounded by these gentlemen, and effected. one of the greatest improrements in the manufacture of object-glasses, by joining, together a plano-concave flint lens and a convex, by means of a transparent cement, Canada balsam. This, he says, ' is desirable to. be taken as a basis for the microscopic object-glass : it diminishes very nearly half the loss of light from reflection, which is considerable at the numerous surfaces of a combination; the clearness of the field: and brightness of the picture is evidently. increased by doing this; and it prevents any dewiness or vegetation from forming on the inner surfaces.' Since this paper appeared, Mr. Ross has been constantly employed in bringing the manufacture of object-glasses to their greatest perfection, and at length they have attained to their present improved manufacture. Having applied Mr. Lister's principles with a degree of success never anticipated; so perfect were the corrections given to the achromatic object-glass, 'so completely were the errors. of sphericity and dispersion. balanced or destroyed, that the circumstance of covering the object with a plate of the thinnest glass. or talc disturbed the corrections, if they had been adapted to an uncovered object, and rendered an object-glass which was perfect under one condition sensibly defective under the other:" Here was another and unexpected difficulty to be overcome, but which was finally accomplished; 
for in a communication made to the Society of Arts in 1837, Mr. Ross stated, that by separating the anterior lens in the combination from the other two, he had been completely successful. The construction of this object-glass will be illustrated and explained in a future chapter.

The rapid progress of improvement in the manufacture of the achromatic compound microscope in this country has been greatly furthered by the spirit of liberality evinced by the late Dr. Goring, Mr. R. H. Solly, and Mr. Bowerbank. To the patronage of the former we owe the construction, by Tulley, of the first triplet achromatic objectglass, of the diamond lens, and of the improved reflecting instrument of Amici by Cuthbert.

To Mr. Solly is due the credit of bringing before the public the improved microscope of $\mathrm{Mr}$. Valentine, the excellent workmanship of Mr. Ross; and by his intimate connection with the Society of Arts, he has been the means of making its Transactions; since 1831, the rehicle through which nearly all the improvements in the construction of telescopes and microscopes have been made known to the world. The achromatic microscopes now manufactured by our London makers, Mr. Ross, Messrs. Powell and Lealand, and Messrs. Smith and Beck, are unequalled in any part of the world. This opinion is confirmed by the reports of the juries on the Exhibition of Works of Industry of all Nations, 1851; at that time the instruments exhibited by the above makers by far excelled those of all other countries: See Juries' Reports for much interesting matter on this subject. 


\section{CHAPTER II.}

MECHANICAL AND OPTICAL PRINCIPLES INVOLVED IN THE CONSTRUCTION OF THE MICROSCOPE, MODE OF ESTIMATING

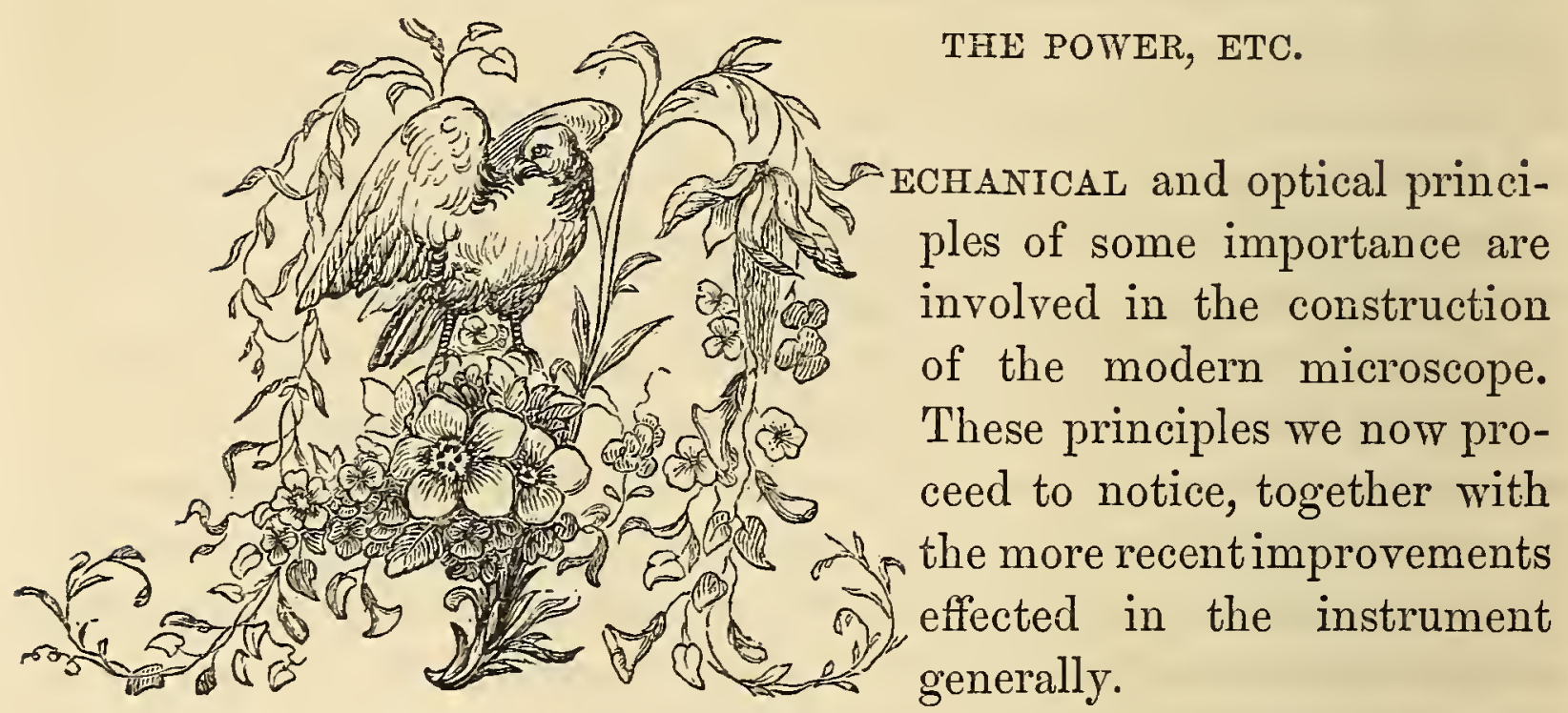

THE POWER, ETC.

The microscope depends for its utility and operation upon concave and convex lenses, and the course of the rays of light passing through them. Lenses are usually defined as pieces of glass, or other transparent substances, having their two surfaces so formed that the rays of light, in passing through them, have their direction changed, and are made to converge or diverge from their original parallelism, or to become parallel after converging or diverging. When a ray of light passes in an oblique direction from one transparent medium to another of a different density, the direction of the ray is changed both on entering and leaving; this influence is the result of the well-known law of refraction,- - that a ray of light passing from a rare into a dense medium is refracted towards the perpendicular, and vice versâ.

Dr. Arnott remarks: "But for this fact, which to many persons might at first appear a subject of regret, as preventing the distinct vision of objects through all transparent media, light could have been of little utility to man. There could have been neither lenses, as now; nor any optical instruments, as telescopes and microscopes, of which lenses 
form a part; nor even the eye itself." Rays of light falling perpendicularly upon a surface of glass or other transparent substance, pass through without being bent from the original line of their direction. Thus, if a ray pass from $k$ perpendicularly to the surface of the piece of glass at $e$ (fig. 3 ), it will go on to $h$ in the right line $k \operatorname{eog} h$. But if the same ray be directed to the surface e obliquely, as from $a$, instead of passing through in a direct line to $b$ in the direction $a e$ $m b$, it will be refracted to $d$, in a direction approaching nearer to the perpendicular line $k h$. The ray $a e$ is termed the ray of incidence, or

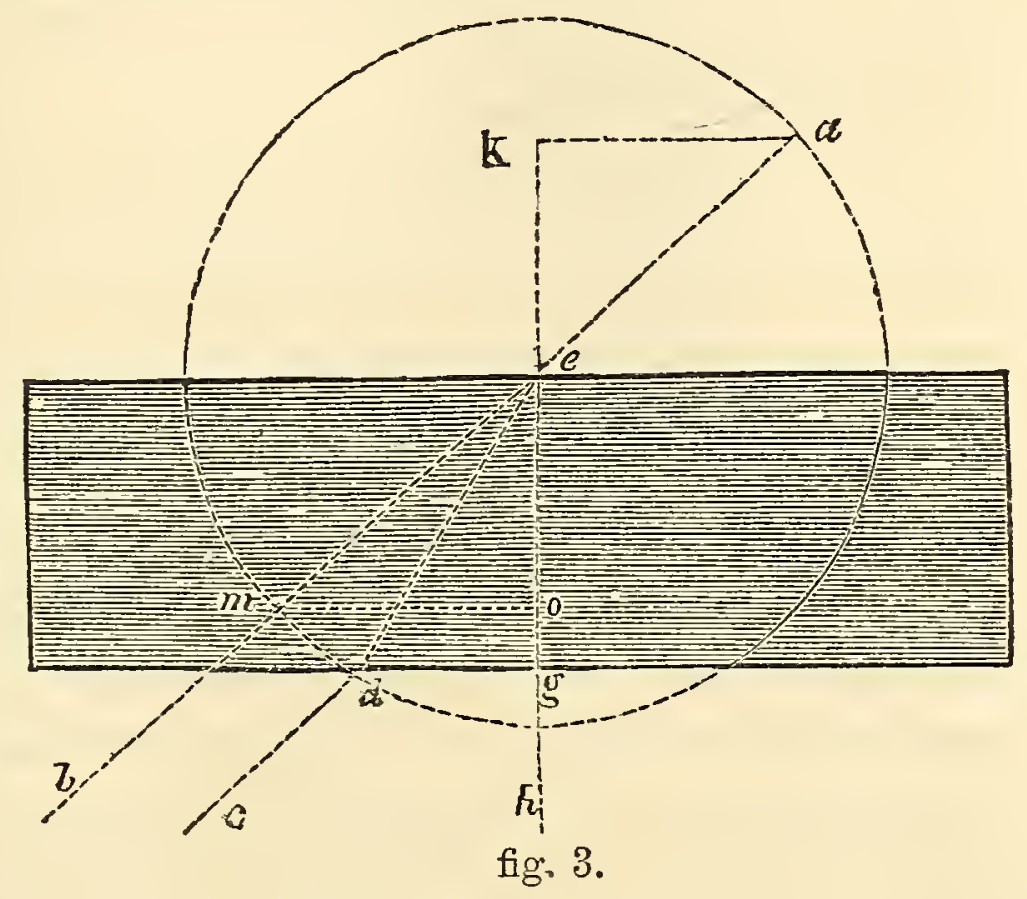
the incident ray; and the angle $a e k$ which it makes with the perpendicular $k \pi$ is called the angle of incidence. That part of the ray from $e$ to $d$ passing through the transparent medium is called the ray of refraction, or the refracted ray; and the angle $d e g$ which it makes with the perpendicular is called the angle of refraction. The ray projected from $a$ to $e$ and refracted to $d$, in passing out of the transparent medium as at $d$, is as much bent from the line of the refracted ray $e d$ as that was from the line of the original ray $a e b$; the ray then passes from $d$ to $c$, parallel to the line of the original ray $a e b$. It follows, then, that any ray passing through a transparent medium, whose two surfaces, the one at which the ray enters, and the one at which it passes out, are parallel planes, is first refracted from its original course; but in passing out is bent into a line parallel to, and running in the same direction as, the original line, the only difference being, that its course at this stage is shifted a little to one side of that of the original. If from the centre $e$ a circle be described with any radius, as $d e$, the arc $g m$ measures the angle of incidence $g e m$, and the arc $g d$ the angle of refraction $g e d$. A line $m o$ drawn from the point $m$ perpendicular to $h k$ is called the sine of the angle of incidence, and the line $d g$ the sine of the angle of refraction. From the conclusions drawn from the principles of geometry, it has been found, that in any particular transparent substance the sine of the angle of incidence $m$ o has always the same ratio to the sine $d g$ of the 
angle of refraction, no matter what be the degree of obliquity with which the ray of incidence $a e$ is projected to the surface of the transparent:medium. If the ray of incidence passes from air obliquely into water, the sine of incidence is to that of refraction as 4 to 3 ; if it passes from air into glass, the proportion is as 3 to 2 ; and if from air into diamond, it is as 5 to 2 .

By the help of glasses of certain forms, we unite in the same sensible point a great number of rays proceeding from one point of an object; and as each ray carries with it the image of the point from whence it proceeded, and all the rays united must form an image of the object from whence they were emitted, this image is higher in proportion as there are more rays united, and more distinct in proportion as the order in which they proceeded is better preserved in their union. The point at which the object must be placed is called the focus of the lens; and the distance from the middle of the lens to the focus is called the focal length, or distance. In every lens the right line perpendicular to the two surfaces is called the axis of the lens, and is seen in the annexed figure; the point where the axis cuts the surface is called the vertex of the lens; the middle point between them the centre; and the distance between them the diameter.

Fig. 4 is intended to represent the different forms of lenses in use :

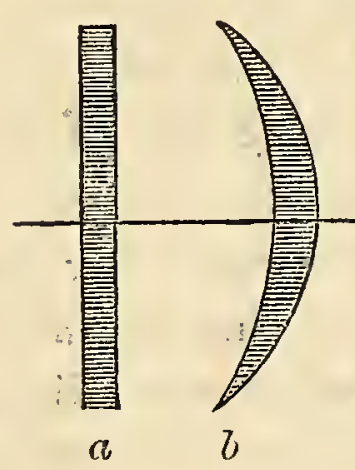

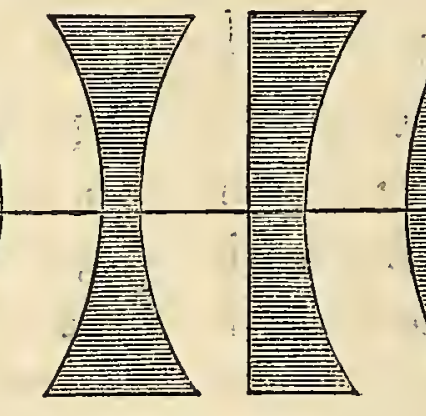

$c$

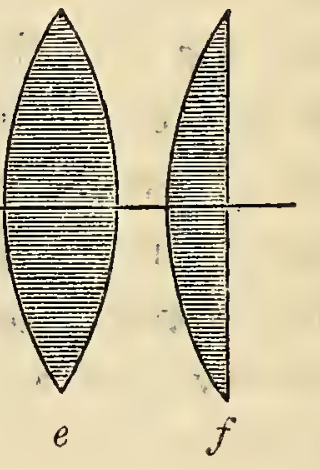
$a$ is a plane glass of equal thickness throughout; $b$, a meniscus, concave on one side, convex on the other ; $c$, a double-concave; $d$, a plano-concave; $e$, a double-convex; $f$, a plano-convex.

fig. 4.

\section{Refraction of Light through Lienses, and Method of tracing the}

\section{Progress of Rays.}

When a ray of light enters the concave surface of a dense medium, or quits a similar surface and enters the convex surface of a rare medium, the method of tracing its progress is shown in fig. 5 , where $m^{\prime} n$ is a dense medium of glass with two concave surfaces, forming a thick concave lens. Let $c c^{\prime}$ be the centres of the two surfaces lying in the axis $c c^{\prime}$, and $h^{\prime} r, h^{\prime \prime} r^{\prime}$ parallel rays incident on the first surface. As $c r$ is per- 
pendicular to the surface at $r, h r c$ will be the angle of incidence; and if a circle is described with a radius $r h, h m$ will be the sine of the angle: the same happens as was shown occurring with parallel surfaces in fig. 3. From a scale on which $h m$ is 1.500 take in the compasses 1 , and find some point, $b$, in the circle, where when one foot of the compasses is placed, the other will fall only on one point, $n$, of the perpendicular, $r c$; the line $r b$, drawn through this point, will be the re-

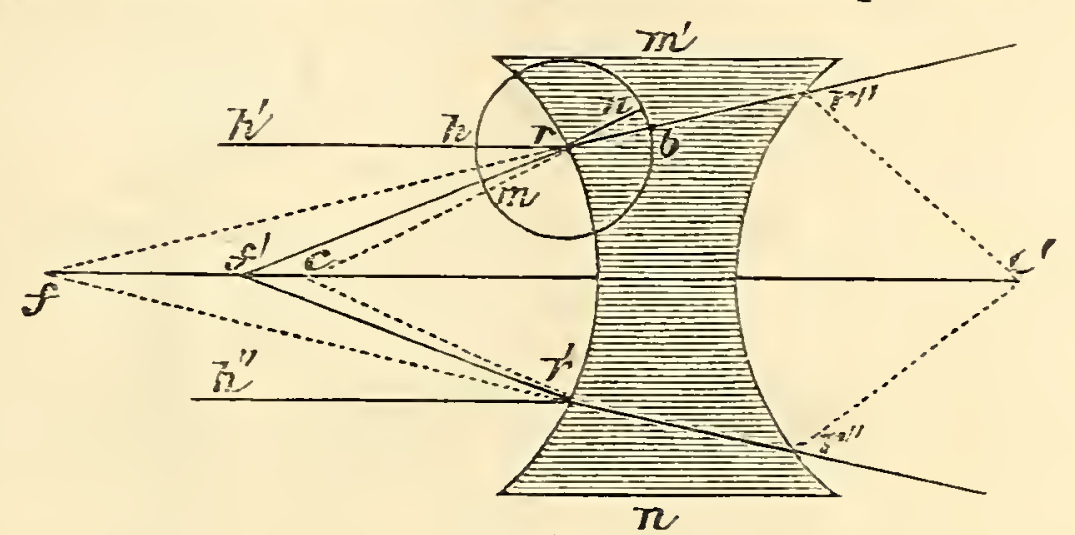

fig. 5.

fracted lay. By continuing this ray, $\delta r$, backwards, it will be found that it meets the axis at $f$. In like manner it will be seen that the ray $h^{\prime \prime} r^{\prime}$ will be refracted in the direction of $r^{\prime} r^{\prime \prime}$, as if it also diverged from $f$. Hence $f$ will be the focus of the parallel rays refracted by a single concare surface, and may be found by the following rule: Divicle the index of refraction by its excess above unity, and the quotient will be the principal focal distance $f^{\prime} c$, the radius of the surface. If, by a similar method, we find the refracted ray $r c$ at the emergence of the ray $r b$ from the second surface $r r^{\prime}$ of the lens, and continue it backwards, it will be found to meet the axis at $f^{\prime}$; so that the divergent rays $r r^{\prime \prime}, r^{\prime} r^{\prime \prime}$ are rendered still more divergent by the second surface, and $c$ will be the focus of the lens $m^{\prime} n$.

Rays of light falling upon a convex lens parallel to its axis are refracted in precisely the same manner as those falling on a sphere; and the refracted ray may be found in the very same way. But as a sphere has an axis in every direction, every incident ray must be parallel to an axis of it; whereas in a lens, which has only one axis, many of the incident rays must be oblique to that axis. In every case, whether of spheres or of lenses, all the rays that pass along the axis suffer no refraction at all, because the axis is always perpendicular to the refracting surfaces.

When parallel rays, $r l, r^{\prime} c, r^{\prime \prime} l^{\prime}$, fig. 6 , fall upon a double-convex lens, $l l^{\prime}$, parallel to its axis, $r^{\prime} f^{\prime}$, the ray $r^{\prime} c$, which coincides with the axis, will pass through without suffering any refraction; but the other rays, $r l, r^{\prime \prime} l$, will be refracted at each of the surfaces of the lens; and the refracted rays corresponding to them, namely $l f^{\prime}, l^{\prime} f^{\prime \prime}$, will be found, by the 'method already given, to nieet at some point, $f^{\prime}$, in the axis. 
But when the rays are oblique to the axis, as $s l, s^{\prime \prime} l^{\prime}, t l, t^{\prime \prime} l^{\prime}$, the rays $s^{\prime} c^{\prime}, t^{\prime} c$, which pass through the centre, $c$, of the lens, will suffer refraction at each surface; but as the two refractions are equal and in opposite directions, the finally refracted rays $c f, c f^{\prime \prime}$ will be parallel

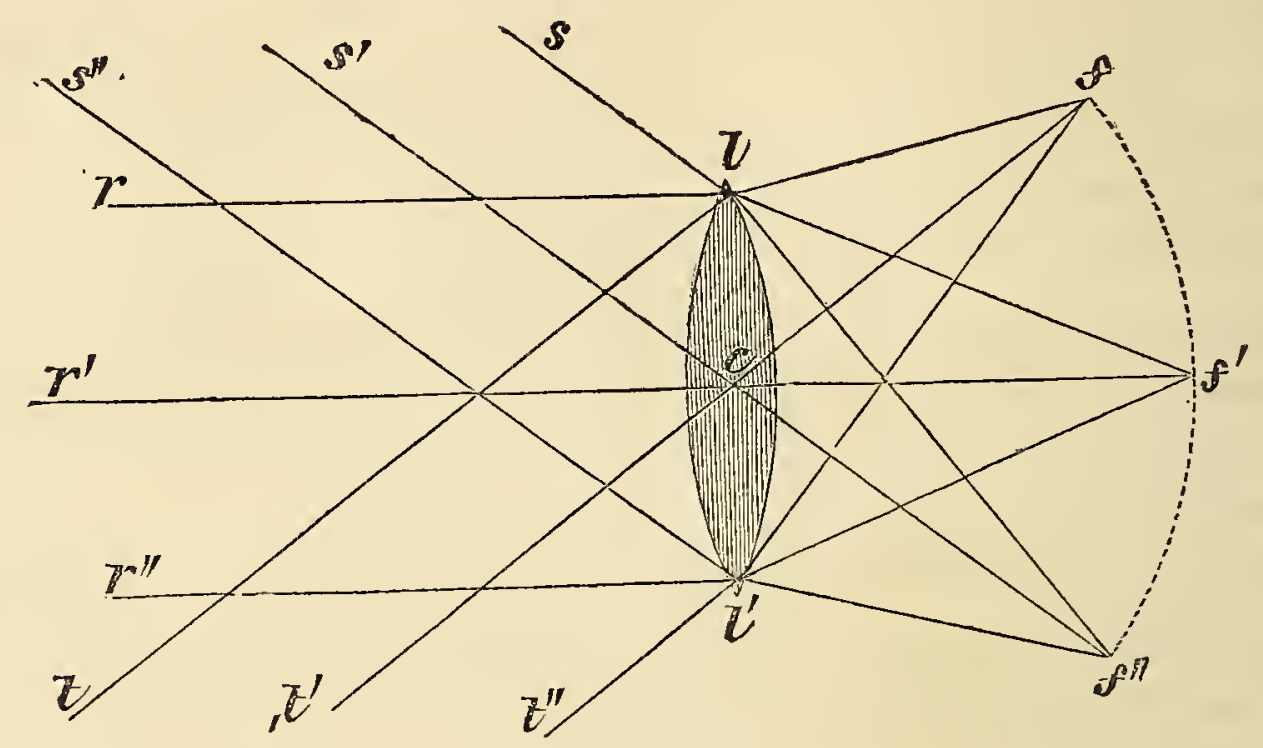

fig. 6.

to $s^{\prime} c, t^{\prime} c$; and it will be found that $s l, s^{\prime \prime} l$ will be refracted to a common point, $f^{\prime \prime}$, in the direction of the central ray $s f^{\prime \prime}$, and $t l, t^{\prime \prime} l^{\prime}$ to the point $f^{\prime \prime}$. When the lens is of glass and equally convex, the focal distance will be equal to the radius. As each ray carries with it the image of the object from whence it proceeded, it follows, that if those rays, after intersecting each other, and having formed an image at their intersection, are again united by refraction or reflection, they will form a new image, and that repeatedly, so long as their order is not disturbed. It follows also, that when the progress of the luminous ray is under consideration, we may look on the image as the object, and the object as the image; and consider the second image, as if it had been produced by the first, as an object, and so on. This is one of the principles involved in the adaptation of these lenses to magnifying objects. It has been shown in fig. 6 , that if the point of light be situated above the line of the axis, the focus will then be below it, and vice vers $\hat{a}$; but the surface of every luminous body may be regarded as comprehending an infinite number of such points, from all of which a pencil of light-rays proceeds, and is refracted according to the general law; so that a perfect but inverted image or picture of the object is formed upon any surface placed in the focus, and adapted to receive the rays.

And if the object be placed at twice the distance of the principal focus, the image being formed at an equal distance on the other side 
of the lens, will be of the same dimensions with the object, as in fig. 7 ;

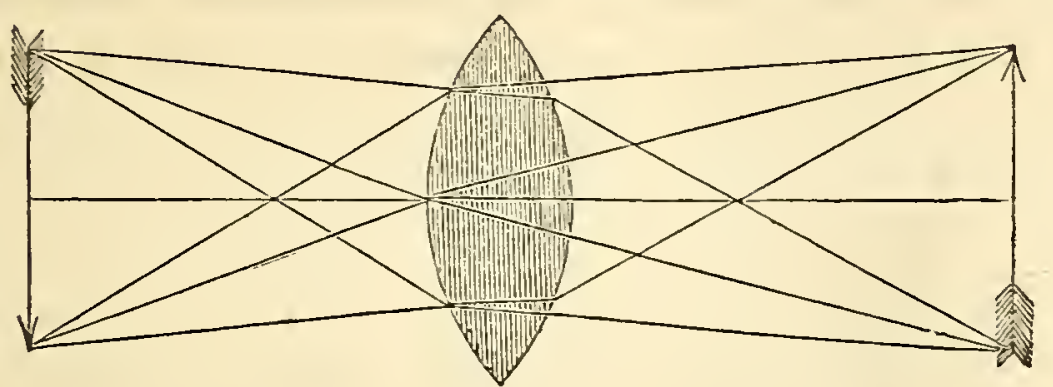

fig. 7 .

but if the object be placed nearer to the lens, the image will be farther from it and of larger dimensions, as in fig. 8 ; and, on the other hand,

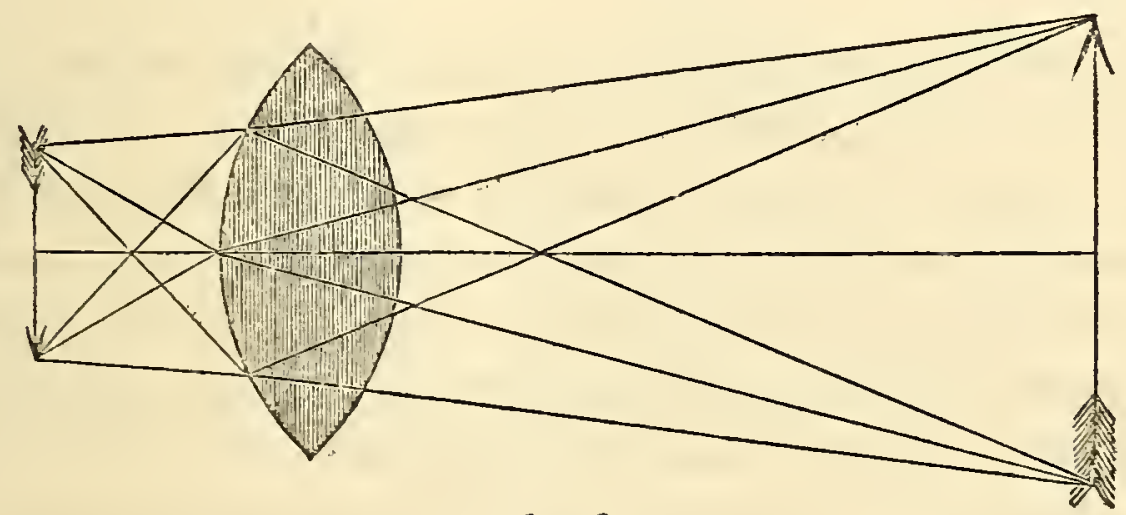

fig. 8.

if the object be farther from the lens, the image will be nearer to it and smaller than itself. But it is to be observed, that the larger the image is in proportion to the object, the less bright it will be, because the same amount of light has to be spread over a greater surface; whilst a smaller image will be much more brilliant.

\section{Spherical Aberration of Lenses.}

We have many imperfections to contend with in our optical arrangements and combinations; one of which results from the spherical aberration of the rays passing through lenses whose curvatures are equal over their whole surfaces. If the course of the rays be observed, it will be seen that they do not all meet exactly in the foci already stated, but that the focus of the rays which have passed through the circumferential portion of the lens is much closer to it than that of the rays which are nearer the line of its axis. This is shown in fig. $9: a b$, the rays, are seen falling on the circumference and coming to a focus, $f$; $a^{\prime} b^{\prime}, a^{\prime \prime} b^{\prime \prime}$ are rays falling nearer the centre, and coming to a more distant focus, $f^{\prime \prime}$; so that if a screen be held at $c d$, the rays which have passed through the central portion of the lens will be stopped at $f$ before they come to a focus, or they will come to it in a state of diverg- 
ence at $c d$ : in either case the image will have a certain degree of indistinctness; and the difference between the focal points of their circumferential and central rays is termed the spherical aberration. It

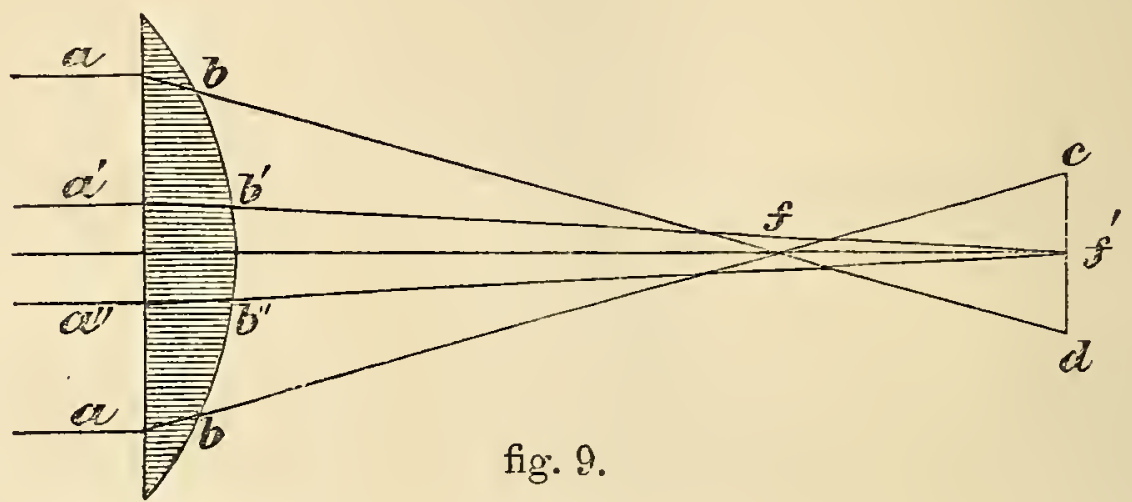

therefore becomes apparent that, to produce the desired effect, the curvature of the lens is required to be increased around the centre, so as to bring the rays which pass through it more speedily to a focus; and to be diminished towards the circumference, so as to throw the focus of the rays influenced by it to a greater distance. This condition is in a measure fulfilled in the meniscus form of lens, which is shown to be the segment of an ellipsoid instead of a sphere.

But the ellipse and the hyperbola are curves of this kind, in which the curvature diminishes from the central ray, or axis, to the circumference $b$; and mathematicians have shown how spherical aberration may be entirely removed by lenses whose sections are ellipses or hyperbolas. This curious discovery we owe to Descartes.

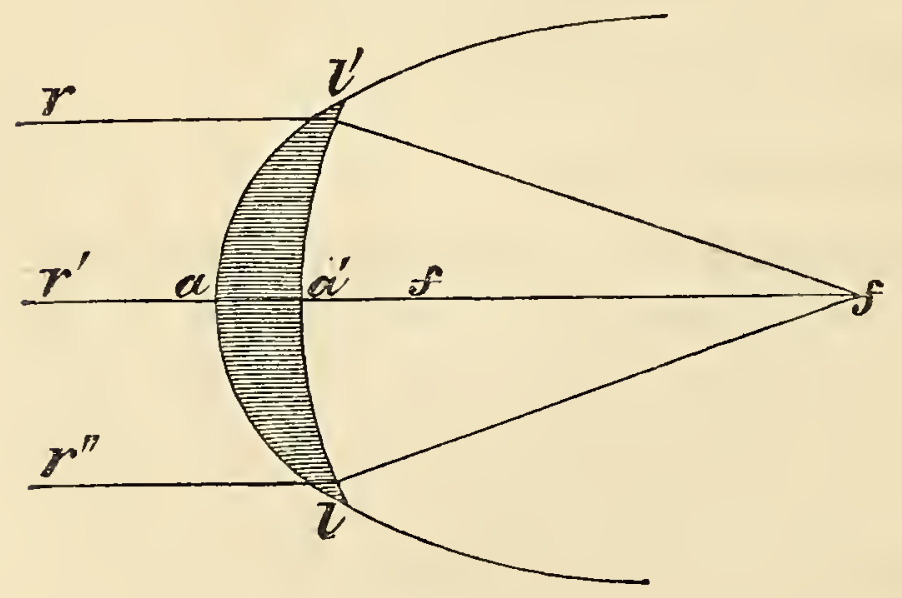

fig. 10 .

If $a l, a l^{\prime}$ for example, fig. 10 , be part of an ellipse whose greater axis is to the distance between its foci $f f$ as the index of refraction is to unity, then parallel rays $r l^{\prime}, r^{\prime \prime} l$ incident upon the elliptical surface $l^{\prime} a l$, will be refracted by the single action of that surface into lines which would meet exactly in the farther focus $f$, if there were no second surface intervening between $l a l^{\prime}$ and $f$. But as every useful lens must have two surfaces, we have only to describe a circle $l a^{\prime} l^{\prime}$ round $f$ as a centre, for the second surface of the lens $l^{\prime} l$.

As all the rays refracted at the surface $l a l^{\prime}$ converge accurately to $f$, and as the circular surface $l a^{\prime} l^{\prime}$ is perpendicular to every one of the refracted rays, all these rays will go on to $f$ without suffering any 
refraction at the circular surface. Hence it should follow, that a meniscus whose concave surface is part of an ellipsoid, and whose concave surface is part of any spherical surface whose centre is in the farther focus, will have no spherical aberration, and will refract parallel rays incident on its convex surface to the farther focus.

In like manner, a concavo-convex lens, fig. 11, $l l^{\prime}$, whose concave surface $l a^{\prime} l^{\prime}$ is a circle described round the farther focus of the ellipse, will cause parallel rays $b l, b^{\prime} l$ to diverge in directions $l r, l^{\prime} r^{\prime \prime}$, which, when continued backwards, will meet exactly in the focus $f$, which will be its virtual focus.

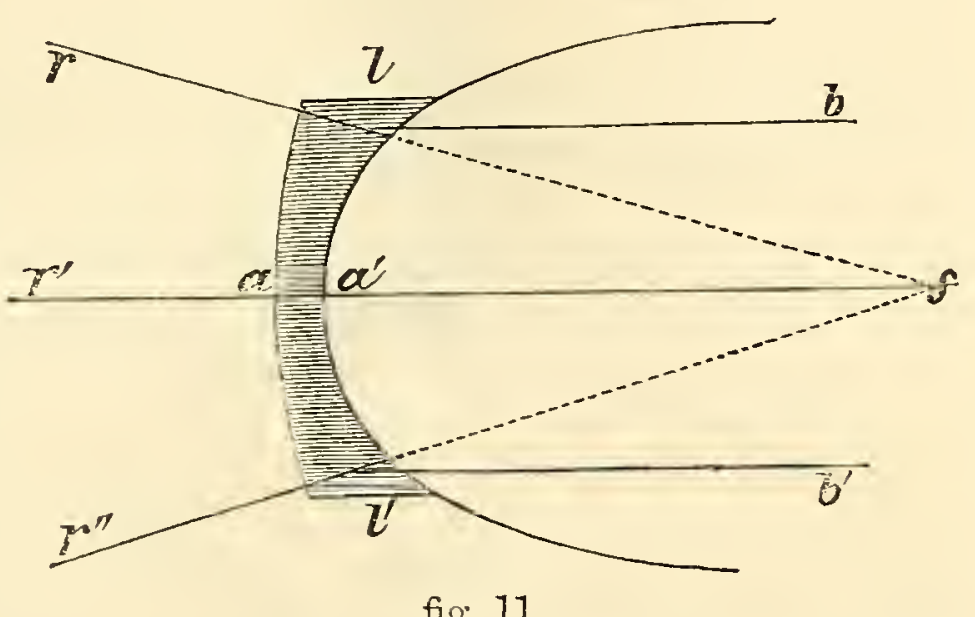

If a plano-convex lens, fig: 12, has its convex surface $l_{a} l^{\prime}$ part of a hyperboloid, formed by the revolution of a hyperbola whose greater axis is to the distance between the foci as unity is to the index of refraction, then parallel rays $r l, r^{\prime \prime} l^{\prime}$ falling perpendicularly in the plane surface will be refracted without aberration to the further focus of the hyperboloid. The same property belongs to a planoconcave lens having a similar hyperbolic surface, and receiving.

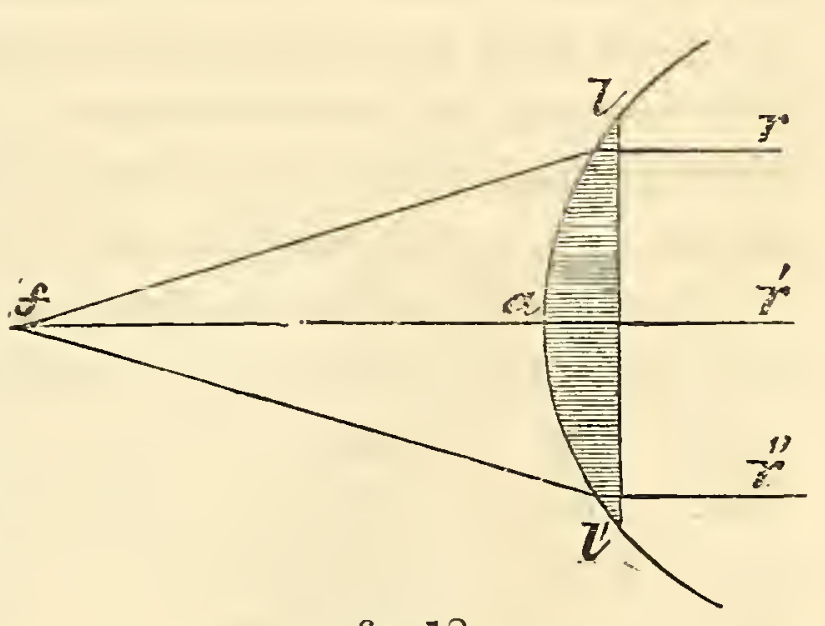

fig. 12. parallel rays in its plane surface.*

When the convex side of a plano-convex lens is exposed to parallel rays, the distance of the focus from the plane side will be equal to twice the radius of its convex surface diminished by two-thirds of the thickness of the lens; but when the plane is exposed to parallel rays, the distance of the focus from the convex side will be equal to twice the radius.

A meniscus with spherical surfaces, fig. 13, has the property of refracting all converging rays to its focus, if its first surface is con-

* It should be borne in mind, that in none of these lenses would the object be corl rectly seen in focus, except at the one point known as the mathematical or geometricaaxis of the lens. 
vex, provided the distance of the point of convergence or divergence from the centre of the first surface is to the radius of the first surface

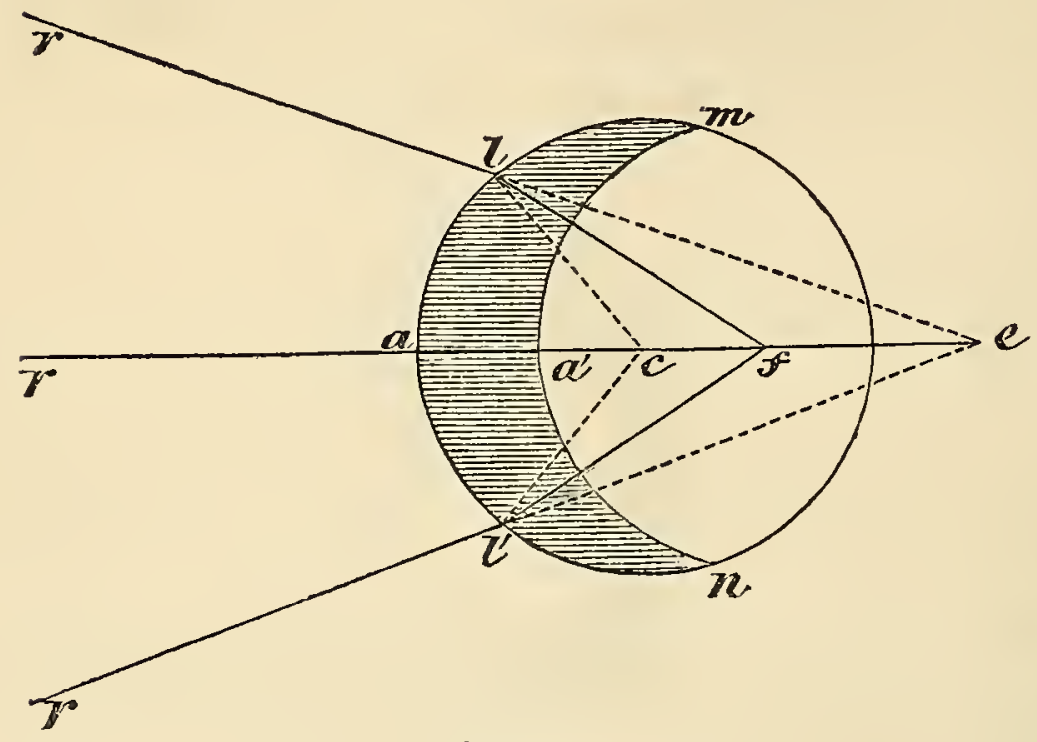

fig. 13. as the index of refraction is to unity. Thus, if $m l$ $l^{\prime} n$ is a meniscus, and $r l$, $r l^{\prime}$ rays converging to the point $e$, whose distance $e c$ from the centre of the first surface $l a l^{\prime}$ of the meniscus is to the radius $c a$, or $c l$, as the index of refraction is to unity, that is, as 1.500 to 1 in glass; then if $f$ is the focus of the first surface, describe, with any radius less than $f a$, a circle $m a^{\prime} n$ for the second surface of the lens. Now it will be found by projection, that the rays $r l, r l$, whether near the axis ae or remote from it, will be refracted accurately to the focus $f$; and as all these rays fall perpendicularly on the second surface $m n$, they will still pass on, without refraction, to the focus $f$. In like manner, it is obvious that rays $f l, f l^{\prime}$ diverging from $f$ will be refracted into $r l, r l^{\prime}$, which diverge accurately from the virtual focus.*

There are certain mechanical difficulties in the way of such lenses as these, but which have to some extent been surmounted by diminishing the working aperture with stops, for correcting their aberration. This is still better effected, or even got rid of altogether, by using combinations of lenses, so disposed that their opposite aberrations shall correct each other, whilst magnifying power is gained. For it is easily seen that, as the aberration of a concave lens is just the opposite of that of a convex lens, the aberration of a

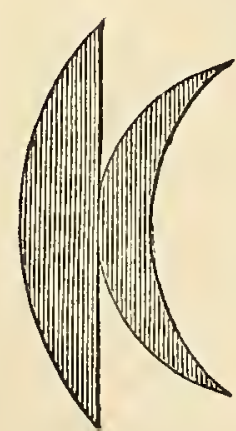

fig. 11 convex lens placed in its most favourable position may be corrected by a concave lens of much less power in its most favourable position. This is the principle of a combination proposed by Sir John F. W. Herschel, fig. 14, consisting of a plano-convex lens and a meniscus; and a doublet of this kind will be found extremely useful and available for microscopic purposes : it affords a large field, like the Coddington lens. Another and serious difficulty arises to the optician in the unequal refrangibility of the different coloured rays which together make up white light, so that they are not

\footnotetext{
* Brewster's Optics.
} 
all brought to the same focus even by a lens free from spherical aberration. It is, indeed, this difference in their refrangibility which causes their complete separation by the prism into a spectrum. This is termed chromaicic aberration, and will be best explained by a reference to fig. 15: $a b$ are rays of white light refracted by a convex lens;

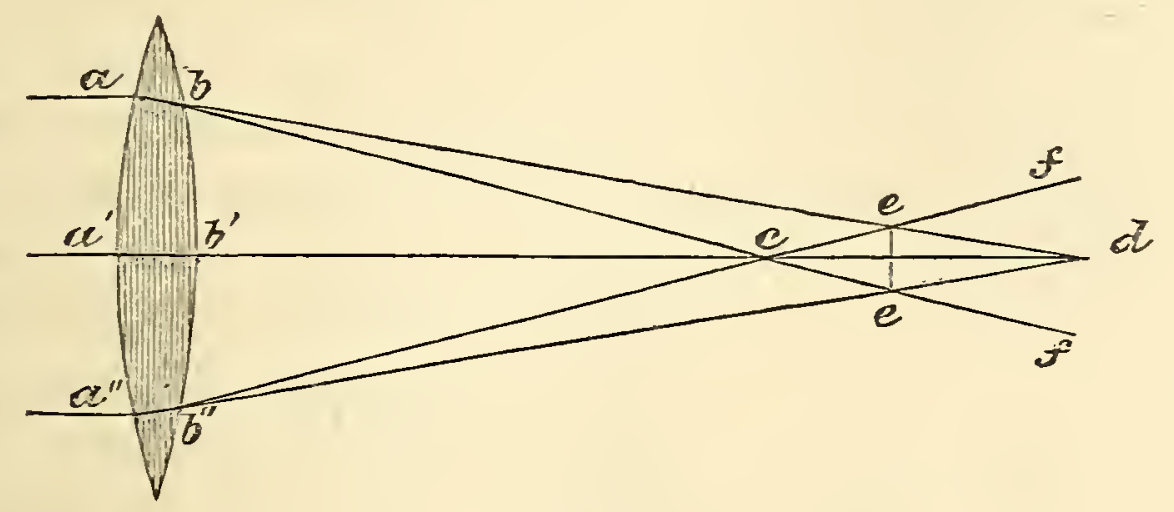

Aig. 15.

$c$ the focus of the violet rays, which then cross and diverge towards $e f ; d$ is the focus of the red rays, which are crossed at the points $e e$ by the violet rays; the middle point, therefore, of this line is the mean focus, or focus of least aberration.

The correction of chromatic aberration has been accomplished by bringing into use the different dispersive powers of various materials which bear no relation to their simple refracting power, -in other words, by a combination of flint and crown glass; and by a most curious series of experiments the dispersive power of flint-glass was found to be so much greater than that of crown-glass, that if the lens a a (fig. 16) be made of crown-glass, whose index of refraction is 1.519 , and dispersive power 0.036 , and the lens $6 b$ of flint-glass, whose index of refraction is 1.589 , and dispersive power 0.0393 , and if the focal length of the conrex crown-glass lens is made $4 \frac{1}{5}$ inches, and that of the concare flint-glass lens $7 \frac{2}{5}$ inches, they will form a lens with a focal length of ten inches, and will bring rays of light, $c c^{\prime}$, to a single focus, $d$, free of colour.

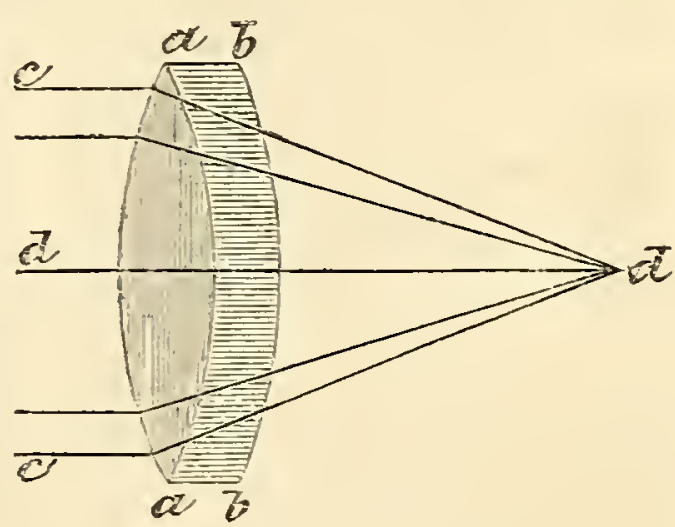

fig. 16. Such a lens is called an achromatic lens: and when used in combination with other glasses in constructing the microscope, it is termed an achromatic microscope.

To assist us in gaining a clearer notion of the mode in which a single lens serres to magnify minute objects, it is necessary to take a 
passing glance at the ordinary phenomena of vision. The human eye is so constituted, that it can only have distinct vision when the rays falling upon it are parallel or slightly divergent; because the retina, on which the image impinges, requires the intervertion of the crystalline lens to bring the rays to an accurate focus upon its surface. The limit of distinct vision is generally estimated at from six to tel inches; objects viewed nearer, to most persons, become indistinct, although they may be larger. The apparent size of an object is, indeed, the angle it subtends to the eye, or the angle formed by two lines drawn from the centre of the eye to the extremity of the object. This will be understood upon reference to fig. 17. The lines drawn from the eye to $\mathrm{A}$

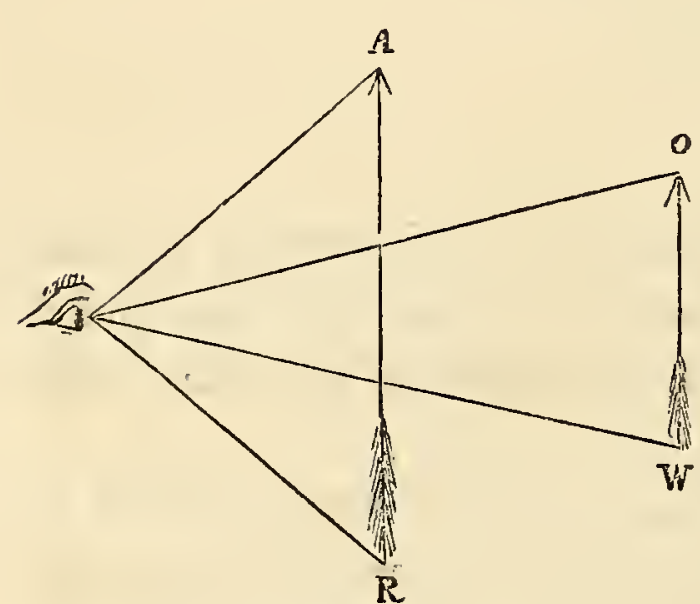

fig. 17 . and $\mathrm{R}$ form an angle, which, when the distance is small, is nearly twice as great as the angle from the eye to $0 \mathrm{w}$, formed by lines drawn at twice the distance. The arrow at $\mathrm{A} R$ will therefore appear nearly twice as long as ow, being seen under twice the angle; and in the same proportion for any greater or lesser difference in distance. This, then, is called the angle of vision, or the visual angle. Now the utility of a convex lens interposed between a near object and the eye consists in its reducing the divergence of the rays forming the several pencils issuing from it; so that they enter the eye in a state of moderate diver-

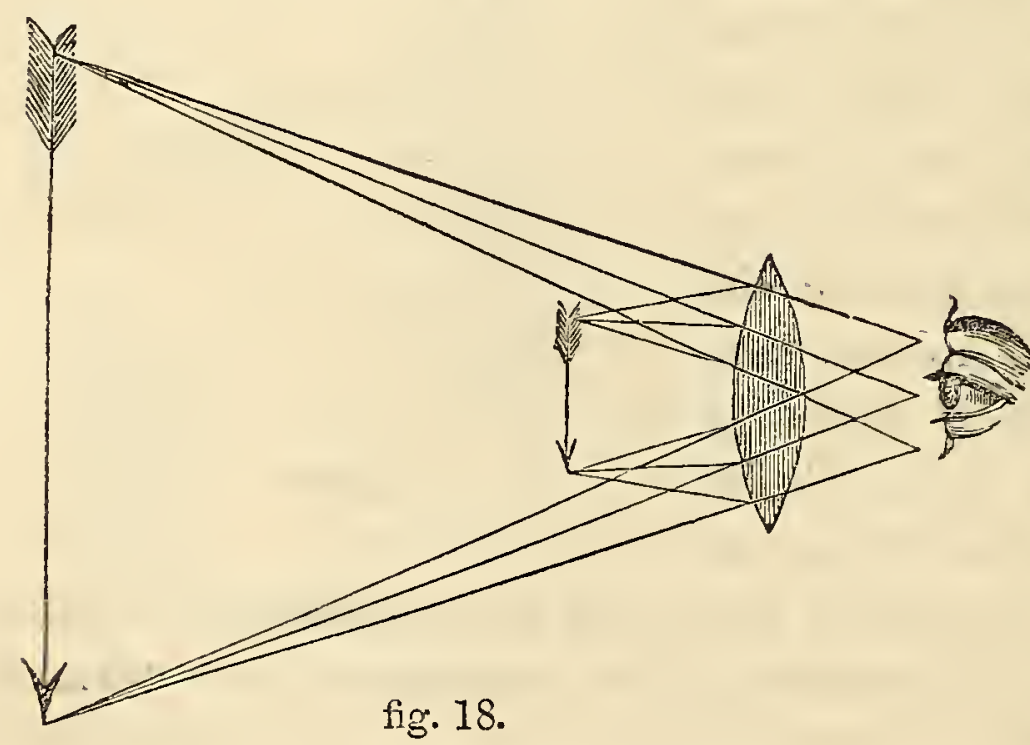
gence, as if they had issued from an object beyond the nearest limit of distinct vision; and a well-defined image is consequently formed upon the retina. In fig. 18 a double-convex lens is placed before the eye, near which is a small arrow, to represent the object under examination; and the cones drawn from it are portions of the rays of light diverging from those points and falling upon the lens. These rays, if permitted to fall 
at once upon the pupil, would be too divergent to allow of their being brought to a focus upon the retina by the optical arrangements of the eye. But being first passed through the lens, they arc bent into nearly parallel lines, or into lines diverging from some points within the limits of distinet vision. Thus altered, the eye receives them precisely as if they had emanated directly from a larger arrow placed at ten inches from the eye. The difference between the real and the imaginary arrow is called the magnifying power of the lens. Fig. 19 will perhaps convey a clearer idea of the increase of the angle of vision by interposing a convex lens: without this lens placed at $f^{\prime} g^{\prime}$, the eye would see the dart at $b^{\prime} c^{\prime}$ under the angle formed by the eye and $b^{\prime} c^{\prime}$; but the rays $b^{\prime} f^{\prime}$ and $c^{\prime} g^{\prime}$ from the extremities of the dart, in passing through the lens, are refracted to the eye in the direction of $f$ and $g$, which causes the dart to be seen under the much larger angle formed with the eye

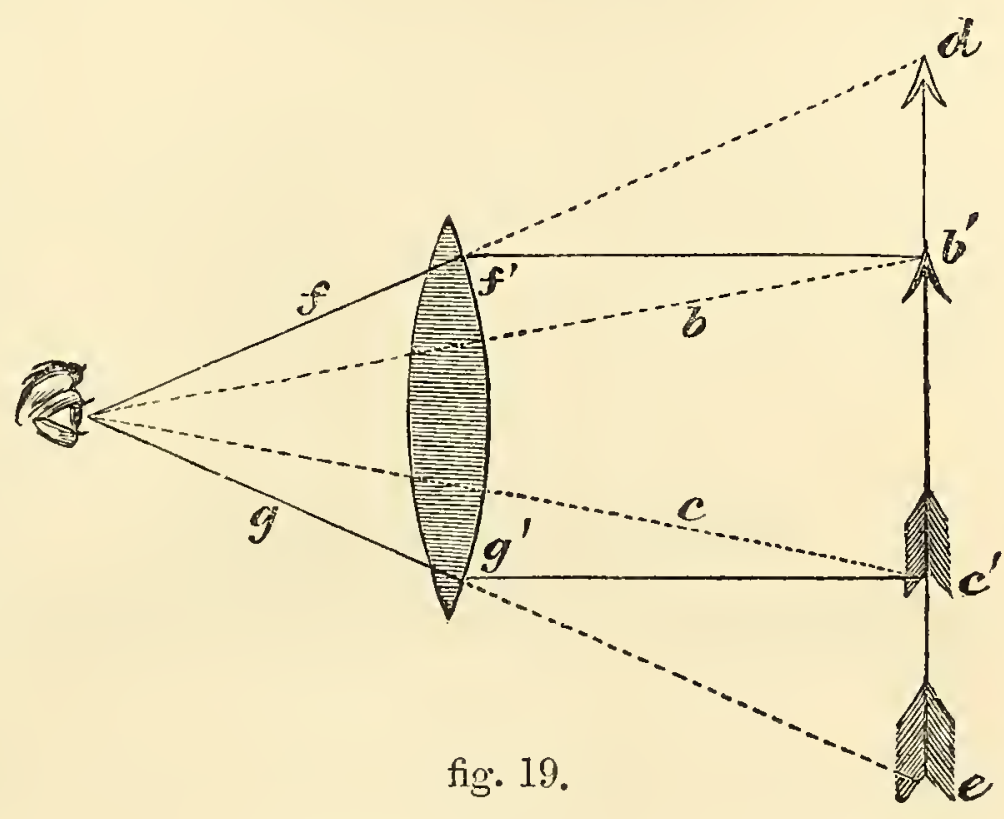
and $d e$; and it therefore appears so much magnificd as to extend from $d$ to $e$. The object, when thus seen, appears to be magnified nearly in the proportion which the focal distance of the lens bears to the distance of the object when viewed by the unassisted eyc; and is entirely owing to the object being distinctly riewed so much nearer to the eye than it could be without the lens. With these preliminary remarks as to the medium by which microscopic power is obtained, we shall proceed to apply them to the construction of a perfect instrument. A microscope, as we have before explained, may be either a single or simple, or a compound instrument. The simple microscope may consist of one, as seen in fig. 18, or of two or three lenses; but these latter arc so arranged as to have the effect only of a single lens. In the compound microscope, not less than two lenses must be employed : one to form the inverter image of the object, which being the nearest to the object, is called the object-glass; and the other to magnify this image, and from. being next the eye of the observer, called the eye-glass. Both these may be formed out of a combination of lenses, as will be hereafter seen.

We have hitherto considered a lens only in reference to its enlarge- 
ment of the object, or the increase of the angle under which the object is seen. A further and equally important consideration is that of the number of rays or quantity of light by which every point of the object is rendered visible; but it at the same time becomes as important that we do not sacrifice definition to this end. Much may be accomplished, as we have before pointed out, by the combination of two or more lenses instead of one, thus reducing the angles of incidence and refraction.

"The first satisfactory arrangement for this purpose was the invention of the celebrated Dr. Wollastonl. His doublet (fig. 20) consisted of

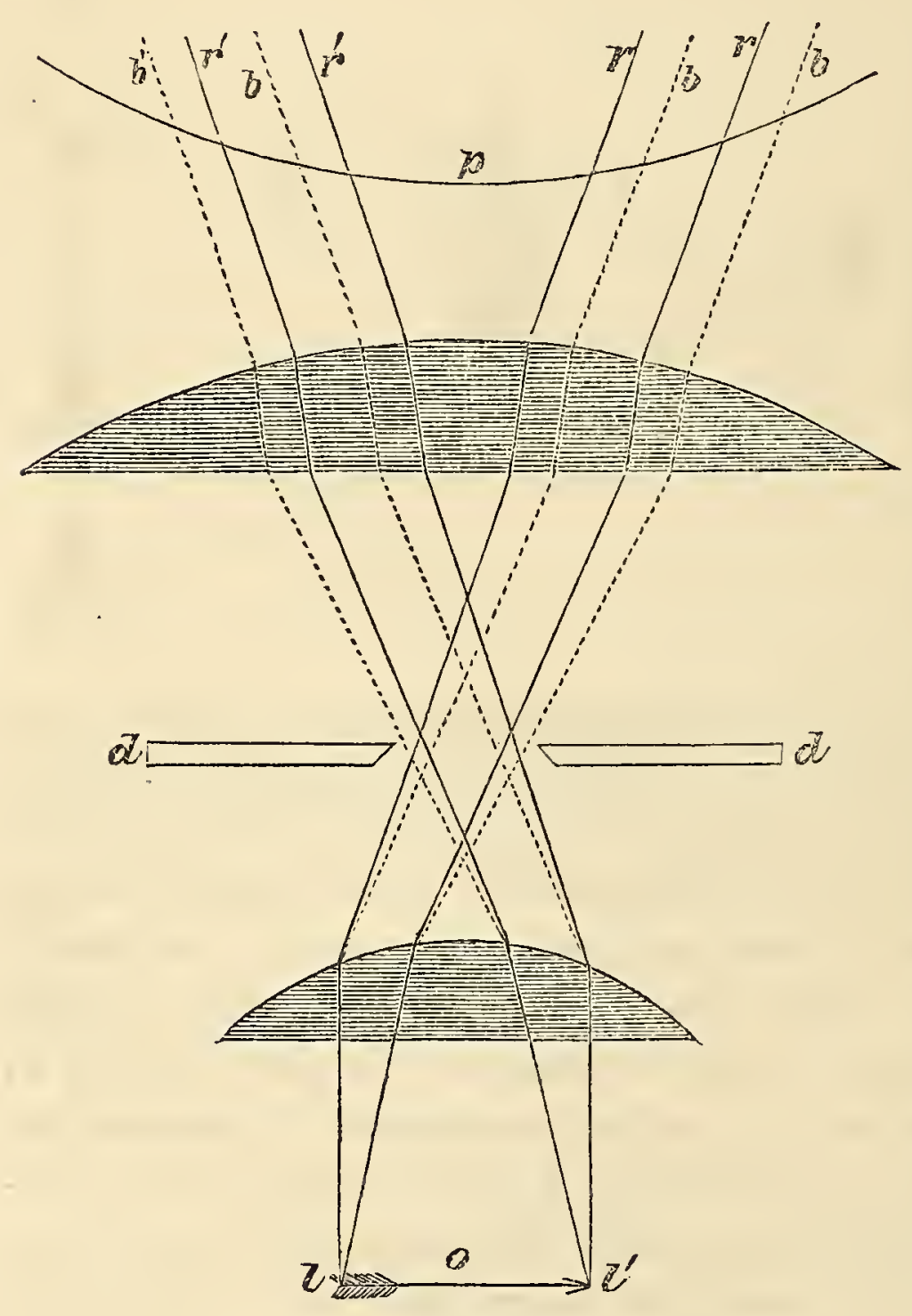

fig. 20 . two plano-convex lenses having their focal lengths in the proportion of one to three, or nearly so, and placed at a distance which can be ascertained best by actual experiment. Their plane sides are placed towards the object, and the lens of shortest focal length next the object.

"It appears that Dr. Wollaston was led to this invention by considering that the achromatic Huyghenean eye-piece, which will be presently described, would, if reversed, possess similar good properties as a simple microscope. But it will be evident, when the eyepiece is understood, that the circumstances which render it achromatic are very imperfectly applićable to the simple microscope, and that the doublet, without a nice adjustment of the stop, would be valueless. Dr. Wollaston makes no allusion to a stop, nor is it certain that he contemplated its introduction; although his illness, which terminated fatally soon after the presentation of his paper to the Royal Society, may account for the omission. 
"The nature of the corrections which take place in the doublet is explained in the annexed diagram, where $l_{0} l^{\prime}$ is the object, $p$ a portion of the pupil, and $d d$ the stop, or limiting aperture.

"Now it will be observed that each of the pencils of light from the extremities $l l^{\prime}$ of the object is rendered excentrical by the stop; and of consequence each passes through the two lenses on opposite sides of their common axis op; thus each becomes affected by opposite errors, which to some extent balance and correct each other. To take the pencil $l$, for instance, which enters the ere at $r b, r b:$ it is bent to the right at the first lens, and to the left at the second; and as each bending alters the direction of the blue rays more than the red, and moreorer as the blue rays fall nearer the margin of the second lens, where the refraction, being more powerful than near the centre, compensates in some degree for the greater focal length of the second lens, the blue rays will emerge very nearly parallel, and of consequence colourless to the eye. At the same time the spherical aberration has been diminished by the circumstance that the side of the pencil which passes one lens nearest the axis passes the other nearest the margin.

"This explanation applies only to the pencils near the extremities of the object. The central pencils, it is obrious, would pass both lenses symmetrically, the same portions of light occupying nearly the same relative places on both lenses. The blue light would enter the second lens nearer to its axis than the red; and being thus less refracted than the red by the second lens, a small amount of compensation would take place, quite different in principle, and inferior in degree, to that which is produced in the excentrical pencils.

"In the intermediate spaces the corrections are still more imperfect and uncertain; and this explains the cause of the aberrations which must of necessity exist even in the best-made doublet. It is, howerer, infinitely superior to a single lens, and will transmit a pencil of an angle of from $35^{\circ}$ to $50^{\circ}$ without any very sensible errors. It exhibits, therefore, many of the usual test-objects in a very beautiful manner."

The next step in the improrement of the simple microscope bears more relation to the ere-piece; this was effected by Mr. Holland: it consists in substituting two lenses for the first in the doublet, and retaining the stop between them and the third. The first bending being thus effected by two lenses instead of one, is accompanied by smaller aberrations, which are, therefore, more completely balanced or corrected at the second bending, in the opposite direction, by the third lens.

Every increase in the number of lenses is attended with one draw- 
back, from the circumstance that a certain portion of light is lost by reflection and absorption each time that the ray enters a new medium.

The combination of three lenses approaches so very close to the object under observation as, indeed, to prevent the use of more than three; and this constitutes a limit to the improvement of the simple microscope,-for it is called a simple microscope, although consisting of three lenses.

Before we proceed to describe the simple microscope and its appendages, it will be well to explain such other points in reference to the form and materials of lenses as are most likely to be interesting.

A very useful form of lens was proposed by Dr. Wollaston, and called by him the Periscopic lens. It consisted of two hemispherical lenses cemented together by their plane faces, having a stop between them to limit the aperture. A similar proposal was made by $\mathrm{Mr}$. Coddington, who, liowever, executed the project in a better manner, by cutting a groove in a whole sphere, and filling the groove with opaque matter. His lens, which is the well-known Coddington lens, is shown at fig. 21: it gives a large field of view, which is equally good in all directions, as it is evident that the pencils $a b$ and $b a$ pass through under precisely the same circumstances. Its spherical form has the further advantage of rendering the position in which it is held of comparatively little consequence. It is therefore very convenient as a hand magnifier; but its definition is, of course, not so good as that of a well-made doublet or achromatic lens. It is generally set in a folding case, as re-

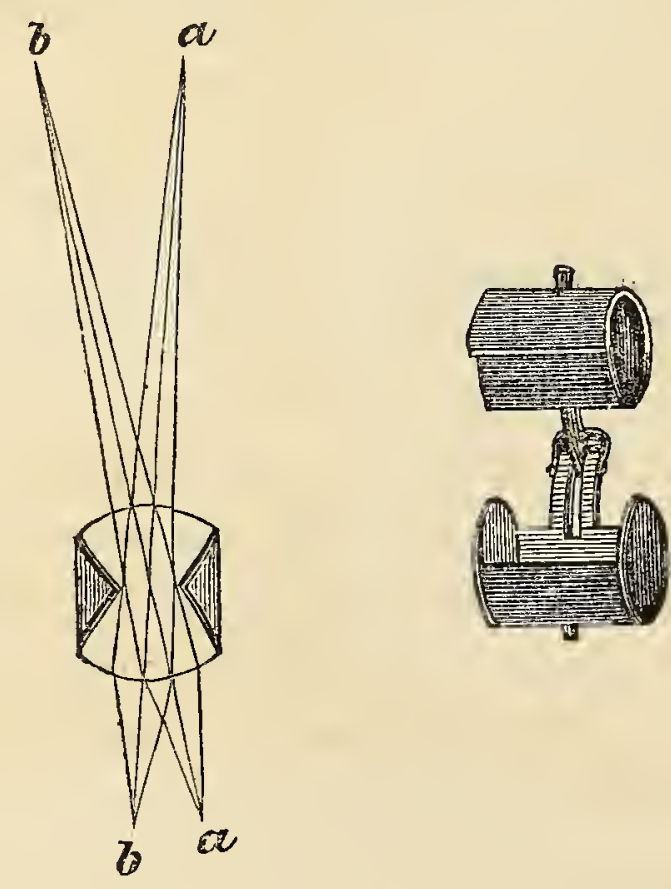

fig. 21.

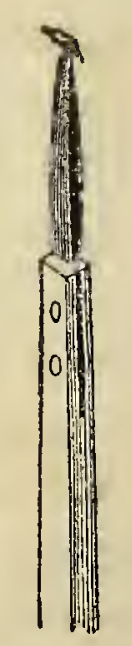

fig. 22.

presented in the figure, and so contrived that it is admirably adapted for the waistcoat-pocket; which, together with the small holder, fig. 22, 
for securing small objects and holding them during examination, are all that is required for a field instrument during a day's ramble. The useful little holder may be purchased in a case of Mr. Weedon, 41 Hart-street, Bloomsbury. The Stanhope lens is similarly constructed, although not so good and convenient as the former, and is but seldom to be purchased properly made.

When the magnifying power of a lens is considerable, or when its focal length is short, and its proper distance from the object equally short, it then becomes necessary to be placed at a proper distance with great precision; it cannot therefore be held with sufficient accuracy and steadiness by the unassisted hand, but must be mounted in a frame, having a rack or screw to move it towards or from another frame or stage which holds the object. It is then called a microscope; and it is furnished, according to circumstances, with lenses and mirrors to collect and reflect the light upon the object, with other conveniences.

The best of the kind was that contrived by Mrr. Ross : it is represented in fig. 23, and consists of a circular foot $e$, from which rises a short tubular stem $d$, into which slides another short tube $c$, carrying

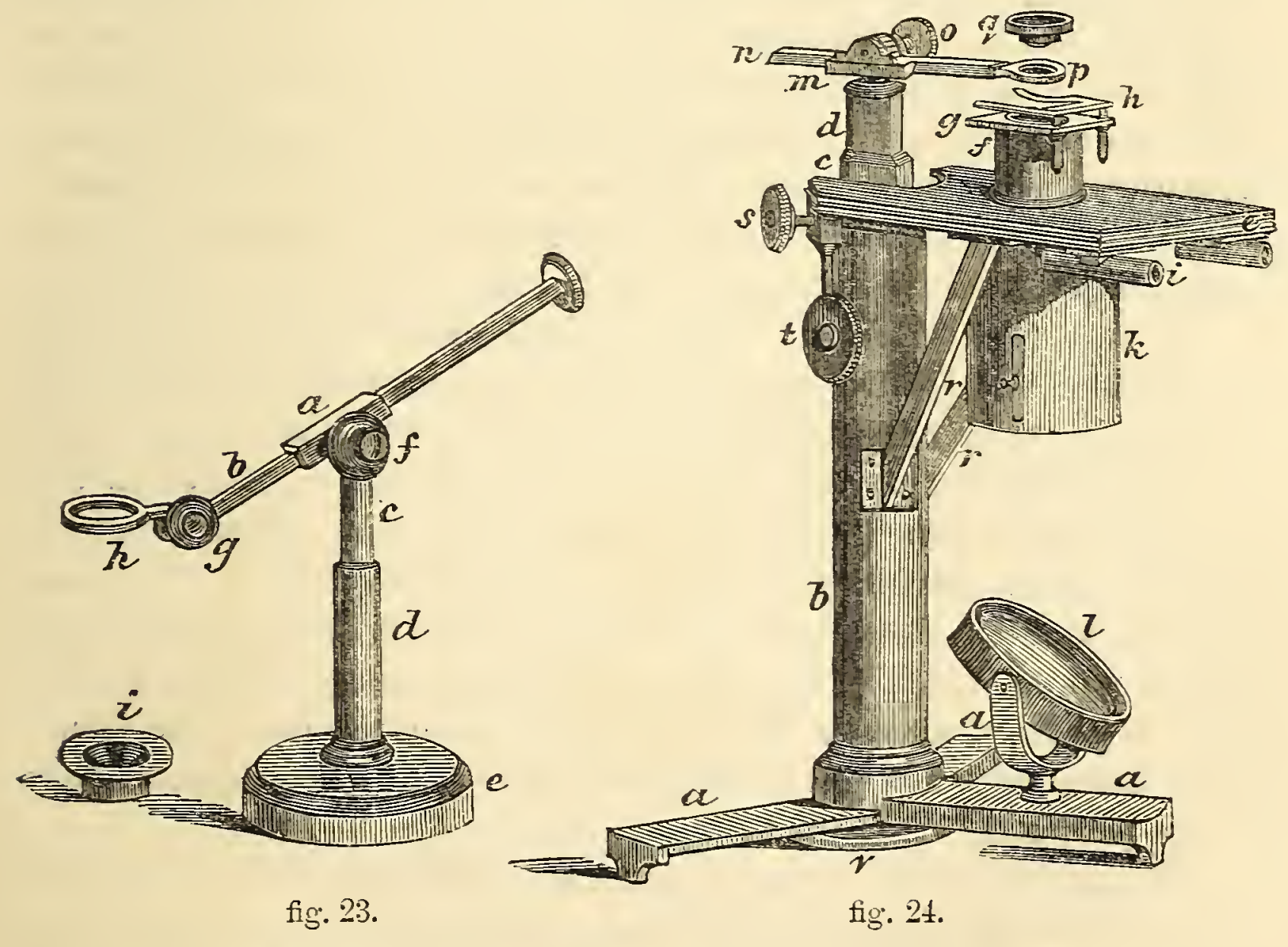

at its top a joint $f$; to this joint is fixed a square tube $a$, through which a rod $b$ slides; this rod has at one end another but smaller joint $g$, to which is attached a lens $h$. By means of the joint at $f$, the square rod can be moved up or down, so as to bring the lens close to the ob- 
ject; and by the rod sliding through the square tube $a$, the distance between the stand and the lens may be either increased or diminished: the joint $g$, at the end of the rod, is for the purpose of allowing the lens to be brought either horizontally or at an angle to the subject to be investigated. By means of the sliding arm the distance between the table and the jointed arm can be increased or diminished. This microscope is provided with lenses of one-inch and half-inch focal length, and is thereby most useful for the examination and dissection of objects. It is readily unscrewed and taken to pieces, and may be packed in a small case for the pocket.

Another highly-useful and more complete simple microscope was contrived by Mr. W. Valentine, and made for him by Mr. Ross in 1831. It is thus described by the latter gentleman, and is represented in fig. 24. It is supported on a firm tripod, made of bell-metal, the feet of which, $a$ a $a$, are made to close up for the purpose of packing it in a box. The firm pillar $b$ rises from the tripod, and carries the stage $e$; this is further strengthened by the two supports $r r$. From the pillar a triangular bar $d$, and a triangular tube $c$, is moved up and down by a screw, having fifty threads in the inch, and turned by a large milled head $v$, which is situated at the base of the pillar: tliis is the fine adjustment. The small triangular base $d$ is moved up and down within the triangular bar $c$, by turning the milled head $t$, forming the coarse adjustment: this bar carries the lens-holder $m n o p$. The stage $e$ consists of three plates; the lowest one is firmly attached to the pillar, and upon this the other two work. The upper one carries a small elevated stage $g$, on which the objects are placed; this stage is mounted on a tube $f$, and has a spring clip $h$ for holding, if necessary, the objects under examination. By means of two screws placed diagonally, one of which is seen at $s$, this elevated stage can be moved in two directions, at right angles to one another; and thus different parts of objects can be brought successively into the field of view. The arm $n p$, for carrying the lenses, is attached to the triangular bar $d$ by a conical pin, on which it is made to turn horizontally, and the arm itself can be lengthened or shortened by means of the rack and pinion $m o$; hence the lens $q$ can be applied to every part of an object without moving the stage.

The mirror $l$ is fitted into the largest of the three legs, and consists of a concave and plane glass reflector. To the under side of the stage is fitted a Wollaston's condensor k; and the lens is made to slide up and down by means of two small handles projecting from the cell in which the lens is set. Two small tubes $i$, with either a condensing lens for opaque objects, or a pair of forceps, may be attached to this side of 
the stage. The magnifiers are either simple lenses or doublets; or it could be easily converted into a compound microscope by inserting a compound body, supported on a bent arm, in the place of the one carrying the single lenses.

\section{THE COMPOUND MICROSCOPE.}

The compound microscope may, as before stated, consist of only two lenses, while a simple microscope has been shown to contain sometimes three. In the triplet for the simple microscope, however, it was explained that the object of the first two lenses was to do what might have been accomplished, though not so well, by one; and the third merely effected certain modifications in the light before it entered the eye. But in the compound microscope the two lenses have totally different functions: the first receives the rays from the object, and bringing them to new foci, forms an image, which the second lens treats as an original object, and magnifies it just as the single microscope magnificd the object itself.

Fig. 25 shows the earliest form of the compound microscope, with the magnified image of a fly, as given by Adams, which he describes as consisting of an object-glass, $l n$, and an eye-glass, $f g$; the object, $b^{\prime} o^{\prime}$, being placed a little further from the lens than its principal focal clistance, the pencil of rays from which converge to a focus, and form an inverted image of the object at $p q$, which image is viewed by the eye placed at $a$ through the eye-glass $f g$. The rays remain parallel after passing out until they reach the eye, when they will converge by the refractive powers of this organ, and be collected on the retina. But the image differs from the real object in a very essential particular. The light being emitted from the

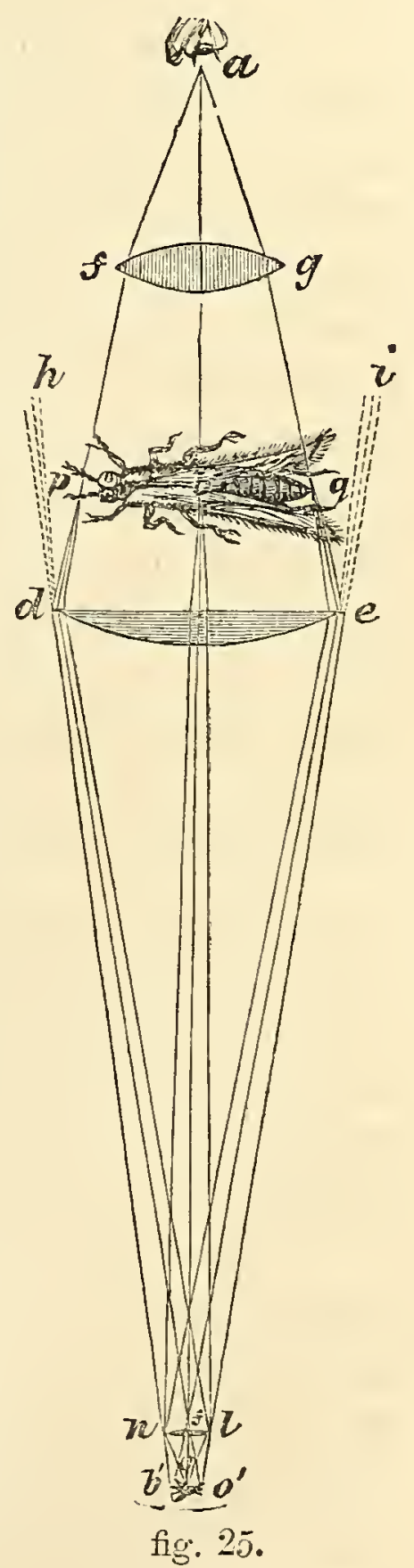
object in every direction, renders it visible to an eye placed in any position; but the points of the image formed by a lens emitting no more than a small conical body of rays, which it receives from the 
glass, can be visible only to the eye situate within its range. Thus the pencil of rays emanating from the object at $o$ is converged by the lens to $f$, cross each other, and diverge towards $h$, and therefore would never arrive at the lens $f g$, without the interposition of the planoconvex lens at $d e$, placed at a smaller distance from the object; and by this means the pencil $d n$, which would have proceeded to $h$, is refracted or bent towards the lens $f g$, having a radial point at $p q$. The object is magnified upon two accounts : first, because if we view the image with the naked eye, it would appear as much longer than the object as the image is really longer than it, or as the distance $f b$ is greater than the distance from the real object to $f$; and secondly, because this picture is again magnified by the eye-glass. The compound microscope, then, consists of an object-lens, $l n$, by which the image is formed, enlarged, and inverted; an amplifying lens, $d e$, by which the field of view is enlarged, and is consequently called the fieldglass; and an eye-glass or lens, by which the eye is permitted to approach very near, and consequently enabled to view the image under a large angle of apparent magnitude. The two, when combined, are called the eye-piece.

Upon the construction of this microscope Mr. Ross observes: "Since the power depends on the ratio between the anterior and posterior foci of the object-glass, it is evident that by increasing that ratio any power may be obtained, the same eye-glass being used; or having determined the first, any further power may be obtained by increasing that of the eye-glass ; and thus, by a pre-arrangement of the relative proportions in which the magnifying power shall be divided between the object-glass and the eye-glass, almost any given distance (within certain limits) between the first and its object may be secured. This is one valuable peculiarity of the compound instrument; and another is the large field, or large angle of view, which may be obtained, every part of which will be nearly equally good; whereas with the best simple microscopes the field is small, and is good only in the centre.

"For upwards of a century-as we have shown in our historythe compound microscope, notwithstanding the advantages above mentioned, was a comparatively feeble and inefficient instrument, owing to the distance which the light had to traverse, and the consequent increase of the chromatic and spherical aberrations. Indeed, the objectglass would not form one image, as has been supposed, but an infinite number of variously-coloured and various-sized images, occupying a midway space between the two glasses. Those nearest the objectglass would be red, anc. those nearest the eye-glass would be blue.

"The effect of this is to produce so much confusion, that the instru- 
ment was reduced to a mere toy, although these errors were diminished to the utmost possible extent by limiting the aperture of the objectglass, and thus restricting the angle of the pencil of light from each point of the object. But this involved the defects already explained of making the picture obscure; so that, on the whole, the best compound instruments were inferior to the simple microscopes of a single lens, with which, indeed, all the important observations of the last century were made.

"Even after the improvement of the simple microscopes by the use of doublets and triplets, the long course of the rays, and the large angular pencil required in the compound instrument, deterred the most sanguine from anticipating the period when they should be conducted through such a path free both from spherical and chromatic errors. Within twenty years of the present period, philosophers of no less eminence than M. Biot and Dr. Wollaston predicted that the compound would never rival the simple microscope, and that the idea of making achromatic object-glasses was hopeless. Nor can these opinions be wondered at, when we consider how many years the achromatic telescope had existed without an attempt to apply its principles to the compound microscope. When we consider the smallness of the pencil required by the telescope, and the enormous increase of difficulty attending every enlargement of the pencil; when we consider further that these difficulties had to be contended with and removed by operations on portions of glass so small that they are themselves almost microscopic objects, - we shall not be surprised that even a cautious philosopher and most able manipulator like Dr. Wollaston should prescribe limits to improvement.

"Fortunately for scienee, and especially for the departments of animal and vegetable physiology, these predictions have been shown to be unfounded. The last fifteen years have sufficed to elevate the compound microscope from the condition we have described to that of being the most important instrument ever bestowed by art upon the investigator of nature. It now holds a very high rank among philosophical instruments; while the transcendent beauties of form, colour, and organisation which it reveals to us in the minute works of nature, render it subservient to the most delightful and instructive pursuits. To these claims on our attention it appears likely to add a third of still higher importance. The microscopic examination of the blood and other human organic matter will, in all probability, afford more satisfactory and conclusive evidence regarding the nature and seat of disease than any hitherto appealed to, and will, of consequence, lead to similar certainty in the choice and application of remedies." 
Mr. Lister, as before stated, first set about investigations which have ultimately proved of the utmost value. The results arrived at by him were published in the Philosophical Transactions; and the principles applied and exhibited by Mr. Hugh Powell and Mr. Andrew Ross, with an unlooked-for degree of success. It is due to the late Mr. Tulley to say, that he constructed an achromatic object-glass of nine-tenths of an inch focal length, composed of three lenses, transmitting a pencil of eighteen clegrees; and as regards accurate correction throughout the field, his combination has not been excelled by any subsequent set of three lenses. By Mr. Lister's combination he was enabled to produce lenses which transmitted a pencil of fifty degrees with a large field correct in every part. In the paper referred to above he enters into many interesting particulars, which, however, are not necessary to the comprehension of our subject. Mr. Ross presented to the Society of Arts, in 1837, a paper on the subject, which was published in the 5lst volume of their Transactions. This being essential to a full understanding of the ultimate refinements of the instrument, we give it in full:

"In the course of a practical investigation, with the view of constructing a combination of lenses for the object-glass of a compound microscope which should be free from the effects of aberration, both for central and oblique pencils of great angle, I obtained the greatest possible distance between the object and object-glass; for in objectglasses of short focal length, their closeness to the object has been an obstacle, in many cases, to the use of high magnifying powers, and is a constant source of inconvenience.

"In the improved combination the diameter is only sufficient to admit the proper pencil; the convex lenses are wrought to an edge, and the concave have only sufficient thickness to support their figure; consequently the combination is the thinnest possible, and it follows that there will be the greatest distance between the object and the object-glass. The focal length is $\frac{1}{8}$ of an inch, having an angular aperture of $60^{\circ}$, with a distance of $\frac{1}{25}$ of an inch, and a magnifying power of 970 times linear, with perfect definition on the most difficult Podura scales. I have made object-glasses $\frac{1}{16}$ of an inch focal length; but as the angular aperture cannot be advantageously increased if the greatest distance between the object and object-glass is preserved, their use will be very limited.

"The quality of the definition produced by an achromatic compound microscope will depend upon the accuracy with which the aberrations, both chromatic and spherical, are balanced, together with the general perfection of the workmanship. Now in Wollaston's doublets and Holland's triplets there are no means of producing a balance of the 
aberrations, as they are composed of convex lenses only; therefore the best thing that can be done is to make the aberrations a minimum. The remaining positive aberration in these forms produces its peculiar effect upon objects (particularly the detail of the thin transparent class), which may lead to misapprehension of their true structure; but with the achromatic object-glass, where the aberrations are correctly balanced, the most minute parts of an object are accurately displayed, so that a satisfactory judgment of their character may be formed. When an object has its aberrations balanced for viewing an opaque object, and it is required to examine that object by transmitted light, the correction will remain; but if it is necessary to immerse the object in a fluid, or to cover it with glass, an aberration arises from these circumstances which will disturb the previous correction, and consequently deteriorate the definition; and this defect will be more obvious with the increase of distance between the object and object-glass.

"If an object-glass is constructed as represented in fig. 26, where the posterior combination $p$ and the middle $m$ have together an excess of negative aberration, and if this be corrected by the anterior combi- $p$ nation $a$ having an excess of positive aberration, then this latter combination can be made to act more or less powerfully upon $p$ and $m$, by making it approach to or recede from them; for when the three act in close contact, the distance of the object from the object-glass is greatest, and consequently the rays from the object are diverging from a point at a greater distance than when the combinations are separated; and as a lens bends the rays more, or acts with

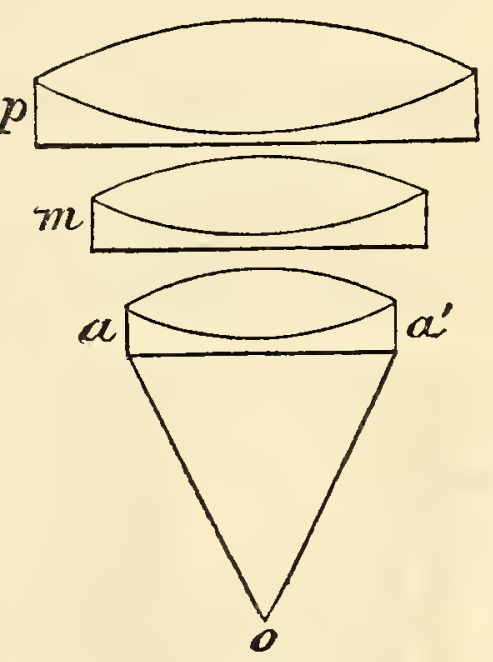

fig. 26. greater effect, the more distant the object is from which the rays diverge, the effect of the anterior combination $a$ upon the other two, $p$ and $m$, will vary with its distance from thence.

"When, therefore, the correction of the whole is effected for an opaque object, with a certain distance between the anterior and middle combination, if they are then put in contact, the distance between the object and object-glass will be increased; consequently the anterior combination will act more powerfully, and the whole will have an excess of positive aberration. Now the effect of the aberration produced by a piece of flat and parallel glass being of the negative character, it is obvious that the above considerations suggest the means of correction, by moving the lenses nearer together, till the positive aberration 
thereby produced balances the negative aberration caused by the medium.

"The preceding refers only to the spherical aberration; but the effect of the chromatic is also seen when an object is covered with a piece of glass : for in the course of my experiments I observed that it produced a chromatic thickening of the outline of the Podura and other delicate scales ; and if diverging rays near the axis and at the margin are projected through a piece of flat parallel glass, with the various indices of refraction for the different colours, it will be seen that each ray will emerge, separated, into a beam consisting of the component colours of the ray, and that each beam is widely different in form. This difference being magnified by the power of the microscope, readily accounts for the chromatic thickening of the outline just mentioned. Therefore, to obtain the finest definition of extremely delicate and minute objects, they should be viewed without a covering: if it be desirable to immerse them in a fluid, they should be covered with the thinnest possible film of talc, as, from the character of the chromatic aberration, it will be seen that varying the distances of the combinations will not sensibly affect the correction; though object-lenses may be made to include a given fluid, or solid medium, in their correction for colour.

"The mechanism for applying these principles to the correction of an object-glass under the various circumstances is represented in fig. $2 \pi$,

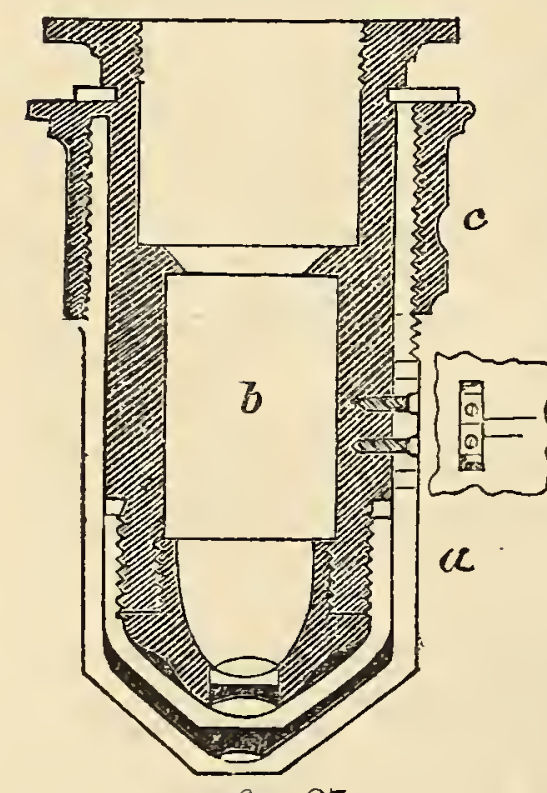

fig. 27 . where the anterior lens is set in the end of a tube $a$, which slides on the cylinder $b$, containing the remainder of the combination; the tube $a$, holding the lens : nearest the object, may then be moved upon the cylinder $b$, for the purpose of varying the distance, according to the thickness of the glass covering the object, by turning the screwed ring $c$, or more simply, by sliding the one on the other, and clamping them together when adjusted. An aperture is made in the tube $a$, within which is seen a mark engraved on the cylinder; and on the edge of which are two marks, a longer and a shorter, engraved upon the tube. When the mark on the cylinder coincides with the longer mark on the tube, the adjustment is perfect for an uncovered object; and when the coincidence is with the short mark, the proper distance is obtained to balance the aberrations produced by glass the hundredth of an inch thick, and such gliss can be readily supplied. This adjustment should 
be tested experimentally by moving the milled edge, so as to separate or close together the combinations, and then bringing the object to distinct vision by the screw adjustment of the microscope. In this process the milled edge of the object-glass will be employed to adjust for character of definition, and the fine screw movement of the microscope for correct focus.

"It is hardly necessary to observe, that the necessity for this correction is wholly independent of any particular construction of the objectglass, as in all cases where the object-glass is corrected for an object uncovered, any covering of glass will create a different value of aberration to the first lens, which previously balanced the aberration resulting from the rest of the lenses; and as this disturbance is effected at the first refraction, it is independent of the other part of the combination. The visibility of the effect depends on the distance of the object from the object-glass, the angle of the pencil transmitted, the focal length of the combination, the thickness of the glass corering the object, and the general perfection of the corrections for chromatism and the oblique pencils.

"With this adjusting object-glass, therefore, we can have the requisites of the greatest possible distance between the object and objectglass, an intense and sharply-defined image throughout the field, from the large pencil transmitted, and the accurate correction of the aberrations; also, by the adjustment, the means of preserving that correction under all the varied circumstances in which it may be necessary to place an object for the purpose of observation."

Fig. 28 represents the body of one of Mr. Ross's compound microscopes with the triple object-glass, where $o$ is an object; and above it is seen the triple achromatic object-glass, in connection with the eye-piece $e e, f f$ the plano-convex lens ; $e e$ being the eye-glass, and $f f$ the field-glass, and between them, at $b b$, a dark spot or diaphragm. The course of the light is shown by three rays drawn from the centre, and three from each end of the object $o$; these rays, if not pre-

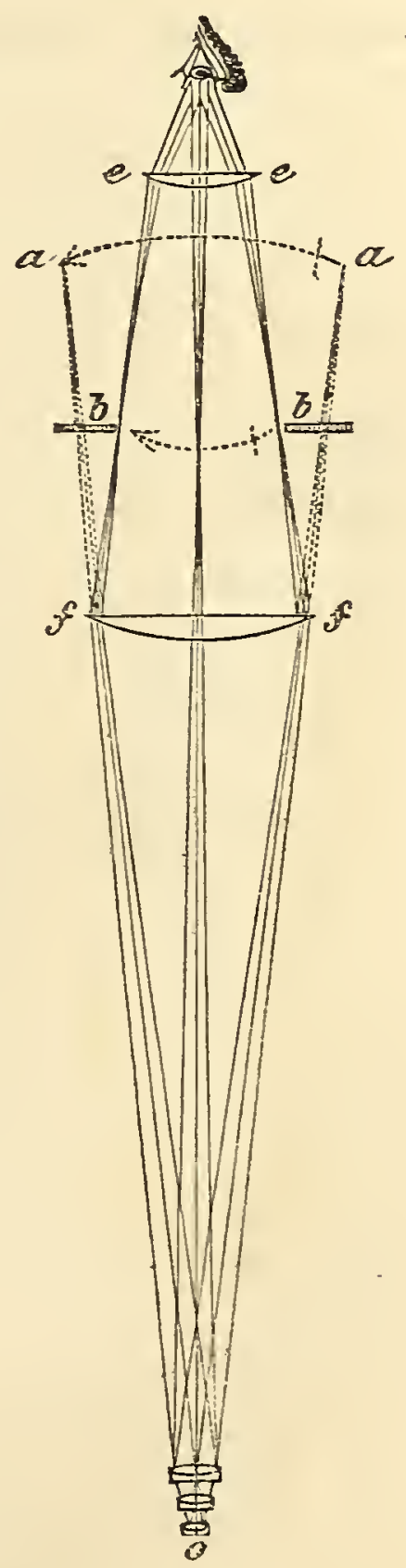

fig. 28. vented by the lens $f f$, or the diaphragm at $b \vec{b}$, would form an image 
at $a a$; but as they meet with the lens $f f$ in their passage, they are converged by it and meet at $b b$, where the diaphragm is placed to intercept all the light except that required for the formation of a perfect image ; the image at $b b$ is further magnified by the lens $e e$, as if it were an original object. The triple achromatic combination constructed on Mr. Lister's improved plan, although capable of transmitting large angular pencils, and corrected as to its own errors of spherical and chromatic aberration, would, nevertheless, be of little service without an eye-piece of peculiar construction.

"If we stopped here," says Mr. Ross, "we should convey a very imperfect idea of the beautiful series of corrections effected by the eyepiece, and which were first pointed out in detail in a paper on the subject, published by Mr. Varley, in the fifty-first volume of the Transactions of the Society of Arts. The eye-piece in question was invented

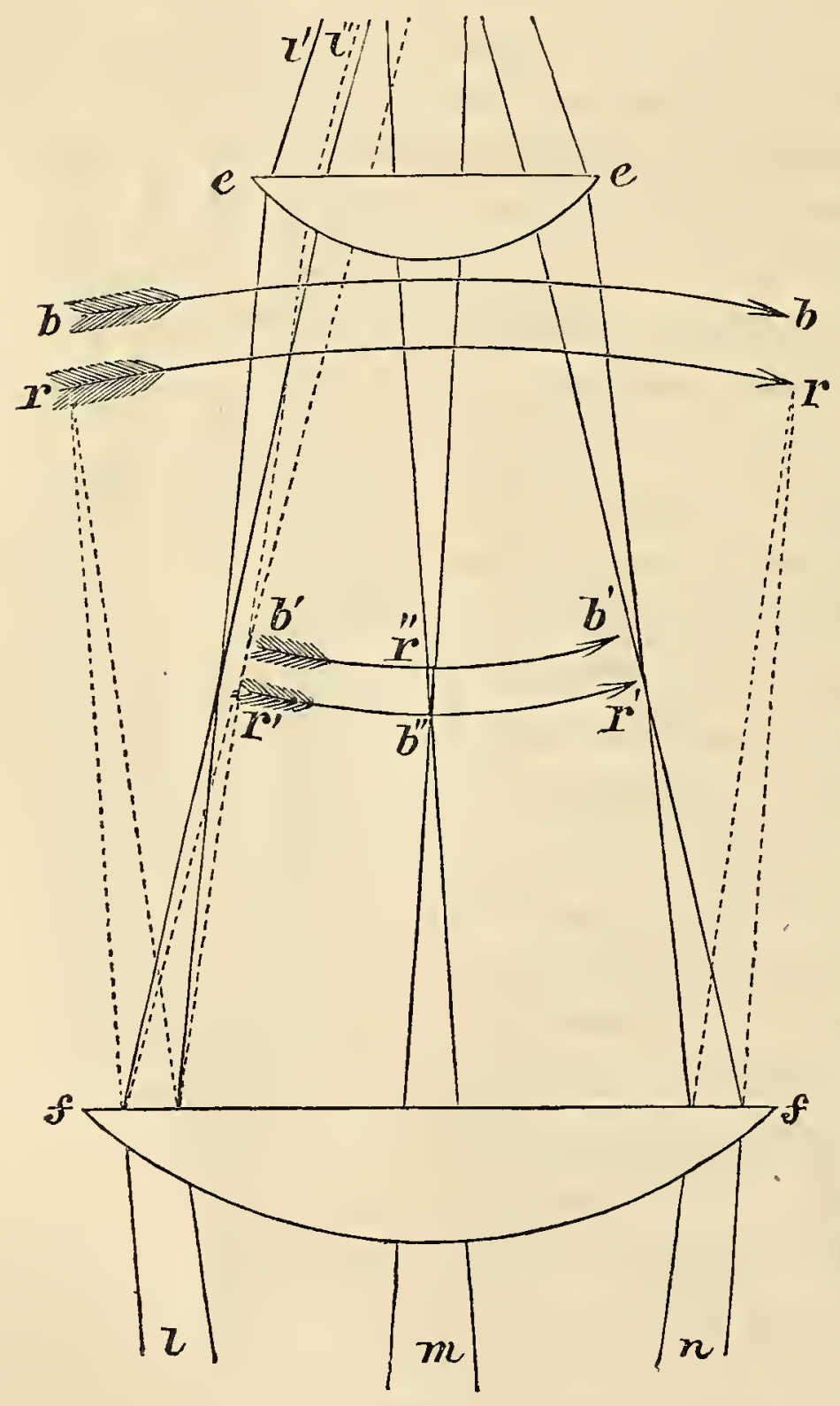

fig: 29 . by Huyghens for telescopes, with no other view than that of diminishing the spherical aberration by producing the refractions at two glasses instead of one, and of increasing the field of view. It consists of two plano-convex lenses, with their plane sides towards the eye, and placed at a distance apart equal to half the sum at their focal lengths, with a stop or diaphragm placed midway between the lenses. Huyghens was not aware of the value of his eye-piece; it was reserved for Boscovich to point out that he had, by this important arrangement, accidentally corrected a great part of the achromatic aberration. Let fig. 29 represent the Huyghenian eye-piece of a microscope, $f f$ being the field-glass, and $e e$ the eyeglass, and $\operatorname{lm} n$ the two extreme rays of each of the three pencils emanating from the centre 
and ends of the object, of which, but for the field-glass, a series of coloured images would be formed from $r r$ to $b b$; those near $r \cdot r$ being: red, those near $b b$ blue, and the intermediate ones green, yellow, and so on, corresponding with the colours of the prismatic spectrum. This order of colours is the reverse of that of the common compound microscope, in which the single object-glass projects the red image beyond the blue.

"The effect just described, of projecting the blue image beyond the red, is purposely produced for reasons presently to be given, and is called over-correcting the object-glass as to colour. It is to be observed also, that the images $b b$ and $r r$ are curved in the wrong direction to be distinctly seen by a convex eye-lens, and this is a further defect of the compound microscope of two lenses. But the field-glass, at the same time that it bends the rays and converges them to foci at $b^{\prime} b^{\prime}$ and $r^{\prime} r^{\prime}$, also reverses the curvature of the images as there shown, and gives them the form bcst adapted for distinct vision by the eyeglass $e e$. The field-glass has at the same time brought the blue and red images closer together, so that they are adapted to pass uncoloured through the eye-glass. To render this important point more intelligible, let it be supposed that the object-glass had not been over-corrected, that it had been perfectly achromatic; the rays would then have become coloured as soon as they had passed the field-glass: the blue rays, to take the central pencil, for example, would converge at $b^{\prime \prime}$, and the red rays at $r^{\prime \prime}$, which is just the reverse of what the eyelens requires; for as its blue focus is also shorter than its red, it would demand rather that the bluc image should be at $r^{\prime \prime}$, and the red at $b^{\prime \prime}$. This effect we have shown to be produced by the over-correction of the object-glass, which protrudes the blue foci $b b$ as much beyond the red foci $r r$ as the sum of the distances between the red and the blue foci of the field-lens and eye-lens; so that the scparation $b r$ is exactly taken up in passing through those two lenses, and the whole of the colours coincide as to focal distance as soon as the rays have passed the eyelens. But while they coincide as to distance, they differ in another respect,-the blue images are rendered smaller than the red by the superior refractive power of the field-glass upon the blue rays. In tracing the pencil $l$, for instance, it will be noticed that, after passing the fieldglass, two sets of lines are drawn, one whole and one dotted, the former representing the red, and the latter the blue rays. This is the accidental effect in the Huyghenian eye-piece pointed out by Boscovich. The separation into colours of the field-glass is like the over-correction of the object-glass,--it leads to a subsequent complete correction. For 
if the differently coloured rays were kept together till they reached the eye-glass, they would then become coloured, and present coloured images to the eye; but fortunately, and most beautifully, the separation effected by the field-glass causes the blue rays to fall so much nearer the centre of the eye-glass, where, owing to the spherical figure, the refractive power is less than at the margin, that that spherical error of the eye-lens constitutes a nearly perfect balance to the chromatic dispersion of the field-lens, and the blue and red rays $l^{\prime}$ and $l^{\prime \prime}$ emerge sensibly parallel, presenting, in consequence, the perfect definition of a single point to the eye. The same reasoning is true of the intermediate colours and of the other pencils."

From what has been stated, it is obvious what we mean by an achromatic object-glass : one in which the usual order of dispersion is so far reversed, that the light, after undergoing the singularly beautiful series of changes effected by the eye-piece, shall come uncoloured to the eye.

The Huyghenian eye-piece, which we have described, is the best for merely optical purposes; but when it is required to measure the magnified image, we use the eye-piece invented by Mr. Ramsden, and called, from its purpose, the micrometer eye-piece. When it is stated that we sometimes require to measure portions of animal or vegetable matter a hundred times smaller than any divisions that can be artificially made on any measuring instrument, the advantage of applying the scale to the magnified image will be obvious, as compared with the application of engraved or mechanical micrometers to the stage of the instrument.

The arrangement may be readily understood upon reference to fig. 30. The eye and field glasses have now their plane faces turned

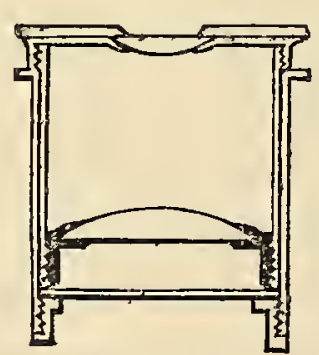

nig. 30 .

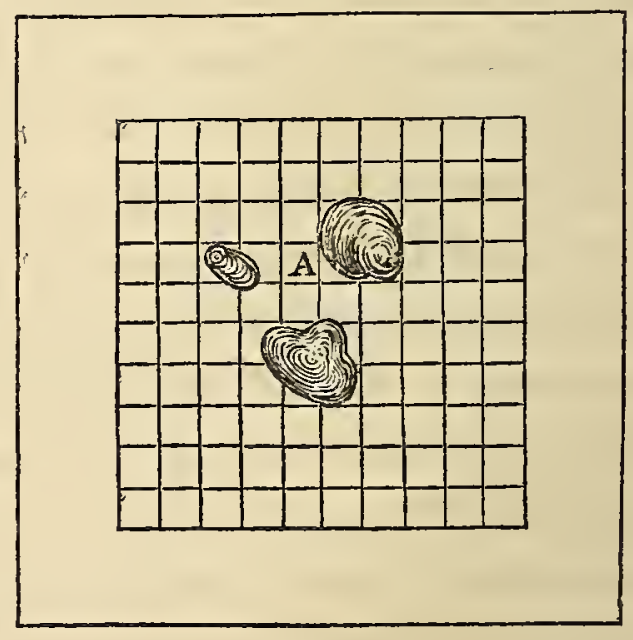

fig. 31.

towards the object; the rays from the object are made to converge 
immediately in front of the field-glass; and here is placed a plane-glass ou which are engraved divisions of 1-100th of an inch or less. The markings of these divisions come into focus, therefore, at the same time as the image of the object, and both are distinctly seen together. The glass with its dvisions is shown in fig. 31 , and at it are seen some magnified grains of starch. Thus the measure of the magnified image is given by mere inspection; and the value of such measures, in reference to the real object, may be obtained thus, which, when once obtained, is constant for the same object-glass.

Mr. Lister placed on the stage of his instrment a divided scale, the value of which was known; and viewing the scale as the microscopic object, observed how many of the divisions on the scale attached to the eye-piece corresponded with one of those in the magnified image. If, for instance, ten of those in the eye-piece correspond with one of those in the image, and if the divisions are known to be equal, then the image is ten times larger than the object, and the dimensions of the object are ten times less than indicated by the micrometer. If the divisions on the micrometer and on the magnified scale were not equal, it becomes a mere rule of-three sum; but in general this trouble is taken by the maker of the instrument, who furnishes a table showing the value of each division of the micrometer for every object-glass with which it may be used.

While on the subject of measuring, it may be well to explain the mode of ascertaining the magnifying power of the compound microscope, which is generally taken on the assumption before mentioned, that the naked eye sees most distinctly at the distance of ten inches.

Place on the stage of the instrument, as before, a known divided scale, and when it is distinctly, seen, hold a rule at ten inches distance from the disengaged eye, so that it may be seen by that eye overlapping or lying by the side of the magnified picture of the other scale; then move the rule till one or more of its known divisions correspond with a number of those in the magnified scale, and a comparison of the two gives the magnifying power.

Mr. Jackson has adopted a: simpler and cheaper form of micrometer, represented in fig. 32, which is thus described by him in the Microscopical Society's Transactions: "It consists of a slip of glass. placed in the focus of the eye-glass, and can be used with the divisions sufficiently fine to have the value of the ten-thousandth of an: inch with the quarter-inch object-glass, and the twenty-thousandth with the eighth; and at the same time the half, or even the quarter of a. division may be estimated, thus affording the means of attaining all 
the accuracy that is really available. It may therefore entirely supersede the more complicated and expensive screw-micrometer, being

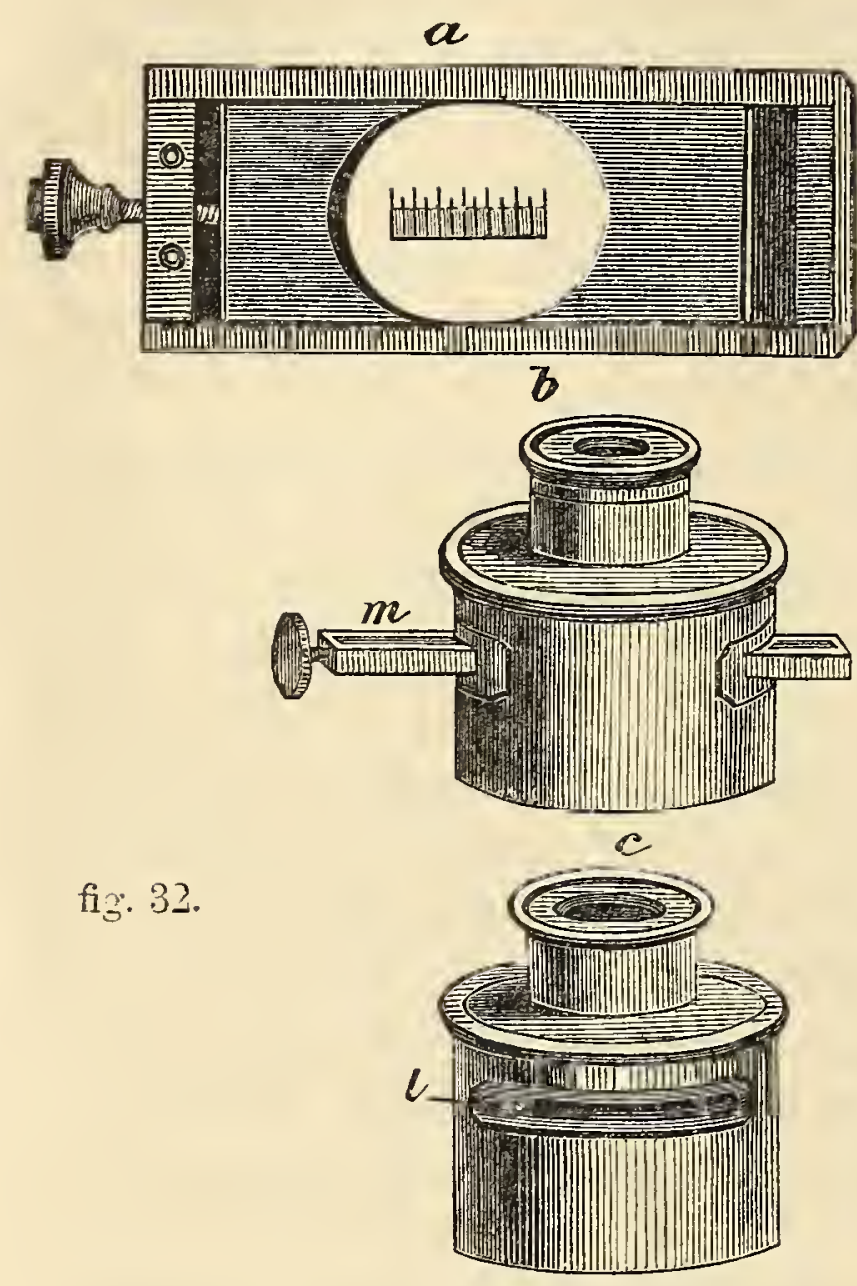
much handier to use, and not liable to derangement in inexperienced hands.

"The positive eye-piece gives the best view of the micrometer, the negative of the object. The former is quite free from distortion, even to the edges of the field; but the object is slightly coloured. The latter is free from colour, but is slightly distorted at the edges. In the centre of the field, however, to the extent of half its diameter, there is no perceptible distortion; and the clearness of the definition gives a precision to the measurement which is very satisfactory. For this reason I give it the preference. I will now proceed to describe the micrometer.

"Short bold lines are ruled on a piece of glass, $a$, fig. 32 ; and to facilitate counting, the fifth is drawn longer, and the tenth still longer, as in the common rule. Very finely levigated plumbago is rubbed into the lines, to render them visible; and they are covered with a piece of thin glass, cemented by Canada balsam, to secure the plumbago from being wiped out. The slip of glass thus prepared is placed in a thin brass frame, so that it may slide freely; and is acted on at one end by a pushing-screw, and at the other by a slight spring.

"Slits are cut in the negative eye-piece on each side, $b$, fig. 32 , so that the brass frame may be pressed across the field in the focus of the eye-glass, as at $m$; the cell of which should have a longer screw than usual, to admit of adjustment for different eyes. The brass frame is retained in its place by a spring within the tube of the eye-piece; and in using it, the object is brought to the centre of the field by the stage movements; and the coincidence between one side of it and one of the long lines is made with great accuracy by means of the small pushingscrew that moves the slip of glass. The divisions are then read off as easily as the inches and tenths on a common rule. The operation 
indeed is nothing more than the laying a rule across the body to be measured; and it matters not whether the object be transparent or opaque, mounted or not mounted; if its edges can be distinctly seen, its diameter can be taken.

"Previously, however, to using the micrometer, the value of the divisions should be ascertained with each object-glass; the mode of doing which I will explain more fully.

"Lay a slip of ruled glass on the stage, and having turned the eyepiece so that the lines on the two glasses are parallel, read off the number of divisions in the eye-piece which cover one on the stage. Repeat this process with different portions of the stage-micrometer, and if there be any difference, take the mean. Suppose the hundredth of an inch on the stage requires eighteen divisions in the eye-piece to cover it; it is quite plain that an inch would require eighteen hundred, and an object which occupied nine of these divisions would measure the two-hundredth of an inch. This is the common mode of expressing microscopical measurements; but $I$ am of opinion that a decimal notation would be preferable, if universally adopted. Take the instance supposed, and let the microscope be furnished with a draw-tube, marked on the side with inches and tenths. By drawing this out a short distance, the image of the stage-micrometer may be expanded until one division is covered by twenty in the eye-piece. These will then have the value of two-thousandths of an inch, and the object which before measured nine will then measure ten; which, divided by 2000 , gives the decimal fraction $\cdot 005$.

"Enter in a table the length to which the tube is drawn out, and the number of divisions on the eye-piece micrometer equivalent to an inch on the stage, and any measurements afterwards taken with that micrometer and object-glass may, by a short process of mental arithmetic, be reduced to the decimal parts of an inch, if not actually observed in them.

"In ascertaining the value of the micrometer with a deep objectglass, the hundredth of an inch on the stage will occupy too much of the field; the two-hundredth or five-hundredth should then be used, and the number of divisions corresponding to that quantity be multiplied by two hundred or five hundred, as the case may be.

"The micrometer should not be fitted into too deep an eye-piece, for it is essential to preserve clear definition. The middle eye-piece of the London-made instruments is for most purposes the best, provided the object-glass be of the first quality; otherwise the eye-piece of lowest power answers better. The lens above the micrometer should not be 
of shorter focus than three-quarters of an inch, even with the best object-glasses.

"It is almost needless to remark, that the eye-piece is not injured by the slit cut in the tube, as this can be closed at any time by a small sliding bar, as at $l$, fig. 32 ; consequently an additional one is not necessary, and the expense is confined to the slip of ruled glass and the fittings. The means, therefore, of measuring minute objects with the utmost accuracy can be applied to any compound microscope for a few shillings. The value of the micrometer should be found with the objectglass set both for uncovered and covered objects; and during this operation the screw of the focal fine adjustment should be placed about midway between the two extremes."

We subjoin the following table of comparative micrometrical measures given by Dr. Hannover, as it may be useful for reference.

\begin{tabular}{|c|c|c|c|c|}
\hline Millemetre. & Paris lines. & Vienna lines. & Rhenish lines. & English inch. \\
\cline { 2 - 3 } & 0.443296 & 0.4555550 & 0.458813 & 0.0393708 \\
2.255829 & 1 & 1.027643 & 1.035003 & 0.0388138 \\
2.195149 & 0.9731 .01 & 1 & 1.9071625 & 0.0864248 \\
2.179538 & 0.966181 & 0.992888 & 1 & 0.0858101 \\
25.39954 & 11.25952 & 11.57076 & 11.65364 & 1 \\
\hline
\end{tabular}

The wonderful tracing on glass, executed by M. Nobert, of Barth, in Prussia, deserves especial notice. The plan adopted by him is, to trace on glass ten separate bands at equal distances from each other, each band being composed of parallel lines of some fraction of a Prussian inch apart; in some they are 1-1000th, and in others only 1-4000th of a Prussian inch separated. The distance of these parallel lines forms part of a geometric series:

0.001000 lines
0.000857,
0.000735,
0.000630,
0.000540,

0.000463 lines.
$0000397 "$,
$0.000340 "$,
$0.000292 "$,
0.000225,

To see these lines at all, it is requisite to use a microscope with a magnifying power of 100 diameters; the bands containing the fewest number of lines will then be visible. To distinguish the finer lines, it will be necessary to use a magnifying power of 2000 , and then the lines which are only 1-47000th of an inch apart will be seen as perfectly traced as the coarser lines. Of all the tests yet found for object-glasses 
of high power, these would seem the most valuable. These tracings have tended to confirm the undulating theory of light, the different colours of the spectrum being exhibited in the ruled spaces according to the separation of the lines; and in those cases where the distance between the lines are smaller than the length of the violet-coloured waves, no colour is perceived; and it is stated, that if inequalities amounting to .000002 line occur in some of the systems, stripes of another colour would appear in them.

The Eye-piece.-To the most complete instruments a set of eyepieces, consisting of three, is generally made. These differ in power; the longest is always the lowest power, and is marked $\mathbf{A}$. Its angular aperture, which determines the size of the field of view, is generally less than that of the others (if constructed on the Huyghean plan), being limited by the diameter of the body. It is usually a little under 30 degrees. The next eye-piece, or middle power, marked $\mathrm{B}$, and the deepest, c, have more than 30 degrees of angular aperture.

For viewing thin sections of recent or fossil woods, coal, the fructification of ferns and mosses; fossil-shells, seeds, small insects, or parts of larger ones; molusca, or the circulation in the frog, \&c., the eye-piece $\mathrm{A}$ is best adapted.

For examining the details of any of the above objects, it will be advisable to substitute the eye-piece $B$, which also should be used in the observation of crystals when illuminated by polarised light, the pollen of flowers, minute dissections of insects, the vascular and cellular tissues of plants, the Haversian canals and lacunæ of bone, and the serrated laminæ of the crystalline lens in the eyes of birds and fishes.

The eye-piece $\mathrm{C}$ is of use when it is requisite to investigate the intimate structure of delicate tissues; and also in the observation of polygastric infusoria, volvox globata, scales from moths' wings, and the raphides found in certain plants, \&c. The employment of this eye-piece, when higher power is required, obviates the necessity of using a deeper object-glass, which always occasions a fresh arrangement of the illumination and focus. It must be borne in mind, that the more powerful the eye-piece, the more apparent will the imperfections of the object-glass become; hence less confidence should be placed in the observations made under a powerful eye-piece, than when a similar degree of amplification is obtained with a shallow one and a deeper object-glass. Achromatic object-glasses for microscopes are of various foci, differing from 2 inches to 1 -16th of an inch. 
Magnifying Power of Mr. Ross's Object-Glasses with his various Eye-Glasses.

\begin{tabular}{|c|c|c|c|c|c|c|}
\hline \multirow{2}{*}{ EYE-GLASSES. } & \multicolumn{6}{|c|}{ ОВJECT-GLASSES. } \\
\hline & 1-inch. & 2-inch. & $\frac{1}{2}$-inch. & $\frac{1}{4}$-inch. & $\frac{1}{8}$-inch. & $\frac{1}{12}$-inch. \\
\hline A & 20 & 60 & 100 & 220 & 420 & 600 \\
\hline B & 30 & 80 & 130 & 350 & 670 & 870 \\
\hline $\mathrm{C}$ & 40 & 100 & 180 & 500 & 900 & 1200 \\
\hline $\begin{array}{l}\text { Value of each } \\
\text { space in the Micro- } \\
\text { meter eye-glass, } \\
\text { with the various } \\
\text { object-glasses. }\end{array}$ & $\begin{array}{c}\frac{3}{400} \\
\cdot 0025\end{array}$ & $\begin{array}{c}\overline{9}^{\frac{1}{70}} \\
\cdot 001031\end{array}$ & $\begin{array}{c}\frac{\lambda}{1900} \\
\cdot 0005263\end{array}$ & $\begin{array}{c}\frac{1}{4 \frac{3}{300}} \\
\cdot 0002325\end{array}$ & $\begin{array}{c}\frac{1}{90 \overline{0} 0} \\
\cdot 0001111\end{array}$ & $\begin{array}{c}\frac{2}{13500} \\
\cdot 000074\end{array}$ \\
\hline
\end{tabular}

\section{Mechanical Arrangements.}

Having now explained the optical principles of the achromatic compound microscope, it remains only to describe the mechanical and accessory arrangements for giving those principles their full effect. The mechanism of a microscope is of much more importance than might be imagined by those who have not studied the subject. In the first place, steadiness, or freedom from vibration, and most particularly freedom from any vibrations which are not equally communicated to the object under examination and to the lenses by which it is viewed, is a point of the utmost consequence. When, for instance, the body containing the lenses is screwed by its lower extremity to a horizontal arm, we have one of the most vibratory forms conceivable; it is precisely the form of the inverted pendulum, which is expressly calculated to indicate otherwise insensible vibrations. The tremor necessarily attendant on such an arrangement is magnified by the whole power of the instrument; and as the object on the stage partakes of this tremor in a comparatively insensible degree, the image is seen to oscillate so rapidly, as in some cases to be wholly indistinguishable. Such microscopes cannot possibly be used with high powers in ordinary houses abutting on any paved streets through which carriages are passing; nor, indeed, are they adapted to be used in houses in which the ordinary internal sources of shaking exist. 
One of the best modes of mounting a compound microscope is that shown, fig. 33, in one of Mr. Ross's smaller instruments, which, although it does not exhibit all the details, will serve to explain the chief features of the arrangement.

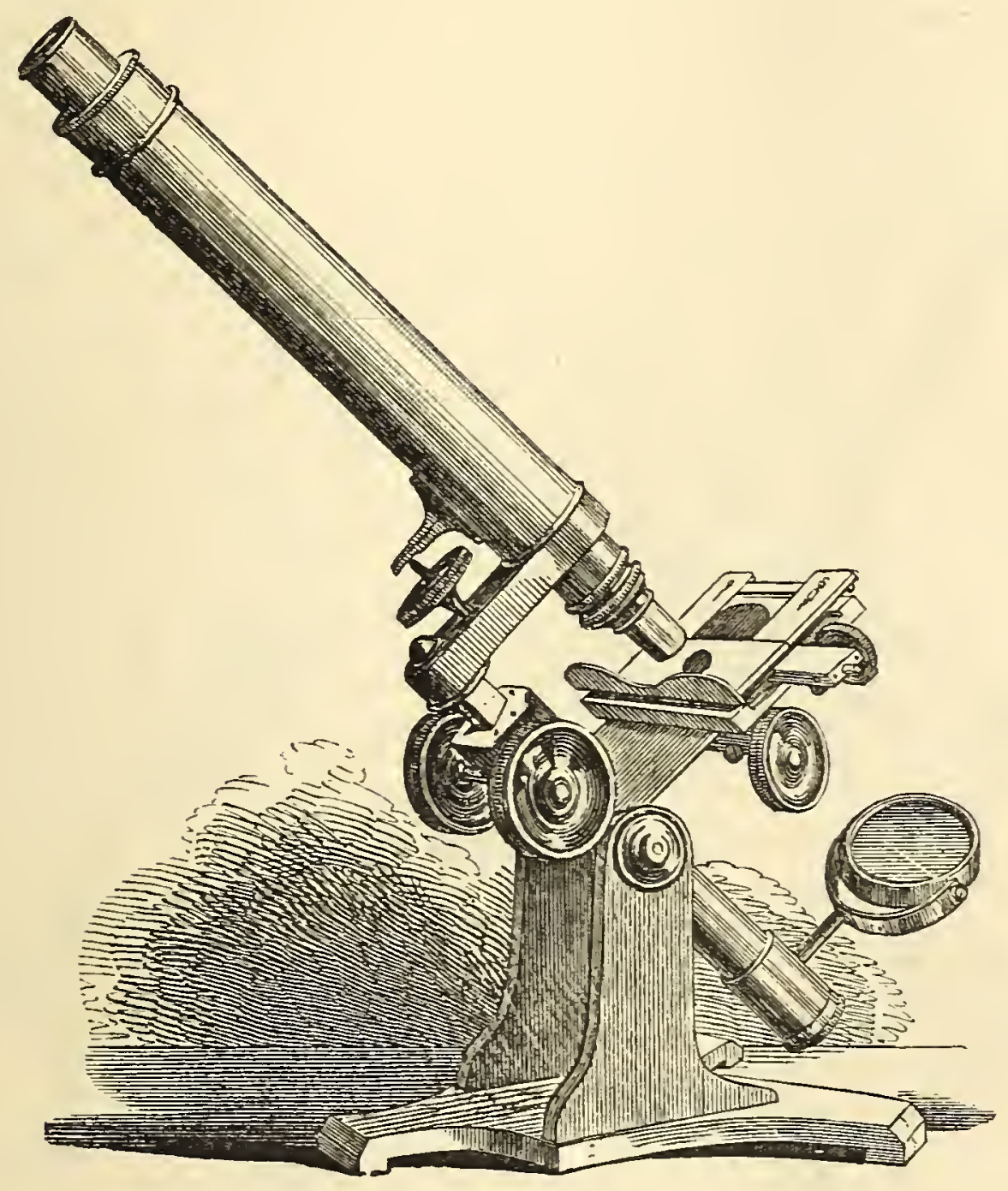

fig. 33. ROSS'S SMALL COMPOUND MIICROSCOPE.

The compound microscope was first described by Mr. Ross, in the London Physiological Journal, in 1843.

"The mechanical construction," says Mr. Ross, "is derived from a practical acquaintance with the various improvements made in the microscope for many years. The general arrangement, which is properly the province of the mechanic, has been contrived to obtain the utmost freedom from tremor, and to afford the greatest facility in using the various movements; while the extent, direction, and number of these have been collected from the experience of the most indefatigable observers in all the rarious branches of microscopic inquiry. 
"The optical part, also, has arrived at such perfection, that points or lines whose distance is such that their separation is bordering on

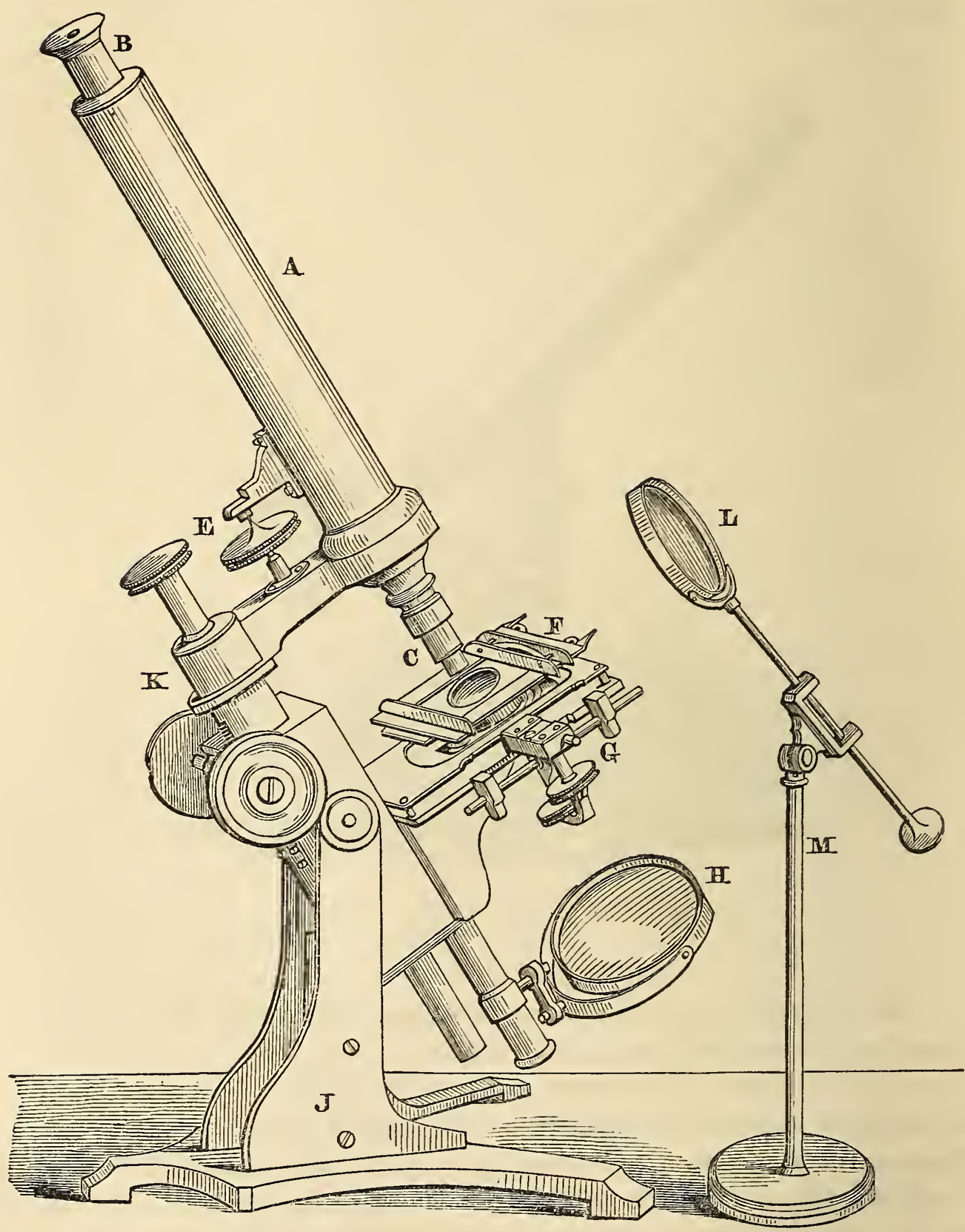


interfering with the physical constitution of light can be distinctly separated, thus insuring a reality in the appearance of objects where the minuteness of the detail approaches the natural limit of microscopic vision."

In the larger instrument, fig. 3t, two uprights, $\mathrm{J}$, are strengthened by two internal buttresses mounted on a strong tripod; at the upper part, and between the uprights, we have an axis, upon which the whole of the upper part of the instrument turns, so as to enable it to take a horizontal or vertical position, or any intermediate inclination,-such, for instance, as that shown in the drawing. This movable part is fixed to the axis near its centre of gravity, and consists of the stage FG; the arm $\mathrm{K}$ is screwed into the triangular bar, and carries the microscope tube or hody $\mathrm{A}$, at the apper end of which is the eye-piece $\mathrm{B}$, and at the lower the object-glasses $\mathrm{C}$. The stage $\mathrm{F} G$ has rectangular movements one inch in extent on the racket-cylinders, and are moved by the pinions connected with the milled-heads at $G$. The triangular bar, together with the arm and microscope tube, is mored by the large milled-heads at $\mathrm{D}$; and a more delicate adjustment of this optical part is effected by the milled-head $\mathrm{E}$. The other milled-head fixes the arm $\mathrm{K}$ to the triangular bar. II is the mirror, which slides up or down the tube to which it is attached; standing near which is the condenser Ir, with its upright support of brass for holding the condensing-lens $\mathrm{L}$,- this is made to unscrew, for the convenience of packing. Such instruments are manufactured by Mr. Ross at prices varying from $10 l$. to $100 l$.

Mrr. Ross's small achromatic compound microscope, represented in fig. 33, like the larger one, is supported on a firm tripod base, from which rise two strong uprights, stipporting at their upper parts the trunnions to which the square frame, carrying the stage and triangular bar, with the body, are attached. Within the tube a smaller one is made to slide up and down by rack and pinion: this forms the coarse adjustment. The fine adjustment consists of a conical-pointed steel screw pressing against the top of a slit in the inner tube, to the end of which the adapter for receiving the object-glasses is fixed. The stage has the usual rectangular motions, that from the side being performed by a screw and nut, by turning the milled-head; whilst the up-and-down morement is performed by a rack and pinion, turned by the milledhead below the other. The stage-plate is provided with a sliding-rest, by which the distance of an object from the central hole in the plate may be regulated before foctissing: this is sometimes made to answer the purpose of the complicated sliding-frame in the more expensive instru- 
ments. At the upper part of this stage-plate there are two holes for the reception of the forceps and side reflector. To the under part of the stage, the achromatic condensers, the diaphragm-plate, and polarising-prism may all be adapted as in the larger instruments; and for convenience of package, the stage itself may be turned on a pivot, so as to be at right angles with the tube. The mirror is mounted in the usual manner, and is capable of being raised up or down the tube on which it is supported. This is particularly to be recommended

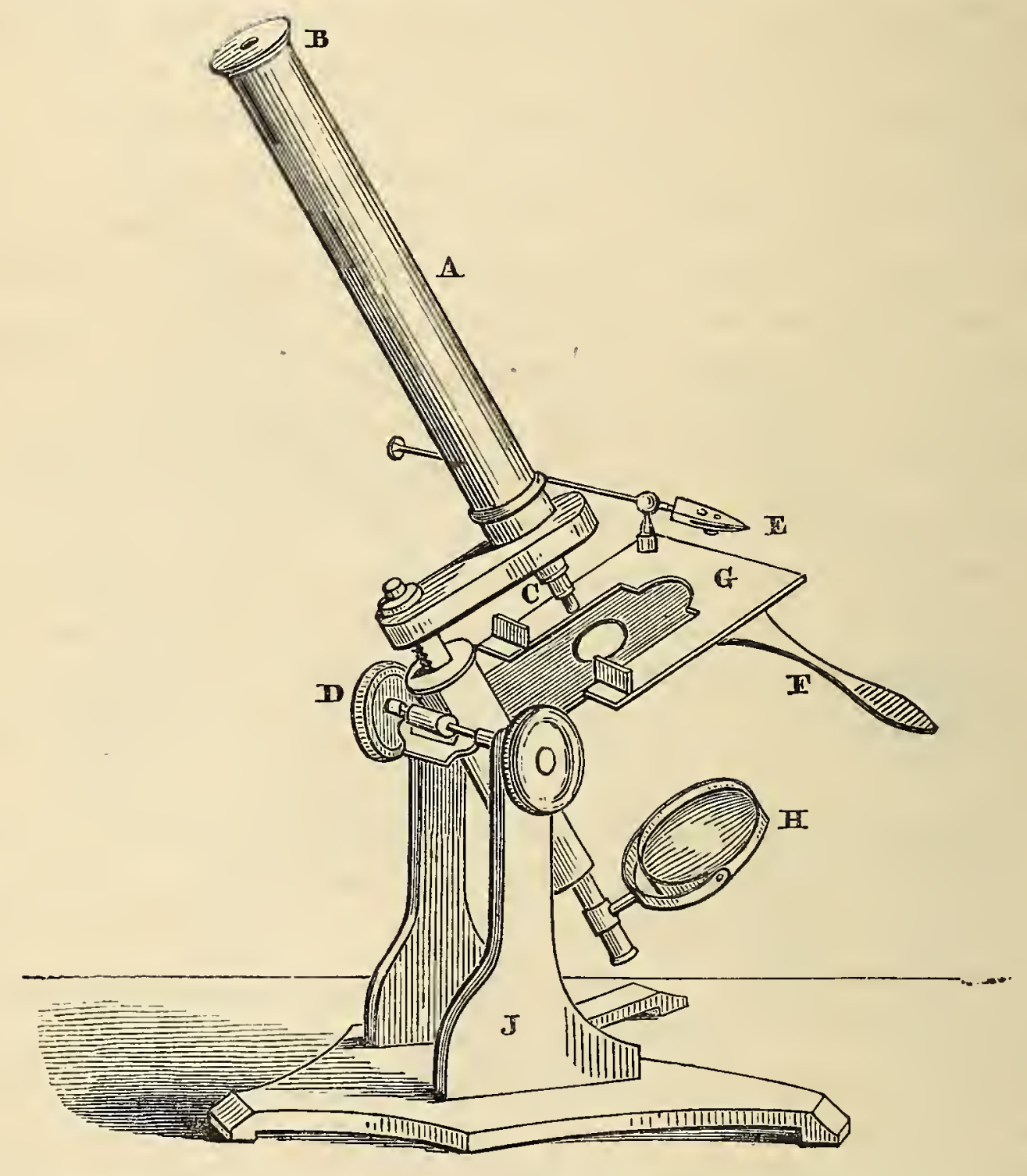

11]

fig. 35 .

to those "whose means are limited, in consequence of the low price, it being of a form which may be added to from time to time, according to the wants of the employer: thus, for instance, a vertical stand, with two eye-pieces, exclusive of the object-glasses, may be procured without the stage movements or the fine adjustment, at the 
small cost of $4 l .10 s$; and as both the stage and the compound body are of the same size as in the more perfect instrument, the fine adjustment and the stage movement may be added to the former at any time, and render it as complete.

A smaller compound achromatic microscope is seen in fig. 35, and, like the others described, is supported on a firm tripod base, into which are firmly screwed two strong uprights $J$, carrying the tube enclosing a triangular bar with rack-and-pinion motion, to which is screwed the brass arm with the body A screwed into it and the eye-piece $\mathrm{B}$; beneath the arm is seen the object-glass $\mathrm{C}$ : this is all moved by the milled-heads at $\mathrm{D}$; $\mathrm{G}$ is the stage, with a lever $\mathrm{F}$ for moving the stage plate; and at $\mathrm{E}$ is the condensing lens, fitted into a small hole in the corner of the stage; the forceps is also fitted into the same; $\mathrm{H}$ is the mirror, made to slide up or down its support. This instrument is particularly adapted for students: it is packed into a neat mahogany case, complete, for the small sum of $4 l$. 10s., by Mr. Baker, 244 Holborn, who likewise furnishes all the requisites for microscopical purposes, with well-selected specimens of mounted objects at a small cost. Messrs. Powell and Lealand, and Messrs. Smith and Beck, supply cheap forms of instruments for beginners, as do many other makers.

The following useful remarks on the microscope are extracted from the Juries' Reports of the Great Exhibition, 1851: "The powers varying from one-inch to a quarter-inch focus, inclusive, are by far the most generally useful in the whole range of microscopic combinations, especially for educational purposes. It must be remarked, that the angle of aperture of the combinations should not be extended to its utmost possible limit when destined for the general purposes of natural history or anatomical investigation. Combinations of high power, and extremely extended angle of aperture, are excellent in developing one class of test objects, viz. minute lines or dots on plane surfaces, and admirably demonstrate the high perfection to which such glasses are capable of being carried by scientific opticians; but such combinations, with a less angle of aperture and more penetrating power, are far more generally useful and valuable to the minute anatomist and the naturalist. In regard to the brass-work, the qualities especially requisite in the stand of a microscope are simplicity of construction, portability, combined with sufficient weight to ensure safety and steadiness, with smoothness and accuracy of action in all the working parts, and such a construction as to distribute any tremor that may be communicated to the instrument equally over its body, stage, and other working parts." 
CHAPTER III.

PRELIMINARY DIRECTIONS - ILLUMINATION - ACCESSORY INSTRUMENTS -PREPARING AND MOUNTING OBJECTS-POLARISED LIGHT-BINOCULAR INSTRUMENT-PHOTOGRAPHIC DRAWING, \&C.

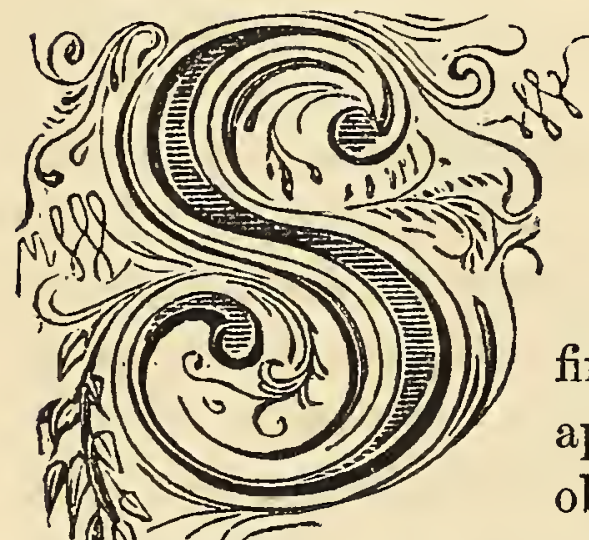

ELECT an apartment with a northern aspect, having, if possible, only one window, and that not overshadowed by trees or buildings: sky-lights are not proper for a microscopic study. In such a room, on a firm, steady table, keep your instruments and apparatus open, and at all times ready for observation. A large bell-glass will be found of great service in keeping dust from a microscope when of set up for use. In winter it will be proper to slightly warm the instrument before it is used, otherwise the perspiration from the eye will be perpetually condensing on the eye-glass, thus greatly impeding vision. Always begin to examine your object with the lowest power you have, unless it be very minute. Remember what was before stated, that, as a general rule, large objects require low powers, and small ones high powers: low powers show the whole or general view of an object, the high ones only its parts in succession; and as the power increases, so does the difficulty of finding the object and adjusting the focus. When you clean the eye-glasses, do not remove more than one at a time, and replace it before you touch another; by so doing you will preserve the component glasses in their proper places: recollect that if intermingled they are useless. Keep a piece of welldusted and very dry chamois leather, slightly impregnated with the finest tripoli or rotten-stone powder in a small box, to wipe your glasses: a small piece of dried elder-pith is preferred by some for the purpose.

When you look through the instrument, be sure to place your eye quite close to the eye-piece, otherwise the whole field of view will not be visible; and observe, moreover, if you see a round disc of light, at least when the object is not on the slider-holder: if you do not, it is a sign that something is wrong; perhaps the body is not placed directly before the aperture of the slider-holder, or may not be truly airected towards 
the light. Use the least amount of light possible, if you work for any length of time. Choose a steady light, with a shade to protect the eyes; one of the old-fashioned fan-shades will be found useful for this purpose. Look at the object with both eyes open, and use the eyes alternately, Sit in a comfortable position, and bring the instrument to the proper angle, which will prevent congestion of the eyes, which is indicated if the microscopist is annoyed with little moving objects apparently. floating before them: if the eye-lashes be reflected from the eye-glass, it is through your being in an improper position; . you are looking upon the eye-glass instead of through it. Take care also that the mirror be properly arranged.

Sir David Brewster's excellent directions for viewing objects, should be made familiar to the microscopist. He observes:

"First. Protect the eye from all surrounding light, letting only the rays which proceed from the illuminated centre of the object fall upon it.

"Secondly. Delicate observations should not be made when the fluid which lubricates the cornea is in a viscid state, or there is any irritation or inflammation about any part of the eye.

"Thirdly. The best position for microscopic observations is with the microscope bent to such an angle with the body, that the head may always remain in a natural and easy attitude; consequently, the worst position would be that which compels us to look downwards vertically.

"Fourthly. If we lie horizontally. on the back, parallel markings and lines on objects will be seen more perfectly when their direction is vertical, or in a contrary direction to that in. which the lubricating fluid descends over the corruea of the eye.

"Fifthly. Only a portion of the object should be viewed at one time, and every other part excluded. The light which illuminates that part should be admitted through a small diaphragm : at night, from the concentrated light of a sperm-oil or gas lamp, having a faint blue-tinted chimney-glass to correct the yellow colour which predominates in all our artificial illumination. If in the day-time, close a portion of the window-shutters.

"Sixthly. In all cases when high powers are used, the intensity of the illumination should be increased by optical contrivances below the object and stage : this is generally effected by using achromatic condensers beneath the stage."

Mr. James Smith contributed the subjoined practical observations on the same subject to the Microscopical Transactions:-

"Much of the beauty of the objects seen depends upon the management of the light that is thrown upon or behind them, which can only be fully mastered by practice. It may be remarked, however, as a 
general rule, that in viewing those which are transparent, the plane mirror is most suitable for bright daylight; the concave for a lamp or candle, which should have the bull's-eye lens, when that is used, so close to it, that the rays may fall nearly parallel on the mirror.

"If the bull's-eye lens is not used, the illuminating body should not be more than five or six inches from the mirror. The latter is seldom required to be more than three inches from the object, the details of which are best shown when the rays from the mirror fall upon it before crossing; and the centre should be, especially by lamplight, in the axis of the microscope. For obscure objects, seen by transmitted light, and for outline, a full central illumination is commonly best; but for seeing delicate lines, like those on the scales of insects, it should be made to fall obliquely, and in a direction at right angles to the lines to be viewed.

"The diaphragm is often of great use in modifying the light, and stopping such rays as would confuse the image (especially with low or moderate powers); but many cases occur when the effects desired are best produced by admitting the whole from the mirror. If an achromatic condenser is employed instead of the diaphragm, its axis should correspond with that of the body; and its glasses, when adjusted to their right place, should show the image of the source of artificial light; or by day, that of a cloud or window-bar in the field of the microscope, while the object to be viewed is in focus. The most pleasing light for objects in general is that reflected from a white cloud on a sunny day; but an Argand's lamp or wax candle, with the bull's-eye lens, is a good substitute. A large proportion of opaque objects are seen perfectly well (especially by daylight) with the side reflector, and the dark bore as a background; and for showing irregularities of surface, this lateral light is sometimes the best: but the more vertical illumination of the Lieberkuhn is usually preferable; the light thrown up to it from the mirror below being, with good management, susceptible of much command and variety."

Mr. Ross very properly remarks, that "the mode of illuminating objects is one on which we must give some further information; for the manner in which an object is lighted is second in importance only to the excellence of the glass through which it is seen. In investigating any new or unknown specimen, it should be viewed in turns by every description of light, direct and oblique, as a transparent object and as an opaque object, with strong and with faint light, with large angular pencils thrown in all possible directions. Every change will probably develop some new fact in reference to the structure of the object, which should itself be varied in the mode of mounting in every possible way." 
It should be seen both wet and dry, and immersed in fluids of various qualities and densities; such as water, alcohol, oil, and Canada balsam, for instance; which last has a refractive power nearly equal to that of glass.

If the object be delicate vegetable tissue, it will be, in some respects, rendered more visible by gentle heating or scorching by a clear fire, placed between two plates of glass.

In this way the spiral vessels of asparagus and other similar vegetables may be beautifully displayed. Dyeing the objects in tincture of iodine, or some one of the dye-woods, will, in some cases, answer this purpose better.

But the principal question in regard to illumination is the magnitude of the illuminating pencil, particularly in reference to transparent objects. Generally speaking, the illuminating pencil should be as large as can be received by the lens, and no larger. Any light beyond this produces indistinctness and glare. The superfluous light from the mirror may be cut off by a screen, having various-sized apertures placed below the stage.

This is known as the Diaphragm, fig. 36. It consists of two plates of brass, one of which is perforated with four or five holes of different sizes; this plate is of a circular figure, and is made to revolve upon another plate, by a central pin or axis; this last plate is also provided with a hole as large as the largest in the diaphragm plate, and corresponds in situation to the axis of the compound body. To ascertain when either of the holes in the diaphragm-

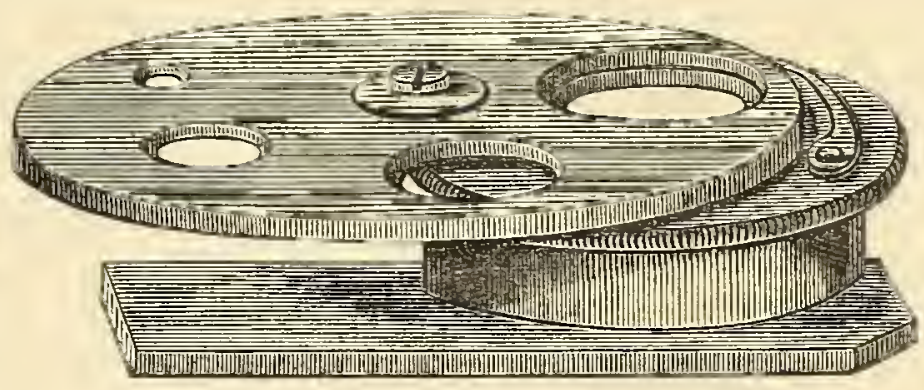

fig. 36 . plate is in the centre, a bent spring is fitted into the second plate, and rubs against the edge of the diaphragm-plate, which is provided with notches. The space between the smallest and largest is great enough to use for the purpose of shutting off all the light from the mirror.

A good mode of imitating artificially the light of a white cloud opposite the sun has been proposed by Mr. Varley: he covers the surface of the mirror under the stage with carbonate of soda, or any similar material, and then concentrates the sun's light upon its surface by a large condensing lens.

Another mode of illumination was that proposed by Dr. Wollaston; it is known as the "Wollaston Condenser." The form now in general use is that made and described by Mr. Ross as the achromatic condenser. 
THE ACHROMATIC ILLUMINATOR.

"When employing this apparatus, the general practice is to insert in it, as an illuminating lens, the object-glass next lowest in power to that which is intended to be attached to the microscope; so that when the one-eighth is used on the microscope, the one-fourth is screwed into the illuminating apparatus; and so, in like manner, with the rest. But when economy is not regarded, a system of three achromatic combinations is supplied, adapted for the illumination of the whole range of the powers of the microscope: the whole system being employed for the highest powers; two of such combinations with the middle powers; and the largest combination by itself for the lowest powers. This illumination is not required for objects when viewed with object-glasses transmitting small pencils of rays, or whose angular aperture is less than thirty degrees; that is, where the object-glass is of greater focal length than half an inch.

"The apparatus is fixed to the under side of the stage of the microscope, in the place of the diaphragm-plate; and before fixing, the proper object-glass, as an illuminating lens, must be screwed on to it. In fig. 37, two tubes are seen sliding one within the other; to the outer one, $b$, is attached a flat plate $a$, which

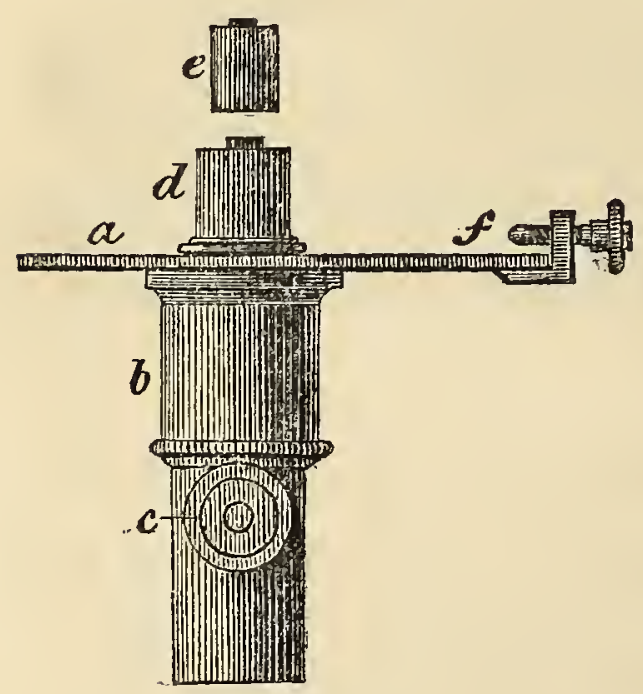

fig. 37 . slides underneath the stage, and is adjusted for distance by the screw $f$; at $c$ the milled-bead is connected to a pinion; and by means of a rack attached, the inner tube, carrying the achromatic combination $d$, is raised or lowered: the upper part of the outer tube is larger than that where the milled-head is seen, for the purpose of allowing the milled ridge of the achromatic to pass up and down. For the $\frac{1}{2}$ or $\frac{1}{4}$ inch, the combination $d$ is used; and for the higher power, $\frac{1}{8}$ or $\frac{1}{12}$, the second: $e$ is slipped over $d$. Place the cbject to ibe viewed upon the stage of the microscope; and when the instrument is not directed at once (as after noticed) to the source of light, such as the flame of a lamp, or a white cloud, arrange the reflector (having the plane mirror upwards) so: as to throw the light up the tube of the apparatus; which may be ascertained by turning aside the microscope tube, and observing when the spot of light appears on 
the object placed on the stage. The microscope tube is then to be replaced as nearly over the spot of light as possible, and vision of the object obtained, disregarding the precise quality of the light.

"The two following important adjustments must next be effected: first, make the image of the source of light (as flame of lamp or light cloud) distinctly seen at the same time that the object on the stage is seen ; and secondly, make the axis of the tube of the illuminating apparatus coincide with that of the tube of the microscope. The first adjustment is accomplished by turning the milled-head screw $f$ on the side of the tube of the apparatus, until the brightest illumination is seen through the microscope; then move the microscope-tube from its central position sideways across the spot of light and back again, to ascertain if the spot of light passes across the centre of the field of vision; if not, it may be corrected by turning the milled-head screw of the illuminating apparatus which presses against the front edge of the stage, and when, by adjustment, it is found that the spot of light passes centrally across the field, then, having adjusted the microscopetube so as to bring the spot of light to the centre of the field of vision, screw the microscope-tube fast in its place. To effect the second adjustment mentioned above, the mirror must be moved about, until an image, more or less distinct, of some adjacent object-such as a window-bar or chimney-pot-crosses the field of the microscope; the'most distinct vision of this object is then to be obtained by turning the milled-head screw on the side of the illuminating apparatus, the microscope-tube remaining stationary with the object in its focus. When these general adjustments are accomplished, the whole may require correction, which must be effected by a repetition of the process. The mirror may then be turned up to the sky, that being the source of light; and its best state for illuminating microscopic objects is by means of white clouds opposite the sun. The utmost perfection of vision is generally obtained by this adjustment for day-light illumination upon delicate objects; but by lamp-light these objects are sometimes seen best by placing the achromatic illuminating lens a little nearer the object than the distance which produces a distinct image of the source of light, at the same time that the object is in focus. Objects having sorne little thickness are best seen when the lens is a little farther off than the distance just mentioned. This last position of the illuminating lens diffuses the light more equally over the field.

"When lamp-light is used, an object-glass of lower power than that which it is intended to employ for observation should be applied to 
the microscope-tube, and the adjustment performed as before directed. Attention must be paid to this additional circumstance-namely, that the image of the lamp be placed in the middle of the illuminated disc of light by means of the mirror: the mirror and lamp are then to remain stationary. But if the mirror slould not be employed, and the light obtained direct from the lamp, then, in order to throw the image of the lamp in the middle of the illuminated disc, the adjustment must be made by a lateral movement of the whole microscope, and by varying its inclination : the image being thus found, the lamp and microscope must remain stationary. The object-glass used in this preliminary adjustment is then to be removed, and the object-glass to be employed for observation screwed on to the microscope-tube in its place, and the object brought into focus, which will also bring the image of the flame of the lamp distinct. But the distinctness, as also the centricity of the image of the flame, may have been in a degree deranged by slight differences in the screws of the object-glass and other minute circumstances. This deviation is to be rectified by moving the microscope-tube sideways and back again across the image of the flame, and, if adjustment be necessary, by turning the milled-head screw which presses against the front edge of the stage, as before described, while the mirror and lamp remain stationary. Slight obliquity of the illumination subdues the glare attendant upon perfectly central and full illumination by lamp-light; and this obliquity may be obtained by slightly altering the position of the mirror ; or if the mirror is not employed, but light is obtained by pointing the microscope-tube directly to the lamp, then the obliquity required may be obtained by a small variation of the inclination of the microscope. It is essential that the mirror and lamp-glass be free from dust and soot."

F. H. Wenham, Esq. (Micros. Trans. 1851) proposed a new illuminator, for the purpose of obtaining perfect definition under high powers. The cause of the markings on test-objects being seen by oblique illumination, which cannot be shown by the ordinary method, appears to be, that in the former case no greater amount of light is allowed to pass through the object into the object-glass than is sufficient to render the structure distinctly visible; consequently the object is not flooded by an intense glare of light, which is sometimes occasioned with ordinary direct illumination. From this it would appear that no rays from the source of light should enter the object-glass by passing. through or around the object, which must be illuminated by very intense light thrown on to it in all opposite directions, at an angle exceeding the aperture of the object-glass, so that the light which enters the 
microscope should be that which radiates only from the object, as if it were self-luminous.

Those who have experimented on the subject may have observed that there is something in the nature of oblique light reflected from a metallic surface particularly favourable for the purpose of bringing out minute markings, which may, in some measure, be attributed to the circumstance of light so reflected being purely achromatic. In order to render this property available, $\mathrm{Mr}$. Wenham contrived a very ingenious metallic reflector, by which the condensation of lateral light may be effected.

"The apparatus is shown in section in fig $38: a a$ is a parabolic reflector, of a tenth of an inch focus, with a polished silver surface, having the apex so far cut away as to bring the focal point at such a distance above the top of the apparatus (which is closed with a screw-cap when not in use), as may allow the rays to pass through the thickest glass commonly used for mounting objects upon before coming to a focus.

"At the base of the parabola is placed a disc of thin glass $b b$, in the centre of which is cemented a dark well, with a flange equal in diameter to the aperture at the top of the reflector, for the purpose of preventing the direct rays from the source of light passing through the apparatus.

"The reflector is moved to and from the object by means of the rack and pinion $c$, and has similar adjustments for centering, and is fixed under the stage of the microscope in the same way as the ordinary achromatic condenser: in addition there is a revolving diaphragm $d$, made to slide on the bottom tube of the apparatus; it has two apertures $e$ e, placed diametrically, for the purpose of obtaining two pencils of oblique light in opposite directions. The effects of the chromatic and spherical aberrations, in the shape of fog and colour about the objects, caused by the glass slides upon which they are mounted, frequently require compensation; for as the parabola has the property of 
throwing parallel rays uncoloured to a point, when used alone, it is most suitable for objects without glass underneath.

"By the addition of a meniscus, this compensation is obtained, and also greater purity and intensity of illumination is procured; and as the silver reflector is now closed with glass, it is hermetically sealed, and permanently protected from dust and damp, and will therefore retain its polish. The light most suitable for this method of illumination is lamp or candle light, the rays of which must in all cases be rendered parallel by means of a large plano-convex lens, or condenser ; the light may then be used direct, or reflected from the plane mirror. The object having been adjusted, the illuminator is moved to and fro till the best effect is produced. For the purpose of viewing some objects, such as the naviculæ, the circular diaphragm should be slid on the extremity of the apparatus, and revolved till the two pencils of light are thrown most suitably across the object.

"All objects either transparent or opaque, with the exception of white, absorb some of the rays of light, and are rendered visible by that portion which they radiate: a predominance of those rays, either primary or compound, of which ordinary white light is composed, is the cause of their various colours, the intensity of which depends upon the quantity radiated; therefore any object at all capable of radiating light will be well shown by this mode of illumination, and, if the light thrown on them is achromatic, in all their natural colours.

"In viewing objects by light transmitted directly through them, we have two sets of rays entering the eye-viz. those emanating from the source of light; and those radiating from the object; the imperfections produced by the former passing through and around the object mingling with the latter, and preventing them from producing their proper effect."

As the method of illuminating microscopic objects by means of a large angular pencil of light, having the central rays obscured, is of recent introduction, we shall mention a few instances where transparent objects are shown, under similar circumstances, with perfect or improved definition.

"If a black wafer be fixed to a window on a bright day, and a large transparent object, such as a feather or the wing of the dragon-fly, be held before it, and examined with a hand magnifier of an inch focus, it will then be entirely illuminated with lateral light; and it will be seen that the object is more beautifully developed than when seen against a bright cloud, the comparison being immediate.

"Those who are in the habit of collecting infusoria will be assisted 
in distinguishing the various species, if a slip of black cloth be pasted to one side of the phial, which should be held against the sky, and the contents examined with a Coddington lens, or hand magnifier. Some objects, especially those of a dark colour, will be best seen on a ground either coloured or slightly illuminated. This circumstance is provided for in the reflector, the bottom of the dark well being movable, so that patches of tissue-paper, more or less diaphanous, or variously coloured, may be used for the background of the object.

"The lateral mode of illumination will be found to possess peculiar advantages in the examination of test-objects and the internal mechanism of infusoria. The markings on most of the test-objects are either depressions or projections by direct light: all parts of an object are illuminated with equal intensity, and delicate colours are in great part destroyed, consequently there is a want of contrast. The effect of an angular pencil of rays of 175 degrees, with the central ones stopped, is, that there is a greater relative amount of light thrown on these prominences, as they intercept the largest portion of the marginal rays near the apex of the reflector, leaving the base of the prominence in comparative shadow, consequently the markings we wish to see are the most strongly lighted. The different organs in the interior of an animalcule may be much of the same colour and transparency, and yet possess a different refraction, according to their density. Direct light will pass through these transparent membranes in straight lines, without being affected by their various refractive powers. The effect of lateral or oblique light on such tissues is, that the rays are more refracted according to their inclination, and proportionate to the various densities of the medium, the most refractive structure transmitting the greatest quantity of light, and being in consequence the most illuminated; and this reason is somewhat confirmed by the circumstance of lateral illumination showing the structure of some objects which, from slight variation in density, were invisible, except by the use of polarised light." Mr. Shadbolt has since made considerable improvements in this reflector, which he denominates "a sphero-annular condenser :" it has superior reflecting arrangements, with less liability of derangement.

Condensing lenses are used either for opaque objects, or to condense the light upon the mirror attached to the microscope. Two lenses, as represented in fig. 39, are sometimes used. A bull's-eye, or plano-convex lens, of three inches focal length, is best suited for the larger; and the mode of employing the two condensers upon an object placed on the stage of the microscope at $a$ is here shown. The bull's-eye lens $c$ slides 
up and down a brass rod, screwed into a steady foot; $b$, the smaller lens, working on a joint, or it may be fixed into the stage of the microscope,

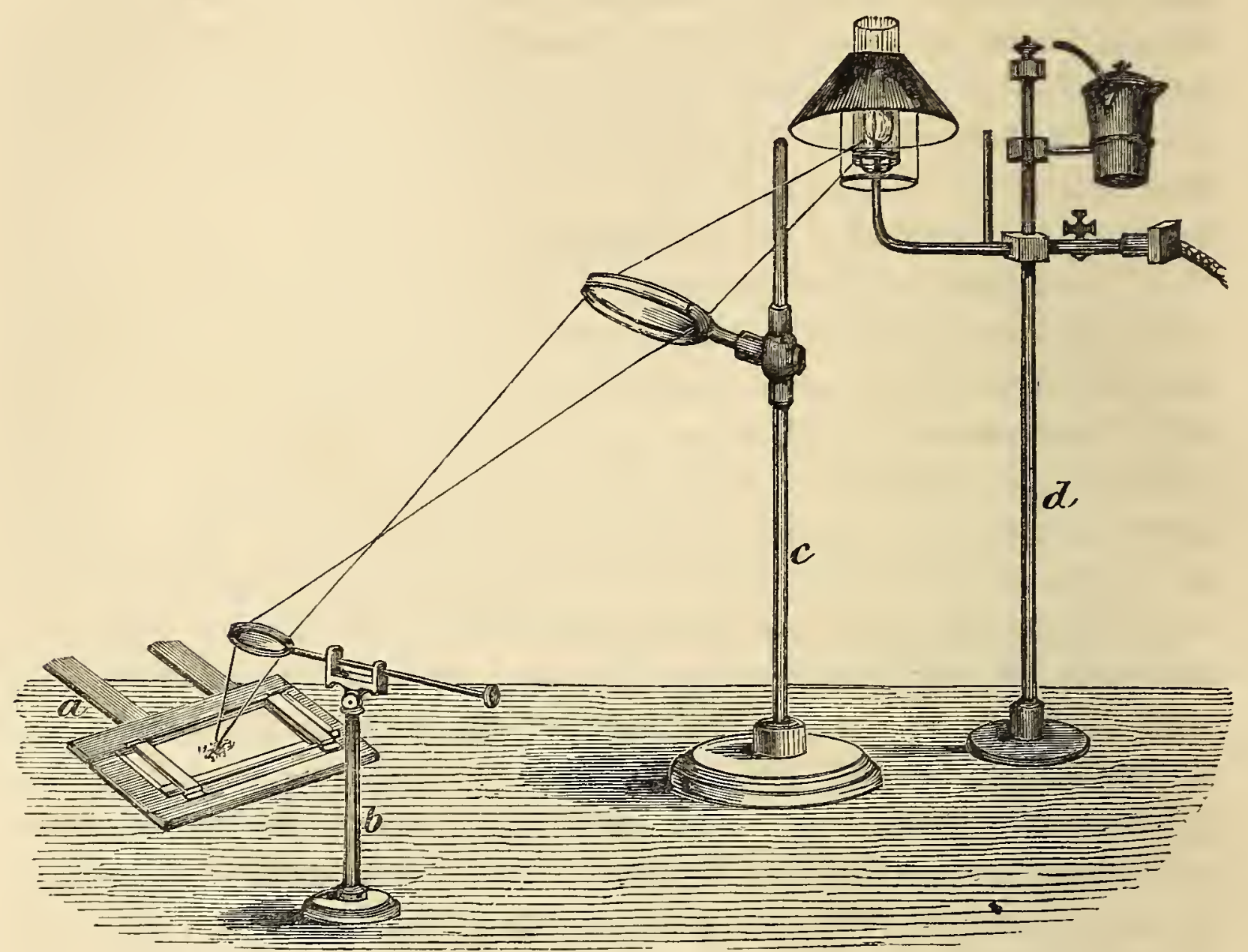

fig. 39.

through which the light is finally concentrated upon the object from the table gas-lamp $c$. Mr. Brooke's method of viewing opaque objects under the highest powers of the microscope (the $\frac{1}{8}$ and $\frac{1}{1} \frac{1}{2}$ inch object-glass) is effected by two reflexions. The rays from a lamp rendered parallel by a condensing lens are received on an elliptic reflector, the end of which is cut off a little beyond the focus; the rays of light converging from this surface are reflected down on the object by a plane mirror attached to the object-glass, and on a level with the outer surface. By these means the structure of the scale of the Podura, and the different characters of its inner and outer surfaces, are rendered distinctly visible. Silver specula, known as Lieberkuhn's, are much employed and preferred by some microscopists. The Lieberkuhn is concave, and attached to the object-glasses, from the two-inch to the half-inch, in the manner represented at fig. 40 , where $a$ exhibits the lower part of the compound body; $b$ the object-glass, over which is slid a tube and the Lieberkubn $c$ attached to it; the rays of light reflected from the mirror are brought to a focus upon an object $d$, placed between it and the mirror. 
The object may either be mounted on a slip of glass, or else held in the forceps $f$; and when too small to fill up the entire field of view, or when transparent, it is necessary to place behind it the dark well $e$.

Each Lieberkuhn being mounted on a short piece of tube, can be slid up and down on the outside of the object-glass, so that the maximum of illumination may be readily obtained. In all the higher powers the end of each object-glass is turned small, and passes through the aperture in the centre of the Lieberkuhn; but in the lower powers, where a great amount of reflecting. surface would be lost on account of the

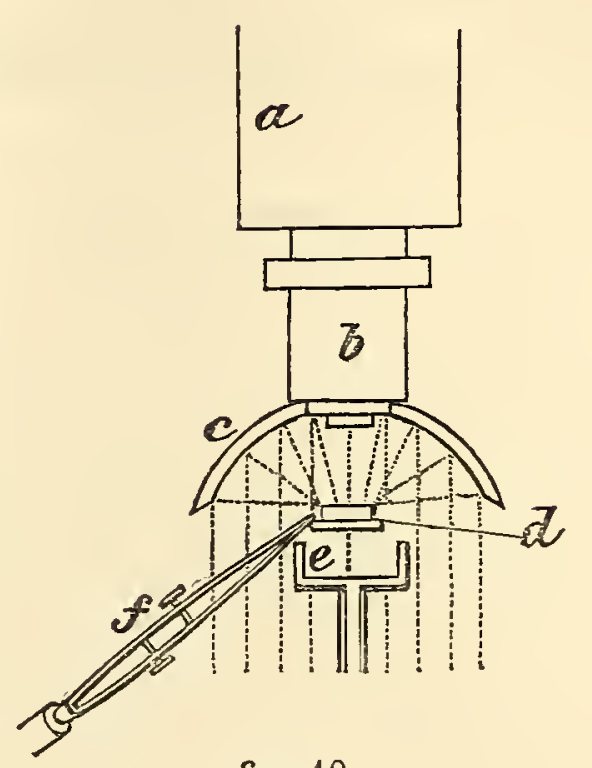

fig: 40. large size of the glasses employed if this plan were adopted, the aperture in the centre of the Lieberkuhn is made to admit as many rays as will fill the field of view, and no more.

Mr. S. Highley, of Fleet-street, invented an achromatic gas-lamp, fig. 41, now much in use for condensing the light on the mirror. Gas, as a source of light, presents great advantages over oil and spirit, on account of cleanliness, being ever ready for use, and affording a perfect control over the flame; but when the ordinary gas-lamps are used for the purpose of illuminating the field of the microscope, a yellow glaring light is given, alike injurious to the eye and the definition of the object under examination. To correct these evils, he has arranged a lamp which is also otherwise useful to the microscopist. It consists of a stage A, supported by a tube and socket, sliding on an upright rod rising from the stand. This carries an argand burner $\mathrm{B}$; a metal cone $\mathrm{C}$ rises to the level of the burner, and is about one-eighth of an inch from its outer margin.

This arrangement gives a bright cylindrical flame. The bottom of the stage $\mathrm{A}$ is covered with wire-gauze, to cut off irregular currents of air, and thus secures a stecdy flame. Over the burner is placed a Leblond's blue glass chimney $\mathrm{D}$. This corrects the colour of the flame to a certain extent; but it is still further rectified by a disc of bluishblack neutral-tint glass $\mathrm{E}$, fitted in a tube $\mathrm{F}$, attached obliquely to the shield G. G is a half-cylinder of metal, which serves to shield the eyes from all extraneous light, but may be rotated on the stage $\mathrm{A}$ by aid of the ivory knot $\mathrm{H}$, when the full light from the flame is desired. A metallic reflector I, fixed on its supports, so as to be parallel to E, con- 
centrates the light. By the combination of the two glasses $D$ and $E$, the yellow rays of the flame are absorbed, and the arrangement affords a soft white light, which may be still further improved by receiving the rays on a concave mirror, backed with plaster-of-Paris, $\mathrm{L}$; and where a very

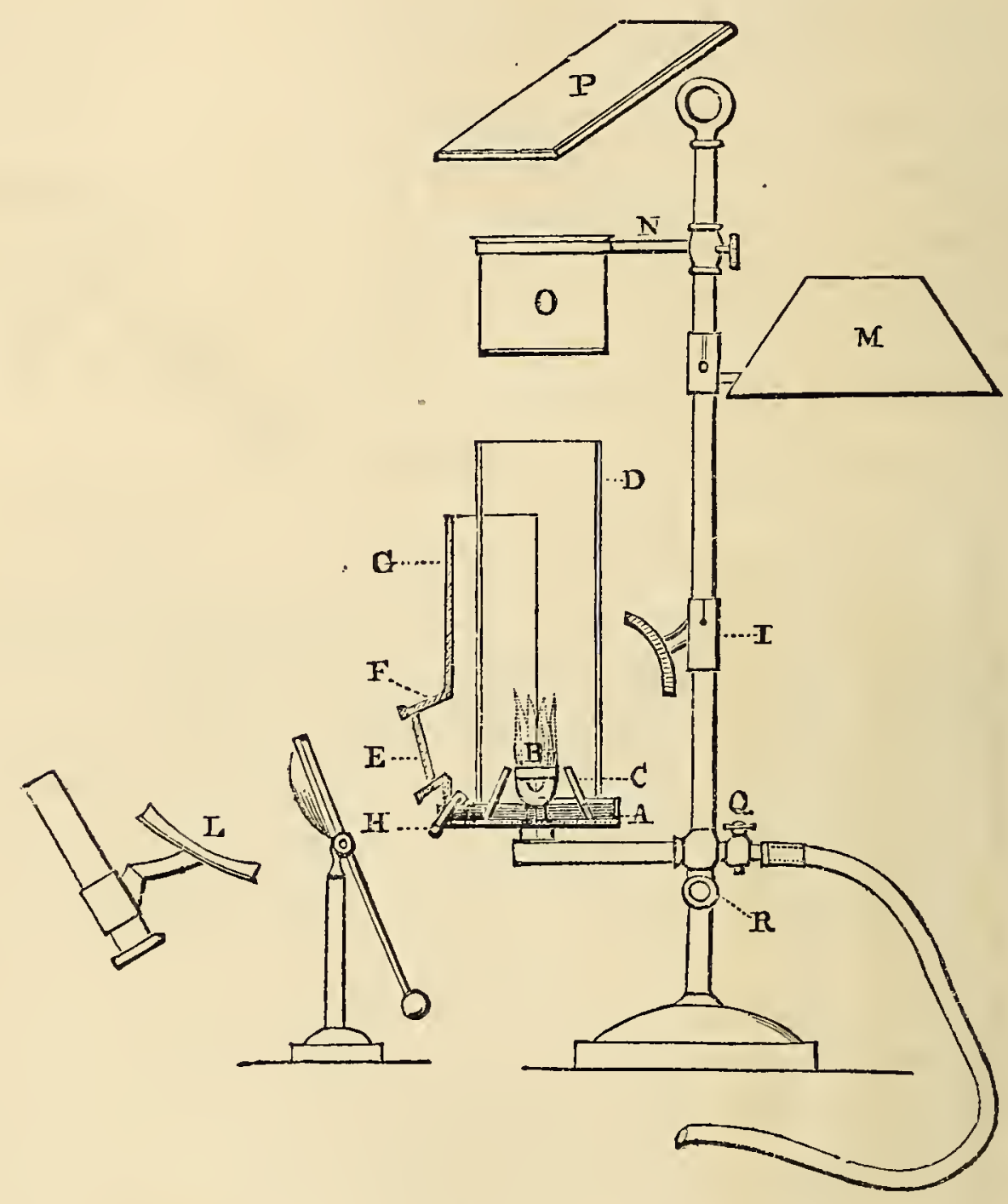

fig. 41.

strong light is required, a condensing lens should be interposed between the lamp and the mirror of the microscope. By removing the shield $G$, and bringing the shade $\mathrm{M}$ over the burner, it may be used as a readinglamp. A retort ring $\mathrm{N}$ supports a water-bath $\mathrm{o}$, or a wrought-iron plate $P, 6$ inches by $2 \frac{1}{2}$ inches, both used in mounting objects. The stop-cock $a$ gives the means of regulating the flame. The screw $\mathrm{R}$ clamps the lamp-head at any height desired. The lamp may be attached to any gas-supply by vulcanised India-rubber tubing.

Forceps.-For holding minute objects, such as parts of plants or insects, to be examined either as transparent or opaque objects, the most useful is represented by fig. 42 . It consists of a piece of steel wire, about three inches long, which slides through a small tube, con- 
nected to a stout pin by means of a cradle-joint; to one end of the wire is attached a pair of blades, fitting closely together by their own

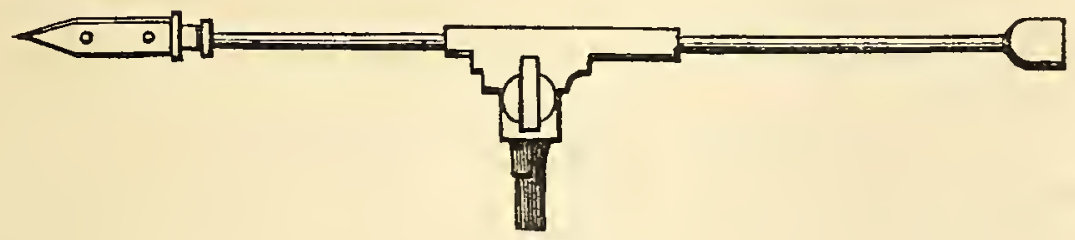

fig. 42.

elasticity, but which, for the reception of any object, may be separated by pressing the two projecting studs; to the opposite end of the wire is adapted a small brass cup, filled with cork, into which pins passed through discs of cork, card-board, or other material, having objects mounted on them, may be stuck.

Dipping-tubes for taking up animalcules.-These are tubes of glass,

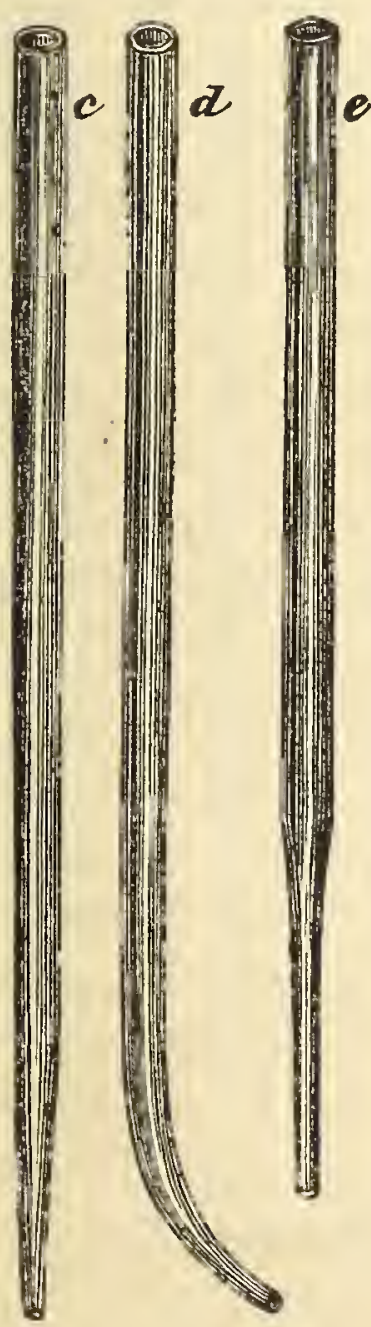

fig. 43 .

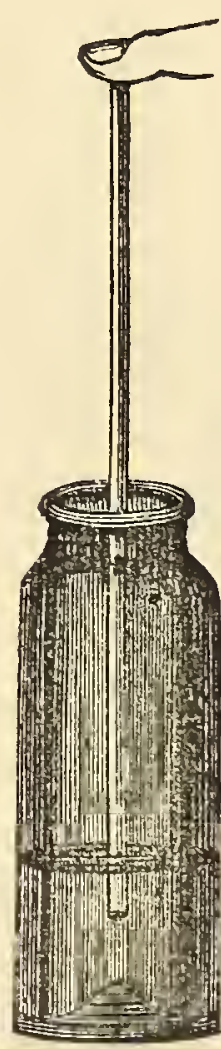

fig. 44.

about nine inches in length, open at both ends, and from one-eighth to one-fourth of an inch in diameter. The ends should be nicely rounded off in the flame of a blow-pipe; some of them may be straight, whilst 
others should be drawn out to a fine point, and made of either of the shapes represented at $c d e$. Mr. Varley describes the method of using them in volume forty-eighth of the Transactions of the Society of Arts. "Supposing the animalcules that are about to be examined to be contained in a phial or glass jar, as in fig. 44, having observed where they are most numerous, - either with the naked eye, if they are large, or with a pocket-magnifier, if they are small,-either of the glass-tubes, having one end previously closed by the thumb or fore-finger, wetted for the purpose, is introduced into the phial in the manner represented by the figure,- this prevents the water from entering the tube; and when the end is near to the object which it is wished to obtain, the finger is to be quickly removed and as quickly replaced. The moment the finger is taken off, the atmospheric pressure will force the water, and with it, in all probability, the desired objects, up the tube. When the finger has been replaced, the tube containing the fluid may be withdrawn from the phial; and as the tube is almost certain to contain much more fluid than is requisite, the entire quantity must be dropped into a watch-glass, which spreads it, and the insect may be again caught by putting the tube over it, when a small quantity of fluid is sure to run in by capillary attraction. This small quantity is to be placed upon the tablet; but should there be still too much for the tablet, if it be touched with the tube again, it will be diminished accordingly. If we wish to place several individuals together on the tablet, it is necessary that each should be taken up with the smallest amount of water : to effect this, Mr. Varley suggests that the tube should be emptied on a slip of glass, in separate drops; and with one of the capillary tubes, but little larger than enough to catch them, they may be lifted out one by one, and be placed on the tablet. Generally speaking, it is necessary to add a small quantity of vegetable matter to animalcules, to keep them alive; and as many species of them are found on confervæ and duck-weed, some instrument is required to take small portions of these plants out of the jar in which they are growing." For this purpose Mr. Varley uses the forceps represented by fig. 45 .

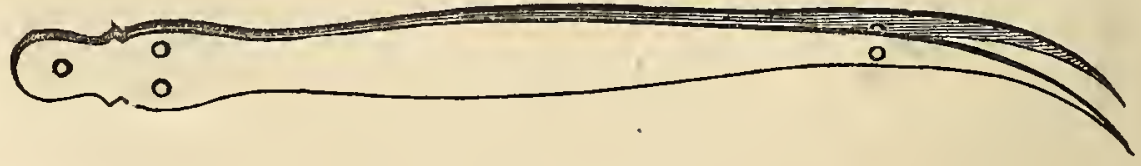

fig. 45 .

They are made of brass, and the points are a little curved; to keep them accurately together, they are provided with a hole and steady-pin. 
This instrument serves very well to put into a phial, and to take out small portions of regetable matter, dc.

\section{COLLECTING ANILALCULæ.}

For collecting the living water animalculæ, the cambric-muslin net, made similar to a landing-net, fig. 46 , will be found very useful ; this should be secured to a brass-ring $a$, and fitted into a socket $b$, by

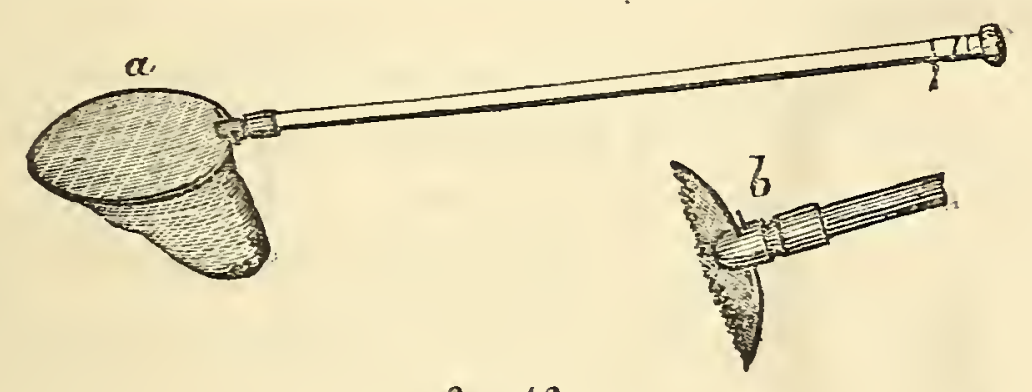

fig. 46 .

which it can be attached to the end of a walking-stick; or; when not in use, the socket may be carried in the pocket; and the net, by contracting the diameter of the ring (which the construction admits of) can be put inside of the hat. Fig. 47 , is a box containing six bottles for holding the animalculæ when caught. These bottles should be filled with the water when you collect the insects, and the larger insects put by themselves. When collecting from different localities, take care not to mix the insects from one brook with those from another, otherwise serious conflicts may take place, and on reaching home you will find the greater part of your stock either dead or dying. Always separate the various sizes and races as speedily as possible. This can be done most easily by emptying each

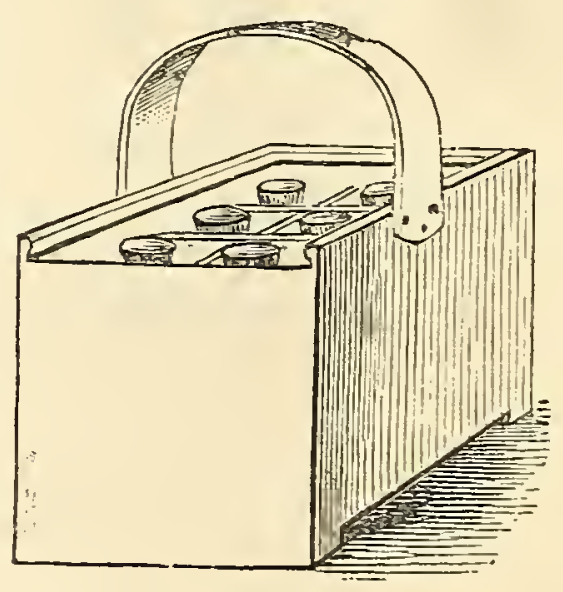

fig. 47 . bottle in its turn into a soup-plate; then with the feather of a pen first lift out the smaller ones, and with the quill-end cut like a scoop lift out the larger, classifying and allotting each species to its separate "fish-pond." One of the best localities in the neighbourhood of London for collecting is Epping Forest.

Mr. Williamson has a very cheap and simple contrivance for converting the end of a walking-stick or umbrella into what he terms a "collecting-stick." In fig. 48, a represents a piece of whalebone, about 
18 inches long, bent round the end of the stick or umbrella $b$, and made fast in that position by one or two rings, $d$, of gutta-percha, india-rubber, or brass. A small wide-

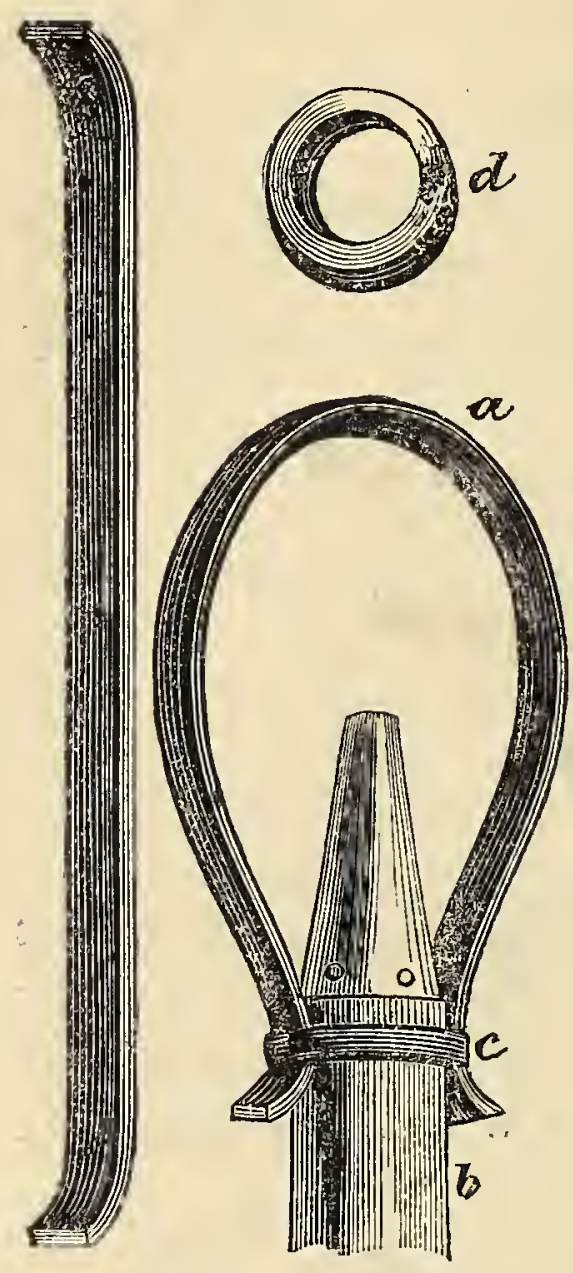

fig. 48. mouthed bottle, having a rim which will prevent its falling through, is now inserted in the loop thus formed, and is held tightly there, by the ends of the whalebone being drawn further through the ring, and thus diminishing the size of the loop. The bottle thus fixed may be used for dipping out the animalculæ. Whalebone can be moulded to any form by placing it for a short time near the fire.

Animalculce cages.-Mr. Varley, in the year 1831, greatly improved the form of this instrument, and gave to it the name of capillary-tablet, or cage. He made a channel all round the object-plate, so that the fluid and the animalculæ in it were retained at the top of the object-plate by capillary attraction; and they then bear turning about in all directions without leaving the top, provided the cage be not suddenly shaken. The cover is made to slide down upon the object-plate. The plate of brass to which the tube supporting the tablet and cover is attached is of a circular form, slightly flattened on two opposite sides for convenience of pack-

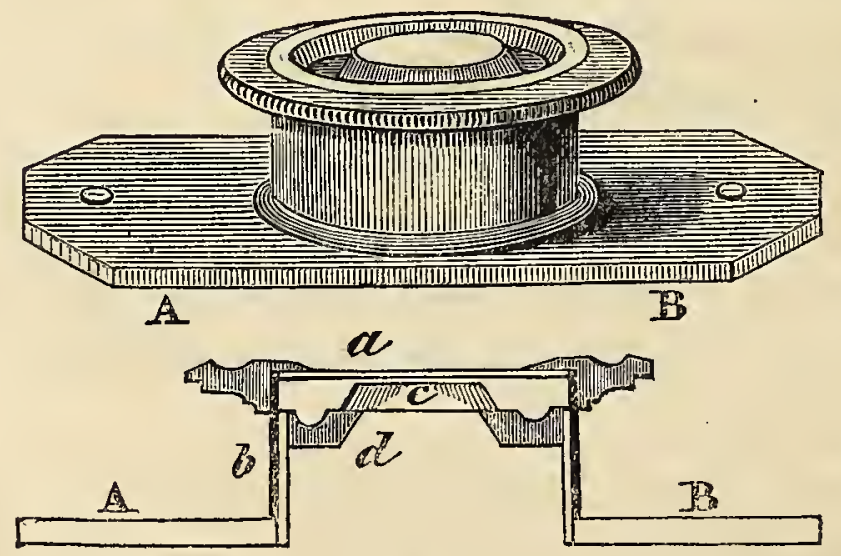

fig. 49 .

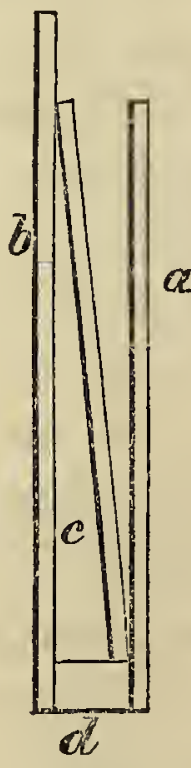

fig. 50 . 
age. One of these instruments, with the present modifications, is seen in elevation and in section, in fig. 49. A B, in both figures, is the flat plate of brass to which the short tube carrying the object-plate or tablet is fixed; $d$ the piece of brass into which the tablet $c$ is fastened; $b$ the tubular part of the cover, into the rim of which the thin plate of glass $a$ is cemented.

Smith and Beck's troughs for chara and polyps, a sectional view of which is shown at fig. 50, are made of three pieces of glass, the bottom being a thick strip, and the front $a$ of thinner glass than the back $b$; the whole is cemented together with Jeffery's marine-glue. The method adopted for confining objects near to the front varies according to circumstances. One of the most convenient plans is to place in the trough a piece of glass that will stand across it diagonally, as at $c$; then if the object be heavier than water, it will sink, until stopped by this plate of glass. At other times, when used to view chara, the diagonal plate may be made to press it close to the front by means of thin strips of glass, a wedge of glass or cork, or even a folded spring. When using the trough, it is necessary that the microscope should be in a position nearly horizontal. A useful trough described by $\mathrm{Mr}$. Varley, in the Transactions of the Society of Arts, consists of a bottom. plate of stout glass, upon which is cemented with pitch and bees'-wax a thin one for the top; slips of glass between it and the bottom-plate forming the sides. The top-plate is not so broad as the bottom, in order that a slip of chara may be more readily placed in the trough, as it can be first laid upon the bottom-plate, and then gradually be slid.

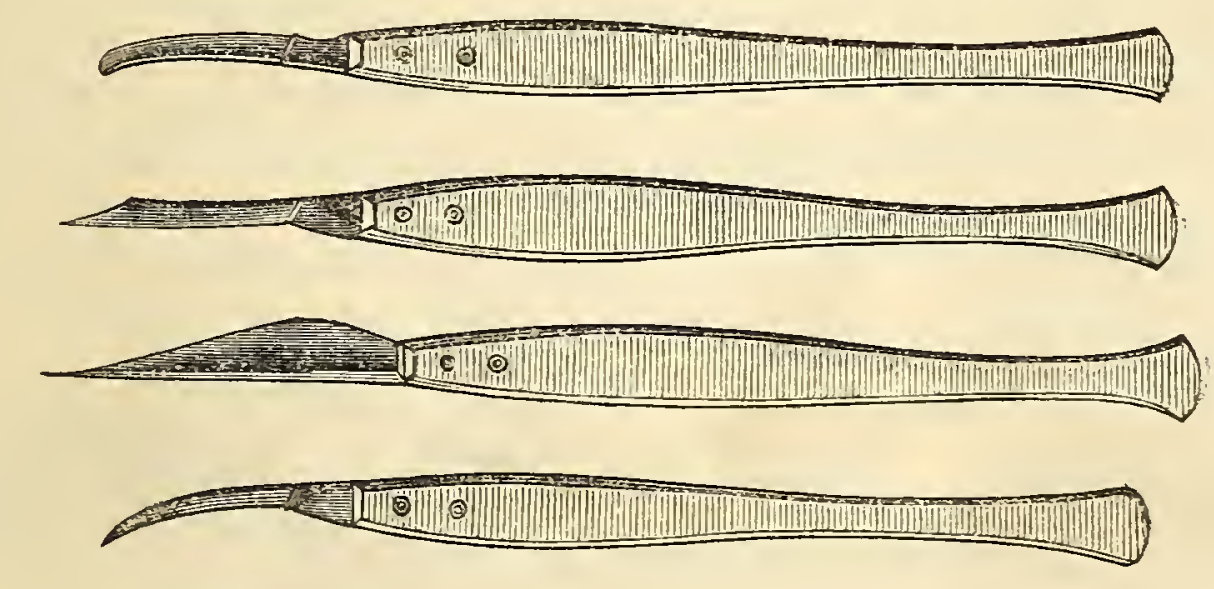

fig. 51.

down between it and the other. In order to render the trough more manageable, it can be cemented to a larger bottom-plate by Canada balsam; but it will be found far more advantageous if the bottomplate itself be large and broad, and the cover cemented to that, and 
not to another plate, as two extra surfaces will then be dispensed with.

Dissecting Knives, \&c.-Knives and needles of various kinds and sizes are required for microscopic dissection: the best for the purpose are represented in fig. 51, being, in fact, the very delicately made knives used by surgeons in operations upon the eye. Dissecting needles may be either straight or curved. They may be fixed, or made to take in and out of their handles. Three of the most convenient are shown in fig. 52; which are made expressly by Mr. Weedon for the use of the microscopist.

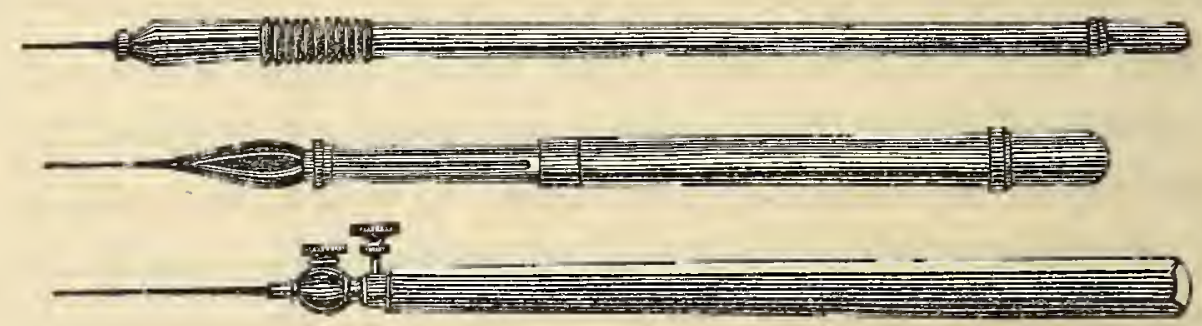

fig. 52 .

In the preparation of objects, no microscopist was ever more successful than Swammerdam: "His chief art seems to have been in constructing very fine scissors, and giving them an extreme sharpness; these he made use of to cut very minute objects, because they dissected them equally, whereas knives, if ever so fine and sharp, are apt to disorder delicate substances. His knives, lancets, and styles, were so fine that he could not see to sharpen them without a magnifying glass."

The mode adopted for breaking up tissues into very small pieces is usually conducted as represented at fig. 53, with a pair of the small needles held firmly between the fore-finger and thumb. The structure must be teazed out; an operation which requires care and perseverance, as most of the animal tissues are very difficult of separation, especially

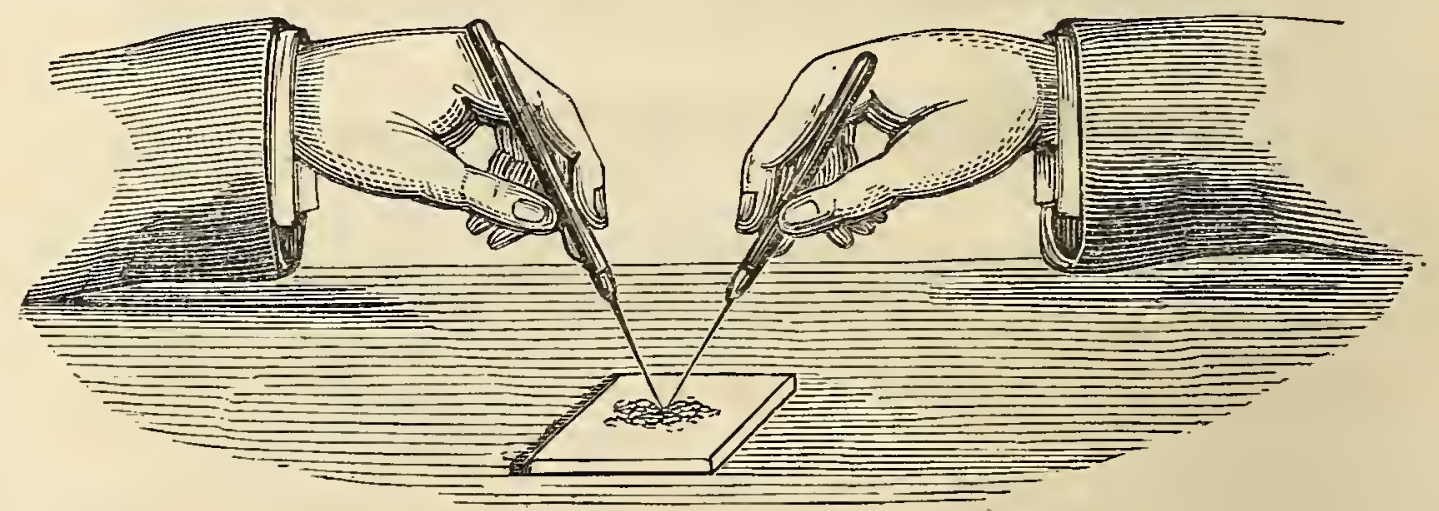

fig. 53 .

when it is wished to examine them under high powers. Muscular fibre and fibrous tissues are of this character, yet well repay the trouble of 
dissecting and dividing them into fibrillce. With other very delicate membranes, and with those of the nervous system of the smaller animals, insects, \&c., it becomes necessary that the investigation should be carried on under water, or in fluid of some sort, the objects being contained in a glass case, and having a very strong light thrown down upon it by the aid of the condensing lens, as represented in fig. 54 . The cells or troughs are generally made of pieces of stout plate-glass, their edges being accurately ground and cemented together with marine-glue or sealing-wax, the size of the trough about three inches square and one inch deep.

If desirable to dissect under the microscope itself, the instrument should be brought over the trough, and the subject adjusted to the focus of an inch or a two-inch magnifier, as it is very difficult to employ a much higher power. The simple microscope, p. 27, fig. 23, is that generally employed for the purpose. If the object be a portion of an injected animal, it is better to pin it out on a piece of cork covered with white wax, and then immerse it in the water-trough; the more delicate the structure, the sooner after death should it be examined, especially in animals. With most vegetable structures, the dissection should be carried on under water. The separation of the woody and vascular tissues, and the spiral ressels, is only effected by maceration and tearing with the fine needles under water.

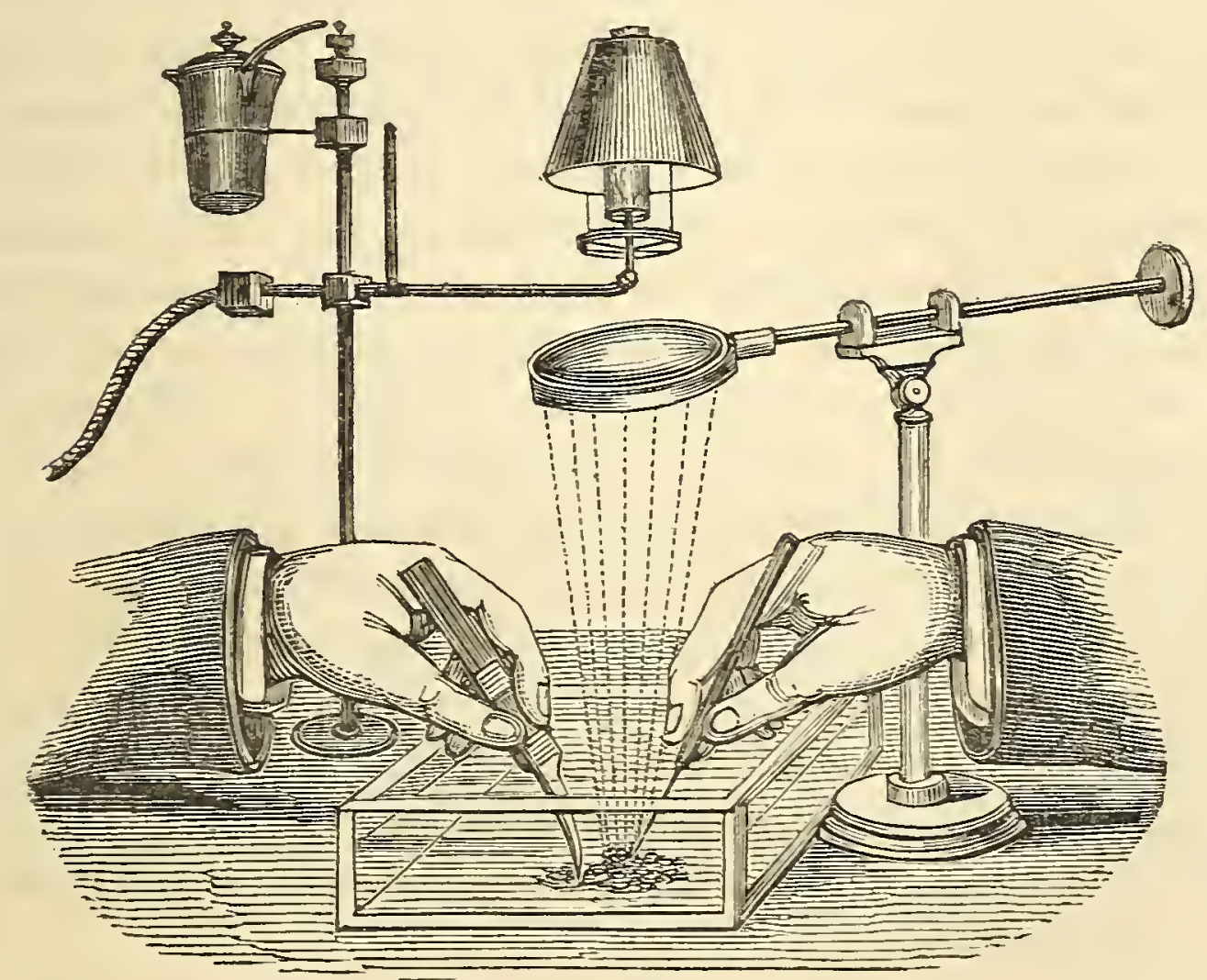

fig. 54. 
The improved Valentin's. Knife.-For making fine sections of large substances, or those soft in structure, such as the liver, spleen, and kidney, the double-bladed knife, the invention of Professor Valentin, may be used with advantage. An improved construction of this knife by Professor Quekett is represented in fig. 55. It consists of two blades, one of which is prolonged by a flat piece of steel to form a handle, and has two pieces of wood riveted to it for the purpose of its

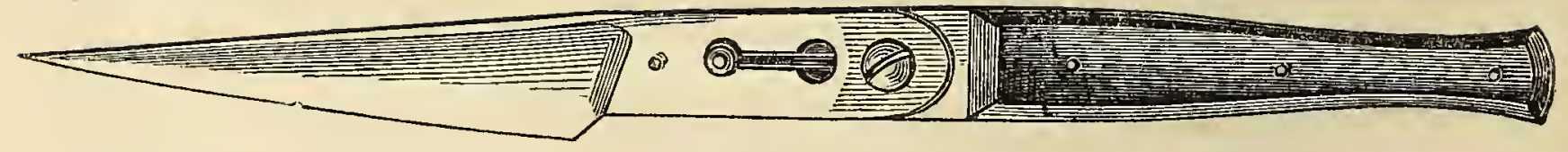

fig. 55.

Deing held more steadily; to this blade another one is attached by a screw; this last is also lengthened by a shorter piece of steel, and both it and the preceding have slits cut out in them exactly opposite to each other, up and down which slit a rivet with two heads is made to slide, for the purpose either of allowing the blades to be widely separated or brought so closely together as to touch; one head of this rivet being smaller than the hole in the end of the slit, can be drawn through it; so that the blade seen in the front of the figure may be turned away from the other in order to be sharpened, or allow of the section made by it being taken away from between the blades. The blades are so constructed that their opposed surfaces are either flat or very slightly concave, so that they may fit accurately to each other, which is effected more completely by a steadying pin seen at the base of the front blade. When the instrument is required to be used, the thickness of the section about to be made will depend upon the distance the blades are apart, and this is regulated by sliding up or down the rivet, as the blades, by their own elasticity, will always spring open and keep the rivet in place; a cut is then to be made by it, as with an ordinary knife, and the part cut will be found between the blades, from which it may be separated either by opening them as wide as possible by the rivet, or by turning them apart in the manner before described, and floating the section out in water.

Dissecting Scissors.-In addition to the forceps and knives, scissors will be necessary for the purposes of dissection: of these the most useful 
are shown in fig: 56. They are either straight or curved; of the first kind, two pairs will be required, one having the extremities broad, and the other sharp-pointed; and if large dissections be undertaken, a still stronger pair, with the extremities broad, and made rough like a file, will also be necessary. In dissecting under the microscope, the curvedpointed pair shown at $f$ will be found most convenient. In all of these

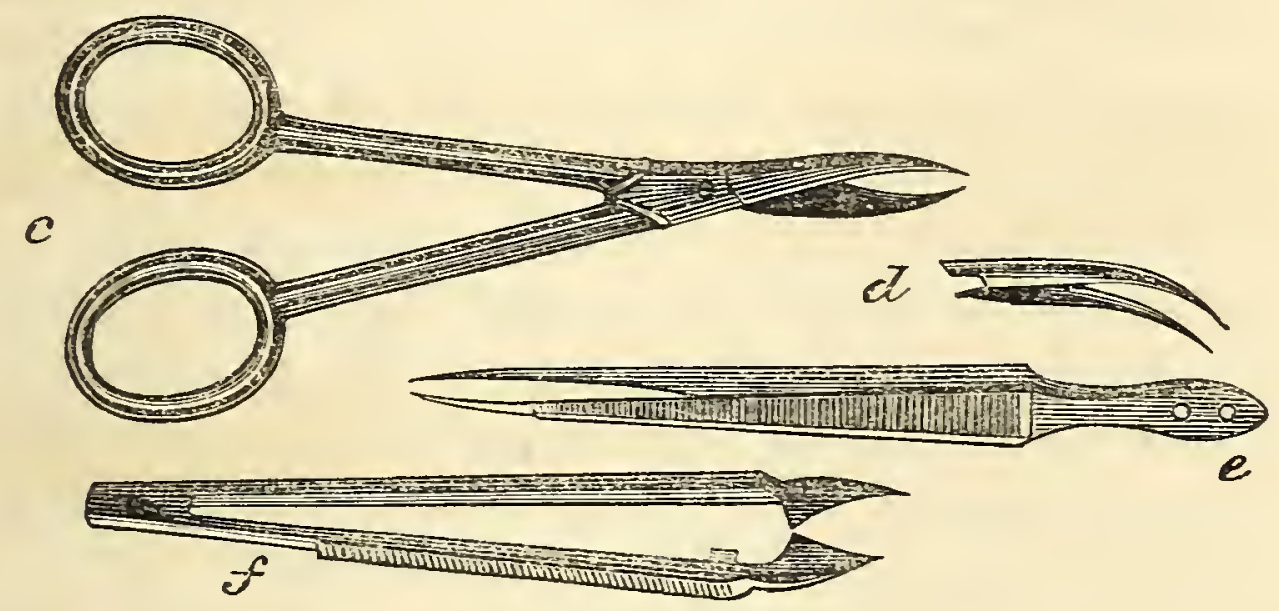

fig. 56.

instruments the points should fit accurately together : sometimes those that are very sharp are apt to cross; this may in a great measure be prevented by having the branches wide at the base where they are riveted. The points can be sharpened on a hone, and a magnifier employed to examine if they fit closely together.

Circular Disc.-For the purpose of cutting glass corers, or making shallow cells with japanners' gold-size for mounting objects, fig. 57 ,

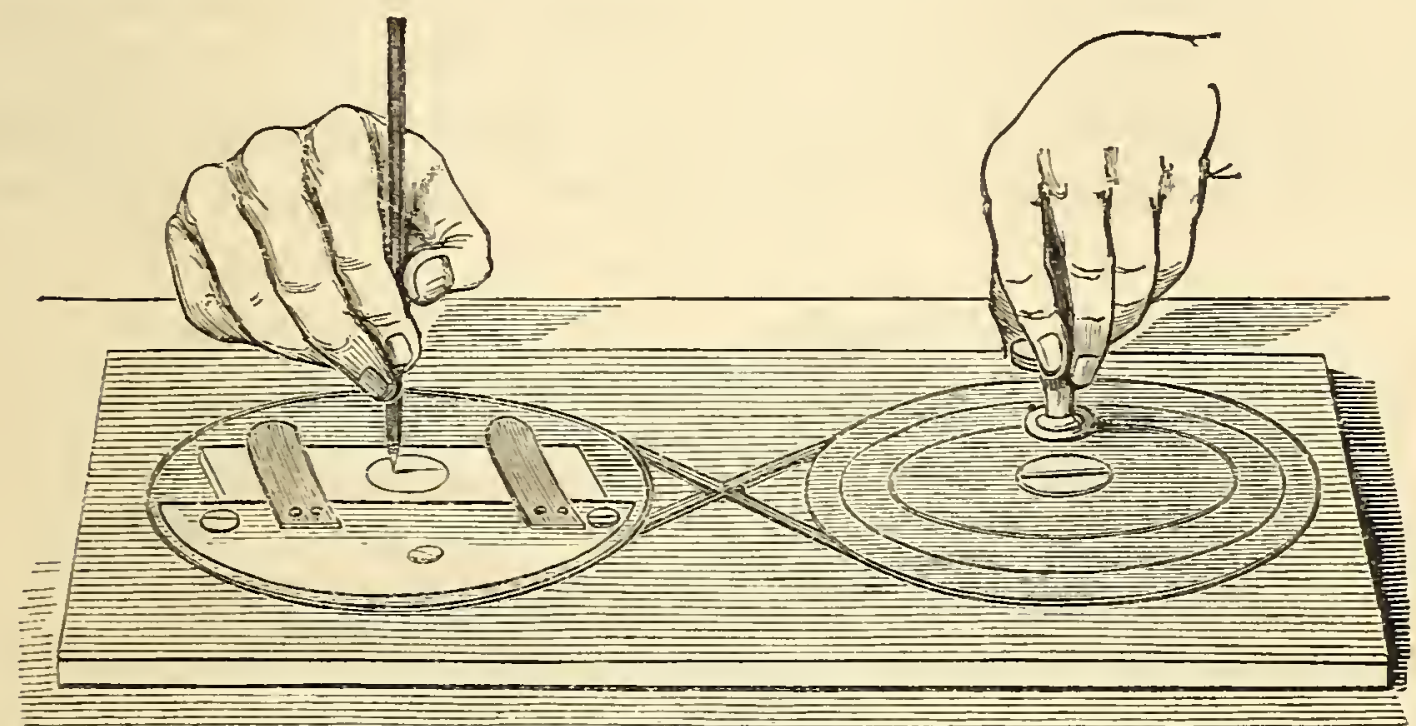

fig. $5 \%$.

is made out of two circular wheels of wood, these being let into a solid block of wood, and secured there by central screws. A handle 
of wood is fixed into the upper part of one, for the purpose of turning it round, the motion being communicated to the other by an endless band of catgut running in the grooved edge of each. On the upper surface of the wheel, under the right hand, are fixed, by means of screws, two strips of brass, which serve as springs for securing the glass-slip; a camels'-hair pencil previously dipped in japanners' goldsize is then taken between the finger and thumb, and held as represented in the woodcut; when the wheel is put in motion, and a perfect circle is rapidly formed; the cell is then removed, and put aside to dry. In the same way, by securing a sheet of thin glass under the brass springs, and substituting for the pencil a cutting diamond, a circular cover may be readily cut out. A cutting diamond is not only useful to the microscopist for the above purpose, but also for writing the names of mounted objects on the ends of the glass slides. A glazier's diamond for cutting glass slides is both convenient and economical : the mode of using it may be learned in any glazier's shop.

On Mounting and Preserving Objects. - Microscopic objects are usually mounted on slips of glass three-inches by one-inch, either dry or immersed in some fluid. The minute structures, such as the tissues of animals, parts of insects, vessels of plants, \&c., must be preserved in thin cells, made as directed above, with a small amount of fluid. Clean the glass with a weak solution of ammonia or potash from all grease, and wipe it dry with a piece of chamois leather; cloth generally leaves behind it small filaments, which are always unsightly when seen near the object; let fall a drop of the preserving fluid or Canada balsam on the centre of the glass, then place the object in it with a small pair of forceps, and spread it out very carefully with the point of one of your fine needles. Select a thin glass cover, previously cleaned, touch its edges with cement, and let it fall gently and gradually down upon the

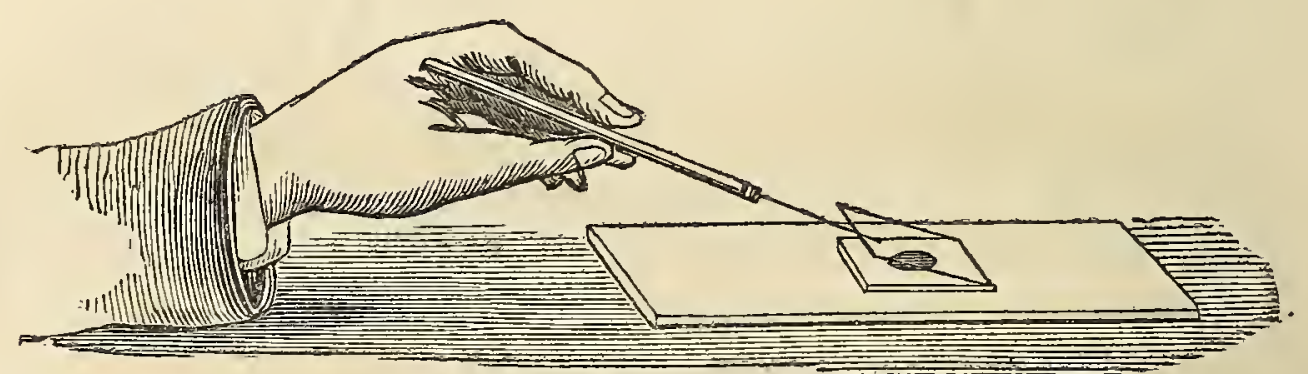

fig. 58.

object, as represented in fig. 58 ; press it lightly to exclude any excess of fluid, which remove with strips of blotting-paper, being careful to do all this with a light hand, that small bubbles of air may not insinuate 
themselves to replace any lost fluid, air-bubbles being at all times unsightly, and liable to spoil an object when allowed to remain; lastly, cement the edges of the cover to the bottom glass with japanners' goldsize of sealing-wax varnish, carefully drawn around the edges with a camels'-hair pencil. Mr. John Gorham has lately proposed the use of a "Brass cementing Pencil," which he has described and represented in the Microscopical Journal. Fig. 59 is a brass tube, six inches long, with a conical bore, having a lid to screw on. A small portion of the cement crumbled into fragments is shot into the tube, which is then ready for use. In using this instrument, the extremity is gently heated in the flame of a spirit-lamp, and when the cement begins to ooze out, holding the pencil like a pen, the point is traced along each side of the cover, leaving a line of cement in the angle. It is thus laid on much easier than with a brush, and after a little manipulation, it will be found that the point will suffice to polish off, instead of using the flattened wire. The cement recommended by $\mathrm{Mr}$. Quekett for cementing deep cells is made by melting together two ounces of black resin, one ounce of bees'-wax, wind one of vermilion. Mr. Hett prefers dark-coloured and old japanners' gold-size for securing the cells of his beautifully dolicate injected preparations.

Mr. Gorham at the same time gave the drawing of a "holder," similar to one long used by the author (shown in fig. 60), for the purpose of pressing together objects mounted in the dry way, and during the drying process, after maceration, which facilitates removal of the object from time to time fig. 59 . for the purpose of examining it. It is made of two stout strips
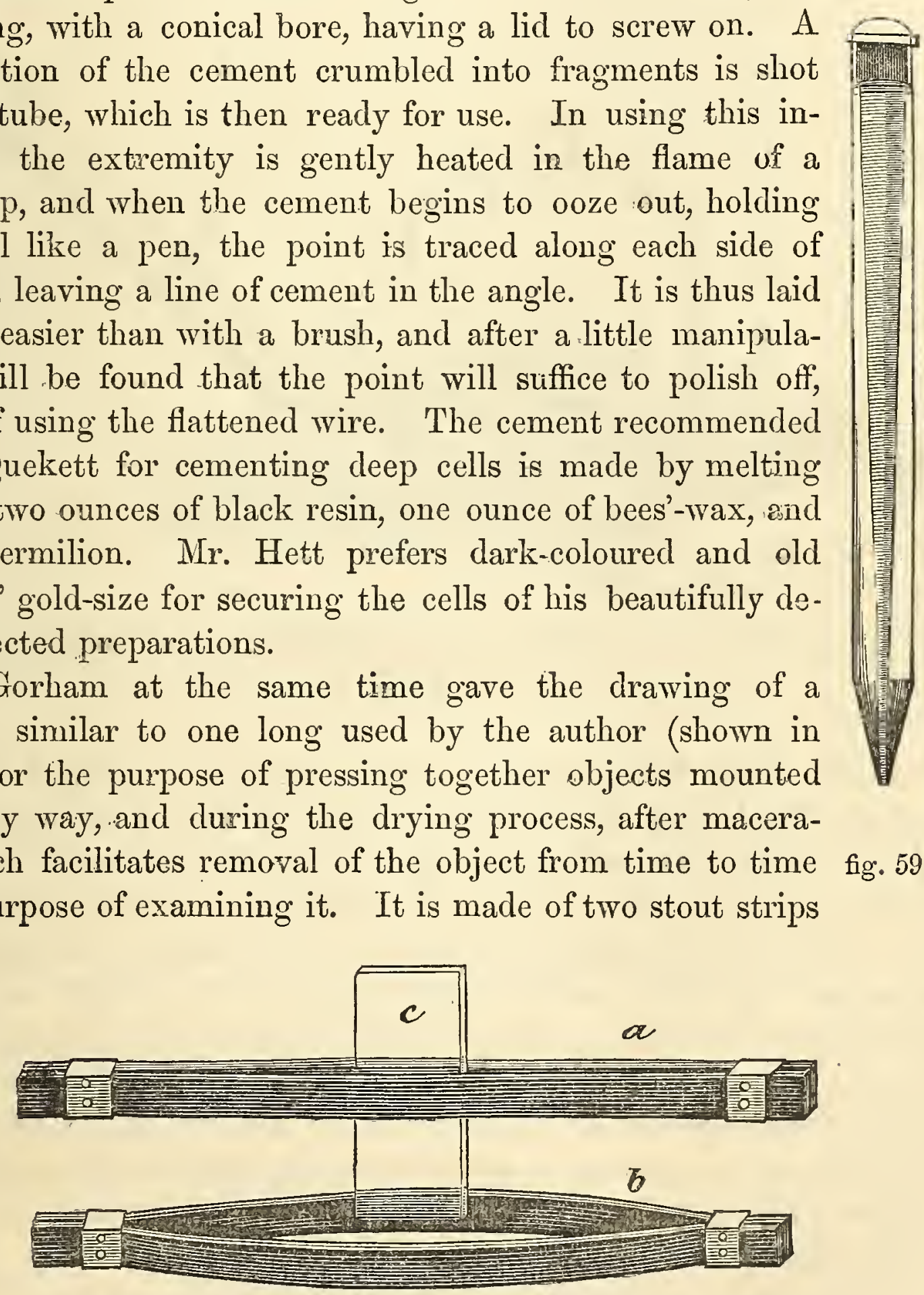

fig. 60 .

of whalebone three or four inches in length, held together at each end with square pieces of brass; these may be moved at pleasure towards the centre, and thus made to exert considerable pressure upon the 
pieces of glass and the object, which are placed crossways between the strips of whalebone.

For the purpose of more effectually removing bubbles of air from the cells before cementing them down, the small air-pump, fig. 61, will be found very serviceable. This may be purchased of Baker, Hol-

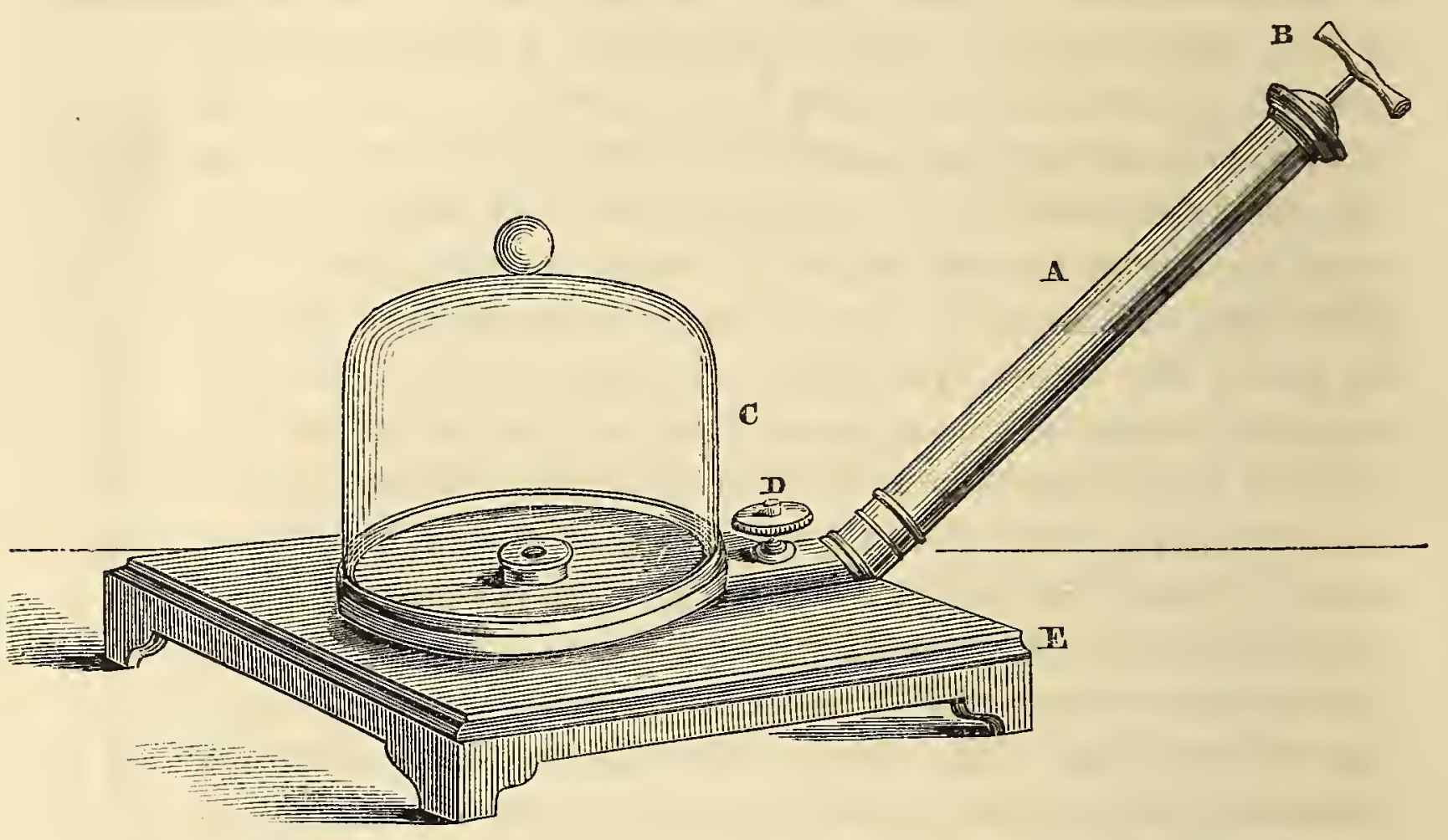

fig. 61 .

born, for a few shillings. The mode of using it, is simply to put the object, after having placed the thin glass cover over it, under the bellglass $\mathrm{C}$; then, by drawing up and pushing down the handle $\mathrm{B}$, pump out the air until the whole of the small bubbles are withdrawn from around the object to be secured and preserved. By turning the small screw at $\mathrm{D}$, you will let in sufficient air to remove the bell-glass; or allow your object to remain under it for several hours until the cement around the edge of the glass-cover becomes perfectly dried and secure, when exposure to the external air will no longer affect it. It will be also found useful in withdrawing the air from the cells of woods or limbs of insects. The pump itself, a, when unscrewed, can be used as an injecting-syringe for fine anatomical injections.

Mrr. Thomas Boys, in his directions fur mounting objects in Canada balsam, says, the first thing to be considered is the apparatus required.

1st. A small single-wick oil-lamp, having a glass chimney about four inches long; the flame to be about the size of that in a handlantern : a spirit-lamp will do even better. 
2d. Slips of glass of required size, and small pieces of thin glass to cover the object, all well cleaned.

3d. A pair of nippers to hold the glass-slips.

4th. A pointed iron-piercer in a wooden handle.

5th. A bottle containing the clearest Canada balsam, diluted with the best spirits of turpentine to a consistency allowing it to drop readily from one end of the iron-piercer, or Mr. Gorham's cementingpencil. The preceding articles being spread before you ready for use, and the object to be displayed well examined for choice of position, and cleaned if necessary, fix the glass-slip in the nippers, dip the piercer into the balsam, and withdraw a full-sized drop to place upon the slide. The centre of the slide should now be rested across the chimney of the lamp until the balsam begins to spread, when it must be immediately withdrawn. The object is then to be placed on this balsam, and at once covered with a second drop, applied in the same way as the first; in this state the slide should remain (covered to exclude the dust) for two or three minutes, that the balsam may have time to penetrate; the thin glass is then to be taken up between the finger and thumb, and placed gently upon the balsam covering the object. The slide being now held by the nippers at one end, place the other extremity over the lamp-chimney, so that the heat may be gradually extended towards the object. The proof of its having done so sufficiently will be that the balsam flows to the edge of the thin glass, taking with it all air-bubbles from that part nearest the heat. The slide is now to be turned, the heated end being placed in the nippers, and the process repeated. The slide should remain flat till nearly cool, when pressure should be made with a small piece of wood perpendicularly on the upper glass; this will expel all superfluous balsam, and with it all extraneous matter. Should any air-bubbles remain, they generally disappear in a few days. If the balsam require hardening, place the slide on the mantel-piece, the gentle heat from which will prove sufficient.-Microscopical Society's Transactions.

Mr. Deane recommends a composition of gelatine for mounting dry or moist animal or vegetable structures in place of Canada balsam, his formula for which is as follows:- "Take of White's patent size, 6 ounces by weight; honey 9 ounces by weight; add a little spirits of wine and a few drops of creosote; mix and filter whilst hot, to render perfectly clear. Or take of pure glycerine, 4 fluid ounces; distilled water, 2 fiuid ounces; gelatine, 1 ounce by weight: dissolve the gelatine in the water made hot, then add the glycerine and mix with care; this need not be filtered. 
"Fresh animal or vegetable structures containing their natural juices require little or no preparation before mounting in this substance. Those contained in water, as the desmidiex, crustaceans, \&c. may, when taken out of their native element, be placed on a proper slip of glass previously warmed, the superfluous water removed with blotting-paper, and then mounted at once by dropping a little warm medium upon them and covering with thin glass. Animalculæ mounted in this way do not appear to alter either in colour or dimensions. The latter may be accounted for in this way, viz. that the contraction of the medium is entirely in thickness, and the pressure of the cover prevents any alteration taking place in the object laterally.

"It is probable that some animalculæ may be better shown if some moisture be allowed to remain in the medium, the evaporation from which may be stopped at any stage, by filling round the edge of the cover with some gold-size varnish, or even boiled linseed-oil.

"For many delicate objects this has a great advantage over Canada balsam, in not possessing the high refractive power of that substance, and the minute hairs and other parts of insects, that are quite obliterated with the balsam, are beautifully shown in this medium."

Glycerine was first introduced by Mr. Warrington as a preservative fluid for mounting organic substances in. He says, "The method I have adopted in the employment of glycerine, is simply to mount the object in the manner it is usually performed when spirit of wine or creosote-water is used as a medium, and having covered the immersed object with the thin glass, and removed all excess of liquid, to cement the margin with a coating of shell-lac varnish; the one I usually employ consists of the ordinary black sealing-wax dissolved in rectified spirit of wine. Care must be taken during this operation to maintain the slider in a flat position, until the varnish has become dry from the evaporation of the spirit, and also until a sufficient number of coatings or layers of the varnish have been applied to render the subject perfectly secure, and prevent any escape of the fluid. Gold-size or copal dissolved in oil of lavender may be employed to effect the same purpose; and the second and third coatings may, with advantage, consist of either of these, which yield a tough varnish above the lac, which is otherwise liable to become brittle.

"The glycerine may be used in its concentrated form or treacly state, or it may be diluted with distilled water to any required extent, according to the object of the operator, and the subject to be mounted; if there be extremely fine markings on the subject, it is better to add about four or five times its volume of water, as otherwise the thick 
fluid may prevent these from being so sharply defined as may be desired. I have a number of slides of Desmidiece, which have been mounted from four to ten months by this means, and they have kept excellently.

"The glycerine may also be used with the addition of a small portion of culinary salt, corrosive sublimate, creosote, or spirit of wine, if considered desirable. While on this subject, I will describe the method adopted for using castor-oil as a medium for mounting crystals of salts and other objects.

"The object required to be mounted is placed on the slider in its dry state, or deposited wet an allowed to dry; or if in solution, a drop of the liquid is to be placed on the slip of glass, and allowed to crystallise by spontaneous evaporation; in the latter case I prefer taking a drop of a warm saturated solution of the salt required, and when a good group of well-defined crystals has been obtained, to break through the marginal ring of crystalline deposit with a small point of wood, and carefully conduct off the uncrystallised portion or mother-liquor to the extremity of the slide, at the same time placing it in a vertical position to drain until it is dry. A small quantity of castor-oil is to be next carefully dropped on the subject, and guided over the field with the point of a needle; in this way it readily displaces the air and occupies the most minute cavities. After a short time the upper glass is to be placed on the surface, taking care to lower it gradually so as to exclude the air; if the field is too full of oil, the excess may be removed by a small piece of bibulous paper; and if, on the contrary, sufficient oil has not been used, an additional portion can be readily introduced by the capillary action between the glasses. I mention these points of practical manipulation, because on them the success of the operation may often depend, as it must be evident that, in the first case the excess would prevent the cell from being properly sealed by the varnish; and in the latter it would be drawn into the field, and the whole operation spoiled. The shell-lac varnish is then to be used as the cementing medium in the same way as has been described, and with the same precautions. I may also observe, that this varnish cannot be replaced by either of the others, as it is actually necessary (and this should always be borne in mind,) that there should exist no affinity between the fluid in the cell and the varnish used to seal it permanently. Hundreds of excellent objects have been lost from this cause, and much valuable time and labour thrown away.

"The reason why oil was selected for this purpose arose from its want of action on most crystalline salts, many of which could not be 
preserved uninjured in any other medium. Castor-oil was chosen in consequence of its not depositing a crystalline stearin by reduction of temperature, as is the case with most oils, and even in some specimens of this material ; it therefore requires to be carefully examined in this respect before it is employed. In the same year I used this medium as a mounting fluid for minute fungi and pediculi with complete success."

Mr. Goadby has succeeded in supplying to microscopists a ready, cheap, and effectual means for mounting animal structures with the greatest possible ease and security. The following are his formulæ :

Take for No. 1 solution, bay salt, $4 \mathrm{oz}$; alum, 2 oz.; corrosive sublimate, 2 grains; boiling water, 1 quart: mix. For No. 2 solution, bay salt, 4 oz.; alum, 2 oz.; corrosive sublimate, 4 grs.; boiling water, 2 quarts : mix.

The No. 1 is too strong for most purposes, and should only be employed where great astringency is needed to give form and support to very delicate structures. No. 2 is best adapted for permanent preparations; but neither should be used in the preservation of animals containing carbonate of lime (all the mollusca), as the alum becomes decomposed, sulphate of lime is precipitated, and the preparation spoiled. For such use the following:

Bay salt, 8 oz. ; corrosive sublimate, 2 grs.; water, 1 quart:- mix.

The corrosive sublimate is used to prevent the growth of vegetation in the fluid; but as this salt possesses the property of coagulating albumen, these solutions cannot be used in the preservation of ova, or when it is desired to maintain the transparency of certain tissues, such as the cellular tissue, the white corpuscles of the blood, \&c.

Mr. Gorham proposes to employ "collodion in the production of transparent membranes for microscopic purposes; and there are reasons for supposing that it will enable us to construct a series of novel and highly interesting preparations, by its presenting the minute tracery observed on the surface of many opaque objects in a transparent form. In this way we can multiply impressions of specimens which are very beautiful and rare. It bids fair also to put us into possession of the general configuration on the surface of certain minute fresh vegetable structures, which become shrivelled and their beauty obliterated in drying. It is best applied as follows: A few chips of red sanderswood are taken up in a drachm or two of good collodion; the surface of the object is then painted over four or five times, and in less than ten minutes the flake or cast of collodion can be peeled off and mounted on a slide under a thin cover as a dry preparation." 


\section{The Iode of Injecting Ninute Vessels, \&c.}

For minute injections the most essential instrument is a proper syringe. This is usually made of brass, of such a size that the top of the thumb may press on the button at the top of the piston-rod when drawn out, while the body is supported between the two fingers. Fig. 62 represents the syringe: $a$ is a cylindrical brass body, with a screw at the top for the purpose of firmly screwing down the cover $b$ after the piston $c$ is introduced into it; this is rendered airtight with leather; the bottom of the syringe $d$ also unscrews for the convenience of cleaning; $e$ is a stop-cock, on the end of which another stop-cock $f$ fits accurately; and on the end of this either of the small pipes $g$, which are of different sizes, may be fixed. The transverse wires across the pipes are intended to secure them more tightly to the vessels into which they may be inserted with thread, so that they may not slip out. In addition to the syringe, a large tin vessel, to contain hot water, with two or three lesser ones fixed in it for the injections, will be found useful.

To prepare the material for injecting :-Take of the finest and most transparent glue one pound, break it into small pieces, put it into an earthen pot, and pour on it three pints of cold water ; let

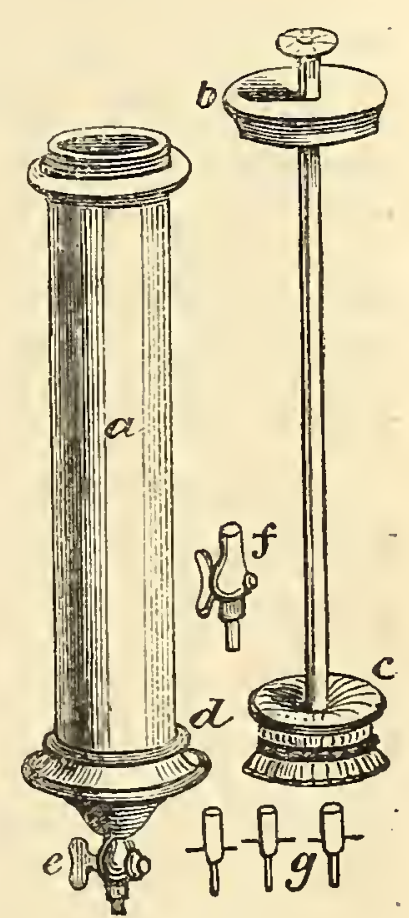

fig. 62 . it stand twenty-four hours, stirring it now and then with a stick; then set it over a slow fire for half an hour, or until all the pieces are perfectly dissolved; skim off the froth from the surface, and strain through a flannel for use. Isinglass and cuttings of parchment make an excellent size, and are preferable for very particular injections.

The size thus prepared may be coloured with any of the following :

For one Red.

$\begin{array}{ll}\text { " } & \text { Yellow. } \\ \text { "White. } & \text { Blue. }\end{array}$

To pint of size, add $2 \mathrm{oz}$. of Chinese vermilion.

, $2 \frac{1}{2}$ oz. of chrome yellow.

, $3 \frac{1}{2}$ oz. of flake white.

, $6 \mathrm{oz}$. of fine blue smalts.

It is necessary to remember, that whatever colouring matter is employed must be very finely levigated before it is mixed with the injection. This is a matter of great importance; for a small lump or mass of colour, dirt, \&c. will clog the minute vessels, so that the injection 
will not pass into them, and the object will be defeated. The mixture of size and colour should be frequently stirred, or the colouring matter will sink to the bottom. Respecting the choice of a proper subject for injecting, it may be remarked, that the injection will usually go farthest in young subjects; and the more the creature's fluids have been exhausted in life, the greater will be the success of the injection.

To prepare the subject, the principal points to be aimed at are, to dissolve the fluids, empty the vessels of them, relax the solids, and prevent the injection from coagulating too.soon. For this purpose it is necessary to place the animal, or part to be injected, in warm water, as hot as the operator's hand will bear. This should be kept at nearly the same temperature for some time by occasionally adding hot water. The length of time required is in proportion to the size of the part and the amount of its rigidity. Ruysch (from whom the art of injecting has been called the Ruyschian art) recommends a previous maceration for a day or two in cold water.

When the size and the subject have both been properly prepared, have the injection as hot as the fingers can well bear. One of the pipes $g$, fig. 62 , must then be placed in the largest artery of the part, and securely tied. Put the stop-cock $f$ into the open end of the pipe $e$, and it is then ready to receive the injection from successive applications to the syringe $a$, leaving sufficient space only for the piston $c$. The injection should be thrown in by a very steady and gentle pressure on the end of the piston-rod. The resistance of the vessels, when nearly full, is often considerable; but it must not be overcome by violent pressure with the syringe. When as much injection is passed as may be thought advisable, the preparation may be left (with the stop-cock closed in the pipe) for twenty-four hours, when more material may be thrown in.

As the method of injecting the minute capillaries with coloured size is often attended with doubtful success, various other plans have been proposed. Ruysch's method, according to Rigerius, was to employ melted tallow coloured with vermilion, to which in the summer a little white wax was added. A material used by some is made of resin and tallow: take of each two ounces, melted and strained through linen, to which add three ounces of vermilion or finely ground indigo, first well rubbed with eight ounces of turpentine varnish. Monro recommended coloured oil of turpentine for the small vessels, after the use of which he threw in the common coarse injection.

Professor Breschet frequently employed with success milk, isinglass, the alcoholic solution of gum-lac, spirit varnish, and spirit of 
turpentine; but he highly commends the colouring matter extracted from Campeachy, Fernambone, or Sandal-woods. He says: "The colouring matter of Campeachy wood easily dissolves in water and in alcohol; it is so penetrating that it becomes rapidly spread through the vascular net-works. The sole inconvenience of this kind of injection is, that it cannot be made to distend any except most delicate vessels, and that its ready penetration does not admit of distinguishing between arteries, veins, and lymphatics." He also recommends a solution of caoutchouc.

Another process, which may be termed the chemical process, was published in the Comptes Rendus, 1841, as the invention of M. Doyers. According to this, an aqueous solution of bichromate of potass is propelled into the vessels; and after a short time, in the same manner and in the same vessels, an aqueous solution of acetate of lead is injected. This is an excellent method, as the material is quite fluid, and the precipitation of the chromate of lead, which takes place in the ressels themselves, gives a fine sulphur-yellow colour.

Mr. Goarby has improved upon the process last named by uniting to the chemical solutions a portion of gelatine. The following is his formula, published in the Lancet:

Saturated solution of bichromate of potash, 8 fluid ounces; water, 8 ounces; gelatine, 2 ounces.

Saturated solution of acetate of lead, 8 fluid ounces; water, 8 ounces; gelatine, 2 ounces.

Mr. Goadby remarks respecting this process: "The majority of preparations thus injected require to be dried and mounted in Canada balsam. Each preparation, when placed on a slip of glass, will necessarily possess more or less of the coloured infiltrated gelatine, (by which he means the gelatine coloured by the blood, which, together with the acetate of potash resulting from the chemical decomposition, may have transuded through the coats of the ressel,) which, when dry, forms, together with the different shades of the chromate of lead, beautiful objects, possessing depth and richness of colour. The gelatine also separates and defines the different layers of vessels. By this injection the arteries are always readily distinguishable by the purity and brightness of the chromate of lead within them, while the veins are detected by the altered colour imparted by the blood.

"Those preparations which require to be kept wet can be preserved perfectly in my B fluid, specific gravity 1,100; the A fluid destroys them.

"I would recommend that the slips of glass employed for the dry 
preparation be instantly inscribed with the name of the preparation, written with a diamond; for, when dry, it is difficult to recognise one preparation from the other, until the operator's eye be educated to the effects of this chemico-gelatinous injection. Where so much wet abounds, gummed paper is apt to come off.

"When dry, it is sufficient, for the purpose of brief examination by the microscope, to wet the surface of a preparation with clean oil of turpentine; immediately after examination, it should be put away carefully in a box, to keep it from the dust, until it can be mounted in Canada balsam.

"The bichromate of potash is greatly superior in colour to the chromate, which yields too pale a yellow; and subsequent experience has proved that the acetate of potash frequently effect: its liberation by destruction of the capillaries, and this even long after the preparations have been mounted in Canada balsam; perhaps this may be owing to some chemical action of the acetate of potash upon them.

"I would suggest the substitution of the nitrate for the acetate of lead, as we should then have, in the liberated nitrate of potash, a valuable auxiliary in the process of preservation.

"Although highly desirable, as the demonstrator of the capillaries of normal tissues, I do not think this kind of injection fitted for morbid preparations; the infiltrated gelatine producing appearances of a puzzling kind, and calculated to mislead the pathologist.

"In preparing portions of dried well-injected skin for examination by the microscope, I have tried the effect of dilute nitric acid as a corroder with very good results. But, probably, liquor potassa would have answered this purpose better.

"When size injection is to be employed, coloured either with vermilion or the chromate of lead, the animal should be previously prepared by bleeding to empty the vessels; for if they be filled with coagulated blood, it is quite impossible to transmit even size, to say nothing of the colouring matter. Hence the difficulty of procuring good injections of the human subject.

"But with the chemico-gelatinous injections no such preparation is necessary; and success should always be certain, for the potash liquefies the blood, while constant and long-continued pressure by the syringe drives it through the parietes of the vessel into the cellular tissue. The large quantity of infiltrated blood - the invariable concomitant of my process-characterises this from all other modes of injecting, and is a distinctive feature of these preparations."

Still another plan has been suggested. It consists in adding a 
quantity of sulphuric ether to the finely levigated colouring matter, which is also first ground or mixed with linseed oil, in the manner employed by painters. Upon this plan (as well as upon the last named) some beautiful injections of the smallest capillaries are made; but sometimes it is a failure, owing to the too rapid evaporation of the ether, and the clogging up of the vessels from the early deposition of the solid colouring matter. Perhaps a solution of gum-mastic in ether, coloured with fine vermilion, will answer the purpose better.

A foetus may be injected by the umbilical vein; the head, by the carotids; the liver, mucous membrane of the intestines, \&c. by the portal vein; an extremity by the principal artery, \&c.

Many parts, after injection, require to be macerated in water, or corroded by dilute muriatic acid, \&c., in order to exhibit the ramifications of the small vessels. They should be very carefully handled, or moved, in the macerating liquor, as the slightest force may break the vessels. When corroded, the pulpy flesh is to be carefully washed away, by placing it under a stream of water, flowing very slowly, or by the use of a syringe with water.

The lymphatics are now usually injected with coloured material instead of quicksilver, as formerly. M. Rusconi employed a small silver syringe, together with a kind of trocar, of which the canula is formed from the quill of the wing-feather of the quail or partridge, - the trocar being a tolerably large-sized surgical needle, the point having three facets. When desirous of injecting the lymphatic system of a lizard, tortoise, \&c., he says: "I seize with a small pair of forceps the mesentery, close to the vertebral column, where the reservoir of the chyle is situated, and I introduce into it the point of the trocar; I then retain the quill, and withdraw the needle from the tube. I then seize with the small forceps the quill, and introduce into it the small extremity of the syringe ; and push the piston with a force always decreasing."

By carefully following out these directions, any one, after a little practice, will be enabled to succeed in making many useful and valuable preparations. Injections are best viewed by the aid of the Leiberkuhn, or under a condensed light with a one or two inch object-glass.

Dr. Schacht recommends the following chemical re-agents and preservative fluids for microscopic uses.

1. "Alcohol, principally for the removal of air from sections of wood and other preparations; also as a solvent for certain colouring matters,

2. Ether, chiefly as a solvent for resins, fatty and other essential oils, \&c. ; also useful for the removal of air.

3. Solution of Caustic Potass, as a solvent for fatty matters; also of use occasionally in consequence of its action upon the rest of the 
cell-contents and thickening layers. This solution acts best upon being heated.

4. Solution of Iodire (iodine one grain, iodide of potassium three grains, distilled water one ounce) for the coloration of the cell-membrane and of the cell-contents.

5. Concentrated Sulphuric Acid, employed chiefly in the examination of pollen and spores.

6. Diluted Sulphuric Acid (three parts acid, one part water), for the coloration of cells previously immersed in the iodine solution. The preparation is first moistened with the iodine solution, which is afterwards removed with a hair pencil, and a drop of sulphuric acid added by means of a glass rod; the preparation is then immediately covered with a piece of glass. The action of the sulphuric acid and iodine, as well as that of the iodised chloride of zinc solution, is not always uniform throughout the whole surface of the preparation. The colour is more intense where the mixture is more concentrated; it frequently happens that many spots remain uncoloured. The colour changes after some time, the blue being frequently changed into red after twentyfour hours.

7. A Solution of Chloride of Zinc, Iodine, and Iodide of Polassium. A drop of this compound solution, added to a preparation placed in a little water, produces the same colour as iodine and sulphuric acid. This solution, which was first proposed and employed by Professor Schulz of Rostock, is more convenient in its application than iodine and sulphuric acid, and performs nearly the same services, while it does not, like the sulphuric acid, destroy the tissues to which it is applied. The precise composition is as follows:-

Zinc is to be dissolved in hydrochloric acid, and the solution, in contact with metallic zinc, is to be diluted to a syrupy consistence, anci the solution must then be saturated with iodide of potassium. Iodine is then to be added, and the solution, if necessary, diluted with water.

8. Nitric Asid, or what is better, chloride of potass and nitric acid, as an agent to effect the isolation of cells. The mode of employing this means, also discovered by Professor Schulz, is as follows:-

The object, a thin section of wood for instance, is introduced, with an equal bulk of chloride of potass, into a long and moderately wide tube, and as much nitric acid as will at least cover the whole.

The tube is then warmed over a spirit-lamp: a copious evolution of gas takes place, upon which the tube is removed from the flame, and the action of the oxydising agent allowed to continue for two or three minutes. The contents of the tube are then poured into a watchglass with water, from which the slightly cohering particles are col- 
lected and placed in a tube, and again boiled in aIcohol as long as any colour is communicated.

They are again boiled in a little water. The cells may now be isolated under the simple microscope by means of needles. The boiling with nitric acid and chlorate of potass should never be carried on in the same room with the microscope, the glasses of which may suffer injury from the vapours.

Thin sections of vegetable tissue are warmed for half a minute; or a minute, in a watch-glass: boiling is here unnecessary. The section is taken out, and treated with water in a watch-glass.

9. Oit of Lemons, or any other essential oil, for the investigation of pollen and spores.

10. A moderately strong solution of Nuriate of Lime (one part dry muriate of lime, and three parts distilled water), for the preservation of microscopical preparations. This is applicable to most things, even for the most delicate preparations, excepting starch. If it is desired to keep a preparation, which is not to be retained permanently, for some days, a small drop of the solution may be placed upon it, and the whole put under a glass cover to keep it fiom dust.

11. Glycerine also for the preservation of microscopical preparations. It is well adapted for the preparation of cells containing starch, which remains uncharged by it. In starch-grains exhibiting a laminated structure, as, for instanee, in potato-starch, the lamination is usually not apparent for the first few hours, but after about twenty-. four hours it becomes more evident.

12. Copal Varnish and Canadcs Balsam, also used for the preservation of microseopic objects. Both substances can only be employed in the case of a few thin sections of wood, especially of fossil woods; both render the object more transparent than the solution of muriate of lime.

Lastly may be enumerated a pretty strong solution of Carbonate of Soda and also Acetic Acid; which latter, however, is more especially useful in the investigation of animal tissues.

To the above may be added a test for protein compounds. This test is composed of sugar and sulphuric acid, and is thus employed: A thin section or portion of the tissue to be examined is placed in a drop of simple syrup. This is then removed by means of a hair pencil; and a drop of the diluted sulphuric acid added; the red colour usually: does not appear till after the lapse of about ten minutes.

In making thin sections of tissues, it is recommended that, in the ease of objects the consistence of which differs in different parts, the section should be carried from the harder into the softer portion. Also 
in making a thin section of a very minute yielding substance, to enclose it between two pieces of cork, and to slice the whole together. It is also useful sometimes to saturate the object with mucilage, which is to be allowed to dry slowly; in this way very delicate tissues may be sliced, or otherwise divided without injury, and with great facility."*

The preceding observations must be received with caution; a very useful warning having been recently given by Dr. Parkes in reference to unusual crystalline forms he has found some of these re-agents to assume, when in use, under the microscope. Without a knowledge of this singular fact, and a perfect recognition of the crystalline forms, errors in micro-chemical research cannot but occur. For example, if a drop of liquor potassæ be allowed to evaporate on a slip of glass, crystals appear, chiefly six-sided tables, precisely like cystine; when in quantity, they are often crowded together, as the cystine plates are, and sometimes exhibit a similar nucleus-like body in their centres. These crystals do not arise from the presence of impurities; at least perfectly pure potash exhibited the same phenomena.

$\mathrm{He}$ also states that the form of the crystals of acetate of potash varies according to the strength of the acid out of which it crystallises; and when formed out of strong acid, very much resembles that of the crystals of uric acid; for, mixed up with other forms, long dagger-like or lancet-shaped crystals are seen, which might well deceive us.

It may also be noticed in this place, that Majendie has lately pointed out that in certain albuminous mixtures, iodine loses the property of colouring starch blue. This difficulty must be got rid of before iodine can be used without fear in micro-chemistry.

The same author recommends a solution of muriate of lime for the preservation of vegetable tissues.

Preservation and Preparation of Algce, \&c.-Mr. Ralf's excellent directions for making preparations of the algæ for the microscope are as follows: "First to obtain a fluid which shall preserve the plant as little altered as possible from its appearance when living; and secondly, to adopt the best means for preventing the escape of this fluid after the object has been mounted in it. With respect to the first point, the fluid which I have found to answer best is made in the following way: to sixteen parts of distilled water add one part of rectified spirits of wine, and a few drops of creosote, sufficient to saturate it; stir in a small quantity of prepared chalk, and then filter; with this fluid mix an equal measure of camphor-water (water saturated with camphor); and before using strain off through a piece of fine linen.

"This fluid I do not find to alter the appearance of the endochrome

* Quarterly Journal of Microscopical Science, October 1852. 
of algæ more than distilled water alone does after some time; and there is certainly less probability of confervoid filaments making their appearance in the preparations; and there would seem to be nothing to prevent such a growth from taking place, when the object is mounted in water only, provided a germ of one of these minute plants happen to be present, as well as a small quantity of free carbonic acid.

"Fluids containing a larger quantity of spirits of wine, and consequently of creosote also, than the one of which I have given the formula, produce a greater change in the appearance of the endochrome.

"My method of making cells in which to mount preparations of algæ is as follows: some objects require very shallow, and others somewhat deeper cells. The former may be made with a mixture of japanners' gold size and litharge, to which (if a dark colour is preferred) a small quantity of lamp-black can be added. These materials should be rubbed up together with a painter's muller, and the mixture laid on the slips of glass with a camel-hair pencil as expeditiously as possible, since it quickly becomes hard; so that it is expedient to make but a small quantity at a time. For the deeper cells marine glue answers extremely well, provided it is not too soft. It must be melted and dropped upon the slip of glass; then flattened, whilst warm, with a piece of wet glass, and what is superfluous cut away with a knife, so as to leave only the walls of the cell; these, if they have become loosened, may be made firm again by warming the under surface of the slip of glass. The surface of the cells must be made quite flat; which can be easily done by rubbing them upon a wet piece of smooth marble, covered with the finest emery-powder.

"When about to mount a preparation, a very thin layer of goldsize must be put upon the wall of the cell, as well as on the edge of the piece of thin glass which is to cover it; before this is quite dry, the fluid with the object is to be put into the cell, and the cover of thin glass slowly laid upon it, beginning at one end; gentle pressure must then be used to squeeze out the superfluous fluid; and, after carefully wiping the slide dry, a thin coat of gold-size should be applied round the edge of the cell; and a second coat so soon as the first is dry; a thin coat or two of black sealing-wax varnish may then be put on with advantage, in order to prevent effectually the admission of air into the cell, or the escape of fluid out of it.

"I at first mounted objects for the microscope without enclosing them in a cell previously prepared for their reception, but merely by laying them on the slip of glass with a drop of the fluid, and then covering them up with a piece of thin glass or talc; and afterwards sur- 
rounding the latter with a border of thick gold-size, in order to prevent the evaporation of the enclosed fluid. Preparations so made will frequently last some considerable time; but eventually the contraction, as it becomes dry, of the outer surface of gold-size forces the remainder, which still continues soft, between the two glasses, and the mounted object is thus injured. I found the same thing frequently to occur when the cells were made of gold-size only, without the litharge; but this inconvenience seems to be completely obviated by the plan I have recommended above.

"I would remark, that the gold-size employed should be of the consistence of treacle; when purchased, it is usually too fluid, and should be exposed for some time in an open vessel; a process which renders it fit for use."

In mounting the Desmidiece, great attention is necessary to exclude air-bubbles, which cannot be avoided, unless the fluid completely fills the cells; and also not to use too much fluid, as in this case the smaller species will often be washed away on the escape of the superfluous portion.

As the cells cannot be sealed whilst any moisture remains on their edge, it should be removed by blotting-paper, in preference to any other mode.

A thin description of glass is manufactured expressly for the purpose of covering specimens when mounted.

Specimens are frequently spoiled by the intrusion of the gold-size into the cell. Experience will best teach how this accident can be avoided. Attention, however, to the following particulars will assist the inexperienced. The cells should be prepared some days before they are used, in order that their walls may become firm. When the cell is closed, the brush should be passed round the edge of the cover, with just sufficient size to prevent the admission of air into the cell; and upon the operator's care in this respect his success will depend.

If too little be used, the air will enter; whereas if too much be put on, or if the cell be not completely filled with fluid, the size will enter, in either case spoiling the specimen.

The fluid which Mr. Topping has used for mounting consists of one ounce of rectified spirit to five ounces of distilled water; this he thinks superior to any other combination. To preserve delicate colours, however, he prefers to use a solution of acetate of alumina; one ounce of the acetate to four ounces of distilled water: of other solutions he says that they tend to destroy the colouring matter of delicate objects, and ultimately spoil them by rendering them opaque. 


\section{On Coliecting Objects.}

The following hints for collecting objects for microscopical examination, by G. Shadbolt, Esq., are exceedingly useful and valuable to the microscopist.

"Having procured a good microscope, it is often a source of perplexity to the novice to obtain a sufficient supply of objects on which to exercise his powers.

"Rivers, brooks, springs, fountains, ponds, marshes, bogs, and rocks by the sea-side, are all localities that may be expected to be productive; some being more prolific than others, and the species obtained differing, of ccurse, in general, to a certain extent, according to the habitat.

"On considering the nature of some of the places indicated, it will be apparent that, in order to spend a day in collecting with any comfort, it will be necessary to make some proxision for keeping the feet dry, for which a pair of India-rubber goloshes will answer, or better still, a pair of waterproof fishing-boots; but without one or other the work is by no means pleasant.

"A dozen or two of small bottles made of glass-tubing, about half an inch in diameter, and without necks, and from one to two inches in length, are the most convenient depositories for the specimens, if intended ultimately for mounting; and it is advisable also to take two or three wide-mouthed bottles of a larger size, holding from one to two fluid ounces, an old iron spoon, a tin box, some pieces of linen or calico, two or three inches square; a piece of string, a slip or two of glass, with the edges ground, such as are used for mounting objects; and lastly, a good and pretty powerful hand-magnifier. Two Coddington lenses mounted in one frame of about half an inch, and onetenth of an inch in local power, are specially convenient.

"Perhaps it will be as well, in pointing out a few localities, to describe some that I have actually visited, with the means of access, and the appearance of the various species, en masse, that I have met with.

"Swanscombe Salt-marsh will be found well worth a visit; and it can be reached by steam-boat or railway from London-bridge to Northfleet. On quitting the railway-station, make towards the almshouses on the top of the hill; and arriving at the road, turn to the left, descend the hill, and cross a sort of bridge over a somewhat insignificant stream. Continue along the main road a little farther, to a point 
where it begins to ascend again, and diverges to the left towards the railway; here quit it, taking your course along an obscure road, nearly in a direct line with the main one; passing a windmill on the right hand, and continuing until you arrive at another still more obscure road, turning off to the right; which road appears as if made of the mud dredged from the bottom of the river, and partially hardened. This is Swanscombe Salt-marsh; and the road just described leads towards Broad Ness Beacon. On either side is a sort of ditch; one containing salt or very brackish water, the other filled with a sort of black mud, about the consistence of cream, the surface being in parts of a slaty grey, with little patches here and there of a most brilliant brown colour, glistening in the sunshine, and presenting a striking contrast to the sombre shade. By carefully insinuating the end of one of the slips of glass under this brilliant brown substance, and raising it gently, it can be examined with the Coddington; and it will probably be found to consist of myriads of specimens of pleurosigma (navicula of Ehrenberg), angulatum, or balticum, or some other species of this genus. The iron spoon is now useful, as by its aid the brown stratum, with little or no mud, can be skimmed off and bottled for future examination. On the surface of the water in the other ditch may be noticed a floating mass of a clark olive colour, which to the touch feels not unlike a lump of the curd of milk, and consists of $C y c l o-$ tella menighiniana, and a surirella or two embedded in a mass of Spimulina hutchinsia; and another mass of floating weed, which feels harsh to the touch, procceding from a quantity of a synedra, closely investing a filamentous alga, and elsewhere Meloseira nummuloides (gallionella of Ehrenberg).

"In a trench by the sec-wall, as it is termed, is a mass of brown matter of a shade somewhat different to any hither'to observed, adhering to some of the parts of the trench, being partially submerged, and having a somewhat tremulous motion on agitating the water. This is a species of schizonema, and it consists of a quantity of gelatinous hollow filaments filled with an immense number of bright-brown shuttleshaped bodies, like very minute naviculce.

"It is not necessary to be particular about collecting the specimen free from mud, as the filaments are so tough that the mud can be readily washed away by shaking the whole violently in a bottle of water, and pouring off the mud without at all injuring the specimen. The Amphisporium alatum conmunicates a somewhat frothy appearance to the otherwise clear water, and to get any quantity of this requires a little management; but by skimming the surface with the 
spoon, and using one of the larger bottles, an abundance may readily be obtained. Between the sea-wall and the river the marsh is intersected in every direction with a number of meandering creeks, being in some places eight to ten feet deep, though in others quite shallow; but it is exceedingly difficult to make one's way amongit them, and I have never found them so prolific any where, on the few occasions of my visiting the place, as in the parts more away from the influence of the tide. It will be observed, that the brilliant brown colour, of a deep but bright cinnamon tint, is one of the best indications of the presence of diatomacece; and though this is by no means universal, the variation is most frequently dependent upon the presence of something which qualifies the tint. The peculiarity of the colour is due to the endochrome contained in the frustule; and this must in general be got rid of before the beautiful and delicate marking can be made out. But it is highly advantageous and instructive to view them in a living state; and this should be done as soon as possible after reaching home with all specimens procured from salt-water localities, as they rapidly putrefy in confinement, and emit a most disgusting odour, not unlike that arising from a box of inferior congreve-matches.

"Washing in fresh water, and then immersing in creosote-water, preserves many of the species in a very natural-looking manner; but they are killed by the fresh water, and the enduchrome becomes much condensed in the pleurosigmata and some other species. The addition of spirits quite spoils the appearance of the frustules, as it dissolves the endochrome.

"There is another salt-marsh a little farther down the same railway, at Higham, which it would be well to explore.

"The most favourable months for procuring cliatomacece, are A pril, May, September, and October ; but some species are found in perfection as early as February, and many as late as November, and a few at all times of the year.

"There is a piece of boggy ground near Keston, beyond Bromley in Kent, where the river Ravensburne takes its rise, where many interesting species of desmidiece and other fresh-water algæ may be procured. From Bromley, walk on towards Keston, passing near Hayes Common and Bromley Common on the right. Continue for about another half-mile along the road, and then turn to the right hand; pass the reservoirs; and approach an open space where there is a bog of about a quarter of a mile in extent; and tending towards the right, make ycur way amongst heaths, ferns, mosses, and the beautiful Droserca rotundifolia (sun-dew), to the lower part of the little stream rippling 
through a sort of narrow trench in the sphagnum, \&c. By working your way up the stream, you avoid the inconvenience which would otherwise be experienced of the water being rendered turbid, in consequence of having to tread in the boggy ground.

"In the centre of the little stream may be observed something of a pale pea-green colour flickering about in the current, which, on your attempting to grasp, most likely eludes you, and slips through the fingers, from being of a gelatinous nature. It consists of a hyaline substance, with a comparatively small quantity of a bright-green endochrome, disposed in little branches, and this is the Draparnaldia glomerata.

"Another object is a mass of green filaments, rather harsh to the touch, and zery slippery. When viewed with a lens of moderate power, each filament is seen to be surrounded with several bands of green dots, looking like a ribbon twisted spirally, and may be recognised as Zygnema nitidum. In various parts there are other species of zygnemce, tyndaridea, mougeotia, mesocarpus, and many others.

"Keeping up. the stream, and occasionally diverging an little on either side of it, amongst the miniature bays and pools formed by the sphagnum, on looking straight down into the water we shall probably see at the bottom a little mass of jelly of a bright green, studded with numerous brilliant bubbles of oxygen-gas. This is the general appearance of most of the desmidiece, as micrasteias, euastrum, closterium, cosmarium, \&c. "The spoon is also a handy tool in this case, though, by practice, the finger will do nearly as well; the chief difficulty arises when the specimen is brought to the surface of the water, it not being easy to get it out without losing a considerable portion of it:

"Little" pools in the bog, made by the footsteps of cattle, are particularly good spots to find desmidiece, many species being in a very contracted space. The most prolific bog is at Tunbridge Wells, near a house known as Fisher's Castle, not far from Hurst Wood. There is also a good one at Esher, at a spot called West-End.

"It must not be imagined that nothing can be obtained in this department of botany without going some distance from town; but assuredly only commoner and fewer species can be met with nearer home. At the West India Docks are Synedra fasciculata, Gomphonema curvata, Diatoma elongata, Diatoma vulgaris, Surirella ovata, \&c.; and at this same place a few objects, not of the botanical class, as Spongilla Auviatilis, Cordylophora lacustris, Alcyonella stagnorum, \&c., are obtainable in abundance in the autumn.

"In the Serpentine may be found Cladophora glomerata and Sphcero- 
plea crispa, two of the filamentous algæ. And in the ornamental water in St. James's Park, Cocconema lanaceolatum, and other species of this genus, Gomphonema crispata, dc. Epping Forest, about the neighbourhood of Leytonstone, Snaresbrook, Wanstead, and Woodford Bridge, are also capital localities for the filamentous algæ, especially the last-named, where Nitella translucens and Chara vulgaris abound."

Localities of microscopic plants and animals. -For the information of microscopical observers resident, in and near the metropolis, the following species, in addition to the commoner ones not necessary to be specified, may be found in the fresh-water and brackish ditches in the parish of Milton, next Gravesend :

Surirella striatula.

,g gemma.

Aneurea squamata.

$$
, \text { striata. }
$$

Nitschia rerersa.

$$
\text { , closterium. }
$$

Gallionella nummuloides.

Cosmarium crenatum.

, unảulatum.

Synedra valens.

, biceps.

„, acicularis.

", radians.

, lunaris.
Srnedra ulna.

Amphora ovalis.

Cymbella Enrenbergii.

Cyclotella Kutzingiana.

Gomphonema olivaceum.

Bacillaria paradoxa.

Leucophrys patula.

Coleps hirtus.

Nassula elegans.

Schizonema.

Euplotes Charon.

$$
\text { , truncatus. }
$$

Pleurosigma Hippocampus.

$$
\text { " elongatum. }
$$

On the north side of the Serpentine, Hyde Park, especially near the bridge, may be found :

Cymbella maculata.

Gomphonema cristatum.

Scenedesmus quadricauda. obliquus.

Ankistrodesmus falcatus.

Pediastrum Heptactys.

Cocconema lanceolatum.
Amphora ovalis.

Cocconeis placentula.

Uvella hyalina.

Gallionella nummuloides.

Euastrum elegans.

Pixidula operculata.

\section{The CAuera Lưdida.}

The Camera Lucida, fig. 63, was inventec by Dr. Wollaston, in 1807: it is a valuable addition to the microscope, for making drawings of structures, and for obtaining, with a micrometer, accurate measurements. It consists of a four-sided prism of glass, set in a brass frame

\footnotetext{
* Quarterly Journal of Microscopical Science.
} 
or case, as represented in the adjoining figure; and by means of a short tube it is slipped over the front part of either of the eye-pieces, its cap having been previously taken off. Mr. Ross "attaches the prism, by two short supports, to a circular piece of brass at the end of the tube; on this it can be slightly rotated, whilst the prism itself can also be turned up or down, by means of two screws with milled heads. So arranged, the camera may be adapted to the eye-piece, the microscope having been previously placed in a horizontal position; if the light be then reflected up through the compound body, an eye placed over the

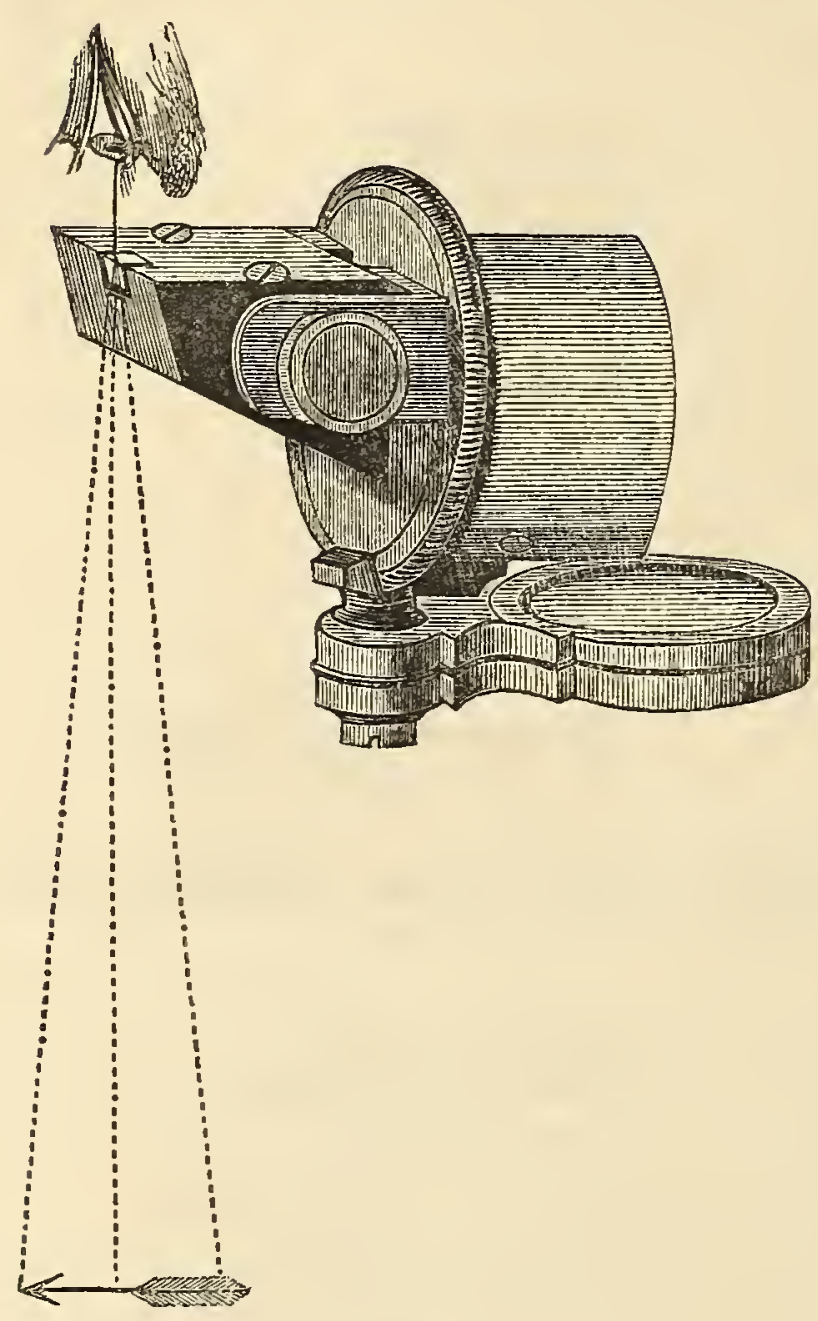

fig. 63 . square hole in the frame of the prism will see the image of any object on the stage upon a sheet of white paper placed on the table immediately below it. But should it happen that the whole of the field of view is not well illuminated, then, either by revolving the circular plate or turning the prism upon the screws, the desired object will be effected. The chief difficulty in the use of this instrument is for the artist to be able to see, at one and the same time, the pencil and the image. To facilitate this in some measure, the one or two lenses below the prism will cause the rays from the paper and pencil to diverge at the same angle as those received from the prism, whereby both object and pencil may be seen with the same degree of distinctness."

Professor Quekett gives the following directions for using the Camera Lucida with the microscope: "The first step to be taken after the object about to be drawn has been properly illuminated, adjusted, and brought into the centre of the field of view, is to place the compound body of the microscope in a horizontal position, and to fix it there. The cap of the eye-piece having been removed, the camera is to be slid on in its stead: if the prism is properly adjusted, a circle of white light, with the object within it, will be seen on a piece of white paper placed on the table immediately under the camera, when the eye of the 
observer is placed over the uncovered edge of the prism, and its axis directed towards the paper on the table. Should, however, the field of view be only in part illuminated, the prism must either be turned round on the eye-piece, or revolved on its axis, by the screws affixed to its frame-work, until the entire field is illuminated. The next step is to procure a hard, sharp-pointed pencil, which, in order to be well seen, may be blackened with ink round the point; the observer is then to bring his eye so near the edge of the prism that he may be able to see on the paper, at one and the same time, the pencil-point and the image of the object. When he has accomplished this, the pencil may be moved along the outline of the image, so as to trace it on the paper. How ever easy this may appear in description, it will be found very difficult in practice; and the observer must not be foiled in his first attempts, but must persevere until he accomplishes his purpose. Sometimes he will find that he can see the pencil-point, and all at once it disappears : this happens from the movement of the axis of the eye. The plan then is to keep the pencil upon the paper, and to move about the eye until the pencil is again seen, when the eye is to be kept stedfastly fixed on the same position until the entire outline is traced. It will be found the best plan for the beginner to employ at first an inch objectglass, and some object, such as a piece of moss, that has a well-defined outline, and to make many tracings, and examine how nearly they agree with each other; and, when he has succeeded to his liking, he may then take a more complicated subject. If the operation is conducted by lamp-light, it will be found very advantageous not to illuminate the object too much, but rather to illuminate the paper on which the sketch is to be made, either by means of the lamp with the condensing. lens, or a small taper placed near it. When the object is so complicated that too much time would be required for it to be completed at one sitting, the paper should be fixed to the table by a weight, or on a board by drawing-pins. An excellent plan to adopt is to fix the microscope on a piece of deal about two feet in length and one foot in breadth, and to pin the paper to the same; there will then be no risk of the shifting of the paper, as, when the wood is moved, both microscope and paper will move with it. In all sketches made by the camera, certain things must be borne in mind: the eye, when once applied to it, should be kept steadily fixed in one position; and if the sketches are to be reserved for comparison with others, the distance between the paper and the camera should be always the same. A short rule or a piece of wood may be placed between the paper and the under surface either of the compound body or the arm supporting 
it, in order to regulate the distance, as the size of the drawing made by the camera will depend upon the distance between it and the paper. It is also very desirable, before the camera is removed, to make a tracing in some part of the paper of two or more of the divisions of the stage micrometer, in order that they may form a guide to the measurement of all parts of the object. Some persons cover the whole of the drawing ower with squares, to facilitate, not only the measurement, but in order that a larger or smaller drawing may be made from it than that given by the camera. It must be recollected that an accurate outline is the only thing the camera will give: the finishing of the picture will depend entirely upon the skill of the artist himself."

\section{On the Polarisation of Light as applied to the Microscope.}

Huyghens and others having observed that a ray of light has not the same properties in every part of its circumference, compared it to a magnet, or a collection of magnets; and supposed that the minute particles of which it was said to be composed had different poles, which, when acted on in certain ways, arranged themselves in particular positions; and thence the term polarisation, a term having neither reference to cause nor effect. It is to Malus, however, who, in 1808, discovered polarisation by reflection, that we are indebted for the series of splendid phenomena which have since that period been developed; phenomena of such surpassing beauty as far to exceed any thing which can be presented to our eyes under the microscope. It has been truly observed by Sir David Brewster, that "the application of the principles of double refraction to the examination of structures is of the highest value. The chemist may perform the most dexterous analysis; the crystallographer may examine crystals by the nicest determination of their forms and cleavage; the anatomist or botanist may use the dissecting knife and microscope with the most exquisite skill; but there are still structures in the mineral, vegetable, and animal kingdoms which defy all such modes of examination, and which will yield only to the magical analysis of polarised light. A body which is quite transparent to the eye, and which might be judged as monotonous in structure as it is in aspect, will yet exhibit, under polarised light, the most exquisite organisation, and will display the result of new laws of combination which the imagination even could scarcely have conceived. In evidence of the utility of this agent in exploring mineral, vegetable, and animal structures, the extraordinary organisation 
of Apophyllite and Analcime may be referred to; also the symmetrical and figurate depositions of silicious crystals in the epidermis of equisetaceous plants, and the wonderful variations of density in the crystalline lenses of the eyes of animals.

"If we transmit a beam of the sun's light through a circular aperture into a darkened room, and if we reflect it from any crystallised or uncrystallised body, or transmit it through a thin plate of either of them, it will be reflected and transmitted in the very same manner, and with the same intensity, whether the surface of the body is held above or below the beam, or on the right side or left, provided that in all cases it falls upon the surface in the same manner; or, what amounts to the same thing, the beam of solar light has the same properties on all its sides; and this is true, whether it is white light as directly emitted from the sun, or from a candle or any burning or self. luminous body; and all such light is called common light. A section of such a beam of light will be a circle, like $a b c d$, fig. 64 ; and we shall
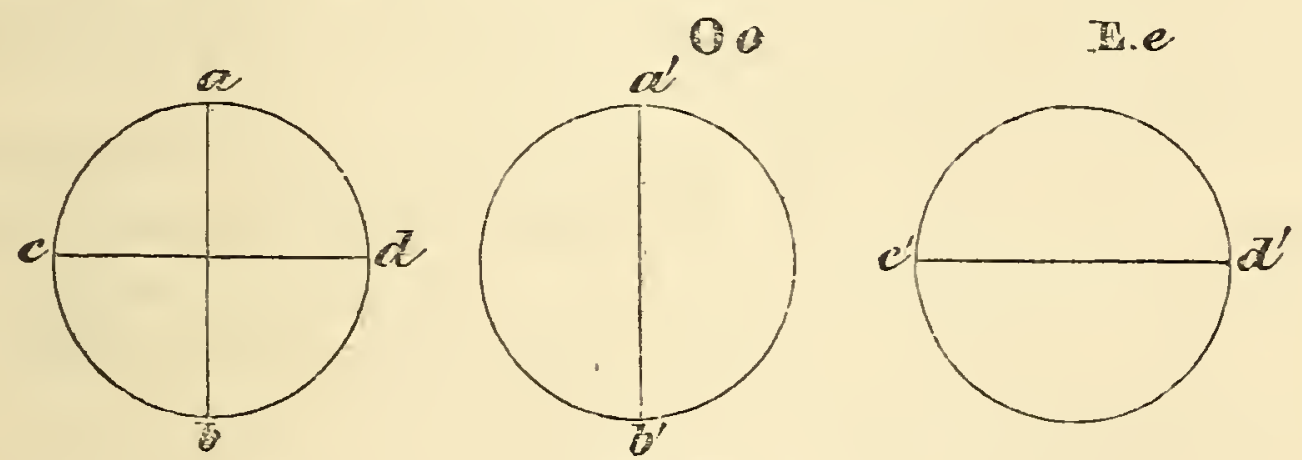

fig. 64 .

distinguish the section of a beam of common light by a circle with two diameters $a b, c d$, at right angles to each other.

"If we now allow the same beam of light to fall upon a rhomb of Iceland spar, and examine the two circular beams, $O o E e$, formed by double refraction, we shall find, 1st, that the beams $O o E e$ have different properties on different sides, so that each of them differs in this respect from the beam of common light.

" 2 d. That the beam $O o$ differs from $E e$ in nothing excepting that the former has the same properties at the sides $a^{\prime} b^{\prime}$ that the latter has at the sides $c^{\prime}$ and $d^{\prime}$; or in general, that the diameter of the beam, at the extremities of which the beam has similar properties, are at right angles to each other, as $a^{\prime} b^{\prime}$ and $c^{\prime}$ and $d^{\prime}$ for example.

"These two beams, $O o, E$ e, are therefore said to be polarised, or" to be beams of polarised light, because they have sides or poles of different properties and plines passing through the lines $a b, c d$; or $a^{\prime} b^{\prime}, c^{\prime} d$, 
are said to be the planes of polarisation of each beam, because they have the same property, and one which no other plane passing through the beam possesses.

"Now it is a curious fact, that if we cause the two polarised beams $O O, E e$ to be united into one, or if we produce them by a thin plate of Iceland spar, which is not capable of separating them, we obtain a beam which has exactly the same properties as the beam $a b c d$ of common light. Hence we infer that a beam of common light, $a b c d$, consists of two beams of polarised light, whose plane of polarisation, or whose diameters of similar properties are at right angles to one another. If $O o$ is laid above $E_{e} e$, it will produce a figure like $a b c d$; and we shall therefore represent common light by such a figure. If we were to place $O o$ above $E e$, so that the planes of polarisation $a^{\prime} b^{\prime}$ and $c^{\prime} d^{\prime}$ coincide, then we should have a beam of polarised light twice as luminous as either $O o$ or $E e$, and possessing exactly the same properties; for the lines of similar property in the one beam coincide with the lines of similar property in the other. Hence it follows, that there are three ways of converting a beam of common light, $a b c d$, into a beam or beams of polarised light.

" 1st. We may separate the beam of common light, $a b c d$ into its component parts $O o$ and $E^{T}$. 2 d. We may turn round the planes of polarisation, $a b c d$, till they coincide or are parallel to each other; or, we may absorb or stop one of the beams, and leave the other, which will consequently be in a state of polarisation."**

The first of these methods of producing polarised light is that in which we employ a doubly reflecting crystal, which was first discovered to exist in a transparent mineral substance called Iceland spar, calcareous spar, or carbonate of lime. This substance is admirably adapted for exhibiting this phenomenon, and is the one generally used by microscopists. Iceland spar is composed of fifty-six parts of lime and forty-four parts of carbonic acid; it is

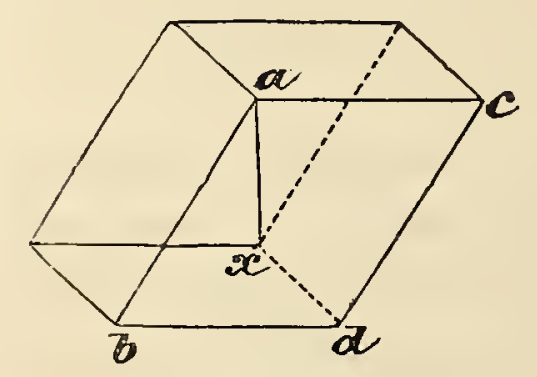

fig. 65 . found in various shapes in almost all countries; but whether found in crystals or in masses, we can always cleave it or split it into shapes represented by fig. 65 , which is called a rhomb of Iceland spar, a solid bounded by six equal and similar rhomboidal surfaces, whose sides are parallel, and whose angles $b a c, a c d$, are $101^{\circ} 55^{\prime}$ and $78^{\circ} 5^{\prime}$. The line $a x$, called the axis of the rhomb or of the crystal, is equally inclined to each of the 
six faces at an angle of $45^{\circ} 23^{\prime}$. It is very transparent, and generally colourless. Its natural faces when it is split are commonly even and perfectly polished; but when they are not so, we may by a new cleaverage replace the imperfect face by a better one, or we may grind and polish any imperfect face.

It is found that in all bodies where there seems to be a regularity of structure, as salts, crystallised minerals, on light passing through them, it is divided into two distinct pencils. If we take a crystal of Iceland spar, and look at a black line or a dot on a sheet of paper, there will appear to be two lines or dots; and on turning the spar round, these objects will seem to turn round also; and twice in the revolution they will fall upon each other, which occurs when the two positions of the spar are exactly opposite, that is, when turned one-half from the position where it is first observed. In the accompanying diagram, fig. 66, the line appears double, as $a b$ and $c d$, or the dot as $e$ and $f$. Or allow a ray of light, $g h$, to fall thus on the crystal, it will in its passage through be separated into two rays, $h f, h e$; and on coming to the opposite surface of the crystal, they will pass out at $e f$ in the direction of $i \hbar$, parallel to $g \hbar$. The plane $l m n o$ is designated the principal section of the crystal, and the line drawn from the solid angle $l$ to the angle $o$ is

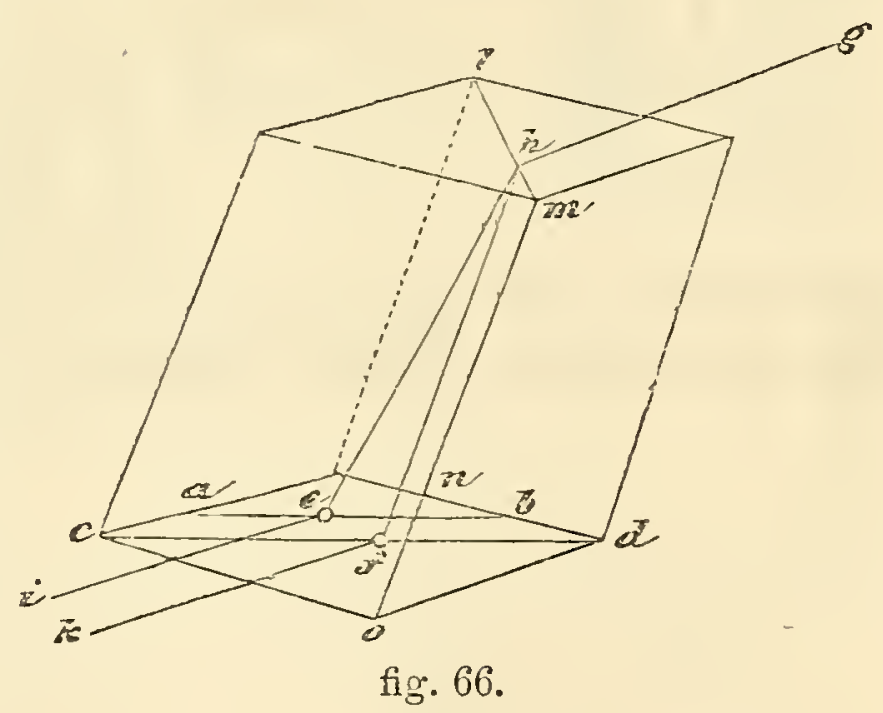
where the axis of the crystal is contained; it is also the optic axis of the mineral. Now when a ray of light passes along this axis, it is undivided, and there is only one image; but in all other directions there are two.

If two crystals of Iceland spar be used, the only difference will be, that the objects seem farther apart, from the increased thickness. But if two crystals be placed with their principal sections at right angles to each other, the ordinary ray refracted in the first will be the extraordinary in the second, and so on vice versâ. At the intermediate position of the two crystals there is a subdivision of each ray, and therefore four images are seen; when the crystals are at an angle of $45^{\circ}$ to each other, then the images are all seen of equal intensity.

Mr. Nicol first succeeded in making rhombs of Iceland spar into single-image prisms, by dividing one into two equal portions. His 
mode of proceeding is thus described in the Edinburgh Philosophical Journal (vol. vi.p. 83): "A rhomb of Iceland spar of one-fourth of an inch in length, and about four-eighths of an inch in breadth and thickness, is divided into two equal portions in a plane, passing through the acute lateral angle, and nearly touching the obtuse solid angle. The sectional plane of each of these halves must be carefully polished, and the portions cemented firmly with Canada balsam, so as to form a rhomb similar to what it was before its division; by this management the ordinary and extraordinary rays are so separated that only one of them is transmitted: the cause of this great divergence of the rays is considered to be owing to the action of the Canada balsam, the refractive index of which $(1.549)$ is that between the ordinary (1.6543) and the extraordinary (1.4833) refraction of calcareous spar, and which will change the direction of both rays in an opposite manner before they

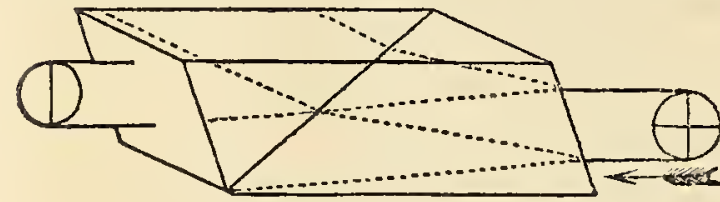

fig. 67 . enter the posterior half of the combination." The direction of rays passing through such a prism is seen by the arrow and fig. 67. This combination is shown mounted and for use, the one under the stage of the microscope, fig. 68 , termed the polariser; the other, fig. 69 , screwed on to and above the object-glasses, is called the analyser.

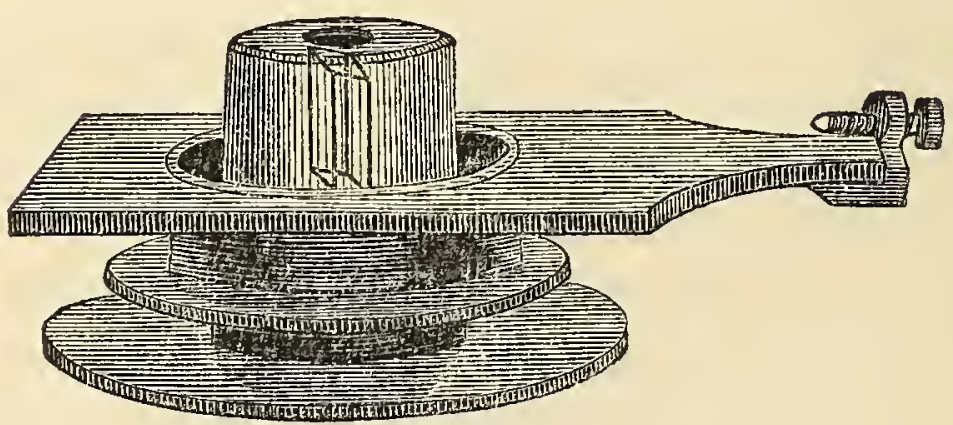

fig. 68 .

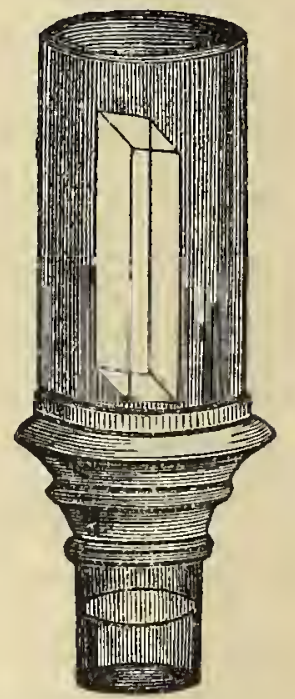

fig. 69.

Method of using the polarising prism, fig. 68. After having adapted it to slide into a groove on the under-surface of the stage, it is held in its place by turning the small milled-head screw at one end: the other prism, fig. 69, is screwed on to the object-glasses, and made to pass into the body of the instrument itself. By this arrangement the whole field is brought into view at the same time; but by placing the analys- 
ing prism above the eye-piece, a very narrow and contracted field of vision results. The light having been reflected through them by the mirror, it becomes necessary to make the axes of the two prisms coincide; this is done by regulating the milled-head screw, until by revolving the polarising prism, the field of view is entirely darkened twice during one revolution. This is also ascertained and carefully corrected by the maker and adapter of the apparatus. If very minute salts or crystals are to be viewed, it is preferable to place the polariser above the eye-piece; it should then be mounted as in fig. 70. Thus the polariscope consists of two parts ; one for polarising, the other for analysing or testing the light. There is no essential difference between the two parts, except what convenience or economy may lead us to adopt; and either part, therefore, may be used as polariser or analyser; but whichever we use as the polariser, the other becomes the analyser.

The tourmaline, a precious stone of a neutral or bluish tint, forms an excellent analyser; it should be cut about $\frac{1}{20}$ th of an inch thick, and parallel to its axis. The great objection to it is, that the transmitted polarised beam is more or less coloured. The best tourmaline to choose "is the one that stops the most light when its axis is at right angles to that of the polariser, and yet admits the most when in the same plane."

The production of colour by polarised light has been most clearly and comprehensively explained by Mr. Woodward, in his "Introduction to the Study

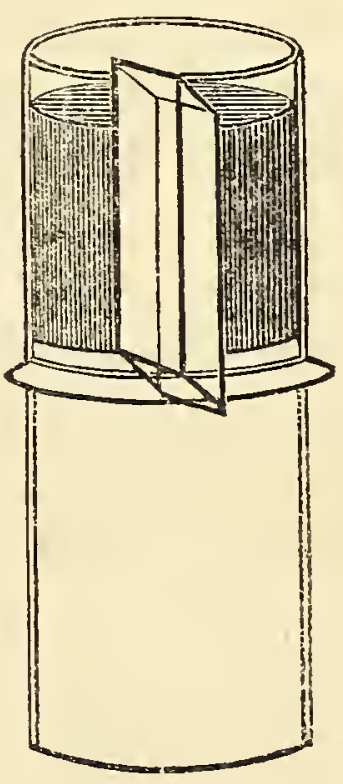

fig. 70 . of Polarised Light." To render the diagram more intelligible, as
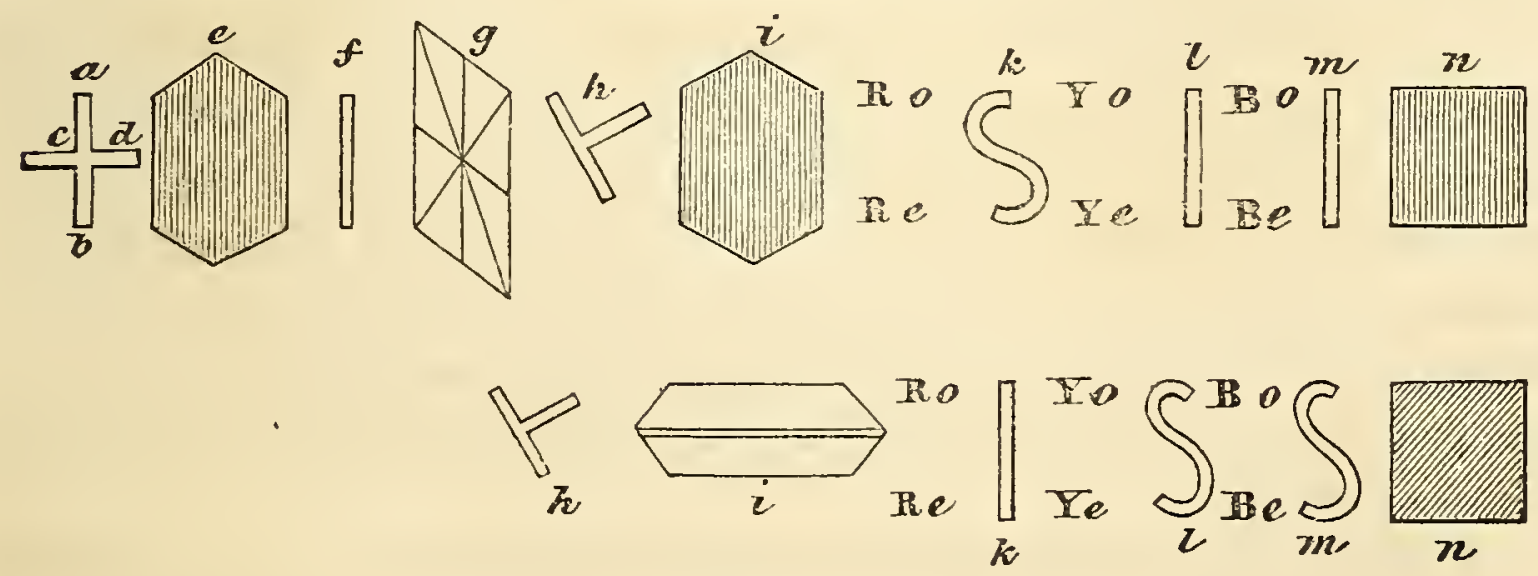

fig. 71 .

we are unable to introduce them in the colours given by the above gentleman, we may state that ordinary light is represented by a cross, 
which denotes that its vibrations are in planes at right angles to each other; whereas when one set of such vibrations only is shown, the light is said to be polarised. In fig. $71, a b c d$ represent the rectangular vibrations of common light; $e$ a plate of tourmaline placed with its axis in a vertical direction-this is called the polariser; $f$ a beam of polarised light obtained from $a b c d$ by the stoppage of plane $c d ; g$ a film of selenite of such a thickness as to produce red or green light; $h$ the polarised beam $f$ split into two planes at right angles to each other; $i$ a second tourmaline or analyser, with its axis in the same direction as that of $e$; by this all the vibrations that are not inclined at a greater angle than $45^{\circ}$ to the axis of the analyser can be transmitted and again brought together; $k$ the waves $R o R e$ of red light, meeting in the same state of vibration, and forming a wave of red light of doubled intensity; $l m$ the waves $Y_{e} Y_{o}$ and $B o B e$ for yellow and blue meeting together, with a difference of an odd number of half undulations, and thus neutralising each other by interference; $n$ the resultant red light.

In the next line of figures may be seen what takes place by turning the analysing tourmaline one quarter of a circle. $\quad h$ represents the polar. ised beam split into two rays; $i$ the tourmaline turned so that its axis is at right angles to that in the preceding figure; $k$ the waves $R o R e$ of red light destroying each other by interference; $l m$ the waves $Y_{o} Y e$ and $B o B e$ for yellow and blue, meeting together in the same state of vibration, and by their coincidences, waves of doubled intensity for yellow and blue light; $n$ green light, resulting from the mixture of the yellow and blue light respectively. By substituting Nicol's prisms for the two plates of tourmaline, and by the addition of the object-glass and eye-piece, the diagrams would then represent the passage of polarised light through a microscope.

"For showing objects by polarised light under the microscope, put upon the stage a film of selenite, which exhibits, under ordinary circumstances, the red ray in one position of the polarising prism, and the green ray in another; and each arc will assume one of these complementary colours, whilst the centre of the field will remain colourless. Into this field introduce any microscopic object; and whatever its structure, it will exhibit the effects of polarised light with great intensity and purity of colour.

"In this way many objects, which in the usual arrangement of the polariscope undergo no change in colour, are made to display the most brilliant effects. Sections of wood, feathers, algæ, and scales, are among the objects best suited for this kind of exhibition. 
"The best power for the purpose is a two-inch object-glass, the intensity of colour, as well as the separating power of the prism, being impaired under much higher amplification; although in some few instances, such as in viewing animalculæ, the inch object-glass is perhaps to be preferred."

Mr. J. King attributes the foregoing phenomenon "to the doubleimage prism separating the constituents of the polarised ray into two planes, and causing them to overlap each other, except at the edges where the light is analysed; and, of course, the combination of complementary colours in the centre of the field produces white light. Any object, therefore, placed in the white field, partakes of the characters of the selenite; one image being refracted into the plane which exhibits the green ray, assumes that colour; whilst the second image being refracted into the plane of the red ray, partakes of that tint. Should the object, however, be so large that actual separation of the images is not effected, the extremities and interstices only will be polarised, whilst the middle will remain dark, or present only its natural hue."

Selenite is the native crystallised hydrated sulphate of lime. A beautiful fibrous variety called satin gypsum is found in Derbyshire. It is found also at Shotover Hill, near Oxford, where the labourers call it quarry-glass. Very large crystals of it are found at Montmartre, near Paris. The form of the crystal most frequently met with is that of an oblique rectangular prism, with ten rhomboidal faces, two of which are much larger than the rest. It is usually slit into thin laminæ parallel to these large lateral faces; the film having a thickness of from onetwentieth to the one-sixtieth of an inch. In the two rectangular directions they allow perpendicular rays of polarised light to traverse them unchanged; these directions are called the neutral axis. In two other directions, however, which form respectively angles of $45^{\circ}$ with the neutral axis, these films have the property of double refraction. These directions are known as the depolarising doubly refracting axis.

The thrckness of the film of selenite determines the particular tint. If, therefore, we use a film of irregular thickness, different colours are presented by the different thicknesses. These facts admit of very curious and beautiful illustration, when used under the object placed on the stage of the microscope. The films employed should be mounted between two glasses for protection. Some persons employ a large film mounted in this way between plates of glass, with a raised edge, to act as a stage for supporting the object, it is then called the "selenite stage." Mr. Darker has constructed a very neat stage of brass for this purpose, and that of producing a mixture of all the colours ioy super- 
imposing three films, one on the other; by a slight variation in their positions, produced by means of an endless-screw motion, all the colours of the spectrum are shown.

Dr. Herapath, of Bristol, has recently described a salt of quinine, which has remarkable polarising properties. The salt was first accidentally observed by Mr. Phelps, a pupil of Dr. Herapath's, in a bottle which contained a solution of disulphate of quinine. The salt is best formed by dissolving disulphate of quinine in concentrated acetic, acid, then warming the solution, and dropping into it carefully, and by small quantities at a time, a spirituous solution of iodine. On placing this mixture aside for some hours, brilliant plates of the new salt will be formed. The crystals of this salt, when examined by reflected light, have a brilliant emerald-green colour, with almost a metallic lustre; they appear like portions of the elytræ of cantharides, and are also very similar to murexide in appearance. When examined by transmitted light, they scarcely possess any colour, there is only a slightly olivegreen tinge; but if two crystals, crossing at right angles, be examined, the spot where they intersect appears perfectly black, even if the crystals are not one five-hundreth of an inch in thickness. If the light be in the slightest degree polarised-as by reflection from a cloud, or by the blue sky, or from the glass surface of the mirror of the microscope placed at the polarising angle $56^{\circ} 45^{\prime}$-these little prisms immediately assume complementary colours : one appears green, and the other pink, and the part at which they cross is a chocolate or deep chestnut brown, instead of black. As the result of a series of very elaborate experiments, Dr. Herapath finds that this salt possesses the properties of tourmaline in a very exalted degree, as well as of a plate of selenite; so that it combines the properties of polarising a ray and of depolarising it. Dr. Herapath also states that he has succeeded in making an artificial tourmaline large enough to surmount the eye-piece of the microscope; so that all experiments with those crystals upon polarised light may be made without the tourmaline or Nicol's prism. The brilliancy of the colours is much more intense with the artificial crystal than when employing the natural tourmaline. As an analyser above the eye-piece, it offers some advantages over the Nicol's prism in the same position, as it gives a perfectly uniform tint of colour over a much more extensive field than can be had with the prism.".*

* Dr. Herapath has given a later and better process for the manufacture of these artificial tourmalines in the Quarterly Journal of Microscopical Science for January 1854. The editors of the journal say the crystals produced are most satisfactory, and. equal to any polarising: arrangement they have ever employed. 
A variety of interesting phenomena have been described by $\mathrm{Mr}$. S. Legg in the Iransactions of the Microscopical Society. He says:

"The following experiments, if carefully performed, will illustrate the most striking phenomena of double refraction, and form a useful introduction to the practical application of this principle.

"A plate of brass, fig. 72, three inches by one, perforated with a series of holes from about one-sixteenth to one-fourth of an inch in

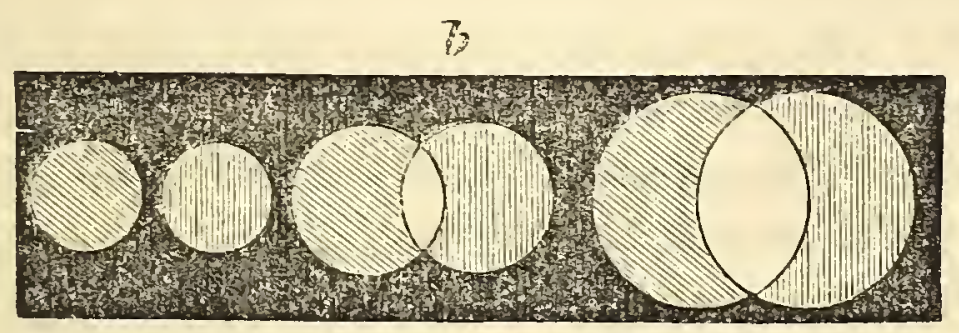

iig. 72.

diameter; the size of the smallest should be in accordance with the power of the object-glass, and the separating power of the double refraction.

"Experiment 1. Place the brass plate so that the smallest hole shall be in the centre of the stage of the instrument; employ a low power ( $1 \frac{1}{2}$ or 2 inch) object-glass, and adjust the focus as for an ordinary microscopic object; place the doubly-refracting crystal over the eyepiece, and there will appear two distinct images; then, by revolving the eye-piece, these will describe a circle, the circumference of which cuts the centre of the field of view; the one is called the ordinary, the other the extraordinary ray. By passing the slide along, that the larger orifices may appear in the field, the images will not be completely separated, but will overlap, as represented in the figure.

"Experiment 2. Screw the Nicol's prism into its place under the stage, still retaining the double refractor over the eye-piece; then, by examining the object, there will appear in some positions two, but in others only one image; and it will be observed, that at $90^{\circ}$ from the latter position this ray will be cut off, and that which was first observed will become visible; at $180^{\circ}$, or one-half the circle, an alternate change will take place; at $270^{\circ}$ another change; and at $360^{\circ}$, or the completion of the circle, the original appearance.

"Before proceeding to the next experiment, it will be as well to observe the position of the Nicol's prism, which should be adjusted with its angles parallel to the square parts of the stage; in order to secure the greatest brilliancy in the experiment, the proper relative position of the selenite may be determined by noticing the natural 
flaws in the film, which will be observed to run parallel with each other; these flaws should be adjusted at about $46^{\circ}$ from the square parts of the stage, to obtain the greatest amount of depolarisation.

"Experiment 3. If we now take the plate of selenite thus prepared, and place it under the piece of brass on the stage, we shall see, instead of the alternate black and white images, two coloured images composed of the constituents of white light, which will alternately change by revolving the eye-piece at every quarter of the circle; then, by passing along the brass, the images will overlap; and at the point at which they do so, white light will be produced. If, by accident, the prism is placed at an angle of $45^{\circ}$ from the square part of the stage, no particular colour will be perceived; and it will then illustrate the phenomena of the neutral axis of the selenite, because when placed in that relative position no depolarisation takes place. The phenomena of polarised light may be further illustrated by the addition of a second double refractor, and a film of selenite adapted between the double refractors. The systems of coloured rings in crystals cut perpendicularly to the principal axis of the crystal, are best seen by screwing the Nicol's prism under the stage, and employing the lowest object-glass: place the crystals over the eye-piece, and use a tourmaline as the analyser."

It was long believed that all crystals had only one axis of double refraction; but Brewster found that the great body of crystals, which are either formed by art, or which occur in the mineral kingdom, have two axes of double refraction as. well as of polarisation.

Nitre crystallises in six-sided prisms with angles of about $120^{\circ}$. It has two axes of double refraction, along which a ray of light is not divided into two. These axes are each inclined about $2 \frac{1}{2}^{\circ}$ to the axes of the prism, and $5^{\circ}$ to each other. If, therefore, we cut off a piece from a prism of nitre with a knife driven by a smart blow of a hammer, and polish two flat surfaces perpendicular to the axis of the prism, so as to leave the thickness of the sixth or eighth of an

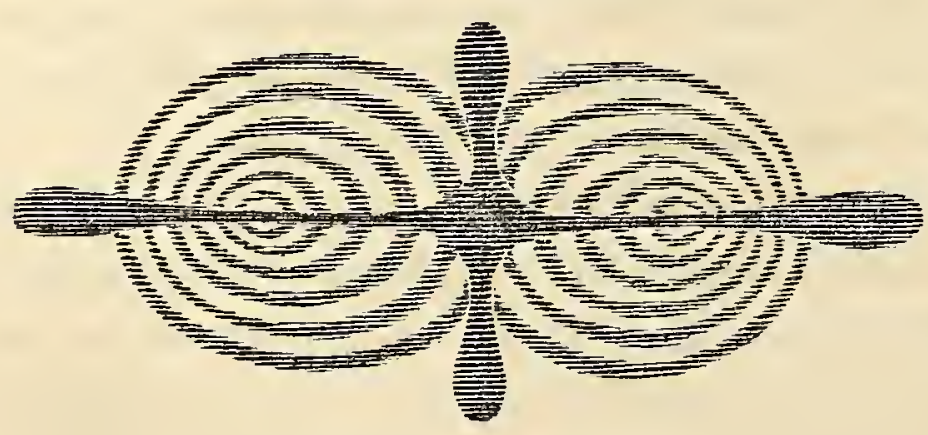

fig. 73 .

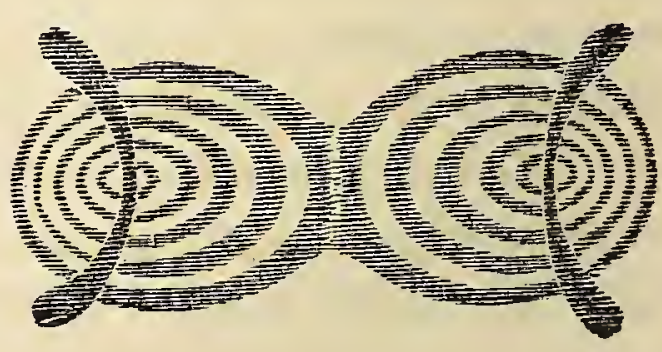

fig. 74 . 
inch, and then transmit a ray of polarised light along the axis of the prism, we shall see the double system of rings shown in figs. 73 and 74 .

When the line connecting the two axes of the crystal is inclined $45^{\circ}$ to the plane of primitive polarisation, the cross seen as first described, on revolving the nitre, opens, and gradually assumes the form of two hyperbolic curres, fig. 74. But if the tourmaline be revolved, the black crossed lines will be replaced by white spaces, and the red rings by green ones, the yellow by indigo, and so on.

These systems of rings have, generally speaking, the same colours as those of thin plates, or as those of a system of rings round one axis. The orders of the colours commence at the centres of each system; but at a certain distance, which corresponds to the sixth ring, the rings, instead of returning and encircling each pole, encircle the two poles as an ellipse does its two foci. When we diminish or increase the thickness of a plate of nitre, the rings are diminished or increased accordingly.

A large number of crystals exhibit this curious and beautiful system of coloured rings. Small specimens of salts may also be crystallised and mounted in Canada balsam for viewing under the stage of the microscope; by arresting the crystallisation at certain stages, a greater variety of forms and colours may be obtained: we may enumerate salicine, asparagine, acetate of copper, phospho-borate of soda, sugar, carbonate of lime, chlorate of potassa, oxalic acid, and all the
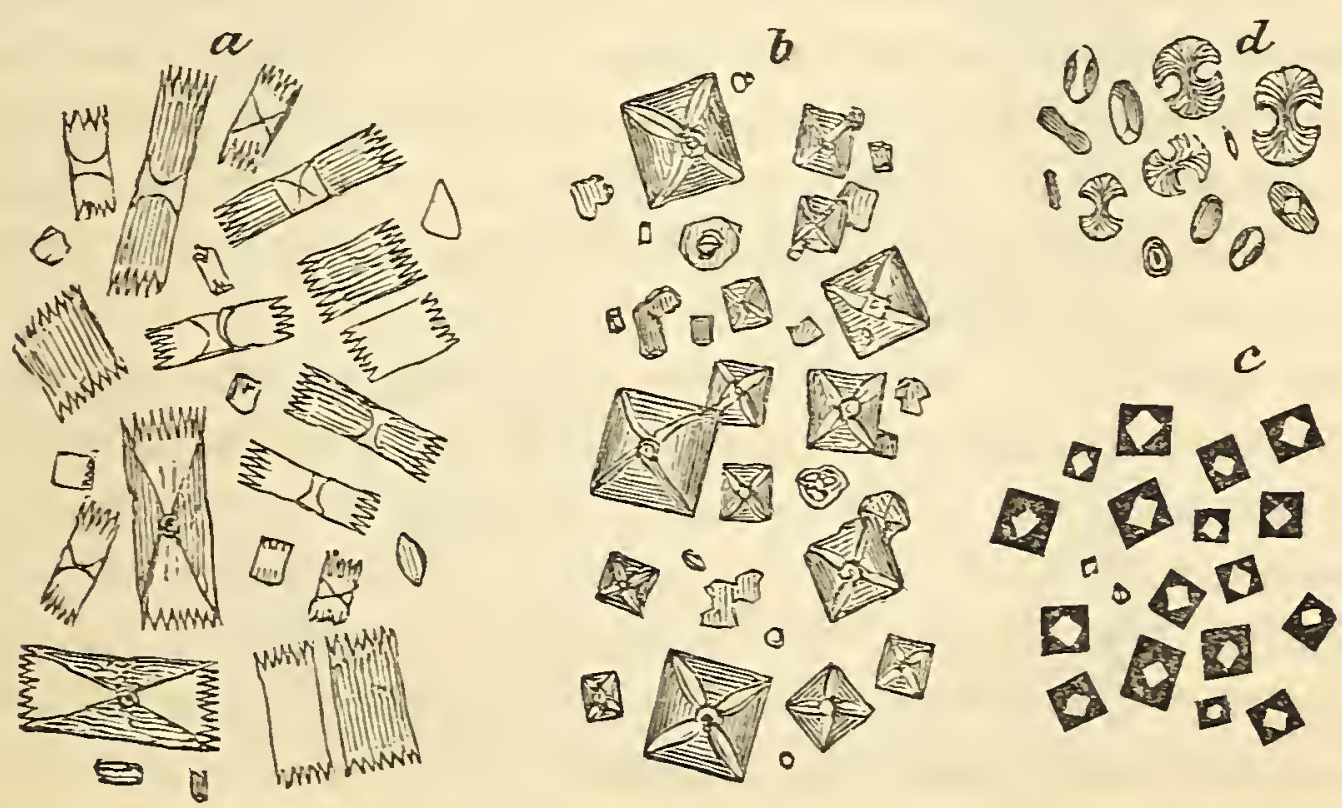

fig. 75. FORMS OF URINART SALTS SEEX BY POI.ARISED LIGHT.

$a$, Uric acid deposit. $b$, Oxalate of lime, octahedral crystals of. c, Oxalate of lime allowed to dry, forming a black cuke. $d_{2}$ Osalate of lime, as it occasionally appears, termed the dumb-bell form. 
oxalates found in urine, with the other salts in the same fluid, some of which are shown in fig. 75 .

Dr. W. B. Herapath has contributed an interesting addition to the, uses of polarised light, by applying it to discover the salts of alkaloids, quinine, \&c. in the urine of patients. He says: "It has long been a favourite subject of inquiry with the professional man to trace the course of remedies in the system of the patient under his care, and to know what has become of the various substances which he might have administered during the treatment of the disease. Whilst some of these remedies have been proved to exert a chemical change upon the circulating medium, and to add some of their elements to the blood for the permanent benefit of the individual, others, on the contrary, make but a temporary sojourn in the vessels of the body, circulating with the blood for a longer or shorter period, but are eventually expelled and eliminated from it at different outlets, and by various glandular apparatus; some of these substances are found to be more or less altered in chemical composition, in consequence of having been subjected to the manifold processes of vital chemistry during their transit through the system; whilst others have experienced no alteration in their constitution, but have resisted all the destructive and converting powers of the animal laboratory, and, by appropriate means, have been again separated from the various excretions by the physiological and pathological chemist in their pristine state of purity.

"It has recently been somewhat more than a subject of conjecture, that, in common with several others of the vegetable alkaloides, quinine may be included in this last class of remedial agents ; and several methods of discovering its presence have emanated from different scientific observers. It has been repeatedly traced in the urine of patients suffering from intermittent fever, to whom large doses of this substance have been necessarily administered.

"The nature of the tests hitherto employed, and the various processes adopted, require large quantities of the fluid for examination; and the imperfection of the evidence resulting from the experiment threw considerable doubt upon the value of the conclusions arrived at.

"Having been struck with the facility of application, and the extreme delicacy of the reaction of polarised light, when going through the series of experiments upon the sulphate of iodo-quinine, I determined upon attempting to bring this method practically into use for the detection of minute quantities of quinine in organic fluids; and after more or less success by different methods of experimenting, I have at length discovered a process by which it is possible to obtain demon- 
strative evidence of the presence of quinine, even if in quantities not exceeding the 1-100,000th part of a grain; in fact, in quantities so exceedingly minute, that all other methods would fail in recognising its existence. Take for

"Test-fuid.-A mixture of three drachms of pure acetic acid, with one fluid drachm of rectified spirit-of-wine, to which add six drops of diluted sulphuric acid.

"One drop of this test-fluid placed on a glass-slide, and the merest atom of the alkaloid added; time given for solution to take place; then, upon the tip of a very fine glass-rod let an extremely minute drop of the alcoholic solution of iodine be added. The first effect is the production of the yellow or cinnamon-coloured compound of iodine and quinine, which forms as a small circular spot; the alcohol separates in little drops, which, by a sort of repulsive movement, drive the fluid away; after a time, the acid liquid again flows over the spot, and the.polarising crystals of sulphate of iodo-quinine are slowly produced in beautiful rosettes. This succeeds best without the aid of heat.

"To render these crystals evident, it merely remains to bring the glass-slide upon the field of the microscope, with the selenite stage and single tourmaline, or Nicol's prism, beneath it; instantly the crystals assume the two complementary colours of the stage; red and green, supposing that the pink stage is employed, or blue and yellow, provided the

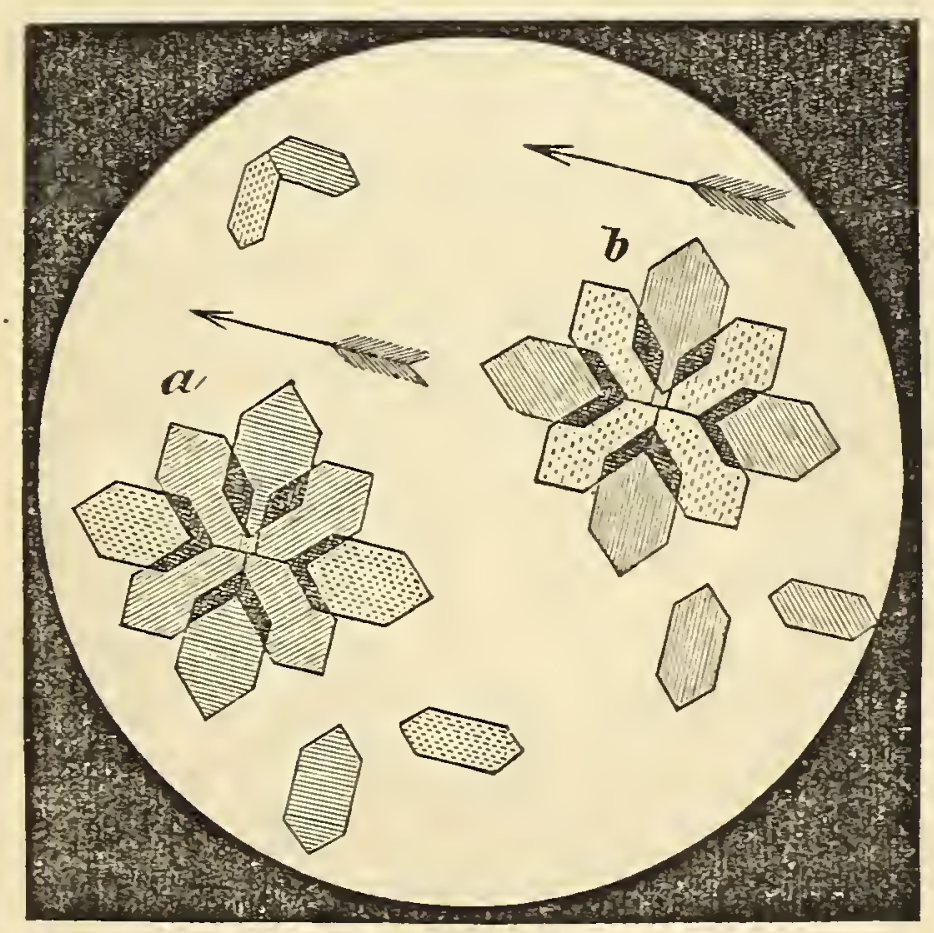

fig. 76. In this figure the heraldic lines are adopted to denote colour. The dotted parts indicate yellow, the straight lines red, the horizontal lines blue, and the diagonal, or oblique lines, green. The arrows show the plane of the tourmaline. $a$, blue stage ; $b$, red stage of selenite employed. 
blue selenite is made use of. All those crystals at right angles to the plane of the tourmaline, producing that tint which an analysing-plate of tourmaline would produce when at right angles to the polarisingplate; whilst those at $90^{\circ}$ to these educe the complementary tint, as the analysing-plate would also have done if revolved through an arc of $90^{\circ} . "$

This test is so ready of application, and so delicate, that it must become the test par excellence for quinine. (See fig. 76, $a$ and $b$.)

Not only do these peculiar crystals act in the way just related, but they may be easily proved to possess the whole of the optical properties of that remarkable salt of quinine, so fully described by me in the Philosophical Magazine for March 1852, and the chemical analysis of which was published in the number for September in the same year. In fact, these crystals are perfectly identical with the sulphate of iodoquinine in every respect.

To test for quinidine, it is merely necessary to allow the drop of acid solution to evaporate to dryness upon the slide, and to examine the crystalline mass by two tourmalines, crossed at right angles, and without the stage. Immediately little circular discs of white, with a well-defined black cross very vividly shown, start into existence, should quinidine be present even in very minute traces. These crystals are shown in fig. 77.

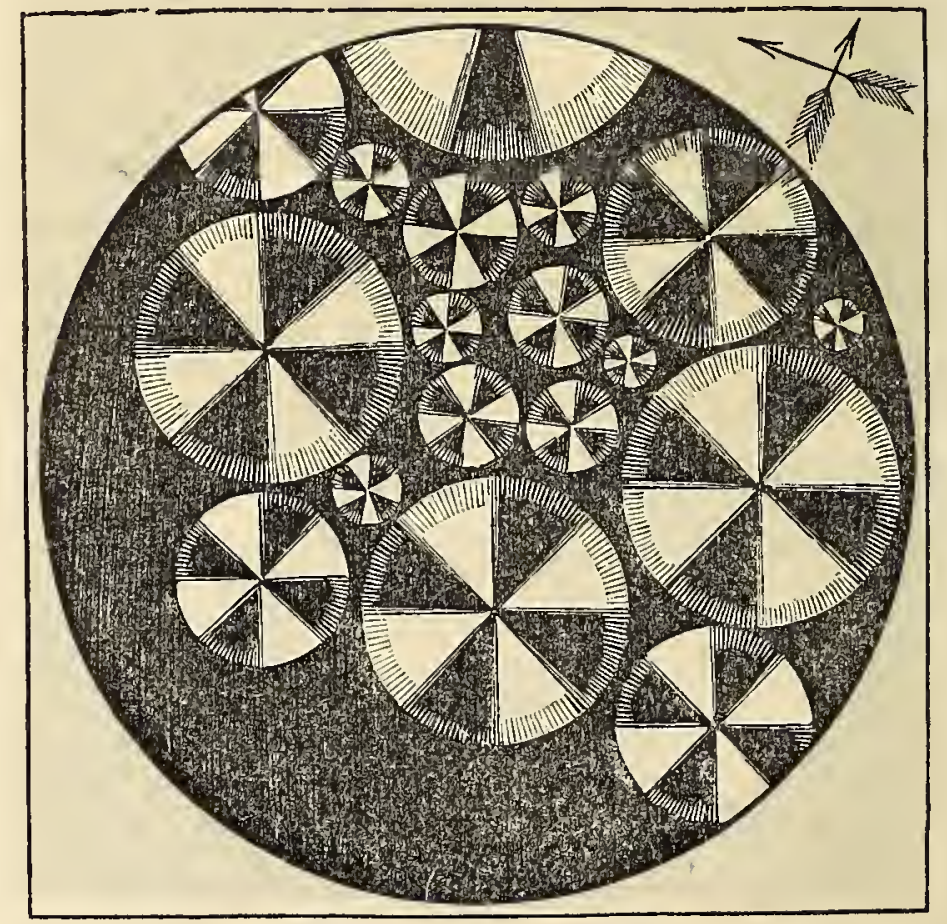

fig. 77 .

If we employ the selenite stage in the examination of this object, 
we obtain one of the most gorgeous appearances in the whole domain of the polarising-microscope; the black cross at once disappears, and is replaced by one which consists of two colours, being divided into a cross having a red and green fringe, whilst the four intermediate sectors are of a gorgeous orange-yellow. These appearances alter upon the revolution of the analysing-plate of tourmaline; when the blue stage is employed, the cross will assume a blue or yellow tint, according to the position of the analysing-plate. These phenomena are analogous to those exhibited by certain circular crystals of boracic acid, and to those circular discs of salicine (prepared by fusion); the difference being, that the salts of quinidine have more intense depolarising powers than either of the other substances; besides which, the mode of preparation effectually excludes these from consideration. Quinine prepared in the same manner as the quinidines has a very different mode of crystallisation; but it occasionally presents circular corneous plates, also exhibiting the black cross and white sectors, but not with one-tenth part of the brilliancy, which of course enables us readily to discriminate the two.-Philosophicai Magazine, 1853.
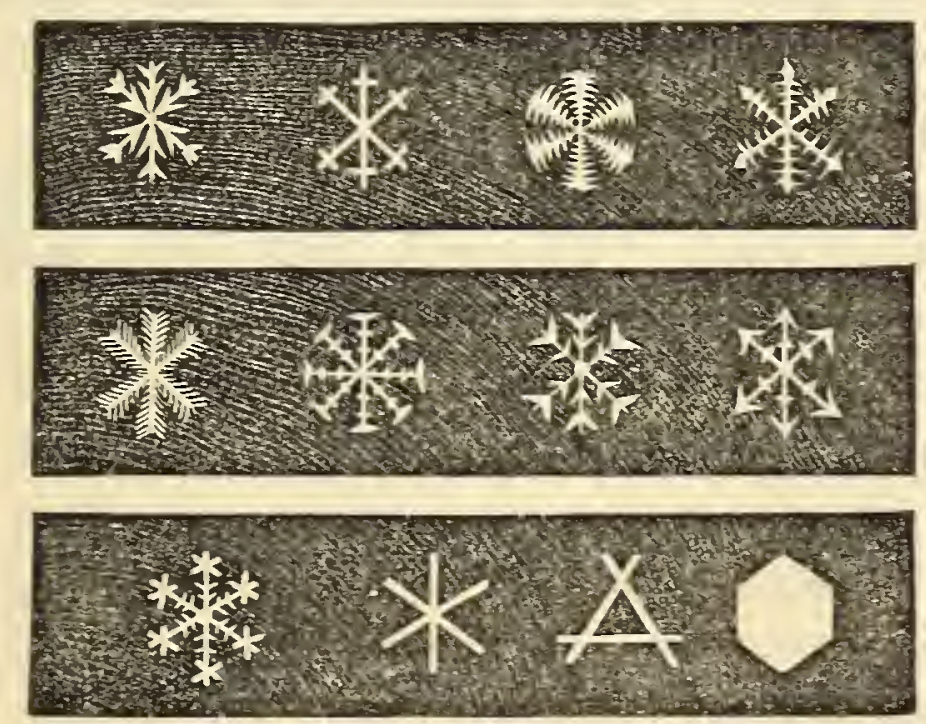

fig. 7S. CRYstals OF Srow.

Ice doubly refracts, while water singly refracts. Ice takes the rhomboidic form, and snow in its crystalline form may be regarded as the skeleton crystals of this system. A sheet of clear ice, of about one inch thick, and slowly formed in still weather, will show the circular rings and cross when viewed by polarised light. Some of the Wenham I ake ice answers very well.

A great variety of animal, vegetable, and other substances possess a doubly refracting or depolarising structure, as: a quill cut and laid out flat on glass; the cornea of a sheep's eye ; skin, hair, a thin sec- 
tion of a finger-nail ; sections of bone, teeth, horn, silk, cotton, whalebone; stems of plants containing silica or flint; barley, wheat, \&c. The larger-grained starches form splendid objects; tous les mois, being the largest, may be taken as the type of all the others. It presents a black cross, as at a, fig. 79, the arms of which meet at the hilum.
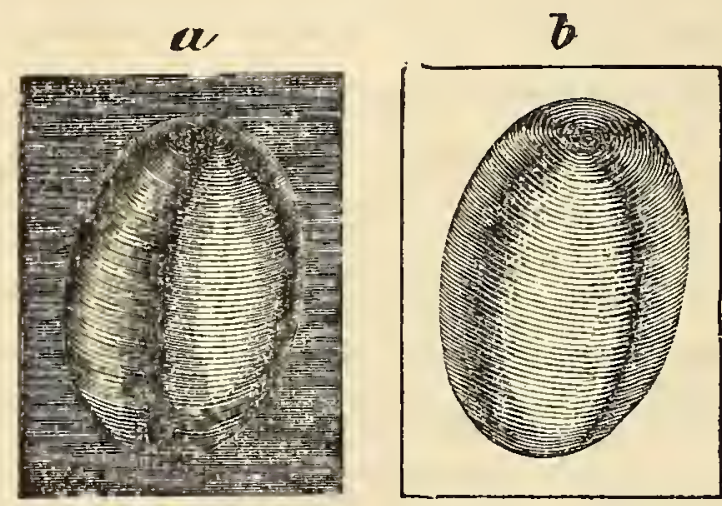

fig. 79 . On rotating the analyser, the black cross disappears, and at $90^{\circ}$ is replaced by a white cross, as at $b$; another, but much fainter black cross being perceived between the arms of the white cross. Hitherto, however, no colour is perceptible. But if a thin plate of selenite be interposed between the starch-grains and the polariser, most splendid and delicate colours appear. All the colours change by revolving the analyser, and become complementary at every quadrature of the circle. West and East India arrow-root, sago, tapioca, and many other starch-grains, present the same appearance; but in proportion as the grains are smaller, so are their markings and colourings less distinct.

Professor Quekett, in pointing out the Advantages of Polarised Light to the Microscopist," says: "The application of this modification of light to the illumination of very minute structures has not yet been fully carried out; but still there is no test of differences in density between any two or more parts of the same substance that can at all approach it in delicacy. All structures, therefore, belonging either to the animal, vegetable, or mineral kingdom, in which the power of unequal or double refraction is suspected to be present, are those that should especially be investigated by polarised light. Some of the most delicate of the elementary tissues of animals, such as the tubes of nerves, the ultimate fibrillæ of muscles, \&c. are amongst the most striking subjects that may be studied with advantage under this method of illumination. It would be impossible, in a work like the present, to give a long list of objects that require the aid of polarised light for their exhibition: every structure that the microscopist is investigating should be examined by this light, as well as by that either transmitted or reflected. Objects mounted in Canada balsam, that are far too delicate to exhibit any structure under transmitted, will often be well seen under polarised light; its uses, therefore, are manifold."

\footnotetext{
* Quekett's Practical Treatise on the Use of the Microscope.
} 
The application of this principle to microscopic purposes seems to have been tried as early as 1677 , by a French philosopher, le Père Cherubin, of Orléans, a Capuchin friar. The following is an extract from the description given by him of his instrument: "Some years ago I resolved to effect what I had long before premeditated, to make a microscope to see the smallest objects with the two eyes conjointly: and this project has succeeded even beyond my expectation; with advantages above the single instrument so extraordinary and so surprising, that every intelligent person to whom I have shown the effect has assured me that inquiring philosophers will be highiy pleased with the communication."

This appears to have long slumbered and been forgotten, and nothing more was heard of the subject until Professor Wheatstone's very surprising invention of the stereoscope, when it again attracted the attention of the above philosopher, who applied to both Ross and Powell to construct him an instrument; but this was not done; and last year a notice appeared in Silliman's American Journal of a birocular instrument constructed by Mr. J. L. Riddell. This even had many disadvantages and inconveniences, which a well-known microscopist of London (F. H. Wenham, Esq.) has succeeded in modifying and greatly improving:

In describing his improvements, he states: "That in obtaining" binocularity with the compound achromatic microscope in its complete acting state, there are far greater practical difficulties to contend against; and which it is highly important to overcome, in order to correct some of the false appearances arising from what is considered the very perfection of the instrument.

"All the object-glasses, from the one-inch upwards; are possessed of considerable angular aperture; consequently, images of the object are obtained from a different point of view, with the two opposite extremes of the margin of the cone of rays; and the resulting effect is, that there are a number of dissimilar perspectives of the object all blended together upon the single retina at once. For this reason, if the object has any considerable bulk, we shall have a more accurate notion of its form by reducing the aperture of the object-glass.

"Select any object lying in an inclined position, and place it in the centre of the field of view of the microscope; then, with a card held close to the object-glass, stop off alternately the right or left hand portion of the front lens; it will be seen that during each alternate change 
certain parts of the object will alter in their relative position. To

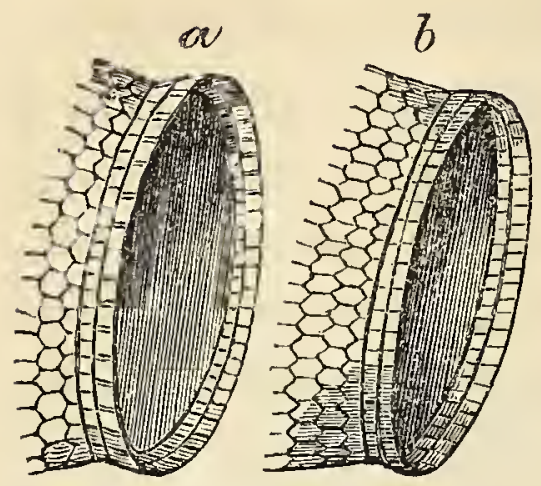

fig. 80 . illustrate this, figs. $80 a b$ are enlarged drawings of a portion of the egg of the common bed-bug (Cimex lecticularis), the operculum which covers the orifice having been forced off at the time the young was hatched. The figures exactly represent the two positions that the inclined orifice will occupy when the right and left hand portions of the object-glass are stopped off. It was illuminated as an opaque object, and drawn under a two-thirds object-glass of about $28^{\circ}$ of aperture. If this experiment is repeated, by holding the card over the eye-piece, and stopping off alternately the right and left half of the ultimate emergent pencil, exactly the same changes and appearances will be observed in the object under view. The two different images just produced are such as are required for obtaining stereoscopic vision. It is therefore evident that if, instead of bringing them confusedly together into one eye, we can separate them so as to bring figs. $80 a b$ into the left and right eye, in the combined effect of the two projections we shall obtain all that is necessary to enable us to form a correct judgment of the solidity and distances of the various parts of the object.

"If a rectangular plate of speculum metal is ground and polished, so as to form two reflecting facets inclined to each other at the required angle, as represented at 4, fig. 81 , and this is placed at an angle of $45^{\circ}$ with the division of the facets intersecting the axis of the objectglass, we shall divide the rays, and reflect them horizontally by one single reflection. Any other direction than a right angle, with respect to the axis of the object-glass, may, of course, be given to the rays, by inclining the reflector more or less. From the simplicity of this contrivance, and the facility with which it may be constructed, I shall take an early opportunity of giving it a trial. The only question I have is, whether a material may not be found that will reflect more light than even speculum metal. I have heard an alloy of cast-steel and platinum well spoken of, but have never seen any of it.

"In considering the aberrations which the thickness of glass contained in the reflecting prisms must inevitably produce, when placed immediately behind the object-glass, it occurred to me that if the same prisms were placed close to the top lens of the eye-piece, these errors, not being magnified, would be less sensibly felt.

"Diagram 3, fig. 81, represents the methods that I have contrived for 
obtaining the effect of bringing the two eyes sufficiently close to each other to enable them both to see through the same eye-piece together.
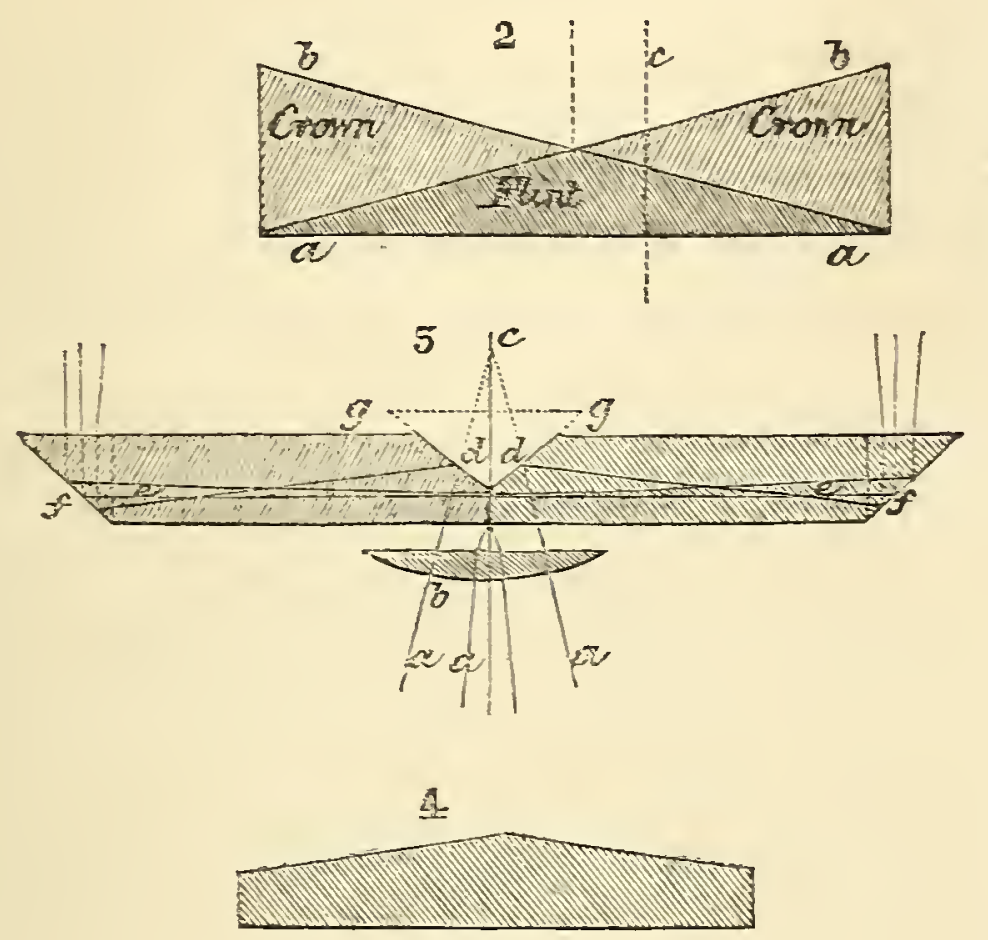

fig. S1.

$a a a$ are rays converging from the field lens of the eye-piece; after passing the eye-lens $b$, if not intercepted, they would come to a focus at $c$; but they are arrested by the inclined surfaces $d d$, of two solid glass prisms. From the refraction of the under incident surface of the prisms, the focus of the eye-piece becomes elongated, and falls within the substance of the glass at $e$. The rays then cliverge, and after being reflected by the second inclined surface $f$, emerge from the upper side of the prism, when their course is rendered still more divergent, as shown by the figure. The reflecting angle that I have giren to the prisms is $47 \frac{1}{2}^{\circ}$. I also find it is requisite to grind away the contact edges of the prisms, as represented, as it prevents the extreme margins of the reflecting surfaces from coming into operation, which can seldom be made rery perfect.

"The definition with these prisms is good; but they are liable to objection, on account of the extremely small portion of the field of view that they take in, and which arises from the distance that the eyes are of necessity placed beyond the focus of the eye-piece, where, the rays being divergent, the pupil of the eye is incapable of taking them all in; also there is great nicety required in the length of the prisms, which must differ for nearly every different observer.

"I have constructed an adjusting binocular eye-piece, not differing in principle from the last. The first reflection is performed by means 
of a triangular steel prism, with the two inclined facets very highly polished; this is represented by the dotted outline $g g$. The rays, after having been reflected at right angles, are taken up by two rectangular glass prisms, shown by the dotted lines at $f f$.

"The loss of light in this is much greater than in the former instance, and the field of view more contracted; for the rays from the eye-piece, after being reflected from the surface of the steel prism, fall to their natural focal distance, instead of being elongated, as in the solid prism; consequently the eye is still further removed from the focus. I had chosen hard steel for the reflector, on account of the property this material possesses of allowing the figure of a small flat surface to be retained, or even perfected, during the operation of polishing. I have also tried a combination of prisms over the field-glass, using two eyelenses ; but with no good result. The best effect that I have yet produced in the way of binocular vision applied to the microscope, is that next to be described, in which I have altogether dispensed with reflecting surfaces, merely using three refracting prisms, which, when placed together, are perfectly achromatic. $a$ a, diagram 2 , fig. 81 , is a single prism of dense flint-glass, with the three surfaces well polished; $b b$ are two prisms of crown-glass of half the length of the under-flint prism, to the upper inclines of which they are cemented with Canada balsam.

"The angle of inclination to be given to the prisms must depend upon the dispersive power of the flint and crown-glass employed. In the combination that I have worked out, I have used, for the sake of simplicity, some flint and crown that Mr. Smith kindly furnished me with, in which the dispersive powers are exactly as two to one; consequently I have had to make the angle of the crown just double that of the flint, in order to obtain perfect achromatism. The refractive power of each must also be known, that we may determine the angles of the prisms suitable for refracting the rays from the object-glass into the two eyes, at a distance of nine inches. $c c$, fig. 82, represents a ray of light incident at right angles upon the under surface of the flint prism. On leaving the second surface and entering the crown prism, it is slightly bent inwards; and on finally emerging, it is refracted outwards in the direction required. On looking through this prism, I could not discover the slightest colour or distortion: it is almost like looking through a piece of plain glass ; and the loss of light is so inappreciable, that it is difficult to distinguish any difference between an object and its refracted image. The base of the compound prism should not be larger than is sufficient to cover the stop of the lowest object-glass, in order that they may be made very thin. 
"The method of applying the prism to the binocular microscope is shown by fig. 82: $a a$ is the object-glass, $b$ the prism placed as close behind it as the fittings will admit. The prism is set in an aperture in a flat disc of brass, which has a horizontal play in every direction, in order that it may be adjusted and fixed in such a position that the junction of the prisms may bisect the rays from the object-glass, and at the same time be at right angles to the tranverse centres of the eyepiece; $c c$ are the two bodies of the microscope, provided with draw tubes, and the usual eye-pieces $d d$. The distance between them should be rather less than the averàge distance asunder of the eyes; and in cases where these are very wide apart, we can pull out the draw-tubes, which will increase the distance between the eye-pieces.

"With this apparatus I obtain the whole of the field of view in each eye ; which circumstance I was not prepared to expect; as this must, in some measure, depend upon the correction of the oblique pencils of the object-glass, for we cannot expect to look

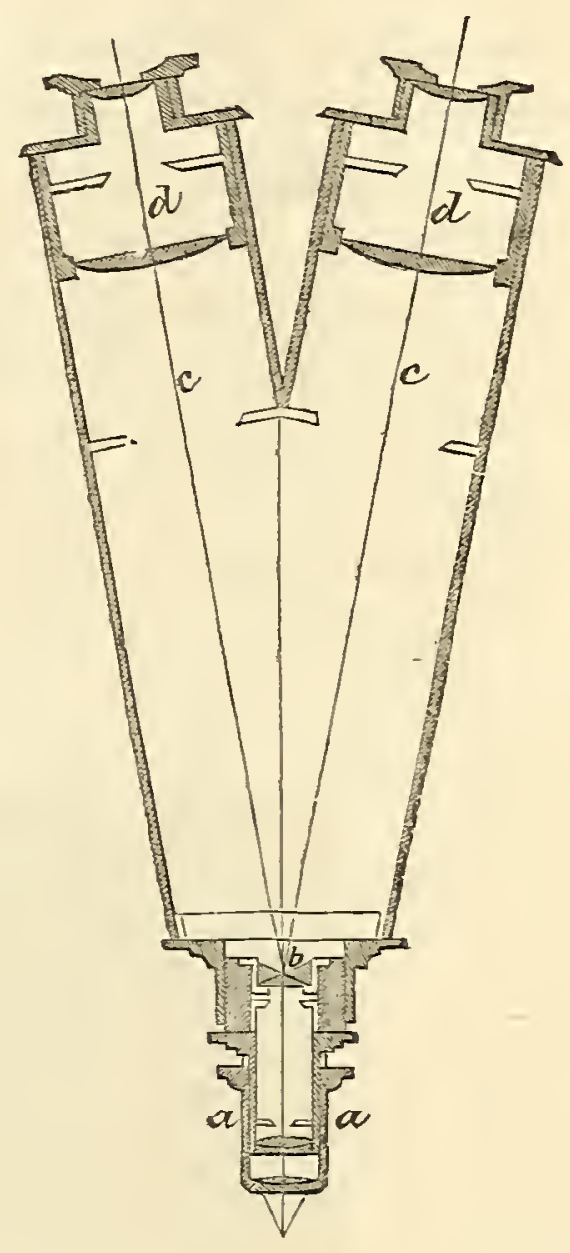

fig. 82. obliquely through the objective of a compound achromatic microscope in the same way as in the single lens arrangement, but can only avail ourselves of such oblique pencils of rays as are corrected for passing through the axis of the microscope."-Quarterly Journal of Microscopical Science, October $18 \tilde{3}$.

\section{PHOTOGRAPHY APPLIED TO THE MICROSCOPE.}

When this book was first projected, it was thought that if the objects so beautifully exhibited under the microscope could be drawn by light on the page of the book; or on the wood-block, so that the engraver might work directly from the drawing thus made, truthfulness would be insured, and we should present to the reader a valuable record of microscopic research never before seen or attempted. But in this we were doomed to disappointment by the existence of a patent, which presented obstacles too great to be surmounted at that time; 
and the idea was abandoned, with the exception of a few drawings then prepared, and now ready to hand: the patent restrictions having been since removed, we have embodied them in our pages. The eye and feet of fly, antenna of moth, paddles of water-beetle, with a few others, were first taken on a film of collodion, and then floated on to the surface of a block of wood; the wood having been previously and lightly inked with printer's ink or amber-varnish; the film was then gently rubbed or smoothed down to an even surface, at the same time carefully pressing out bubbles of air or fluid.

Mr. S. Highley has published the best mode of adapting an objectglass to the ordinary camera, for the purpose of taking microscopic objects on collodion and other surfaces.

Fig. 83 gives a sectional view of his arrangement, which is very

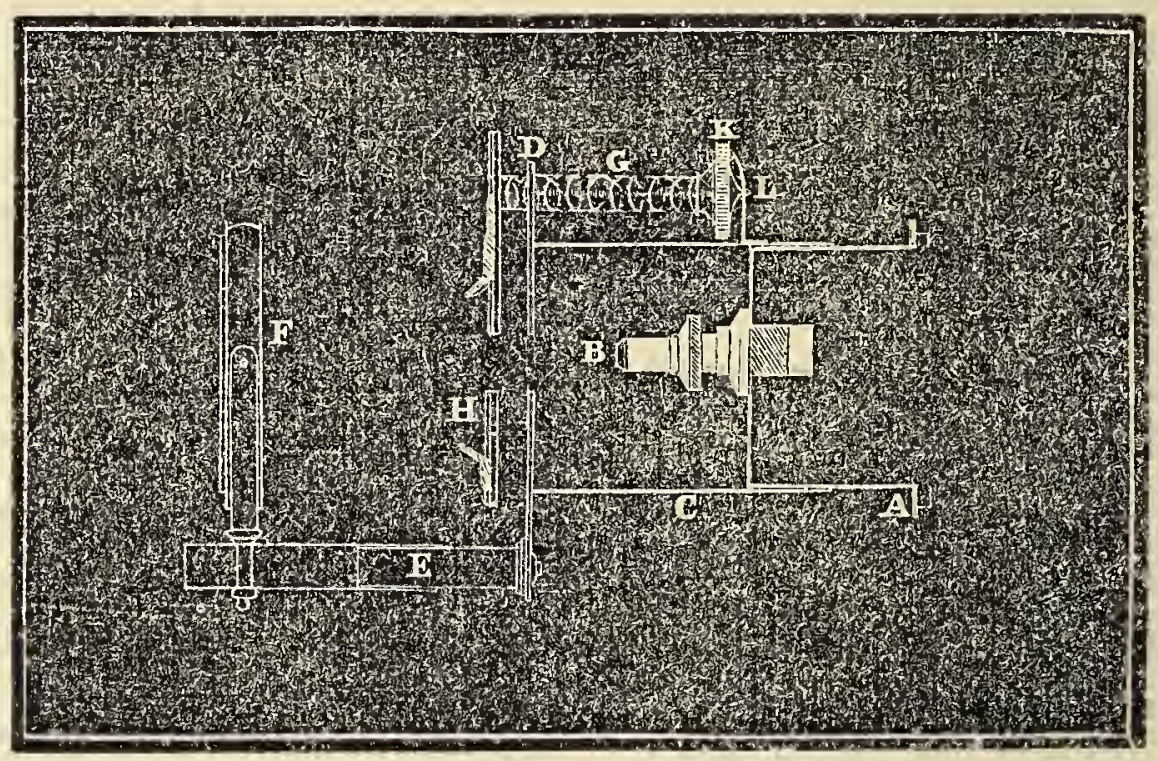

fig. 83 .

compact, steady, and ever ready for immediate use. The tube, A, screws into the flange of a camera which has a range of twenty-four inches; the front of this tube is closed, and into it screws the object-glass, B. Over A slides another tube, $\mathrm{c}$; this is closed by a plate, $D$, which extends beyond the upper and lower circumference of $\mathrm{C}$, and carries a small tube, $\mathbf{E}$, on which the mirror, $\mathbf{F}$, is adjusted. To the upper part of $D$ the fine adjustment $G$ is attached; this consists of a spring wire coil acting on an inner tube, to which the stage-plate, $\mathrm{H}$, is fixed, and is regulated by a graduated head, $\mathbf{k}$, acting on a fine screw, likewise attached to the stage-plate, after the manner of Oberhauser's microscopes. An index, $\mathrm{L}$, is fixed opposite the graduated head, к. The stage and clamp slides vertically on $\mathrm{H}$; and by sliding 
this up or down, and the glass object-slide horizontally, the requisite amount of movement is obtained to bring the object into the field. The object being brought into view, the image is roughly adjusted on the focussing-glass by sliding $\mathrm{C}$ on $\mathrm{A}$; the focussing is completed by aid of the fine adjustments, $\mathrm{G}, \mathrm{K}$, and allowance then made for the amount of non-coincidence between the chemical and visual foci of the object-glass. The difference in each glass employed should be ascertained by experiment in the first instance, and then noted. By employing a finely-ground focussing-glass greased with oil, this arrangement forms an agreeable method of viewing microscopical objects with both eyes, and is less fatiguing. As a very large field is presented to the observer, this arrangement might be advantageously employed for class demonstration."

Two exquisitely delineated negative objects obtained in this way by Mr. Delves were afterwards printed as positives, for the purpose of illustrating an excellent paper on the "Application of Photography to Microscopy," by S. Highley, jun., in No. 3 of the Quarterly Journal of Microscopical Science. 


\section{PART II.}

\section{CHAPTER I.}

A GLANCE INTO THE MICROSCOPIC WORLD. HISTORY OF INFUSORIAL ANIMALCULE. DESCRIPTION OF CILIA. POLYGASTRIA, ROTIFERA, VORTICELLA, STENTORS, HYDRA, ZOOPHYTES, SPONGES, FOSSIL INFUSORIA, FORAMINIFERA, CRUSTACER, MOLLUSCA, ETC.

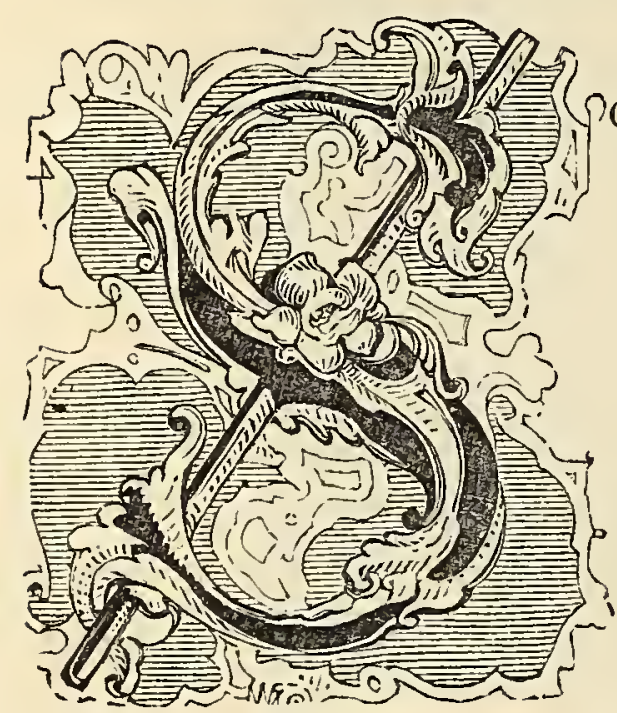

CIENCE has defined matter, space, time, and natural history, so infinitesimally, that the brain becomes bewildered from want of some familiar object to convey to the mind by comparison the results exhibited.

The precious metal gold can be reduced to a film the four-millionth part of an inch in thickness by manual ingenuity; space measured and divided the hundred-thousandth part of an inch with truthfulness by a machine; electricity proved to pass along a wire with the velocity of five hundred and seventy-six thousand miles in a second, and an object of the most elaborate workmanship seized in every detail, and faithfully portrayed, by electricity and the camera in the one-millionth part of the fraction of time denominated a second. At the least, a million of living creatures of delicate structure may be seen in active pursuit of prey in a space not larger than a mustard seed, and a single one, increasing in twenty-four hours to the enormous number of one hundred and forty millions, is demonstrated by means of the philosophical instrument, the microscope, the construction of which we have just described.

That sparkling gem, the eye, which adorns the human form, conveying so many delightful sensations, and acting as the outward index of the workings of the secret emotions, is, like the other organs of sense 
apportioned to man, limited in its powers. The restless curiosity of the human intellect led to the invention of the telescope, by which man daringly pierced the mysterious and illimitable space above us; revealing to his understanding a great and wonderful series of worlds lost to his unaided powers of vision. While, by the microscope, he has discovered an animal, vegetable, and mineral kingdom, of which he was previously ignorant, on account of its minuteness placing it beyond the keenest observation of the naked eye. In this last-named new and amazing world there is displayed a beauty, a perfection, adaptation, and reproduction, surprisingly surpassing those objects with which we are familiar in every-day life. With the microscope we search into the mysteries of creation, and detect many of the secret workings of nature. We see the utility of this busy, multitudinous, invisible world of auimal life, to the health, comfort, and preservation of human-kind; and the unbounded love of God in the admirable secret provisions for the unceasing changes in the form of matter. The more powerful the instrument, the more astounding its revelations; until we marvel in what sized atom organic matter ceases.

The practised microscopist will display to the novice a small slip of glass, on which the eye in vain seeks to detect the most minute speck; but when properly adjusted in the instrument, and the eyelit is looked through, there may be presented to the delighted sense of sight a shell the most perfect in formation, the most exquisite in its markings. The mortar of the builder, and the white cliffs of Albion, are almost entirely composed of the flinty covering of a minute animal, that once possessed sensation and volition, and sported with the activity of life.

Slates, flints, sandstones, vast tracts of country, and chains of mountains, are by the microscope proved to be immense masses of the shells of invisible animals: such are some of the revelations read in the book of nature by means of magnifying glasses; showing facts stranger than fiction, far beyond the imaginings of the most poetic brain.

The microscope is an instrument imperative to all who would cultivate their minds by the possession of a store of interesting facts, and rank well as intelligent beings in society. It aids the studies of the botanist, anatomist, physiologist, geologist, zoologist, and investigator of inorganic or organic matter; giving as it were to man a new sense, and thus adding to the enjoyments of life. In medical science its value is inappreciable; it becomes a daily auxiliary to the scientific inquirer into the causes and effects of the diseases or disorganisation of the body. 


\section{HISTORY OF ANIMALCULE.}

The astronomer turns his telescope from the earth, and ranges over the vast vault of heaven, to detect and delineate the beautiful objects of his pursuit. The naturalist turns his microscope to the earth, and in a drop of water finds a wondrous world of animated beings, more numerous than the stars of the milky way; and these he classifies into genus, and families, and catalogues in his history of the invisible world.

Numerous and extensive, indeed, must be these objects of his study, when there is no condition of water, from the most fetid to the most crystal, but teems with animated nature. Thus the stagnant pond, the limpid stream, the bubbling spring, the extensive watershed, the majestic river, the broad lake, the mighty ocean, the deep mine, the unstable bog, the torrid zone, the arctic regions, the forest-leaves, the lowly herb, the lovely flowers, the living plants, the aerial ocean, the guano of Shabac, the skin and interior of animals, as well as that of haughty man,-are all invested with living animalculæ; many having even parasites existing on them more minute than their own invisible bodies.

Providence, in His Almighty wisdom, has formed them for carrying out the scheme of creation with harmony, freshness, beauty, and continuity. The revolutions in matter which the little beings create are incessant and immense, and, in Divine love to man, beneficial to his well-being:

One of the most interesting and important organs possessed by the infusorial animalcule is scientifically known by the term cilium, which is the Latin word for eyelash, the plural being cilic. Its appearance is that of a minute delicate hair.

The cilium is not only useful in the act of progression, but also as an assistance in procuring food; the two duties being performed at the same time, the motion of the organs that propels it forward causing a current to set towards the mouth, which carries with it the prey on which the animal feeds. From the cilia being found in the gills or beard of the tadpole, the oyster, and mussel, it would appear that they are serviceable as organs of respiration, by imbibing oxygen, and emitting the carbonic acid generated in the blood during its circulation through the body; they are also believed to be the medium of taste and touch. It is not only at the mouth, but over the whole body, that cilia are discovered; and it is now satisfactorily shown that cilia exist also in the internal organs of man and other vertebrated animals; and are agents by which many of the most important functions of the 
animal economy are performed. The anatomist discovers them on the bronchial tubes, ventricles of the brain, the trachea, the mucous membrane of the laryns, the pericardium of the heart, and the peritoneum of the intestines, as well as in other parts of the animal economy in all classes; their offices seem to be to promote the passage of the fluids of the body by their motions. They rary in size from the 1000th to the 10,000th of an inch in length. These minute organs would often be invisible, were it not from the water being coloured when placed under a microscope; then the little currents made by the action of the cilia are easily perceived; and when the water is evaporated, the delicate tracing of their formation may be observed on the glass. They are differently placed, and vary in quantity in the numerous species of infusoria. In some they are in rows the whole length of the body, in others on the base; many have them over the whole of the body; sometimes they fringe the mouth, form bands around projections on the body; and many have but two projecting from the mouth, as long as the body of the creature. Ehrenberg says they are fixed at their base by a bulb moving in a socket, in a similar manner to a man's outstretched arm; and by their moving round in a circle, they form a cone, of which the apex is the bulb. Poison, galvanism applied to the animal, and death, do not immediately stop the motion of the cilia, as they will continue in action some hours afterwards; even longer than nervous or muscular action can be sustained, until the fluids dry up and they stiffen.

Nothing certain is known of their muscular derelopment, from their extreme minuteness no glass yet constructed being porerful enough to reach this structure; but there can be no doubt of its existence. Now in the wheel animalculæ the cilia are in circular rows; and each revolves around its bulb, giving a singular appearance, seeming to more together like a wheel upon its axle, whence their name Rotifera; in some of these muscles have been traced. The cilia must not be mistaken by joung microscopists for the stiff hairs and bristles found on some animalculæ, serving the purpose of locomotion in crawling or climbing.

Professor Quekett, in his valuable work on the microscope, describes the method of viewing the ciliary movement:- "If the roof of the mouth of a living frog be scraped with the end of a scalpel, and the detached mucous membrane placed on a glass slide, and examined with a power of 300 diameters, the ciliated epithelium-cells will be well seen; when a number of these are collected together, the movement is effected with apparent regularity; but in detached scales it is often so violent, that the scale itself is whirled about in a similar manner to an animalcule provided with a locomotive apparatus of the same description, and 
has frequently been mistaken for such. The animals more commonly employed for the examination of the cilia are the oyster and the mussel; but the latter are generally preferred. To exhibit the movement to the best advantage, the following method must be adopted :-open carefully the shells of one of those mollusks, spilling as little as possible of the contained fluid; then with a pair of fine scissors remove a portion of one of the gills (branchiæ); lay this on a slide, or the tablet of an animalcule cage, and add to it a drop or two of the fluid from the shell, and by means of the needle-points separate the filaments one from the other; cover it lightly with a thin piece of glass, and it is ready for examination. The cilia may then be seen in several rows beating and lashing the water, and producing an infinity of currents in it. If fresh water instead of that from the shell be added, the movement will speedily stop; hence the necessity of the caution of preserving the liquid contained in the shell. To observe the action of any one of the cilia, and its form and structure, some hours should be allowed to. elapse after the preparation of the filaments above given, the movements then will have become sluggish; if a power of 400 diameters be used, and that part of the cilia attached to the epithelium scale carefully watched, each one will be found to revolve a quarter of a circle whereby a 'feathering' movement' is effected, and a current in one direction constantly produced. In the higher animals the action of the cilia can only be observed a short time after death. In a polypus of the nose, when situated at the upper and back part of the schneiderian membrane, the cilia may be beautifully seen in rapid action some few hours after its removal; but in the respiratory and other tracts, where ciliated epithelium is found, it would be almost impossible ever to see it in action, unless the body were opened immediately after death. In some animals it may be seen in the interior of the kidney, as was first discovered by Professor Bowman in the expanding extremity of the small tube surrounding the network of bloodvessels forming the so-called Malpighian body; in order to exhibit the ciliary action, the kidney should have a very thin slice cut from it; and this is to be moistened with the serum of the blood of the same animal; the vascular and secreting portions of the organ may then be seen with a power of 250 diameters, and also the cilia in the expanded extremity of each tube, as it passes over to surround the vessels ; the epithelium. of the tubes themselves is of the spheroidal or glandular character."

The cilia of the mussel, when viewed through a good microscope, appear as delicate elegant lines fringed with numerous cylindrical sharp-pointed filaments in unceasing motion. It is generally sup: 
PLATE II.
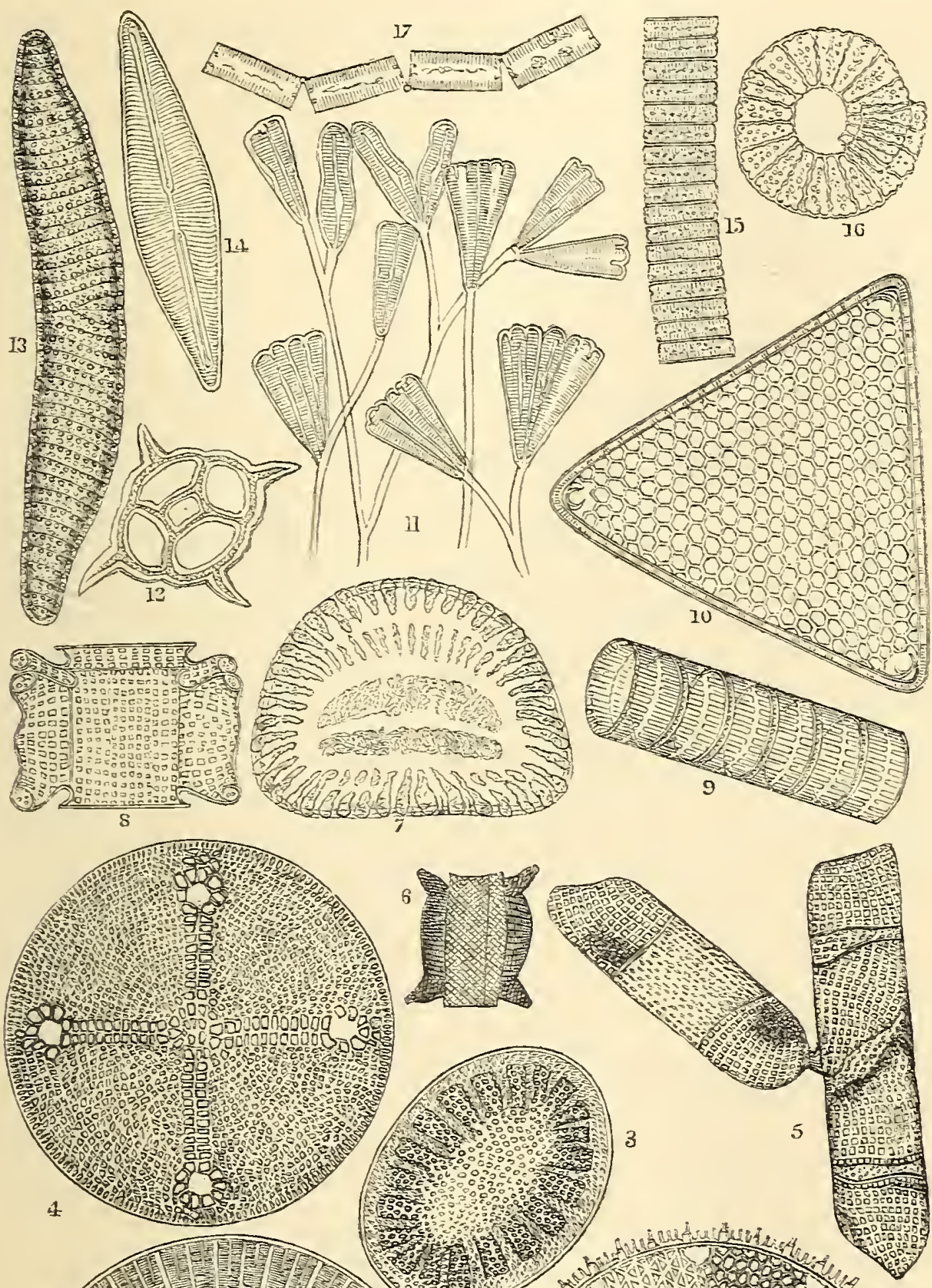

4

14
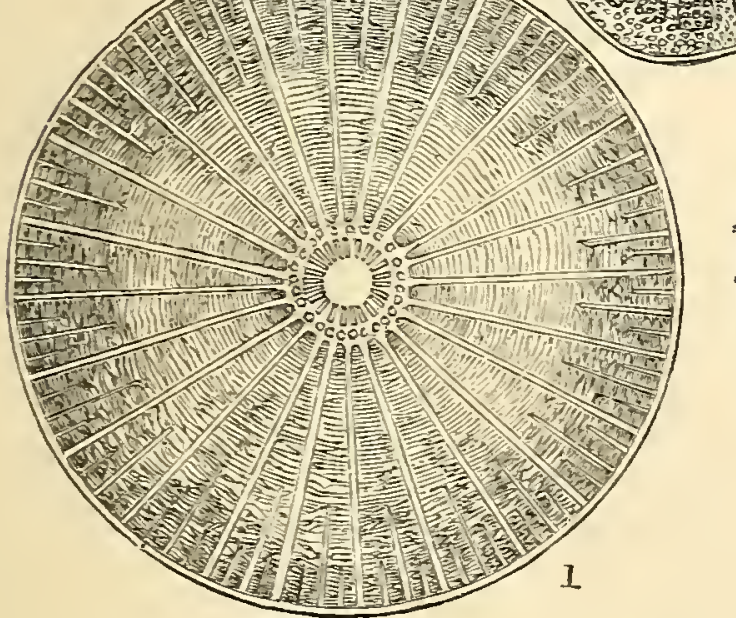

\%od 4 .

\% 3000 .
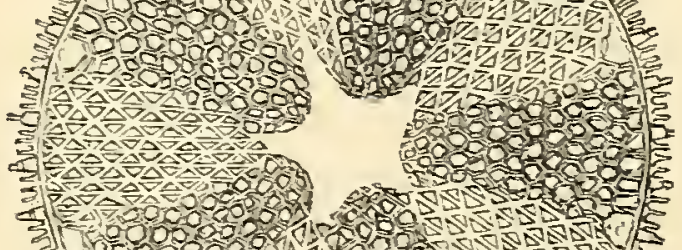

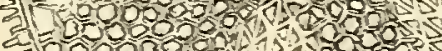

20000000 \%

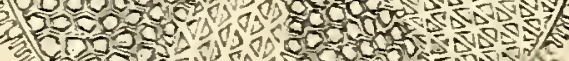

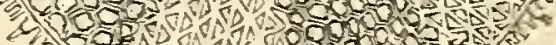

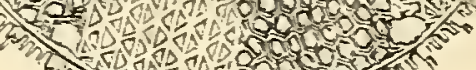

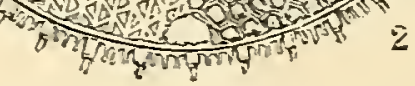

INFUSORIAL ANIMLALCUL王. 

posed, from the motion of the cilia, that their form is that of an oar, being flattened in one direction, which would at once account for the peculiar actions they perform.

\section{INFUSORIAL ANIMALCULE.}

The Polygastria of Ehrenberg comprise the smallest forms of animal life yet discovered by the aid of the microscope. They have, as their name implies (many stomachs), numerous little globular bladder's, or sacs, connected together for the purpose of receiving and digesting their food. The term Iniusoria is applied to them because they were first discovered in water where vegetable matter was decomposed, and therefore the infusion was considered necessary for their production. Now, however, it is an established fact, that they are in a higher state of organisation when taken from pure streams and clear ponds than from putrid and stagnant waters. A little bundle of hay, or sage leaves, left for about ten days in a mug containing some pure rain water, caught before entering a butt, produces the common wheel-animalculæ, which are found adhering to the sides of the mug near to the surface of the water. The only use of the vegetables seerns to be to facilitate the development of the latent life of the atoms of organic matter, and perhaps as the first sources of their food.

The Infusoria are a mighty family, as they frequently, in countless myriads, cover leagues of the ocean, and give to it a beautiful tinge from their vivid hue. They are discovered in all climes, have been found alive sixty feet below the surface of the earth, and in the mud brought up from a depth of sixteen hundred feet of the ocean. They exist at the poles and the equator, in the fluids of the animal body and plants, and in the most powerful acids. Above seven hundred and eighty-six species are enumerated in the catalogue of the naturalist, having different forms, habits, developments, and modes of continuing

\section{Description of Plate II.}

1. Shell of Arachnoidiscus. 2. Shell of Actinocyclus (Bermuda). 3. Shell of Cocconeis (Algoa Bay). 4. Shell of Coscinodiscus (Bermuda). 5. Shell of Isthmia enerris. 6. Zygoceros rhombus. 7. Campylodiscus clypeus. 8. Amphitetras. 9. Gallionella sulcata. 10. Triceratium, found in Thames' mud. 11. Gomphonema geminatum, attached to their stalks. 12. Dictyocha fibula. 13. Eunotia. 14. Cocconema. 15. Fragillaria pectinalis, 16. Meridion circulare. 17. Diatoma flocculosum. 
their distinct races. A brotherhood will be found in a little transparent shell, to which a drop of water is a world; and within these are sometimes other communities, performing all the functions granted them by their Creator, and eagerly pursuing the chase of others less than themselves as their prey.

The forms of the Infusoria are endless; some changing their shape at pleasure, some resembling globes, eels, trumpets, serpents, boats, stars, pitchers, wheels, flasks, cups, funnels, fans, and fruits.

The multiplication of the species is effected in some by spontaneous division or fissuration, in others by gemmation or budding; whilst some species are oviparous, and others viviparous. The first step in the process by which infusorial animals are eliminated, is the formation of globular corpuscles or cells, which, by their aggregation in some cases, and individual evolutions in others, give birth to the organisms which subsequently appear.

On examining the structure of infusorial animalculæ, some are found to have a soft yielding skin, so elastic as to stretch when food or other circumstances render it necessary, returning again to its previous condition as the cause of distension ceases; these are designated illoricated, which signifies shell-less. Others are termed loricated, from being covered with a shell, which is beautifully transparent, and flexible like horn. When the delicate and soft substance in which the functions of life perform their allotted duties perishes, the shell that protected it from injury during its hours of existence remains as a token of the past labours of nature; this sometimes consists entirely of flint, and in other cases of lime united with oxide of iron, destructible in some instances by fire, and in others not so. As millions upon millions are born, attain perfection, and die in a few days, these invisible shells form tracts of country which have served man as a foundation for his cities; they cover the bottoms of seas, lakes, and rivers; aid in filling up marshes, swamps, and morasses; they entirely constitute the tripoli, or polishing slate, the iron of bogs, chains of hills ; in fact, are the ceaseless silent agency renovating a great portion of the surface of the earth.

Some of these minute beings have apportioned to them setoe, or bristles; these stiff hairs, attached to the surface of their bodies, do not rotate, but are movable, and appear to be a means for the support of their bodies, as aids in climbing over obstacles that present themselves, or as feelers. Others are possessed of unci, or hooks, projecting from the under part of the body, which are capable of motion; and by their means the animalcule can attach itself to any particular 
thing that it desires. Some, again, have styles, which are a kind of thick bristle, jointed at the base, possessing a movement, but not rotary; they are in the shape of a cone, large at their base, and delicate at their summit. Many, also, can extend and withdraw their bodies at pleasure, in a similar manner to the snail or leech. Thus are they adapted for the various phases of their existence.

We find these invisible atoms of life have various periods allotted to them for the enjoyments of existence; some accomplish their destiny in a few hours, others in a few weeks. The watchful devotee in this branch of science has traced an animalcule through a course of existence extending to the old age of twenty-three days. The vital spark flies instantaneously in general; but in those of a higher organisation there is a spasmodic convulsion, as if the delicate and intricate machinery renderer life so exquisite, that the parting" with the "heavenly flame" was reluctant and painful. The most surprising circumstance attendant on the nature of some of the Infusoria is that of apparent death. When the water or mud in which they have sported in the fulness of buoyant health becomes dried up, they lie an inanimate speck of matter; but after months, nay years, a drop of water being applied, their bodies will be resuscitated, and in a short time their frames be active with life. Leuwenhœck kept some in a hard and dry condition, and restored them to life after a sleep of death of twenty-one months. Professor Owen saw an animalcule that had been entombed in a grave of dry sand four years reborn to all the activity of life. Spallanzani tried the experiment of alternate life and death, and accomplished it in some instances on the same object fifteen times; after which nature was exhausted, and refused further aid in this miraculous care of those minute objects of her wonderful works.

The following interesting account of these animals we extract from Baker's excellent treatise on the Employment for the Microscope: "If the water standing in lead gutters, or the slimy sediment it leaves behind, has any thing of a reddish colour, one may almost be certain of finding the wheel-aninals therein; and if in summer, when all the water is dried up, and nothing but dust remains, that dust appears red or of a dark brown, one shall seldom fail, on putting it in water, to disclose multitudes of minute reddish globules, which are, indeed, the animals themselves, and which will soon change their appearance; for in the space of half an hour a languid motion begins, the globule turns itself about, lengthens by slow degrees, and becomes in the form of a transparent caterpillar, and appears lengthening out its body considerably sometimes, and contracting it as much at others. Its mo- 
tion from place to place is likewise then performed in the manner of such creatures ; fixing first its tail, and extending its whole body; then fastening its head, and drawing up its tail to it, and so on ; by which it gets along pretty nimbly. But one shall often behold it changing its appearance in an instant, and assuming a form extremely different; for its snout being pulled somewhat inward, the front end becomes clubbed, and immediately dividing, exhibits most surprisingly to view a couple of semicircular instruments before unseen, round the edges whereof many little cilia move themselves very briskly, sometimes with a kind of rotation, and sometimes in a vibrating or trembling manner. An opening or mouth also appears in the middle, between these two semicircles. When in this condition, the animal is often seen to unfasten its tail, and swim along with a great deal of swiftness, seemingly in pursuit of prey. When the fore part of the creature first appears to open or divide, the instruments above described, which when fully protruded resemble a pair of wheels having a quick rotation-coming then but a little way beyond their tubular cases, and being in that condition like round pieces of paper folded in the middle, or funnels whose sides are flattened almost together-seem only like a couple of semicircular parts, about the edges whereof what are seen afterwards like the teeth of wheels appear only as little spicula, having a nimble and continual vibrating motion upwards and downwards; whereby the water becomes greatly agitated, and is brought to the animal from several times the distance of its own length. As the creature is capable of thrusting out these wheels or drawing them in, as snails do their horns, the figure of them is different in their several degrees of extension or contraction, or according to their position to the eye of the observer."

To this description of these curious little animals little more has been added up to the present time. The infusorial animalculæ vary in their capabilities of the endurance of heat and cold, some of them retaining life in water $200^{\circ}$ Fah., while others expire from the heat of a warm room. Many die when the mercury is as low as $80^{\circ}$ Fah., while others survive. They have been found embedded in ice at the arctic regions, surrounded by a little water, which Ehrenberg supposes is not frozen from the natural heat of their bodies. Air is as necessary to their existence as it is to superior animals, for if placed in a tightly corked bottle in water, where oil is poured on its surface, or underneath the receiver of an air-pump, they are killed. They will live in water where poisons which mingle mechanically, not chemically, are infused. But all sudden transitions are destructive, as the mixture 
of sea with fresh water; still, if the change be gradual, they will adapt themselves to the new element and survive. The phosphorescent appearance of the ocean, arising chiefly from the presence of animalculæ, is thus described by Darwin: "While sailing a little south of the River La Plata, on one very dark night, the sea presented a wonderful and most beautiful spectacle. There was a fresh breeze, and every part of the surface which during the day is seen as foam, now glowed with a pale light. The vessel drove before her bows two billows of liquid phosphorus, and in her wake she was followed by a milky train. As far as the eye reached, the crest of every wave was bright; and the sky above the horizon, from the reflected glare of these livid flames, was not so utterly obscure as over the vault of the heavens. Near the mouth of the Plata some circular and oval patches, from two to four yards, shone with a steady but pale light, while the surrounding water only gave out a few sparks. The appearance resembled the reflection of the moon or some luminous body, for the edges were sinuous from the undulations of the surface. The ship, which drew 13 feet of water, passed over without disturbing these patches; we must therefore suppose that some of the luminous marine animals were congregated together at a greater depth than the bottom of the vessel, or 13 feet beneath the surface of the sea." The author has witnessed a similar appearance in the sea from the Nore to Ramsgate, and watched it with the same delight.

Naturalists consider the phosphoric light of the marine animalcule to be the effect of vital action. The sparks are intermittent like the fire fly; they measure from the 12,000th to 100th of an inch in size. Captain Scoresby found that the broad expanse of waters at Greenland was nearly all discoloured by animalculæ, and computed that of some species, one hundred and fifty millions would find ample room in a tumbler of water. Mr. Gosse thus describes the luminous appearances presented by these minute animalculæ: "Some weeks afterwards I had an opportunity of becoming acquainted with the minute animals, to which a great portion of the luminousness of the sea is attributed. One of my large glass vases of sea-water I had observed to become suddenly at night, whell tapped with the finger, studded with minute but brilliant sparks at various points on the surface of the water. I set the jar in the window, and was not long in discovering, without the aid of a lens, a goodly number of the tiny jelly-like globules of Noctiluca miliaris, swimming about in various directions. They swam with an even gliding motion, much resembling that of the Volvox globator of our fresh-water pools. They congregated in little groups, and a shake of the 
vessel sent them darting down from the surface. It was not easy to keep them in view when seen, owing rather to their extreme delicacy and colourless transparency than to their minuteness. They were, in fact, distinctly appreciable by the naked eye measuring from $\frac{1}{50}$ th to $\frac{1}{50}$ th of an inch in diameter." With a power of about 200 diameters they are seen of various forms and stages of growth as represented in fig. 84 .
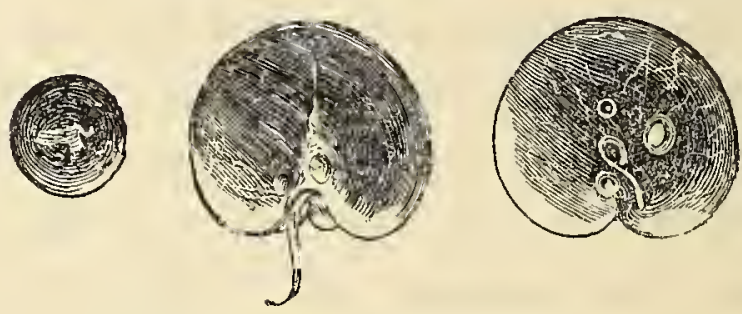

fig. 84. Noctiluca miliaris.

"Awaked before the rushing prow,

The mimic fires of ocean glow,

Those lightnings of the wave;

Wild sparkles crest the broken tides, And, flashing round the vessel's sides,

With elfish Iustre lave;

While far behind their livid light

To the dark billows of the night

A gloomy splendour gave."

ScotT.

The Infusoria have no night in their existence ; they issue into life in a state of activity, and continue the duration of their being in one ceaseless state of motion; their term is short, they have no time for rest, and therefore have but one day, which ends only with their death and decomposition. Nevertheless, they appear to love that which promotes life,- - the light of heaven; but others, born in the bowels of the earth, and who never partook of the blessing, still, like the ignorant among mankind, have their own contracted round of unenlightened joys; they perform their mechanical duties, and expire hidden and unknown.

Polygastric Animalculce.-In the valuable voluminous treatises on the subject of Infusorial Animalculæ, the first class is denominated Polygastric; and that class is divided into several families. As our object is to give a popular account of the wonders disclosed by the microscope, we will not closely follow the path of the industrious and learned investigators of this branch of science; and hence will avoid as much as possible the adoption of those terms that so alarm the uninitiated; culling, as we pass, only those objects of most interest, likely 
to create a desire for further knowledge, whereby there will be fênd a future pleasure in overcoming the obstacles by which the learned render so mysterious and repugnant to the popular mind the stores of their gathered wealth.

\section{MONADS.}

Monads:-The smallest atom of matter possessing the mysterious principle of life, discernible by the extraordinary magnifying power of the microscope, is the monad. Minute, however, as it is, no one can say but that it derives its sustenance by preying on animals even less than itself, as larger ones of the same species do upon it; still, with all the aids of the brilliant achievements of science, this is the limit at present set to man's discoveries in the minutiæ of the invisible world.

A drop of water only a tenth of an inch in diameter may glitter like a diamond from its translucency, and yet under the microscope be seen to hold 500 millions of these animated beings; an amount about equal to the whole human family existing on the surface of the globe. Vainly does man, with all the subtlety of his mind, endeavour to conceive an object the twenty-four thousandth of an inch in length; yet this is the size of the monad: some have been discovered twice this length, but still they are inconceivably small when attempted to be defined by comparison. If each be allowed three times its bulk to move about in, a cubic inch of water will then contain 800,000 millions of these organised beings. Nay, some philosophers say this family of Infusoria are seen only the one forty-thousandth of an irch in length. What incalculable numbers of animalculæe must swarm the waters of creation !

Monads vary in their colours, some being red, green, yellow, and others colourless; in shape they are round or oval (see 5 and 6 , fig. 87), and possessed of immense activity, having one or more parts devoted to the purpose of locomotion. The microscope has not detected the organ of vision in all, but in some a bright red spot is discoverable, evidently an eye, and hence there must be a nervous system for the performance of its functions.

These little specks of animated nature are polygastric (many-stomached). It may excite some surprise how it is possible to detect the internal structure of these animals, but therein consists the difference of the philosopher and the common observer; the one devotes the energies of his mind to the elucidation of truths, the other merely enjoys the fruits collected by skilled industry: 
Ehrenberg* says: "All true Infusoria, even the smallest monads, are organised animal bodies; some consist of a homogeneous jelly, and are distinctly provided with at least a mouth, and internal nutritive apparatus." Perceiving small round spots within the bodies of some ani-malculæ, he judged them to be stomachs, in contradiction to the supposition of the former great philosopher in this branch of science, Müller, in whose work, published in 1773, they were stated to be the animal's eggs. To test the truth of his idea, and convince the world, Ehrenberg fed the little things with colouring matter diffused in the water which contained them. If the water be clear in which the animalcule is living, the stomachs are transparent, more so than the other parts of the body; but are rendered visible by tinting the water with pure sap green, carmine, or indigo. Some of one of these colours is rubbed on a piece of glass, then a few drops of water added; a portion of the water is then allowed to run off by tilting the glass on one side, and a little of the remainder of the coloured matter dropped into the water containing the animalcule. Portions of the coloured fluid are swallowed by the animalcule, when the stomachs, from their transparency, are distinctly seen of the same colour as the liquid, while the other portions of the body remain unchanged. Some species of the polygastria have upwards of a 100 stomachs, others only four. Sap green is the colour most easily imbibed by the tiny beings; carmine shows development better than any other; whilst the indigo, which Ehrenberg found to answer his purpose most satisfactorily, is rather difficult to manage. Care has always to be observed that the colours are not those that chemically combine with water; but only such as are diffusible through the fluid in a state of minute subdivision, as otherwise they are poisoned by it. This important discovery of feeding the little things on colour set aside the opinion of previous naturalists, that they effected nutrition by cuticular absorption; it also led to a classification, not, as formerly, by shape, but structure.

Monas Crepusculum, or Twilight Monads, are round in shape, and of a bright transparent appearance; a peppercorn occupies more space than fifteen hundred millions of these organised beings. As far as can be ascertained, they live upon animal matter, at least they are found in water containing it; and as it decomposes, the life of the monads ceases,

* Christian Gottfried Ehrenberg, medical counsellor and professor at Berlin, was born at Delitzoch in 1795, and educated at Schulpforta and Leipzig. In 1820-25, he, in company with Hemprich, visited Egypt and Nubia at the expense of the Berlin Academy ; and in 1829 he accompanied Alexander von Humboldt to the Ural Mountains. The results of these journeys he published in various invaluable works, which will hand his name down to posterity with undying honour. 
and the bodies float to the surface in myriads, appearing to the naked eye a colourless jelly-like mass. The animals move about by means of cilia that project from their mouths, aided by a slight muscular motion of the body, by which they are capable of great activity.

Monas Termo, or End Monad.-The name given these animalculæ is from their appearing, under the greatest power of the microscope, as mere encls or points; in fact, to catch a glimpse of them is very difficult, as they are round in shape, and of a bright transparent appearance.

Monas Atomus. - The atom monad has always a round body, and varies from the 6000th to the 3000 th of an inch in diameter; it is of the colour of water. In this little being is first recognisable the mode of continuing its species, which it does by a division of its own body. Its shape first becomes distorted, then a line is seen across its middle, two animals gradually become formed, when they separate, each to enjoy a separate existence.

Monas Grandis. - The great monad appears in a variety of bright colours; it is oval in form, and when floating on the tops of ponds and ditches, the water seems encrusted with slime.

Monas Mica.-The grain monad is about the 1500th of an inch in size, and two oval-shaped bodies are perceptible in it; but that which renders it an object of interest when viewed through the microscope, is a beautiful halo that surrounds it, which is supposed to proceed from cilia, or hair in motion; it often revolves on its own axis.

Monas Uva. - The grape monad is so named from their sometimes forming themselves into clusters resembling a bunch of grapes. They are oval in form, have two cilia, and are wondrously active in pursuing and devouring their lesser brethren, several of whom they consume at a meal, having no less than twelve stomachs to fill. They multiply both by eggs and self-division; scientifically, they are termed oviparous and gemmiparous. When they propagate in the latter mode, their bodies clivide into four parts, and thus become this number of distinct animals.

The Gonium, or Tablet Monad.-Enclosed in a flat transparent shell, irregular in shape, and not larger than one three-hundredth of an inch in length, is discovered a happy community of sixteen green-coloured monads, of a size from the five-hundredth to the one-thousandth of an inch, with one mind influencing the actions of all. These are sometimes called The Breast-plate Animalculce (see fig. 85, No. 3). They move about in all directions, upwards and downwards, forwards and backwards, and rolling on the edge like a wheel. Twenty-four of these 
cilia projecting from the sides, and eight from the centre, are actively engaged in satisfying that first law of nature, self-preservation by food. They are bound to each other, not only by mutual will, but by six threads or tubes. The four centre animals are usually bigger than the others. When they have all attained their growth, the shell divides into four parts, leaving four monads on each; these four grow in size, and each again divide into four, hence arises their magic number of sixteen; then, as soon as the sixteen are of a mature size, they divide into a community of four; and thus go on dividing and subdividing, endlessly fulfilling their appointed destiny in links of creation.

The Proteus.-There is found a yellow jelly-like mass, about oneseventeenth of an inch in length, which, from its assuming a multiplicity of shapes, is denominated the Proteus. This singular animalcule has many stomachs; it protrudes at pleasure, out from various parts of its body, limbs, that may act as legs, arms, or feelers. Ten or twelve of these at one time are observable; they are rapidly withdrawn, and with equal facility are made to appear in other parts; from its form ever changing, what its true shape may be is unknown. Thus it is honoured with the name of a fabled god, who could be either animal, vegetable, or elemental in his nature.

Enchelis, or Flask Animalcule (fig. 85, No. 2). These are described by Müller as simple invisible animalculæ of a cylindrical form. On the surface of the waters of ponds and ditches is often seen a kind of green scum, from which people are accustomed to turn with disgust, and ascribe to it some injurious property. When this is brought under the powers of the microscope, the water is seen to be pure and clean, and the green found to consist of innumerable slender cylindrical-formed animalculæ, whose stomachs, or interiors, impart the colour from being distended with vegetable matter. The wise and loving decrees of Providence are here exemplified, as, by the innate wants and tastes of this growing and living speck, varying in size from 1-1200th to 1-400th of an inch, the decaying and putrefying matter is removed, and the noxious effects on man and beast prevented. Others, of the same species of these industrious and useful mites in the animal economy of nature, with their little active ruby-coloured eye, whose masses alone render them visible to the human eye as a coloured substance, exhibit much variety in form and habit.

Enchelis Pupa, or Puppet-flask Animalcule, attains the size of the 1-400th of an inch. Its stomach extends the whole length of its body, and, like the boa constrictor, it swallows its prey whole, distending and distorting its little body with the huge lumps, which it contrives 
to get within its stomach. When, by the motions of its cilia, it has drawn its victim to its mouth, it retains it there, swimming actively about to assist in the engorgement. Gradually the food, after being got inside the animal, lessens near the mouth, progressing in its decay downwards; and as this is accomplished the body resumes its natural shape.

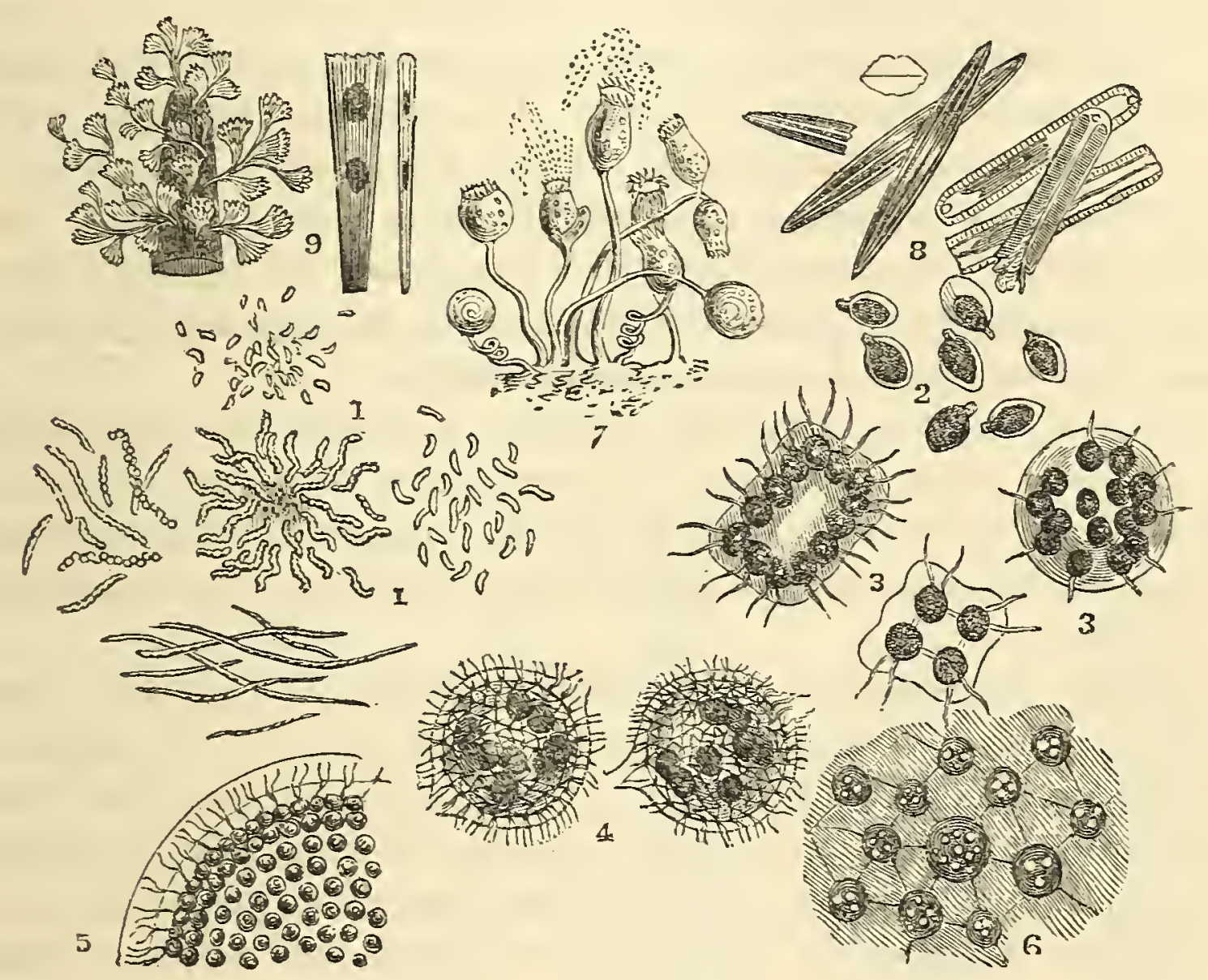

fig. 85. Infusortal Animalcule, \&c.

1. Group of the Vibrio Spirilla. 2. Enchelis, or Flask Animalcula. 3. Gonium pectorale, or Breast-plate Animalculæ. 4. Volvox globata. 5. A section showing the ciliated edge of the cell. 6. A portion more highly magnified, to show the young Volvox with nuclei and attachments. 7. The Vorticella cyanthina, or bell-shaped animalculæ. 8. Berg-mehl, or Mountain-meal Animalculæ. 9. Echinella, or Fanshaped Animalculæ; an enlarged view of one is seen by the side of the cluster.

Vibrio Spirilla, or Trembling Animalculce, when motionless, appear as extremely minute hairs; but when they exert the powers of locomotion, they take a spiral form, like the threads of a fine screw, and by undulations wind themselves through the water with rapidity. Each apparent hair is a collection of animals bound together by a pliant band; thus, as they are individually so small, little is known of their structure. Still, they form very interesting objects to view; their very minuteness claiming attention, while their activity and motions 
excite surprise. The species are numerous, as represented at No. 1, fig. 85. One in particular has been the especial subject of investigation by the medical microscopist : it is somewhat of an oval shape, and found in many forms of diseased structure; doubtless it precedes or leads to the entire destruction of the tissues it is found in. This shape of the curious little animalcule, it should be observed, is confined to animal substances; whereas the long, or hair-shaped, is generally to be met with in the disorganisation of vegetable matters.

These hair-like animalcul æe were very accurately described by Baker, who ascribes the discovery of them to Mr. Anderson. He says: "They were discovered in a large ditch running into a river near Norwich, the bottom of which was covered with them to some thickness; when first examined, being motionless, they were taken for vegetable fibres; but on keeping them under the microscope, and occasionally viewing them, they were seen to move in various forms."

Astatia.-Astatia, signifying without a station, in contradistinction to those living in groups, is the term given to a kind of crimsoncoloured animalcule the 318 th of an inch in length, that exist in enormous numbers, and give the waters in which they live the appearance of their bodies.

Astatia Homatodes. - The blood-like astatia is first of a green colour; but as it matures, becomes the tint by which it is designated. Its form is that of a fish having a long pointed tail and cilia, affording it the power of motion; some species have the gift of changing their form, by withdrawing their tail and contracting their bodies.

Astatia Viridis.-On the surface of ponds and stagnant waters is sometimes seen a crimson covering, which, when examined by a microscupe, is found to consist of a mass of oblong blood-red eye animalculce the 300th of an inch in length. Ehrenberg states, that in the early part of their existence they are green; and that the red and green spots on their bodies are caused by the condition of the eggs at different periods in their stomach-cells. A sparkling red eye is possessed by the living atom; and a cilium proceeding from its mouth gives it motion, sometimes in a straight line, at others rolling about in all manner of ways. When two cilia are seen, then the animal is about to divide into two perfect and separate beings, to proceed again in the career of its ariginal. They seem to have the power of changing their shapes at will; at one time they have a rolling-pin form, at another that of a fish without a tail, and are also seen with their bodies extended at the side like wings.

Bacillaria, derived from the Latin word bacillum, a little stick, is 
classed by Ehrenberg among the infusorial animalculæ, while Meyen affirms "that much observation is yet wanting, ere we can hope satisfactorily to determine that the bacillaria are true animals." Some observers consider them as belonging to the Algæ, or Desmidiaceæ order, rather than that of the Infusoria; in this uncertainty they were termed existences, and supposed to be a link between the vegetable and animal kingdoms. They comprise a large class, and are found both in the waters of the ocean and land. The microscopist is delighted and enthusiastic in his examination of them, as they afford some of the most beautiful objects for observation.

\section{NAVICULR.}

The Navicula Animalculce.-Navicula is the Latin word for ship, and has been applied to this kind of Infusoria from their resemblance in form to a long ship or boat.

In the catalogue of the microscopist there are twenty-four different species named, fourteen of which are at the present day found alive (see fig. 86 , Nos. 1 , 2, and 3). From their beauty and minuteness, they are used as test-objects for microscopes ; that is, if the glasses define the exquisite and delicate markings on the shells, they are deemed sufficient for scientific purposes, and adopted; but if the lines are not clearly seen, the power is considered indifferent and imperfect, and should be discarded. By this test one microscope is compared with another, and the value estimated accordingly. It was only in 1841 that Mr. Harrison, of Hull, discovered the beautiful longitudinal and transverse strice (groovings) on the
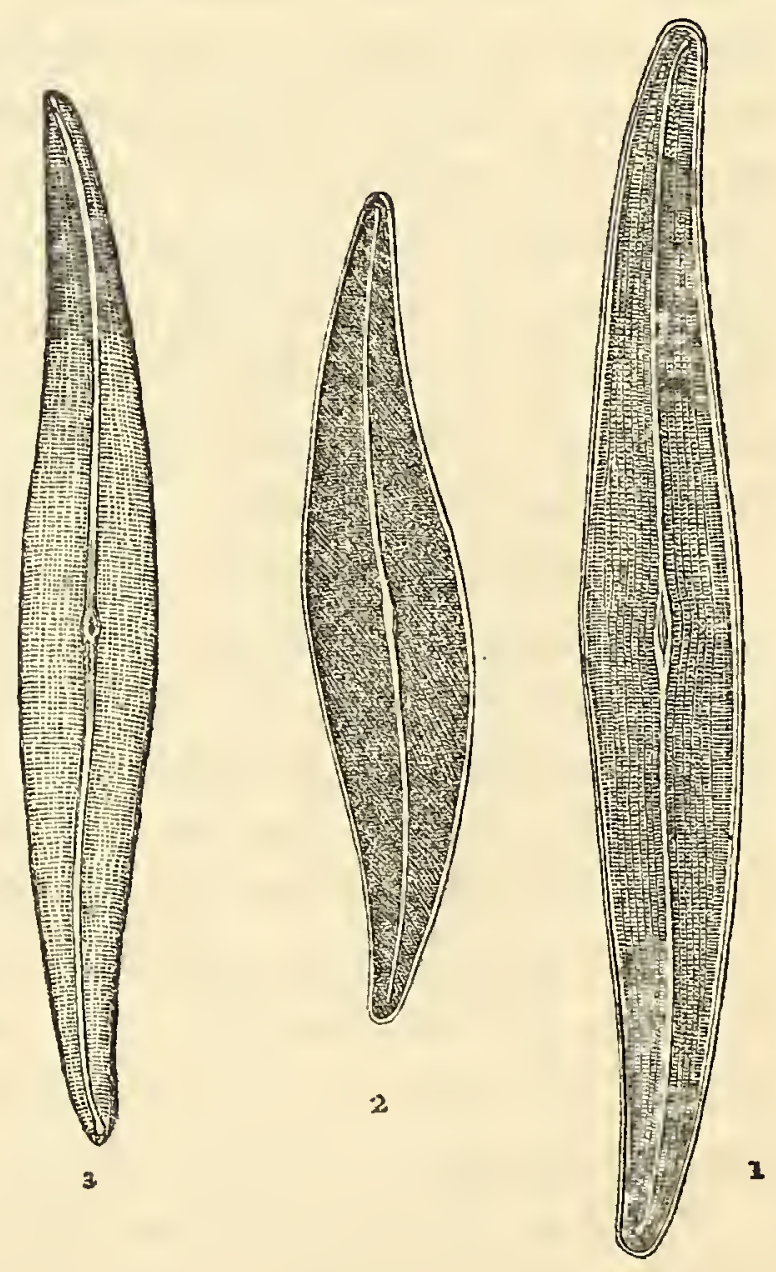

fig. 86 .

1. Navicula hippocampus. 2. Navicula angulata, magnified 250 diameters. 3. Navicula Spencerii, magnified 350 diameters. Navicula Hippocampus or seahorse ship, No. 1: a curved graceful line runs down the shell, in the 
centre of which is an expanded oval opening, and smaller ones at each end. Near to the central opening, the dots elongate crossways, presenting the appearance of small short bands. The Navicula angulata, No. 2, or cornered little ship, was first discovered in the Humber; the lines upon its surface resemble the most elegant tracery, which are resolvable into raised minute dots. The markings are seen to be longitudinal, transverse, and oblique. Man boasts of the fineness and perfection of his handicraft; his manufacture will be so small that a magnifying-glass is required to observe it; he will proudly display a piece of lace of most fragile material: but when he once gazes through the microscope at these objects, invisible to his naked eye, and sees the perfection of Nature's works, he feels abashed, and sinks, conscious of the futility of his attempts to rival the accuracy and completeness given by a guiding power to the most insignificant of its creations.

The animated atoms are of a jelly-like substance; and specks of green, yellow, and brown matter are perceived jealously enclosed, which, by naturalists, are named eggs. The shell is durable, thin, and siliceous ; it divides with the little inhabitant into four parts, at portions resembling ribs.

The Green Navicula was found by Dr. Mantell in a pool on Clapham Common; it is about the 100th part of an inch in length; it has as many as 15 stomachs in every 1200 th of an inch of its length. In this specimen the ribbed division is distinctly seen, and extends the whole extent of its shell.

The Golden Navicula is another beautiful species, and was so named from the numerous points within the shell giving it a bright yellow appearance. The shell is an oblong oval, and has upon it numerous delicate and regular flutings.

The Eunotia is of the Navicula species. A furrow is seen the entire length of the shell, from which spring numerous ribs tending towards the edges; of these, eight may be counted in the 1200th of an inch; there can also be traced, in the length, a line, by which, when at maturity, the animal divides, and in this manner preserves its generation from age to age. See Plate II. No. 13.

Professor Siebold believes Ehrenberg to be in error when he states that in Navicula there are six rounded openings upon the dorsal and abdominal surfaces, - four at each end, and two in the middle. Of the four openings at the ends, the two on the abdominal surface are oral openings, and the two on the dorsal, respiratory ; whilst the central opening of the abdominal surface is stated to be for the protrusion of the foot. 
Siebold says: "I have been unable to detect these six openings in the Navicula; and precisely at the spots which Ehrenberg and others suppose they have seen openings, the siliceous cell-membrane becomes thickened, and forms rounded eminences which project internally. On the same two surfaces upon which the thickenings of the siliceous shield of Navicula are placed, there may be observed four lines passing along the middle of the surfaces from one thickening to another. These lines-which have been long known, but hitherto little noticed - are to be referred to a suture, fissure, or rather gap, in which no siliceous matter is deposited; so that in these places the delicate primordial membrane which lines the siliceous shield can be brought in close relation with the outer world. It is exactly at these four sutures or fissures that the water surrounding the Navicula is set in motion. The existence of this current is readily demonstrated if some minute solid particles be added to the water in which are some fresh Naviculæ. When water coloured with indigo has come to a state of rest on the object-glass, it will soon be perceived by the microscope that those particles which come in contact with the living Naviculæ are set into a quivering motion, though previously quite still. It will also be perceived, that only particles of indigo are set in motion, which are in contact with the four sutures of the siliceous shield; whilst the particles adherent to the other parts of the shield remain altogether motionless. Another striking motion is perceptible in these particles when they come in contact with the sutures of the siliceous shield, being forced rapidly up and down upon it. Those particles which are propelled from the terminal towards the two central eminences, are never ebserved to pass beyond the latter; at this point there is always a quiet space, from which the particles are again repelled towards the extremities. This proves that the linear sutures do not extend over the central eminences of the shield. At these clefts the current is sometimes so strong, that comparatively large bodies are set in motion by it."

\section{VORTICELLÆ,}

Proceeding through the waters of swamps, most generally is discovered a beautiful group of deep bell-formed animalculæ, attached to each other like a delicate branch of a shrub, about the hundred-andtwentieth of an inch in length, which are the Carchesium polypinum.

The Vorticella Cyanthina, fig. 85, No.7: a fringe of cilia surrounds the lips of the cup, and the food is drawn to the mouth, and taken into the several stomachs of the little being. A single individual is seen, which increases in breadth; it then divides, and two perfect animals, joined 
by a stem, undergo the same change; and their progeny again, until the family be complete; when one is cast off into the world to found its race, as its parent had done previously. It possesses a bright red eye.

Another beautiful branch of this family is the Carchesium, or Tree Vorticella, which appears like a tender exotic, having a fragile stem with branches spreading out at the head. When alarmed, the stalk assumes a spiral shape, and the little family cluster together in the form of a ball. The whole are united together by a muscle that proceeds up the stem, and extends its powers to each of the living group; which allows also of the coiling and uncoiling its own stalk. The size of each of these beings is about the five-hundredth of an inch.

Vorticella Convallaria, or Lily-shaped Vorticella.-In his searches for objects of study, the microscopist is sure to meet with some twig, leaf of a plant, or perhaps other animal, to which is attached a slimy-looking substance, which, when placed under a microscope, is discovered to be the animalcule named above. The bell-formed body is surrounded at the edge by cilia; and at the opposite extremity is a long stalk, by which it attaches itself to an extraneous object. With an astounding velocity they can contract the stalk, and shrink down; uncoiling it and stretching it to its utmost length when in search of prey. They generally are found in communities, and very busy they seem to be; some reaching in one direction, and others in another; while some are contracting their stalk like a coil of rope. By times the entire family will cower down in confusion; and after the danger is past, gradually one by one recommence their active search for food. They are also about the five-hundredth of an inch in size, and propagate by self-division and budding. When one is broken off from its stem, this part remains inactive and lengthened out, as if dead; but the animal swims away with the divided part first, which becomes a head; and from the former head springs another stem.

The Vorticella Anastatica differs in shape, being more round than bell-form; they branch from the top of a stem, which does not contract; and on the second or third day the young separate from the parent stem. Mr. Trembley saw in twenty-four hours one of these round buds surrounded by a family of 100 produced by division and subdivision. Although the stem has no contractile power, the branches have; and sympathy with each other excites the entire family; for if one is threatened, they all participate in the fear, and cluster together.

STENTORS.

Belonging to the bell-shaped family are the Trumpet Animalculoe, 
or Stentors (fig. 87, No. 4), so named from the shape in which they exist. They are of various colours, white, blue, yellow, red, and green; they swim in a parallel line to their sides, sometimes tail first, rotating on their own axis. They attach themselves to objects by a sucker at the lower part. Some of them have cilia over their entire bodies, and a good fringe over their gaping mouths, which is a type of their ravenous nature. The prey may be seen in their row of stomachs, as if strung like a strap of beads up and down its interior. They differ from many gluttons by possessing great activity, moving swiftly through the water. They are said to increase both by eggs and self-division. There are many species, differing in size, shape, and colour. Usually they are about the hundredth part of an inch in size. When found, they appear a mass of green jelly encircling a twig. Often, when swimming, they take the form of a cup, having their tails drawn within their bodies.

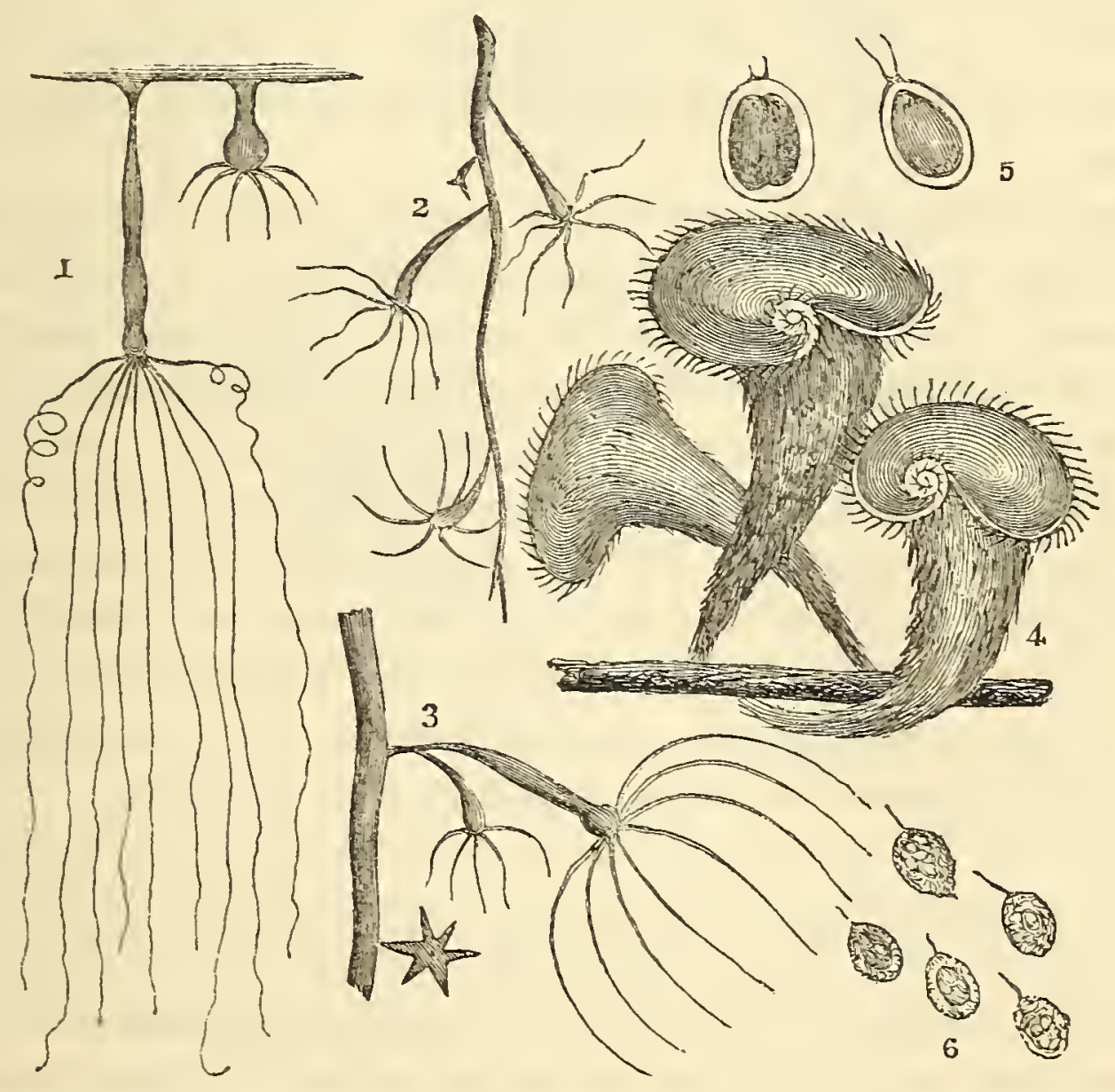

fig. 87.

1, 2, 3. Hydrex, or Fresh-water Polypes, attached to the stem of a plant. 4. A group of the Stentor polymorphus, or Multi-shaped Stentor. 5, 6. Monads, Viviparous and Cloak Monads.

Stentor Comuleus, or Blue Stentor, is remarkable from having a crest extending along its body; it assumes a peculiar shape when swimming, 
appearing to possess a thick tail nearly one half the breadth and length of its body.

Before proceeding to the details of a higher type of these wonderful creations, we may mention that Ehrenberg enumerates other genera in this division of animalculæ. The Cyclidium, a minute, brilliant, crystallike object, flat, round, or ovate in shape. The Paramoecium, flat, oval, and membraneous; in some about 20.0 stomachs have been seen. The Kolpoda Müller describes as "an invisible, very simple, pellucid, flat, crooked animalcule." The Bursaria, a simple, hollow, membraneous animalcule, having longer cilia at its mouth than over its body; its stomachs resemble purses (hence its name), which are attached to its inside by slender threads. The Cercaria are transparent objects having a tail. The Leucophrys, ciliated and pellucid. The Trichoda, transparent, and partially covered with cilia. The Kerona, which have hooks and bristles; and the Himantopus, that possess a cluster or tuft of hair. Although these are placed in the lower scale of animalculæ, yet many of them are possessed of such higher organisations as to entitle them to rank with the next class in advance of the Rotifera.

Upon quitting these animalculæ, we may remark this peculiarity, that when they are procured from infusions, the first instances of life are generally the Monads, which in a very short period increase to a most extraordinary extent. These afterwards gradually decrease, larger and more perfect creatures supplying their place, as the Cyclidia, Paramesia, Kolpodæ, and others; and these, again, are supplanted by the Vorticellæ and Branchioni. Though the generations thus rise up, there is no regular order in their doing so, even in the same infusions.

The next great aivision of the animalcule kingdom, though coming under the genus of Vorticella, claims a distinct notice; as the machinery by which life is rendered active is plainly distinguishable.

THE ROTIFERA, ROTATING OR WHEEL-ANIMALCULIE.

This higher grade of the Infusoria derives its name from the appearance presented by the motion of its circles of cilia on the superior part of its body, which resembles the turning round of a wheel, as they rapidly vibrate. Many have been the speculations as to the mechanism of this beautiful movement: some have considered it as a magnetic or electrical force; and as one passes out of sight while the next appears, adding to the optical illusion, a philosopher of considerable note was led to look upon the whole as a deception of the sight, and affirmed they 
had no existence. The cilia processes appear but in one part of the Rotifera; whereas in the Polygastria, cilia are seen in various parts of the body.

These animalculæ are only discovered in water, which is their native element; still, they are found in damp earth, and are indwellers of the cells of moss and sea-weed. They possess but one stomach, and generally have teeth and jaws to supply its wants. They can elongate and contract their bodies; and some species have at their extremity a kind of tail with a sucker, by which they affix themselves to extraneous

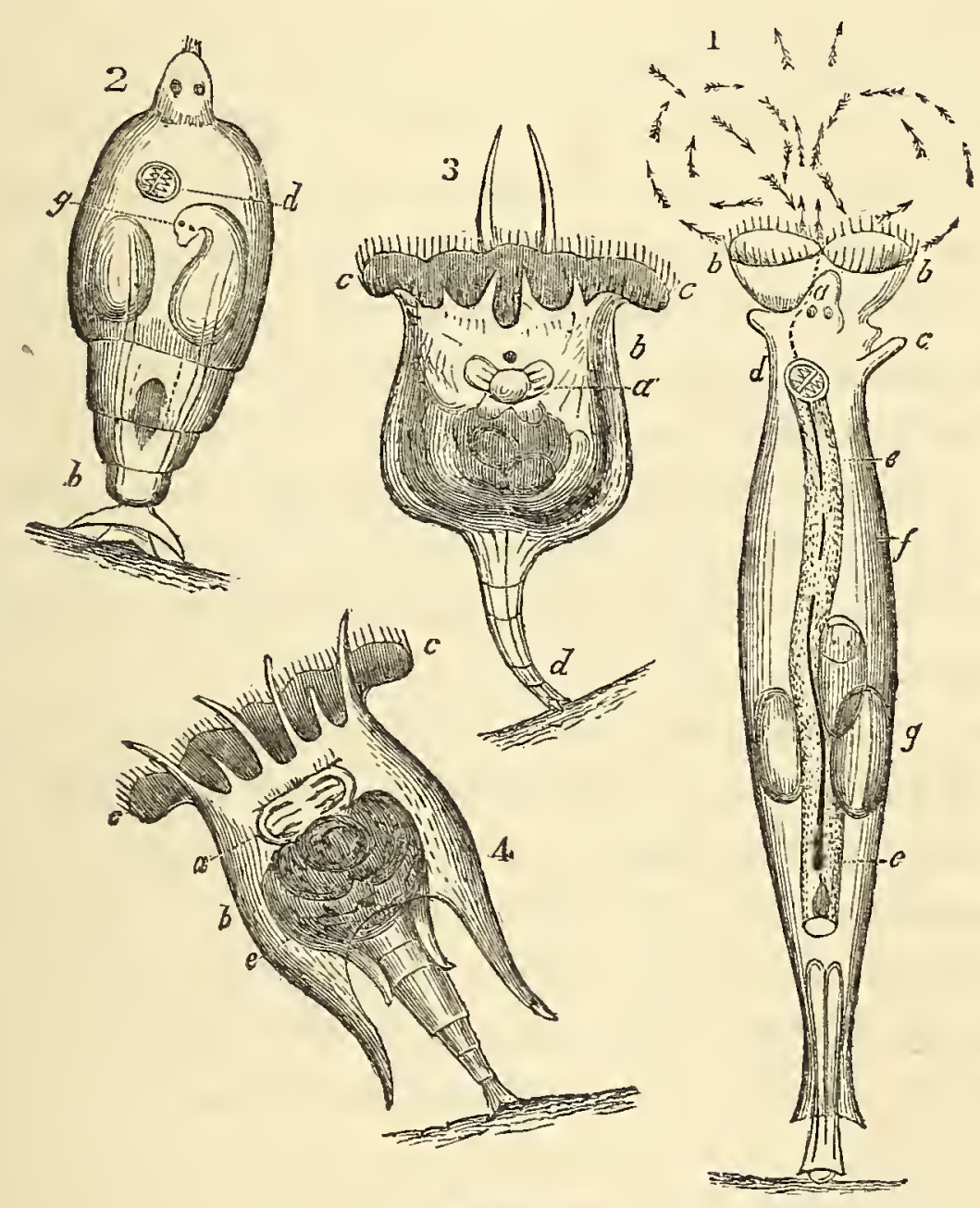

Fig. 88.

1. The common Wheel-Animalcule, with its cilia or rotators pointed. 2. The same in a contracted state and at rest; at $g$ is seen the development of the eyes in the young. 3. The Pitcher-shaped Branchionus : $a$ the jaws; $b$ the shell; $c$ the cilia, or rotators; $d$ the tail. 4. Baker's Branchionus: $a$ the jaws and teeth; $b$ the shell; $c$ the rotators; $e$ the stomach.

substances while the cilia is in rapid motion, and thus prevent the superior portion of the body being drawn in by the force of the rotatory action. They multiply by eggs; but some bring forth their young alive. Both the animal and its eggs possess extraordinary tenacity of life, and will undergo the most opposite extremes of circumstances without destruction to the living principle. 
In the atmosphere the eggs may often be discovered whirling along by the force of the wind to some resting-place, where, when circumstances admit, they spring into active life, and fulfil their appointed destiny. Ehrenberg accurately described the upper part of a common wheel-animalcule, with the cilia, jaws, teeth, eyes, as seen under a magnifying power of 200 diameters, represented at 1, fig. 88 .

The small arrows show the direction of the currents produced by the cilia turning on their base. At the will of the animal a change is made in the direction in which the wheels appear to revolve, and they have the power of withdrawing, with the quickness of thought, the whole of the wheel-work; when this is done, the head is elongated, and with a telescopic appearance, as if capable of sliding within itself. On assuming this form, a cluster of hairs appears at the extremity, that do not revolve, and are considered different from the cilia: as they are usually protruded when the creature is moving from place to place, their functions have been imagined to be that of feelers, shown in No. 2 .

The eyes of the Rotifera are generally of a bright red colour; but the number and arrangement of these organs vary. In some kinds there have been discovered as many as eight, sometimes placed on each side of the head, in a row, in a circle, or in clusters; and in others, in a triangular shape.

Ehrenberg, from actual observation, found that the Rotifera laid four eggs a day; that the young, when two days old, followed the same law as their parents; consequently, a single one in ten days had a family of $1,000,000$, in eleven days 4,000,000; and in twelve days the venerable progenitor was surrounded by 16,000,000 of an active, happy', energetic race, ceaseless in search of prey, and a famous feast for a larger animal.

The Rotifera delight in the sunshine; and when the bright luminary is hidden behind clouds, the animals sink down to the bottom of the water, and there remain. When the water of their haunts is becoming much evaporated, they rise to the top, and give a bright-red tint to it; but when caught and placed in a.jar, their beautiful colour fades in a few days. Locomotion is performed by swimming, the rotatory action of the crowns of cilia impelling it forward; in other instances it bends its body, then moves its tail up towards the head, which it can do from having two processes that serve as feet near to the tail; it then jerks its head to a further distance, again draws up its tail, and so proceeds on its journey. Another peculiarity they possess of drawing in the head and tail until nearly globular, from remaining in this condition fixed hy the sucker; at other times they become a complete ball, and can be rolled about by any agitation in the water. 
The eggs are of an oval form, and from twenty to thirty may be seen in an animal. Some are of a brown colour, and others of a delicate pink and deep golden yellow. In those of a light colour, the young may be seen with their cilia in active vibration.

The body of the wheel-animalcule is of a whitish colour; its form will be seen by the engraving; and its tail has six points. The tube for respiration appears to allow of water passing to the inside. On the food being drawn by the currents to the cup part of the wheels, it passes down canals in the neck to the mouth, which is situated at the lower extremity of that part of the body. The food is crushed by the teeth on the plates of the jaw, with an action like a hammer. From this it passes on to the alimentary canal for the sustenance of the animal.

\section{BRACHIONFA.}

The eighth family of the Infusoria in Ehrenberg's work is the genus Brachionus, or Spine-bearing Animalcule. They are truly interesting. from their perfect, high, and complex organisation. Some are entirely enclosed in a shell, and others only partially covered. Their structure is so beautiful and symmetrical as to cause them to be favourites with those who delight in the studies of microscopic observation.

Brachionus Striatus, or Striped Shell Animalcule (No. 3, fig. 88), is of an elegant jug-like form, the transparent shell being fluted lengthwise, and having six scallops at the upper part, whence the pale citroncoloured inhabitant protrudes itself. Two horn-like processes are appended to its under side. As occasions require, it sinks firmly and securely within its crystal home, through the sides of which its organisation can be distinctly seen. Its progress is effected by means of its rowing cilia.

Brachionas Pala, or Bent Horn Animalcule, Anurcea Cervicornis, is possessed of double rotatory organs, and four long processes, that project above the oval shell. It measures the 90 th part of an inch.

Brachionus Ovalis, or Egg-shaped Brachionus, is remarkable for the strength of its transparent shell, which is beyond that of other shelled creatures. Its projecting tail, as well as head, can at pleasure be withdrawn into its powerful case. It attains to about the 250th of an inch in size.

Brachionus Tripos, or Three-spined Brachionus, has its shell in two parts, united by a joining at the back; it ends in three spines, and between the two first projects its cleft tail.

Brachionus Dentatus, or Toothed Brachionus. This active, bright, 
pink-eyed little creature, the 90th part of an inch in size, is enclosed in a two-valved shell, having each end indented so as to form two pair of teeth. Mr. Pritchard says: "In addition to the rotatory organs for supplying it with food, I have observed it attached to a stem of confervæ, and abrading it with its teeth fixed in the bulbous æsophagus, which, during the operation, oscillates quickly; the rotatory cilia at the same time move rapidly, which makes it highly probable that they perform some office connected with the organs of respiration, as their motion seems altogether unnecessary while the creature is feeding in this manner."

Brachionus Urceolaris, or Flower-pot Brachionus, resides in a shell one-half its own length, from which it thrusts out its tail at one end, and its vibrating cilia at the other : at this latter part it has sharp indentations at one side, and flowing scallops at the other.

Brachionus Bakeri, or Baker's Brachionus (fig. 88, No. 4), is a curious and beautifully-formed animal. At the points of a half-circle are situated the rotatory organs and cilia, between which rise some long spines, each side of the shell proceeding to a point in the lower part, while a square seems taken out of its body, forming thus two spines; from the centre projects a long tail. The eggs are sometimes attached to the spines, and in other instances to the tail.

Notommata Aurita, the Eared Notommata.-The anatomy of this animal, of the Rotifera class, was most lucidly explained and beautifully illustrated by P. H. Gosse, Esq., in the Microscopical Society's Transactions.

Mr. Gosse states, that his specimens were found in a jar of water obtained in the autumn from a pond near Walthamstow, the jar having stood in his study-window through the winter; and from a swarm in the succeeding February he selected one the 70th of an inch in length when extended, but its contractions and elongations rendered its size variable.

"Its form viewed dorsally is somewhat cylindrical, but it frequently becomes pyriform by the repletion of the abdominal viscera. Viewed laterally, the back is arched, gibbous posteriorly, with the head somewhat obliquely truncate, the belly nearly straight. The posterior extremity is produced into a retractile foot, terminating in two pointed toes; this, both in function and structure, is certainly analogous to a limb, and must not be mistaken for the tail, which is a minute projection higher up the body. When not swimming or rotating, the head assumes a rounded outline, displaying through the transparent integument an oval mark on each side, within which a tremulous motion 
is perceived; but at the pleasure of the animal a semi-globular lobe is suddenly projected from each of these spots by evolution of the integument. These projections have suggested the trivial name of aurita. Each lobe is crowned with a wheel of cilia, the rapid rotation of whose waves forms the principal source of swift progression in swimming. The protrusions of these lobes are evidently erersions of the skin, ordinarily concealed in two lateral cavities. They may be protruded by pressure, and are then seen to be covered with long but firm and closeset cilia, which are bent backward, and move more languidly, as death approaches. The whole front is also fringed with short vibratile cilia, which extend all along the face, as far as the constriction of the neck. The whole body is clear and nearly colourless; but its transparency is much hindered by the net-work of dim lines and corrugations that are every where seen, particularly all about the head:"

Mr. Gosse, on putting carmine into the water, saw the jaws working slightly, the points opening a little way, and then closing; the rods of the hammers were drawn towards the bottom for opening, and upwards for closing. A little mass of pigment was soon accumulated beneath the tips of the jaws, which spread itself over a rounded surface, but did not pass farther; nor did an atom at this time go into the stomach.

After entering into further minute detais of the little animal, he observes: "They possess organs that many others do not, and want some that others possess. They prove that the minuteness of the animals of this class does not preclude them from having an organisation most elaborate and complex; and therefore it justifies the belief that the Rotifera occupy a place in the scale of animal life much higher than that which has been commonly assigned to them."

Of the manners of the species, this careful observer states, that, "in a phial, it frequently clings to the sides of the glass, and appears sluggish; but when put into a live-box it is active, especially if there be any conferva, or leaves of chara, or roots of lemna, in the water. Among these it crawls about nimbly and impatiently, like a caterpillar, pushing itself in every direction, by means of its two-toed foot, and by the elongation and contraction of its body, but without any definite course.

"It commonly keeps its ear-like lobes concealed while crawling; but will often suddenly protrude them, and in the same instant shoot off through the water with considerable rapidity, and with a smooth, gliding motion, partially revolving on the longitudinal axis as it proceeds.

"Like most of the class, this notommata is predatory. Mr. Gosse once saw one eagerly nibbling at the contracted body of a sluggish 
Rotifera vulgaris; the mouth was drawn obliquely forward, and the jaws were protruded to the food, so as to touch it. It did not appear, however, to do the rotifer much damage. It seemed chiefly to feed on monads."

\section{FLOSCULARIÆ.}

The Stephanoceros, or Crowned Animalcule.-This beautiful little creature is about the 36th of an inch in length. It is enclosed in a transparent cylindrical flexible case, over which it protrudes five long arms in a graceful manner, which, touching at their points, give the form from which it derives its name. These arms are furnished with several rows of short cilia, and retain the prey brought within their grasp until it is swallowed. The case is attached to the animal on the part we may term the shoulders; so that when it shrinks down in its transparent home, the case is drawn inwards. To the bottom of its home it is fastened by an elongation of the body; and this part, as well as the body, contracts instantly on the approach of danger, the arms coming close together and into the sheath. Its mouth differs a little from the common wheel-animalcule, as it has two distinct sets of teeth, with which it tears and crushes its food. The eggs of the Stephanoceros, after leaving the animal's body, remain in the crystal-like shell until hatched, when they escape from the lower part. A cluster of eggs may be generally seen in the ovisac. Dr. Mantell, from close observation, found that about eighty hours elapsed before their organs were all developed.

Limnias Ceratophylli, or Water Nymph.-This beautiful object is of the family called Floscularia, or flower-shaped animalculæ; about onetwentieth of an inch in size, residing in a:white transparent cylindrical case, one-half the length of the animal, which being glutinous, becomes of a browrish colour, from the adhesion of extraneous matter. Its rotatory apparatus is divided into two lobes, possessing vibrating cilia, as well as a singular projecting angular chin.

The Water Nymph, when young, has two red eyes; but they fade away, as the term of their existence draws to a close. The body of the animal is fixed to the bottom of its little horn; and, by means of the attachment, it can retire on the slightest symptom of danger, or protrude itself out of it in search of that sustenance requisite to its existence. In the rows of little eggs seen in the body. of the parent, may clearly be distinguished many of the particular organs in a state of activity. From its fondness for hornwort, it often receives the rame of that plant.

Floscularia Ornata, or Elegant Flescularia, is a beautiful type of this 
family, and has its rotatory organs divided into several parts; when it draws itself into a small compass, its transparent covering becomes wrinkled. This forms an interesting abject, as its internal structure can be well seen through the translucent sheath that constitutes its dwelling. The little beings are very rapacious, although but the 108th part of an inch in size.

Floscularia Proboscidea, or Horned Floscularia, has six lobes fringed with cilia shorter than in the preceding kind. The name that distinguishes it arises from a kind of horn or proboscis, also having cilia, that is placed in the centre of the lobes. The eggs cast off by the parent are seen in the sheath, and are very pretty objects for microscopic observation. In fact, the tinted case, the light ethereal frame of the tiny animal, and the various colours of the food in the stomach, combine in rendering it deeply interesting.

Melicerta Ringens, or Beaded Melicerta.-Among the Melicerta, or honey floscularia, the most beautiful is the beaded, which belongs to the same family of Infusoria as the stephanoceros. Its crystalline body is first enclosed in a pellucid covering, wider at the top than the bottom, of dark, yellow, or reddish-brown colour, which gradually becomes encrusted with zones of a variety of shapes; glued together by some peculiar exudation that hardens in water: it is these little pellets that, appearing as rows of beads, give the name to the animal.

To the Microscopic Society's Transactions, Mr. Gosse contributes an, excellent account of the "architectural instincts of Melicerta Ringens," which is not only truly surprising, but full of interest. He says "it is an animalcule so minute as to be with difficulty appreciable by the naked eye, inhabiting: a tube composed. of pellets, which it forms and lays one by one. It is a mason, who not only builds up his mansion brick by brick, but makes his bricks as he goes on, from substances which he collects around him, shaping them in a mould which he carries upon his body.

"The animal, as it slowly protrudes itself from its ingeniously-formed mansion, appears a complicated mass of transparent flesh, involved in many folds, displaying at one side a pair of hooked spines, and at the other two slender short blunt processes projecting horizontally. As it exposes itself more and more, suddenly two large rounded discs are expanded, around which, at the same instant, a wreath of cilia is seen performing its surprising motions. Often the animal contents itself with this degree of exposure; but sometimes it protrudes farther, and displays two other smaller leaflets opposite to the former, but in the same plane, margined with cilia in like manner. The appearance is 
not unlike that of a flower of four unequal petals, from which circumstance Linnæus gave it the name ringens, by which it is still known.

"Below the large petals on the ventral aspect, and just above the level of the projecting respiratory tubes, is a small circular disc or aperture, within the margin of which a rapid rotation goes on. This little organ, which seems to have hitherto escaped observation, he, Mr. Gosse, can compare to nothing so well as to one of those little circular ventilators which we sometimes see in one of the upper panes of a kitchen-window, running round and round, for the cure of smoky chimneys.

"The gizzard, or muscular bulb of the gullet, is always very distinct, and its structure is readily demonstrated. It consists of two sub-hemispherical portions, or jaws, each of which is crossed by three developed teeth, which are succeeded by three or four parallel lines, as if new teeth might grow from thence. The teeth are straight, slender, swelling towards their extremity, and pointed. These armed hemispheres work on each other, and on a V-shaped or tabuliform apparatus beneath, common to most of the Rotifera, but in this genus very small."

The pellets composing the case are very regular in form and posi.tion: in a fine specimen, about 1-28th of an inch in length when fully expanded, of which the tube was 1-36th of an inch, Mr. Gosse counted about fifteen longitudinal rows of pellets at one view, which might give about thirty-two or thirty-four rows in all.

In November 1850, Mr. Gosse found a fine specimen attached to a submerged moss from a pond at Hackney; this he saw engaged in building its case, and at the same time discovered the use of the curious little rotatory organ on the neck. "When fully expanded, the head is bent back at nearly a right angle to the body, so that the disc is placed nearly perpendicularly, instead of horizontally; the larger petals, which are the frontal ones, being above the smaller pair. Now, below the large petals (that is, on the ventral side) there is a projecting angular chin, which is ciliated; and immediately below this is the little organ in question. It appears to form a small hemispherical cup, and is capable of some degree of projection, as if ou a short pedicle. On mixing carmine with the water, the course of the ciliary current is readily traced, and forms a fine spectacle. The particles are hurled round the margin of the disc, until they pass off in front through the great sinus, between the larger petals. If the pigment be abundant, the cloudy torrent for the most part rushes off, and prevents our seeing what takes place; but if the atoms be few, we see them swiftly glide along the facial surface, following the irregularities of outline with beautiful pre- 
cision, dash round the projecting chin like a fleet of boats doubling a bold headland, and lodge themselves one after another in the little cuplike receptacle beneath. Mr. Gosse, believing that the pellets of the case might be prepared in the cup-like receptacle, watched the animal; and presently had the satisfaction of seeing it bend its head forward as anticipated, and after a second or two raise it again, the little cup having in the meantime lost its contents. It immediately began to fill again; and when it was full, and the contents were consolidated by rotation, aided probably by the admixture of a salivary secretion, it was again bent down to the margin of the case, and emptied of its pellet. This process he saw repeated many times in succession, until a goodly array of dark-red pellets were laid upon the yellowish-brown ones, but very irregularly. After a ccrtain number were deposited in one part, the animal would suddenly turn itself round in its case, and deposit some in another part. It took from two and a half to three and a half minutes to make and deposit a pellet. Some atoms of the floating carmine now and then passed down the gullet into the gizzard, and thence into the stomach; but these were quite independent of, and unconnected with, the pellets, which were composed exclusively out of the torrent that had passed off the disc. On one occasion the cup was brought down to the margin, but, from some cause or other, failed to deposit its pellet; it was raised for a moment, and then a second attempt was made, which was successful."

Such is the vivid picture and perfect description of the wondrous mechanism and ingenuity of this singular and beautiful animal.

We have taken a rapid survey of some of the marvellous creations in the busy invisible world ; every glimpse inspiring awe, from the immensity, variety, beauty, and minuteness of the organised habitants.

Immensity, in its common impression on the mind, hardly conveys the idea of the myriads upon myriads of Infusoria that have lived and died to produce the tripoli, the opal, the flints, the bog-iron, ochre, and the limestone of the world.

Man even uses their remains as food; for the berg-mehl, or mountain-meal (represented in fig. 85, No. 8), found in Swedish Lapland, and which, in periods of scarcity, the poor are driven to mix with their flour, is principally composed of the flinty shells of the Gallionella sulcata, Navicula viridis, and Gomphonema gemminatum. Dr. Trail, on analysing it, found it to consist of 22 per cent of organic matter, 72 of silica, 6 of alumina, and 0.15 of oxide of iron. This would seem to be the same substance described by M. Laribe the missionary, and put to a similar use in China: "This earth," he says, " is only used in seasons of 
great dearth. One of our Christians, who, at the period of the last famine, fed upon this substance, with five other individuals composing his family, informed me, when they made use of it, they bruised it into a very fine powder, mixing three parts with two of rice-powder, or, better, the flour of wheat, to make small cakes, which were seasoned with salt or sugar. Recourse was only had to this in times of great want; and this being over, no one ever dreamt of making use of it as an article of food. Those accustomed to partake of it complained of a weight at the stomach and constipation. They could subsist for two months on this material formed into cakes; whilst without its assistance, their provisions would only suffice as food for one month. Those persons who employed the fossil-flour without mixing it with vegetable meal, scarcely ever escaped disease."

Professor Owen beautifully explains the uses of this vast amount of animalculæ life :

"Consider their incredible numbers, their universal distribution, their insatiable voracity; and that it is the particles of decaying vegetable and animal bodies which they are appointed to devour and assimilate. Surely we must, in some degree, be indebted to these everactive, invisible scavengers, for the salubrity of the atmosphere and the purity of water. Nor is this all; they perform a still more important office in preventing the gradual diminution of the present amount of organised matter upon the earth. For when this matter is dissolved or suspended in water, in that state of comminution and decay which immediately precedes its final decomposition into the elementary gases, and its consequent return from the organic to the inorganic world; these wakeful members of nature's invisible police are every where ready to arrest the fugitive organised particles, and turn them back into the ascending stream of animal life. Having converted the dead and decomposing particles into their own living tissues, they themselves become the food of larger Infusoria, and of numerous other small animals, which in their turn are devoured by larger animals; and thus a food, fit for the nourishnent of the highest organised beings, is brought back, by a short route, from the extremity of the realms of organised matter. These invisible animalculæ may be compared, in the great organic world, to the minute capillaries in the microcosm of the animal body; receiving organic matter in its state of minutest subdivision, and when in full career to escape from the organic system, and turning it back, by a new route, towards the central and highest point of that system."

Such, then, seem to be some of the purposes for which are created 


\section{PLATE III.}

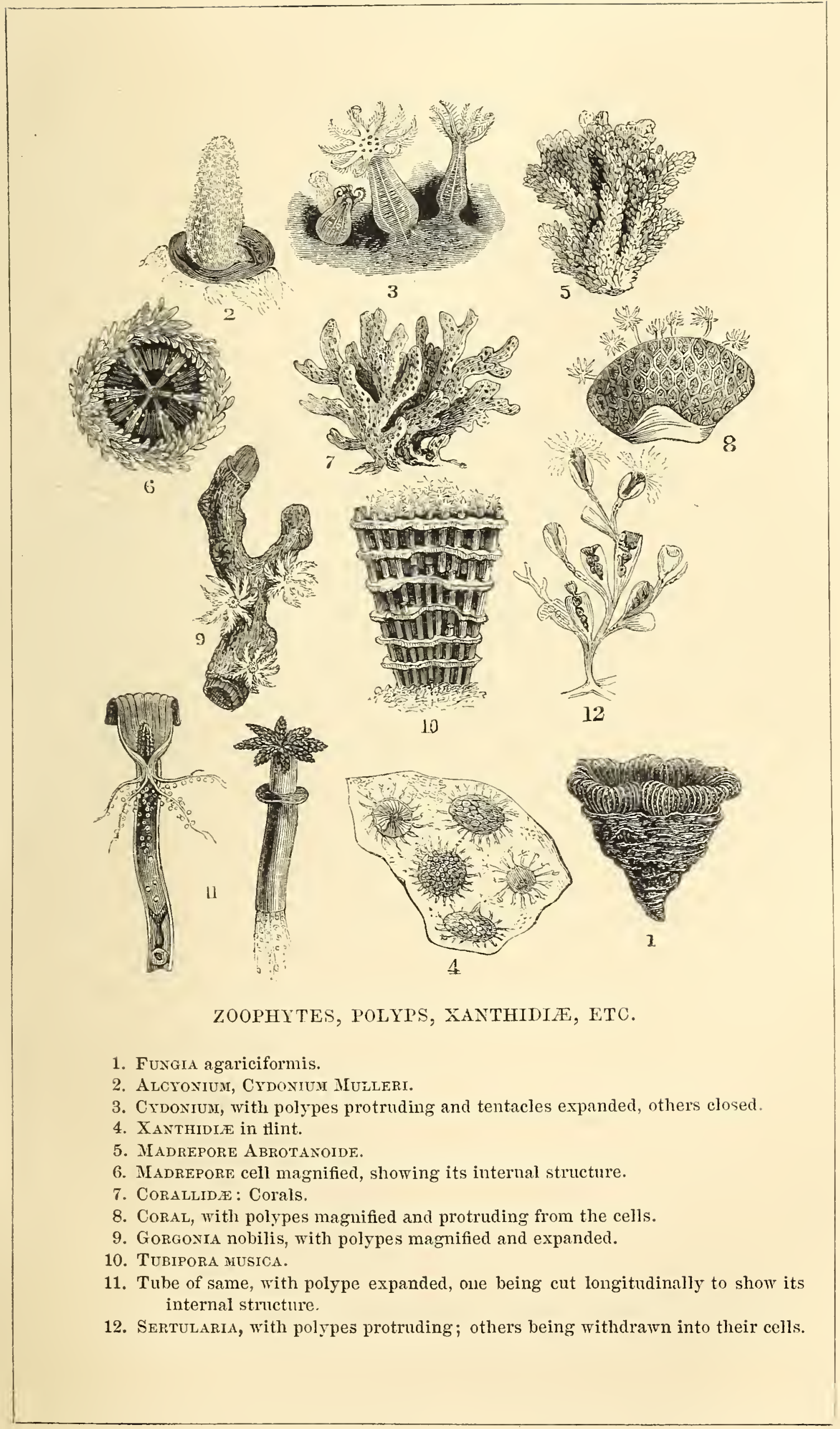



the wonderful invisible myriads of infusorial animalculæ. In words of Holy Writ: "All these things live and remain for ever for all uses; and they are all obedient. All things are double one against another; and He hath made nothing imperfect. One thing establisheth the good of another; and who shall be filled with beholding His glory?"

\section{POLYPIFERA.}

\section{ZUOPHYTES, OR POLYPES.}

Br the term Zoophytes, taken in its strictly limited sense, is understood a class of creatures which, in their form, or most remarkable characters, recall the appearance of a vegetable, or its leading properties. Others, more especially foreign naturalists, give a much wider range to the term, and include in it all animals whose structure emanates from a common centre, and possesses a circular arrangement of its radiating parts. They also include in it sponges and corallines, which other naturalists positively refuse so to class, and insist upon a new mode of classification. The most obvious character common to this vast race of animals is, that their mouths are surrounded by radiating tentacula arranged somewhat like the ray of a flower; and hence the term Zoophyte. So plant-like, indeed, are their forms, that the ancients regarded them as vegetating stomes, and invented many theories to explain their growth.

The mass of scattered information that the investigators of this branch of natural history had published has been collected and arranged, with refreshing enthusiasm, by Dr. George Johnston, of Berwick-uponTweed, under the title of A History of the British Zoophytes; in which work the ardent naturalist has not only combined "the whole under a system more in harmony with the anatomy of the objects than has hitherto been done," but enriched it by many personal researches and observations on the species found in his own neighbourhood. Of the Polypes, Dr. Johnston gives the following definition: "Animals avertebrate, inarticulate, soft, irritable, and contractile, without a vascular or separate respiratory or nervous system ; mouth superior, central, circular, edentulous, surrounded by tubular, or more commonly by filiform tentacula; alimentary canal variable; where there is an intestine, the 
anus opens near the mouth; asexual; gemmiparous; aquatic. The individuals (Polypes) of a few families are separate and perfect in themselves; but the greater number of Zoophytes are compound beings, viz. each Zoophyte consists of an indefinite number of individuals or polypes, organically connected and placed in a calcareous, horny, or membranous case or cells, forming, by their aggregation, corals, or plant-like Polypidoms *** which is the house or support of the polypes, and which, though commonly present, is yet not necessary to the existence of a Zoophyte."

Opposed to all our common ideas of animal life is this singular creation. If we cut a limb off a tree, or sever that of an animal, these parts will wither and decompose, by passing into other forms of matter. Cut a tree across its middle, and its natural symmetry is irreparably disfigured; slit it down its centre, and it is destroyed : all animals so treated suffer instant death, with the sole exception of the polype; for it will put forth new limbs, form a new head or tail; and if slit, become two separate existences.

THE HYDRA, OR FRESH-WATER POLYPE. Fig. 87. Nos. 1, 2, 3.

It received its name from its several long arms being supposed to resemble the fifty-headed water-serpent called Hydra, and destroyed by Hercules in the lake of Lerna, as we are informed in fabulous history.

Leeuwenhock, in 1703, was the first to draw attention to the hydra; and in 1739, M. Trembley, from the Hague, most accurately described the habits of the animal.

In ponds and rivulets, adhering to the leaves of aquatic plants, or twigs and sticks that have fallen into the water, are found the Hydræ. When stretched out, they resemble pieces of hair, from a quarter to three quarters of an inch in length. Some are of a light-green colour, and others brown or yellow; that is, the five varieties found in England. The substance of their body is jelly-like, and tapers in form from the head to the tail. When they contract themselves, they appear as a little round ball of jelly, about the tenth of an inch in diameter, studded with little teats, which are the ends of the contracted long arms that surround the mouth, and are protruded to catch prey. These arms vary in number from six to twelve, and are slender tubes, filled with a fluid capable of being stretched out to a considerable length; and another extraordinary circumstance is, that a polype can extend an arm in any part of its whole length, without doing so throughout; and can swell or lessen its diameter either at the root, at the extremity, 
in the middle, or where it pleases; which occasions a great variety of appearances, making it sometimes terminate with a sharp point, and at other times blunt, knobbed, and thickest at the end.

The extraordinary attenuation of the arms, until they can with difficulty be detected, renders it surprising that they possess strength sufficient to cope with the struggles of large worms and insects, round which the hydra wraps them; but, as the victim seems powerless when once in the fatal coil, it has been thought they possess some electric power similar to the eel and torpedo, that stun or destroy their prey by electrical force; and this belief is more especially entertained, as the little creature rests, like the above-mentioned fish, before regaining power to resume its pursuit of prey.

The polypes are not vegetarians, and were fed by M. Trembley on minced fish, beef, mutton, and veal; they are voracious and active in seizing worms and larvæ much larger than themselves, which they devour with avidity. They carefully and adroitly bring their food towards their mouth; and when near, pounce upon it with eagerness. To make up for the want of teeth, the mouth enlarges to receive the food brought to it by the arms that have twined round the sacrifice. The red worm that tinges the mud of the Thames appears to be the dainty dish they like best to have set before them. Dr. Mantell saw the lasso of a polype thrown over two worms at the same time; yet they could not escape, and lost all power of motion.

"It is a fine entertainment," says Baker, "to behold the dexterity of a polype in the mastering its prey, and observe with what art it evades and overcomes the superior strength or agility thereof. Many times, by way of experiment, I have put a large worm to the very extremity of a single arm, which has instantly fastened on it with its invisible claspers. Then it has afforded me inexpressible pleasure to see the polype poising and balancing the worm, with no less seeming caution and judgment than a skilful angler shows when he perceives a heavy fish at the end of a single hair-line, and fears it should break away. Contracting the arm that holds it, by very slow degrees, he brings it within the reach of his other arms, which eagerly clasping. round it, and the danger of losing it being over, all the former caution and gentleness are laid aside, and it is pulled to the polype's mouth with a surprising violence."

Dr. Johnston writes: "Sometimes it happens that two polypes will seize upon the same worm, when a struggle for the prey ensues, in which the strongest gains, of course, the victory; or each polype begins quietly to swallow his portion, and continues to gulp down his half, 
until the mouths of the pair near, and come at length into actual contact. The rest that now ensues, appears to prove that they are sensible of their untoward position, from which they are frequently liberated by the opportune break of the worm, when each obtains his share; but should the prey prove too tough, woe to the unready! the more resolute dilates the mouth to the requisite extent, and deliberately swallows his opponent: sometimes partially, so as, however, to compel the discharge of the bait; while at other times the entire polype is engulfed! But a polype is no fitting food for a polype, and his capacity of endurance saves him from this living tomb; for, after a time, when the worm is sucked out of him, the sufferer is disgorged with no other loss than his dinner."

This fact is the more remarkable, when it is contrasted with the fate which awaits the worms on which they feed. No sooner are these laid hold upon than they evince every symptom of painful suffering; but their violent contortions are momentary, and a certain death suddenly follows their sapture. How this effect is produced, is mere mat-' ter of conjecture. Worms, in ordinary circumstances, are most tenacious of life, even under severe wounds; and hence one is inclined to suppose that there must be something eminently poisonous in the Hydra's grasp; as it is impossible to believe, with Baker, that this soft, toothless creature can bite and inject a venom into the wound it gives. " I have sometimes," says Baker, "forced a worm from a polype the instant it has been bitten (at the expense of breaking off the polype's arms), and have always observed it to die very soon afterwards, without one single instance of recovery." Trembley states as a fact, that fishes cannot be made to swallow hydræ, seeming to prove the presence of some irritating quality in the latter.

The body of the polype is an assemblage of cells, consisting of green and brown matter, held together by a membranous substance. The stomach occupies the entire inside, which absorbs the nutritious portion of the food, as seen by the cells becoming of the colour of the thing eaten; the body then contracts, and the grosser parts of the food, after some hours' digestion, are again ejected by the mouth.

The polype increases rapidly: a portion of the body swells, a young one puts forth its head from the part, its arms begin to grow, it then is industrious in catching food, its body communicating with that of its parent, and participating in the fears and actions of its progenitor; finally, it is cast off to wander the world of waters. Betimes, ere yet free from parental attachment, it has two generations on its own body. Four or five offspring are thus produced weekly. But the most ex- 
traordinary circumstance in respect to this creature is thus described by M. Trembley: "If one of them be cut in two, the fore part, which contains the head and mouth and arms, lengthens itself, creeps, and eats on the same day. The tail part forms a head and mouth at the wounded end, and shoots forth arms more or less speedily, as the heat is favourable. If the polype be cut the long way through the head, stomach, and body, each part is half a pipe, with half a head, half a mouth, and some of the arms at one of its ends. The edges of these half pipes gradually round themselves and unite, beginning at the tail end; and the half mouth and half stomach of each becomes complete. A polype has been cut lengthways at seven in the morning, and in eight hours afterwards each part has devoured a worm as long as itself."

Still equally wonderful is the fact, that if turned inside out, the parts at once accommodate themselves to their new condition, and carry on all their functions as before the accident. Indeed, this animal seems so peculiarly endowed with the germs of vitality in every part of its body, that it may be cut into ten pieces, and every one will become a new, perfect, living animal. This seems bordering on the vegetable kingdom, in which it is comnoon to propagate by means of slips from the mature shrub.

The polype affices itself by its tail to various substances, and frequently is a parasite to other animals, which it annoys, while benefited itself by taking advantage of their locomotive powers: this is more especially the case with the water-snail, on the body of which may generally be found the polype.

Dr. Roget, in his Bridgewater Treatise, copying Trembley, says: "The position in which they appear to take inost delight is that of remaining suspended from the surface of the water by means of the foot alone; and this they effect in the following manner. When the flat surface of the foot is exposed for a short time to the air, above the surface of the water, it becomes dry, and in this state exerts a repulsive action on the liquid; so that when dragged below the level of the surface, by the weight of the body, it still remains uncovered, and occupies the bottom of the cup-shaped hollow in the fluid, thereby receiving a degree of buoyancy sufficient to suspend it at the surface. The principle is the same as that by which a dry needle is supported on water, in the boat-like hollow which is formed by the cohesive force of the liquid, if care be taken to lay the needle down very gently on the surface. If, while the hydra is floating in this manner, suspended by the extremity of the foot, a drop of water be made to fall upon that part, so as to wet 
it, this hydrostatic power will be destroyed, and the animal will immediately sink to the bottom."

Hydra Vulgaris, or Common Polype (No. 3, fig. 87), is often found upon plants, branches of trees, pieces of wood, rotten leaves, stones, and other substances in the water. They are of an orange-brown or oilgreen colour; and round in shape. The number of its arms, or as they are technically called, tentacula, vary, and are usually a little longer than the outstretched body of the animal itself. On moving from place to place, they dispose themselves in an arched position, grasping some object with their arms; they then draw the tail towards the head, fix it, throw their head out again, and seizing some other substance, thus proceed onwards. A young one is seen sprouting from the body of this Hydra.

Hydra Viridis, or Green Polype (No. 2, fig. 87), differs little from the common polype, except as regards its beautiful light-green colour, and having shorter arms.

Hydra Fusca, Brown or Long-Armed Polype.-This is, like other polypes, composed of a jelly-like matter, formed into cells, which perform certain functions, with a large mouth and a sucker at the opposite extremity; the difference between it and the others named consists in the length of its arms, which will elongate several inches (see No. 1). In the extraordinary foresight of nature, it is provided that during the cold weather the polype shall continue its propagation not by budding, but by eggs; the latter being better calculated to protect the tender thread of life within against the destructive frosts of winter, than could possibly be resisted by the delicate construction of a new-born fragile creature thrust into its nipping influence.

There are other British kinds: the Hydra Verrucosa, which is of a pale ashy colour, with six moderately long arms ; and the Hydra Lutea, a marine species, with a large head and ten very short arms.

Every reflecting person who reads even the slight sketch we have given of this polype tribe must be struck with astonishment at a creature. so primitive in structure, possessing the actions, sensations, and powers of higher organised beings. The stomach is but one simple structureless membrane, or cell, the external surface-cells condensed so as to form a kind of double skin; and the inside a mere wall of cells running crosswise, possessed of a velvet-like surface, the substance being red or brown grains held together by a sort of gluey substance.

This singular formation for the functions of animal life has led to many learned surmises and discussions tending to the most important results in the science of physiology. 


\section{MARINE ANIJIAL-PLANTS.}

It is now a century since the industrious Ellis, when forming a collection of sea-plants at Brighton, discovered from the cells of many of the corallines, the protrusion of arms or feelers, apparently engaged in seizing prey and drawing it to the interior of its home. This led to the discovery of their animal nature, and subsequent arrangement into classes and families: those more generally recognised are the Alcyonidæ, the Corallidæ, the Madreporidæ, the Madrephyllidæ, the Zoanthidæ, the Actiniadæ, the Pennatulidæ, the Tubularidæ, the Tubiporidæ, the Sertularidæ, the Bryozoæ, \&c.* (Plate I.)

We have already described the lowest order of polypes, so ranked from their simple organisation, and called Polypiaria Nuda, or Naked Polypes; they are classed as Hydrozoa, or hydra-like animals.

Rising in the scale of organic structure in the family of polypes and hydroida, next come the anthozoa or flower animals, from their similarity to little bushes, trees, and stems of flowers, bearing the delicate and tinted animals like the blossoms that bedeck and render lovely the gardens of the earth. Those that flourish within the tropical seas, by their rapid formation of habitations on habitations, named polypidom or corallium, create the dangerous coral reefs, and ultimately the lovely islands of the vast and bright Pacific Ocean.

\section{ALCYONID必.}

The family of the Alcyoium derives its name from Alcyone or Halcyone, the daughter of Neptune and wife of Ceyx, who, hearing of her husband's death at sea, cast herself into it; and was, with her husband, changed into birds of the same name, to keep the waters calm while they sit in their nests of sea-foam for the space of seven, eleven, or

\section{* Description of Plate I. (Frontispiece.)}

1. Bryozoa Bowerbankia, or Bowerbank polype, showing its internal structure: near it is a similar animal completely withdrawn into its cell. 2. Eschara cerricornis, or Sea-moss polype: the animal is seen drawn out from its shell or chalky covering. 3. Plumularia pinnata, or Feather polype. 4. Campanularia rolubilis, or Twining polype. These Polypes are all represented magnified. 5. Actinia rubra, or Seamarigold, near which is one shown retracted into its cell. 6. Actinia Bellis, or Daisy sea-anemone; a side view of the animal. 7. The same animal seen with its crown of tentacles fully expanded. 8. Doris tuberculata. 9. Laniogerus Elfortii. 10. Aplysia; all species of water-snails, the last without a shell covering. Fronds of Algæ are represented growing amongst the rocks. In the title-page many other specimens of Marine Polypes, Actiniæ, \&c. are represented. 
fourteen days. Thus Alcyon signifies kingfisher, $n$ s sea-foam. The term Lobularia is sometimes applied to this family, but generally rejected, from its being a botanical appellation.

Alcyonium Digitatum, which signifies having fingers, toes, or claws, is one of the commonest polypes (PI. III. No. 2) found in the sea, being almost always attached to every stone or shell brought up from the bottom of the ocean. Sometimes it is very small; but when larger is named by the fishermen Cow's-paps, and others, differing a little in form, Dead Men's Toes, or Dead Men's Hands. In the description of Asteroida will be found an account of its internal structure. The spicula is calcareous or silicious crystalline, in the form of a cross toothed at the sides; and lies scattered through the jelly-like mass, there being no solid axis.

"The ova," says Professor Grant, "when placed under the microscope, and viewed by transmitted light, appeared as opaque spheres surrounded with a thin transparent margin, which increased in thickness when the ova began to grow, and such of the ova as lay in contact united and grew as one ovum. A rapid current in the water immediately around each ovum, drawing along with each all the loose particles and floating animalculæ, was distinctly seen moving with an equal velocity as in other ciliated ova; and a zone of very minute vibrating cilia was perceptible, surrounding the transparent margin of all the ova. The progressive motion of the ova, always in a direction contrary to that of the current created by their cilia, was very obvious, though less rapid than in any other zoophyte in which I have observed the same remarkable phenomenon. The specimen, suspended in a glass jar filled with pure sea-water, I now brought so close to the transparent side of the vessel, that I could examine through it, with the assistance of a powerful lens, and without disturbing the animal, the motions and progress of the groups of ova passing through the colourless bodies of the polypi. To the naked eye, at first sight, all appeared motionless. The deep vermilion hue of the small round ova, and the colourless transparency of the outer covering of the polypi, formed a beautiful contrast with the pure white colour of the delicate longitudinal folds, the central open canal, and the slender filaments whieh wind down from its sides towards the clusters of white ova at the base: but the living phenomena discovered within were even more admirable than the beautiful contrast of colours, the elegant forms, and the exquisite structure of all the parts; when observed with a lens, the ova were seen to be in constant motion, and quite free within the bodies of the polypi. They moved themselves backwards and forwards, and frequently contracted their sides, as if irritated or capable of feeling. I could observe none 
passing upwards between the stomach and the sides of the polypi. They never assumed the appearance of a string of beads inclosed in a narrow, short, curved tube, as represented by Spix, but swam freely in the water which distended the polype, as figured by Ellis. Their motions in the polypi, though circumscribed, were so incessant, that, by watching attentively, I could observe them with the naked eye; and they became more conspicuous as the ora advanced to the open base of the stomach. From their restlessness, as they approached the last passage which separates them from the sea, they seemed to feel the impulse of a new element, which they were impatient to enjoy; and by following the direction of that impulse, they appeared to find their way into the lower extremity of the stomach, without any organic arrangement to lead them into that narrow canal. In their passage through the stomach, which was effected very slowly, the spontaneous motions of the ova were arrested, unless some imperceptible action of their cilia, or some contraction of their surface, might tend to irritate the sides of that canal, and thus direct or hasten their escape."

Alcyonidium Gelatinosum.-Attached to old stones and shells is this jelly-like transparent spongy zoophyte, growing to a height of nearly a foot, and sometimes much longer. It is branched, and of a brown or yellow colour, dotted with polypes, which are attached to the cells. Through angular openings they protrude their arms or feelers. Dr. Farre states, "the tentacula are sixteen in number (occasionally fifteen); fully two-thirds the length of the body of the animal, and extremely slender and flexible. When expanded, they are frequently seen to roll up closely upon themselres, even down to their base, the revolution taking place either inwardly or outwardly, and in one or more arms at the same time. Their full expansion affords a more perfect companulate form than is usually met with in this class, each of the arms having a sligbt curve outwards towards its extremity, which gives to the whole a very elegant appearance. It is remarkable that in some specimens the arms are much shorter on one side of the body than on the other."

A very singular organ was frequently observed, consisting of a little flask-shaped body situated between the base of two of the arms, and attached to the tentacula-ring by a short peduncle. The carity of its interior is lined with cilia, which vibrate downwards towards the outer, and upwards towards the inner side; it has a narrow neck and a wide mouth, around which a row of delicate cilia are constantly playing. No flow of fluids could ever be detected through it, nor did the use of carmine assist in showing with what parts the cavity in its in- 
terior might communicate. From the circumstance that it is more frequently absent than present, it cannot be an organ of vital importance to the animal; and it is too intimately blended with the sides of the tentacula, and too constant in its position, to be regarded as a parasite.

Alcyonidium Echinatum.-This parasite "incrusts dead univalve shells exclusively, and is about the one-twentieth of an inch in thickness. When first taken out of the water it is soft and spongy, but becomes rigid on drying." They have sharp-pointed spinous little nipple-like protuberances.

The whole family of Alcyonium, or Alcyonidium, have the distinguishing characteristic of lobed, or crustaceous fleshy polypidoms; the cells being imbedded of a pentagonal shape, with horny-fibrous sides, the aperture is terminal and contractile. This portion of the cell, or that which forms the base of the polype, Dr. Farre states to be composed of a series of stout short bristles.

ASTEROIDA.

The next order of Zoophytes is named Asteroida, from the polypes presenting the form of a star, on the surface of the fleshy mass in which they reside. (In title-page various species are distributed over the rockwork at the base.) Their organisation is superior to those previously described; and there exists this vast difference, that instead of the animal domiciling in a hard cell, they exist in a fleshy tough crust, which is supported by hard calcareous spicula, and others with thick branching processes, performing the part of the skeleton in the human frame. This central internal support is usually denominated the axis. The fleshy mass or covering is possessed of sensation, and ramified by various tubes and canals for the sustenance and other functions of life of the polype.

When the polype is seen to issue from its cell, it has a longish round appearance, and eight short feelers or arms, hollow and toothed like a comb. The stomach is situated centrically, supported by eight membranes, that divide the space between the outer surface of the stomach and the inner skin into eight parts. At the lower part of the stomach is another opening similar to the animal's mouth, from which suspend into a cavity below the stomach eight slender threads, either loose or attached to a continuation of the thin membraneous substance that sustains the stomach. From the cavity is a canal that enters the large fleshy mass, and branches into other polype canals, and minute 
ressels, that, like beautiful net-work, fill up the intervening parts, and closely envelope the bone or solid parts: thus each polype communicates with the entire family, and the water swallowed by one passes through the whole. On the animal drinking water, part of it passes into the eight compartments in connection with the stomach, which fluid rising upwards, enters and fills the hollow arms or feelers and cilia; while another portion, flowing into the connecting canals, the entire mass becomes double its former size; and thus, at pleasure, the animal either distends or contracts its body, as it has the power of ejecting or imbibing the fluid. When thrown on the sea-shore, and dried by the sun, the skeleton weighs but a few grains. From this it would appear that the whole is but an animal having numerous mouths, stomachs, and other organs. The position of the animals is seen by the surface of the mass being marked by the semblance of stars, which open out on the animals protruding themselves. When stretched out of the cell, the polype is a delicate transparent cylinder; the outer covering thickens towards its base, which, joining with that of the neighbouring inhabitant, forms the party-wall that constitutes each individual cell. In the greater part this last-named division secretes the carbonate of lime that forms the skeleton frame we have just noticed. In the different families of this order, the parts where the young are generated appear to vary. The eggrs of one kind first appear like a minute smooth wart, which gradually rises up from the surface.

\section{CORALlide. (Plate III. Nos. 7, 8, 9.)}

The family of Zoophytes necessarily embraces many corals; they are as interesting as they are important to man, being, during the span of their brief existence, the original founders of many countries, which, nature clothing with luxuriant verdure, the human family reap the fruit, and enjoy the abundance. As we glance at the map of the world, and think of the profusion of fragrant regetation and delicious food almost spontaneously produced on the lovely sunny islands of the broad Pacific, how startling does it seem when we reflect, that these islands, bearing on their bosoms gardens of Eden, are entirely formed by the cells of deceased polypi, which, rising up in beautiful and delicate forms, displace the mighty ocean, defy its gigantic strength, and display a shelly bosom to the expanse of day! The regetation of the sea, cast on its surface, undergoes a chemical change; the deposit from rains aids in filling up the little gaping catacomb, the fowls of the air and the ocean find a resting-place, and assist in clothing the rocks; mosses carpet the 
surface, seed brought by birds, plants carried by the oceanic currents, animalculæ floating in the atmosphere, live, propagate, and die, and are succeeded, by the assistance their remains bestow, by more advanced vegetable and animal life; and thus generation after generation exist and perish, until at length the coral island becomes a paradise filled with the choicest exotics, the most beautiful birds and delicious fruits, among which man may indolently revel to the utmost desire of his heart.

The species best known from its skeleton is Corallium rubrum, or Red Coral. It is the coral of commerce, and is an inhabitant of the Mediterranean and adjacent seas. In appearance it resembles a tree devoid of leaves and small branches. It requires about ten years to arrive at maturity, and is then about a foot in length. Its skeleton or axis is the polished brilliant red stone used in ornamentations. It is solid, channelled, ramified, and fixed by a broad base, with a thin external, fleshy covering, of a pale blue colour, and studded over with starlike polypi, that extend their feelers to grasp at prey. When brought into the air, the pulpy body of the animal soon decays, when its remains are prepared for market, and the neck or arm of matchless beauty is endeavoured to be rendered more attractive by being adorned with the pretty skeleton forms of the dead animal.

The composition of both the Madrepores' and Corals' internal skeletons consists of masses of carbonate of lime, with a little of the phosphate. When a section of the Corallium rubrum is examined, it is seen to be a series of undulating layers of red-coloured calcareous matter, devoid of cellular structure, and rather of a crystalline nature.

Classed in the Tubiferæa is that handsome coral, Sarcinula musicalis, or Organ-pipe Coral. The coral is composed of parallel tubes, united by lateral plates, or transverse partitions, placed at regular distances; in this manner large masses, consisting of a congeries of pipes or tubes, are formed. When the animalculæ are alive, each tube contains a polype of a beautiful bright green colour, and the upper part of the surface is covered with a gelatinous mass, formed by the confluence of the polypes. This species occurs in great abundance on the coasts of New South Wales, of the Red Sea, and of the Molucca Islands, varying in colour from a bright red to a deep orange. It grows in the shape of large hemispherical masses, and from one to two feet in circumference; these first appear as small specks adhering to a shell or rock; as they increase, the tubes resemble a group of diverging rays, and at length the other tubes are produced on the transverse plates; thus filling up the intervals, and constituting a uniform tubular mass; the surface being 
covered with a green fleshy substance, beset with stellular animalculæ.

When a section is made of the Myriapora, Nulliporc Alciformis, it is seen to have a reticulated structure, the diameter of each cell of which is about the 1300th of an inch. A beautiful representation is seen in fig. 92, No. 1.

The axis of the Virgularia Mirabilis is a brittle calcareous matter, having a series of minute wavy canals in its interior, open on the free surface, and passing in curved lines towards the centre of the stem (fig. 92, No. 2). Allied to the Turbinolia and Caryophyllia are the Fungia, or Sea Ilushrooms (Plate III. No. 1). These elegant forms are found in a great variety; the corals are white, of a flattened round shape, made up of thin plates or scales, around which is a translucent jelly-like substance, and amidst it a large polype; for, unlike others, they exist as individuals: the lower part is of a stony nature, by which the animal is affixed to the rock whereon it lives.

In Ellis's Zoophytes is the following passage, quoted from Rumphius, in regard to the Fungia agariciformis: "The more elevated folds or plaits have borders like the denticulated edge of needleworklace. These are covered with innumerable oblong vesicles, formed of a gelatinous substance, which appear alive under water, and may be observed to move like an insect. I have observed these radiating folds of the animal, which secrete the lamellæ, and which shrink between them when the animal contracts itself on being disturbed. They are constantly moving in tremulous undulations; but the vesicles appeared to me to be air-vessels placed along the edges of the folds, and the vesicles disappeared when the animal was touched."

Pavonic are a large and powerful class of polypes, living in deep and separate cells. A transrerse section of the bony axis or skeleton of Pavonaria quadrangularis is found to be of a square figure with concave margins, and consists of a series of laminæ of fibro-calcareous matter, arranged around a central cross ; the outer portion or crust is so extremely hard, that it can scarcely be cut with a knife. If the calcareous matter be removed, a distinctly fibrous structure remains.

In the British Museum there is a splendid specimen of the Brainstone Coral, or Meandrina cerebriformis, so named by the appearance of its surface resembling the convolutions of the medular matter of the human brain. In a living state the mass is invested with a fleshy substance, variously coloured, and having numerous short, conical polypiform, confluent cells, arranged in rows between the ridges. It attaches itself by a strong stony secretion to rocks; and as one genera- 
tion passes away on the shelly remains, another arises; and thus the imperishable charnel-houses are built upon and increased in magnitude.

Dr. Maccullock, in his Highlands and Western Islands, observes: "Their plants are made of stone, and they construct islands and continerts for the habitation of man. The labours of a worm, which man can barely see, form mountains like the Apennines, and regions to which Britain is as nothing. The invisible, insensible toil of an ephemeral point, conspiring with others in one great design, working unseen, unheard, but for ever guided by one volition,- - by that One Volition which cannot err,- - converts the liquid water into the solid rock, the deep ocean into dry land; and extends the dominions of man, who sees it not, and knows it not, over regions which even his ships had scarcely traversed. This is the Great Pacific Ocean, destined at some future day to be a world. That same Power, which has thus wrought, by means which blind man would have despised as inadequate, by means which he has just discovered, here too shows the versatility, the contrast of its resources. In one hour it lets loose the raging engines, not of its wrath, but of its benevolence; and the volcano and the earthquake lift up to the clouds, the prop and foundation of new worlds, that from those clouds they may draw down the sources of the river, the waters of fertility and plenty."

Ehrenberg; on beholding the coral-beds in the Red Sea, exclaimed: "Where is the paradise of flowers that can rival in variety and beauty these living wonders of the ocean?"

In the Narrative of the Surveying Voyage of H.M.S. Fly, J. B. Jukes, Esq., naturalist of the expedition, thus writes: "In a small bight of the inner edge of the coral-reef was a shattered nook, where the extreme slope was well exposed, and where every coral was in full life and luxuriance. Smooth round masses of meandrinæ and astreæ were contrasted with delicate, leaf-like, and cup-shaped expansions of explanariæ, and with an infinite variety of branching madreporæ, and some with mere finger-shaped projections; others with large branching stems; and others again exhibiting an elegant assemblage of interlacing twigs, of the most delicate and exquisite workmanship. Their colours were unrivalled: vivid greens, contrasting with more sober browns or yellows, mixed with rich shades of purple, from pale pink to deep blue. Bright red, yellow, and peach-coloured millepores clothed those fleshy masses that were dead, mingled with beautiful pearly flakes of escharæ and reteporæ; the latter looking like lace-work in ivory. Amidst the branches of the corals, like birds among trees, floated many beautiful fish, radiant with metallic greens or crimsons, or fantastically banded 
with black and yellow stripes. Patches of clear white sand were seen here and there on the floor, with dark hollows and recesses, beneath overhanging masses and ledges. All these, seen through the clear crystal water, the ripple of which gare motion and quick play of light and shadow to the whole, formed a scene of the rarest beauty; and left nothing to be desired by the eye, either in elegance of form or brilliancy and harmony of colouring."

Captain Basil Hall thus describes a coral-reef near Loo Choo: "When the tide has left the rock for some time dry, it appears to be a compact mass, exceedingly hard and rugged; but as the water rises, and the waves begin to wash over it, the polypes protrude themselves from holes which were before invisible. These animals are of a great variety of shapes and sizes, and in such prodigious numbers, that in a short time the whole surface of the rock appears to be alive and in motion. The most common form is that of a star, with arms, or tentacula, which are moved about with a rapid motion in all directions, probably to catch food. Others are so sluggish, that they may be mistaken for pieces of the rock, and are generally of a dark colour. When the coral is broken above high-water mark, it is a solid hard stone; but if any part of it be detached at a spot where the tide reaches every day, it is found to be full of polypes of different lengths and colours; some being as fine as a thread, of a bright yellow, and sometimes of a blue colour. The growth of coral appear's to cease when no longer exposed to the washing of the sea. Thus a reef rises in the form of a cauliflower, till the top has gained the level of the highest tides, above which the animalculæ have no power to adrance; and the reef, of course, no longer extends upwards."

Of the myriads upon myriads of organised beings created to work out the grand designs of Providence, all calculation seems futile; as the result would be beyond the grasp of our comprehension. The Polynesian Archipelago, now dubbed one of the great divisions of the globe, has its foundation formed of coral reefs, the spontaneous growth of onceliving zoophytes. Of the immense extent of the geographical changes effected by the tiny polypes, Dr. Mantell observes: "We may form some idea, from the facts stated by competent observers, that in the Indian Ocean, to the south-west of Malabar, there is a chain of reefs and islets 480 geographical miles in length; on the east coast of New Holland, an unbroken reef of 350 miles long; between that and New Guinea, a coral formation which extends upwards of 700 miles; and that Disappointment Islands and Duff's Group are connected by 600 miles of coral reefs, orer which the natives can travel from one island to another." 
Nothing can be more impressive than the manner in which these diminutive creatures carry out their stupendous undertakings, which we denominate instinct, intelligence, or design. Commencing betimes from a depth of a thousand or fifteen hundred feet, they work upwards in a perpendicular direction; and on arriving at the surface form a crescent, presenting the back of the arch in that direction from which the storms and winds generally proceed; by which means the wall protects the busy millions at work beneath and within. These coral breakwaters will resist more powerful seas than if formed of granite; rising as they do in a mighty expanse of water, exposed to the utmost powers of the heavy and tumultuous billows that eternally lash against them.

\section{GORGONIE.}

The family of the Gorgonia are named after the three celebrated sisters, daughters of Phorcus and Ceto, who turned to stone all on whom they fixed their eyes, and one of whom had her hair turned into serpents. They are of a large size, rising to a foot or more in height, and being from fifteen to sixteen inches in wiath (see Plate III. No. 9, and branches surrounding the title-page).

A widely diffused class are the Gorgoniæ in every sea; and though they naturally seem to dwell in deep water, yet when found in that more shallow, their colours are richer, deeper, and brighter. They are flexible, and seem like plants growing from the rocks to which they are fixed. Some are branching, covered with lace-like work; others like a feather or fan; whille sorne, again, are straight, and others of a drooping form. The stems flat, angular, or round, of a dark colour, with an outer crust of a soft substance full of pores, out of which the polypes thrust themselves. The flesh when dry is earthy and friable, a considerable proportion of carbonate of lime entering into its composition; but in a recent state it is soft and fleshy, and excavated with numerous cells for the lodgment of the polypes. When a portion of a branch is macerated in a weak acid, the lime is entirely removed; but the branch retains its original size and figure, and shows the framework to be an irregular close texture of corneous fibres, the interstices of which had been, probably, filled with a gelatinous fluid.

The Gorgonia Patula of Ellis and Solander is beautiful from its bright red colour ; on its opposite sides it has holes projecting forwards, through which the polypes protrude in search of food.

Gorgonia Flabellum, sometimes called the Sea-fan, Flabellum Veneris, 
or Venus's Fan, may often be seen of the height of fiye feet. It grows in the form of a net, with its branches compressed inwardly; the flesh is yellow, sometimes purple, with small mouths placed irregularly, having polypes with eight tentacles; the bone is black, horny, and slightly striated on the large branches. When alive, the colour is most beautiful, generally yellow with red spots, and is of a tough nature; but it varies much both in shape and colour, presenting some of the most delicate and graceful forms that can be conceived. Its elegant skeleton is generally seen decorating the houses of seafaring persons. Ray, referring to the fan-shape of some marine objects, says, "That the motion of the water descends to a good depth, I prove, from those plants that grow deepest in the sea, because they all generally grow flat, in the manner of a fan, and not with branches on all sides like trees; which is so contrived by the providence of nature, for that the edges of them do in that posture with most ease cut the water flowing to and fro; and should the flat side be objected to the stream, it would soon be turned edge-wise by the force of $i t$, because in that site it doth least resist the motion of the water; whereas, did the branches of these plants grow round, thesy would be thrown backward and forward every tide. Nay, not only the herbaceous and woody submarine plants, but also the lithophyta themselves affect this manner of growing, as I have observed in various kinds of corals."

In the British family there are also $G$. verncosa, $G$. placomus, $G$. anceps, G. lepadifera, G. umbraculum, G. nobilis.

The Isis belongs to this class of animals. They are small, but nunerously scattered in the soft fleshy integument. Isis Hippuris is the type by which this family is illustrated ; it has a jointed stony stem, which rises into many loose branches. The bone or support of the animal consists of white, cylindrical, stony channelled joints, conrected together by black, contracted, horny intermediate ones. The flesh is whitish, plump, and full of minute vessels; the surface of it is full of the little mouths of the cells, which are disposed in a quincunx order, corering the polypes with eight claws.

\section{CRISIAD压.}

The next family, signifying a separation, are generally parasitical. Crisia cormuta, or Goat's-horn Coralline of Ellis, have the cells linked in a single series, as well as C. chelata, or Bull's-horn Coralline; the latter look like a number of shoes that come close to the ancle, joined by the toe-part to the heel of others. Ellis says: "This beautiful coral- 
line is one of the smallest we meet with. It rises from tubuli growing upon fuci, and passes from thence into sickle-shaped branches, consisting of single rows of cells, looking when magnified like bull's horns inverted, each one arising out of the top of the other. The upper branches take their rise from the fore-part of the entrance of a cell, where we may observe a stiff, short hair, which seems to be the beginning of a branch. The opening of each cell, which is in the front of its upper part, is surrounded by a thin circular rim; and the substance of the cells appears to consist of a fine transparent shell or coral-like substance."

Crisia Eburnea, or I'ufted Ivory Coralline, attains the height of an inch, and displays its beautiful white, bushy tufts, with often a dash of light-red intermingled. Its " cells are loosely aggregated, cylindrical, bent and tubular orifices, free." While the Crisia aculeata has "cells closely aggregated, cylindrical, nearly straight, with long slender spines springing from the margin of every cell, giving it a delicate and pretty appearance."

Notamia, or Back-cell, are so named from the cells being exactly opposite, and united back to back with a thick partition, and having a joint above and below each pair. They are plant-like, and much branched.

Notamia Loriculata, or Coat-of-mail Coralline.-Ellis remarks, that the cells are so placed that the "pair together resemble a coat-of-mail or pair of stays; and the entrances of the cells look like the places for the arms to come out of. It grows from two to four inches long, is bushy, and the series of paired cells are divided by a joint. The polypes have ten arms or feelers, and are without gizzards, but supplied with all the necessary formation for the digestion of food.

In some species of the Flustrce the interior of the cell is protected by a lid which bears some appearance to the head and beak of a bird, and hence it is termed the bird's-head process. This has long been a subject of investigation by naturalists; but no definite discovery has yet been made, so as to explain the structure and functions of these mysterious organs. In the Transactions of the Microscopical Society for 1849 there is an admirable paper, by George Busk, Esq., F.R.S.,"* on the Notamia bursaria, or Shepherd's Purse Coralline (represented in fig. 89, Nos. 1 and 3), which adds to our knowledge of this process, and from which we quote: "This most beautiful pearl-coloured coralline adheres by small tubes to fuci, from whence it changes into flat cells; each single cell, like the bracket of a shelf, broad at top and nar-

* Mr. Busk has more recently and fully described the bird's-head process of this singular zoophyte in the Quarterly Journal of Microscopical Science for January 1854. 
row at bottom: these are placed back to back in pairs, one above another, on an extremely slender tube that seems to run through the middle of the branches of the whole coralline. The cells are open at top. Some of them have black spots in them; and from the top of many of them a figure seems to issue out like a short tobacco-pipe, the small end of which seems to be inserted in the tube that passes through the middle of the whole. The cells in pairs are thought by some to have the appearance of the small pods of the plant shepherd's purse, by others the shape of the seed-vessel of the veronica, or speedwell."

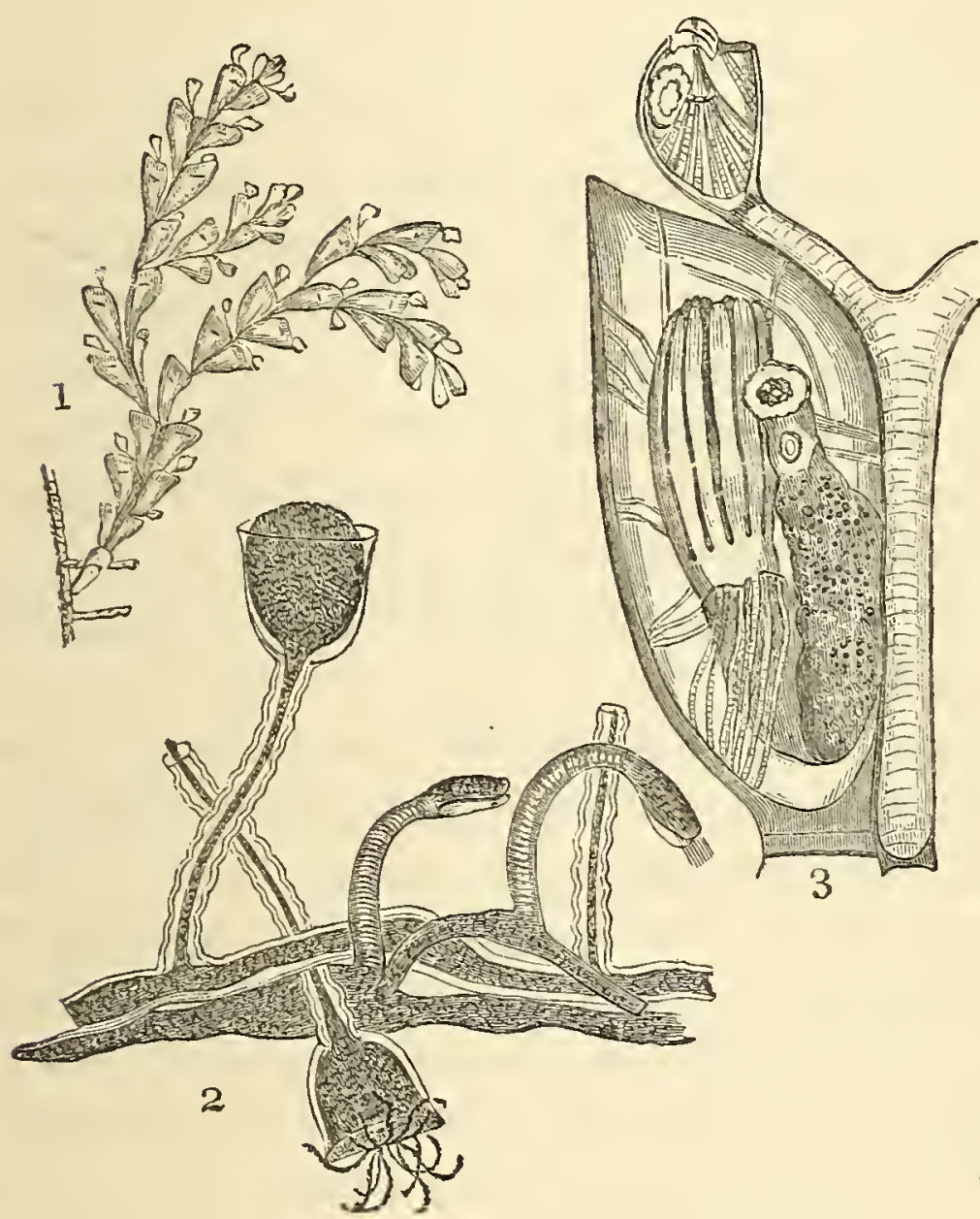

fig. 89 .

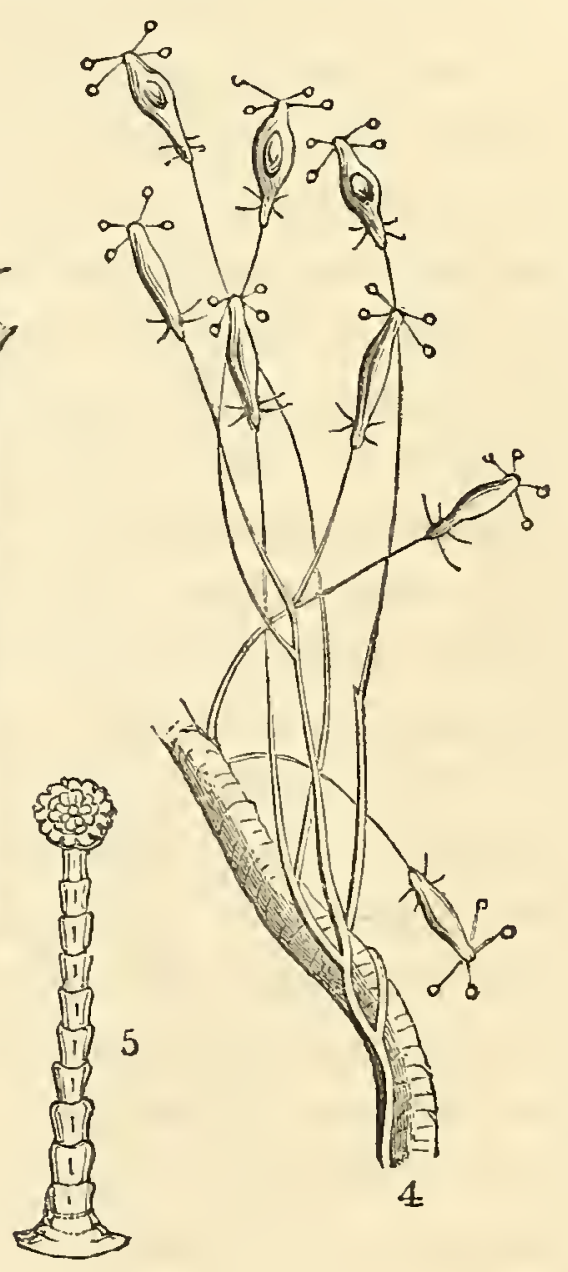

1. Notamia bursaria, or Shepherd's Purse Coralline. 2. Anguinaria spatulata, or
Snake Coralline, growing with the Campanularia integra. 3. The Shepherd's Purse Animalcule drawn into its cup, the internal organism of which is shown greatly magnified. 4. Coryne stauridia, or Slender Coryne. 5. One of the tubercles detached and magnified.

"The polypidom of this bryozoon, like those of most of its congeners, may be said to consist of a radical portion, by which it is affixed to the objects upon which it grows, and of a celliferous portion or branches, upon which the polypes themselves are lodged. The radical portion in the present species consists of a central discoid body of a nearly circular form, and of branches radiating from the periphery of 
the disc, which thence exhibits something of the aspect of the body of an ophiura. The radical tubes or branches, springing from the margin of the disc, are usually five or six in number, and they are given off at pretty regular distances apart; but besides these radical tubes, one or more celliferous branches are not unfrequently seen to arise immediately from the upper surface of the discoid portion.

"The central disc, and the radical tubes arising from it, exhibit a similar structure, and are formed of a thick, firm, apparently horny envelope, containing a coarse granular matter, of a yellowisl-white colour, and which in some portions of the tubes assumes the form of distinct irregularly-globular masses, of nearly uniform size. The central disc is subdivided into distinct compartments by septa of considerable thickness, and each radiating branch arises from one of these distinct compartments; so that there appears to be no communication between one radical branch and another. The radical branches give off at irregular distances secondary branches, which ultimately become celliferous. Each of these secondary branches, however, arises from a distinct compartment, as it were, of the tube from which it springs. This compartment is formed, like those of the central disc, by a thick septum, which shuts off the origin of the secondary branch from the main cavity of the primary one.

"The celliferous branches vary infinitely in number in different individuals, and differ also extremely in length, some being nearly an inch long; but in the majority of individuals they are about half an inch in length when laid straight. When the zoophyte is living or recent, and unaltered by the fluid in which it may have been placed, the celliferous branches are always much curled, reminding one in their habit, thence derived, of the vernation of the fronds of many ferns; and the whole zoophyte from this acquires a peculiar and very elegant aspect. The branches divide diclıotomously at tolerably regular intervals. Above almost every pair of polypiferous cells is to be noticed a pair of smaller cells, not unaptly compared by Eilis to the bowls of tobacco-pipes with short stems. The larger, or polypiferous cells, Mr. Busk proposes to term cells, and the smaller tobacco-pipeshaped organs cups, the latter being usually above the former throughout the polypidom, "excepting immediately below each fork, where the cup is invariably absent above one of the cells of the pair from between which the fork springs.

"The polype-cells are several times larger than the cups, and their walls are much thinner; in fact, sufficiently transparent to allow of the contents of the cell being pretty well seen, without any prepara- 
tion, even during the life of the animal. In shape they are inversely conical, and the outer and upper angle is usually produced into a prominent, sharp point. From the internal and upper angle arises the tubular prolongation going to form the next cell or cup, as the case may be, in succession. They are entirely closed at the top, contrary to what is stated in all previous notices; and, as has been shown, there is no connection whatever between the cell and the cup placed immediately above and behind it. The aperture of the cell is on the anterior face, and towards the upper margin; it is of a crescentic form, and placed obliquely, as it were, across the upper and internal angle of the cell, with the convexity of the curve directed upwards and inwards. The lips of the aperture are strengthened by thin bands of horny material; and, under favourable circumstances, indications of short, muscular fibres, for the purpase of opening or closing the aperture, may be observed."

The shell, which Mr. Busk believes to be entire at the bottom, though closed only by a rery delicate membrane, contains an ascidioid polype, of the usual typical form of that class. "It has ten tentacula, and no gizzard. Two sets of muscular fibres, at least, may be distinguished as appertaining to the polype. The most important of these are the retractor muscles, which, arising from the bottom of the cell, in the form of long, somewhat flattened, transversely striped, isolated fibres, about the one ten-thousandth of an inch in width, are inserted, some of them at the base of the tentades, and others lower down the body of the polype. With respect to the mode of development of the cells and tubes at the extremities of the growing branches of the polypidon, it may be stated that the posterior and anterior connectingtubes of the cells, and the two lateral cups, constitute at the end of each branch four distinct buds, containing a fine granular matter, and appearing to be gradually developed into their respective complete forms, in the mode common to all hitherto observed bryozoa."

When we consider the minuteness of the delicate little sprig which is the natural size of this polype, we cannot but wonder at the triumphs of the microscope in giving such precise details as Mr. Busk relates of the Notamia bursaria. Its beautiful and perfect organisation makes us more awe-stricken at the power of Divine intelligence, and careful provision for the safety and engagements of the minute being that forms a slender portion of animated nature.

The next of the family of corallines we have to notice is the Anguinaria, from the Latin anguis, a snake. An account of the Anguinaria spatulata, or Snxke Coralline, appeared in the Transactions of the 
Microscopical Society, by the same able investigator before quoted, $\mathrm{Mr}$. Busk. He corrects many errors before existing respecting this zoophyte. This polype is parasitical upon fuci, and is not unfrequently associated with other kinds on the same plants, as in fig. 89, No. 2 ; it is there associated with Campanularia integra. The $A$. spatulata " as a whole, consists, like all its congeners, of two distinct portions, one usually termed the radical, and another which constitutes the proper polypecells. In the present instance, the arrangement of these parts is in some respects very peculiar and curious; but it will be found upon strict examination to accord accurately with the universal type. The origin, or base, as it may be termed, of the zoophyte, is a more or less rounded disc of small size, probably divided into compartments, as in the Notamia, from each of which arises a primary radical branch, in this species very short; these primary radical branches or tubes are directly continued into a polype-cell; but the cavities of the tube and cell are not continuous, being separated by a distinct dissepiment, so that the coarsely-granular contents of the radical tube have no communication with the polype-cell. The polype-cell is at first continued in a straight line with the radical tube from which it originates, but soon turns up at a right-angle, and stands erect upon the object on which the bryozoon is growing. After ascending in a nearly straight line for a certain distance, it curves with a gentle sweep, and terminates in a slightly-expanded extremity, at the apex of which, and not at the side, is the opening through which the polype protrudes. The continuation of the radical portion of the zoophyte, or of that creeping fibre, as it appears, which is adnate to the foreign base, is effected by the formation, at the angle where the polype-cell turns up, of a bud containing granular matter, which becomes a narrow tube in all respects similar to the primary radical tube, and which, like it, after running a short distance, terminates in a second polype-cell resembling the first, and so on continuously to an uncertain number. The growth of the polype-cell appears to precede in some degree that of the radical tube continuous from it; but it is not necessary that the growth of the former should be completed before the latter makes its appearance. The development of these two parts seems to be carried on in the usual way, viz. first, in the appearance of a rounded bud filled with granular matter, which gradually increases in length, and the contents of which are finally moulded or resolved into the proper contents of the cell, of which the bud constitutes the origin. In some cases, more than one bud of a radical tube arises at the angle of the polypecell; and in this way arise the apparent branches of the creeping stem. 
The walls of the radical tubes and of the polype-cells consist of a thin transparent horny material, which is insoluble in weak acids, and strengthened or rendered rigid, except in one part, by the deposit of calcareous matter. In the radical tubes, and on the dorsal or upper surface of the dilated extremity of the polype-cell, represented at No. 2, this earthy matter is deposited in the form of minute angular or rounded particles, presenting faint traces of a linear arrangement; but in the main body of the polype-cell, or the upright portion, the calcareous material is arranged in beautifully regular rings, giving that part of the zoophyte a peculiarly elegant appearance under the microscope. This calcareous ingredient is sufficiently abundant to render the contents of the radical tubes and polype-cells indistinct; and to obtain a satisiactory view of these parts it is necessary to remove the earthy matter by some weak acid. When this is done, it will be found that the contents of the radical portion are, as before mentioned, coarsely granular, and the wall rather thicker than those of the proper polype-cell. The latter contains an ascidian polype, which has about twelve tentacula, and no gizzard. The polype, as far as Mr. Busk has observed, is always lodged in the upright portion of the cell; but the long retractor muscular fibres arise near the commencement of the horizontal portion of the cell, and from its upper wall, nearly at one point.

"The expanded portion of the cell, besides the special muscles of the aperture, contains other muscular fibres, in all respects resembling those described by Dr. Farre, as conducing to the extrusion of the polype in Bowerbankia, and which are also very distinct in the Notamia; but which, in the present instance, would seem to have for their chief function the drawing-up or corrugation of the membraneous portion of the polype-cell. These muscular fibres have a distinct central nucleus or thicker portion, as is the case in the analogous muscles in the other cases just cited."

From the above description, the student in natural history will perceive the discoveries of $\mathrm{Mr}$. Busk so far differ from the accepted account of this zoophyte as to remore it from the family in which it is generally placed, as well as Gemellaria loricata and Notamia bursaria.

\section{CELLEPORIDE.}

This family have calcareous polypidoms, cellular, irregularly-lobed, or branched, formed of pitcher-shaped cells, heaped together or arranged in quincunx, the five on playing-cards. 
On the British shores are found C.pumicosa, C. ramulosa, C. Skenei (named after the rodest and talented Dr. David Skene), C. cervicornis, and C. loevis. This last, Dr. Flening says, is "in height an inch and a quarter, diameter one-tenth; the branches are smooth, with the orifices of the cells smooth and concave; towards the extremities the branches are rough with the forming cells, and the orifices are more declining, circumscribed, a little prominent, with a blunt process at the proximal margin."

Lepralia, sea-scurf, from the Greek for marine leprosy, is the name given to this branch of the Cellepora by Dr. Johnston.

Lepralia Nitida, found attached to shells, is thus described: "Crust spreading circularly, closely adherent, rather thin, greyish white, calcareous; cells contiguous, in radiating rows, large, subalternate, ovate, ventricose, silvery, the walls fissured with six or seven cross slits which are on the mesial line; aperture subquadrangular, depressed, terminal; anterior to it there is often found a globular, pearly, smooth, oviferous operculum, with a round even aperture. The remarkable structure of the cells renders this one of the most interesting species under the microscope. There is sometimes an appearance of a spine on each side of the lower angle of the mouth, which is merely the commencement of the walls of the next cell."

L. coccinea, L. variolosa, L. ciliata, L. trispinosa, and L. immersa, are the other British species.

Membranipora is a thin incrusting species. M. pilosa, or hairy polype, which is found on the smaller sea-weeds and shells. Polypidom incrusting, membrano-calcareous, irregular, following the shape and form of the objects it grows upon; straw-yellow, thickish, porous, hairy; cells short, somewhat tubulous, with large roundish apertures ossified and toothed on the margin; the teeth short, sharp, and rigid, and behind the mouth of each cell there is a very long tubular-bristle, which issues from a hollow base like a hair from its bulb. Polypes, with twelve tentacula, long in proportion to the body, thick and rather clumsy. This species is sometimes seen springing into sponge-like leaves, full of cells, and rough on both surfaces.

The family Cellularia, or little cells, have mostly that wonderful provision of nature for their protection, an operculum, a lid or cover over the apertures of their cells. Cellularia ciliata is parasitical, branching, calcareous, white and tufted; grows about half an inch in height, and on the oblique open aperture is armed on the outer edge with four or five long hollow spines. The operculum is pearly, and near the base there is that singular appendage, described as the bircl's- 
head process. Its beauty and transparency render it a favourite object with microscopists.

Cellularia Avicularia has been more accurately described by Mr. Gosse, from his own observations upon specimens secured on the Devonshire coast, during a residence there. He says.: "Well does it deserve the name of Bird's-head Coralline, given to it by the illustrious Ellis; for it presents those curious appendages that resemble vulture's heads in great perfection. All my specimens were most thickly studded with them; not a cell without its bird's head, and all see-sawing, and snapping, and opening their jaws with the most amusing activity; and what was marvellous, equally so in one specimen from whose cells all the polypes had died away, as in those in which they were still protruding their lovely bells of tentacles. The stem ascends perpendicularly from a slender base, which is attached to the rock, or to the cells of a Lepralia growing from the rock. The central part of the spine is most expanded; the diminution above and below being pretty regular; during life, the usual colour is a pale buff, but the cells become nearly white in death. When examined microscopically, it is, however, that the curious organisation of this zoophyte is discovered, especially when in full health and vigour, with all the beautiful polypes protruded and expanded to the utmost, on the watch for prey. It seems to me as poor a thing to strain one's eyes at a microscope over a dead and dry polypidom, as it does to examine a shrivelled and blackened flower out of a herbarium; though I know well that both are often indispensable for the making out of technical characters. But if you want to get an insight into the structure and functions of these minute animals, or if you would be charmed with the perception of beauty, or delighted with new and singular adaptations of means to an end,-or if you desire to see vitality under its most unusual, and yet most interesting phases, - or if you would have emotions of adoring wonder excited, and the tribute of praise elicited to that mighty Creator who made all thing's for his own glory, - then take such a zoophyte:as this, fresh from the clear tide-pool, take him without inflicting injury; therefore detach with care a minute portion of the surface-rock, and drop your prisoner, with every organ in full activity, into a narrow glass cell with parallel sides, filled with clear sea-water, and put the whole on the stage of the microscope, with a power of not more than 100 linear, at least, for the first examination. I greatly mistake if you will not confess that the intellectual treat obtained is well worth-ay, ten times more than worth-all your trouble."

Of the Retepora, or porous net-work family, reticulata is described 
by the Rev. W. Borlasse: "Polypidom latticed, wavy and convolute; the upper side warty and very porous." Dr. Fleming says of this rare coralline, that it is found "expanding to the extent of two or three inches; more or less cup-shaped, waved, uniting; the lobes are oval, regular; the intervening spaces supporting two or three pores in oblique rows. This species is very distinct from Retepora cellulosa, with which it has been confounded.

\section{MADREPORID $\pi$.}

Madrepores, or Motherpores, differ from other corals in not having a smooth skeleton, but one inducted by numbers of small cells for the residence of the living animal ; these are very visible in the Madrepore muricata, when the polype is dead and decomposed; but most distinct in the Oculina ramea, or Abrotanoide, as they are situated at the apparently broken stumps that branch from the trunk of the skeleton. (Plate III., figs. 5 and 6.) Every branch is seen to be covered with multitudes of small pits or dots, scarcely visible to unassisted vision; but when viewed under the microscope, will be found to be cells of the most beautiful construction, remarkable alike for their mathematical regularity and the exquisite fineness of the materials employed in their composition. A magnified drawing of one of the cells is seen at 6 . The living polypes are most beautiful in their native waters; their varying colours adding to the richness of the hues covering the plains of the ocean.

Caryophyllia, or Nut-leaf. The Caryophyllia Smithii is found tightly fixed to rocks; it is round, of a dirty white colour. Dr. Fleming says of the plates: "The lamellæ are disposed in fours, and may be distinguished into three different kinds. The first are the highest and the broadest at the margin; hut as they descend into the disc, they become narrower before they join the central plate. The second kind are narrower than the preceding at the margin, but towards the middle they suddenly enlarge and join the middle plate. The third lind are the smallest, and terminate before reaching the middle plate. The space included between a pair of the first kind of plates contains one of the second kind in the middle, with one of the third kind in each of the lateral spaces. Those on the sides are rough, with small scattered tubercles, and their margins are curled. This last circumstance occasions the roughness externally, where the longitudinal strix are the remains of the gills. The plate which occupies the bottom of the cavity is smooth, variously twisted, and connected with the base of the lateral plates." 
Dr. Coldstream, writing to his friend Dr. Johnston, gives the following interesting account of the animal: "When the soft parts are fully expanded, the appearance of the whole animal resembles very closely that of an Actinia. When shrunk, they are almost entirely hid amongst the radiating plates. The specimens I have seen have varied in size from three-tenths to half an inch in height. They are found pendent from large boulders of sand-stone, just at low-water mark; sometimes they are dredged from the middle of the bay (Torquay). Their colour varies considerably; I have seen the soft parts white, yellowish, orange-brown, reddish, and of a fine apple-green. The tentacula are usually paler. During expansion, the soft parts rise above the level of the calcareous disc to about twice its height. The tentacula are pushed forth very slowly, but sometimes are as long as the whole height of the body. They are terminated by a rounded head. The mouth has the appearance of an elongated slit in the centre of the disc; it is prominent, and the lips are marked with transverse striæ of a white colour. When a solid body is brought into contact gently with the tentacles, they adhere pretty strongly to it, just as the Actinia do; but when they are rudely touched, they contract very quickly; and if the irritation be continued, the whole soft parts sink within the calcareous cup."

Dr. Johnston, in a specimen he had, counted as many as twenty lamella; but Dr. Fleming says he has observed as many as forty. The top of the polypidom is hollowed into the shape of a star-like cup.

\section{tUdUlaride, tUbiporid}

They are generally known as Tubular or Vaginated Polypes, and are of an arborescent appearance, the animals living near the end of the branches, and found attached to stones, sea-weed, and shells. The Tubularia indivisa, or individed tubes, rise up like a tuft of herbage, of a horn colour, to the height of twelve inches. Ellis says, "they seem part of an oat-straw, with the joints cut off." At the summit protrudes the scarlet-coloured polype, well furnished with tentacula, and connected with a pinkish fluid that fills the tubes. It was in this that Dr. Roget discovered the singular peculiarity of a circulation, similar to that in many plants, carried on without the mechanism necessary in higher organised animals : he says, "In a specimen of the Tabularia indivisa, when magnified one hundred times, a current of particles was seen within the tubular stem of the polype, strikingly resembling, in the steadiness and continuity of its stream, the regetable 
circulation in the chara. Its general course was parallel to the slightly spiral lines of irregular spots on the surface of the tube, ascending on the one side, and descending on the other; each of the opposite currents occupying one-half of the circumference of the cylindric cavity. At the knots, or contracted parts of the tube, slight eddies were noticed in the currents; and at each end of the tube the particles were seen to turn round, and pass over to the other side. In various species of sertulariæ, the stream does not flow in the same constant direction; but, after a time, its velocity is retarded, and it then either stops, or exhibits irregular eddies, previous to its return in an opposite course; and so on alternately, like the ebb and flow of the tide. If the currents be designedly obstructed in any part of the stem, those in the branches go on without interruption, and independently of the rest. The most remarkable circumstance attending these streams of fluid is, that they appear to traverse the cavity of the stomach itself, flowing from the axis of the stem into that organ, and returning into the stem, without any visible cause determining these movements.

"The particles carried by it present an analogy to those of the blood in the higher animals on one side, and of the sap of vegetables on the other. Some of them appear to be derived from the digested food, and others from the melting down of parts absorbed; but it would be highly interesting to ascertain distinctly how they are produced, and what is the office they perform, as well as the true character of their remarkable activity and seemingly spontaneous motions; for the hypothesis of their individual vitality is too startling to be adopted without good evidence." Dr. Johnston cautions his readers against confounding this sort of circulation "with those aqueous currents which flow over the surfaces of the external organs of the ascidian polypes;" he adds, that the innumerable cilia that "clothe the surfaces of their tentacula, and by their rapid vibrations drive a constant equable stream of water along one side, which returns along the other in an opposite direction; and by this means the purposes of respiration are effected, and the nutrient fluid fitted for assimilation with the body."

Respecting the singular property of the head dropping off, Sir J. G. Dalyell says of the Tubularia indivisa, "the head is deciduous, falling in general soon after recovery from the sea. It is regenerated at intervals of from ten days to several weeks, but with the number of external organs successively diminishing, though the stem is always elongated. It seems to rise within this tubular stem from below, and to be dependent on the presence of the internal tenacious matter with which the tube is occupied. A head springs from the remaining stem, cut 
off very near the root; and a redundance of heads may be obtained from artificial sections, apparently beyond the ordinary provisions of nature. Thus twenty-two heads were produced through the course of 550 days from three sections of a single stem."

Tubularia Larynx is a most beautiful object; it is so named from its slender, clustered tubes, which are of a horny texture, having the appearance of the windpipe of a bird. They are about three inches high, and the polype of a delicate red colour, with white arms; one circle around the body, and the other-round the oral opening.

In this family are the T. ramous, $T$. ramea, perfect trees in miniature; and the Hermia glandulosa of Dr. Johnston, who says: "I found the name in Shakspeare:

\section{- What ricked and dissembling glass of mine, Made me compare with Hermia's sphery eytue?"}

The fancy that the glands which surround the heads were the guardians of the animal, its "sphery eyne," suggested the name here adopted. These polypes are adherent by a tubular fibre, which creeps along the surface of the object on which they grow, seldom an inch in height, irregularly branched; the stem filiform, tubular, horny, subpellucid, wrinkled, and sometimes ringed at intervals, especially at the origin of the branches; each of which is terminated with an oval or club-shaped head of a reddish colour, and armed with short scattered tentacula, tipt with a globular apex. The ends of the branches are not perforated, but completely covered with a continuation of the horny sheath of the stem. The animal can bend its armed hands at will, or give to any separate tentaculum a distinct motion and direction; but all its morements are very slow and leisured."

We likewise have the Coryne squamata, and the Coryne stauridia, or Slender Coryne (fig. 89, No.4), a sea-water polype, thus described by Mr. Gosse: "It was found by me adhering to the footstalk of a Rhodymenia, about which it creeps in the form of a white thread; by placing both beneath the microscope, this thread appeared cylindrical and tubular, perfectly transparent, without wrinkles, but permeated by a central core, apparently cellular in texture, and hollow; within which a rather slow circulation of globules, few in number and remote, is perceived. It sends off numerous branches; the terminal head of which is oblong, cylindrical, rounded at the end. At the extreme point are fixed four tentacula of the usual form, long, slender, and furnished with globular heads; one of which is shown at No. 5, detached, and more highly magnified. It is much infested with parasites; a vorti- 
cella grows on it, and a sort of vibrio; the latter in immense numbers, forming aggregated clusters here and there; the individuals adhering to each other, and projecting in bristling points in every direction. These animalculæ vary in length; some being as long as $\frac{1}{80}$ inch, with a diameter of $\frac{1}{700} \overline{0}$ inch. They are straight, equal in thickness throughout, and marked with distinct tranverse lines; they bend themselves about with considerable activity, and frequently adhere to the polype by one extremity, while the remainder projects freely."

Tubipora Truncata.-Fleming says they are "about an inch in height, the branches scarcely exceeding one-eighth; the branches are short, pierced by numerous pores, the openings of cells converging. towards the centre; the head is stellate; the rays are highest in the middle of their course, diminishing towards the centre and lower margin of the head; each ray is compressed, and consists of two rows of tubular cells, united, crowded, with sub-angular orifices; the tubes have a central direction, and give to the sides of the plates a striated appearance. This species has probably been referred to as an inhabitant of the North seas, under the title of Millepora truncata; but it differs widely from the Myriozoos of Donati, to which the term was restricted by Pallas."

The other established British Tubipora are T.patina, T. serpens, and T. obelia.

\section{PENNATULIDA:}

The first family in this order derives its name from penna, a quill, which the animal much resembles; magnified sections are seen in fig. 92, No. 3. Naturalists call them Sea-pens; and if they had in general applied such simple, appropriate terms to the objects of nature, they would have removed a bar to the study of science, and overcome a repugnance commonly felt on entering the enchanting field of $\mathrm{know}$ ledge. The crabbed, far-fetched, and learned cognomens usually give an air of mystery and difficulty where none ought to exist. The wonderful discoveries in nature ought to be a book open to all, and like herself, simple and unadorned. Before a student can commence his pursuits in any branch of science, it is imperative that he should be a linguist, which the great body of the people have not opportunities to become; and hence the young and plainly educated are driven to idle, irregular reading, when they might have adorned a science, and brought honour to their country.

The polypiferous mass of the Pennatula is fleshy or jelly-like; and 
the polypes are always found on the margin of the appendages of the polypidom. The skin is tough and leathery, and has numerous calcareous spicula in a parallel position to each other, which Dr. Coldstream is of opinion are solid; and when connected with the body of the animal, seem to be red; but a slight degree of heat is sufficient to bleach them : they consist of phosphate and carbonate of lime, making thus a near approach to the bone of vertebrate animals.

On many parts of the coast, when the fishermen haul in their lines, and more especially if baited with mussels, there are found attached to the bait a number of polypes, which the boatmen call Cockscombs, but naturalists, Pennatula phosphorea; they are from two to four inches long, of a purplish-red colour, except at the base of the smooth stalk, which is a pale ye'low, from, as the fishermen say, this part being imbedded in the mud at the bottom of the sea. They are built up in the same manner as the former. "The papilla on the back of the rachis, and between the pinnæ, are disposed in close rows, and do not differ from the polype-cells except in size. The latter are placed along the upper margin of a flattened fin; they are tubular, and have the aperture armed with eight spinous points, which are movable, and contract and expand at the will of the animated inmates. These are fleshy, white, provided with eight rather long retractile tentacula, beautifully ciliated on the inner aspect with two series of short processes, and strengthened moreover with crystalline spicula, there being a row of these up the stalk, and a series of lesser ones to the latter cilia. The mouth, in the centre of the tentacula, is somewhat angular, bounded by a white ligament, a process from which encircles the base of each tentaculum, which thus seems to issue from an aperture. The ova lie between the membranes of the pinnæ; they are globular, of a yellowish colour, and by a little pressure can be made to pass through the mouth." (Johnston.)

Dr. Grant writes, "a more singular and beautiful spectacle could scarcely be conceived than that of a deep purple Pennatula phosphorea, with all its delicate transparent polypi expanded and emitting their usual brilliant phosphorescent light, sailing through the still and dark abyss, by the regular and synchronous pulsations of the minute fringed arms of the whole polypi." The power of locomotion is doubted by other writers, and the pale blue light is said only to be emitted when under the influence of some painful irritation.

Virgularia Mirabilis. - This is sometimes called the Seca-rush instead of "the admirable little rod;" it is from six to ten inches long. Sowerby describes them as like a quill stripped of its feathers. The 
base has some resemblance to a pen, as in the other species, swelling a little from the end; and then tapering. The upper part is thicker, with alternate semicircular pectinated swellings, larger towards the middle, tapering upwards, and terminating in a thin bony substance, which passes through the whole." Professor Grant writes, "their axis is calcareous, solic, white, brittle, flexible, cylindrical, of equal thickness throughout, and exhibits no mark of attachment at either end. When broken, it exhibits a radiated surface, like the broken spine of an echinus. The axis appears to have little connexion with the fleshy part; and to consist of concentric layens deposited by the soft parts surrounding it. When a portion of the axis is broken off from either extremity, the animal retracts at that part, so as continually to expose a fresh naked portion of the axis; hence we can take out the axis entirely from its soft sheath, and we always find the lower pinnæ of the animal drawn up closely together, as if by the frequent breaking of the base. These very delicate and brittle animals seem to be confined to a small circumscribed part of the coast, which has a considerable depth and a muddy bottom; and the fishermen accustomed to dredge at that place believe, from the clearness of the Virgularix when brought to the surface, that they stand erect at the bottom with one end fixed in the mud or clay. Muller's specimens were likewise found on a part of the Norwegian coast with a muddy bottom. The polypi much resembling those of the common Lobularia digitata, are long, cylindrical, transparent, marked with longitudinal white lines, and have eight tentacula, which present long slender transparent filaments or cilia on each of the lateral surfaces when fully expanded. The polypi are easily perceived extending through the lateral expansions or pinnæ, to near the solid axis, where we observe two transverse rows of small round white ova placed under each pinna, and contained within the fleshy substance. These ova appear to pass along the pinnæ, to be discharged through the polypi, as in the Lobularia, Gorgonia, Caryophyllea, Alcyonia, \&c."

Thus it will be observed there are no small spicula, but a solid axis, or skeleton. The polype mass is free, supporting towards the upper extremity, the dwarfish monn-shaped lobes embracing the stem obliquely and bearing a row of cells on their margin. The lower part is uncovered, and has a deep indent from one end to the other, giving the appearance of a division into two equal parts. 
Sertulariade. (Plate III. No. 12.)

The Sertularice are a numerous, interesting, and beautiful family. They are easily attainable, being found on our own sea-shores. Linnæus made a large genus of them; but Lamarck considerably reduced his classification. The usual type presented is the beautiful Sertularia pluma, fig. 90, which Dr. Fleming proposes to divide into two groups, according as the stems are simple or compound.

The Sertularia polypes have arms or feelers, which are abundantly supplied with cilia, with pitcher-shaped, dwarfish cells, arranged alternately, or in pairs obliquely, not exactly opposite, on the stem and branches of the polyparium, which is horny, fistulous generally, but. attached in ziz-zag radiant fibres.

Such are the general characteristics. drawn by Lamarck; Dr. Johnston includes them in the Zoophyta Hydroida.

Within this family come the Thoo.

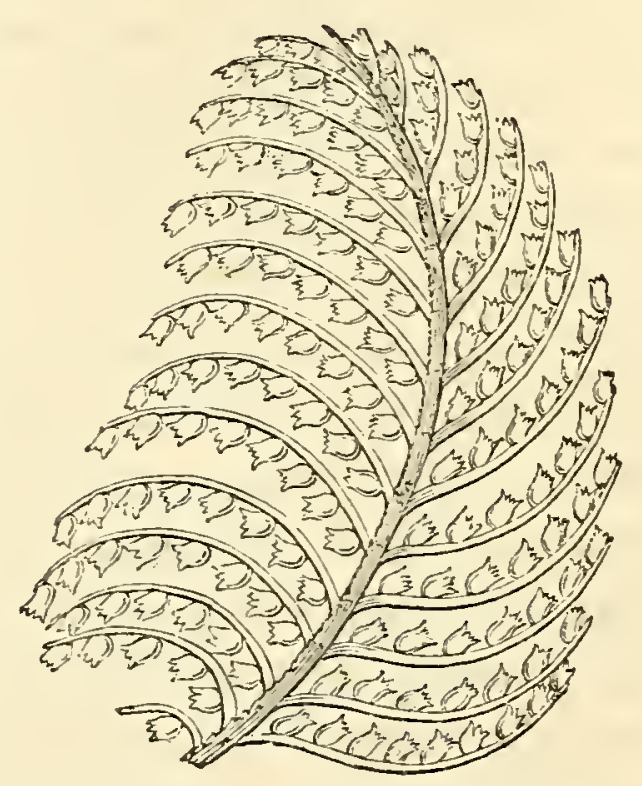

fig. 90. A single branch of Sertulariadæ. of Lamouroux, of which there have been several kinds found in Great Britain. The name is supposed to be derived from the Greek word for sharp; but we think with Dr. Johnston, that it more probably is a mis-spelling of Thoe, one of the Nereids, nymphs of the sea. They are generally of a brown and yellow. colour, branched, and from an inch and a half to six inches in height. The Thoa fabilis has received the name of 2 . Beanii, in honour of the discoverer.

Sertularia Pumila. - This is a parasite found near the sea-shore. It spreads its brown-coloured shoots over various fuci and sea-shells; but rarely attains more than half an inch in height. The little inhabitant of the cell has fourteen arms to entrap its prey, while it reaches its body beyond the edge of the cell to which it is chained during life. Stewart says, "This species, and probably many others, in some particular states of the atmosphere, emits a phosphorescent light in the dark. If a leaf of the above fucus (serratus), with the Sertularia upon it, receives a smart stroke in the dark, the whole coralline is most beautifully illuminated, every denticle seeming to be on fire." Sertularia Hibernica is thus named from being found near Donag- 
hadee. Templeton says, "The branching of this species is somewhat peculiar, each of the primary and secondary branches springing out at an angle of $40^{\circ}$ or $50^{\circ}$. That part of the stem which bears the denticles is waved so as to bear each denticle on the projecting part; the denticles are eliptic, and the mouth of each apparently a little hollowed inwards, perhaps arising from the extremity being fractured; the vesicles are ovate, with four or five blunt teeth surrounding the mouth, and divided into six or eight portions by annulated undulating lines. It might be classed among the large and strong Sertulariæ, the principal shoot being of the thickness of a sparrow's quill at its base, and four inches or five inches long. The branches shoot forth from opposite sides, the whole coralline thus assuming a flat form, to the extent of four inches or five inches."

On the south-eastern coast of England the most common kind found is the Sertularia setacea, which, after rough weather, is cast on to the shore attached to sea-weed. The stem and branches seem composed of separate pieces, fitting into each other as some foreign trees do, and terminate in a star-like head, from which radiate the feelers or arms. Dr. Mantell states he was present on one occasion when Mr. Lister was observing a living specimen, a little globular animalcule swam rapidly by one of the expanded polypes, the latter immediately contracted, seized the globule, and brought it to the mouth or central opening by its tentacula; these gradually opened again, with the exception of one, which remained folded, with its extremity on the animalcule. The mouth instantly seemed filled with cilia, which, closing over the prey, was carried slowly down its stomach; here it was imperfectly seen, and soon disappeared.

Appertaining to this family are the Thuiaria, so named from resembling a cedar-tree, but some kinds look more like a knobbed thorn-stick with a bottle-clearer at the top; others resemble a fir-tree. Antennularia are so called from resembling the lobster's antenna. They are found fixed to shells and rocks, are plentiful on the north-eastern coast of England and the coast of Ireland. They are of a brown colour, and covered with hair-like little branches ; and as the hairy process is continued up its jointed stem, it is sometimes denominated Sea-beard.

The Plumularia, so named from the shoots and offsets being plumous, are an extensive and beautiful branch of this family. Professor Grant thus decribes the Plumularia falcata: "This species is very common in the deeper parts of the Frith of Forth; its vesicles are very numerous, and its ova are in full maturity at the beginning of May. The ova are large, of a light-brown colour, semi-opaque, nearly spherical, composed 
of minute transparent granules, ciliated on the surface, and distinctly irritable. There are only two ova in each vesicle; so that they do not require any external capsules, like those of the Campanularia, to allow them sufficient space to come to maturity. On placing an entire vesicle, with its two ova, under the microscope, we perceive through the transparent sides the cilia vibrating on the surface of the contained ova, and the currents produced in the fluid within by their motion. When we open the vesicles with two needles, in a drop of sea-water, the ova glide to and fro through the water, at first slowly, but afterwards more quickly, and their cilia propel them with the same part always forward. They are highly irritable, and frequently contract their bodies so as to exhibit those singular changes of form spoken off by Cavolini. These contractions are particularly observed when they come in contact with a hair, a filament of conferva, a grain of sand, or any minute object ; and they are likewise frequent and remarkable at the time when the ovum is busied in attaching its body permanently to the surface of the glass. After they have fixed, they become flat and circular, and the more opaque parts of the ova assume a radiated appearance; so that they now appear, even to the naked eye, like so many minute grey-coloured stars, having the interstices between the rays filled with a colourless transparent matter, which seems to harden into horn. The grey matter swells in the centre, where the rays meet, and rises perpendicularly upwards surrounded by the transparent horny matter, so as to form the trunk of the future zoophyte. The rays first formed are obviously the fleshy central substance of the roots; and the portion of that substance which grows perpendicularly upwards, forms the fleshy central part of the stem. As early as I could observe the stem, it was open at the top; and when it bifurcated to form two branches, both were open at their extremities; but the fleshy central matter had nowhere developed itself as yet into the form of a polype. Polypi, therefore, are not the first formed parts of this zoophyte, but are organs which appear long after the formation of the root and stem, as the leaves and flowers of a plant."

Attached to fuci and sea-side shells in abundance on the southern coast of England, is found Plumularic cristatc. It is affixed by a horny, branching, interlacing, tubular fibre to the object on which it grows. At different parts there are plumous shoots, usually about a little more than an inch in height. The cells are of a yellow colour, set in the stalk, of a bell-shape, and are compared to the flower of the lily of the valley; the rim is cut into eight equal teeth; the polype minute, delicate; tentacula ten, annulated; mouth infundibuliform.

"Each plume," says Mr. Lister, in reference to a specimen of this 
species, "might comprise from 400 to 500 polypi " "and a specimen," writes Dr. Johnston, "of no unusual size before me has twelve plumes, with certainly not fewer cells on each than the larger number mentioned; thus giving 6000 polypes as the tenantry of a single polypidom! Now, many such specimens, all united too by a common fibre, and all the offshoots of one common parent, are often located on one sea-weed, the site then of a population, which nor London nor Pekin can rival."

Plumularia Pinnata, or Feather Polype (represented magnified in Plate I. No. 3), "is as remarkable for the elegance of its form, as its likeness to the feather of a pen." It serves not among the denizens of the deep the same purpose as its earthly prototype. Nature writes her works in hieroglyphics formed by the objects themselves. It is plumous, and the cells in a close row, cup-like, and supported on the under side by a lengthened spinous process.

An interest pervades the valuable work of Dr. Johnston, arising from the circumstance that the plates and woodcuts which adorn the volume are, with few exceptions, engraved from drawings made for it by Mrs. Johnston, who also engraved several of them ; and the Doctor states he could not have undertaken the history without such assistance. From this devotion to, and understanding of the subject, it was natural, when an opportunity presented itself, to write in the catalogue of Zoophytes a lasting memorial of his "colleague :" and thus is written the graceful compliment of the beautiful "Plumularia Catharina; stem plumous, the pinnæ opposite, bent inwards; cells distant, campanulate, with an even margin; vesicles scattered, pear-shaped, smooth. Found on old shells, corallines, and ascidia, in deep water. At Scarborough it is rare. In Frith of Forth by Dr. Coldstream, and frequently in Berwick Bay. This equals $P$. pinnata in size and delicacy, but differs from it very obviously in having opposite pinnæ, which, instead of being arched, are bent inwards, so as to render the general form of the coralline concave on a front view; an appearance produced by the pinnæ originating, not from the sides, but from the anterior face of the stem. The stem itself is straight or slightly bent, jointed, pellucid, filled with a granular fluid matter; and in which it differs from its congeners, bearing cells, there being always one at the base and between the insertion of the pinnæ, and generally another on the interval between them. Between the cells there is a series of minute tubular or tooth-like cells, visible only with a high magnifier. The ovarian vesicles are produced in summer; they are stalked, shaped like a pear or vase, solitary, scattered, and originating always at the base of a polype-cell. 
From the intermediate cellules, particularly from the one next the polype-cell, there often grows up a small trumpet-like tube; and I have seen in one specimen, all the ends of the branches terminated by four of these tubes, diverging in pairs."

The next in this family is the Laomedea, of which the Laomedea dichtoma is found in great abundance on the south-west coast of England, and seems most curiously contrived, from its structure, to resist the violence of the waves, all its joints being furnished with springs. Sir J. G. Dalyell says "it rarely produces vesicles. When present, they contain from 20 to 30 greyish corpuscules with a dark central nucleus. At first, all are immature and quiescent, but motion at length commences; the corpuscules become more distinct; several slender arms protrude from the orifice of the vesicle, which are seen in vehement action, and, after many struggles, an animated being escapes. But this has no relation either to the planula of the Sertularia, or the corpusculum of the Flustra, Alcyonitim, or Actinia. It might be rather associated with the Medusaria. Before ascertaining its origin, I had named it Animatculum tintinnabulum, from its general resemblance to a common hand-bell, for the purpose of recognition. This creature is whitish, tending to transparency, about half a line in diameter; the body is like a deep watch-glass, surmounted by a crest rising from the centre, and fririged by about twenty-three tentacula pendent from the lip below. These are of muricate structure, or rough, and connected to the lip by abulb twice their own diameter. The summit of the crest unfolds occasionally into four leaves, and four organs prominent on the convexity of the body appear at its base. When free, the animal swims by jerks, or leaps through the water, or drops gently downwards; it is invited to move by the light, and it has survived at least eight days. Then it disappears, at least I have not been able to pursue its history longer. No other preduct has ever issued from the vesicles of the Sertularia dichotoma."

The last in this family are the-Campanularia, also frequently found on our shores; they possess a simple circle of cilia on their feelers or arms, witl pitcher-shaped cells on stalks that branch, twist, or climb on an axis.

The Campaniiaria Volubilis, or Truining Polype (Fl. I. No. 4), is the common type: it is parasitical, and infests the antennæ of the crab to a great extent; its stem is filiform, and at the end of its slender branches are situated the cells containing the polypes. The polype itself is slender when protruded, as seen in the figure, and becomes dilated at the base into a sort of foot which spreads over the dia- 
phragm, widening at the top, where it fills the mouth of the cell, and gives origin to about twenty slender tentacula, set in two or three series. From the central space, surrounded by the tentacula, a large fleshy mouth protrudes, somewhat funnel-shaped, with lips, endowed with the power of protrusion and contraction; these appear to be very sensitive. $\mathrm{Mr}$. Gosse found them in great abundance round Smallmouth Caves.

The Campanularia Gelatinosa has beautiful bell-shaped cells, out of which the animal protrudes, giving it the semblance of a green flower with a delicate pink stalk. It is indeed an interesting object for observation, as the currents in the tubes may be detected. Dr. Johnston says, "On Saturday, May 29th, 1837, a specimen of Campanularia gelatinosa was procured from the shore, and after having ascertained that the polypes were active and entire, it was placed in a sancer of sea-water. Here it remained undisturbed until Monday afternoon, when all the polypes had disappeared. Some cells were empty, or nearly so; others were half-filled with the wasted body of the polype, which had lost, however, every vestige of the tentacula. The water had become putrid, and the specimen was therefore removed to another vessel with pure water, and again set aside. On examining it on the Thursday, June 1st, the cells were evidently filling again, although no tentacula were visibly protruded; but on the afternoon of Friday, June 2d, every cell had its polype complete, and displayed in the greatest perfection. Had these singular facts been known to Linnæus, how eagerly and effectively would he have impressed them into the support of his favourite theory! Like the flowers of the field, the heads, or 'flores,' of these polypidoms expand their petaloid arms, which after a time fall like blighted blossoms off a tree: they do become 'old in their youth,' and rendered hebetous and unfit for duty or ornament by age or accident; the common trunk throws them off, and supplies its wants by ever-young and vigorous growths. The phenomena are of those which justly challenge admiration, and excuse a sober scepticism, so alien are they to all we are accustomed to observe in more familiar organisms. Faithful observation renders the fact undeniable; but besides that, a reflection on the history of the Hydra might almost have led us to anticipate such events in the life of these Zoophytes. 'Verily, for mine owne part, the more I looke into nature's workes, the sooner am I induced to beleeve of her even those things that seem incredible !'-Baker."

ACTINIADR. (Frontispiece, Nos. 5, 6, and 7.)

All accustomed to wander by the sea-shore, have admired the livid, 
green, and dark little jelly-masses adhering to the rocks, called Actina, from a Greek word signifying a ray, when left in some little pool by the ebbing tide; living as they do principally within high and lowwater mark, and expanding their broad surfaces and fringing feelers, the finger of inquisitive youth is often thrust into the centre to feel the effect of the suction, as the poor animal draws itself up in the form of a little fleshy hillock. They have the power of secreting some adhesive substance at their base, by which they affix themselves to rocks and other objects; but can release their bodies when necessary, and, either by creeping on their feelers, or filling their bodies with water, and gliding along, change their location. Miuch interest has been excited by the exhibition of many of this class in the tanks in the Zoological Gardens. These Zoophytes are more familiarly known by the name of Sea Anemonies.

In their appetites they are roracious, and seize the little shelled inhabitants of the sandy shores; while it would appear, from the rejection of the innutritious portions, to require twelve hours to digest its prey. Dr. Johnston says: "I had once brought me a specimen of Actinia gemmacea, that might have been originally two inches in diameter, and that had somehow contrived to swallow a valve of Pecten maximus, of the size of an ordinary saucer. The shell, fixed within the stomach, was so placed as to divide it completely into two halves; so that the body, stretched tensely over, had become thin, and flattened like a pancake. All communication between the inferior portion of the stomach and the mouth was of course prevented; yet, instead of emaciating and dying of atrophy, the animal had availed itself of what had undoubtedly been a very untoward accident, to increase its enjoyments and its chances of double fare. A new mouth, furnished with two rows of numerous tentacula, was opened up on what had been the base, and led to the under-stomach : the individual had, indeed, become a sort of Siamese twin, but with greater intimacy and extent in its unions." The following curious observations made by Dicquemare upon these rivals of the weather-wise leeches, may be useful to some residents at the sea-shore: "My very earliest observation showed that the sea-anemonies feel, and prognosticate within doors, the different changes of temperature in the atmosphere. I had not leisure at that time to form tables of their various indications; but I have since done it. This fact, if applied to practice, might be of use in the formation of a sea-barometer; an object of no small importance; which several ingenious men have hitherto endearoured in vain to furnish us with. I should prefer the anemonies of the third species for 
this purpose, their sensation being very quick ; they are also easily procured, and may be kept without nourishment. Five of them may be put in a glass vessel four inches wide and as many in depth, in which they will soon cleave to the angle formed by the sides and the bottom. The water must be renewed every day; and as they do not require a great quantity of it, as much may be fetched from the sea (if they be kept on land) as will supply them for several days; its settling some time will only improve it. If the anemonies be at any time shut or contracted, I have reason to apprehend an approaching storm; that is, high winds and an agitated sea. When they are all shut, but not remarkably contracted, they forbode a weather somewhat less boisterous, but still attended with gales and a rough sea. If they appear in the least open, or alternately and frequently opening and closing, they indicate a mean state both of winds and waves. When they are quite open, I expect tolerably fine weather and a smooth sea. And lastly, when their bodies are considerably extended, and their limbs divergent, they surely prognosticate fixed fair weather and a calm sea. There are times when some of the anemonies are open, and others shut; the number must then be consulted; the question is decided by the majority. The anemonies used as barometers should not be fed; for then the quantity of nourishment might influence their predictions. Anemonies of this, and of the first species, live and do well for several years without taking any other food but what they find disseminated in the sea-water; but should a respite of some days be granted them, they might then be fed with some pieces of muscles, or soft fish, and thus restored to their original vigour. Whenever the vessel is sullied by the sediments of salts, slime, the first shoots of sea-plants, \&c. the animal may, on changing the water, be cleansed, by wiping it with a soft hair pencil, or even with the finger, carefully avoiding to rub or press hard on the anemonies. Should any of them drop off during this operation, they may be left at, liberty; for they will soon of their own accord fix themselves to some other place. Should any of them die, which will soon be discovered by the milky colour of the water, and an offensive smell on changing it, it must be taken out, and on the first opportunity another of the same species be put in its place; those of a moderate size are the most eligible."

The Actiniæ bring forth their young alive; one or more appear first in the tentacula, from whence they can be withdrawn and transmitted to others by the parent, and are at last produced by the mouth. In the course of six years, one specimen was found to produce above 276 young. 
Like the Hydra polypes, the Actinix may be cut and its members lopped off without injury, the parts becoming perfect animals, or the limbs rebudding; in fact, the tentacula will be reproduced as often as the sympathising experimenter may choose to perform amputation. If the animal be divided in two, separating the base from the head, the former will become a perfect animal, and the latter industriously swallow food; which passes freely through it, but in time is retained, and a finished stomach effects the necessary function of digestion. Upon trying this experiment, there have been instances of a new head coming at the part where the separation from the base was effected; thus at each end the active and powerful feelers captured prey and conveyed it to the stomach. Hot water may blister its skin, and a transparent palace of ice encase its borly, or an air-pump deprive it of air, the animal will not die; but fresh-water is the swift and sure messenger of death.

The commonest kind of this family, Actinia mesembryanthemum, or Smooth Anemonie, is of a dark flesh or olive colour; some are streaked with white, blue, or green, or spotted. The feelers, when stretched out, are about the same length as the body; the animal generally measures about an inch and a half in diameter. Dr. Johnston says: "The tubercles within the margin of the oral disc are formed by papillary projections of the parenchyma-spongy or porous flesh — of the body, covered over on the top with a thick layer of dense blue matter : in it, as well as in the skin generally, minute fusiform calcareous spicula, some slenderer than other's, may be detected in abundance with the microscope."

Actinia Rubra and Bellis (Nos. 4, 5, 6, and 7 , Plate I.) generally live together in groups of four or five. Gœrtner thus describes them: "From the small base arises a cylindric stalk, which supports the roundish body of the animal, and from whence afterwards the calyx, being a continued membrane of the body, draws its origin. The stalk or pedunculus of the polype is quite smooth, and its colour inclines towards the carnation. The outside of the calyx, and the body of this animal, are marked with a number of small white protuberances resembling warts, to which fragments of shells, sand-grains, \&c. adhere, and hide the beautiful colour of these parts, which, from that of carnation, are insensibly changed towards the border of the calyx, first into purple, then violet, and at last into a dark brown. The inside of the calyx is covered with the feelers that grow in several ranges upon it: they differ considerably in length; those that are near the edge of the calyx being but small papillix, in proportion to those that surround the disc, or the central part of the body. They are almost transparent, and some of them of a pale ash colour, with brown spots; others, on the contrary, are of a chestnut 
colour, marked with white spots. The disc is formed like a star, which, according to the figure that is traced out by the innermost row of the feelers, consists of many angles. The colour of this part of the body is a beautiful mixture of brown, yellow-ash colour, and white, which together form variegated rays, that, from the centre or the mouth of the animal, are spread over the whole surface of the disc. The polype, contracting itself, changes its body into an irregular hemisphere, which is so covered with the several extraneous bodies that stick to it, that it is extremely difficult to know the animal in this state, and to discern it from the rubbish that commonly surrounds it."

Actinia Gemomacea is larger than those previously named; it has three or four rows of thick short feelers around the oral disc, which have suckers at the ends, by which they seize and hold their unfortunate victims. This is a large and varied species of different colours, the feelers being usually variegated with red and white rings. Its body is rough, with glandular warts; and being covered by the sand, shells, or gravel surrounding it, is hidden from objects that may prey upon it, or unobserved by those upon which it preys. So singular is nature in adapting its productions to the circumstances by which they are surrounded, that when this species becomes an inhabitant of waters that do not leave it dry, the masquerading-dress is cast aside, the warts almost disappear, and it stands forth in all its beauty, proving the words of the poet, that "when unadorned, adorned the most."

What will not man venture to eat, in hope of tickling the appetite with some new excitement! Dicquemare says: "Of all the kinds of sea-anemonies, I would prefer this for the table; being boiled some time in sea-water, they acquire a firm and palatable consistence, and may then be eaten with any kind of sauce. They are of an inviting. appearance, of a light silvery texture, and of a soft white and pinkish hue. Their smell is not unlike that of a warm crab or lobster."

Actinia Maculata. - This is a curious and interesting object, as it is found enveloping a snail-like or periwinkled shell, having the superior part of a species of small crab projecting from the inside of the shell. Its body is of a reddish colour, marked with beautiful purple spots, and in its thickest part is not more than three-tenths of an inch; it is about three inches in diameter, and furrowed longitudinally. It does not quite cover the pointed end of its adopted residence; while near the aperture of the shell is a large oval mouth, with short irregularly-placed tentacula surrounding it. It is fixed to the shell by a thin horny membrane, extending generally over the whole, but most strongly fixed at the open edges of the shell. 
Among the largest of the British species of Actinia is one found on the north-east coast, which, when contracted, usually measures three inches in length. Dr. Johnston, as sponsor, gives it the name of Actinica Tuedice, the ancient appellation of the maritime parts of Berwickshire, to indicate the place of its first discovery, where it is often dragged from deep water by the fishermen. It is of a uniform reddish or brownishorange colour, and either smooth or contracted at pleasure into circular folds. The base is smooth and orange-coloured. The mouth is ever rarying in size and form, and there are often protruded from it resicular-like lobes of a reddish colour, scored with fainter lines. When fully expanded, the oral disc is not less than four inches across; there is a smooth space between the mouth and tentacula, which are very numerous, and placed in several rows around the circumference; those of the inner row are larger than the others, measuring frequently two inches in length; and they become gradually shorter in their exterior series. They are of a chestnut or reddish flesh-colour, often darker coloured towards the bases, but never variegated with rings of different hues; thick and clumsy, tapered towards an obtuse point, marked longitudinally with distinct lines or impressed strix, tubular; perforated at the ends, and constricted at their insertions. The creature has no power of withdrawing them within the oral aperture, nor does it seem capable even of shortening them in any considerable degree; but it twists them in a wreathed or spiral form, or gires the whole circle a greater or less degree of expansion.

Anthea, a flower, is a name applied by Dr. Johnston to a branch of the order Helianthoida. Anthea Cereus is of a pretty light-brown colour, haring a somewhat cylindrical furrowed body. About 200 feelers arise from the disc of the animal, which, when expanded, are longer than the body, and of a bright sea-green colour tipped with red. But in some kinds this colour varies. They are common on the Cornwall coast.

Ellis says, "Their tentacula being disposed in regular circles, and tinged with a variety of bright lively colours, very nearly represent the beautiful petals of some of our most elegantly fringed and radiated flowers, such as the carnation, marigold, and anemone."

In the Transactions of the Leeds Philosophical and Literary Society there is a very excellent paper "On the Anatomy of Actinia coriacea, by Mr. T. P. Teale," that expresses the characteristics of this order"; an account which is corroborated by the investigations of Dr. Johnston.

"The appearance of the body of the Helianthoida is that of a short cone or cylinder, flat at the base and dimpled, with the oral opening at the top. Different shaped tentacula spring from between the lip and the border of the disc. When the animal contracts, and sinks as it 
were within itself, this border hides the mouth and feelers, leaving only a slight indentation visible. From the mouth there is a short wide throat to the stomach, which is a large plaited bag, divided into two directly downwards by a deep smooth furrow; these furrows are," says Mr. Teale, "produced on each side by the firm adherence of the gastric membrane to a pair of very dense, fleshy, but narrow, leaflets throughout their whole extent, or, in other words, from the top to the bottom of their internal border. These depressions divide the animal into two lateral halves, constituting a bi-lateral symmetry in Actinia, as has been observed by M. Agassiz in other supposed radiated animals." The animal possesses the singular property of being able to thrust out its stomach beyond the mouth, and there it hangs like a bladder over the exterior of its body. There appears to be no other outlet from the stomach than the mouth; and from it issues the refuse of the food covered over with a gummy substance. Between the stomach and the skin of the animal are thin plates of a muscular texture parallel to, and radiating from, the centre, like the gills of a mushroom on its stalk,-a comparison the more exact, as some only of the lamellæ reach and touch the stomach, the rest coming more or less short, and forming consequently imperfect interseptal spaces.

"The breadth of the leaflets varies considerably, some extending scarcely a line from their external attachment, others reaching as far as the stomach, being nearly half an inch in breadth. The height generally corresponds with the height of the animal; a few, however, of the narrowest leaflets, extending upwards from the base, terminate obliquely in the sides, without being prolonged as high as to the lip or roof." The use of this delicate structure seems to be that of giving the various shapes to the animal, to hold the ovaries, and some white alender threads whose purposes are unknown: these latter are no doubt tubular; but Mr. Teale says, under the microscope they appear simply as a round, solid, translucent cord.

"The epidermis forms a thin layer of unorganised matter spread over the whole extent of the corium, and may be traced into the stomach. The external surface of epidermis is dense and membraneous; internally, when examined by the lens, it appears as a pulpy substance. Intimately intermixed with it, in irregular patches, and not constituting a distinct or separate layer, is a pigment varying in colour in different parts of the same animal, and in different individuals. This colouring matter is extensively distributed over the base, sides, tentacula, and roof; but I have never observed any trace of it in the stomach." "The surface," writes Dr. Johnston, "is either smooth, or studded over with glandular warts; which, having an adhesive quality, 
enable the creatures the more completely to conceal themselves, by enduing the body with an extraneous coat formed of the sand, gravel, and broken shells, which lie around their peculiar localities. This is exchanged in the madreporous tribes for the more perfect defence which a hard coral affords, into whicb the soft parts are withdrawn at will. When a helianthoid polype is at rest and unalarmed, it can dilate the body to fully twice its ordinary bulk by imbibing water through the mouth or tentacula, the bases of which open in the spaces between the perpendicular lamellæ. These spaces being filled, the water is then made to permeate the rim of the oral disc, which is full of carities and cells for its reception; and the tentacula are in the same manner distended, the water being forced into them from behind, while the little opening on their tips is held close. The whole animal is thus distended to a wonderful degree, and every organ stretched out and displayed; the tentacula spread out in quest of prey, the skin rendered alnost clear from very fulness; and the stomach, pushed beyond its natural bounds, lies orer the sides in swollen diaphanous lobes. The water thus introduced is doubtless subservient to the purposes of respiration; and to aid this, the vermiform filaments, and the interual surface of the stomach and tentacula, are clothed with vibratile cilia, exciting and directing currents orer the surfaces. Since, too, the contents of the stomach must be fully exposed to the influence of the water, the nutrient parts may be by its means fitted for more immediate assimilation; for as there is neither circulating nor lymphatic systems, the absorption of the nutrient fluids must be made directly from the stomach itself. By the contraction of the periphery of the body, this water is again expelled at pleasure through the tentacula in a continuous stream or in jets; and if the contraction is sudden and strong, the water may be thrown out with such force as to rise to the height of at least a foot. It is remarkable that the water does not escape from all or the greater number, but only from a few of the tentacula. Whether any part escapes by the mouth is doubtful."

The ova, when examined by the microscope, are found of raried forms; after being ejected by their parent, they move swiftly about by means of their cilia for a few days, when, their organs of active motion being lost to them, they become fixed to a spot, where they attain maturity.

\section{LUCERAARIA.}

These beautiful and singular animals may be seen swimming quickjy through the waters, generally adhering to sea-weed, and spreading 
out and contracting their bodies as they seize their prey; for as soon as the suckers at the end of their feelers have got a little animal within its power, it is carried to the mouth, and the body contracts to enclose and consume it.

They are of a jelly-like nature, with a smooth and thickish skin; their bodies are arborescent, with bell-shaped cells, having a small sucker at the bottom, and are divided interiorly into eight compartments as others of the Actinix.

Lucernaria Campanulata.-This graceful animal is about an inch in height, of a bell-shape, terminating in a sucker resembling the stand of a stalked drinking-glass. The upper part is indented by eight short processes or arms, stretching upward, and terminated by a delicate tuft of a blossom-like appearance; these, about sixty in number, are glands or suckers, by which prey is caught. Its colours are various and rich. The interior is hollow like a flower, in the centre of which a square mouth is seen; from this seems to spread four leaves, which add to the beauty of the appearance.

Dr. Johnston mentions in the British family of Lucernaria, $L$. fascicularis and L. auricula; they differ but little from L. campanulata. They propagate by ova, which are seen as two rows of spots in the arms that extend around the mouth; but in what manner they are launched forth is not known.

\section{BRYOZOZ.}

This order of Zoophytes is placed by Dr. Johnston under the head Ascidioida; in the generality of works they are named Bryozoa, and the individual, Bryozoon; derived from the Greek words ßpviov,

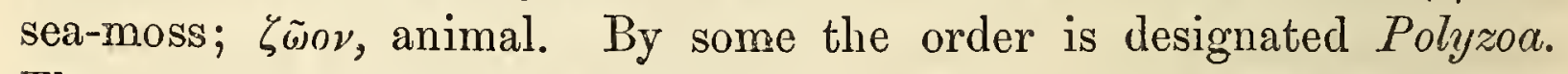
The grand distinction between it and those already described, consists in the polypidom being a living portion of the polype, while all others are unorganised; and that, whilst all other species we have examined are devoid of cilia, in the Bryozoc these are most bountifully supplied. The play of the cilia is most energetic, for the purpose of securing an abundant supply of food, almost without exertion on the part of the creature itself. From this most marked characteristic, Dr. Farre was induced to give them the name of Ciliobrachiata. (Plate I. Nos. 1, 2.)

"The connection of the polypidom with the soft portion of the polype is effected," says Dr. Johnston, "by means of an inner tunic, which, after inclosing the polype's body as in a pouch, is afterwards reflected over the aperture of the cell,-the reflected portion be- 
coming exterior and solidified, either by calcareous depositions in its texture, or by a mutation of its thin membraneous character into a horny investment better suited to the office it has now to perform, of protecting the sentient body from a too rough contact of the medium in which the animals live, and from worse foes. From this mode of connection it results, that when the polypes retire within, they at the same time must close the aperture to their cells; for that portion of the inner tunic which is pushed outwards by their exit, in their withdrawal follows the body by a process of invagination, becoming at one and the same time a sheath for the column of tentacula, and a plug to the aperture, which, when of a flexible material, has its margins also dratin tighter and closer together."

Milne Edwards, in writing on the Eschares, remarks, "that the cell in which it is said the polype retires as into a shell is a component part of the aumal itself, in which it conceals itself, if we may use the comparison, as the hedgehog enters into the thorny skin of his back. It is not a calcareous crust, which is moulded on the surface of its body, but a portion of the general tegumental membrane of the skin of the polype, which, by a molecular deposit of earthy matter in the meshes of its tissue, ossifies as the cartilages of superior animals ossify, without ceasing to be the seat of the nutritive movement."

Bryozoc are never found in a separate condition, and are always clothed with hardy corerings or polypidoms. They subsist ou animal bodies, and differ from mollusca in being able to protrude themselves from their cells. When the animals draw themselves within their protective homes, to the bottom of which they are attached by a sinewy ligament, they double themselves up by bending the lower part of the body upwards. When the creature stretches forth, it presents a beautiful sight, from its blossom-like appearance and busy cilia; its protrusion and retraction are performed with surprising quickness, as it has two sets of muscles for the purpose, one acting on the body of the animal, the other upon its cell. The oral extremity is surrounded by a circle of long tubular tentacula corered with cilia; at each of these feelers or arms there is an aperture, the one at the base communicating with a canal that passes round the edge of the oral aperture or mouth. There are no jaws or teeth, and the food passes down a long gullet, that contracts during the process of swallowing. At the end of this is an orifice that opens into what appears to be a gizzard, having two bodies opposite to each other, with a rough surface, as if for the comminution of food, moved by muscular fibres. Those of the species without this gizzard have a digestive stomach that secretes a coloured fluid. From the upper part 
of the stomach near the entrance from the gizzard arises an intestine, having a narrow opening surrounded by cilia that proceeds upwards, ending in an orifice near to the tentacula, from which the refuse food is ejected. "No trace of either nerves or ganglia," says Dr. Farre, " could be detected; yet the attributes of a nervous system were so clearly exlibited as to leave no doubt but that this must exist, and probably in some degree of perfection. Not only was the delicacy of their sense of touch very strongly marked, but the operations also consequent upon the enjoyment of such a sense were sometimes singularly striking. This is seen in the instant retiring of the animal on the slightest alarm, and the caution which it sometimes shows before emerging again from its cell ; in the obvious selection of its food, and in the pertinacity with which it refuses to expose itself to water that has become in the least degree deteriorated." Ellis, in reference to a branch of Vesicularia that he examined, says "he could plainly distinguish that the internal hollow part of the whole coralline was filled with the substance of the parent polype, which appeared to be of a tender gelatinous nature; and upon the least extension or contraction of the young polypes, this tender fleshy substance was visibly affected; for each one was united to it by their lower part or tail."

The cells are of various shapes, and from one, grow into a family of millions, budding forth from the sides; and though the living matter disappears, the catacombs exist for the foundation of their families, branching out and enduring for ages.

Bryozoa Bowerbankia received its name from Dr. Arthur Farre, in honour of the well-known microscopist, Mr. Bowerbank; who has distinguished himself in more than one department of natural history. A magnified representation of the expanded polype is seen in the frontispiece, Plate I. No. 1.

Dr. Farre gives the following description of Bowerbankia densa: "When fully expanded, it is about one-twelfth of an inch in length. In its retracted state, it is completely inclosed in a delicate horny cell, sufficiently transparent to admit of the whole structure of the contained animal being seen through its walls. The cells are connected together by a cylindrical creeping stem, upon which they are thickly set, sessile, ascending from its sides and upper surface. The animal, when completely expanded, is seen to possess ten arms of about one-third the length of the whole body; and each arm being thickly ciliated on either side, and armed at the back by about a dozen fine hair-like processes, which project at nearly right angles from the tentacula, remaining motionless, while the cilia are in constant and active vibration." 
FLUSTRE. ESCHARÆ.

These are very interesting families, well deserving the great attention they have received from naturalists.

The most common polypes found on our coast are named Flustrce by Linnæus, from the Saxon word Flustrian, to weave; commonly called a Sea-mat, they are like fine net-work spread over stones, rocks, shells, and marine-plants. This species, when submitted to the powers of the microscope, is found to be a cluster of cells, in each of which dwells an animal, that protrudes its feelers when searching for food, and sinks into its little home when tired, or fearful of danger.

Dr. Grant estimates that a single Flustra has as many as four hundred millions of these useful and restless appendages. The feelers vary from ten to twelve; their organisation consists of a long gullet, a gizzard, a stomach and intestines; the body being of a transparent substance. Some take the form of a delicate minute tree, having cells in all parts; and vary in colour, inliabiting every sea. Lamouraux says: "When the animal has acquired its full growth, it flings from the opening of its cell a small globular body, which fixes near the aperture, increases in size, and soon assumes the form of a new cell; it is yet closed, but through the transparent membrane that covers its surface, the motions of the polype may be detected; the habitation at length bursts, and the tentacula protrude; eddies are produced in the water, and conduct to the polype the atoms necessary for its subsistence."

Dr. Graut writes: "The aperture of the cells is formed by a semicircular lid, convex externally and concave internally, which folds down when the polype is about to advance from the cell. The opening of this lid in the F. truncata, where it is very long; appears through the microscope like the opening of a snake's jaws; and the organs by which this motion is effected are not perceptible. The lids of the cells open and shut in the Flustræ without the slightest perceptible synchronous motion of the polypi."

In the formation of their stony skeletons, the animals appear to take no part, "being secreted by the integuments or membranes, with which it was permeated and invested, in like manner as the bones and nails in man are secreted by the tissues designed for that purpose, and acting without his knowledge or control. From an analysis of the stony corals, it appears that their composition is very analogous to that of shells. The porcellaneous shells, as the cowry, are composed 
of animal gluten and carbonate of lime, and resemble, in their mode of formation, the enamel of the teeth; whereas the pearly shells, as the oyster, are formed of carbonate of lime and a gelatinous or cartilaginous substance, the earthy matter being secreted and deposited in the interstices of a cellular tissue, as in bones. In like manner, some corals yield gelatine upon the removal of the lime, while others afford a substance in every respect resembling the membraneous structure, obtained by an analysis of the nacreous (pearly) shells. A recent elaborate analysis of between thirty and forty species of corals, by an eminent young American chemist (Mr. B. Silliman), has shown, contrary to expectation, that they contain a much larger proportion of fluorine than of phosphoric acid."

Flustra Foliacea is the broad-leaved Horn-wrack of Ellis; it is about four inches high, brown, and the polypidom is horny. The cells are small, in alternating rows; sometimes covered by a lid that opens downwards. Hook says: "For curiosity and beauty, I have not, among all the plants or vegetables I have yet observed, seen any one comparable to this sea-weed." Flustra truncata is abundant in deep water, and grows to a height of about four inches; it is of a delicate yellow colour, and bushy. The cells have lines in an oblong direction; it is the narrow-leaved Horn-wrack of Ellis.

Flustra Chartacea.-Ellis states: "The cells of this sea-mat are of an oblong square figure, swelling out a little in the middle of each side. The openings of the cells are defended by a helmet-like figure; from hence the polype-shaped suckers extend themselves. This seamat is of a slender and delicate texture, like a semi-transparent paper of a very light straw colour. It was first found on the coast of Sussex, adhering to a shell. I have since met, on the same coast, about Hastings, in the year 1765, with several specimens, whose tops are digitated, and others that were very irregularly divided."

Flustra Carbasea grows out in a leaf-like manner, gradually widening to the end. It is found on shells of a yellowish-brown colour; on one of the sides the cells are both large and smooth. The animals have about twenty-two arms or feelers, which Dr. Grant, who has written a most careful paper on these polypes, says, are "nearly a third of the length of the body; and there appear to be about fifty cilia on each side of a tentaculum, making 2,200 cilia on each polype." In this species there are more than eighteen cells in a square line, or 1800 in a square inch of surface; and the branches of an ordinary specimen present about ten square inches of surface; so that a common specimen of the $\mathrm{F}$. carbasea presents more than 18,000 polypi, 396,000 tentacula, and 
39,600,000 cilia. The ova of the F. carbasea make their first appearance as a small yellow point, a little below the aperture of the cell, and behind the body of the polype; they are unconnected with the polype, and appear to be produced by the posterior wall of the cell, in the same manner as the axis, or common connecting substance of polypi, produces them in other zoophytes. In this rudimentary state they are found in the same cells with the healthy polypi; but, before they arrive at maturity, the polypi of such cells perish and disappear, leaving the entire cavity for the development of the ovum. There is never more than one ovum in a cell, and it occupies about a third of the cavity, when full-growr and ready to escape. When first visible, it has a round or slightly oblong and regular form; when mature, it is ovate, with the small end next the aperture of the cell. The ova do not appear in all the cells at one time; nor is there any discernible order as to the particular cells which produce ova, or the part of the branch which contains them. Cells containing ova are found alike on every part of the branches, from the base to within two or three rows of the apex occupied only by young polypi.

"In the empty cells from which the ova have escaped, we frequently observe a few remains of the former polype lying at the place where the body of the polype bifurcated, and where the principle connection seems to exist between the polype and the axis; we likewise perceive numerous monades and other animalculæ busily employed in consuming the remains of the dead polype. The ovum, even before arriving at maturity, exhibits very obvious signs of irritability, frequently contracting different parts of its surface, and shrinking backward in its cell; the cilia on its surface are likewise observed in rapid motion within the cell, as in the ciliated ova of other zoophytes. The mature ova are often found with their small end projecting from the opening of the cells, and their final escape is aided by the incessant vibrations of the cilia covering their surface, by the ova contracting themselves in their lateral direction, by the waves agitating the branches of the flustra, and by the same incomprehensible laws which regulate the formation and growth of the ova, and the whole economy of this zoophyte."

When liberated, the ova seem by their motions endowed by both will and perception, as they commence their career in the wide watery world. Dr. Grant, in writing more especially of the F. carbasea, says : "They are very irritable, and are frequently observed to eontract the circular margin of their broad extremity, and to stop suddenly in their course when swimming; they swim with a gentle gliding motion, 
often appear stationary, revolving rapidly round their long axis, with their broad end uppermost, and they bound straight forward, or in circles, without any other apparent object than to keep themselves afloat till they find themselves in a favourable situation for fixing and assuming the perfect state. The transformation of the ova, from that moving, irritable, free condition of animalculæ, to that of the fixed and almost inert zonphytes, exhibits a new metamorphosis in the animal kingdom not less remarkable than that of many reptiles from their first aquatic condition, or that of insects from their larva state."

Flustra Avicularis. - This is another of the little beauties of the deep found usually on old shells, an inch in height, spreading itself fanlike, and of an ashy colour, deeply divided in a dichotomous manner into narrow, thin, plane segments ; truncate at the end, formed of four or five series of oblong cells, capped with a hollow, globose, pearly operculum seated between the spines, of which there is one on either side of the circular aperture. The opercula are so numerous that they give to the upper-surface the appearance of being thickly strewn with orient pearls: the under-surface is even and longitudinally striated, the number of striæ corresponding to the number of rows in which the cells are disposed. Dr. Johnston describes, amiongst many other British species, $F$. membranacea, "a gauze-like incrustation on the frond of the sea-weed, spreading irregularly to the extent of several square inches."

The Escharce resemble Flustrix in most points, except in their having a skeleton dense and stony, containing in its substance a great quartity of earthy matter. In the frontispiece, No. 2, the animal itself is drawn out of its abode, with its long tentacles protruded around its mouth to seize its prey: to these tentacles succeeds a long digestive tube, with a perfect muscular structure given off on either side.

Eschara Foliacea._-"This curious polypidom," writes Dr. Johnston, " attains a large size, being often three or four inches high, and from twelve to twenty inches in diameter. It may be described as a broad membrane, twisted into winding folds, leaving large sinuosities and cavernous interstices; it is very light, and floats in water; crisp when dry, membrano-calcareous, cellular, of a yellowish-brown colour, roughish, and punctured with the numerous pores which open on both sides. The membrane is less than a line in thickness, and consists of two layers of cells, separated behind from one another by a thin plate down the middle. The cells open obliquely by contracted roundish apertures disposed in a quincunx order on the surface, and which, more especially when recently formed, are often covered by a small operculum. When 
a portion is macerated in diluted muriatic acid, it retains the original form, but becomes soft and flaccid from the subtraction of the carbonate of lime. The cells are liable to all the changes of form, resulting from age, upon these polypidoms in general."

Eschara Fascialis.-This is the Italian coral of Ellis. Solander says: "This millepore grows in very irregular masses, but always preserves the same habit of growing; that is, the branches are flat, narrow, and regularly subdivided: they coalesce, twist, and branch out again leaving certain hollow spaces between them; their cells are much smaller, though of the same shape, with the cells in the foliaceous millepore (E. foliacea)."

\section{VESICULARIAD河.}

This family is so named from some supposed resemblance to a bladder. Dr. Farre thus describes them in the Philosophical Transactions: "The transparent horny cell which closely embraces the body of the animal is nearly unyielding in its lower two-thirds, but terminates above by a flexible portion, which serves to protect the upper part of the body when the whole is expanded, in which state it is of the same diameter as the rest of the cell ; but when the animal retracts, is folded up and drawn in after it, and completely closes the mouth of the cells.

"The flexible part consists of two portions, the lower half being a simple continuation of the rest of the cell; the upper consisting of a row of delicate bristle-shaped processes or setæ, which are arranged parallel with each other round the top of the cell, and are prevented separating beyond a certain distance by a membrane of excessive tenuity, which surrounds and connects the whole. This mode of termination of the cell is one of constant occurrence, as will be described in other species, and is evidently a provision for allowing of the freest possible motion of the upper part of the body in its expanded state, to which it affords at the same time support and protection."

Vesicularia Spinosa appears as a beautiful delicate slender tree, full of tubular jointed branches, and rising to a height of from four to eight inches. The polypidoms are rooted by a fibrous base, and three oval transparent cells are placed between each joint, which fall off like the leaves of a tree. The little animals have eight tentacula furnished with cilia. The branches, says Dr. Johnston, "are perforated with a single series of rather distant holes, with a raised rim, as if they had been bored from within outside." Ellis remarks: "The motion of the intestines of the young polypes was rery distinguishable till the water became 
putrid; and then both vesicles and polypes dropped off like blighted blossoms off a tree; and the substance of the parent polype, though seeming to fill the whole cavity of the branch before, as soon as the water became improper for its support, shrivelled up immediately, so as scarce to be visible."

Valkeria have cells that are clustered in some, and scattered in others; and the polypes have no gizzards. $V . u v a$, is usually found on fuci and other corallines. When seen by the microscope, there appear clusters in several parts like bunches of grapes. Ellis says: "Among many other marine substances received fresh from the sea, in September 1753, this object happened to present itself under my magnifying-glasses ; when, to my surprise, I found those grape-like bodies were a cluster of polypes, each having eight claws or tentacula, very lively, extending themselves about in pursuit of prey; and upon their dying, the animals contracted themselves into their vesicles, which closed at the top."

The other British Zoophytes named of this family by Dr. Johnston, are V. cuscuta, $V$. imbricata, and $V$. pustulosa.

The wonders we have brought to view in our glance at the families constituting the zoophytic kingdom, must produce astonishment in those to whom the book of nature has hitherto been closed. We have seen how wonderfully and fearfully they are made; we have seen the surprising faculties with which they are endowed; we have seen the perfection and order bestowed upon them; we have seen the singular faculties they possess to perform the allotted duties of their destiny; and seeing all these things, we read in plain intelligible language the wisdom, power, and goodness, of an almighty and beneficent Being. We may here state, that we have taken a somewhat more extended view of this department of animated nature, from the very circumstance of its presenting a wider field of deeply-interesting and curious subjects for inquiry to the microscopist; and the more especially have these subjects, during the past year, attracted great public attention, from the spirited attempt on the part of the Zoological Society of London, to afford us the opportunity of more closely and frequently observing their remarkable habits, in what has been called marine vivaria. In our frontispiece we have attempted to represent one of these glass cases, merely adding a few magnified figures of the Bowerbankii and allied species. There the visitor may see the Sabellce, the Actinice, of brilliant hues, and many kinds; Mollusca, both shelled and naked; Crustacea, Annellida, \&c. ; all pursuing their various avocations, and enjoying themselves without restraint, under circumstances scarcely distinguishable from 
those of nature. Mr. Warrington and $\mathrm{Mr}$. Gosse made the experiment about the same time; and from Mr. Gosse's recent delightful book, entitled A Naturalist's Rambles on the Deronshire Coast, we extract the plan he adopted to preserve his marine animals for many months in London. He says: "The following facts may be considered as established: marine animals and plants may be kept in health in glass rases of sea-water for a period of greater or less length, provided they be exposed to the influence of light. The oxygen given off by healthy regetation under this stimulus, is sufficient for the support of a moderate amount of animal life; and this amount can be ascertained by experiment.

"But another element in the question soon obtrudes itself. The Actiniæ and other animals habitually throw off a mucous epidermis, and other excretions, which fall to the bottom of the ressel, or accumulate around them. The process of actual decay also continually goes on in the older fronds of the algæ; after a while the presence of this substance becomes too manifest in the offensire odour of the water; in very short time this leads to disease, and the death of the animal. To prevent this, it is absolutely necessary to present the water to the action of the atmosphere in a divided state, which can only be effected by passing it in driblets, or in a slender stream, from another vessel suspended above that which contains the animals and plants. Another advantage is secured by this process, viz. the aeration of the water; for though the requisite oxygen may be supplied by the agency of the plants alone, the mechanical admixture of the atmospheric air with the water by artificial aeration is highly conducive to the health and comfort of the animals."

We must, as a fitting addenda to the above, give the plan pursued by Mr. Gosse, to establish his marine virarium on a small scale. "The first thing to be done, is to obtain the alga in a growing state. As they have no proper roots, but are generally found closely attached to the solid rock, from which they cannot be torn without injury by laceration, I have always used a hammer and chisel to cut away a small portion of the rock itself, haring ready a jar of sea-water, into which I dropped the fragment with its living burden, exposing it as little as possible to the air. The red sea-weeds I have found most successful; the Fuci and Laminarice, besides being unwieldy, discharge so copious a quantity of mucus as to thicken and vitiate the water. The Utrce and Enteromorphce, on the other hand, are apt to lose their colour, or become a colourless membrane, and decay from their attachments. The species most capable of being preserved are Chondrus 
crispus, the Delessaria, and Iridea edulis. The last named is the best of all, and next to it the Delessaria sanguinea, for maintaining the purity of the water; while the colours and forms of these render them very beautiful objects in the vase. Into this were placed specimens of Anthea cereus, Actinia, Crisia denticulata, Coryne, Pedicellina Belgica, Mernbranipora pilosa, Doris, Polycercb, Serpula, Acarida, Entomostraca, Infusoria, Grantia nivea, and other smaller zoophytes and sponges." Most of these appeared to enjoy existence, and afforded, no doubt, months of most interesting microscopic examinations; at the same time they form a beautiful and attractive ornament for the conservatory or drawing-room.

Mr. Warrington, in his trials, found that it was not sufficient that there be plants alone; but where the higher animals, such as fish, are kept, it is necessary that some beings should be introduced for the purpose of feeding on the decaying vegetable matter. This desideratum is supplied by the various forms of phytophagous mollusca. So that, to maintain perfect health, it is necessary to place in the glass seaweeds, mollusca, fish, \&c.; but those should not be put together which will devour each other. The sea-water should be kept near the same temperature always, about $60^{\circ} \mathrm{Far}$; and the loss by evaporation supplied by adding rain or distilled-water from time to time. All dead animal or vegetable matter should be removed, and the glass vase kept near the window, exposed to the influence of light.

\section{PORIFERA.}

\section{SPONGES.*}

The term Porifera, or Canal-bearing Zoophytes, was applied by Professor Grant to designate the remarkable class of organised beings

\section{* Description of Plate IV.}

1. Portion of sponge, showing siliceous spicula imbedded in a soft matrix. 2. Skeleton of sponges of the acerate-form, covered with rows of spines. 3. Showing rings of growth, and a portion of horny fibre, enclosing a bundle of spicula of the genus Verongia. 4. Sphero-stellate spicula of Tthea. 5. Portion of sponge mounted in Canada balsam, showing the spicula more plainly. 6. Tricuspid-anchorate spicula, sphero-stellite. 7. Acuate-biclavate, double recurvo-ternate, expando-ternate, detriradiate spicula. 8. Gemmules of Geodia. 9. Gemmules of Spongilla fluviatitis enclosed in spicula. 10. Clavate spicula, covered with short spines. 11. Gemmules of Geodia in an advanced stage of growth. 12. Birotulate spicula from the Fluviatilis. 13. Gemmules of Spongilla fluviatilis immersed in acid, to show its coating of birotulate spicula. 
PLATE IV.
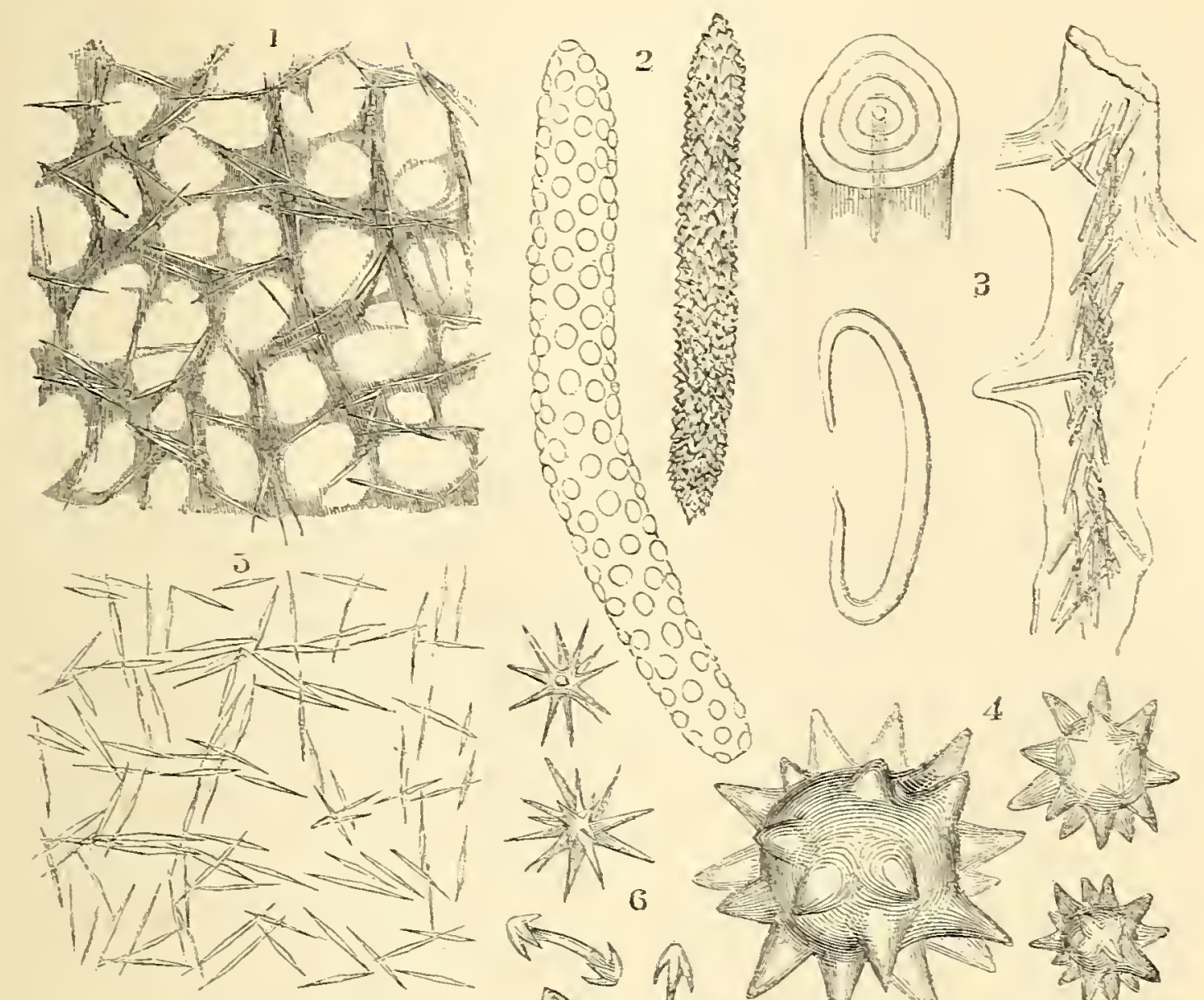

a
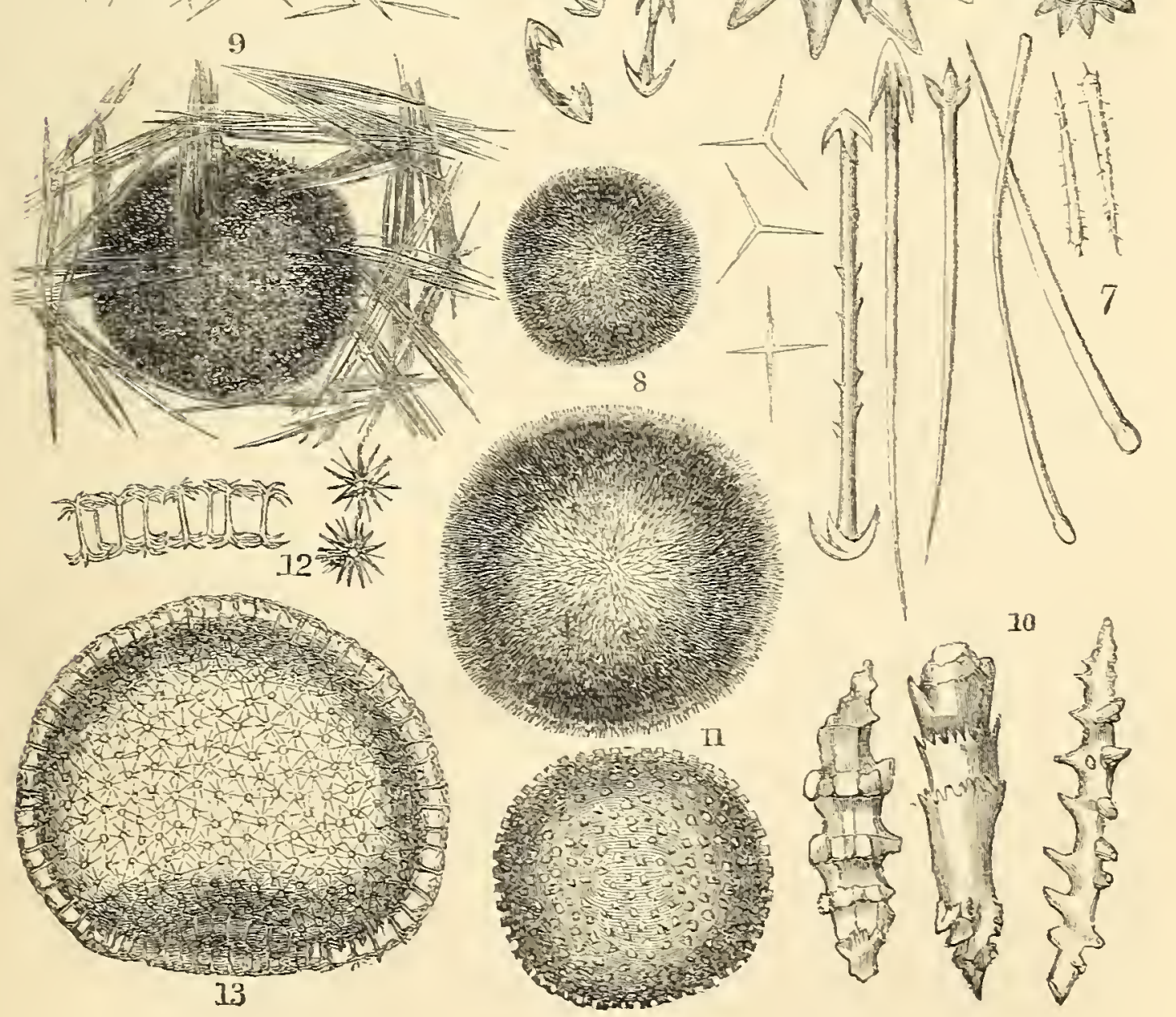

SPICUL玉 FROMI SPONGES. 

usually known as sponges, which are met with in most seas, growing in great abundance on the surface of rocks.

Ellis, in the course of his investigations, was astounded by discovering that sponges possessed a system of pores and vessels, in which sea-water passed, with all the appearance of the regular circulation of fluids in animal bodies, and a seeming purpose of conveying animalculæ to the animal for food. Professor Owen gives it as his opinion that sponges should be placed in the vegetable kingdom: "The living sponge, when highly magnified, exhibits a cellular tissue, permeated by pores, which unite into cells or tubes, that ramify through the mass in every direction, and terminate in larger openings. In most sponges the tissue is strengthened and supported by spines, or spicula of various forms; and which, in some species, are siliceous, and in others calcareous. The minute pores, through which the water is imbibed, have a fine transverse.gelatinous net-work and projecting spicula, by which large animalculæ or noxious particles are excluded; water incessantly enters into these pores, traverses the cells or tubes, and is finally ejected from the larger vents. But the pores of the sponge have not the power of contracting and expanding, as Ellis supposed; the water is attracted to these openings by the action of instruments of a very extraordinary nature (cilia), by which currents are produced in the fluid, and propelled in the direction required by the economy of the animal."

On the disputed point of sponges being animals, Dr. Johnston remarks: "Although I agree with the advocates of the animality of zoophytes in general, I cannot go the length of Ellis in considering it proved that sponges and corallines belong to the same class. Ellis, we have seen, knew that no polypes were to be found in sponge; and their existence in the pores of corallines was inferred merely from the structure of these latter, and their chemical composition. They have been examined by subsequent naturalists fully competent to the task; and under the most favourable circumstances-in particular by Cavolini and Schweigger-and the result has been a conviction that these productions are truly apolypous. Now this fact, in my opinion, determines the point; for if they are not the productions of polypes, the zoologist who retains them in his province, must contend that they are individually animals; an opinion to which I cannot assent, seeing that they have no animal structure or individual organs, and exhibit no one function usually supposed to be characteristic of that kingdom. Like vegetables, they are permanently fixed; like vegetables, they are non-irritable; their movements, like those of vegetables, are extrinsical 
and involuntary; their nutriment is elaborated in no appropriate digestive sac; and, like cryptogamous vegetables, or algæ, they usually grow and ramify in forms determined by local circumstances; and if they present some peculiarities in the mode of the imbibition of their food, and in their secretions, yet even in these they evince a nearer affinity to plants than to any animal whatever."

Müller writes on this question: "If, therefore, it is still a matter of doubt, whether certain simple organised beings, such as the Sponges and several so-called Alcyonella, are animal or vegetable, the absence of all voluntary motion in these bodies, whether of the whole or of individual parts of it, must determine the question; and they must more properly be numbered among the regetable marine structures. It may certainly be said, that the embryo of sponges (as Dr. Grant has shown), like the embryo of polypes and corals, moves by means of ciliæ; but the distinctive marks between the embryo of sponges and marine infusoria are by no means certain, and similar motions have been many times observed in the embryo of true vegetables,-of the algæ for example."

Again, we read that "M. Dujardin having repeated his observations on spongillæ or fresh-water sponges, as well as others on marine sponges, thinks he has proved that these ambiguous beings are positively groups of animals, capable of contraction and extension. If a piece be detached from a living sponge, and submitted to a microscope, it will be seen to group itself into irregularly-rounded masses, and change the form of its edges incessantly: isolated portions, detached from the general mass, move slowly in the liquid, and creep along by means of their alternate contraction and expansion."

The description given of sponges by Dr. Jolnnston is, that they are "organised bodies growing in a variety of forms, permanently rooted, unmoving and irritable, fleshy, fibro-reticular, or irregularly cellular; elastic and bibulous, composed of a fibro-corneous axis or skeleton, often interwoven with siliceous or calcareous spicula, and containing an organic gelatine in the interstices and interior canals; they are reproduced by gelatinous granules called gemmules, which are generated in the interior, but in no special organ. All are aquatic, and with few exceptions marine." The same author says: "Mr. J. Hogg, in a letter dated June 25, states that the green colour of the fresh-water sponge (spongilla fluviatilis) depends upon the action of light,- -as he has proved by experiments which showed that pale-coloured specimens became green when they were exposed for a few days to the light and full rays of the sun; while, on the contrary, green specimens were blanched by being made to grow in darkness or shade. He therefore infers the 
vegetable nature of this sponge; but leans to the opinion that the sea sponges are animals."

Dr. Lankester has given a somewhat better definition for the determination of animal and vegetable life than other investigators. $\mathrm{He}$ proposes that the general fact of vegetables giving off oxygen gas and absorbing carbonic acid, whilst animals absorb oxygen and give off carbonic-acid gas, should be generally admitted to constitute the true line of demarcation between the two, and would thus remove the objections raised by those who will not admit the presence of starch as sufficient to determine the point. Professor Grant has more recently examined the sponges; and from his patient and careful observations of them, they have been finally classed amongst the animal creation. $\mathrm{He}$ ascertained that the water was perpetually sucked into the substance of the sponge through the minute pores that cover its surface, and again expelled through the larger orifices. His own account is so very interesting, that we cannot resist giving, in his own words, the results arrived at in these investigations: "Having placed a portion of live sponge (Spongia coalita, fig. 91, No. 1) in a watch-glass with some sea-water,
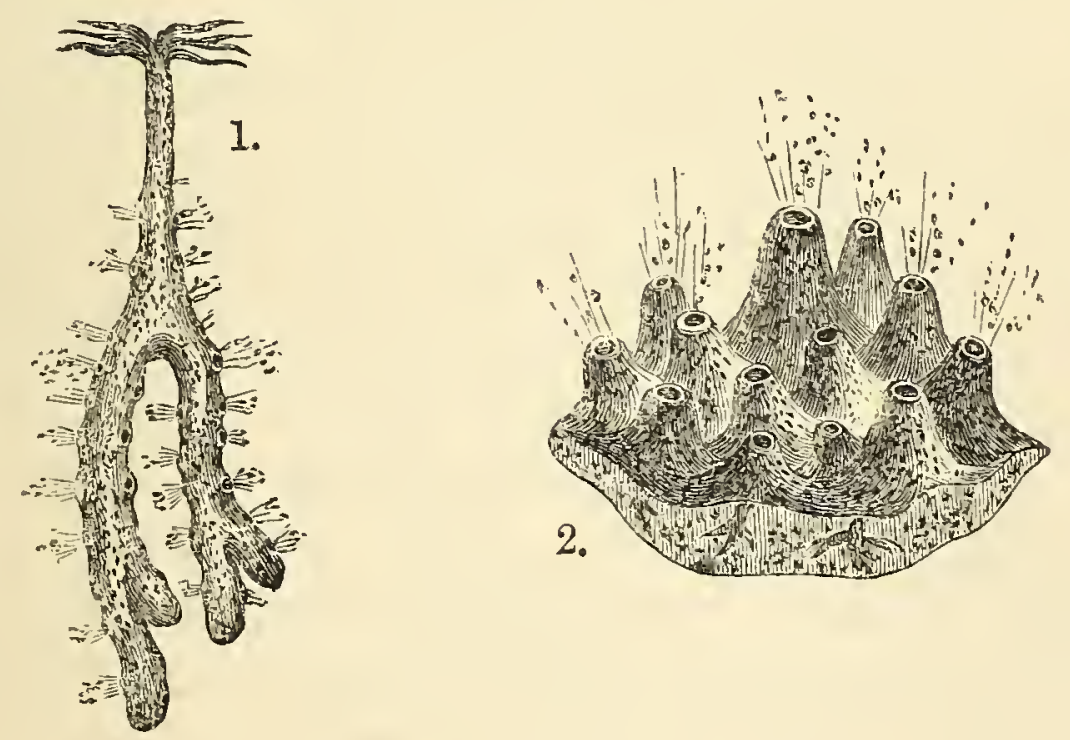

fig 91. I. Sipongia coalita. 2. À piece of Spongia panicea highly magnified:

I beheld for the first time the splendid spectacle of this living fountain, represented in fig. 91, No. 2, vomiting forth from a circular cavity an impetuous torrent of liquid matter, and hurling al@ng in rapid succession opaque masses, which it strewed every where around. The beauty and novelty of such a scene in the animal kingdom long arrested my attention; but after twenty-five minutes of constant observation, I was obliged to withdraw my eye from fatigue, without having seen the torrent for one instant change its direction, or diminish the rapidity of 
its course. In observing another species (Spongia panicea), I placed two entire portions of this together in a glass of sea-water, with their orifices opposite to each other at the distance of two inches; they appeared to the naked eye like two living batteries, and soon covered each other with the materials they ejected. I placed one of them in a shallow vessel, and just covered its surface and highest orifice with water. On strewing some powdered chalk on the surface of the water, the currents were visible to a great distance; and on placing some pieces of cork or of dry paper over the apertures, I could perceive them moving, by the force of the currents, at the distance of ten feet from the table on which the specimen rested."

Alcyonella. - This term distinguishes a sponge-genus found in masses inhabiting stagnant waters, and having "vertical aggregated membranaceous tubes opening on the surface;" sometimes it is discovered floating, and at other times incrusting pieces of wood and other substances.

Alcyonella Stagnorum is a dark green, shapeless, porous, jelly-like mass, filled with tubes, which have a thin lid or cover. Rows of ova appear in the tubes to a most extraordinary extent. Mr. Teale says this polype "is organically connected with the mass, the tube forming its tunic, from which the animated body issues by a process of evolution similar to that which developes the horn of a snail. When developed, the head projects a short way, and is crowned with a beautiful expansion of tentacula, about fifty in number, arranged in an unbroken circle, which is, however, depressed into a deep concavity on one of its sides, so as to produce the appearance of a double row of tentacula, in a horseshoe form. About 1,600 polypes are situated on a square inch of surface of the mass, consequently the number of polypes, in one specimen, which weighed 17 ounces, and measured $14 \frac{1}{2}$ inches in circumference, 'may be computed at 106,000, and the tentacula at 5,320,000!'"

Trembley gave an excellent and interesting account of one of the family of Alcyonella, as far as the powers of the microscope at that time allowed. He says: "This is one of the many kinds of water animals which live as it were in societies; of which some sorts hang together in clusters, but can detach themselves at pleasure; whilst others are so intimately joined and connected together, that no one seems capable of moving or changing place without affecting the quiet and situation of all the rest. But this creature forms as it were an intermediate gradation between the other two, dwelling in the same general habitation with others of its own species, from whence it cannot entirely separate itself; and yet therein it appears perfectly at liberty to exert its own 
roluntary motions; and can either retire into the common receptacle, or push itself out from thence, and expand its curious members, without interfering with or disturbing its companions.

"They dwell together from the numbers of ten to fifteen (seldom exceeding the latter or falling short of the former number,) in a filmy kind of mucilaginous or gelatinous case, which out of the water has no determined form, appearing like a lump of shime; but when expanded therein, resembles nearly the figure of a bell with the mouth upwards, and is usually about the length of half an inch, and one quarter of an inch in breadth or diameter. This case being very transparent, all the motions of its inhabitants may be discerned through it distinctly. It seems divided internally into several apartments, or rather, to contain several smaller Sacculi, each of which encloses one of these animals. The openings at the tops of these Sacculi are but just sufficient to admit the creature's head, and a very small part of its body, to be thrust beyond them, the rest remaining always in the case. The animal can, however, when it pleases, draw itself down entirely within the case, which is an asylum to secure it from its enemies (for it is not unlikely many of the larger aquatic insects prey upon it), and a safe and agreeable retirement, wherein to perform the functions of digestion, sleep, and the other necessary calls of nature. This case it can, I say, retire into at pleasure; and it never fails to do so when any sudden motion of the water or of the vessel it is in disturbs it, as also when it has seized with its arms any of the minute insects which serve for its food.

"The arms are set round the head to the number of forty, having each the figure of a long $f$, one of whose hooked ends is fastened to its head; and altogether, when expanded, compose a figure somewhat of a horse-shoe shape, convex on the side next the body, but gradually opening and turning outwards, so as to leave a considerable area within the outer extremities of the arms. And when thus extended, by giving them a vibrating motion, the creature can produce a current in the water, which brings the animalcules, or whatever other minute bodies are not beyond the sphere of its action, with great velocity to its mouth, whose situation is between the arms; where they are taken in if liked, or else, by a contrary current, which the creature can excite, they are carried away again; whilst at the same time other minute animalcules or substances that, by lying outside the enclosure made by the arms, are less subject to the force of the stream, are seized by them; for their sense of feeling is so exquisite, that on being touched ever so slightly by any such little body, it is caught immediately and conveyed to the mouth. However, one may observe, the creature is 
sometimes disappointed in its acquisition; for, after drawing down one of the arms suddenly inwards towards the mouth; it may be perceived slowly extending itself again without the creature's retiring into its case; which it fails not to do on meeting with any thing worth the while.

"The body of this animal consists of three parts or divisions, in the uppermost whereof all the afore-mentioned intestines are contained, though they are not to be distinguished when the creature is hungry; but after it has eaten they become distended and opaque, and may very plainly be discovered. The other two divisions (the lowermost of which I take to be fixed to the bell or outward case) seem of no other service than to give the creature power of contraction and extension.

"The arms seem not able, like those of the common polype, to contract or shorten themselves; but instead thereof, when the animal retires into his case, they are brought together in a close and curious order, so as easily to be drawn in. Their general figure, when expanded, is that of a cup, whose base and top are of a horse-shoe form; but they present sometimes a very different appearance, by separating into four parts, and ranging themselves in such sort as to represent four separate plumes of feathers.

"Besides the particular and separate motion each of these creatures is able to exert within its own case, and independent of the rest, the whole colony has together a power of altering the position, or even of removing from one place to another, the bell or common habitation of them all. Hence this bell is seen sometimes standing perfectly upright, sometimes bending the upper part downwards.

"It has been mentioned already, that between ten and fifteen of these animals dwell together, as it were a little community, in one bell-like case, or common habitation; but their numbers increasing, this bell may be observed to split gradually, beginning from about the middle of the upper or anterior extremity, and proceeding downwards towards the bottom, till they separate at last entirely, and form two complete colonies, independent of each other; one of which sometimes removes itself to another part of the vessel.

"The bells or colonies of these animals are to be found adhering to the large leaves of duckweed and other aquatic plants; and may easiest be discovered by letting a quantity of water, with duckweed in it, stand quietly for three or four hours in glass vessels, in some window or other place where a strong light comes; for then, if any are about the duckweed, they will be found, on careful inspection, extending themselves out of their cases, spreading their plumes, and making an elegant appearance. 
"They are extremely tender, and require no little care to preserve them; their most general disorder is a kind of slime or mouldiness, which will sometimes envelope them in such a manner as to prove mortal. The best way of curing this is by gently pouring a large quantity of water (perhaps two or three quarts) into the ressel where they are kept, and letting it run off slowly; by which means the sliminess will gradually be loosened, and carried away with the water.

"As to food, if fresh water be given them daily, they will find sufficient for themselves; and it is dangerous to try any other way of feeding them; for the smallest worms, or other visible insects one can think of giving them, will tear their delicate frame in pieces."

\section{LIMNIADES.}

Of this family, the only one observed is the Cristatella mucedo, or Crested IInstiness, by Sir J. G. Dalyell, who thus describes it: "Perfect specimens occur from six lines to twenty-four in length, by two or three in breadth; of a flattened figure, fine translucent green colour, and fleshy consistence. Some of the shorter, tending to an elliptical form, may be compared to the external section of an ellipsoid; but those of the largest dimensions are linear, that is, with parallel sides and curved extremities. The middle of the upper and the whole of the under surface are smooth; the former somewhat convex, occasioned by a border of 70 or 80 , or even of 350 individual polypi, disposed in a triple row.

"Each of the numerous polypi, though an integral portion of the common mass, is a distinct animal, endowed with separate action and sensation. The body, rising about a line by a tubular fleshy stem, is crowned by a head, which may be circumscribed by a circle as much in diameter, formed as a horse-shoe, and bordered by a hundred tentacula. Towards one side, the mouth, of singular mechanism, seems to have projecting lips, and to open as a valve, which folds up within, conveying the particles which are absorbed to the wide orifice of an intestinal organ, which descends, perhaps in a convolution, below, and returns again, to terminate in an excretory canal under the site of the tentacula. Probably the whole race of Cristatellæ is distinguished by a similar conformation.

"Twenty, thirty, or more lenticular substances, of considerable size, and in the most irregular arrangement, imbedded in the flesh, are exposed through the translucent green of the animal. Its death and decomposition towards the end of autumn liberate them to float in the water. Subjected to the microscope, or indeed to the naked eye, their 
convex-surfaces prove brown, the circumference yellow, and begirt with a row of spines, terminating with double hooks. Each is an ovum of the Cristatella, with a hard shell, and occupied by yellowish fluid contents."

Cliona are found in little holes in old oyster-shells. Dr. Grant says : "I have termed this genus cliona (from the Greek) from its most obvious and remarkable property of retracting and shutting the papillæ when irritated; and the Cliona celata, the only one I have met with, is named celata from its concealed and secure habitation within the substance of oyster-shells.

"The cliona in the living state consists of a soft, fleshy, granular and distinctly irritable substance, of a greenish-yellow colour, traversed, like many other zoophytes, with minute and regularly-formed spicula. Its form depends on that of the cavities which it fills ; it insinuates itself into their minutest ramifications, and adheres so closely to their smooth parietes, that it cannot be separated without tearing. The parts of the cliona which project through the holes on the surface of the cell are tubular; and on removing the outer layers of the shell, we can perceive several empty canals winding and ramifying from these tubular papillæ through the body of the zoophyte. During the months of March and April, when these observations were made, numerous small yellow ova were seen in the vicinity of the canals, agreeing much in their form, colour, size, and mode of distribution with those of the Spongia papillaris and Spongia panicea, which were then nearly in the same stage of advancement. The projecting tubular papillæ possess a complicated structure, and a high degree of contractile power, and exhibit a singular series of appearances, when the zoophyte is attentively examined while at rest in pure sea-water. When under water, the papillæ are seen projecting from the apertures of the shell, sometimes to the length of a line and a half; they present a wide circular opening in their centre, and a rapid current of water issues constantly from them, conveying occasional flocculi of a grey membranaceous matter. But on being touched with a needle, or withdrawn from the water, the opening gradually closes, the current ceases, and the whole papillæ continuing slowly to contract, is withdrawn completely within the aperture of the shell. The papillæ, viewed in their contracted state, present a smooth, rounded, shut extremity; but when they begin to advance beyond the surface of the shell, their extremity becomes flat and slightly dilated, assumes a villous appearance, with open fissures, radiating from the centre to the margin of the papillæ; and at length a minute circular opening is perceived in the centre of the 
villous surface. The papillæ advance from the shell, and its central opening enlarges in proportion to the healthy state of the zoophyte, and the purity and stillness of the water; its flat, downy, radiated surface gradually diminishes by the widening of the central opening, till only thin margins are left around the orifice, and the current is again seen to play briskly from it. In recent specimens of the cliona, dredged from an oyster-bed near the shore at Preston-Pans, and examined under the most favourable circumstances on the coast, I have twice observed polypi of extraordinary minuteness and delicacy placed around the margin of the orifice, and which kept in constant motion, advancing and withdrawing themselves into the substance of the papillæ, while the current flowed from its central opening. The polypi were perfectly invisible to the raked eye in an ordinary light and position; but by suspending the cliona in a crystal jar with clean water, and placing it between the eye and candle, or the sun, they were seen like filaments of silk or asbestos, constantly rising and sinking on the margin of the papillæ. On cutting off a papilla, and placing it under a microscope in sea-water, the polypi continued their motions, and were seen to consist of a long, slender, transparent, cylindrical, tubular, fleshy body, at the further extremity of which were placed about eight short broad tentacula, slightly dilated at their free ends, which were constantly inflecting and extending themselves irregularly, while the polypi advanced or retreated. In two entire and fresh specimens, the polypi continued visible and in motion for more than twenty-four hours in a jar of water at Preston-Pans ; but $I$ have not yet succeeded in perceiving them in any of the numerous specimens which $I$ have preserved alive in the water procured from Newhaven. . . . . The spicula are about the fourth of a line in length, and appear through the microscope as minute curved pins, spread irregularly through the whole fleshy substance of the animal ; . . . . slender, cylindrical, tubular, slightly-curved, shut at both ends, a little fusiform in the middle, acutely pointed at one end, and terminated by a small, hollow, round head at the other."

\section{SKELETONS OF ZOOPHYTES.}

In the account we have rendered of the important and large families of zoophytes, we have frequently spoken of the calcareous spicula that form a hard crust over the flesh of the Gorgonidæ and Alcyonidæ; and of some of the latter also, as well as Sertulariæ, possessing horny filaments or tubes as skeletons. That the eye may aid the mind in contemplating these curious structures, we have had drawings made of 
some of the more interesting. We have already remarked that these skeletons possess no blood-vessels; that they are secreted by the fleshy mass of the animal, and some of them are partially formed before the birth of the polypi which have to become their inhabitants.

The skeletons of some of the Campanularia consist of a tubular granular matter, encased in a horny translucent substance, which is enlarged at the end of each branch into cells for the domiciliation of the polypi. The outline is waved from the cells with a jointed appearance, which allows of a certain elasticity to the cells.

Alcyonirm Digitatum are branched, and have tubes; some are curved, others straight, and many take the form of a cross. The dark-red margin of Gorgonia petechialis is a mass of large, red, flattened, angular spicula; while inside they are small and in bundles, forming a network around the pores or canals, thin and slightly-curved, with a small swelling in the centre.

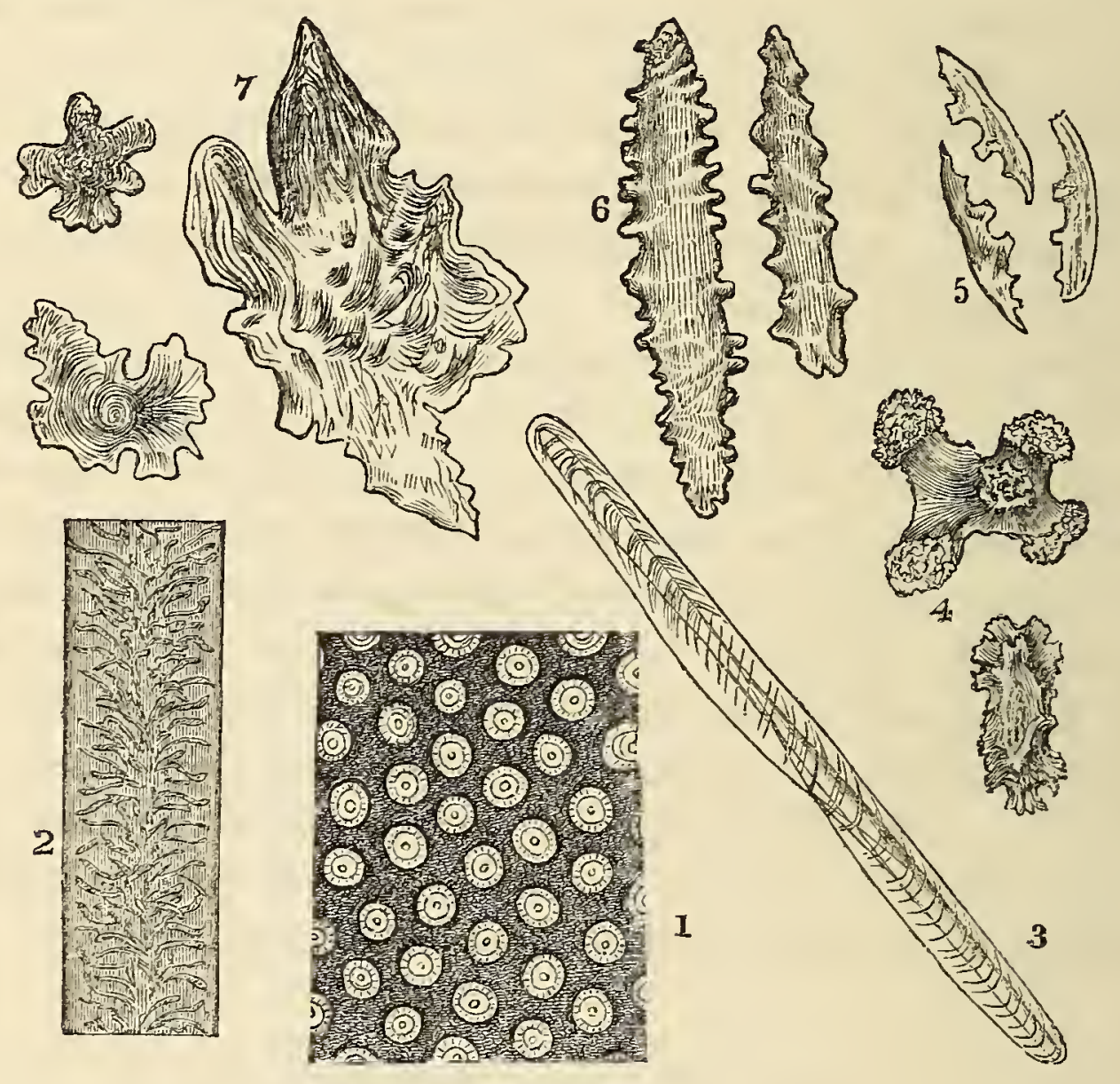

fig. 92.

1. Transverse section of a branch of Myriapore. 2. A section of the stem of Virgularia mirabilis. 3. A spiculum from the outer surface of a Sea-pen. 4. Spicula from crust of Isis hippuris. 5. Spicula from Gorgonia elongata. 6. Spicula fiom Alcyonium. 7. Spicula from Gorgonia umbraculum.

In a New Zealand specimen of Alcyonium the spicular are calcareous, of a brown colour, shaped like a cucumber, having all parts of their 
outer surface studded with conical tubercles; some of them exceed the 1-8th of an inch in length, others are as small as the 1-200th of an inch. (Fig. 92, No. 6.)

The purple-coloured spicula of the outer surface of the Sea-pen (Renilla Americana) are cylindrical, a little twisted, and seem to have a central canal; they are the 1-70th of an inch long, by the 800 th in the short diameter. (Fig. 92, No. 3.)

The dull lake or dirty-white spicula of the crust of Gorgonia Elongata are bowed with projectings from one side (fig. 92, No. 5); but they are commonly elongated, covered with tubercles arranged in rings.

Muricca Elongata, are yellowish-brown, of a pine-apple shape; one extremity covered with short sharp-pointed tubercles, the other expanded with spines; the free extremities of which all point in one direction.

Spicula from the fleshy crust of Isis Hippuris, "mostly of a quadrate or clavate figure, covered with large modulated tubercles," are represented in fig. 92, No. 4.

The Gorgonia Umbraculum present two kinds, one found in the crust, of large stellate angular figure and rich brown colour; the other much smaller, and covered with modulated tubercles. (Fig. 92, No. 7.)

Professor Quekett states that the skeletons of sponges are composed principally of two materials, the one animal, the other mineral; the first of a fibrous horny nature, the second either siliceous or calcareous. The fibrous portion consists of a net-work of smooth, and more or less cylindrical threads, of a light yellow colour, and, with few exceptions, always solid; they frequently anastomose, and vary considerably in size; when developed to a great extent, needle-shaped siliceous bodies termed spicula (little spines) are formed in their interior; in a few cases only one of these spicula is met with, but most commonly they occur in bundles. In some sponges, as those belonging to the genus Halichondria, the same horny kind of material is present in greater or less abundance, but its fibrous structure has become obscure; the fibres, however, in these cases are represented by siliceous needle-shaped spicula, and the horny matter serves the important office of binding them firmly together, as shown in Plate IV. No. 1. There is, however, one remarkable exception to this rule, viz. Dictyochalix pumiceus, described by Mr. S. Stutchbury, in which the fibrous skeleton is composed of threads of silex quite as transparent as glass.

The mineral portion, as before stated, consists of spicula composed either of silica or carbonate of lime; the first kind is the most common 
and likewise most variable in shape, and presents every gradation in form, from the acuate or needle-shaped to that of a star. The calcareous spicula, on the contrary, are more simple in their form, being principally acicular, but not unfrequently branched or even tri or quadriradiate; the two kinds, the siliceous and calcareous, according to Dr. Johnston, not having hitherto been detected co-existent in any native sponges.

The spicula exhibit a more or less distinct trace of a central cavity or canal, the extremities of which are closed, or hermetically sealed; in their natural situation they are invested by an animal membrane, which is not confined to their external surface ; but in many of the large kinds, as pointed out by Mr. Bowerbank, its presence may be detected in their central cavity, by exposing them for a short time to a red heat; when the animal matter will become carbonised, and appear as a black line in their interior.

Many authors have described the spicula as being crystalline, and of an angular figure, and have considered them analogous to the raphides. in plants; but it requires no great magnifying power to prove that they are always round, and according to their size are made up of one or more concentric layers, as shown in Plate IV. No. 3. The spicula occupy certain definite situations in sponges; some are peculiar to the crust, others to the flesh, others to the margins of the large canals, others to the fibrous network of the skeleton, and others belong exclusively to the gemmules. Thus, for instance, in Pachymatisma Johnstonia, according to Mr. Bowerbank, the spicules of the crust are simple, minute, and fusiform, having their surfaces irregularly tuberculated, and their terminations very obtuse; whilst those of the flesh are of a stellate form, the rays varying in number from three to ten or twelve.

Silica, however, may be found in one or more species of sponge of the genus Dysidea, not only in the form of spicula, but as grains of sand of irregular shape and size, evidently of extraneous origin, but so firmly surrounded by horny matter as to form, with a few short and slightly-curved spicula, the fibrous skeleton of the animal. In these sponges the spicula are of large size, and are disposed in lines parallel with the masses of sand.

Spicula are not exclusively confined to the body of sponges, but occasionally form the skeleton of the gemmules, and may be situated either on the external or internal surface of these bodies. A good example of the former kind occurs in the common fresh-water sponge (Spongilla fluviatilis), represented in Plate IV. Nos. 9 and 13. The spicula are of very minute size, and are disposed in lines radiating from 
the centre to the circumference, the markings on the outer surface of the gemmule being the ends of the individual spicula. In all the young gemmules the spicula project from the outer margin as so many spines, but in process of growth the spines become more and more blunt, until at last they appear as so many angular tubercles."

Turkey sponge, or Spongia Officinalis, is brought from the Mediterranean, has a horny network skeleton rather fine in the fibres, solid, small in size and light in colour. (Plate IV. No. 1.) In some larger specimens there is a single large fibre, or a bundle of smaller ones.

In Halichondria Simulans the skeleton is a framework of siliceous needle-shaped spicula, arranged in bundles kept together by a thick coat of horny matter.

Other species of Hcilichondria have siliceous spicula pointed at both extremities - acerate (Plate IV. No. 7); while the spicula of some are round at one end and pointed at the other-acuate (see No. 7); and also spicula round at one end, the former being dilated into a knobspinulate; some have a spinous surface.

From the South Seas, specimens are found having spicula with both ends rounded; cylindrical ; some curved, others straight.

In an unclassed genus, the spicula are discovered rather cylindrical in form, pointed at both ends, having the surface covered with spines placed in an annular form (Pl. IV. No. 2), and another in which the spicula exhibit many curious forms; within the circle may be seen one spiculum of remarkable beauty, being of large size, rounded at both ends, and slightly bent; its outer surface is covered with rows of tubercles of circular figure, which project some little distance beyond the free margin.

In the Halichondria genus there are found some with the spicula of the acuate form covered with spines, discovered at New Zealand; they are blunt at one end and sharp at the other; the spines are small, without order in their situation, but greater in number at the middle.

In the genus Pachymatisma some spicula are sharp at one extremity and expand into two points at the other: expando-binate; they are large, and their purpose that of connecting the crust and the fleshy matter compactly together. The P. Johnstonia spicula are sharp at one end, and expand into three points at the other. Expandoternate; arranged at angles at $45^{\circ}$ to the other part of the stem (Pl. IV. No. 7 ) ; there is also in this kind a variety having spicula sharp at one end and expanding into three branches at the other, each of which again divides into two dichotomo-expando-ternate kind.

In the genus Tethea there are spicula having hooks at both ex- 
tremities, bi-recurvo-ternate: at one end they are not so large or so numerous as at the other; the stem is a little spinous.

In Tethea Lyncurium the ends of the branches of the spicula are recurved, forming two, three, or four hooks, which serve to anchor the crust to the soft central fleshy part; their term is recurvo-binate, ternate, \&c. Of the recurvo-ternate wa give a specimen from a species of Pachymatisma. (Plate IV. No. 6.)

Among the genus Grantia, Geodia, and Levant sponge, are found spicula of a large size, radiating in three directions-triradiate. In the Levant specimen, a central communicating cavity can be distinctly seen. Some Smyrna sponge, and a species of Geodia, have four rays -quadriradiate.

There are spicula in P. Johnstonia and Geodia that have as many as ten rays-multiradiati ; but vary in the quantity. (No. 12.)

In some species of Tethea, the spicula consist of a central spherical body, from which short conical spines proceed-stellate spicula. (Plate IV. No. 4.) These are also found in some of the genus Geodia.

Branched spicula, some covered with spines, have been taken from sponge brought from Ceylon; and from sponge from the same place, spicula more or less curved-curvate.

Spicula having both extremities bent alike-bicurvate-have been obtained from Trieste sponge. (See Plate IV. No. 3.)

Some South Sea sponges have spicula twice bent, and have extremities like the flukes of an anchor-bicurvate-anchorate; sometimes the flukes have three pointed ends. (Plate IV. No. 6.)

The gemmules, or seed-like bodies found in the inside of some sponges, are very interesting objects, and merit therefore a passing notice. Mr. Quekett says: "They are occasionally provided with a skeleton composed of a series of very minute siliceous spicula, arranged in a more or less compact radiated form, and having in their fully-developed state, a pore or funnel-shaped opening leading into their interior. In the early stages of growth, the spicula project from the free surface in the form of spines; but in full-grown specimens; as shown at Plate IV. No. 11, their free extremities are more or less angular and blunt. In some of the fresh-water sponges, the gemmules are of large size; and spicula of a bi-rotulate form give a firm coating to their external surface. The internal part of these gemmules contains a series of small circular cells, which make their escape when the containing envelope is ruptured. The gemmules in fresh-water sponges are generally found in the oldest portions near the base, and each one is protected by a framework of bundles of acerate spicula of the flesh, as shown in Plate 
IV. No. 9 ; but in many marine species, as Geodia and Pachymatisma, they are principally confined to the crust. In the fresh-water sponges, the amount of animal matter in the gemmules is considerable; but in Pachymatisma, Geodia, and many other marine species, a very small quantity only is ever to be found, the substance of each gemmule being almost entirely composed of minute siliceous spicula; and if they be viewed when taken fresh from the sponge, and after boiling in acid to remove the animal matter, a slight increase in transparency is the only perceptible difference of appearance in these two opposite conditions."

In the journal of the Bombay branch of the Royal Asiatic Society for 1849 , Surgeon H. J. Carter gives a minute and able account of the fresh-water sponges in the Island of Bombay. Of five species that he discovered, one was the Spongilla friabilis (Lam.) the others he named Sp. cinerea, Sp. friabilis, Sp. alba, Sp. meyeni, Sp. plumosa.

Spongilla Cinerea is stated to present on its surface a dark, rusty, copper colour, lighter towards the interior, and purplish under water. It throws up no processes, but extends horizontally in circular patches, over surfaces two or three feet in circumference, or accumulates on small objects; and is seldom more than half an inch in thickness. It is found on the sides of fresh-water tanks, on rocks, stones, or gravel. Vents numerous, and situated in the depressions. Texture compact, fine, friable; structure confused-fibro-reticulate; fibres perpendicular, densely-aggregated, and united by transverse filaments. Seed-like bodies spheroidal, about 1-63d of an inch in diameter, presenting rough points externally. Spicula of two kinds, large and small ; large spicula, slightly curved, smooth, pointed at both ends, about 1-67th of an inch in length; small spicula, slightly curved, thickly spiniferous, about 1-380th of an inch in length.

Spongilla Friabilis. - The vents are large and erater-like, situated on its low eminences; it is of a bright green colour on the surface, faintly yellow towards the interior. Growing in circumscribed masses, on fixed bodies, or enveloping floating objects; seldom attaining more than two inches in thickness. It seldom throws up projections much beyond its surface; does not appear to be inclined to spread much, and is matted and confused in its structure towards its base and round its seed-like bodies. From the other sponges it is distinguished by the smooth spicula which surround its seed-like bodies, and the matted structure.

Spongilla Alba has large scattered vents; is of a yellow colour, and grows horizontally in circumscribed masses or patches; seldom more than an inch in thickness. Its texture is coarse and open; structure 
reticulated. The investing membrane abounds in minute spicula; has seed-like spheroidal bodies about 1-30th of an inch in diameter, with rough points externally. The large spicula are slightly curved, smooth pointed at each end, about 1-54th of an inch in length; the small spicula are slightly curved, thickly spiniferous, or pointed at both ends; the former, pertaining to the seed-like bodies, are about 1-200th of an inch in length; the latter, pertaining to the investing membrane, are more slender, and a little less in leugth; these last numerous small spiniferous spicula when dry present a white lacey appearance, from which Mr. Carter gives them the name of alba.

Spongilla Meyeni is massive, having large lobes, mammillary eminences, or pyramidal, compressed, obtuse or sharp-pointed projections, of an inch or more in height; also low, wavy ridges. It is of a yellow colour, and grows in circumscribed masses about three inches in height. The texture is fine, friable, soft, tomatose towards the base, with a structure fibrous, reticulated, and radiated. Its seed-like bodies are spheroidal, about 1-47th of an inch in diameter, studded with little toothed disks. The spicula around the seed-like bodies are cemented together by a shapeless siliceous deposit.

Spongilla Plumosa.-This is much the same in appearance and colour as the last, but looser and coarser in its texture and structure.

It would seem these sponges are for about six months covered with water ; and that after the first heavy rains of the S. W. monsoon, the tanks becoming filled, the sponges appear to undergo a partial state of putrescency, during which gas is generated in them, and accumulates in globules in their structure; through which it must burst, or tear them from their attachments, and force them to the surface of the water.

Mr. Carter states: "The fresh-water sponge is composed of a fleshy mass, supported on a fibrous, reticulated, horny skeleton. The fleshy mass contains a great number of seed-like bodies in all stages of development, and the horny skeleton is permeated throughout with siliceous spicula. When the fleshy mass is examined by the aid of a microscope, it is found to be composed of a number of cells imbedded in, and held together by an intercellular substance.

"The granules are round or oviod; translucent, and of an emerald or yellowish-green colour, varying in diameter below the 12,000th part of an inch, which is the average linear measurement of the largest. In some cells they are so minute and colourless as to appear only under the form of a nebular mass; while in others they are of the largest kind, and few in number." 
The hyaline vesicles, on the other hand, are transparent, colourless, and globular; and although variable in point of size, like the green granules, are seldom recognised before they much exceed the latter in diameter. They generally possess the remarkable property of slowly dilating and contracting themselves, and present in their interior molecules of extreme minuteness in rapid commotion.

The intercellular substance, which forms the bond of union between the cells, is mucilaginous. When observed in the delicate pellicle, which, with its imbedded cells and granules, it forms orer the surface, and throughout the canals of the sponge, it is transparent; but when a portion of this pellicle is cut from its attachments, it collapses, and becomes semi-opaque. In this state, the detached portion immediately evinces a tendency to assume a spheroidal form; but whether the intercellular substance participates in this act, or remains passive while it is wholly performed by the habit of the cells which are imbedded in it to approximate themselves, Mr. Carter has not been able to determine.

The seed-like bodies occupy the oldest or first-formed portions of the sponge, near its periphery. They are round or oviod, according: to the species, and each presents a single infundibular depression on its surface, which communicates with the interior. At the earliest period of development in which Mr. Carter recognised the seed-like body, it has been composed of a number of cells, united together in a globular or oriod mass, according to the species, by an intercellular substance similar to that just described. The seed-like body passes from the state just mentioned into a more circumscribed form; then becomes surrounded by a soft, white, compressible capsule ; and finally thickens, turns yellow, and develops upon its exterior a firm crust of siliceous spicula.

Thus matured, its cells, which were originally unequal in size, now become nearly all equal, almost motionless, and a little exceed the average diameter of the largest sponge-cells; while their germs, which in the first instance so nearly resembled the gemmules of the spongecells, are now four or five times larger; and vary in diameter below the 3000 th part of an inch, which is the average linear measurement of the largest of their kind.

The capsule passes from a soft, white state, into a tough, yellow, coriaceous membrane, presenting in meyeni and plumosa a hexagonallytessellated appearance, on the divisions of which rest asteroid discs of the vertically-placed spicula which surround it.

In the two species just mentioned, the spicula are arranged per- 
pendicularly to the surface of the capsule; and the interval between them is filled up with a white siliceous amorphous matter, which keeps them in position. Each spiculum extends a little beyond this matter, and supports on its free end a toothed disc, similar to the one on its fixed end, which rests on the capsule; so that the external surface of the seed-like body in meyeni and plumosa is studded with little stellated bodies; while in the other species, where there appears to be no such regular arrangement of these spicula, a number of smooth or spiniferous points is presented.

On the development of Spongilla Mr. Carter states: "When the cells of the seed-like body are forcibly expelled from their natural cavity under water, they are irregular in form and motionless, but soon swell out (by endosmose?), become globular, and after a few hours burst. At the time of bursting, their visible contents, which consist of a mass of germs, occupying about two-thirds of the cavity of the cell, subside, and afterwards gradually become spread over the bottom of the vessel in which they are contained. They are of various diameters below the 3000 th part of an inch, which is the average linear measurement of the largest, and appear to be endowed with the power of locomotion in proportion to their size."

PRESERVATION OF THE POLYPIDOMS OF ZOOPHYTES.

An excellent and very simple plan for preserving Zoophytes as wet preparations, so as to retain the polypes and their tentacular arms in situ, has been proposed by Dr. Golding Bird. "For this purpose a lively specimen should be chosen, and then plunged into cold pure water; the polypes are killed almost immediately, and their tentacula often do not retract; proper-sized specimens should then be selected, and preserved in weak alcohol. Little phials about two inches long: should be procured, made from thin flat glass tubes, so as to be half an inch wide, and about a quarter of an inch, or even less, from back to front. The specimens should be fixed to a thin platinum wire, and then placed in one of these phials (previously filled with weak spirits), so as to reach half-way down. When several are thus arranged, they should be put on a glass-cylinder, and removed to the air-pump. On pumping out the air, a copious ebullition of bubbles will take place; and many of the tentacula previously concealed will emerge from their cells. After being left in vacuo for a few hours, the bottles should be filled up, closely corked, and tied over, like anatomical prepara- 
tions in general. For all examinations with a one or two-inch objectglass these bottles are most excellent, and afford cheap and useful substitutes for the more expensive and difficultly-managed cells. In this manner specimens of the genera Cycloum, Membranipora, Alcyonidium, and Crisia, exhibit their structure most beautifully.

"A few dozen of these little bottles hardly occupy any room, and would form a useful accompaniment to the microscopist by the seaside. Any one visiting the caverns in St. Catherine's Island at Tenby, could reap a harvest which would afford amusement and instruction for many weeks. These caverns are so rich in zoophytes and sponges, that they are literally roofed with the Laomedeæ, Grantiæ, and their allies; whilst the elegant Tubularia afford an ornament to the shallow pools on the floor; and the walls are wreathed with the pink, yellow, green, and purple Actiniæ.

"When these objects are examined by polarised light, most interesting results are produced. For this purpose, let a piece of selenite be placed on the stage of the microscope, and the polarising prisms arranged so that the ray transmitted is absorbed by the analyser.

"If a specimen of Sertularia operculata be placed on the selenite stage, and examined with a two-inch object-glass, the central stem is shown to be a continuous tube, assuming a pink tint throughout its whole extent. The cells appear violet in colour; their pointed orifices are seen much more distinctly than when viewed with common light. The vesicles are paler than the rest of the object; and their lids, which so remarkably resemble the operculum of the theca of a moss, are beautifully distinct, being of orange-yellowish colour.

"This zoophyte is often covered with minute bivalve shells, distinguished by the naked eye from the vesicles only by their circular form; and these, when present, add much to the beauty of the specimen, presenting a striated structure, and becoming illuminated with most beautiful colours.

"Sertularia Filicula forms an interesting object; the waved stem becoming of a dusky red, whilst the cells assume but little colour, renders their mutual relation very obvious. Sertularia abietina is also a fine object, especially when loaded with vesicles, as it often is in the autumn. Plumularia falcata acquires fresh beauty under polarised light, the cells being a pale green, whilst the tubular stem becomes of a crimson hue; thus presenting a feathered appearance.

"The most splendid tints are exhibited by the calcareous structure of the Polyzoa, and of these the Flustra truncata, when viewed on the selenite stage. The Cellularia avicularia is very brilliant when 
viewed in the same way; its cells being covered with plates of carbonate of lime, it presents a fine display of beautiful tints, especially its bird's-head appendages."

\section{FOSSIL INFUSORIA.}

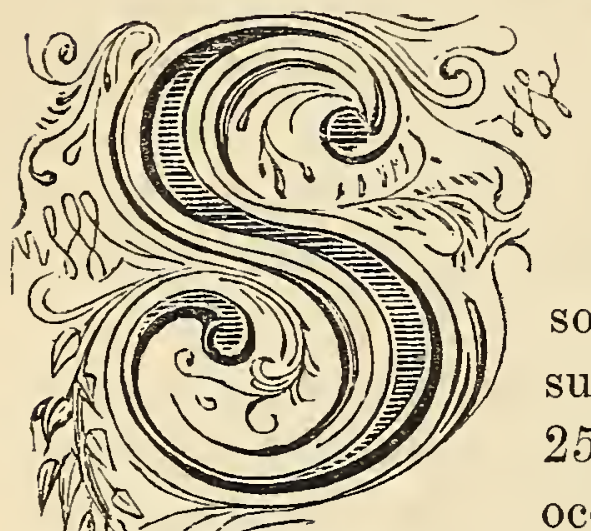

TARTLING and almost $i$ icredible as the assertion may appear to some, it is none theless a fact, established beyond all question by the aid of the microscope, that some of our most gigantic mountain-ranges, such as the mighty Andes, towering into space 25,250 feet above the level of the sea, their base occupying so vast an area of land; as also our in 2 massive limestone rocks; the sand that covers our boundfeserts and the soil of many of our wide-extended on plains; are principally composed of portions of invisible "The remains of such minute animals have added much more to the mass of materials which compose the exterior crust of the globe, than the bones of elephants, hippopotami, and whales."

The stratum of slate, fourteen feet thick, found at Bilin, in Austria, was the first that was discovered to consist almost entirely of minute flinty shells. A cubic inch does not weigh quite half an ounce; and in this bulk it is estimated there are not less than forty-thousand millions of individual organic remains! This slate, as well as the Tripoli, found in Africa, is ground to a powder, and sold for polishing. The similarity of the formation of each is proved by the microscope; and their properties being the same, in commerce they both pass under the name of Tripoli. One merchant alone in Berlin disposes annually of twenty tons weight. The thickness of a single shell is about the sixth of a human hair, and its weight the hundred-and-eighty-seven-millionth part of a grain.

The berg-mehl, or mountain-meal, we have already described: in Norway and Lapland it has been found thirty feet in thickness; in Saxony twenty-eight feet thick; and it has also been discovered in Tuscany, Bohemia, Africa, Asia, the South Sea Islands, and South 


\section{PLATE V.}
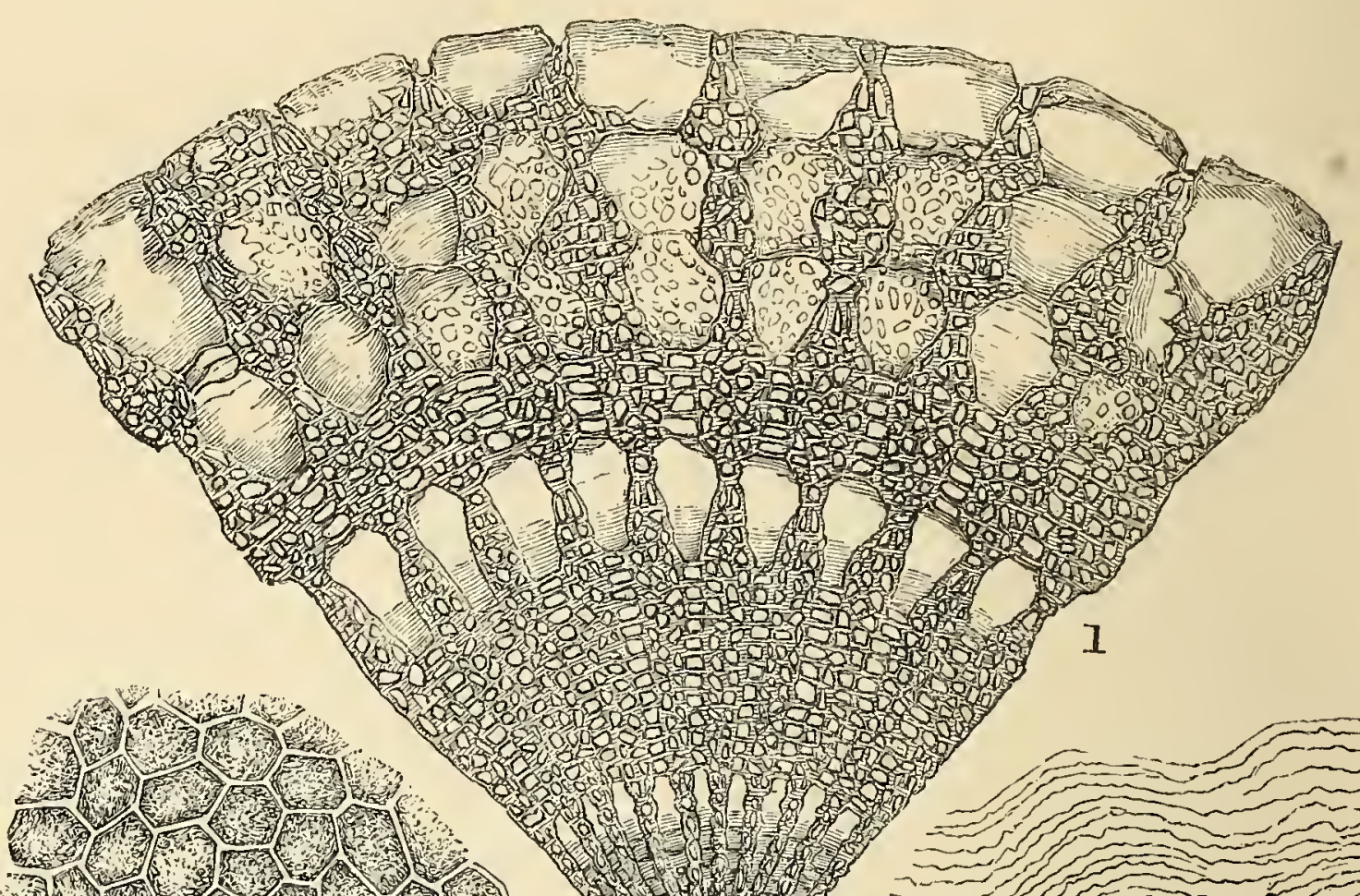

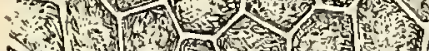

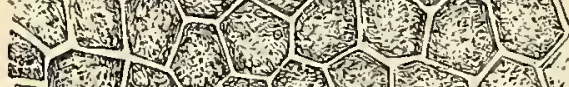

2.

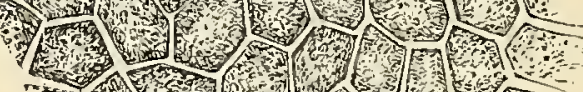

5

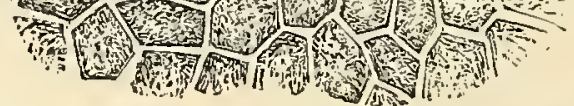

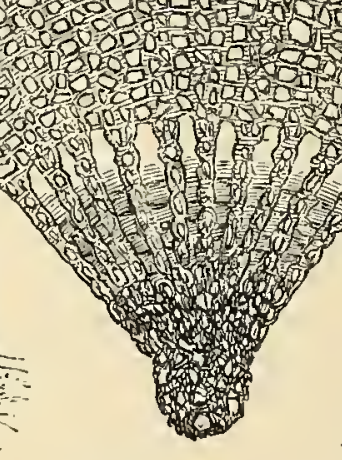

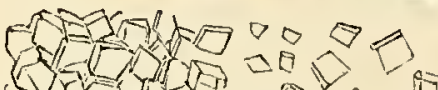
(1) ence a o o minion An An fincin 0 is कan an a
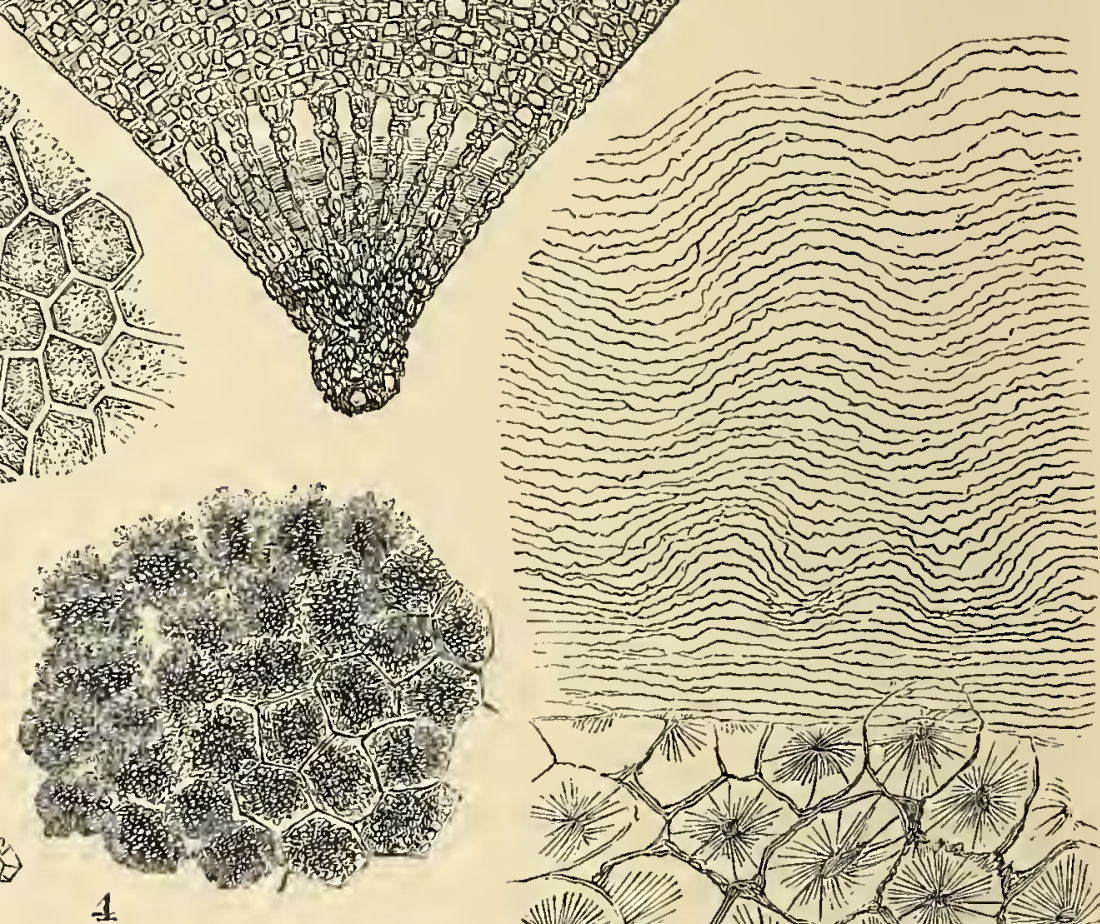
I 400 a

1


SHELL OF GASTEROPODA, ECHINO-DERMATA, ETC.

1. Portion of a transverse section of the Spine of an Echinus. 2. Portion of a transverse section of the Spine of an Echinus, exhibiting reticulated structure, the calcareous portion having been dissolved out by acid. 3. Horizontal section of sliell of Haliotis splendens, with stellate pigment in the interior. 4. Portion of Shell of a Crab, ture 6 . Horizes beneath the articular layer. 5. Another portion of same Shell, showing its hexagonal strucsection of shell of Pinna ingens. 8. Crystals of Carbonate of Lime, from the outer brown shell of the oyster. 
America; of this, almost the entire mass is composed of flinty skeletons. That in Tuscany and Bohemia resembles pure magnesia, and consists entirely of a shell called campilodiscus, about the two-hundredth of an inch in size.

Dr. Darwin, writing of Patagonia, says : "Here along the coast for hundreds of miles, we have our great tertiary formation, including many tertiary shells, all apparently extinct. The most common shell is a massive, gigantic oyster, sometimes even a foot in diameter. The beds composing this formation, are covered by others of a peculiar soft white stone, including much gypsum, and resembling chalk; but really of the nature of pumice-stone. It is highly remarkable, from its being composed, to at least one-tenth of its bulk, of Infusoria; and Professor Ehrenberg has already recognised in it thirty marine forms. This bed, which extends for five hundred miles along the coast, and probably runs to a considerabily greater distance, is more than eight hundred feet in thickness at Port St. Julian." Ehrenberg discovered in the rock of the volcanic island of Ascension many siliceous shells of freshwater Infusoria; and the same indefatigable investigator found that the immense oceans of sandy deserts in Africa, were in great part composed of the shells of animalculæ. The mighty Deltas, and other deposits of rivers, are also found to be filled with the remains of this vast family of minute organisation. At Richmond in Virginia, United States, there is a flinty marl many miles in extent, and from twelve to twenty-five feet in thickness; almost wholly composed of the shells of marine animalculæ; for in the slightest particles of it they are discoverable. On these myriads of skeletons are built the towns of Richmond and Petersburg. The species in these earths are chiefly the Navicula; but the most attractive, from the beauty of its form, is the Coscinodiscus, or sieve-like disc, found alike near Cuxhaven, at the mouth of the Elbe, in the Baltic, near Wismar, in the guano, and the stomachs of our oysters, scallops, and other shell-fish. Another large deposit is found at Andover, Connecticut; and Ehrenberg states, "that similar beds occur by the river Amazon, and in great extent from Virginia to Labrador." The chalk and flints of our sea-coasts are found to be principally shells and animal remains. Ehrenberg computes, that in a cubic inch of chalk there are the remains of a million distinct organic beings. The Paris Basin, one hundred and eighty miles long, and averaging ninety in breadth, abounds in Infusoria and other siliceous remains. Ehrenberg, on examining the immense deposit of mud at the harbour of Wismar, Mecklenburg-Schwerin, found one-tenth to consist of the shells of animalculæ; giving a mass of animal remains amounting 
to 22,885 cubic feet in bulk, and weighing forty tons, as the quantity annually deposited there. How vast, how utterly incomprehensible, then, must be the number of once living beings, whose remains have in the lapse of time accumulated! In the frigid regions of the North Pole no less than sixty-eight species of the fossil Infusoria have been found. The guano of the island of Ichaboe abounds with fossil Infusoria, which must have first entered the stomachs of fish, then those of the sea-fowl, and become ultimately deposited on the island, incrustating its surface; whence they are transported, after the lapse of centuries, to aid the fruition of the earth, for the benefit of the present race of civilised man. The hazy and injurious atmosphere met with off Cape Verde Islands, and hundreds of miles distant from the coast of Africa, is caused entirely by a brown dust, which, upon being examined microscopically by Ehrenberg, was found chiefly to consist of the flinty shells of Infusoria, and the siliceous tissue of plants : of these Infusoria, sixtyfour proved to be fresh-water animalculæ, and two were denizens of the ocean. From the direction of the periodical winds, this dust is reasonably supposed to be the finer portions of the sands of the desert of the interior of Africa.

The deposit of the beneficent Nile, that fertilises so large a tract of country, has undergone the keen scientific scrutiny of Ehrenberg; and he found the nutritive principle to consist of fossil Infusoria. So profusely were they diffused, that he could not detect the smallest particle of the deposit that did not contain the remains of one or more of the extensive but diminutive family that once revelled in all the enjoyment of animal existence.

Before entering on any details of the fossil Infusoria, we would first state how they may be prepared for microscopic examination: "A great many of the infusorial earths may be mounted as objects without any previous washing or preparation; some, such as chalk, however, must be repeatedly washed, to deprive the Infusoria of all impurities; whilst others, by far the most numerous class, require either to be digested for a long time, or even boiled in strong nitric or hydrochloric acid, for the same purpose. Place a small portion of the earth to be prepared in a test-tube, or other convenient vessel, capable of bearing the heat of a lamp; then pour upon it enough diluted hydrochloric acid to about half fill the tube. Brisk effervescence will now take place, which may be assisted by the application of a small amount of heat, either from a sand-bath or from a lamp : as soon as the action of the acid has ceased, another supply may be added; and the same continued until no further effect is produced. Strong nitric acid should 
now be substituted for the hydro-chloric, when a further effervescence will take place, which may be greatly aided by heat; after two or three fresh supplies of this acid, distilled water may be employed to neutralise all the remains of the acid in the tube; and this repeated until the water comes away perfectly clear, and without any trace of acidity. The residuum of the earth, which consists of silica, will contain all the infusorial forms; and some of this may be taken up by a fishing-tube, laid on a slide, and examined in the usual manner. Should perfect specimens of the Coscinodiscus, Gallionella, or Naricula be present, they may be mounted in Canada balsam; if not, the slide may be wiped clean, and another portion of the sediment taken, and dealt with in the same way; or, if good, after being dried, may be mounted in Canada balsam."

Mr. Redfern has given us an excellent mode of isolating Naviculæ and other test objects. He says: "Having found the methods ordinarily employed very tedious and frequently destructive of the specimens, I adopted the following plan: Select a fine hair which has been split at its free extremity into from three to five or six parts, and having fixed it in a common needle-holder by passing it through a slit in a piece of cork, use it as a forceps under a two-thirds of ar inch objective, with an erecting eye-piece. When the split extremity of the hair touches the glass-slide, its parts separate from each other to an amount proportionate to the pressure, and on being brought up to the object, are easily made to seize it, when it can be transferred as a single specimen to another slide without injury. The object is most easily seized when pushed to the edge of the fluid on the slide. Hairs split at the extremity may always be found in a shaving-brush which has been in use for some time. Those should be selected which have thin, split portions so closely in contact that they appear single until touched at their ends. I have also found entire hairs very useful when set in needle-holders in a similar manner, any amount of flexibility being given to them by regulating the length of the part of the hair in use."

We now proceed to notice in detail some of the most interesting of the fossil Infusoria.

DIATOMACEA, OR BRITTLETORTS.

Amongst the organic beings whose existence the microscope has brought to light, few possess a higher interest than the group now known as the family of Diatomacer. Appearing every where as the

* Professor Quekett, op. cit. 
first-born of life, and wherever inorganic matter is found in a condition fit for its development, these beings were looked upon suspiciously by early observers; and whilst regarded by some as doubtful in their nature, were by others accepted as evidences of spontaneous generation. Their siliceous structure, which was early known, seemed to give them a strange resemblance to the mineral world; whilst, although endowed with motion, they presented no traces of organic tissues. The labours, however, of Ehrenberg have set at rest the question of their belonging to the mineral world, and he decidedly regarded them as members of the animal kingdom. Their relationship to the Desmidiex is very apparent, and therefore many authors contend for placing them among the Algce, the simplest form of regetable life; yet as Ehrenberg classed them as polygastric Infusoria of the family Bacillaria, and propagating as they do by spontaneous division, and having siliceous skeletons that are found abundantly with fossil Infusoria, we will notice them under this division of the present work, to which we believe they truly belong. Recent specimens are of a green colour, mingled with a tinge of brown; their form is generally that of a prism, or four-sided; the flinty remains consist of one or more pieces, and in many cases it is only with the highest powers of the microscope that their beauties can be revealed. The delicate lines are often found to be minute raised dots, and the fineness of the tracery sets at defiance the most wonderful efforts of imitation. They are found both in the inland waters and the ocean, generally in large assemblies.

Professor J. W. Bailey, of New York, has enriched the Museum of the College of Surgeons with several valuable specimens of the skeletons of Infusoria; among them is a fresh-water Bacillaria, named Meridion circulare, which Professor Quekett in the Historical Catalogue describes as " consisting of a series of wedge-shaped bivalve siliceous loricæ arranged in spiral coils; when perfect, and in certain positions, they resemble circles; each lorica is articulated by two lateral surfaces. It is asserted that they can creep about when free from the stalk-plate. (Plate II. No. 16.)

Cocconema Boëckii is composed of two lanceolate flinty cases, that taper towards their ends, one of which is attached to a little foot. Each lorica has a line marked in its centre, and transverse rows of dots on both sides; Ehrenberg says there are twenty-six rows in the one-hundredth of a line. (Plate II. No. 14.)

Achnanthes Longpipes have at the margins two coarse convex pieces roughly dotted, and two inner pieces firmly grooved; the inside seems filled with green matter. At one corner they are affixed to a jointed. 
pedicle, which in many specimens contain green granules. They propagate by self-division.

In a specimen of a fossil Eunotia, found in some Bermuda earth, the flinty case is in four parts; it is of a half-lanceolate shape, and a little indented on both margins; two of them have curved rows of dots, and the other two are partly grooved with finer rows. Ehrenberg' says they have four openings, all on one side (Plate II. No. 13), presenting a row of dots varying very much in number; minute striæ in some cases extend from each dot towards the middle of the lorica; and on the circumference there are two of these dots. The spirals and the individual lorica are very fragile, and therefore easily separated from each other.

Of a glistening whiteness is the ribbon-like flinty case of Fragitlaria pectivalis, which consists of a number of bivalve parts; on the articulating surface there are small grooves, represented in Plate II. No. 15.

A singular class of objects are Diatomce flocculosce, being rather oblong-looking, and joined to each other at opposite corners; sometimes they are grooved on each side. (Plate II. No. 1.7.)

A recent specimen of the flinty, wedge-shaped Gomphonema geminatum (No. 11), fixed on a horny stalk, is in the Museum of the College of Surgeons. Ehrenberg states they have two openings at their hemispherical or indented broad ends. The surfaces are grooved crossways. There is another kind, commonly called the Swollen Eunotia, which is generally about from the eleventh to the two-hundredth of an inch in length. A groove, that is widest in the centre, and tapers off to the ends, passes along its centre on both sides, with curved lines proceeding from it. So wonderfully close are these lines or ribs, that as many as eight of them have been counted in the space of the twelve-hundredth of an inch. They are usually found when alive adhering to a branch of some weed that forms the green coating orer stagnant waters. They propagate by self-division; a slight line running down the centre marks where the separation will occur, on each becoming perfectly developed as a distinct object; and thus from age to age they grow and separate, filling the earth with their finty shells. The earth found in the river Upper Baun, Ireland, contains an abundance of Gallionellce distans, so plentiful in the State of Bilin, and the berg-mehl of Sweden, with which, as we have explained, the starving peasantry adulterate their food to fill their stomachs, that the pangs of hunger may be allayed.

Gallionella Sulcate is found in many parts of North America; it somewhat resembles the cylindrical box for spices, which was at one 
time so common among good housewives; scientifically, it is described as consisting of "chains of cylindrical bivalve loricæ, having their outer surfaces marked or furrowed with longitudinal striæ; short joints may occasionally be seen having their ends uppermost, the depth of the furrows being shown on the margin; within the margin is a thin transparent rim having radiating striæ." Sometimes as many as forty will be found joined together, see Plate II. No. 9. The Gallionella received its designation from a celebrated French naturalist named Gaillon, and is often termed the Box-chain Animalcule; and when the flinty case is seen lying on its face, it much resembles a coin. These animalculæ are found in almost all waters, and are stated to be so rapid in their growth, that one hundred and forty millions will by self-division be produced in twenty-four hours. A species named the Striped Gallionella has been discovered by Dr. Mantell near London, having yellow and green granules in the interior; the same species is also found in the ocean. Sometimes the chains will be found three inches long; the size is from the 14th to the 400th part of an inch.

The beautiful Navicula, or Little Ship, is found in all parts of the world; and from being met with most readily near London, that great hive of scientific industry, has been minutely examined and carefully described. We cannot do better than give the careful account furnished by Professor Quekett, in the catalogue we have before referred to: "Earth from Bohemia, particularly rich in fossil specimens of Navicula viridis, which consists of four prismatic loricæ, two ventral and two lateral; the former having round, the latter truncate extremities; and both provided with two rows of transverse markings and dots, longer and more marked on the ventral than on the lateral surfaces. The specimens having their ventral surfaces uppermost, exhibit a longitudinal marking in the centre, with a slight dilitation or knob at each extremity ; this marking is interrupted in the middle of the lorica, and a diamond-shaped spot is left; if one of the lateral loricæ be examined, two of the same spots will be seen, one on each side; they are of triangular figure, and appear to be thicker parts of the shell, and not holes, as Ehrenberg has imagined. Four smaller triangular spots may be observed in the same lorica, one being situated at each corner; these also have been considered as openings by Ehrenberg: their length varies considerably; some exceed 1-100th, whilst others are even smaller than 1-1000th of an inch."

Isthmia Enervis (No. 5) is found attached to sea-weed. It is in three parts, and of a trapezoid shape. The centre part appears like a band passing over, and is bounded by broad straight lines. "Its outer surface 
is covered with a network of rounded reticulations, arranged in parallel lines. Among the most remarkable are Amphitetras antediluviana; they are of a cubical or box-like figure, and consist of three portions, the one in the centre being in the form of a band, as shown at Plate II. No. 8, and the two lateral ones having four slightly-projecting angles, with an opening into each. When viewed in detached pieces, the central one is like a box, and the two lateral portions resemble the cover and bottom. The former may be readily known, as consisting merely of a square frame-work with striated sides; but both the latter are marked with radiating reticulations. When recent, they are found in zigzag chains, from their cohering only by alternate angles. In some instances, as in Biddulphia and Isthmia, two young specimens may be found within an old one."

Cocconeis is oval, and is marked with eight or ten lines proceeding from the inner margin to the centre ; between which are dotted furrows, which leave a small elder spot in the centre. (Plate II. No. 3.)

Campylodiscus Clypeus is oval, and curved in opposite ways at the long and short diameters. On the margin there are two series of dots, sometimes joined; and on the oval centre there are also dots about the margin, while the middle is nearly plain. (Plate II. No. 7.)

Actinocyclus has a round bivalve flinty case, with numerous cells formed by radiating partitions. Sometimes only every alternate cell is on the same plane. The specimen in the Museum of the College of Surgeons is exquisite in its markings; it was found in some Bermuda earth, and has "a beautifully raised margin, and a five-rayed star in the centre ; the number of cells is ten, five being on one plane, and five on another. One set has the usual hexagonal reticulations crossed with diagonal lines, the other has the same lines, with a much smaller series of triangular reticulations, so disposed that they appear to form with each other parts of minute circles." One of the valves of this specimen is represented in Plate II. No. 2.

Artists who design for art-manufacturers might derive many useful hints from the revelations of the microscope, as evidenced in the arrangement of the shell last noticed, and in that of the pretty genus Coscinodiscus, another handsome object; the shells are marked with a network of cells in a hexagonal form, arranged in radiating lines or circles; they vary from 1-200th to 1-800th of an inch in diameter. A specimen found in Bermuda earth has on one of its valves two parallel rows of oval cells that form a kind of cross ; they are gradually larger from the centre to the margin. The angles of the cross are filled with the hexagonal cells previously noticed. (Plate II. No. 4.) 
The unskilled manipulator may for some time endeavour to adjust a slide, having a piece of glass exposed not larger in size than a pea, on which he is informed an invisible object worthy his attention is fixed, before he is rewarded by a sight of Triceratium favus, extracted from the mud of the too-muddy Thames. The hexagonal markings or cells are beautiful, and at each corner there is a curved projecting horn or foot. (Plate II. fig. 10.) In Bermuda earth there is a small species found, which has its three margins curved; and also a curious species, which resembles a triradiate spiculum of sponge.

It is remarkable how, in these minute and obscure organisms, we find ourselves met by the same difficulties concerning any positive laws governing the formation of any generic types, as in the larger and more complex forms of animal and vegetable life. It appears as if we could carry our real knowledge little beyond that of species; and when we attempt to define kinds and groups, we are encountered on every side by forms which set at nought our definitions.

Zygoceros Rhombus is in three parts; one central, which is like a broad band, the others lateral, of a rhomboidal shape, and curved; while at each corner is a projecting piece like a spine. It is entirely marked with very small dots. (Plate II. No. 6.)

As well as the beautiful shell of the Coscinodiscus, found both in a fossil and recent state, there is one of exquisite elegance and richness of the genus Arachnoidiscus, so named from the resemblance of the markings of the shell to the slender fibres of a spider's web. (Plate II. No. 1.) This is met with in the guano of Ichaboe; it is also found in the United States, as well as among the sea-weed from Japan, and the algæ of the Cape of Good Hope. From the latter place some specimens were presented to George Shadbolt, Esq., who, after submitting them to a most careful examination, gave the results in a paper to the Microscopical Society. Mr. Shadbolt says: "These shells are not, strictly speaking, bivalves, although capable of being separated into two corresponding portions; but are more properly multivalves, each shell consisting of two discoid portions, and two annular valves exactly similar respectively to one another.

"Each of the discoid valves is also capable of a further separation into two circular but dissimilar portions; which separation, however, is by no means easy of accomplishment, owing to the very fragile nature of the substance of the shell; in fact, I have only been able to effect this separation by accident, although this I have done sufficiently often to satisfy me that all the discoid valves are composed of the two portions adverted to; and this fact is capable of very distinct verification. . . 
"The two parts of the disc consist of, first, a very thin membrane, somewhat flexible and elastic, and capable of resisting the action of boiling nitric-acid; and on this membrane are the characteristic spider's-web-like markings, which have given rise to the name of the genus. It is situated externally in both discs of each individual.

"Secondly, each disc is composed of a siliceous frame-work, admirably adapted to support and strengthen the outer membrane; and bears a very close resemblance to a circular Gothic window.

"Each of these portions I have been able to obtain separately by boiling the discs for a considerable time in nitric acid, and then carefully washing them in distilled water; but it is only here or there that the separation is effected, and still less frequently that the siliceous framework is obtained entire; but that each disc really consists of these two portions is easily demonstrable. . . . .

"When in sit $\hat{\imath}$, these openings (central apertures) are partially covered internally by a delicate cup-like process, so as to form a species of valve; hence, when the shells are attached to the algæ, and are viewed even as opaque objects previcusly to their undergoing any sort of manipulation, it is not satisfactorily shown that the apertures exist in every specimen. I consider it highly probable that at these openings the nutriment is taken in; and, from the peculiar radiated arrangement of the siliceous parts immediately around them, it is not improbable that the animals may be provided with organs somewhat resembling in arrangement the tentacula of Madrepores. This is supposing the objects to be of an animal nature; of which, I confess, I think there is very little doubt.

"With regard to the two annular valves previously mentioned, they are situated between the two discoid ones; and thus the whole shell, when in its natural state, resembles, like those of some other genera, a circular snuff-box. These valves consist of a siliceous ring, within which (extending a slight distance towards the centre) is an annular membrane; and when in situt the valves are placed so that the membranes of each valve are in contact, and thus the space included between the two discoid valves is partially divided into two (not three) chambers. The membrane is so thin, that when mounted in balsam it is not visible; I have, however, been able to detect it upon one or two occasions, when it has got accidentally discoloured. It can, however, be readily seen by breaking away the upper valve from a specimen when attached."

Collecting and preserving Diatomacece.-The following directions are given by the Rev. W. Smith for collecting and preserving the Dia- 
tomaceæ, which may prove useful to the student: "Let him provide himself in the first place with the necessary applaratus for the field: this includes a good stock of small wide-mouthed bottles, that each gathering may be kept perfectly distinct; a long rod or stick, to which can be attached a small muslin net; a cutting-hook of about three inches in length, and a broad, flat spoon; the first, to collect such specimens as float upon the surface, or are held in suspension by the water; the second, to remove the larger algæ which may be covered with parasitic Diatorus ; and the third, to skim the surface of the mud for those which lie at the bottom of the pool.

"He will probably find, notwithstanding every care, that his specimens are mixed with much foreign matter, in the form of minute particles of mud or sand, which impair their value, and interfere with observation, especially with the higher powers of his instrument. These substances the student may remove in various ways: by repeated washings in pure water, and at the same time profiting by the various specific gravities of the Diatorus and the intermixed substances, to secure their separation; but, more particularly, by availing himself of the tendency which the Diatomaceæ generally have to make their way towards the light. This affords an easy mode of separating and procuring them in a tolerably clean state; all that is necessary being to place the gathering which contains them in a shallow vessel, and leave them undisturbed for a sufficient length of time in the sunlight, and then carefully remove them from the surface of the mud or water.

"Having performed these operations, which a little practice will render comparatively easy and generally successful, the next proceeding is to preserve the specimens in such a manner as to render them suitable for examination by the microscope at any future time. This may be done in various ways, according to the nature of the species and the precise object desired.

"The simplest method, and the one most generally useful to the scientific observer, is simply to dry the specimens upon small portions of talc, which can at any time be placed under the microscope, and examined without further preparation; and this mode possesses one great advantage, - that is, that the specimens can be submitted without further preparation to a heat sufficient to remove all the cell contents and softer parts, leaving the siliceous epiderm in a transparent state."

In the vicinity of Hull many very interesting varieties of Diatomaceæ. have been found, the beauty of the varied forms of which are such as to delight the microscopist; and, at the same time, some of them are highly useful, as forming that class of test objects which are 
best calculated of all others for determining the excellence and powers of our object-glasses. It has been shown by Mr. Sollitt that the markings on some of the shells were so fine as to range between the 30,000 th and 60,000th of an inch; the Plurosigma strigitis having the strongest markings, and the Navicula acus the finest. It is very remarkable that at Holderness, in digging out a submerged forest on the coast, numbers of fossil fresh-water Diatomacer have been discovered, although the sea flows over the part at every tide.

\section{XANTHIDIÆ.}

Tunthidice, or Double-bar Animalculce, have long been classed among the Infusoria. The name Xanthidia is derived from a Greek word signifying yellow, that being the prevailing hue of these animalcula. They are found plenteously in a fossil state, imbedded in flint, as many as twenty being detected in a piece the twelfth of an inch in diameter; in fact, it is rare to find a gun-flint without them. As living animals they are described as having a round transparent shell, from which proceed spikes varying in shape in different species. The green substance seen within them has been supposed to be ova; and it was stated that they propagated by self-division, and lived sometimes in pairs, though more generally singly. One kind was found by $\mathrm{Dr}$. Bailey in the United States of an oval shape, the 288th of an inch in length; and another kind, round in form, by the late Dr. Mantell, at Clapham: both these kinds were of a beautiful green colour. Specimens of the Branched Xanthidium, found in flint by Dr. Mantell, were from the 300th to the 500th of an inch in diameter. (See a representation of Xanthidia in Plate III. No. 4.) Mr. Ralfs says: "That the orbicular spinous bodies so frequent in flint are fossil Sporangia of Desmidiex, cannot, I think, be doubtful, when they are compared with figures of recent ones. Indeed, the celebrated geologist, Dr. G. Mantell, who, in his ILeclals of Crection, without any misgiving, had adopted Ehrenberg's ideas concerning them, has changed his opinion; and in his last work regards them as having been reproductive bodies, although he is still uncertain whether they are of vegetable origin."

Ehrenberg and his followers describe these bodies as fossil species of Xanthidium; but no doubt erroneously, since their structure is very different. For the true Xanthidium has a compressed, bipartite, and bivalved cell; whilst these fossils have a globose and entire one.

The fossil forms vary like recent Sporangia, in being smooth, bristly, or furnished with spines, which in some are simple, and in others 
branched at the extremity. Sometimes, too, a membrane may be traced, even more distinctly than in recent specimens, either covering the spines, or entangled with them. Some writers describe the fossil forms as having been siliceous in their living state; but Mr. Williamson informs us that he possesses specimens which exhibit bent spines and torn margins; and this wholly contradicts the idea that they were siliceous before they were imbedded in the flint. In the present state of our knowledge, it would be premature to attempt identifying the fossil with recent species; it is better, therefore, at least for the present, to retain the names bestowed on the former by those who have described them.

Near to Sydden Spout and the Round Down Cliff on the Dover beach, H. Deane, Esq. cut out a piece of pyrites with the adherent chalk, which, on examination, "exposed to view bodies similar to, if not identical with, the Xanthidia in flints; and clearly recognised $X$. spinosum, ramosum, tubiferum, simplex, tubiferum recurvum, malleoferum, and pyxidiculum, together with casts of Polythalamia, and other bodies frequently found in flints." Mr. Deane says : "In shape they are somewhat flattened spheres, the greater part of them having a remarkable resemblance to some gemmules of sponge, and having a circular opening in the centre of one of the flattened sides. The arms or spines of all appear to be perfectly closed at the ends, even including those which have been considered in the flint specimens to be decidedly tubiferous; showing that if the arms are tubes, they could afford no egress to a ciliated apparatus, similar to those existing among Zoophytes. On submitting them to pressure in water between two pieces of glass, they were torn asunder laterally, like a horny or tough cartilaginous substance; and the arms in immediate contact with the glass were bent. Some specimens, put up after several weeks' maceration in water, were so flaccid, that, as the water in which they were suspended evaporated away, the spines or arms fell inclined to the glass. These circumstances alone seem clearly to disprove the idea of their being purely siliceous. The casts of the Polythalamia, portions of minute crustaceans, \&c. appeared also to be, like the Xanthidia, some modification of organic matter; and in the case of the Polythalamia, the bodies are so perfectly preserved, that in some the lining membranes of the shells are readily distinguishable."

These investigations corroborate Mr. Ralfs, and also show that the same fossil remains so abundant in flint, are to be found in the beds of chalk by which it is surrounded.

Mr. Wilkinson, who examined some Tanthidia found in the Thames 
mud and slime on piles and stones at Greenhithe, gives it as his opinion, that they are not siliceous, but "of a horny nature, similar to the wiry sponges, which Mr. Bowerbank describes as being very difficult to destroy without the action of fire."

$\mathrm{He}$ also met with a peculiarity in a $X$. spinosum, which he had never seen in any other species; it was in a piece of a gun-flint. There appeared, as it were, a groove or division round the circumference, similar to that formed by two cups when placed on each other, so as to make their rims or upper edges meet. There also seemed to be a peculiarity in the arrangement of the tentacula: the tentacula of Xanthidic generally pass from the body in a direction perpendicular to its centre, without any apparent fixed arrangement of position; but in this instance, both in the upper and lower portions, there were two circlets of tentacula; one placed round each extremity, and another round that part where the specimen appeared to be separated. The size was the 325 th part of an inch in diameter, and the number of tentacula about twenty-five.

The other fossil Infusoria found most abundantly in the chalk and flint of England, are the Rotalia or wheel-shaped, and the Textutaria or woven-work animalculæ; the latter having the appearance of a cluster of eg's in a pyramidical form, the largest being at the base, and lessening towards the apex.

In the earth taken from the shores of Lough Mourne, county Antrim, Ireland, there are found great numbers of various species of the flinty cases of Diatomacer. It is the opinion of the Rer. W. Smith that this deposit is of a recent date, and that most of the species are to be found alive in the waters of the lake. When fresh, the earth is of a delicate cream colour, but becomes perfectly white on being boiled in acid, and pulverized; it is an excellent polishing-powder for silver plate.

As important in a geological point of view, as closely appertaining to this division of our work, and as interesting to all striving for knowledge, we will quote from a paper read at the Microscopical Society in 1844: "On Animals found in Chalk, still to be found in a living state in the Stomachs of Oysters. By the Rev. J. B. Read." He says :"It has been discovered, or rather asserted, that there is a break in the great geological chain of organised beings; and that a link is wanting to connect the cretaceous and antecedent series with a series of subsequent formations. In the primary and secondary formations, and again in the tertiary and recent, there is, in some cases, an absolute identity of organic structure in their respective animal or regetable remains, which is supposed to be altogether wanting between the 
secondary and tertiary. 'Below the tertiary formation,' says Professor Phillips, 'are no recent species.' From this point, therefore, the work of creation is dated anew; and upon this supposition is founded Sir Charles Lyell's arrangement of the subdivision of the tertiary system; his Eocene period, or dawn of our present animal and vegetable kingdoms, being coincident with the third geological era. About five years ago, Dr. Mantell evidently felt some misgivings as to the accuracy of these views. He adopts Sir C. Lyell's arrangement as one of great utility in the present state of our knowledge; but he adds, 'it appears probable that it may require considerable modification, or perhaps be afterwards altogether abandoned with the progress of geological research; for it cannot be doubted that strata in which no recent species have been found may yield them to more accurate and extended observation.' It is remarkable that the converse of this supposition has actually obtained; and our modern seas have yielded living forms hitherto characteristic of the chalk formation, and regarded as antediluvial. The merit of this discovery rests with Professor Ehrenberg. . . . .

"My own observations confirm all the important conclusions arrived at by Ehrenberg, and are fortunately capabie of easy verification. It will not be necessary to send for sea-mud from Norway, or for Peruvian or Mexican sea-water; half a dozen native oysters, or, in truth, I may say, the stomach of a single oyster, will often afford us ample proof of the uniformity and identity of organic life in distant ages of the earth, and leave us in no doubt that the dawn of the organic creation, coexistent with ourselves, reaches further back into the history of the earth than has hitherto appeared.

"The well-known ciliary currents in the fringes of the oyster induced me to examine the contents of the stomach, under the expectation of finding some minute forms of Infusoria; for it seemed but reasonable to infer that the absence of locomotive power, and the consequent inability of seeking for food, might be compensated by so beautiful a contrivance for insuring constant nourishment. My expectations were fulfilled and surpassed. In the stomach of every oyster I examined, and in the alimentary canal, I found myriads of living Monads, the Vibrio also in great abundance and activity, and swarms of a conglomerate and ciliated living organism, which may be named Volvox ostrearius, somewhat resembling the Volvox glabator, but of so extremely delicate a structure, that it must be slightly charred to be rendered permanently visible.

"This result, though highly interesting in itself, and marking so 
clearly the especial function of those instruments which fit the oyster for its peculiar place in the scale of created beings, is nevertheless of inferior importance, in a geological point of view, to some other results to which the presence of other Infusoria in the stomach of the oyster conduct us. These Infusoria I will briefly enumerate. They have silicenus loricæ, and belong to the family of the Bacillariæ; and some of these in their fossil state constitute the chief bulk of the chalk. They are the following: Actinocyclus senaris, Ceratoneis fasciola, Coscinodiscus minor, C. patina, C. radiatus, Dictyocha aculeata, D. fibula, D. speculum, Gallionella sulcata, Navicula entomon, Tripodiscus argus, Xanthidium furcatum, X. hirsutum, Zygoceros rhombus, Z. surirella, and two new species of this genus.

"All the above, together with some well-known recent species of the same extensive family of the Bacillariæ, I have found in the stomachs of oysters. That they are not the detritus of chalk or chalk-marl, although identical as to species with the fossils of the chalk, is decided by the fact of my finding several of them alive; and even in the dead specimens there are the same distinct remains of organic details, which enabled Ehrenberg to pronounce with perfect accuracy, that many similar dead forms had been recently in a living state. If further proof be required, it may be furnished by the process of charring, which immediately blackens the yellow membraneous integument of several of the species.

"We must not expect to find the same species of Infusoria in this new locality during every portion of the year. Like creatures of a larger growth, oysters live on the food which is successively in season; and I find, in point of fact, that even a different shore is marked by a decided difference in the infusorial contents of the stomach. The 'Scotch Natives' are characterised by innumerable minute circular forms, resembling the Coscinodiscus. Our own oysters, on the contrary, are nearly destitute of these living rotatory discs, but they are much richer in more interesting species; and in addition to the siliceous-shelled Infusoria, which are received into their stomachs, they also occasionally furnish examples of calcareous Polythalamia adhering to the inner surface of their shells. These latter microscopic cephalopods form part of the proper food of shrimps; and accordingly I find that very minute Rotalia pass into the stomachs of shrimps entire."

Mr. Read then proceeds to say that the comparison which Ehrenberg conducted so successfully between the chalk and recent Infusoria, he attempted to extend one step lower in the oolitic series, and to embrace in the investigation the Infusoria of the Kunmeridge clay; the large 
oyster-beds in this stratum in his own locality, the vale of Aylesbury, very naturally inviting the search for the food of these ancient molluscs. On examination, he found in this clay vast numbers of the circular discs, which he has alluded to as existing in living oysters, and, in addition, decided examples of the Cosinodiscus and portions of calcareous shells of Polythalamia. Thus he suspects an absolute identity between the genera and species of some of the minute and simple occupants of the ancient and the modern seas, though larger forms of more complicated mechanism have successively risen and disappeared.

"It is my chief object, however," adds Mr. Read, "to show, with Ehrenberg, that animals of the chalk are still found in a living state; and having done this, it is needless, with Ehrenberg's paper in our hands, to point out, at any length, the importance of these researches. They bear directly upon mountain masses, and seem to make the vast chalkrange overstep its boundary, and connect itself rather with the tertiary and recent, than with the primary and transition formations. Beyond all doubt the bulk of the cretaceous series is composed of organisms still living, as I have now shown, in our British seas, and, as Ehrenberg observes, still capable, under the concurrence of favourable circumstances, of giving rise to the greatest changes in the distribution of the solid crust of the earth. The material, and not the magnitude, of the Infusoria, is the proper element in these calculations. We must shut our eyes to the minuteness of each individual atom of life, and look rather at the marvellous activity of its law of increase, and at its indestructible shield, which sets at defiance the two great reducers of organic structure, death and fire. A very few nutshells would hold all we should leave of an elephant if reduced to its ultimate elements by fire, but no known intensity of heat would reduce the siliceous mass of Infusoria. Fusion, under certain conditions, would be the utmost result, and thence would originate other mountain masses, which, to say the least, would be very nearly allied to those which we term granitic. The question, then, is not unreasonable,-was granite originally an infusorial earth?-and we accept Ehrenberg's hypothesis as the reply: 'Since of the four as yet well established great geological periods of the earth's formation, the quaternary, tertiary, and secondary formations contain recent organisms, it is as three to one more probable that the transition or primary formation is not differently circumstanced, but that, from the gradual longer decomposition and change of many of its organic relations, it is more difficult to examine and determine." 


\section{FORAMINIFERA.}

The Foraminifera are a division of animalculæ having minute perforated shells, as if covered with pores; they are found in great abundance in a fossil state in every part of the globe, and numbers of species corresponding to the structure of past generations are discovered alive, still adding to the calcareous portions of the world. The great Pyramid of Egypt, covering eleven acres of ground, is based on blocks of limestone consisting of Foraminifera, nummulites or stone coin, and other fossil animalculæ. The nummulites vary in size, from that of a most minute object to that of a crown-piece, and many appear like a snake coiled in a round form. A chain of mountains in the United States, 300 feet high, seems to be wholly formed of one kind of this fossil-shell. The crystalline marble of the Pyrenees, and the lime-stone ranges at the head of the Adriatic gulf, are composed of small nummulites. Vast deposits of Foraminifera have been traced in Egypt and the Holy Land, on the shores of the Red Sea, Arabia and Hindostan, and, in fact, may be said to spread over thousands of square miles from the Pyrenees to the Himalayas.

In an interesting paper " on Foraminifera, their organisation, and their existence in a fossilised state in Arabia, Sindh, Kutch, and Khattyawar," by Surgeon H. J. Carter, published in the Journal of the Bombay Branch of the Royal A siatic Society, 1849, the author says: "The Poorbandar limestone derives its specific denomination from the place near which it is quarried in Khattyawar, and is imported at Bombay in the shape of blocks and flags for building purposes. It is of a brownish white colour, uniform in structure, granular, and composed of oolitic particles of calcareous sand united together in a firm compact rock. The granular structure first attracted my attention, and knowing, from the discoveries of Ehrenberg, that most of the cretaceous deposits were almost entirely composed of the remains of microscopic Foraminifera, and that Dr. Mantell, in testing the truth of Ehrenberg's observations in examining the 'chalk and flint of the south-east of England,' had not only confirmed his statements, but had moreover found that the softer and more perishable parts of the bodies of these animals had also become mineralised, I thought it probable that this deposit also might be composed of the remains of them, and that certain yellow specks which it contained might be the mineral which Dr. Mantell had described as supplying casts or fac-similes of their bodies. To ascertain this, a portion of it was reduced to coarse powder, and the yellow par- 
ticles having been liberated from their connexion with the carbonate of lime by an acid solvent, they were found to be what I had expected, from Dr. Mantell's observations, viz. the casts of the interior of microscopic foraminiferous shells of the most exquisite beauty in their forms and symmetrical development ; and the more highly they were magnified, the more minutely could be distinguished the innermost recesses of the cells or chambers they represented. The larger of these casts seldom approach to or exceed the twenty-fifth part of an inch, so that their forms, in detail, are entirely invisible to the unassisted eye. Occasionally the figures of the fossilised shells which enclose them can be partially seen; but this is seldom the case, they having become transformed, by partial dissolution and recrystallisation, into the oolitic grains of which the rock is composed." Mr. Carter discovered that the Poorbandar limestone was almost entirely composed of the remains of a variety of Foraminifera.

"The fossilised Foraminifera in the Poorbandar limestone, although occasionally reaching the twenty-fifth, do not average more than the hundredth part of an inch in diameter; so that more than a million of them may be computed to exist in a cubic inch of the stone. They may be separated into two divisions-those in which the cells are large, the regularity of their arrangement visible, and their bond of union consisting of a single constructed portion between each; and those in which the cells are minute, not averaging more than the 900 th part of an inch in diameter, the regularity of their arrangement not distinctly seen, and their bond of union consisting of many thread-like filaments. To ascertain the mineral composition of the amber-coloured particles or casts, after having found that it was carbonate of lime with which they were surrounded, I placed them for a few moments in the reducing flame of a blow-pipe, and observed that on subsequently exposing them to the influence of a magnet, they were all attracted by it. Hence, in a rough way, this rock may be said to be composed of carbonate of lime and oxide of iron."

Truthfully does Lamarck say of the Foraminifera: "Their smallness renders their bodies contemptible to our eyes; in fact, we can hardly distinguish them; but we cease to think thus when we consider that it is with the smallest objects that nature produces the most imposing and remarkable phenomena. Now, it is here again that we have one of the numerous instances which attest that, in her production of living bodies, all that nature appears to lose on one side in volume, she regains on the other in the number of individuals, which she multiplies to infinity." 
Besides the name of Foraminifera, they are often called by that of Polythalamia, a word signifying many-chambered, from the sections of their shells; to these designations some have added that of Rhizopoda, from the tentacula, or feelers, having root-like prolongations.

Dujardin, in a paper published by the French Royal Academy of Science, in 1835, says that the chambers of the cells of the shells are filled with a red or orange-coloured animal matter, of a slimy consistence, capable of extension into minute threads filled with irregular granulations, but without organs. He adds, that by means of its filaments it exercised locomotion by creeping along; and as the filaments extended like roots, he proposed the name of Rhizopoda.

Little is known of the habits and structure of the animals which inhabited the shells of the Foraminifera; but the best account we have yet met with, is in a most pains-taking paper by W. C. Williamson, Esq., of Manchester, published in the Microscopical Society's Transactions, March 1849, and to this we beg to refer the reader.

\section{ECHINO-DERIIATA.}

These creatures form an extensive class, to which the above name has been applied, as well as that of Echinidce, sea-urchins, or sea-eggs. The animals belonging to this class are, for the most part, characterised by having rough and spiny skins, in which the radiated forms of the Asteriadæ seem wholly lost, and the calcareous crust is more or less transformed into a globular case of wonderful workmanship. The class of Echino-dermata is the only one among radiated animals in which shells are met with, and these are by no means universally present throughout the group. If a small portion be broken away from the thin margin of the shell of any species, say the Pinna, and placed without any preparation under a low magnifying power, it presents on each of its surfaces, when viewed by transmitted light, very much the appearance of a honeycomb (Plate V. No. 7); whilst at the broken edge, it exhibits an aspect which is evidently fibrous to the eye, but, when examined with reflected light, resembles that of basaltic columns.

The Echini may be found in abundance upon our sea-shores, lurking among the rocks, where they entrap their prey. The shell in the fresh animal is covered with a thin fleshy skin, filled with calcareous points; and projecting beyond these points, are innumerable suckers, which he uses as feet, or as a mode of progression, even to the climbing of rocks, in order to feed upon the corallines and other zoophytes; he 
marches along with ease where apparently no footing could be found, or digs a hole with his spines to bury himself in the sand, to escape his pursuer, or to hide himself from observation. Immediately beneath the fleshy skin is placed the shell, which, when denuded of its spines, would seem to be an ordinary shell, having its outer surface covered with small projecting spines or tubercles, regularly arranged in circular lines.

The skeleton of these animals generally consists of an assemblage of plates, or joints, of calcareous matter. The minute structure of which presents more or less a reticulated character; and the solid parts are usually composed of a series of superimposed laminæ or scales. The openings, or areolæ, in one layer being always placed over the solid cell-walls of the layer beneath it, the spines are situated on the external surface of the shell; they are generally of a conical figure, and are articulated to the tubercles by a ball-and-socket joint. When a thin transverse section of one of these spines is examined with the naked eye, it appears to be made up of a series of concentric layers, varying considerably in number; not however with the size of the spine, but with the distance from the base at which the section was made; when a section taken from the middle of the spine is examined with a power of fifty diameters, it will be seen that the centre is occupied by a reticulated structure; around the margin of this may be observed a series of small structureless spots, arranged at equal distances apart (Plate V. No. 2); these are the ribs or pillars, and indicate the external surface of the first layer deposited; passing towards the margin, other rows of larger pillars may be seen, giving it a beautiful indented appearance; all the other parts of the section are occupied by the usual reticulated tissue. In the greater number of spines the sections of the pillars present no structure, in others they exhibit a series of concentric rings of successive growth, which strongly remind us of the medullary rays of plants; occasionally they are traversed by reticulated structures, as represented in Plate V. No. 1. When a vertical section of a spine is examined, it will be found to be composed of a series of cones placed one over the other; the outer margin of each cone being formed by the series of pillars. In the genus Echinus, the number of cones is considerable, while in that of Cidaris there are seldom more than one or two; so that from such species transverse sections may be made having no concentric rings, and in which only the external row of pillars may be seen.

"In whatever direction we slice the shell of the Echinus, we always meet with a sort of reticulated structure; for, if one section be 
parallel to the surface, it passes vertically through a series of laminæ, and in the direction of the pillars that connect them, which thus constitutes an areolar structure of a tolerably regular form. The testa is thus of an extremely porous character, the areolæ having the freest communication with each other; even in the living state, however, the areolæ appear to be empty, the ingress of the fluid with which the surface of the shell is in contact being prevented by the delicate membrane that covers it. At the same time, it possesses a remarkable degree of strength, in proportion to the amount of solid matter employed in its construction.

"The skeleton of the Echino-dermata contains very little organic matter. When it is submitted to the action of very dilute acid, to dissolve out the calcareots matter, the residuum is very small in amount. When obtained, it is found to possess the reticular structure of the calcareous shell; the meshes or areolæ being bounded by a substance in which a fibrous appearance, intermingled with granules, may be discerned under a sufficiently high magnifying power, as was first pointed out by Professor Valentine. This tissue bears a close resemblance to the areolar tissue of higher animals; and the shell may probably be considered as formed, not by the consolidation of the cells of the epidermis, as in the mollusca, but by the calcification of the fibroareolar tissue of the true skin. This calcification of areolar or simply fibrous tissue, by the deposit of mineral substance, not in the meshes of areolæ, but in intimate union with the organic basis, is a condition of much interest to the physiologist; for it presents us with an example, even in this low grade of the animal kingdom, of a process which seems to have an important share in the formation and growth of bone, namely, in the progressire calcification of the fibrous tissue of the periosteum membrane covering the bone." "

The above description is characteristic of spines generally, including the echinites, \&c.; they will all be found to be constructed on a nearly uniform plan.

From their peculiarity of structure, they may be said to be almost imperishable. Their shells exist abundantly in all our chalky cliffs, innumerable specimens of which may be obtained, exhibiting the same wondrous forms and characters as those which now frequent our shores.

* Dr. Carpenter, Cyclopædia of Anatomy and Physiolog. 


\section{ACALEPHÆ.}

In great variety of form and colour, swimming freely about the waters of the ocean, are found in abundance the beautiful Acalephæ. From some of them having a remarkable stinging property, they have derived their name of Sec-nettles, while others, from their gelatinous nature, are commonly called Sec-jelly, or Jelly-fish; one of which is shown in the title-page of this work, depending from the top.

These interesting animals were arranged in three orders: A. stabiles (fixed), A. liberce (free), and A. hydrostatica (hydrostatic). Cuvier classed them in two orders: A. simplices, and A. hydrostaticæ. They are now, however, classed into groups, according to the peculiar mode in which they effect their locomotion.

The Medusce spread on the surface of the water a beautiful jellylike mass, that in form resembles an umbrella; and by a continual contraction and opening out of this part, they pass along in the path they desire. The Beroë, like many of the Infusoria, propel themselves with active ciliated arms. The Physalidoe have an organ common to fishes,swimming bladders, - by filling or emptying which they rise or sink, and move along in their watery home.

The Portuguese Men-of-war have a large bladder, which, when filled with air, rises above the surface of the waves, and is propelled by the wind; a contrivance something similar to, though more successful, we suspect, than the proposition to drag along land-carriages by means of balloons. The Man-of-war in its voyages deals out death to its prey.

Velella also hoists a sail, but of a different construction, it being a delicate membrane, which the animal stretches forth to the favouring breeze. This sail is supported by a horny matter, a preparation of which is to be seen in the Museum of the Royal College of Surgeons, and is thus described in the catalogue: "A horizontal and vertical plate of transparent horny matter, forming the skeleton of Velella limbosa. The former is of an oval figure, concave below, and marked with concentric lines of growth; whilst the latter is not only much thinner than the horizontal one, but is firmly attached to it in a rectangular position, and rises above it in the form of a crest. When examined with a power of a hundred diameters, neither of the plates presents any trace of cells or reticulations; the concentric lines, which are even visible to the naked eye, are the only parts that can be said to exhibit any variety in structure. Some specimens of this animal are occasionally met with as small as $\frac{1}{8}$ th of an inch, whilst others even exceed two inches 
in length." The same valuable work describes "the flat circular horny disc, forming the skeleton of Propita gigantea. To the naked eye it exhibits both radiating and concentric markings; and when examined with a power of 40 diameters, its upper surface is found to be furrowed, and two rows of small projecting spines occur upon the ridges between the furrows, the ridges being the radiating fibres above noticed. The under-surface, or that to which the greater portion of the soft parts of the animal are attached, is more deeply furrowed; and plicæ or folds of the mantle fit accurately into the furrows, from which they can easily be removed by the application of a gentle force. The concentric markings have in all cases small scalloped edges; they occur at certain regular intervals, and are so many indications of the lines of growth. In the centre there is a circular depression; and between its circumference and that of the first concentric marking, there are eight flattened radii. If the under-surface be examined with a power of 100 linear, the ridges will all be found to have small jointed tubular processes like hairs projecting from them. The precise office these processes perform cannot be here stated, as the soft parts were separated from the skeleton before it was submitted to examination. In no part of this horny tissue is there a trace of a cellular or reticular structure."

The beautiful transparency of the tissues renders the Acalephæ delightful objects for the microscope. In the publications of the Royal Society, Professor Forbes gives a very full and accurate account of the Medusæ; and this numerous class of pulmograde animals he divides into two classes, those having a protective appendage to the eyes, or that part supposed to be the eyes, and those that have no such appendage, and are called "naked-eyed," or the Gymnophthalmata. To the Transactions of the Microscopical Society, George Busk, Esq. has contributed an excellent paper on the anatomy of two species of naked-eyed Medusæ.

\section{ANNELID E.}

The lowest members of the class Annelidæ exhibit, in many respects, habits analogous to the vermiform Echino-dermata. Most of them are marine, living in the vicinity of the shore, where they burrow in the sand, much in the same manner as the Siponculi. In many instances, they are found to line the holes in which they live with a calcareous deposit exuded from the surface of their bodies, or some of them construct factitious tubes of foreign substances, or even residences, composed of true shell. Most persons may remember having noticed that 
shell-stones, fragments of pottery, or any similar substances, which have been immersed for any length of time in the sea, become covered over with irregularly-twisted calcareous tubes, which sometimes accumulate in great abundance; and not unfrequently similar masses are brought up from considerable depths by the lines of the fisherman.

If, while the contained animals are alive, these be placed in a vessel of sea-water, a very pleasing spectacle may soon be witnessed. The mouth of the tube is first seen to open, by the raising of an exquisitelyconstructed door, and then the creature cautiously protrudes the anterior part of its body, spreading out at the same time two beautiful fanlike expansions of a rich purple or scarlet colour, which float elegantly in the surrounding water, and serve as branchial or breathing organs. The Serpula, for such is the name of the worm which fabricates these tubes, when withdrawn from its residence (fig. 93), is seen to have the

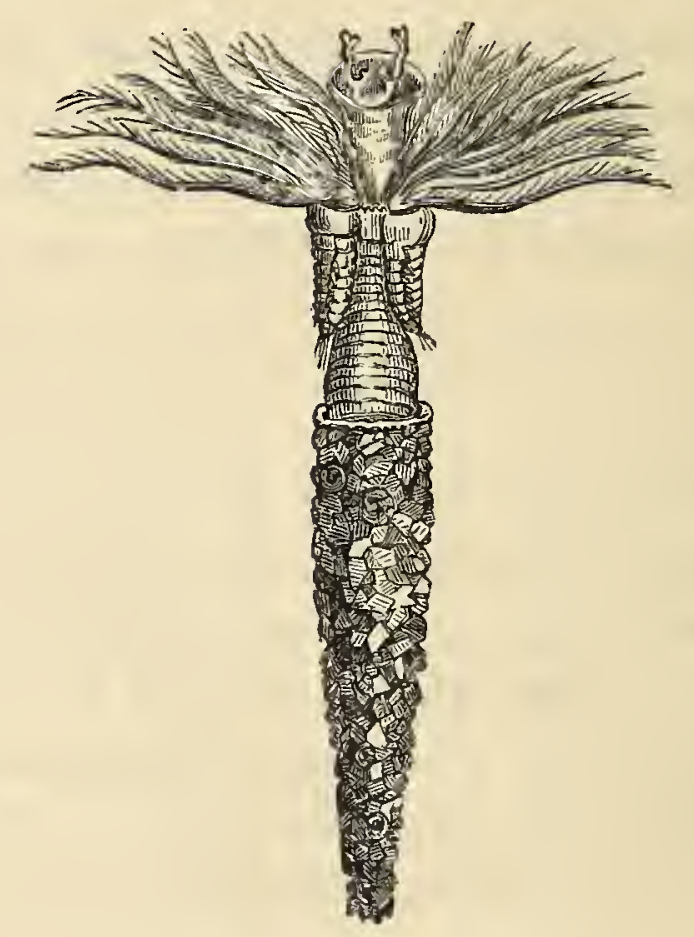

fig. 93. The Serpula in its calcareous tube.

hinder part of its body composed of a series of flattened rings, entirely destitute of limbs, or any other appendages. The tube itself is calcareous, being formed by an exudation from the creature's body, which soon hardens into a shell, and in which the animal remains during its whole lifetime fishing for its food.

Another important group of the Annelida is represented by the Medical Leech; it is one of a race termed Suctorial Annelides. The most interesting part of the anatomy of the leech, to microscopists, is the structure of the mouth. "This piece of mechanism is a dilatable 
orifice, which would seem at first sight to be but a simple hole. It is not so; for we find that just within the margin of this hole three beautiful little semicircular saws are situated, arranged so that their edges meet in the centre. It is by means of these saws that the leech makes the incisions whence blood is to be procured, an operation which is performed in the following manner: no sooner is the sucker firmly fixed to the skin, than the mouth becomes slightly everted, and the edges of the saws are thus made to press upon the tense skin; a sawing morement being at the same time giren to each, whereby it is made gradually to pierce the surface, and cut its way to the small bloodressels beneath. Nothing could be more admirably adapted to secure the end in view than the shape of the wound thus inflicted, the lips of which must necessarily be drawn asunder by the very contractibility of the skin itself; and that the enormous sacculated stomach, which fills nearly the whole body of the leech, was designed to contain its greedily devoured meal, there can be no reasonable question. The leech, in its native element, could hardly hope for a supply of hot blood as food; and, on the other hand, its habits are most abstemious, and it may be kept alive and healthy for years, with no other apparent nourishment than what is derived from pure water frequently changed; eren when at large, minute aquatic insects and their larræ form its usual diet."*

\section{CRUSTACEE.}

The skeletons of Crustacea are external to the soft parts; in a great number of species it is thin and membraneous, in others it is of a horny material, thickened with calcareous matter, having a distinct series of pigment-cells of a stellate figure, all howerer supplying beautiful objects for microscopic examination.

A crustaceous animal consists of three parts: the head, the body, and the carapace, which is covered with one entire shell, and is popularly called the tail, consisting of seren rings, or joints. There are properly fourteen rings in that part of the body which is called the carapace; but they are only used when the animal changes its shell. The joints in the tail are to enable the animal to spring forward, which it does frequently when it wishes to change its position. It can also crawl; but it mores in this manner awkwardly, and in an oblique direction. The river craw-fish belongs to the same genus (Astucus) as the lobster, and both have long tails, which are spread out when they crawl, and nume-

* Professor T. Rymer Jones, Cyclopædia of Anatomy and Physiology. 
rous legs and claws, with which they can pinch severely when they wish to defend themselves. The crab has a short tail, and belongs to the genus Cancer. The shrimp, though it has no claws, properly so called, has two feet larger than the others, each of which has a hooked jointed nail. The prawn, which is quite different from the shrimp, is nearly allied to the crawfish, or thorny lobster. All the Crustaceæ have the power of renewing their claws if they are torn off at a joint, and they change their shells every year. The new shell is at first quite soft, and at that period the fish are unwholesome to eat. The females spawn in July and August, and soon after great numbers of the little Crustaceæ may be found swimming about, not more than half an inch in length.

Dr. Carpenter describes the shell of the crab and lobster as " being composed of three layers, viz. the epidermis or cuticle, the rete-mucosum, or pigment, and the corium. The epidermis is of a horny nature, being generally more or less brown in colour, and under the highest magnifying powers presenting no trace of structure (seen in Plate V. No. 7, a section of the Pinna shell); it invests all the outer parts of the shell, and has in many instances large cylindrical or feather-like hairs developed from certain portions of its surface. The rete-mucosum, or pigment-cells, consist of either a series of hexagonal cells, forming a distinct stratum, or of pigmental matter diffused throughout a certain thickness of the calcareous layer." (Plate V. No. 5.) In the crab and lobster it is very thin, but in the cray-fish it occupies in some parts more than one-third of the entire thickness of the shell; when examined by the microscope, this portion appears to be composed of a large number of very thin laminæ, which are indicated by fine lines taking the same direction on the surface of the shell, the number of lines being the greatest in the oldest specimens ; these layers, even in the cray-fish, are covered by a thin stratum of very minute hexagonal cells, without any trace of cell matter in their interior. The corium is the thickest layer of the three, being the one on which the strength of the shell depends, in consequence of the calcareous material deposited in it. (Plate V. No. 4.) When a vertical section of the shell of the crab is examined, it is found to be traversed by parallel tubes, like those in the dentine of the human tooth; these tubes extend from the inner to the outer surface of the shell, and are occasionally covered by wavy lines, probably those of growth, shown in a portion of No. 3, plate V. If a horizontal section of the same shell be made so that the tubes be divided at right-angles to their length, the surface will clearly exhibit their open mouths, surrounded by calcareous matter. In shrimps and 
very small crabs, the deposition of the calcareous matter takes place in concentric rings, like those of agate; and occasionally small centres of ossification somewhat similar to No. 3, with radiating stria, are to be met with in the former animal. If the calcareous portion of the shell be steeped in hydro-chloric acid, a distinct animal structure or basis is left behind, and the characters of the part will be very accurately preserved. The calcareous matter, like that of bone, generally presents a more or less granular appearance (as at No. 4), and so angular in figure as to resemble certain forms of rhomboidal crystals, as shown at $N_{0}$. 8, from the outer brown shell of the oyster. The beauty of all these structures is much increased when viewed by polarised light on the selenite stage.

The microscopic animalculæ, known as Cyclops quadricornis, Daphnia pulex, Cypris pubera, are generally classed by naturalists among the Crustacea. They are found in vast numbers in all the water supplied to this metropolis, and may be seen most actively darting about in water that has stood by for a few days. The appearance of some of these singular little creatures, inclosed in a shelly covering, is calculated to reward the microscopist. The leg's of some are furnished with branchial fringes, which appear in perpetual motion. Their number varies,-some have sis, others twenty, or even a hundred. Many are so much like minute shells, that their true place in the animal kingdom would scarcely be suspected; microscopic plants and animalculæe serve them as food, while they, in their turn, are the prey of the hydra and small fishes.

\section{MIOLLUSCA.}

The term Mollusc is derived from the Latin, and signifies soft; the body of the animal being fleshy and unjointed, having a shell partly or entirely covering it, and attached to it by means of muscles. The shells are of two kinds; those of an epidermal character being formed upon the surface of a filmy cloak-like organ called a mantle, answering to the true skin of other animals; and those of a dermal character being concealed within the substance of the mantle, frequently moulded into a great diversity of forms, and coloured with various tints.

The animals belonging to the molluscous sub-kingdom are divided into six classes, viz. Tunicata, Brachiopoda, Branchiata, Gasteropoda, Pteropoda, and Cephalopoda; of these, all, except the Tunicata and a few of the Pteropoda, are provided with a hard calcareous shell, some- 
what the same as the Crustacece. Mr. Bowerbank has shown that they possess a more or less distinct organic structure, and which, like the epidermis and other outer coverings of the higher animals, is developed from cells that in process of growth have become hardened by the deposition of calcareous matter in the interior. This earthy matter of shell consists principally of carbonate of lime, deposited in a crystalline state; and in certain shells, as in that of the common oyster (Plate V. No. 8), from the animal-cell not having sufficiently controlled the mode of deposition of the earth particles, they have assumed the form of perfect rhomboidal crystals.

The oyster itself is a molluscous animal of the kind called Acephalus, or non-headed, as it has no distinct head. The gills, or breathing apparatus, form what is commonly called the beard of the oyster. The creature is attached by strong muscles to its shell, which, as it consists of two parts, or valves, is called a bivalve, to distinguish it from those that are in one part, like that of the snail, and which are called univalves. The mouth of the oyster is a mere opening in the body, without jaws or teeth; and its food consists of nourishing substances which may be in the water, and which are washed into the shell when it is open. Oysters attach one of their valves to rocky ground, or some fixed substance, by means of a mucilaginous liquid, which soon becomes as hard as the shell. Oysters generally spawn in May; and their growth is so rapid, that in three days after the deposition of the spawn, the shell of the young oyster is nearly a quarter of an inch broad; and in three months it is larger than a shilling. The spawn is a very interesting object for microscopic examination, especially when viewed under polarised light. The oyster appears to be extremely inanimate : it fixes itself to any object that may be near, being sometimes found attached to the back of a living lobster or crab, and frequently to the roots of trees.

\section{PEARLS.}

At one time a most extravagant value was set upon pearls: for one of these molluscous secretions, Tavernier paid the sum of $110,000 l_{0}$; it was found at the Catifa fishery, off the coast of Arabia. At the Bahrein Islands, Persian Gulf, the produce of the two months' fishing is averaged at the value of $90,000 \mathrm{l}$. annually.

Pearls are usually found in the shells of Meleagrince, or pearloysters, as they are commonly called; and the choicest in a large one named Avicula, or Meleagrina Margaritifera; also in a mussel 
termed Mya Margaritifera. An inferior kind of pearl is also found in many mussels of the rivers of Great Britain ; and, at one time, the pearlfishery of Ireland was greatly celebrated. The oysters on our coasts have frequently a dull common kind of pearl within their shells.

Naturalists somewhat differ in their opinions as to the mode in which pearls are formed. Some think that they are caused by particles of sand having got into the stomach, when the animal, to prevent the roughness of these particles from injuring its delicate structure, covers them over with a secretion from its body, thus continually increasing their size by continual additions. It is now, however, pretty generally admitted to be a disease; and it is quite certain that the pearls are matured on a nucleus, consisting of the same matter as that from which the new layers of shell proceed at the edge of the mussel or oyster. The finest kind are formed in the body of the animal, or originate in the pearly part of the shell. It is by the size, roundness, and brilliancy of pearls, that their value is estimated. They are found either in the mantle of the animal, or attached to the inner surface of the shell.

Pearls have been produced in an artificial manner.-Apollonius, the philosopher of Tyana, who is supposed to have died at an advanced age in the year 97 , being the first to notice such a practice, on the borders of the Red Sea. He says: "The Indians dived into the sea, after they had rendered it calm, and perhaps clearer, by the pouring in of oil; they then induced the mussels, by means of some attractive baits, to expand their shells; and having pricked them with a sharppointed instrument, the liquor which exuded from the wound was received into a perforated iron, where it hardened gradually, and formed pearls of the finest water." Of this method, if correct, we now know nothing; certainly, Sir Joseph Banks had specimens of some Chinese mussels, in which were small pieces of iron covered with a pearly substance, that at first seemed to give countenance to such a story; but there is a greater probability that the presence of these pieces of wire resulted from a practice which caused considerable sensation on its announcement in Europe in 1773, and which was stated to be employed by the Chinese, and was recommended to be tried on the oysters of European production. It consisted in taking living oysters, and perforating their shells with a wire or awl, so as not to hurt their vitality, and introducing into the holes a small iron wire, having knobs at a small distance from each other. The oysters were then to be replaced in their beds; and by the time the season came round again, a concretion of pearly matter would be found to have 
covered the knobs of the wire, and would be the larger and the more perfect the longer the oysters were suffered to remain. Buffon had some time before proposed the formation of pearls by puncturing the shells of oysters; but, from the character he had acquired of a visionary speculator, he was unheeded.

Mussels, in which these artificial pearls had been formed by the Chinese, have been recently brought to this country by Mr. Rawson, from one of which the accompanying drawing was made (fig. 94); it is represented of the natural size, with the simple pearls adhering to the shell.

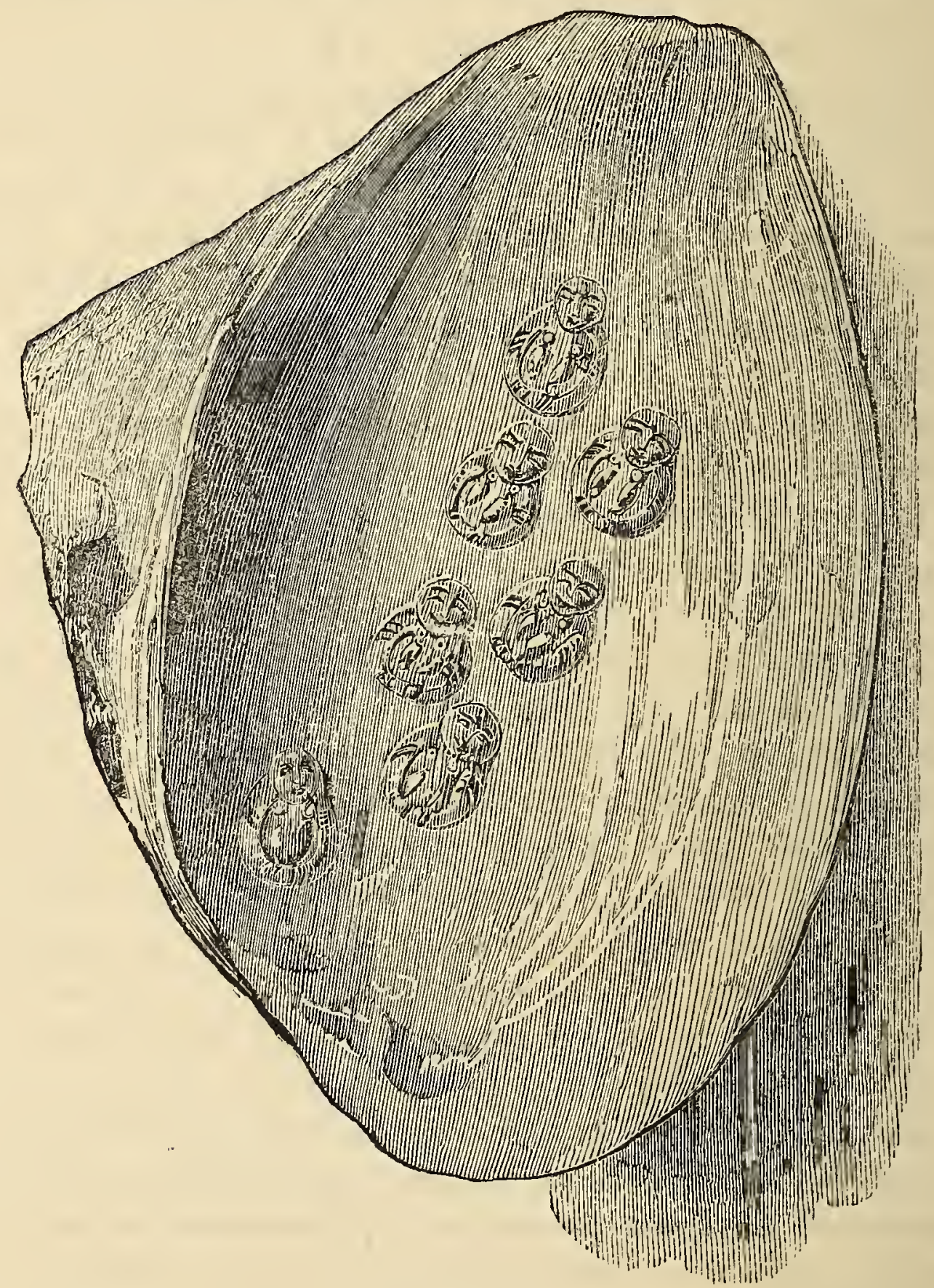

fig. 94 .

ArtificiaI PearIs in the shell of Mya Margaritifera.

The account furnished by this gentleman at the same time is, "that they are only obtained near Ning-po. The Hermes steamer being on a visit to 
that place, he was able to obtain a few living specimens; in which, on being opened, several pearls, as many as eighteen or twenty, were found in the course of formation. It appears they are formed by introducing some pieces of wood or baked earth into the animal while alive, which, irritating it, cause it to cover the extraneous substance with a pearly deposit. Little figures made of metal are frequently introduced; and, when covered with the deposit, are valued by the Chinese as charms. These figures generally represent Buddha in the sitting position, in which that image is most frequently portrayed." This, it will be observed, nearly coincides with the account given by Sir Joseph Banks of these artificial productions.

It is said that the fishermen of the Thames reaped a harvest by

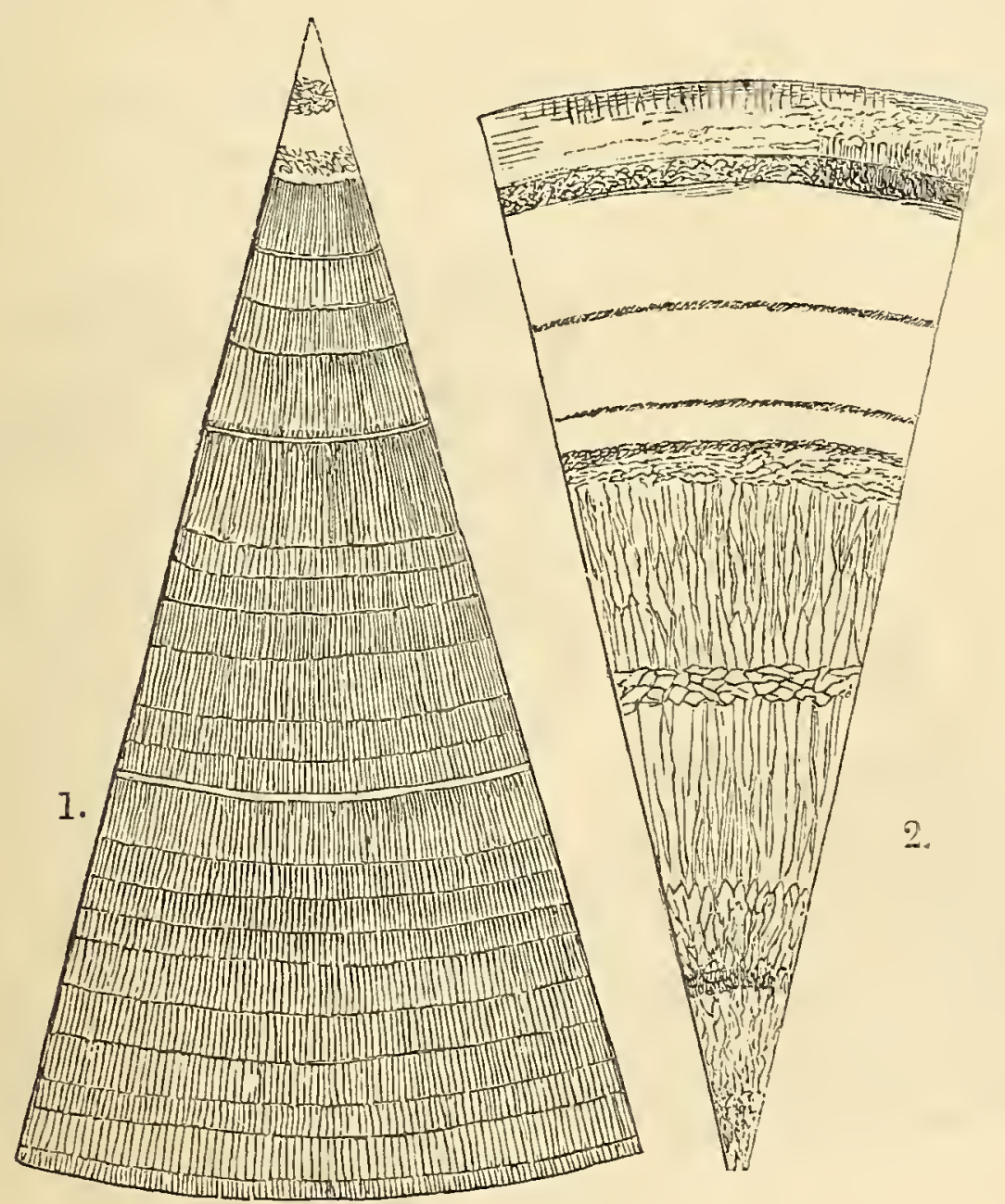

fig. 95.

1. A transverse section of a Pearl from the oyster, showing its prismatic structure. 2. A transverse section of another Pearl, showing its central cellular structure and outside rings of true pearly matter. Magnified fifty diameters.

catching the bleat, or blag; and the price of the fish rose to a guinea per quart, from their value in forming artificial pearls under the hands of certain Jews. While the fish were alive and struggling, the fine sil- 
very scales were scraped from the sides; and then the unfortunate fish were cast again into their element, in the hope of affording a prize at some future day to avarice aided by cruelty. The scales were washed and rubbed, then suffered to settle in water. The sediment was of an oily consistence; a little of it was then dropped into a hollow glassbead of a bluish tint, and shaken so as to cover all the inner surface of the bead; after this the bead was filled up with white wax, to give the pearl solidity and weight.

The microscope discloses the different structure of pearls: those having a prismatic cellular structure have a brown horny nucleus, surrounded by small imperfectly-formed prismatic cells; then there is a ring of horny matter, followed by other prisms, and so on, as represented in fig. 95, which are transverse sections of pearls from oysters, showing successive rings of growth, or deposits.

In a horizontal section (fig. 96) of another pearl, the prismatic structure and the transverse grooves of the prisms are very well

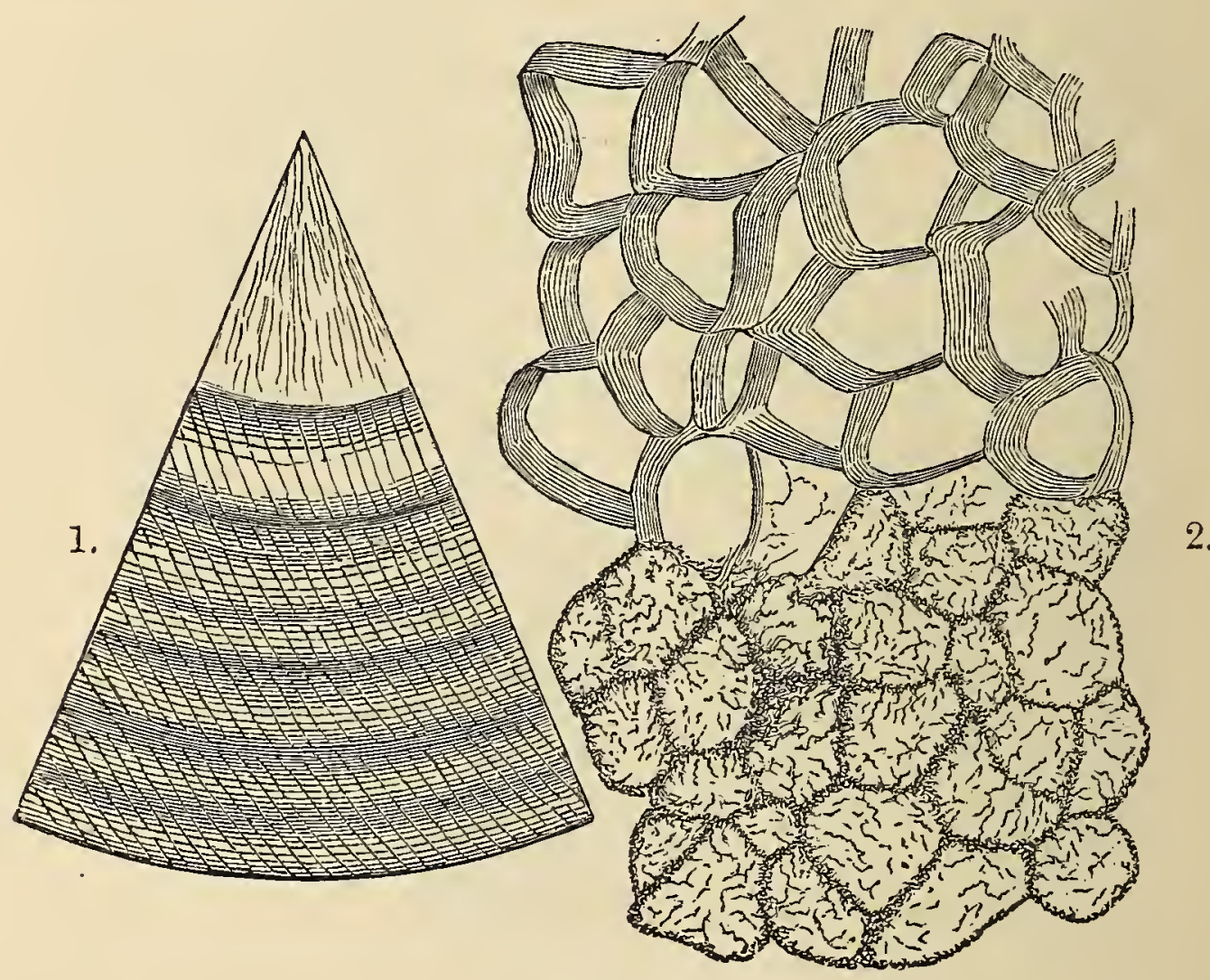

2.

fig. 96.

1. A transverse section of a small Pearl from a species of Mytilus. 2. A horizontal section of a Pearl magnified 250 diameters, showing its prismatic structure, and the transverse strix of the prisms.

shown. The prisms are smallest in the centre, and nearly filled with dark matter, as are also some nearer the edge. In a segment of a transverse section of a small purple pearl from a species of Mytilus, all 
trace of prismatic structure has gone, and only a series of fine curved or radiating lines are seen. It consists of a beautiful purple-coloured series of concentric laminæ (fig. 96). Many of this kind of pearls have a series of concentric zones, and some are of a yellow tint.

The true pearls are composed entirely of nacre, and are exquisite in their colouring. Their structure is shown highly magnified at fig. 96. Some, again, are composed of nacre and prismatic cellular structure; the centre having the prismatic formation, banded outside by two rings of the true pearly matter - nacre.

\section{GASTEROPODA.}

The Gasteropodc are characterised by their being provided with a fleshy disc, serving as a foot, upon which to creep. The back is covered with a cloak, in or upon which the shell is secreted, and may consist of one or more pieces. All the shells are remarkable for the small amount of the animal as compared with that of the earthy matter, so that they are extremely brittle, and their fractured surfaces have a crystalline appearance. In some the shell is of a horny texture, for example, in the Aplysia, Sect-hare (Plate I. No. 10); it is also transparent and flexible.

Not less curious than beautiful is the internal layer of different kinds of bivalves, which present a nacreous or iridescent lustre, the result of its surface being varied with a series of grooved lines running nearly parallel to each other. One of the most remarkable is the well-known Earshell, Haliotus splendens; this has been ascertained to consist of numerous plates, resembling tortoiseshell, forming a series of hexagonal cells, in the centre of which the stellate pigment is deposited (Plate V. No. 3), alternating with thin layers of pearl or nacre; and this exhibits, when highly magnified, a series of irregular undulating folds, represented in the upper portion of the section. "The iridescent lines are often extremely pleasing; and if a piece be submitted to the action of diluted nitric acid, until the calcareous portion of the nacreous layers be dissolved out, the plates of animal matter fall apart, each one carrying with it the membraneous residuum of the layer of nacre that belonged to its inner surface. But the nacre and membrane covering some of these horny plates remain undisturbed; and their folded or plaited surfaces, although divested of calcareous matter, exhibit iridescent hues of the most gorgeous description. But if the membrane is spread out with a needle, and the plates unfolded to a considerable extent, the iridescence is no 
longer seen; a fact which clearly demonstrates that the beautiful effects presented by the nacreous portions of shells, commonly called mother-of-pearl, are produced solely by the disposition of single membraneous layers in folds or plaits, lying more or less obliquely to the general surface." * The majority of the Gasteropoda are furnished with a shell, which has been denominated spirivalve. The cause of this spiral arrangement is said to be owing to the shape of the body of the animal inhabiting the shell, which, as it grows, principally enlarges its shell in one direction; thus, of course, making it form a spine, modified in shape according to the degree in which each successive turn surpasses in bulk that which preceded it. It would rather appear that this is principally owing to the ciliary motion imparted to the early stage of the embryo ; the first deposit of calcareous matter forming the axis, the tube continues to rotate upon its axial pillar, or columella, as it is called; and by reason of some other peculiar vital tendency, the shell is gradually deposited in a series of cells ; thus enlarging its conical form, and winding obliquely from right to left. Mr. Bowerbank has satisfactorily proved that the formation of each layer of shell is a progressive operation, new matter being added to its interior after the exterior has become consolidated. It is also highly organised, and has a vascular communication with the soft parts. Every turn around the axis is technically termed a whorl; and when the columella is hollow, it is said to be umbilicated. In the spirivalve-shelled Gasteropoda, we find a difference in structure between that part of the mantle which envelopes the viscera, and which is always concealed within the cavity of the shell, and the more vascular portion placed around its aperture.

The polishing material, which lines the interior, causing it to appear like enamel or pearl, may be likened to the glazing of an earthenware vessel, and is a varnish produced from the general surface of the mantle, by some modification of its secretions, spread in successive coatings over the coarser calcareous matter, wherever such a polish becomes needful. But even this secretion may be over-excited by the introduction of some poison; when it is over-secreted, it then becomes a disease; an instance of which has been given in the pearl found in the oyster.

The Terebratula present little or no structure, except a series of large, dark oval spots, which in a vertical section will be seen to be perforations, or tubes running obliquely from the inner to the outer surface of the shell, and having a series of radiating lines on the edge

* Dr. Carpenter, Cyclopædia of Anatomy and Physiology. 
(Plate V. No. 6); the outer layer has been removed, to show the perforations of the colourless prismatic cells.

The mouth of such of the Gasteropoda as devour vegetable matter consists of a strong muscular cavity, with a single crescent-shaped horny tooth, furnished along its upper edge with sharp points, separated by semicircular cutting spaces, admirably adapted for the division of vegetable food, and furnishing beautifully-curious objects for the microscope-especially under polarised light. Several kinds of molluscous animals are to be found in shallow water, in brooks, and ditches. One of the most common of these is commonly known as the horny coilshell, or Planorbis corneus. The shell of this creature at first sight looks like that of one of those little flat snails which are sometimes
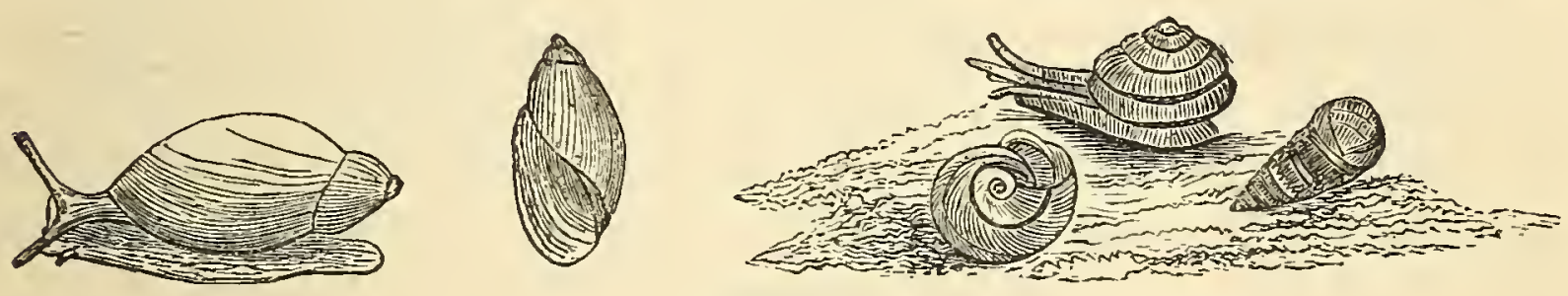

fig. 97 .

The Amber Snail. Physa fontinalis. Moor Snail and Mountain Bulimus.

ound in cellars; but, on examination, it will be found to differ from these creatures in being exactly the same on both sides; or, in the language of a naturalist, having neither spine nor column. The animal belonging to this shell is extremely like a snail, when it is crawling with its tentacula extended; but it is much smaller in all its parts. It is found in ditches and ponds. The amber snail (Succinea amphibia), has a beautiful transparent shell of a light amber colour, and it is from this that it derives its scientific name-as Succinum signifies amber. The puddle-mud snail (Lymaea peregra) is also very frequently found in this country. Its shell bears considerable resemblance to that of Succinea; but it is less transparent, and has a more horny look. The shells of all the species of Lymoea have the aperture on the right hand, and the plait on the left hand, which distinguishes them from Succinea. Another kind of pond-snail, called the stream-bubble shell (Physa fontinalis), is distinguished from Lymoea by its opening being on the left hand instead of the right. All the pond-snails have a singular manner of appearing to crawl under the surface of the water with their shells downwards. Some of them also let themselves down in the water with a thread, in the same way as some kinds of caterpillars let themselves down in the air. The shells of the marsh-snails are found 
abundantly in the river Colne at Uxbridge, in the Thames, and in the rivers of Cambridgeshire, Oxfordshire, Essex, and Suffolk; but they are never found in the north of England, or near the sea. The river limpet (Ancylus fuviatilis) is a very small shell, found in streams and rivulets attached to stones. The animal is greyish, and very lively. The shell is almost transparent, with a blue tinge inside, and a pointed top, which is on one side, and slightly curved downwards. These animals are sometimes seen swimming in the water, just below the surface, with the shell downwards, like the pond-snail.

Land suails shut themselves up for the winter by means of what is called an operculum; this is a shell-like substance just large enough to fill the opening of the shell, to which the snail glues it with a strong cement, having previously fixed herself to a wall or tree by a portion of the same glutinous substance; and in this state the snail remains without either air or food, till recalled to life by the warmth and moisture of spring. In the countries where snails are eaten, they are only used while in this state of hybernation. They are fattened in what are called snail-gardens; that is, in broad shallow pits sunk in the ground. In these the snails are kept and fed with fresh leaves, bran, and potatoes during the summer; and in the winter, when they fix themselves against the walls of the pits, they are collected, packed in casks, and sent to market. It is said that four millions of snails are exported every year from the city of Ulm alone; and yet there are snail-gardens in various other parts of Germany. The common garden-snail (Helix aspersa) is never eaten; it is only the large apple-snail (Helix pomatia) which is used as food. This large snail is found at Dorking, and other places in England.

There is a kind of snail (Helix virgata), common in Devonshire, which is so small as to be generally found sticking to the blades of grass, together with a species of Bulimus; and these molluscous animals, being eaten by the sheep with the grass, are said to afford a most fattening nourishment, and to make the mutton remarkably sweet. Many persons are not aware how many different kinds of snail are to be found even in Great Britain. In different parts of the world there are 250 living species known and described, and sixteen fossil kinds. Some are very beautiful, their shells being spotted with various brilliant colours.

It is extremely curious to watch the development of the spawn of these animals under a magnifying-glass. The spawn of the water-snail is usually found attached to the surface of stones, pieces of weed, or other matters, under the water; and generally connected together in long 
ribands or delicate ova-sacs of a curious and beautiful form. The mass of eggs deposited by the Doris resembles a frill of lace of great beauty. In the Aplysia the spawn resembles long strings of vermicelli, of varying
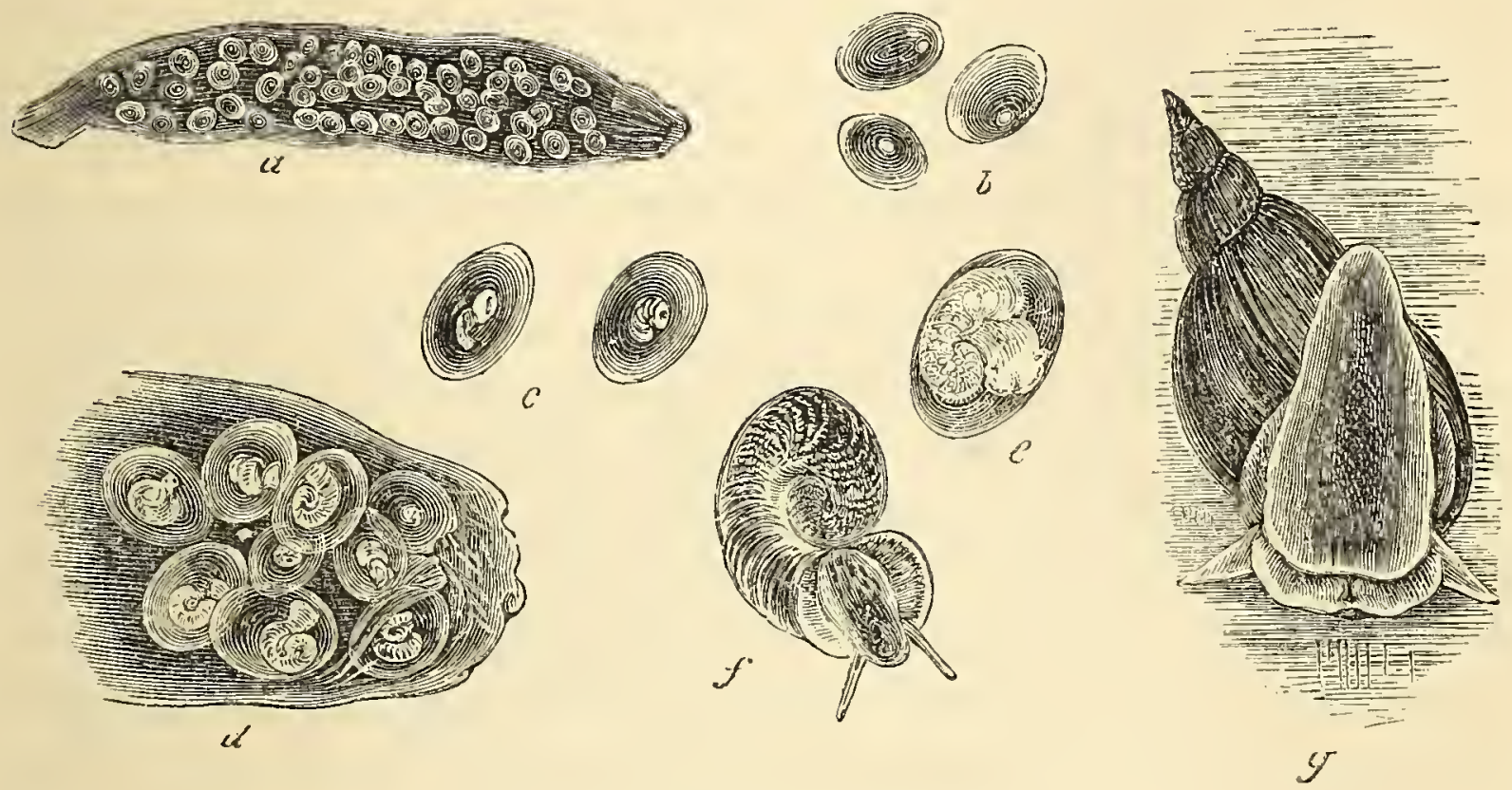

fig. 98. Lymnoea stagnalis.

tints throughout the different parts of the thread. In the Lymoea stagnalis it is deposited in small sacks, containing from fifty to sixty ova; one of which is represented at $\alpha$, fig. 98 . If examined soon after they are deposited, the vesicles appear to be filled with a perfectly clear fluid; at the end of twenty-four hours, a very minute yellow spot may be detected adhering to one side of the cell-wall. In about fortyeight hours afterwards, this small spot is seen to have a smaller central spot of a rather deeper colour; - this is the nucleolus. On the fourth day the spot or nucleus may be observed to have changed its position, and enlarged to double the size: a magnified view is shown at $b$; upon viewing it closely, a transverse fissure or depression may be seen, which on the eighth day most distinctly divides the small mass into shell and soft part of the future animal, as at $c$. It then becomes detached from the side of the cell, and moves with a rotatory motion around the whole of the cell-interior; the direction of this motion is from the right to the left, and is always increased when sunlight, and consequently heat, is thrown upon it. The increase is gradual up to the sixteenth day, when the greater half appears to be the shell portion, as seen magnified at $d$. The spiral axis, around which the calcareous lamella are depositing, can be traced and seen by oblique light, from its darker colour; offering a striking difference in appearance to the soft parts. On the eighteenth day, these changes are more 
distinctly visible, and the ova crowd down to the mouth of the ovasac; when, by using a higher magnifier, a minute black speck, the future eye, may be seen protruded with the tentacles, as at $e$. Upon closely observing it, a fringe of cilia will be seen in motion near the edge of the shell; it now became apparent that the rotatory motion first observed must have been in a great measure due to this; a current, no doubt, being kept up in the fluid contents of the cell by the ciliary fringes. For days after the young animal has escaped from the egg, this ciliary motion is carried on, not alone by the fringe surrounding the mouth, but by cilia entirely surrounding the tenticles themselves, which brings the whole supply of nourishment required; at the same time, and by the same means, the proper æration of the blood is effected. Whilst in the ova, it is, no doubt, by this motion that the cell-contents are converted into the various tissues of the body and shell. From the twenty-sixth to the twenty-eighth day, it appears actively engaged near the side of the egg, using all its force to break through the cell-wall, which it at length succeeds in doing; leaving the shell in the ova-sac, and immediately attaching itself to the side of the glass-vase, to recommence the ciliary motion; it then appears in the advanced stage of life seen at $f$. It is still some months before it grows to the perfect form represented at $g$, where the animal is drawn with its sucker-like foot adhering closely to the side of the glass-vase. One of these animals will deposit from two to three of these ova-sacs a week; thus producing, in the course of six weeks or two months, from 900 to 1000 young, upon which the smaller kinds of fish feed.

The shell itself is deposited in minute cells, that take up a circular position around the axis, on the under-surface of which a hyaline membrane is secreted, soon to become permeated with vessels; at the same time the integument expands, and at various points an internal colouring-matter or pigment is deposited ; the point of one cell being in contact with those of others, thus form the ribs seen in the shell.* The increase of the membrane goes on until the expanded foot is formed, the outer edge of which is rounded off and turned over by a condensed tissue having the form of a twisted wire, enclosing a network of small vessels filled with a fluid in constant and rapid motion. The course of the blood or fluid, as it passes from the heart, may be traced through the large branches to the respiratory organs, consisting of branchial-fringes placed above the mouth; the blood may also be seen returning through other trunks. The heart itself is a

* See further researches by the author, publisked in the Microscopical Journal, 1854. 
strong muscular apparatus. It is pear-shaped, with a pericardium or external wall-enveloping membrane, extremely thin and pellucid. Affixed to the sides of the heart are muscular bands of considerable strength, the action of which appears very like the alternate to-and-fro motion occasioned by drawing out bands of India-rubber, they being analogous to the muscular cords of the mammal heart. The organ beats or contracts at the rate of about sixty times a minute. The heart is placed rather far back in the body, and towards the terminal portion of the shell.

The nervous system is made up of many ganglia, or nervous centres, distributed through the different portions of the body, but connected with each other by cords of communication; the nerve-fibres proceeding to the different parts of the body from the ganglia.

The singular arrangement of the eye must not be omitted; it appears in the early stage to be situated within the tentacle, and consequently capable of being withdrawn with it. In the adult animal, the eye is found to be at the base of the tentacle; and although it can be protruded at pleasure for a short distance, it seems to be dependent upon the tentacle only for an external cover-lid; as it invariably draws it down over the eye whenever that organ needs protection. The eye itself is pyriform, somewhat resembling the round figure of the human eye-ball, with its optic-nerve attached. In colour it is very dark, with a single central pupillary-opening for the admission of light. The tentacle, which is rounded in the young animal, becomes flat and triangular in shape in the adult. The young animal is for some time without teeth; consequently, it does not very early betake itself to a vegetable sustenance: in place of teeth, it has two rows of cilia, as before stated, which drop off when the teeth are fully formed, The lingual band bearing the teeth, or, as it is termed, the "tongue" of the mollusc, consists of several rows of cutting spines, pointed with silica, which form a most interesting object when seen under the microscope.

Should any injury occur to the shell, or a portion of it become broken off, the calcareous deposit is quickly resumed, in order to replace the lost part; the cells being only half the size of those first deposited in the original formation. This may be cited as another proof of the principle laid down by Professor Paget in his lectures delivered at the Royal College of Surgeons, 1852, - " that, as a rule, the reparative power in each perfect species, whether it be higher or lower in the scale, is in an inverse proportion to the amount of change through which it has passed in its development from the embryonic to the per- 
fect state. And the deduction to be made from them is, that the powers for development from the embryo are identical with those exercised for the restoration from injuries; in other words, that the powers are the same by which perfection is first achieved, and by which, when lost, it is recovered. Indeed, it would almost seem as if the species that have the least means of escape or defence from mutilation, were those on which the most ample power of repair has been bestowed; an admirable instance, if it be only generally true, of the beneficence that has provided for the welfare of even the least of the living world, with as much care as if they were the sole objects of the Divine regard."

The primordial cell-wall of each cell does not appear to enter into the formative process of the embryo-the cell-contents alone nourishing the vital blastema of the nucleus. A gradual cycle of progressive development, once set up, goes on until the animal is sufficiently matured to break through the cell-wall, and thus escape from the ova-sac. At the same time it may be inferred, that all this is in some measure aided by the process of endosmose; and in this way certain gases or fluids may become drawn into the interior, and thus aid in the supply of nourishment for the growth of the animal.

The cell-wall would appear to bear the same relation to the future perfect animal, that the egg-shell of the chick does to it; it is, in fact, but an external covering to a certain amount of gaseous and fluid matter, thus placing the germ of life in a more favourable state for development, assisted by an increase of temperature, usually the resultant of a chemical action, set up or once begun in an organism and a medium. In this one fact we are furnished with a wellmarked line of demarcation between the animal and the vegetable kingdoms. In the development of the animal, the cell-wall appears to take no part in the formative process; it is but an envelope required for a time, and then thrown off; on the contrary, in the vegetable tribes it enters largely into their formation, and the ultimate development of all their tissues; it is ever to be found growing with their growth, cell-wall upon cell-wall intact, with or without their earliest contents.

The ovum destined to become a new creature originates from a cell enclosing gemmules, from which its tissues are formed, and nutriment is assimilated, and which eventually enables "the animal to successively renew its organs, through a series of metamorphoses that give it permanent conditions, not only different, but even directly contrary to those which it had primitively." 
"Oh, there are curious things of which men know

As yet but little! secrets lying hid

Within all natural objects. Be they shells, Which ocean flingeth forth from off her billows

On the low sand, or flowers, or trees, or grasses, Covering the earth; rich metals or bright ores

Beneath the surface. He who findeth out

Those secret things hath a fair right to gladness;

For he hath well performed, and doth awake

Another note of praise on Nature's harp

To hymn her great Creator."

\section{THE MODE OF SHOWING THE STRUCTURE OF SHELLS.}

We may exhibit the structure of shells by using an acid solvent in the following manner. If a sufficient quantity of nitric acid, considerably diluted with water (say one part acid to twenty-four of water), be poured upon a shell contained in a glass vessel, it will soon exhibit a soft floating substance, consisting of innumerable membranes, which retain the figure of the shell, and afford a beautiful and popular object for the microscope. In analysing shells of a finer texture than such as are generally submitted to the test of experiment, the greatest circumspection is necessary. So much so, that M. Herissant, whose attention was particularly devoted to the subject, after placing a porcelain shell in spirits of wine, added, from day to day, for the space of two months, a single drop of spirits of nitre, lest the air, generated or let loose, by the action of the nitric acid on the earthy substance, should tear the net-work of the fine membranaceous structure. This gradual operation was attended with complete success, and a delicate and beautifully reticulated film, resembling a spider's web in texture, rewarded the patience of the operator; the organisation of which film, from its extreme fineness, he was not, however, able to delineate. In shells of peculiar delicacy, even five or six months are sometimes necessary for their complete development; but in others of a coarser texture, the process is soon completed.

\section{REMARKS ON COLLECTING SALT-WATER SPECIMIENS.}

"Nothing," as Dr. Harvey says, "can exceed the beauty of a clear rock-pool, seen under strong sunlight, and through a calm surface, tenanted by its various animated tribes all fufilling the duties allotted to their several kinds. Careful examination with a lens will generally detect a multitude of minute shells, some of very strange shapes, and others possessing structures of great elegance." These are the various 
species of Foraminifera. We should recommend these species to be studied in a living condition, whenever opportunity presents, as it will prove a study of great interest. The drift-sand will often be found to contain a wonderful variety of minute spiral univalve shells, though these are scarcely of so small a size as to come within the list of microscopic objects. Others may be obtained by the gatherers of seaweeds, with little trouble, if they will only preserve the sediment that collects in the water in which the sea-weeds are washed. When the sea-weeds are plunged into fresh water, these minute molluscs (Rissoæ) are quickly killed, and fall to the bottom, and may then be secured by simply straining the water through a piece of canvas. Many other minute and curious animals, and sometimes Diatomacece, may be collocted in a similar way.

"Having thus surveyed the rocks, sands, and weeds of the shore above low-water mark, if we launch upon the deep itself, a similar abundance of minute and interesting forms is still presented to us.

"A small muslin bag, the mouth of which is kept open by a wire ring about four inches in diameter, towed slowly behind a boat, on a calm and bright day, in any sheltered bay or inlet, will be found to have gathered multitudes of creatures of the most beautiful forms, and occasionally of the most brilliant colours, - creatures whose crystalline substance affords to our wondering gaze a ready insight into many things connected with the structure of the lower animals, which will in rain be sought elsewhere. In this way are collected the numerous species of minute Naked-eye Medusa.

"Nothing can be conceived more elegant and graceful than the motions of these minute crystalline bodies in a glass of water. On almost every part of the coast, besides the beautiful Tunis neglecta and the allied Beroes, the towing-net will gather innumerable specimens of a creature resembling a slender spicula of glass, about an inch in length, but which is so slender and transparent as to be almost invisible except in a particular direction of the light: this is the Sagitta bipunctata; and its simple structure affords an excellent subject for microscopic research. When fishing for objects of this kind, it is best to have in the boat a large white basin half filled with sea-water; and into this the towing-net is to be inverted and gently shaken every now and then. In this way the delicate creatures it contains will come out of it without injury; and though themselves perhaps at first wholly invisible, their shadows will be seen with great distinctness at the bottom of the basin; and thus many forms which might otherwise escape observation be rendered evident. 
"The microscopic wonders of the sea, however, are still far from being exhausted; it presents as many, if not more, curiosities at the bottom, where its depths are never opened to view, than at the surface. The best and most convenient mode of obtaining these, is by the use of an instrument, with which all perhaps are acquainted in one shape or another,-we mean the dredge. The construction of an instrument of this kind is very simple, and may be effected, if need be, by almost any country blacksmith.

"The essential qualities of a microscopist's dredge are, a small and convenient size, with sufficient weight to ensure its sinking to, and keeping at, the bottom, even when at a considerable depth and drawn with some velocity through the water. The dredge we should recommend is made of cast-iron, which reduces the cost considerably; and it is, in practice, found to be sufficiently strong. It is about 18 inches in length, and the opening is about 4 inches wide, the two sides diverging outwards at a slight angle, and coming to a sharp edge."*

We append a short list of some of the most interesting specimens of the foregoing objects for microscopic examination.

Chalk from Meudon.

Chalky mar] or clay from Caltanisetta.

Calcareous earth of Cara, St. Giorgio.

Berg-mehl from Lillhaggsion in Lapland.

Berg-mehl from Eger.

Sertularia cupressina.

Calcareous spiculæ of Pennatula rubra.

Silicious spiculæ of sponge.

Calcareous spinioles of Gorgonia muricata.

Section of skin of Holothuria.

Calcareous frame-work of skin of Holothuria.

Calcareous body from the oral tentacula of Holothuria.

Calcareous body of an Euryale.

Calcareous body of a star-fish.

\author{
Suctorial pedicles of sea-urchin. \\ Spine of sea-urchin. \\ Pedicellaria of sea-urchin. \\ Ptilota phimosa. \\ Calcareous body of Synapta. \\ Spongia, \\ Sijicious structures of sponge. \\ Echinorhynchus (intest, worm). \\ Serpula. \\ Hook of Serpula. \\ Rudimentary foot of a Nereis. \\ Crisia eburnea. \\ Flustra. \\ Flustra upon Algæ (transrerse section). \\ Cirrhipedia (feet). \\ Cirrhipedia (cibarial apparatus). \\ Ghost-crab (Caprella).
}

* Quarterly Journal of Microscopical Science. 


\section{CHAPTER II.}

INSECTS-THEIR STRUCTURE-HABITS-PARASITES-AND TEACHINGS.

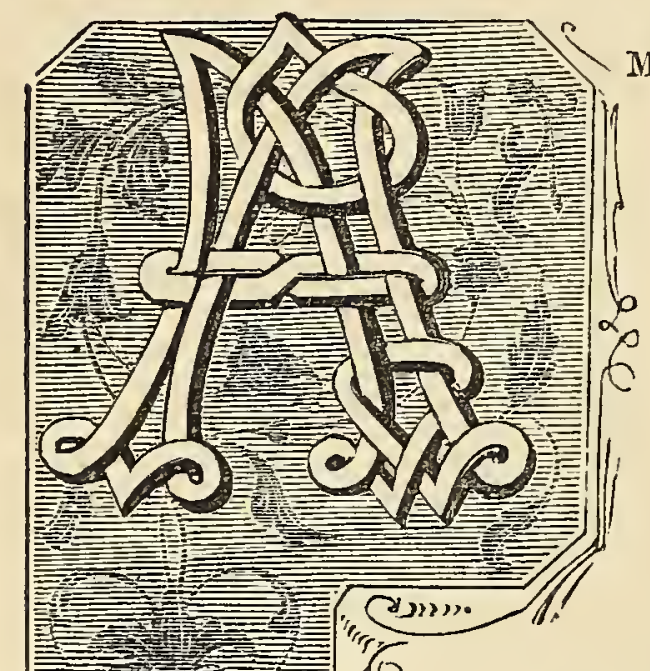

MONGST the numerous objects which engage the attention of the microscopist, the insect tribes in general are far from being the least interesting; and their curious and wonderful economy is a subject well deserving especial investigation. Earth, air, and water teem with the various tribes of insects, for the most part invisible to the unassisted eye of man, but presenting, when viewed with the microscope, the most beautiful mechanism in their frame-work, the most perfect regularity in their laws of being, and exhibiting the same wondrous adaptation of parts to the creature's wants, which, throughout all creation, furnishes traces of the love and wisdom that so strongly mark the works of God.

"I cannot," says the excellent Swammerdam, "after an attentive examination of the nature and structure of both the least and largest of the great family of nature, but allow the less an equal, perhaps a superior degree of dignity. Whoever duly considers the conduct and instinct of the one, with the manners and actions of the other, must acknowledge all are under the direction and control of a superior and supreme Intelligence; which, as in the largest it extends beyond the limits of our comprehension, escapes our researches in the smallest. If, while we dissect with care the larger animals, we are filled with wonder at the elegant disposition of their limbs, the inimitable order of their muscles, and the regular direction of their veins, arteries, and nerves, to what a height is our astonishment raised when we discover all these parts arranged in the least, in the same regular manner! How is it possible but what we must stand amazed, when we reflect that those little animals, whose bodies are smaller than the point of the dissecting-knife, have muscles, veins, arteries, and every other part common to the larger animals! Creatures so very diminutive, that our hands are not delicate enough to manage, nor our eyes sufficiently accite to see them. 


\section{PLATE VI.}

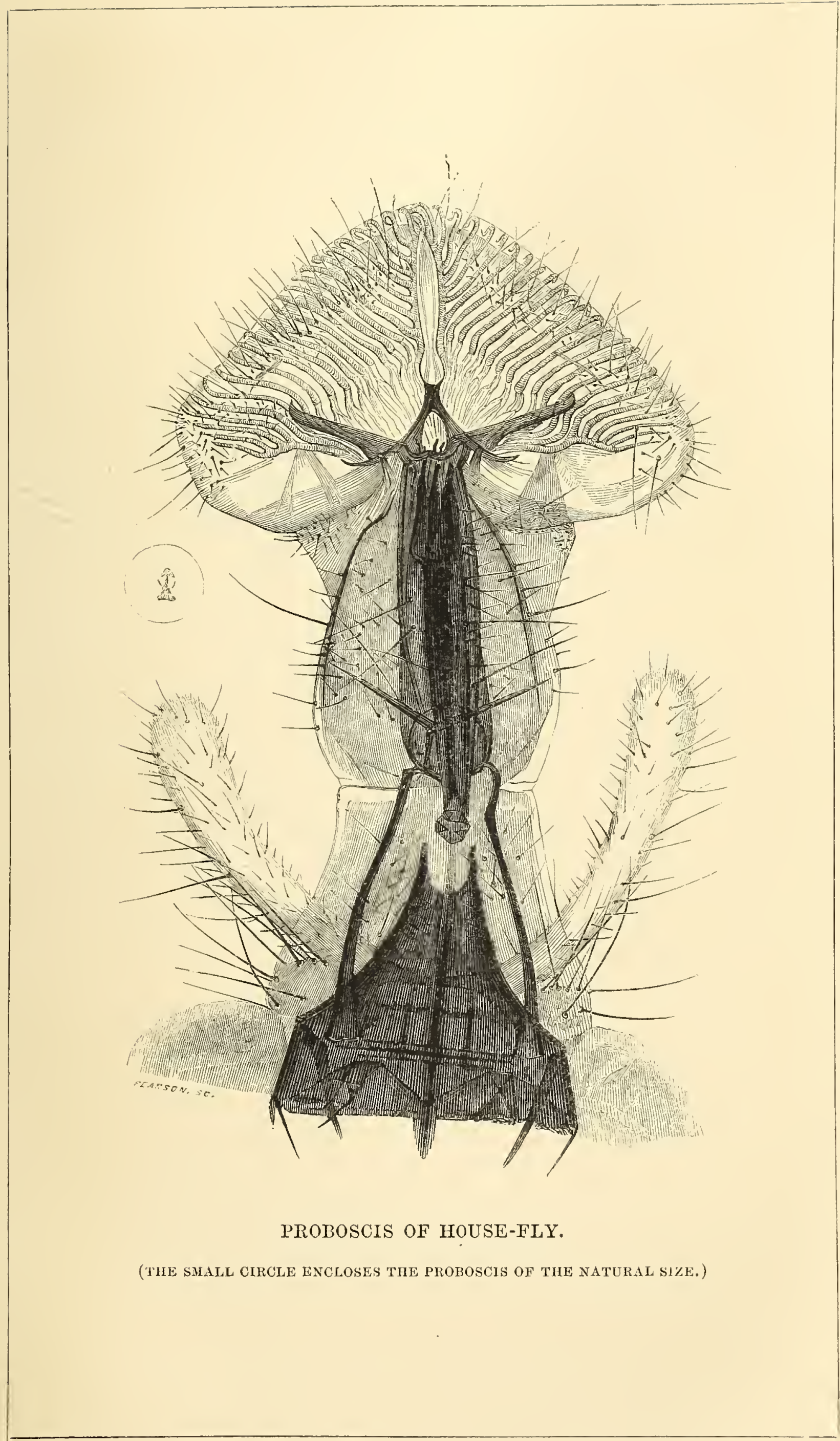



"Insects constitute the most beautiful, most active, and most highly organised of any of the invertebrated classes. Like birds, they are inhabitants of the air, the earth, and the water; and the dominion of some of them is even extended to the bodies of other animals. The number of species is greater than is known in any other division of the animal kingdom, and is only exceeded, as in fishes, by the almost countless myriads of individuals which every species produces. The metamorphoses which most of them undergo, before they arrive at the perfect state and are able to fulfil all the ends of their existence, are more curious and striking than in any other class; and in the greater number of species the same individual differs so materially at its different periods of life, both in its internal as well as external conformation, in its habits, locality, and kind of food, that it becomes one of the most interesting investigations of the physiologist to ascertain the manner in which these changes are effected, to trace the successive steps by which that despised and almost unnoticed larva, that but a

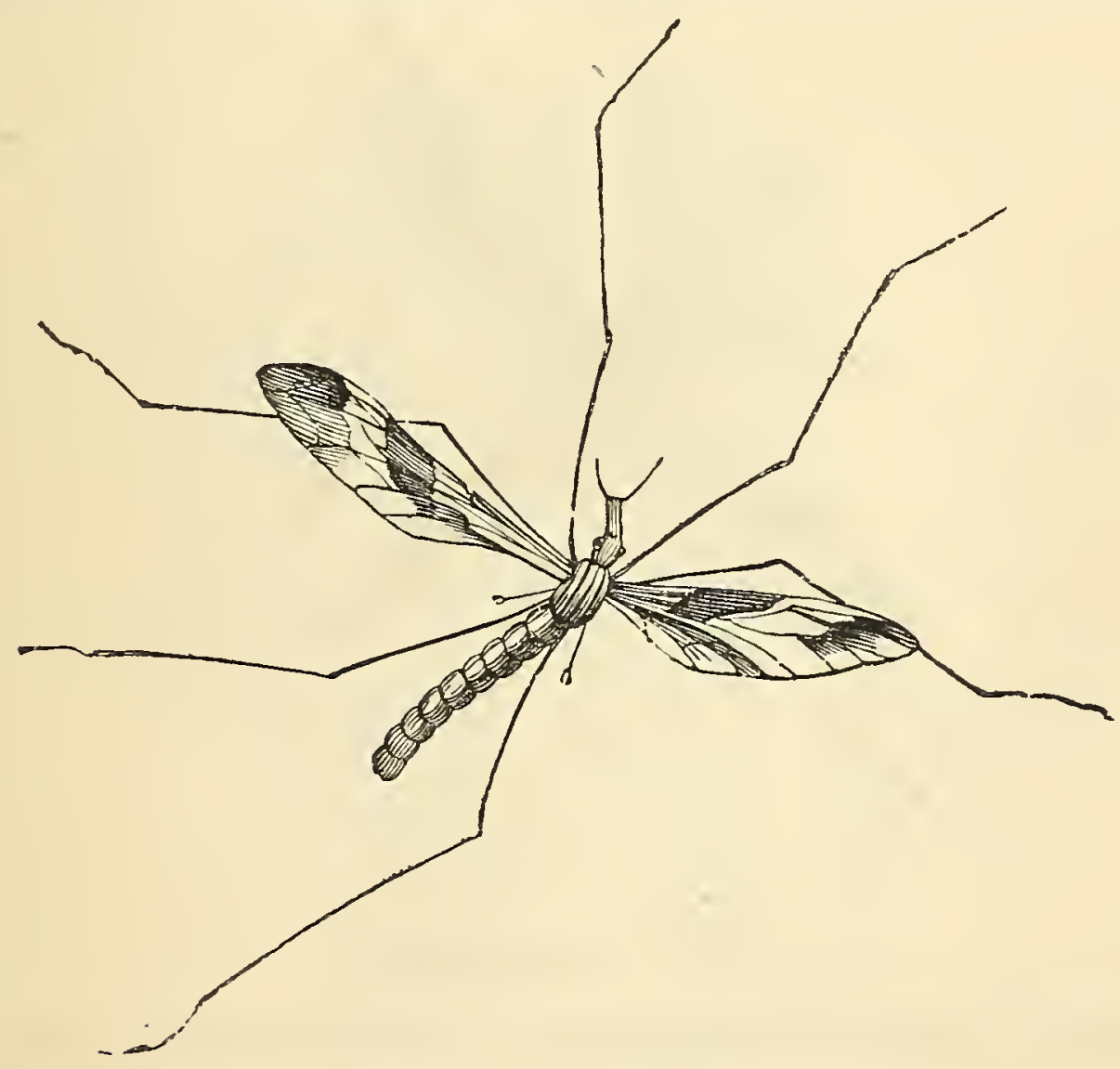

fig. 99. Male Crane-fly (Tipula hortorium).

few days before was grovelling in the earth, with its internal organisation fitted only for the reception of, and assimilation of, the grossest vegetable matter, has had the whole of its internal form so completely changed, as now to have become an object of admiration and delight, and able to 'spurn the dull earth,' and wing its way into the open atmospnere, with its internal parts adapted only for the reception of the 
purest and most concentrated aliment, now rendered absolutely necessary for the support and renovation of its redoubled energies."

The name 'insect' is appropriately intended to designate the sections or divisions into which the bodies of the greater portion of them are divided; and of these we have usually four principal parts, viz. the caput or head, the thorax or trunk, the abdomen or belly, and artus or limbs. Our space will not admit of an entomological examination of the whole; we must therefore content ourselves with noticing only such parts as present some curious point of interest to the microscopist. These parts will be more easily recognised by referring to the accompanying illustrations of the common Crane-fly (figs. 99 and 100). The insect, properly so called, has its body constructed with a view to its. being furnished with wings for the purpose of poising itself in the air.

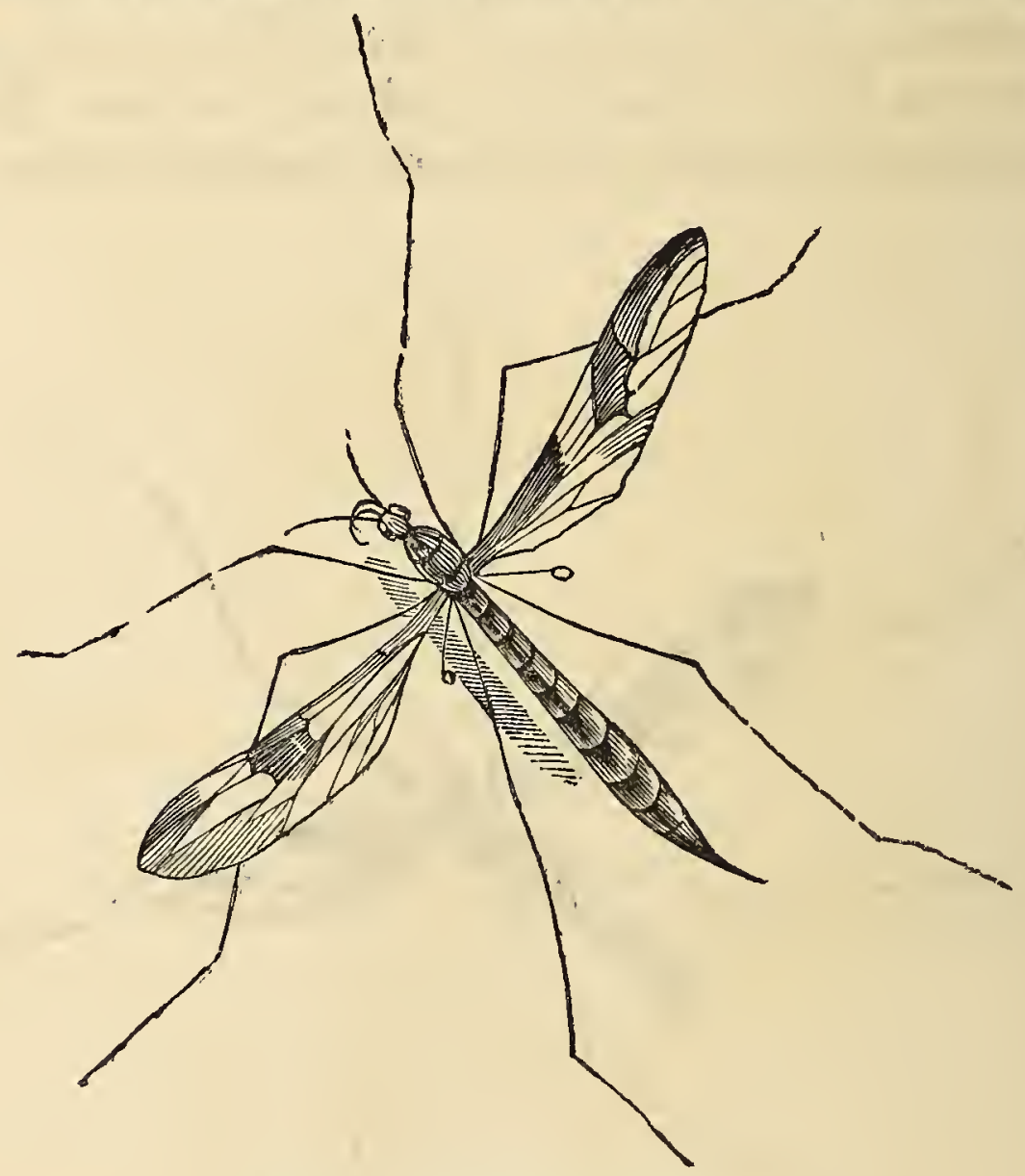

fig. 100. Female Crane-fly.

The head is affixed to the thorax by a species of articulation called the neck, and is furnished with a moutb, eyes, antennæ, \&c. While in some species the head expands and grows, the body does not; in others, when the body increases, the head remains of the same size The heads of all kinds of insects are good objects for the microscope. This will be seen by reference to fig. 108, which shows the head of a gnat, detached from the thorax, and drawn under a magnifying power of 50 diameters, by the aid of the camera lucida; the eyes cover nearly 
two-thirds of the head; and from the fore part is projected the proboscis, with lancets, antennæ, \&c.

In the mouth and tongue of insects, the most admirable art and wisdom are displayed; and the diversity of form is almost as great as the variety of species. The mouth is usually placed in the fore part of the head, extending somewhat downwards. Many have their mouths armed with strong jaws or mandibles, provided with muscles of great power, with which they bruise and tear their food, answering to the teeth of the higher animals; and in their various shapes and modifications serving as knives, scissors, augers, files, saws, trowels, pincers, or other tools, according to the requirements of the insect.

The tongue is generally a compact instrument, used principally to extract the juices on which the insect feeds, varying greatly in its length in different species; it is capable of being extended or contracted at the insect's pleasure; sometimes dexterously rolled up; taper and spiral, as in the butterfly; tubular and fleshy, as in the wasp. In

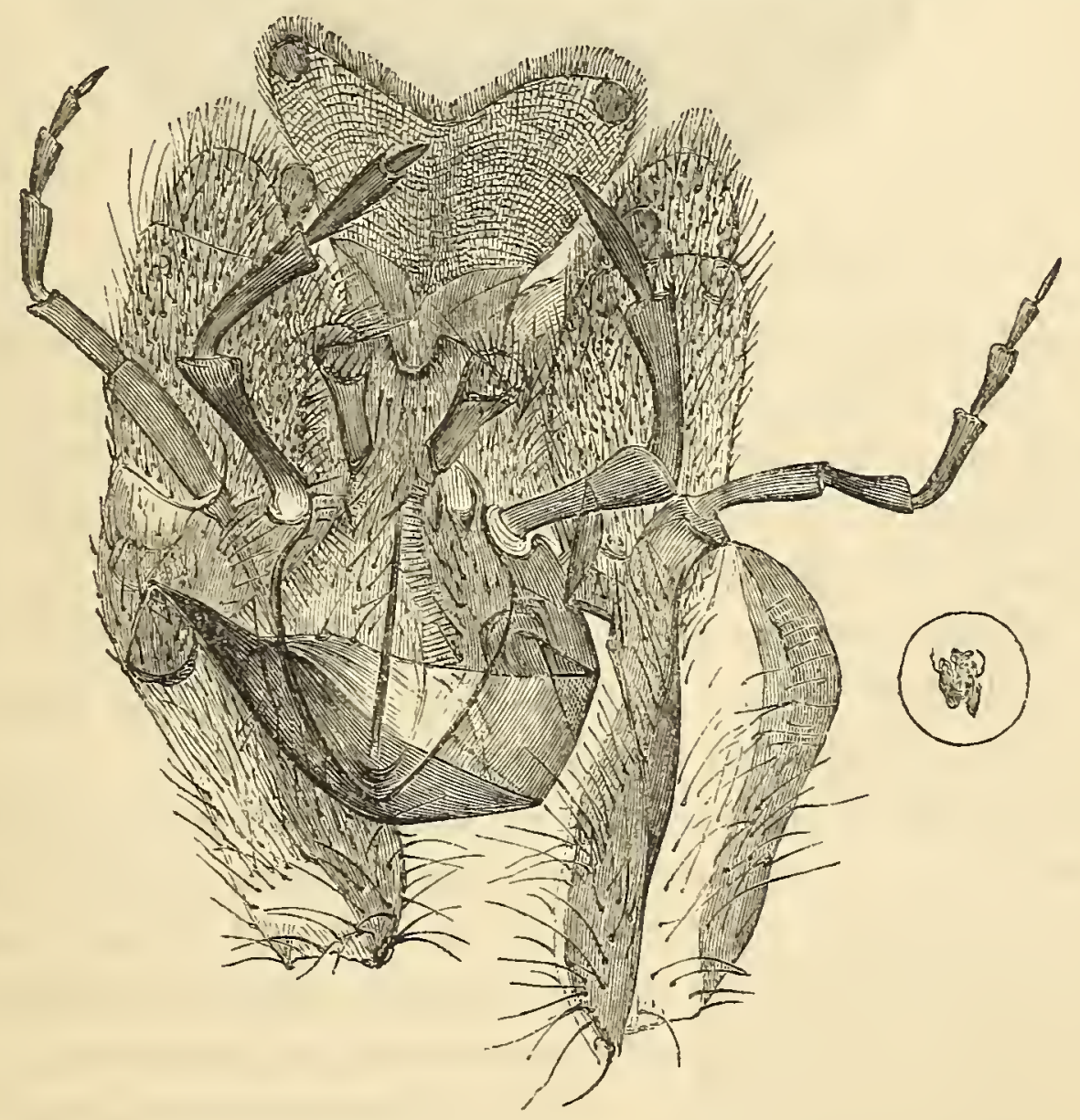

fig. 101.

Under-surface of a Wasp's Tongue, Feelers, sc. Within the circle it is intended to represent the life-size of same.

fig. 101 the under-lip of the wasp is shown, with its brush on either side; above which are two jointed feelers (palpi labiales), the use of 
which is probably to examine the food before it is taken into the mouth, and to clean the tongue. Near these feelers the antennæ or horns are placed, curious in form as they are delicate in structure.

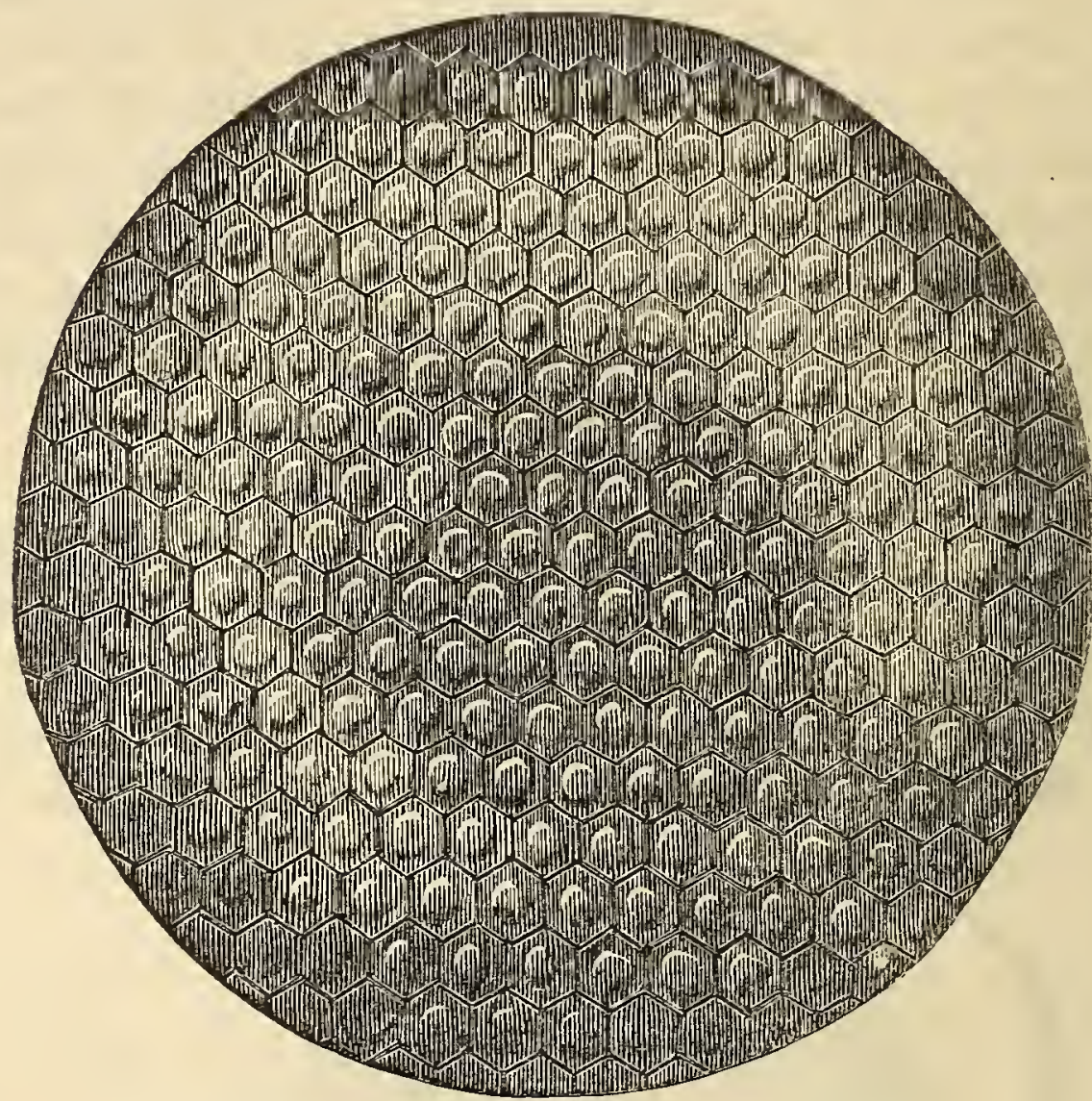

fig. 102. Eye of Fly, magnified 100 diameters.

The greater number of insects have but two antennæ; those of the male generally differing from those of the female: some writers have believed them to be the organs of smell or hearing; others that they are solely intended to add to the perfection of their touch or feeling, being sensible to the least motion or disturbance of the air. They are one of the most interesting and distinguishing characteristics of insects; and seem always employed by them for the purpose of examining every object they alight upon.

The structure of the eye is in all creatures a most admirable piece of mechanism: in none more so than in those of the insect tribe.

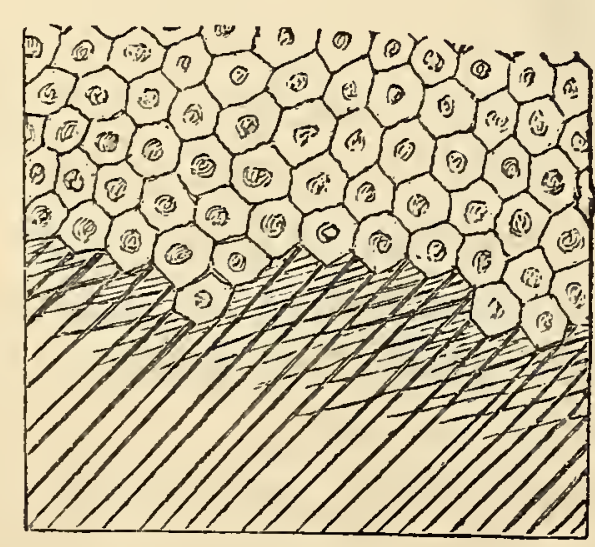

fig. 103.

The eyes are different in each species; varying in number, situation, figure, simplicity of construction, and in colour.

Fig. 102 represents a small portion of the eye of the common fly, drawn by the light of the sun upon a prepared photographic surface of wood ready for the engraving, not a line being added by the hand of the draughtsman; as were other objects given 
in this book. Fig. 103 represents a side view of the eye when thrown down, and showing the compound eye to be made up of a large number of cylindrical tubes.

"On examining the head of an insect, we shall find a couple of protuberances, more or less prominent, and situated symmetrically one on each side.

"Their outline at the base is for the most part oral, elliptical, circular, or truncated; while their curved surfaces are spherical, spheroidal, or pyriform. These horny, round, and naked parts, seem externally to represent the corner of the eyes of insects; at least they are with propriety so termed, from the analogy they bear to those transparent tunics in the higher classes of animals. They differ, however, from these; for when viewed by the microscope, they display a large number of hexagonal facets, which constitute the medium for the admission of light to as many simple eyes. Under an ordinary lens, and by reflected light, the entire surface of one of these corner presents a beautiful reticulation, like very fine wire gauze, with a minute papilla, or at least a slight elevation, in the centre of each mesh. These are resolved, however, by the aid of a compound microscope, and with a power of from 80 to 100 diameters, into an almost incredible number (when compared with the space they occupy) of minute, regular, geometrical hexagons, well defined, and capable of being computed with tolerable ease, their exceeding minuteness being taken into consideration. When viewed in this way, the entire surface bears a resemblance to that which might easily and artificially be produced by straining a portion of Brussels lace with hexagonal meshes over a small hemisphere of ground glass. That this gires a tolerably fair idea of the intricate carving on the exterior, may be further shown from the fact, that delicate and beautiful casts in collodion may be procured from the surface, by giving this three or four coats with a eamel-hair pencil. When dry, it is peeled off in thin flakes, upon which the impressions are left so distinct, that their hexagonal form can be discovered with a Coddington lens. This experiment will be found useful in examining the configuration of the facets of the hard and unyielding eyes of many of the Coleoptera, in which the reticulations become either distorted by corrugation, or broken by the pressure required to flatten them. It will be observed also, that by this method perfect casts can be obtained without any dissection whatever: and that these artificial exuvice-for such they really are-become available for microscopic investigations; obviating the necessity for a more lengthened or laborious preparation. The dissection of the 
cornea of an insect's eye is by no means easy. I have used generally a small pair of scissors, with well-adjusted and pointed extremities, and a camel-hair pencil, having a portion of the hairs cut off at the end, which is thereby Hattened. The extremity of the cedar handle should be cut to a fine point, so that the brush may be the more easily revolved between the finger and thumb; and the coloured pigment on the interior may be scrubbed off by this simple process. A brush thus prepared, and slightly moistened, forms, as far as my experience goes, by far the best forceps for manipulating these objects preparatory to mounting; as, if only touched with any hard-pointed substance, they will often spring from the table from mere elasticity, and thus the labour of hours be lost in a moment. It does not appear to me desirable to attempt to flatten an entire cornea by pressure and maceration, although this is generally recommencled, as it does not either aid in developing the beauty, or counting the number of its lenses. On the contrary, the rounded membrane becomes, if the margin remains intact, corrugated; and so one hexagon overlaps the other. It will be useful, therefore, to make two preparations of the eyes of one insect: the one entire, retaining its natural curved form, not having: been subjected to pressure; the other nicked at its margin, or cut into small fragments, and pressed between two slides.

"Each hexagon forms the slightly horny case of an eye. Their margins of separation are often thickly set with hair, as in the bee; in other instances naked, as in the dragon-fly, house-fly, \&c. The number of these lenses has been calculated by various authors, and their multitude cannot fail to excite astonishment. Hooke counted 7000 in the eye of a house-fly; Leeuwenhoek more than 12,000 in that of a dragonfly; and Geoffry cites a calculation, according to which there are 34,650 of such facets in the eye of a butterily."**

The trunk is situated between the head and the abdomen; the legs and wings are inserted into it. The thorax is the upper part of the trunk; the sides and back of which are usually armed with points or hairs. The abdomen forms the posterior part of the body, and is generally made up of rings or segments, by means of which the insect can lengthen or shorten itself. Running along the sides of the abdomen are the spiracles, or breathing apparatus, fig. 104, communicating directly with the internal respiratory organs. Pure air being thus freely admitted to every part, and the circulating fluids kept exposed to the vivifying influence of the atmosphere, the necessity for more 
complicated and cumbersome breathing organs is at once obviated; and thus the whole body is at the same time rendered lighter. They are usually nine or ten in number, and consist of a horny ring, gene-
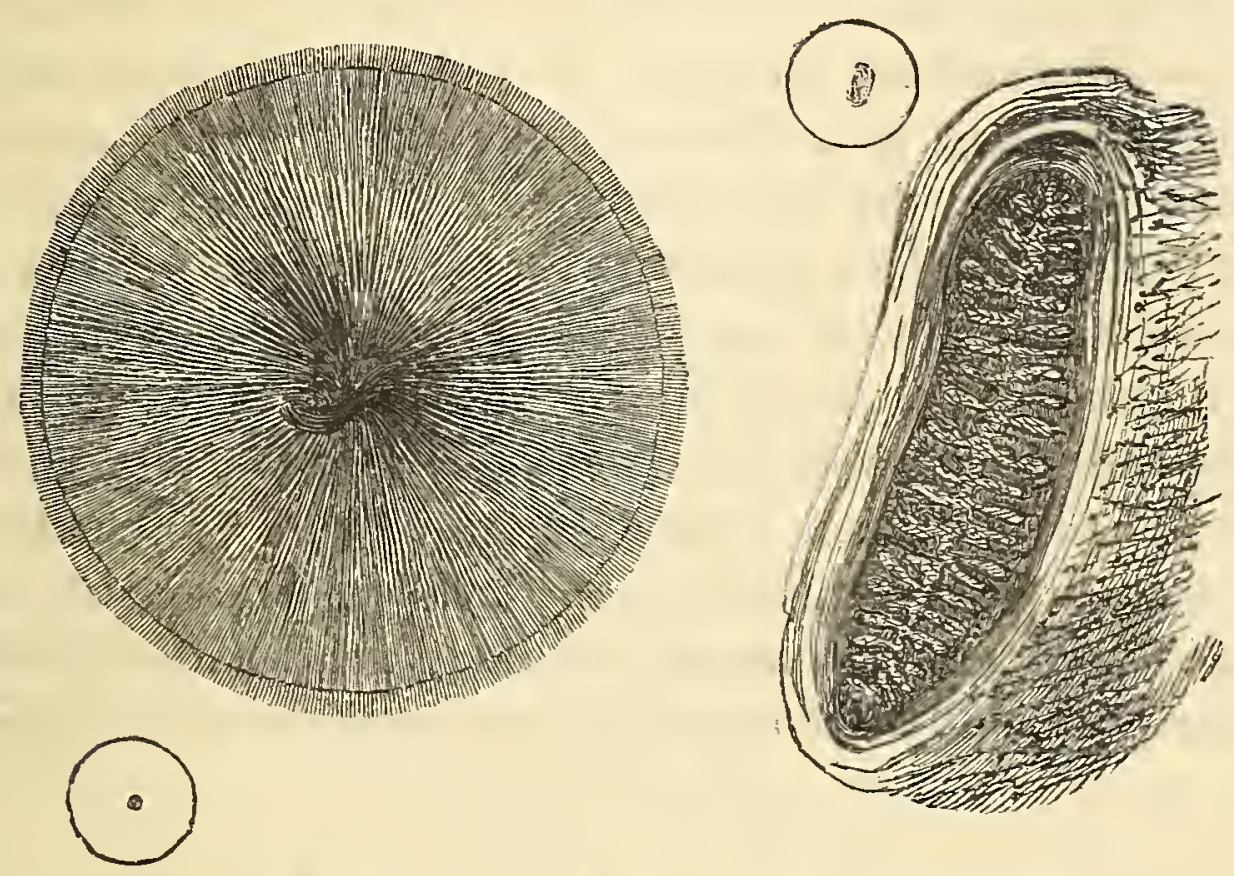

fig. 104.

Sucker on the under-surface of foot of Fly, magnified 150 diameters. The circle encloses the foot of natural size.

\section{Breathing-aperture or spiracle of Silk-worm, magnified 50 diameters. The circle en- closes the object of natural size.}

rally of an oval form. The air-tubes are exquisitely eomposed of two thin membranes, between which a delicate elastic thread, or spiral fibre, is interposed, forming a cylindrical pipe, and keeping the tube always in a distended condition; thus wonderfully preserving the sides from collapse or pressure in their passage through the air, which would occasion suffocation. No. 4, Plate VIII. represents the beautiful mechanism of a portion of the tracheæ of the silk-worm moth. Fig. 105 is a small portion of a tracheæ, highly magnified, and showing the peculiar arrangement of the spiral tubes, giving elasticity and strength to the air-tubes of the water-insect Hydrophitis.

The legs of insects are extremely curious and interesting, each leg consisting of several horny cylinders, connected by joints

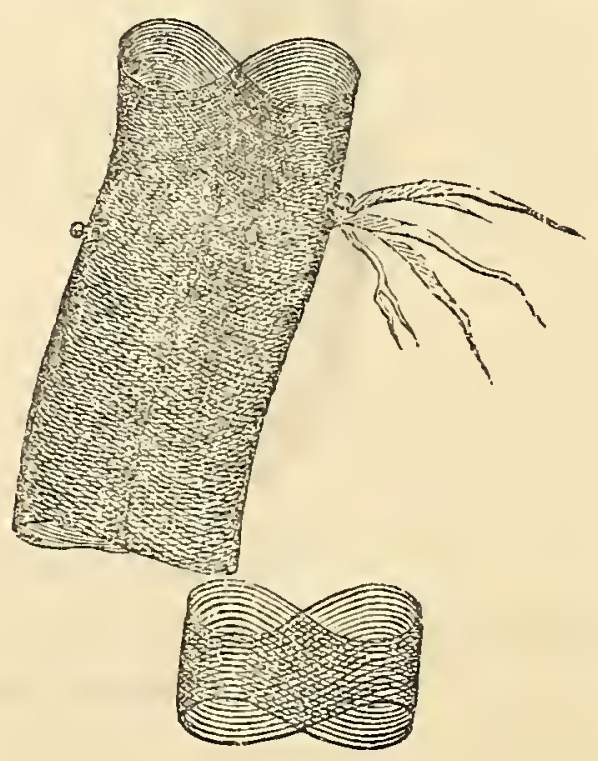

fig. 105.

Highly magnified portions of the trachea of the Hydrophilis, showing the spiral tubes, and their arrangement. 
and ligaments; enclosing within them sets of powerful muscles, whereby their movements are effected. The first is called the hip; it is short, and is connected by a ball-and-socket joint to the thorax; the second, called the trochanter, is connected with the former by a tough membrane, fitting to a corresponding socket; the third, the femur, or thigh, is the largest and strongest, serving as a fulcrum, upon which the leg depends for strength and motion; the fourth is named the tibia, or shank; this is connected to the thigh by a beautiful hinge-joint, which permits of both bending and extension; to the end of this is attached the tarsus, or foot, generally terminated by two horny hooks or claws, whereby the insect holds to the object upon which it moves; between these hooks, in most species, is situated a cushion, sucker, or broad flaps, wherewith to take hold of a smooth surface. In fig. 106 the lower joints, with the feet of flies, are beautifully represented, marking peculiarities of structure in each; and in fig. 104, the admirably-constructed sucker attached to
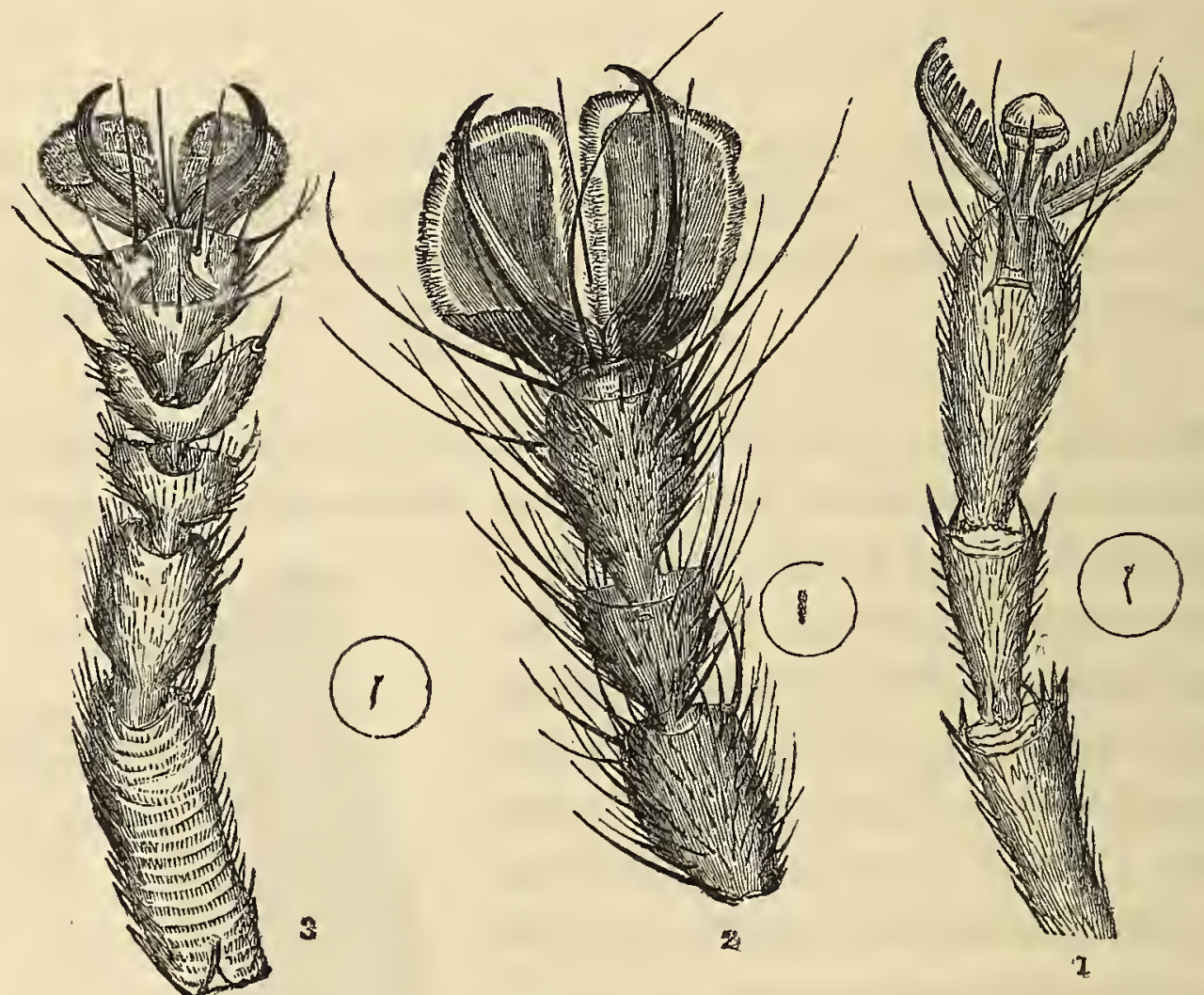

fig. 106. Foet and Legs, magnified 150 diameters.

1. Foot and leg of a small Spider. 2. Foot and leg of Blow-fly. 3. Foot and leg of Drone-fly. The small circle encloses each object of the natural size.

the under-surface of the foot of house-flies, by means of which "they tread the ceiling or inverted floor, and from its precipice depend secure." It was formerly supposed, from the experiments of Sir Everard Home, that flies. were enabled to walk against glass, and with the back 
downwards in various situations, solely by the formation of a vacuum under the soles of their feet, if they may be so termed; as it was observed that the margins of the feet were closely applied to the glass, while the central part was drawn up. It has, however, now been discovered that this hypothesis was not correct, as Mr. Blackwall (a gentleman residing. in Manchester, and an acute observer of nature) noticed that flies remained attached to the sides of an exhausted glass receiver of an airpump, even after they had entirely lost the power of locomotion, and an evident distension of the body had been occasioned by the exhaustion of the air. To detach them from these stations, Mr. Westrood adds, the employment of a small degree of force was found requisite. "In prosecuting this subject, clean phials of transparent glass, containing spiders and various insects in the larva and imago (perfect) states, capable of walking on their upright sides, were breathed into, till the aqueous vapour expelled from the lungs was copiously condensed on their inner surface. The result was remarkable; the moisture totally prevented those animals from obtaining any effectual hold on the glass; and the event was equally decisive if a small quantity of oil was substituted for the aqueous vapour." In fact, it was found that powder, or any substance on the inside of the phials, prevented the flies from climbing; and the idea naturally suggested itself that some glutinous substance was emitted by the feet of the flies, which enabled them to adhere to the glass. The next point to be determined, therefore, was, whether spiders and insects in the larva and perfect states were found to leave any visible track behind them when they crawled over glass; and, by the aid of powerful magnifying-glasses, it was found that traces were left of an exceedingly minute quantity of glutincus matter, which appeared to have been emitted by the feet of these creatures; and subsequent experiments proved that the hair-like appendages which form the brushes of spiders and flies are all tubular. It has often been observed that flies that have been half drowned, if taken out of the milk or water into which they have fallen, take a great deal of time in cleaning their feet before they can walk; and this, no doubt, is to clear out the brushes of their feet, and to bring them into a proper state for emitting the glutinous fluid.

Plate VII. represents the tongue and that part of the apparatus of the Tabanus with which it pierces the skin of horses and cattle. This remarkable compound structure, together with the admirable form and exquisite beauty of the apparatus, must strike the mind with wonder and delight, and lead the observer to reflect on the weakness and impotence of all human mechanism, when compared with the skill 
and inimitable finish displayed in the object before us. The fleshy outer case which encloses it has been removed for the purpose of viewing the several parts, which consist of two spongy guards or feelers, covered with short hairs, united to the head by muscles; these feelers appear to be merely used as a preservative apparatus, in guarding the organs from external injury. The two lancets seen above them are formed somewhat like a cutlass, or the dissecting knife of the anatomist, and purposely intended for making a deep and sharp cut, and also for cutting vertically with a sweeping stroke. The other and larger cutting instrument appears to be intended to enlarge the wound, if necessary; or it may be for the purpose of irritating and exciting the part around, thereby increasing the flow of blood to the part, being jagged or toothed at the extremity. The larger apparatus, with its three peculiar prongs or teeth, is tubular, to permit the drawing up of the blood and conveying it to the stomach; it is enclosed in a case which entirely covers it. The spongy tongue itself projects some distance beyond this apparatus, and is composed of a beautiful net-work of soft muscular spiral fibres, forming a series of absorbent tubes; and these are moved by powerful muscles and ligaments, the retractile character of which may be there seen in the drawing of the proboscis of the fly, Plate VI. : by the aid of these hooklets he is enabled to draw in and dart out the tongue with wonderful rapidity. The striated appearance of another set of muscles may be seen at the root of the whole.

"In the organisation of the mouth of various insects we have a modification of form, to adapt them to a different mode of use; as in the Muscidce, or common house-flies. When the food is easily accessible, and almost entirely liquid, the parts of the mouth are soft and fleshy, and simply adapted to form a sucking tube, which in a state of rest is closely folded up in a deep fissure, on the under-surface of the head. The proboscis at its base appears to be formed by the union of the lacinia above, and the labium below, the latter forming the chief portion of the organ, which is tenanted by dilated muscular lips. In the Tabanus these are exceedingly large and broad, and are widely expanded, to encompass the wound made by the insect with its lancetshaped mandibles in the skin of the animal it attacks. On their outer surface they are fleshy and muscular, to fit them to be employed as prehensile organs, while on their inner they are more soft and delicate, but thickly covered with rows of very minute stiff hairs, directed a little backwards, and arranged closely together. There are very many rows of these hairs on each of the lips; and from their being arranged in a similar direction, they are easily employed by the insect in scraping or. 


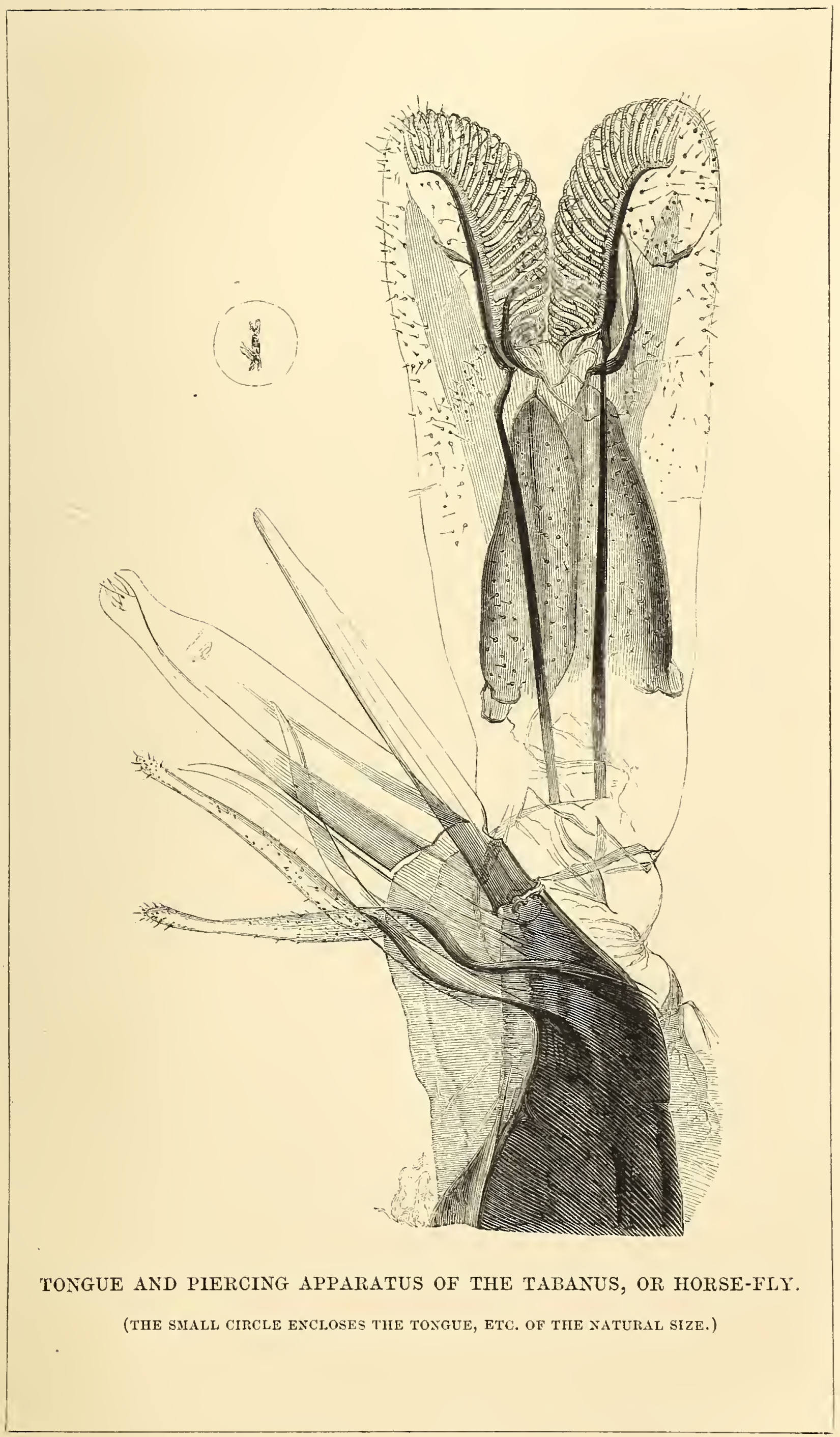



tearing delicate surfaces. It is by means of this curious structure that the busy house-fly often occasions much mischief to the covers of our books, by scraping off the white of egg and sugar varnish used to give them the polish, leaving traces of its depredations in the soiled and spotted appearance which it occasions on them. It is by means of these also that it teases us in the heat of summer, when it alights on the hand or face, to sip the perspiration as it exudes from and is condensed upon the skin. The fluid ascends the proboscis, partly by a sucking action, assisted by the muscles of the lips themselves, which are of a spiral form, arranged around a highly elastic, tendinous, and ligamentous structure, with other retractile additions for rapidity and facility of motion." " The beautiful form of the spiral will be best seen under a magnifying power of 250 diameters, or quarter-inch object-glass.

The common blow-fly is hairy, black, with its abdomen shining, and deposits its eggs on animal flesh, either fresh or putrid. The eggs are hatched in a few hours, and the maggots, when full grown, which is in five or six days, are of a yellowish-white colour, with a slight tinge of pale red. This maggot is of a lengthened shape, with a pointed front, in which the mouth is situated; and from this the body gradually increases to the other end, which is broad and flat, and on which are two specks resembling eyes; so that a person might take this for its head, and the head for the tail. The insect afterwards changes to a chrysalis; the skin drys round it, and the whole becomes of an oval form. In ten days more the young fly emerges.

These insects are of great service in the economy of nature, their province being the consumption of decaying animal matter, given out in such small quantities, that they are not perceptible to common observers, neither removable by the ordinary means of cleanliness, even in the best-kept apartments, in hot weather. It was asserted by Linnæus, that three of these flies would consume a dead hor'se as quickly as a lion. This was, of course, said with reference to the offspring of such three flies; and it is possible the assertion may be correct, since the young begin to eat as soon as they are born. A single blow-fly has been known to produce twenty thousand living maggots; and each of these continues eating so voraciously, that in twenty-four hours it has increased its own weight above two hundred times; and in five days it has attained its full size. When the maggots attain their full size, they change into the pupa state, and remain in that only a few days; they then become flies, ready to produce thousands more maggots, and afterwards flies, till 
the whole brood is destroyed by cold. The blue-bottle fly lays eggrs, as does the common house-fly. These eggs are generally deposited either in dunghills or other heaps of rubbish, from whence they issue in great quantities on a warm day. One kind of small two-winged fly lays its eggs on the leaf of the sow thistle, and the maggots live entirely upon the cellular tissue of the leaf, without touching the outer skin, either on the upper or under side. These maggots generally commit their ravages in the maggot state, early in the month of October; and appear in their fly state towards the close of that month.

We cannot resist an apt quotation on this wonderful little insect: "A fly on the wing is no less curious an object than one on foot; yet, when do we trouble our heads about it, except as a thing which troubles us? The most obvious wonder of its flight is its variety of direction, most usually forwards, with its back upwards like a bird, but on occasions backwards, with its back downwards, as when starting from the window and alighting on the ceiling. Marvellous velocity is another of its characteristics. By fair comparison of sizes, what is the swiftness of a race-horse clearing his mile a minute to the speed of the fly cutting through her third of the same distance in the same time? And what the speed of our steaming giants, the grand puffers of the age, compared with the swiftness of our tiny buzzers; of whom a monster train, scenting their game afar, may even follow partridges and pheasants on the wings of steam in their last flight as friendly offerings? But, however, with their game the flies themselves would be most in 'keeping' on the atmospheric line; a principal agent in their flight, as well as in that of other insects, being the air. This enters from the breathing organs of their bodies in the nerves and muscles of their wings, from which arrangement their velocity depends, not alone on muscular power, but also on the state of the atmosphere. How does a fly buzz? is another question more easily asked than answered. 'With its wings, to be sure,' hastily replies one of our readers. 'With its wings as they vibrate upon the air,' responds another, with a smile, half of contempt, half of complacency, at his own more than common measurement of natural philosophy. But how, then, let us ask, can the great dragon-fly, and other similar broad-pinioned, rapid-flying insects, cut through the air with silent swiftness, while others go on buzzing when not upon the wing at all? Rennie, who has already put this posing query, himself ascribes the sound partially to air, but to air as it plays on the "edges of their wings at their origin, as with an Eolian harpstring,' or to the friction of some internal organ on the root of the wing nervures. Lastly, how does the fly feed? The busy, curious, 
thirsty fly, that 'drinks with me,' but does 'not drink as I,' his sole instrument for eating or drinking being his trunk or suck; the narrow pipe by means of which, when let down upon his dainties, he is enabled to imbibe as much as suits his capacity. This trunk might seem an instrument convenient enough when inserted into a saucer of syrup, or applied to the broken surface of an over-ripe blackberry, but we often see our sipper of sweets quite as busy on a solid lump of sugar, which we shall find on close inspection growing. 'small by degrees' under his attack. How, without grinder's, does he accomplish the consumption of such crystal condiment? A magnifier will solve the difficulty, and show how the fly dissolves his rock, Hannibal fashion, by a diluent, a salivary fluid passing down through the same pipe, which returns the sugar melted into syrup." **

The wings, those delicately-formed organs by which the insect is enabled to poise and dart with such amazing rapidity through the air, are of great variety in form and structure; the beauty of their colouring, the art with which they are connected to the body, the curious manner in which some are folded up, the fine articulations provided for this purpose, with the rarious ramifications by which the nourishing fluids are circulated and the wing strengtbened, all afford a fund of rational investigation highly entertaining, and exhibiting, when examined under the microscope, beautiful and wonderful design in their formation. Take the dragon-flies as an example, whose wings, with their horny frame-work, are as elegant, delicate, and as transparent as gauze, often ornamented with coloured spots, which, at different inclinations of the sun's rays, show all the tints of the rainbow. It has been observed of these insects by Kirby and Spence, "that, without turning, they can fly in all directions, backwards, and to the right and left, as well as forwards. This ability to fly all ways without turning must be very useful to them when pursued by a bird. Leeuwenhoek once saw a swallow chasing an insect of this tribe, which he calls a Mordella, in a menagerie about 100 feet long. The little creature flew with such astonishing velocity, to the right, to the left, and in all directions, that this bird of rapid wing and ready evolution was unable to overtake and entrap it, the insect eluding every attempt, and being generally six feet before it. Indeed, such is the power of the long wings by which dragon-flies are distinguished, and such the force of the muscles that move them, that they seem never to be wearied with flying. One species (Libellula virgo, fig. 107) may be seen

* "Episodes of Insect Life," a charming buok, published by Reeve, 18551. 
sailing for hours over a piece of water, all the while chasing, capturing, and devouring the various insects that come athwart its course, or

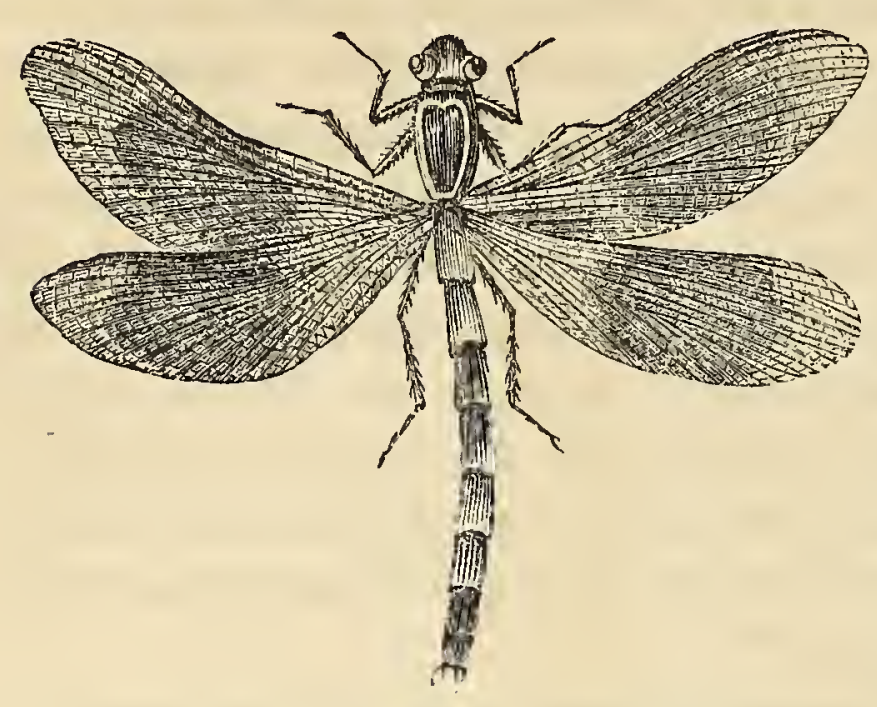

fig. 107. The Dragon-fly (Libellula virgo). driving away its competitors, without ever seeming tired or inclined to alight."

In fine weather, the female dragon-flies deposit their eggs, which they lay in water, making a strange noise, as though they were beating the water; and the eggs themselves look like a floating bunch of small grapes. The larva, when hatched, live in the water; and it is scarcely possible to fancy more strange-looking creatures. They are short, comparatively thick, and their motions are heavy and clumsy. They shed their skins, and become pupæ; still continuing to live in the water. The pupa differs from the larva principally in having four small scales on its sides, which conceal the future wings. While the dragon-fly continues in its aquatic state, both as larva and pupa, it devours all the insects it can catch; but as it can only move slowly, it is furnished with a very curious apparatus to its head, which it can project at pleasure, and use as a trap. This apparatus consists of a pair of very large, jointed, movable jaws, which the insect keeps closely folded over its head, like a kind of mask, till it sees its prey. When it does, it creeps softly along till it is sufficiently near, and then darts out those long, arm-like jaws, and seizing the insect it had marked, conveys it to its mouth. When the dragon-fly emerges from its pupa-case, it places itself on the brink of the pond, or on the leaf of some water-plant which is sufficiently strong to bear its weight, and there it divests itself of its pupa-case. When the insect first appears, it has two very small wings; these gradually swell out, the veins fill with coloured liquid globules, and then two other wings gradually appear. As soon as the wings are fully'expanded, and have attained their beautiful gauze-like texture, the dragon-fly begins to dart about, and to catch any insect that may fall in its way. A dragon-fly may sometimes be seen flying about with an insect in its mouth, so much larger than its own head, that it is difficult to imagine how it will contrive to swallow it. The mouths and stomachs of dragon-flies are gifted with an extraordinary power of distension; and thus, however 
large the captured fly, moth, or butterfly, may be, it disappears, as though by magic.

Equally rapid, exactly steered, and unwearied in its flight, is the gnat. The wing of a gnat has been calculated, during its flight, to vi-

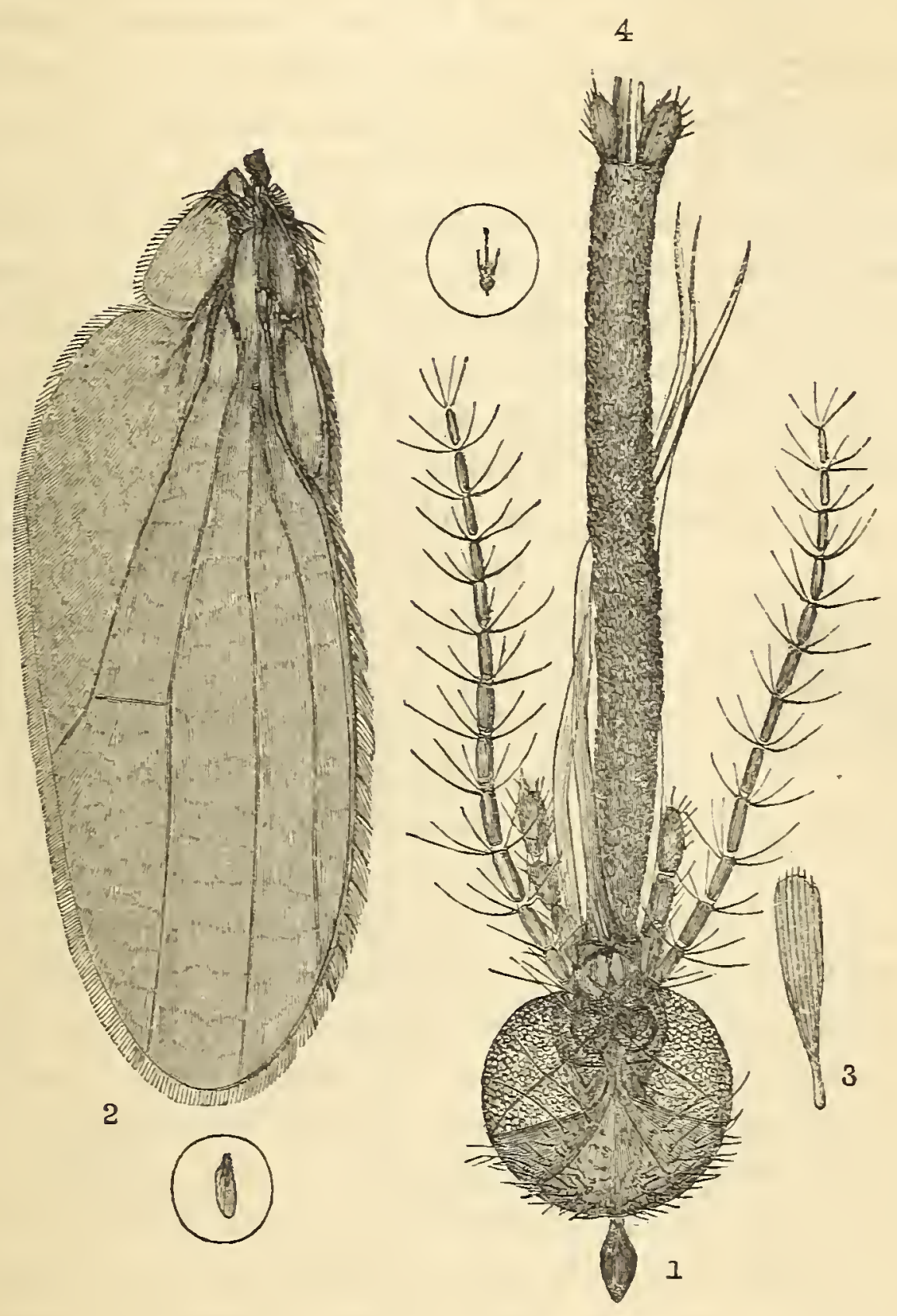

fig. 108. Head and Wing of Gnat.

1. Head of Gnat, detached from the body. 2. Wing of Gnat. 3. A Scale from the Proboscis. 4. The Proboscis and Lancets. The reticulation on each side of the head shows the space occupied by the eyes. The small circles enclose the objects of natural size. The head and wing are magnified 50 diameters; the feather or scale from proboscis 250 diameters.

brate 3000 times in a minute: these wonderful wings are corered on surface and edge with a fine down or hair.

The alternations of bright sunshine and rain which are common in 
March, are extremely favourable to the appearance of gnats. The first that appear are called the winter midges (Trichocerc hyemalis). As the spring advances, these midges are succeeded by others of a different species; and as the weather becomes warmer, the true gnats appear. The sting of the gnat (Culex pipiens) is well known; though gnats themselves are generally so rapid in their movements, and so much dreaded, that very few people care to examine the delicacy and elegance of their forms. The sting is very curiously formed; see fig. 108.) The sucker is enclosed in a sheath, which folds up after one or more of the six lancets have pierced the flesh; it thus inflicts a severe though minute wound, the pain of which is increased by an acrid liquor injected into it through the curiously-formed proboscis, which is covered with feathers or scales. A magnified view of one of these feathers is seen at No. 3, fig. 108; and another, magnified 600 diameters, at No. 7, Plate IX. The proboscis is protected on either side by antennæ, or feelers. Those who will take the

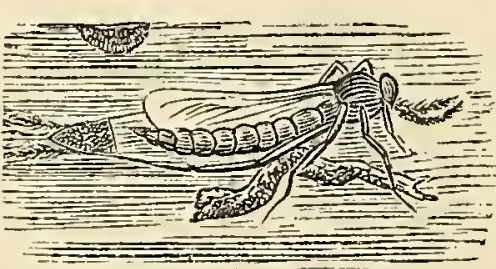

fig. 109. Female Gnat depositing her eggs. trouble to watch the operations of the female (fig. 109), when she is about to make her nest, will be very much struck with the ingenuity and admirable instinct which this little creature displays. The eggs of the gnat are pointed at the upper end and much broader below; and they are so heavy, that if laid singly in the water they would sink to the bottom. The difficulty, therefore, is to contrive some mode of keeping them floating; and this the gnat performs by making her eggs into a kind of boat-shaped raft. To perform this, the mother gnat fixes herself by her fore-legs to a floating leaf, branch, or any thing else that may be in the water, with her body resting on the surface, except the last ring of her tail, which is a little raised; "she then crosses her two hind-legs in the form of the letter $\mathrm{X}$, the inner opening of which is intended to form the scaffolding of her structure: She accordingly brings the inner angle of her crossed legs close to the raised part of her body, and places in it an egg, covered, as is usual among insects, with a glutinous fluid. On each side of this egg she places another; all of which adhere firmly together, by means of their glue, and form a triangular figure for the stern of the raft. She proceeds in this manner to add egg after egg in a vertical (not a horizontal) position, carefully regulating the shape by her crossed legs; and, as her raft increases in magnitude, she pushes the whole gradually to a greater distance; and when about half finished, she uncrosses her legs and places them parallel, the angle being no longer necessary for shap- 
ing the boat. Each raft consists of from 250 to 350 eggs; which, when all laid, float on the water secure from sinking, and are finally abandoned by the mother. They are hatched by the heat of the sun in a few days, the grubs issuing from the lower end; but the boat, now composed of the empty shells, continues to float till it is destroyed by the weather.

The bodies of insects are covered with a hard skin; this answers the purpose of an internal skeleton, and is one of the chief characteristics of insects. All animals, and most fishes, have an internal skeleton of bones, to which the muscles are fixed; but the interior of an insect is composed of a soft mass, and the muscles are affixed to the exterior casing or horny skin, which answers all the purposes of bone, connecting the various parts, and maintaining them in their proper places; at the same time it is a perfect covering to the body. In some insects this horny skin is remarkable for its strength, as in the beetle tribe, many of which are exceedingly curious objects. In the water-

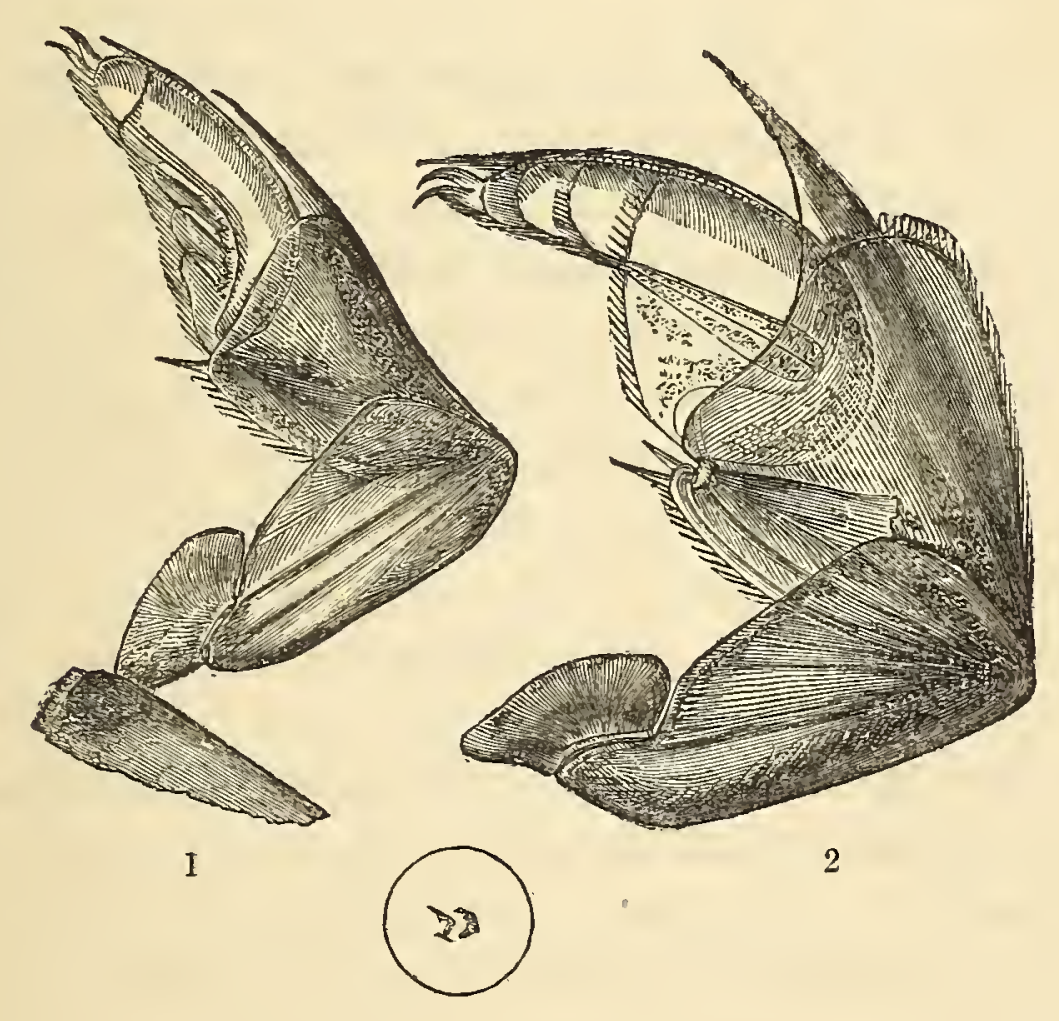

fig. 110 .

1. Legs of Water-beetle (Dytiscus, or Diver). 2. Legs with paddle expanded, as when in the act of swimming. The small circle encloses the object of the natural size.

beetle, we have combined contrivances to facilitate the creature's movements in the element it frequents. The hinder legs are converted into a pair of oars of remarkable efficiency, the point of their connection with the body being adapted with great precision to insure the most effec- 
tual application of the propelling power; and as they are struck out behind in the act of swimming, there is a membranous expansion, which enables the beetle to move about with great rapidity; while, on their being drawn back towards the body, the membrane closes up again, and thus offers but a small resistance to the water: this will be seen upon reference to No. 1, fig. 110 ; and in 2 the swimming apparatus is shown expanded. If the beetle wishes to remain below the surface of the water, he employs the small hooks at the extremity of the leg, for the purpose of adhering to the roots or stem of some aquatic plant. All the species are extremely voracious, greedily devouring the larvæ of gnats, ephemeræ, dragon-flies, \&c.

The horny covering and muscular apparatus under the wing-case of the Cricket (Acheta domestica, fig. 111), offer many curious points for

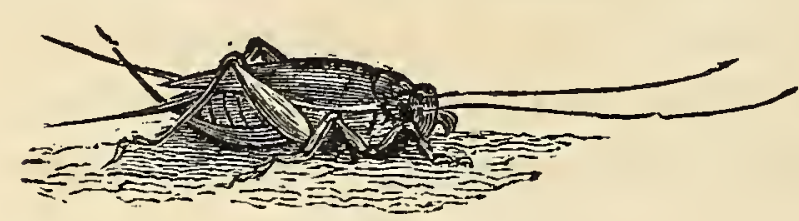

fig. 111. The Cricket. microscopic examination. When we hear the chirp of a cricket, we naturally suppose that it is a sound uttered from the mouth; but this is by no means the case. The cricket has two wings, which are covered with wing-cases of a leathery consistency, near the base of which is a horny ridge, having transverse furrows, exactly resembling a rasp or file; this the cricket rubs against its body with a very brisk motion, whereby it produces its sound. It has been remarked, that the chirp becomes louder in proportion as the heat increases; and it is extremely difficult to silence the crickets in any way but by putting out the fire near which the little animal is chirping.

\section{BEETLES.}

The common Black-beetle (Blatta orientalis, fig. 112) is not, strictly speaking, one of the beetle tribe, but more nearly allied to the cricket and grasshopper. All the insects belonging to this class are very destructive, as they continue eating through all their transformations. The female black-beetle does not lay her eggs singly, but always sixteen at a time; and these eggs are enclosed in a capsule, which resembles a small oblong box (see upper part of cut). The mother carries this capsule about with her for a long time, the half of it protruding from her body, until by degrees the sides of it have attained a proper firmness. The outer part of this capsule is at first white; by degrees it becomes brown. If this receptacle for the eggs is more closely examined, it will be seen that one of the two longer margins is very finely 
toothed, and is composed of two layers, and so constructed that the teeth of one of the layers easily go into the spaces between the teeth of the other. This margin is also so firmly united by means of a gummy substance, that it might be easier opened at any other part than at the toothed edge. As soon as the young are hatched and have quitted the egg, they emit a fluid from their mouths, by which they soften the cement that united the two layers of the capsule together; and thus they contrive to open the door of their prison-house. The anxious mother lays the capsule containing her eggs on clothes, leather, and even on walls, taking abundant care to cover it with a portion of the same kind of material as

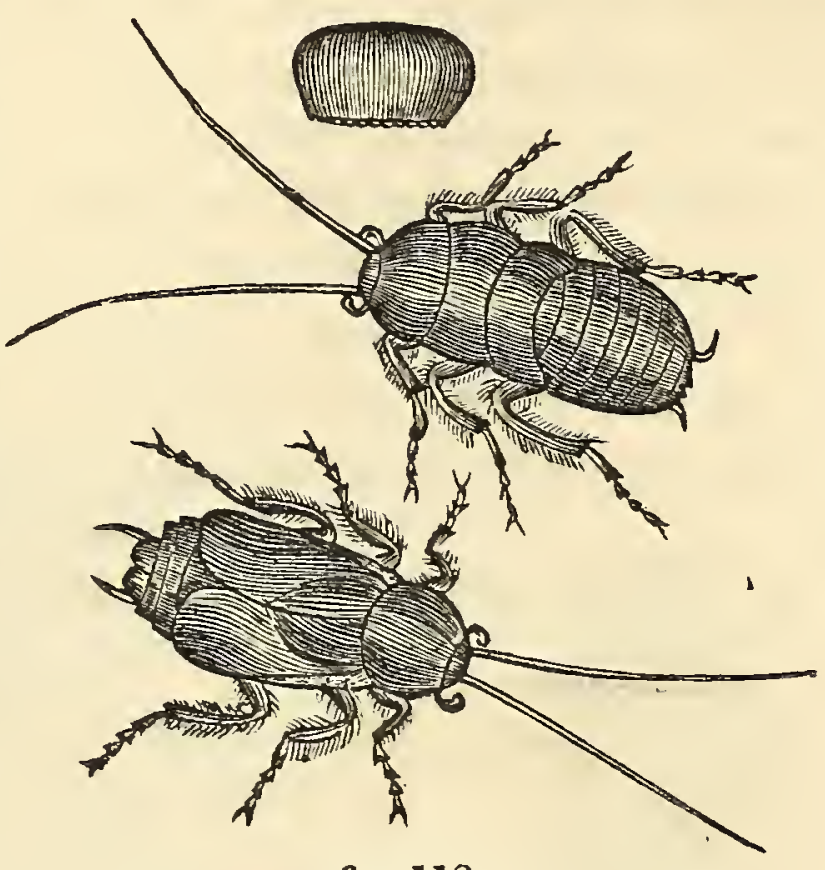

fig. 112.

Male and female Blatta orientalis. that on which she has laid it. She even carries this feeling so far as to scrape the lime from the wall, and to spread it over the capsule. Black-beetles are fond of warm places, and they are found in the greatest abundance in kitchens and bakehouses. Their favourite food is bread and flour; but they will eat almost any thing. They avoid the light, and hide themselves in dark places during the day, coming out of their hiding-places in the evening to feed. The wings and wing-cases of the male are one-third shorter than the body. The female is without wings, and has only very short rounded wing-cases, which are separated from each other.

The insect vulgarly called the Death-watch, fig. 113, is a species of Anobium, a genus of small beetles. These insects live entirely upon wood: the eggs are deposited near some crack in a piece of furniture, or on the binding of an old book. As the larvæ are hatched, they begin to eat their way into the furniture on which they have been deposited; and when they have attained a sufficient depth, they undergo their transformations, and return, by another passage, as beetles. In furniture attacked by them, little round holes, about the size of the head of a pin, may be seen; and these are the holes that have been

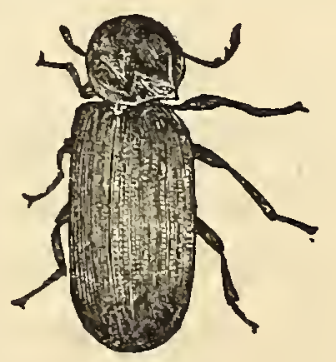

fig. 113.

The Death-watch (Atropus). made by the beetles. The noise, which has given rise to the name of 
death-watch, is made by the insect striking its head against the wood. The larva is called a book-worm when it attacks books; and old books that are seldom used are often found bored through by it; as, though it prefers the cover, when it has finished one side it searches for the other, and takes the nearest way to it, by boring through the leaves of the book, however thick the volume may be. Kirby and Spence mention, that in one case twenty-seven folio volumes were eaten through, in a straight line, by this insect. The beetle is very small, and almost black. The head is particularly small; and from the prominence of the thorax, looks as if it were covered with a hood. Another insect of the same genus (Anobium puniceum, fig. 114) attacks dried objects of natural his-

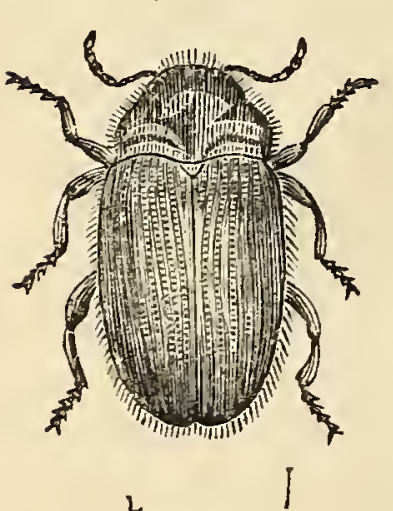

fig. 114. tory, and all kinds of bread and biscuits, particularly sailors' biscuits, in which its maggots frequently abound. In collections of insects, it first consumes the interior; and when the larva assails birds, it is generally the feet that it devours first; and in plants, the stem or ligneous part. The larva is a small white maggot, the body of which is wrinkled, consisting of several segments covered with fine hairs. The jaws are strong and horny, and of a dark brown. Anobium puniceum. The pupa is white, but so transparent, that all the parts of the perfect insect may be seen through it. The beetle is of a reddish-brown, covered with fine hairs.

The Bacon-beetle (Dermestes lardarius, fig. 115) is one whose ravages are very extensive. The larva of this insect is particularly partial to the

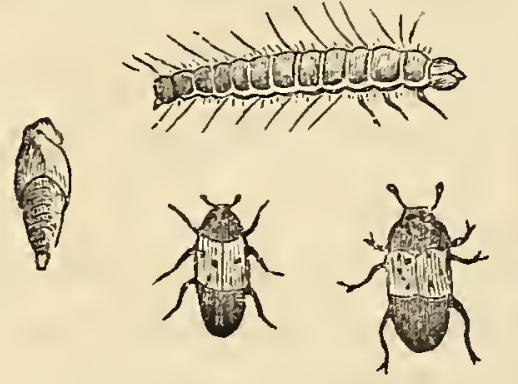

fig. 115. Dermestes lardarius. skin of any animal that falls in its way; and consequently it destroys stuffed animals and birds in collections of natural history, whenever it can gain access to them. It attacks hams and bacon for their skin; but as it is very gluttonous, it extends its ravages to the flesh. The larva is long and slender, its thirteen segments, blackish-brown in the middle, and white at the edge. The whole body is furnished with bristle-shaped reddish-brown hairs. The beetle is black at the head and tail, with an ash-grey band across the back, having three black spots on each wing-case. Sometimes this band takes a yellowish tinge; and the whole beetle is furnished here and there with tufts of ash-grey or yellowish-grey hairs. The beetle is frequently seen in December and January, but is most destructive in spring. The larvæ are very seldom seen, as they conceal themselves 
in the bodies they attack; and their presence can only be guessed by finding occasionally their cast-off skins, as they change their skins several times while in their larva state. Whenever, therefore, little rolls of black skin are found near the places where ham and bacon are kept, or in cases containing objects of natural history, it is probable the bacon-beetle has attacked them. The small scales covering many species of Dermestes, as well as the hairs, are most beautiful microscopic objects, and are very generally used for testing the power of an object-glass.

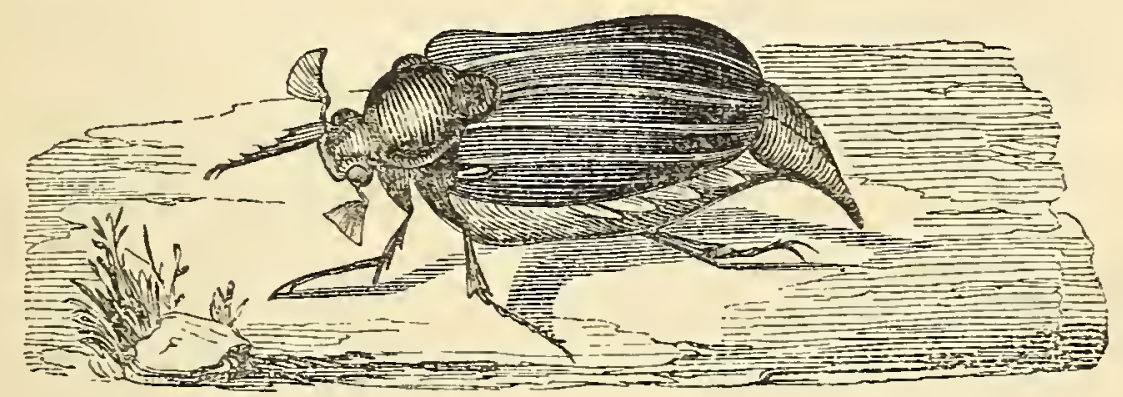

fig. 116. The Cockchafer.

Melolontha Vulgaris, or Cockchafer, is very abundant in our island, and it has a variety of names, - the brown tree-beetle, blind-beetle, May-bug, chaffer, May-bob, or oak-web, jack-horner, geffry-cock, acrebob, as it is termed in different parts of the country.

Its colour is brown; thorax hairy; tail inflected; a triangular white spot at each incisure of the abdomen. The larva is soft and grey, with the head and legs protected by a shelly covering of a yellow-brown colour. While in the larva state, which continues for a space of two or three years, it devours the roots of corn, grass, and other vegetables. The larvæ of this insect are much sought after by crows, rooks, and other birds, as well as animals. It is this that is so frequently turned up by ploughing, and in quest of which crows are often seen following the tracks of the ploughshare. Children are also employed to follow the plough, and collect the white worms, as they are called.

The egg's are laid in small detached heaps, beneath the surface of some clod; and the young, when first hatched, are scarcely more than one-eighth of an inch in length, gradually increasing in their growth, occasionally changing their skins, until they are of the size of two inches or more. At this time they descend to the depth of two feet, where they construct an oval cell, very smooth in the inside; and after a certain time, divest themselves of their last skin, and appear in the chrysalis form, in which they continue till the succeeding spring, when they assume the perfect beetle; but remain for a considerable time in a 
weak state, not venturing out till the fine days of May or the beginning of June, at which time the beetle emerges from its retirement, and commits its depredations on the leaves of trees, \&c., breeds and deposits its eggs, after which its life is short. The antennæ have a remarkable comb-shaped appearance, and are, with other parts of this insect, generally found in the microscopist's list.

The elytra or wing-cases of the diamond beetles are amongst the most brilliant of all opaque objects. Some are improved by being mounted in Canada balsam, whilst others are more or less injured by it: a trial of a small portion, by first touching it with turpentine, will decide this point.

\section{BUTTERFLIES AND MOTHS.}

Butterflies and moths are divided into three distinct genera, viz. Butterfly, Sphinx, and Moth.

Who has not seen and admired the elegant butterfly, fluttering over flowers, which they frequently excel in splendour of colour, and at length resting on them with a touch so light as not to appear to be resting there? Who has not seen them, whilst reposing on the flower, opening and shutting their beautiful wings, alternately erecting and depressing their long and slender antennæ, popularly called horns? and who has not seen the beautiful apparatus by which they extract the nectar from the flowers?

All butterflies and moths proceed from caterpillars, which afterwards change into chrysalides; out of which, after a certain time, proceed the perfect insects. The female butterfly deposits her eggs upon such substances as are proper to nourish the caterpillars which proceed from them: thus, the common cabbage-butterfly places them on cabbage; the peacock-butterfly on nettles; the swallow-tailed butterfly on fennel or rue; the atalanta-butterfly on nettles, \&c. These eggs are simply attached by some glutinous secretion to leaves or stems; in the same way are the eggs of moths placed, except that the latter are enclosed in down.

The distinguishing characteristics of butterflies are, that the horns terminate in small knobs; and the wings, when the insects are at rest, are so placed that they meet upwards. Moths, on the contrary, have sharp-pointed horns, which in many are simple, in others beautifully feathered along the sides; while the wings, when at rest, lie in a horizontal position.

The Sphinx or Hawk moths are a genus distinguished by the antennæ or horns tapering at each end, and which are generally short in 


\section{PLATE VIII.}

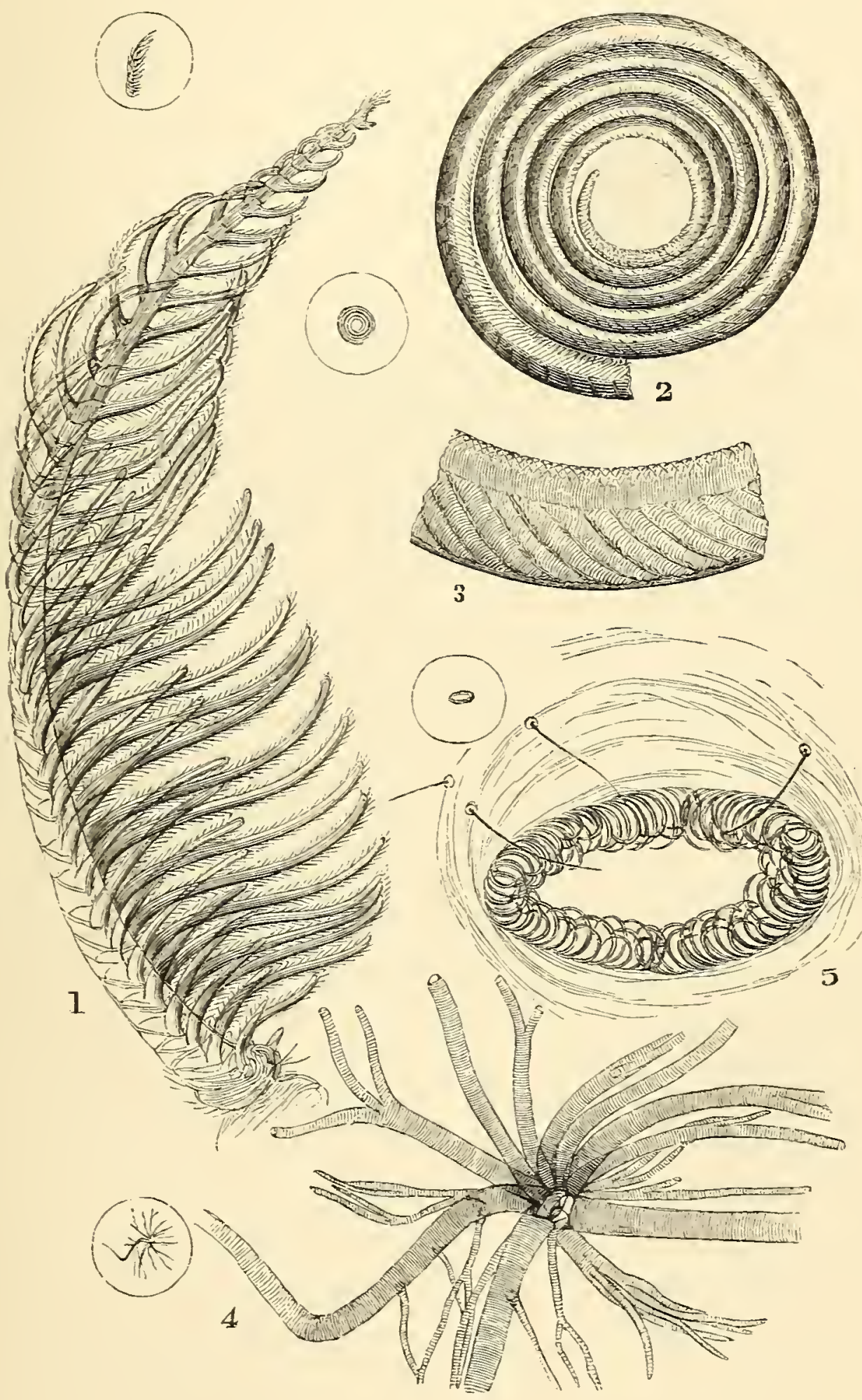

SILKTORM-NIOTH.

1. The Antenna. 2. The Tongne. 3. A portion of the tongue highly magnified, showing circular muscular fibre. 4. The Tracher of Silkworm. 5. The Foot of Silkworm.

(THE SMALL CIRClF. EXCLOSE THE OBJECTS OF THE NATURAL SIZE.) 

proportion to the animal; and by the thickness of their bodies, which in most terminate in a point, as is seen in fig. 117.

There are nearly 200 different species of this genus: they fly about only in the morning and evening, are slow on the wing, and often make a humming noise. The name of Sphinx is applied on account of the posture assumed by the caterpillars of the larger species, which are often seen with their foreparts risen from, and the rest of the body applied flat to the surface on which they are situated; an attitudemuch resembling the Egyptian Sphinx. Many of the species are of great beauty and elegance. Most of these caterpillars descend a considerable depth

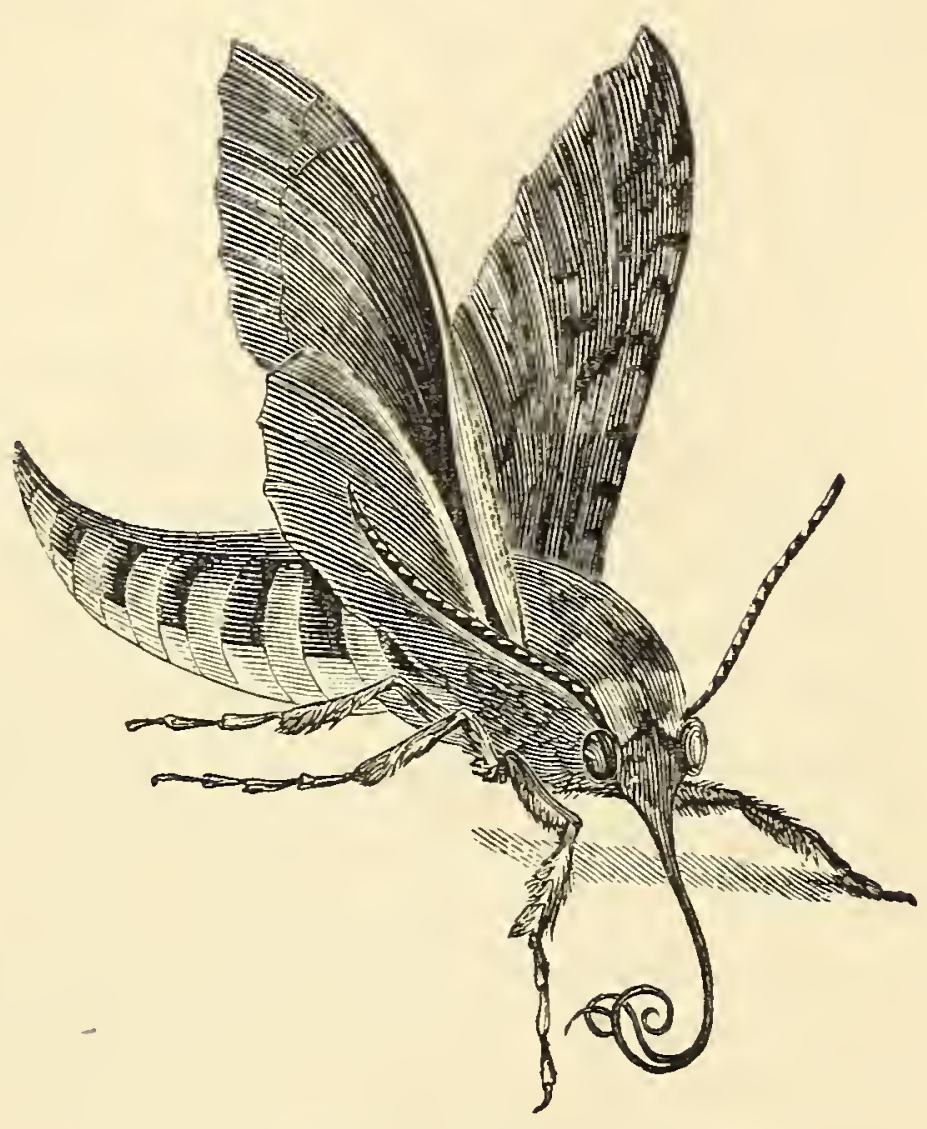

fig. 117. Sphinx Moth. beneath the surface of the earth.

Moths and butterflies supply the microscopist with some of the most beautiful objects for examination. What can be more wonderful in their adaptation than the antennæ of the moth, represented in Plate VIII., No. 1, with thin finger-like extremities almost supplying the insect with a perfect and useful hand, moved throughout its extent by a muscular apparatus, the whole being of a feathery construction! The tongue, No. 2, for the purpose of dipping into the interior of the flower, and extracting the honey, is endowed with a series of muscles: an enlarged view of a portion of the same is seen at No. 3 .

The inconceivably delicate structure of the rnaxillæ or tongues (for there are two) of the butterfly, rolled up like the trunk of an elephant, and capable like it of every variety of movement, has been carefully examined and described by Mr. Newport. "Each maxilla is convex on its outer surface, but concave on its inner; so that when the two are approximated, they form a tube by their union, through which fluids may be drawn into the mouth. The inner or concave surface, which forms the tube, is lined with a very smooth membrane, and extends throughout the whole length of the organ; but that each maxilla is hollow in its interior, forming a tube 'in itself,' as is generally described, is a 
mistake; which has doubtless arisen from the existence of large tracheæ, or breathing-tubes, in the interior of each portion of the proboscis. In some species, the extremity of each maxilla is studded externally with a great number of minute papillæ, or fringes-as in the Vanessa Atalanta-in which they are litstle elongated barrel-shaped bodies, terminated by smaller papillæ at their extremities." Mr. Newport supposes that the way in which the insect is enabled to pump up the fluid nourishment into its mouth is this : "on alighting on a flower, the insect makes a powerful expiratory effort, by which the air is expelled from the interior air-tubes, and from those with which they are connected in the head and body; and at the moment of applying its proboscis to the food, it makes an inspiratory effort, by which the central canal in the proboscis is dilated, and the food ascends it at the same instant to supply the vacuun produced; and thus it passes into the mouth and stomach: the constant ascent of the fluid being assisted by the action of the muscles of the proboscis, which continues during the whole time that the insect is feeding. By this combined agency of the acts of respiration and the muscles of the proboscis, we are also enabled to understand the manner in which the humming-bird sphinx extracts in an instant the honey from a flower while hovering over it, without alighting; and which it certainly would be unable to do, were the ascent of the fluid entirely dependent upon the action of the muscles of the organ."*

The caterpillar's foot is made up of a series of hooklets, which enable him to cling with ease to the surface of a leaf or stalk of a plant. A magnified view of one is seen, No. 5, Plate VIII. In the wings of moths and butterflies we have a combination of strength with lightness; the wing itself is covered with scales or feathers, carefully overlapping each other, as the tiles cover the tops of our houses. The iridescent variety of colouring on the wings arises from the peculiar wavy arrangement of these scales. They have always been favourite objects with the microscopist, and are generally used to test the power of penetration and definition of the instrument itself. In Plate IX. are seen magnified representations of a few of them. No. 1 is a scale of the Morpho Menelaus, taken from the side of the wing, of a pale-blue colour : it measures about 1-120th of an inch in length, and exhibits a series of longitudinal stripes or lines, between which are disposed cross-lines or strix, giving it the appearance of brick-work. The microscope should be enabled to make out these markings with the spaces between them clear and distinct, as shown at No. $1 a$.

Polyommatus Argiolus, or Azure-blue, Nos. 2 and 6, are large and * Cyclop. Anat. and Physiol., article "Insecta." 
small scales taken from the under-side of the wing of this beautiful blue butterfly; the small scale is covered with a series of spots, and exhibits both longitudinal and transverse strix, which should be most clearly defined, and the spots separated: it is a very good test of the defining power of a quarter-inch object-glass.

No. 3. Hipparchia Janira, or Common Meadow Brown Butterfly: on this may be seen a number of brown spots of irregular shape and longitudinal strix. Arnici used this as a test-object.

No. 4. Pontia Brassica, or Cabbage-butterfy, affords an excellent criterion of the penetration and definition of a microscope: it is provided at its free extremity with a brush-like appendage. With a high porrer, the longitudinal markings appear to be rows of little beads.

No. 5. Podura Plumbea, or Lead-colour Springtail: a small apterous insect, frequenting damp places. These insects are usually found in wine-cellars among the sawdust, leaping about like fleas, and are therefore difficult to take. The following is generally the plan resorted to: sprinkle a little oatmeal on a piece of black paper near their haunts; after a few hours, remore it carefully to a large glazed basin, so that when they leap from the paper, as they will when brought to the light, they may fall into the basin, and thus become separated from the meal. The best way of separating the very fine scales from their bodies is, to put them into a small bottle, cork it and shake it about, and in a few shakings a sufficient number will fall out; the mouth of the bottle should then be turned down, and shaken gently orer a slip of glass, to which they will readily adhere. The markings cannot be seen with a lower power than a quarter-inch object-glass. Under a power of 500 diameters, the surface appears to be covered with extremely delicate wedge-shaped dots or scales, as seen at No. $\tilde{5}$ a, so arranged that they form both longitudinal and transverse wary markings. The smaller scales are much more difficult to resolve than the larger; and they form a good test of the defining power of a 1-Sth or 1-12th object-glass. The feather or scale from the gnat's proboscis, No. 7, and No 8, a portion of a large scale of Lepisma Saccharina, are good test-objects, showing the longitudinal markings.

The Tinea Vestianella, or Clothes-moth, possesses very delicate and unique scales: two of these are imperfectly represented near the Acarus found on one of these moths, at page 325. The feathers from the under-side of the wing are the best, requiring some management of illumination to bring out the lines sharp and clear.

The common clothes-moth generally lays its egg's on the woollen or fur articles it intends to destroy; and when its larva appears, it begins 
to eat immediately; and with the hairs or wool it has gnawed off, it forms a silken case or tube, under the protection of which it devours the substance of the article on which it has fixed its abode. This tube is of parchment-like consistence, and quite white. It is cylindrical in its shape, and furnished at both ends with a kind of flap, which the insect can raise at pleasure, and crawl out; or it can project the front part of its body with its fore-feet through the opening, so as to crawl about without removing the rest of its body from the tube, which it drags after it. There are several kinds of clothes-moths; and the caterpillars of some of them bury themselves in the article on which they feed, instead of making themselves a silken tube. The moths also differ very much in appearance : the commonest kind is of a light buff ; one species, Tinea Tapetzella, fig. 118, is nearly black, with the tips of its larger wings white, or pale grey.

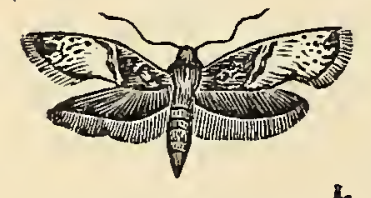

fig. 118.

The Black Clothesmoth.

Mr. Topping, the excellent preparer of microscopic objects, generally furnishes three kinds of test-objects, which he carefully covers with the thinnest glass, in order that object-glasses of the highest powers may be used to examine them. The following list contains his best:

SCALES.

Azure blue.

Pontia brassica.

Morpho Menelaus.

Alucita pentadactyla.

Fruca vestianella.

Lepisma saccharina.

Podura plumbea.

Hipparchia janira.

Plumed Gnat.

INFUSORIA.

(These are much more difficult to define than the former.)

Navicula hippocampus.
Navicula Spenceri.

", angulata.

Tripoli, from Kritchelberg.

Berg-mehl, \&c.

HAIRS.

Bat, Indian.

Mole.

Mouse.

Rabbit.

Squirrel.

Larva of Dermestes.

Dr. Goring says, that "the study of the manner in which these objects are exhibited is of great importance; since in proportion to the excellence of the instrument will the darkness and blackness of the lines be increased, and the transparency of the spaces between them be augmented. Cylindrical or spherical bodies appear the best suited for ascertaining the goodness of an instrument as regards definition; for this reason the Infusoria are considered most valuable; and in illuminating them, oblique diverging rays appear to be essential for 


\section{PLATE IX.}
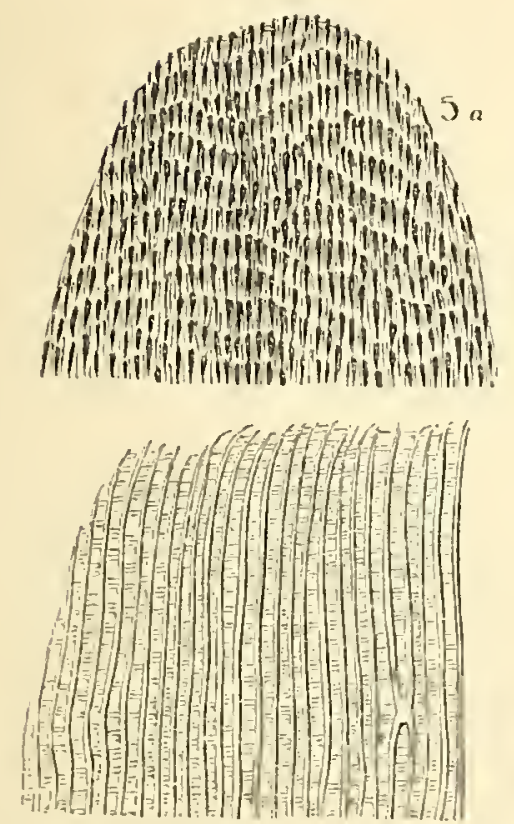

Ia
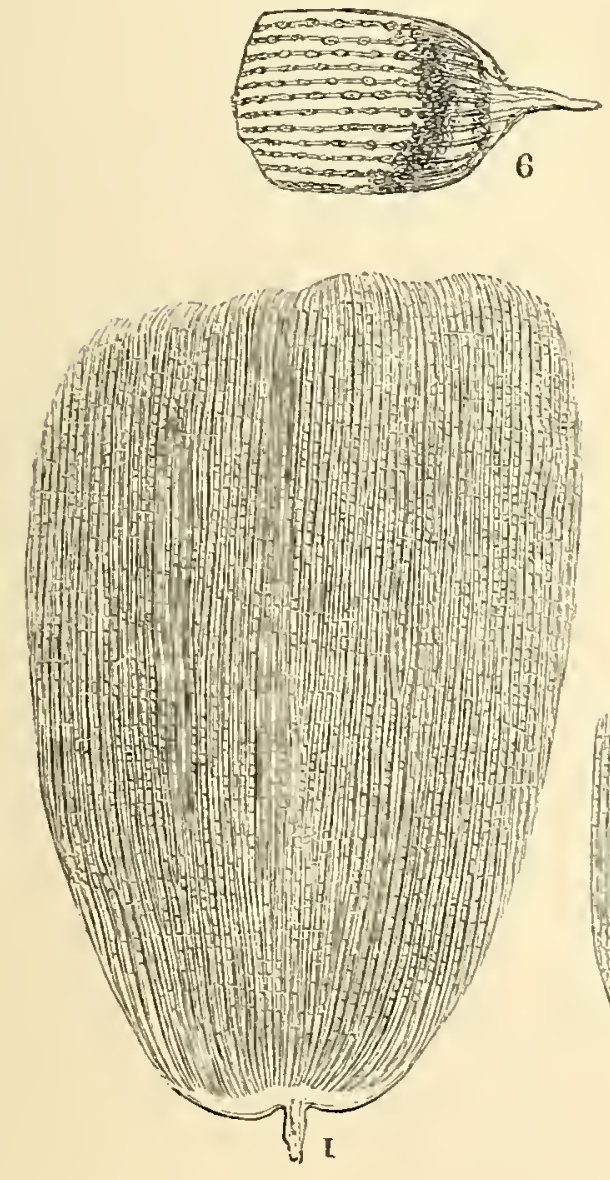
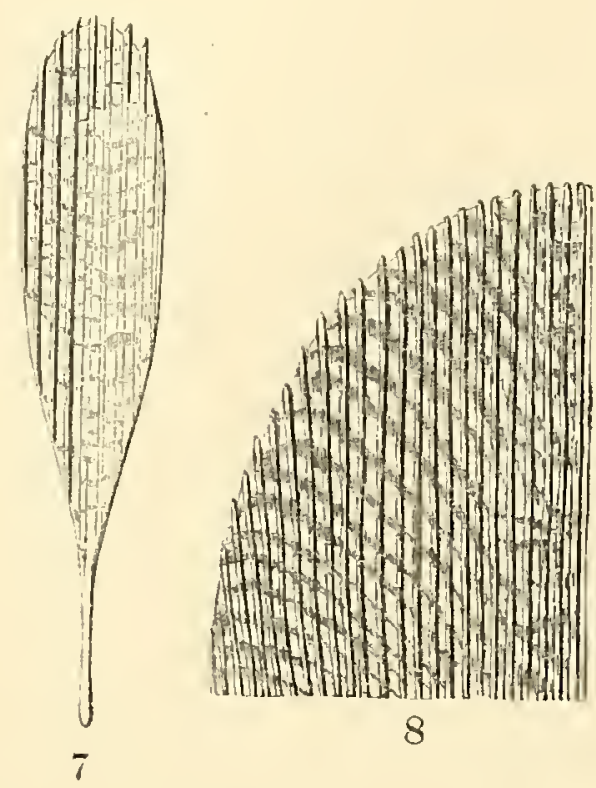

8
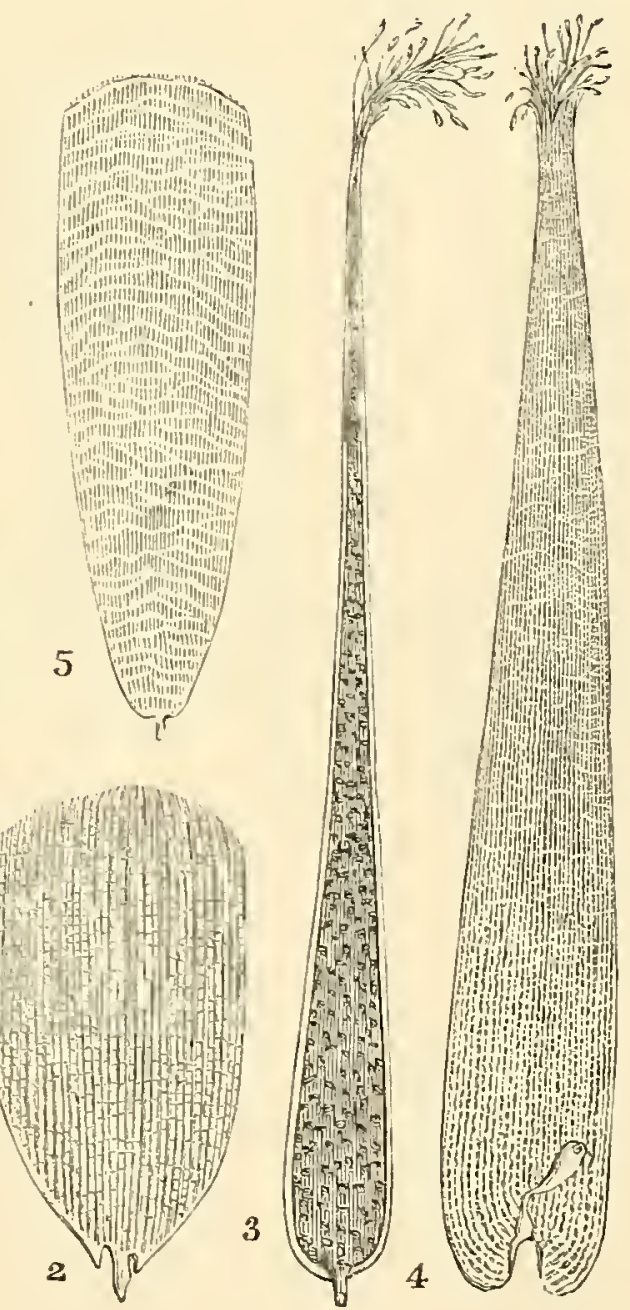

\section{SCALES FROII BUTTERFLY'S AND MOTH'S WINGS.}

1. Scale of Morpho Menelaus, magnified 250 diameters. 1a. Portion of Scale of Morpho Menelaus, magnified 500 diameters. 2. Large Seale of Polyommatus Argiolus, azure blue, magnified 250 diameters. 3. Hipparchia Janira Argiolus, magnified 250 diameters. 4. l'ontia Brassica, magnified 250 diameters. 5. Podura Plumbea, magnified 250 diamcters. $5 \%$. Portion of Large Scale of Podura Plumbea, magnified 500 diameters. 6. Small Scale of Azure Blue, magnified 250 diameters. 7 . Scale from the Wing of Gnat, magnified 500 diameters. 8. Portion of a Large Scale of Lepisma Saccharina, magnified 500 diameters. 

the development of their structure, the degree of obliquity varying with the different lined specimens. The extremes of this variation, however, are the Podura and the Brassica; the delicacy of the former requiring almost central light, while the latter requires it very oblique. From this cause, artificial illumination is to be preferred to daylight for this class of objects, as the light of a lamp or candle gives the rays diverging without any apparatus whatever. It will be found generally preferable to employ a small quantity of intense light rather than a larger portion of weak light; and if possible, avoid the use of lenses or mirrors for condensing or changing the direction of the light."

\section{THE EGGS OF INSECTS.}

Insects supply the microscopist with many other objects of great interest. The eggs of some are very curious; and these may be found in great abundance, though they are generally so carefully concealed as only to be recognised by a naturalist. The twigs of several kinds of trees will be found to have rings of what look like beads upon them, but which are in fact the eggs of the lackey-moth (fig. 119), glued so firmly together that they cannot be separated without the aid of a penknife. The ergs of the gipsy-moth are covered with little tufts of

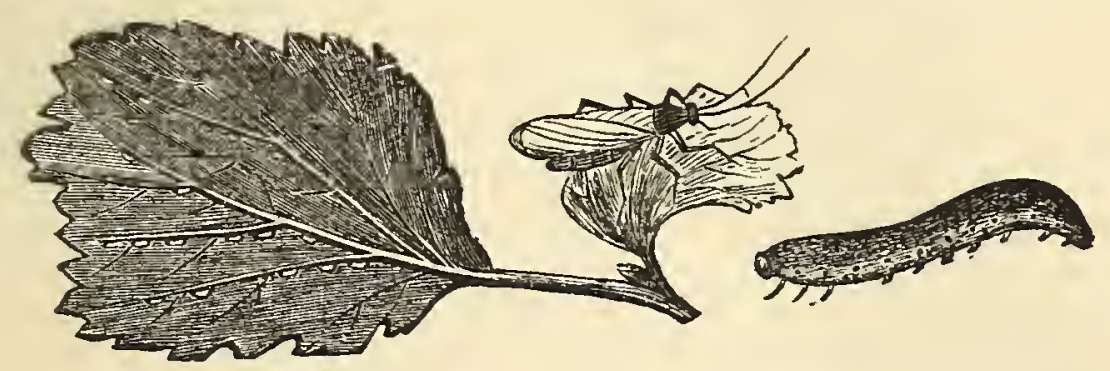

The Saw-fly of the Gooseberry.

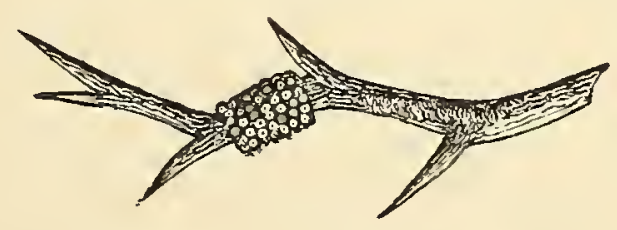

Eggs of the Lackey-moth.

fig. 119.

down; and those of the vapourer are found on the outside of the weblike bag which served the female for her cocoon.

The $S a w-f y$, so destructive to the gooseberry-bushes, is remarkable for the manner in which the female provides for the safety of her eggs. This fly has a flat yellow body, and four transparent wings, the outer 
two of which are marked with brown on the edge. The female lays her eggs on the under-side of the leaf, on the projecting veins; and they are so firmly attached, that they cannot be removed without crushing them. The instrument which the little insect uses for the purpose of cutting the leaf, is one of the most remarkable pieces of perfect mechanism : it is securely lodged, when not in use, in a long narrow slit beneath the hinder part of the abdomen, bounded by two horny plates. At first these appear to consist of a single piece; but upon closer inspection four plates are found to enter into their construction: namely, two saws placed side by side, as in fig. 120; and two supports, very like the saws in shape. A deep groove runs along the thick edge of the

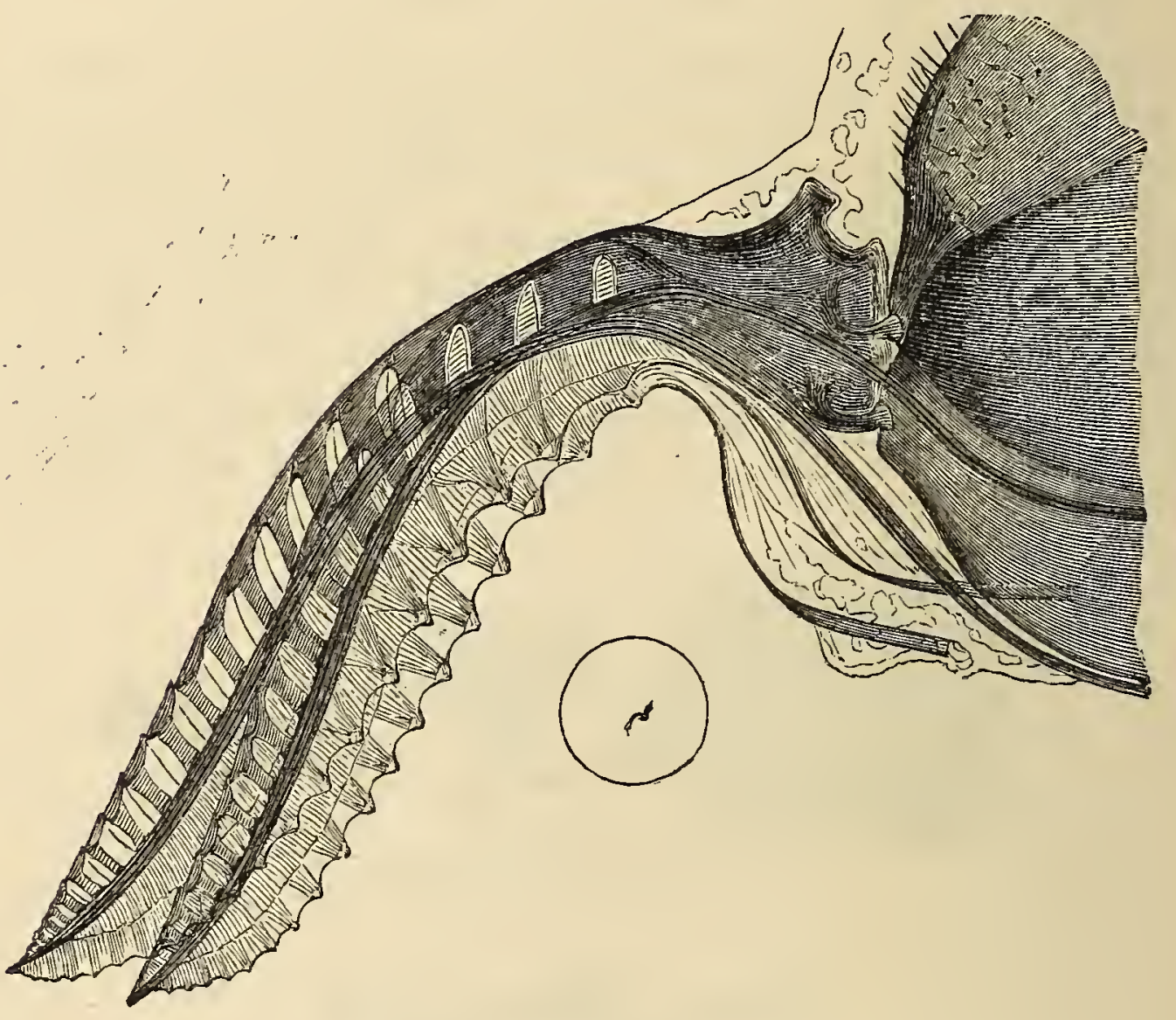

fig. 120. Saws of Saw-fly.

In the small circle it was intended to represent the comparative size of these instruments; but they have been drawn rather too small.

latter, which is so arranged that the saws run backwards or forwards, without the possibility of getting out of the groove. When the cut is made, the four are drawn together; and through the central canal, which is now formed by combining the whole, an egg is protruded into the fissure made by the saws in the leaf. The cutting edges of the saws are 
provided with about eighteen or twenty teeth, having sharp points of extreme delicacy; together forming a good serrated edge of exactly the form given to the finest and best-made surgical saws. In the summertime the proceedings of this little insect may be watched, and the mode of using this curious instrument seen, by the aid of a hand magnifyingglass; as they are not easily alarmed when busy at this work. Other insects of the same kind are to be met with, and seen at work, on the leaves of the alder, osier, rose-tree, dc.

Many other insects are provided with instruments for boring into the bark or solid wood itself; the Uraceridce are of this kind. The Cynip bores a hole into the side of the oak-apple, for the purpose of depositing its larra, whence, as well as being comfortably lodged, it receives its early food. When full grown, it eats its way out of the nut; and dropping to the ground, it assumes its pupa form, which in a short time is exchanged for that of the perfect fly. The most important of these insects is the Cynip gallce tinctorice, which causes the formation of the gall-nut, so extensirely employed in the manufacture of ink and for dying purposes. Thousands of pounds worth of these nuts are annually imported into this country.

MI. Lacare and Riche instituted a curious experiment in their examinations of these little hymenopterous insects, to determine the comparative quantity of fat assimilated in the growth of animals. The gall is found to be a series of concentric layers enreloping the alimentary mass, in the centre of which the insect grows, feeding on this mass of cells, containing fecula or starch. They analysed this alimentary mass on which the insect fed, and compared it with the analysis of the insect. In the gall of one plant it was found that the mass contained 0.236 milligrammes of fatty matter, whereas the insect contained $5 \cdot 010$ milligrammes; showing an excess of more than 4 milligrammes, which it must hare formed from the fecula.

The Cuckoo-spit, or Froth-fy, is an insect rery curious in its habits and structure. The names of cuckoo-spit and froth-fly both allude to the peculiar habit of the insect, when in the larra state, of enveloping itself in a kind of frothy secretion, somewhat resembling saliva; and which, indeed, was formerly supposed to be the saliva of the cuckoo, it being found on the young shoots of plants just about the time that the cuckoo is heard in the woods. The frothy secretion is supposed to be intended to preserve the tender body of the insect from the orerpowering effects of the sun, as it has been observed to be produced in exact proportion to the heat of the weather. It is not known exactly how the froth is produced. When by any chance it becomes con- 
densed, it drops like rain from the trees on which the insect is found. It is only in its larva or infant state that it produces the froth. The larva and the pupa resemble the perfect insect, except that the larva has no wings, and the pupa has very small ones. The perfect insect, however, has both wings and wing-cases; and it has the power of flying to a considerable distance. Sometimes, indeed, these insects are seen in vast multitudes on the wing. One of the peculiarities of this insect is its power of leaping, which is so great that, being assisted by its wings, it will sometimes leap a distance of five or six feet; which, as Messrs.

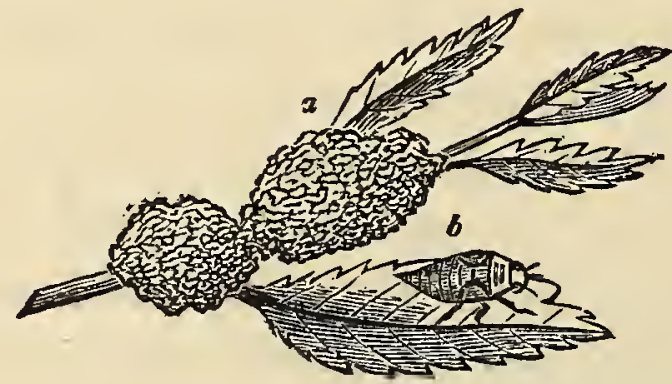

Cuckoo-spit.

$a$. The frothy substance. $b$. The pupa.

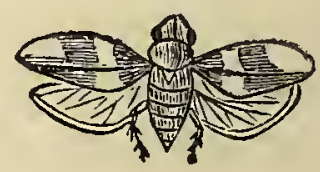

Perfect Insect ot the Cuckoo-spit.

fig. 121.

Kirby and Spence remark, is more than two hundred and fifty times its own length, or as much as if a man were to take a leap a quarter of a mile high. This extraordinary activity appears to be principally occasioned by the great length of the thighs of the insect, which are also furnished on their outer margin with a fringe of stiff hairs or strong spines, which are of great use to the insect in leaping. The insect, when about to leap forward, places its hind thighs nearly erect, keeping them close to the body; it next, with great violence, kicks them out backwards, so as to stretch the leg in a right line, and to press the spines upon the ground; the spines then lay hold of the surface, and by their pressure enable the body to spring forwards. The great assistance afforded by the spines is clearly shown by the fact that, when the insect is on glass, of which the spines cannot catch hold of the surface, it cannot leap more than six inches.

The Aphides, or Plant-lice. The habits and structure of these insects are very curious. They are somewhat allied to the above insect. The sucking-apparatus of the aphides consists of a proboscis made up of several jointed parts, which shut up into each other, much after the 
manner of the telescope. It is armed at its extremity with a sharp point, which the insect plunges into the young shoots of the plant on which it lives, to suck up its juices. A close relationship is said to exist between the aphides and the ants.

The Ant deserves a passing notice. The ants generally seen are little black creatures, with long legs, large heads, and very slender bodies. But these are only the working part of the community; and many people are probably not aware that in the month of August, and sometimes later, "the habitations of the rarious species of ants may be seen to swarm with winged insects, which are the males and females, preparing to quit for ever the scene of their nativity and education. Every thing is in motion; and the silver wings, contrasted with the jet bodies which compose the animated mass, add a degree of splendour to the interesting scene. The bustle increases, till at length the males rise, as it were by a general impulse, into the air, and the females accompany them; the whole swarm alternately rising and falling, with a slow movement, to the height of about ten feet: the males flying obliquely, with a rapid zig-zag motion; and the females, though they follow the general movement of the column, appearing suspended in the air, like balloons, seemingly with no individual motion, and having their heads

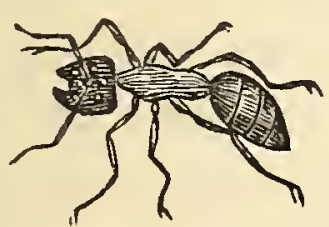

Common Ant.

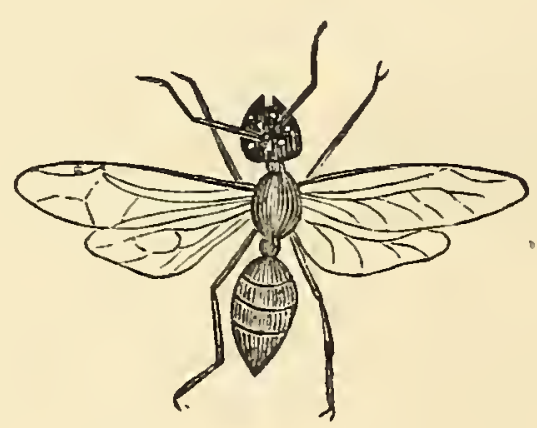

Winged Ant,'

fig. 122.

turned towards the wind." "Sometimes the swarms of a whole district," continue Messrs. Kirby and Spence, "unite their infinite myriads, and rising with incredible velocity, in distinct columns, they soar above the clouds. Each column looks like a kind of slender net-work, and has a tremulous undulating motion, which has been observed to be produced by the regular alternate rising and falling just alluded to. The noise emitted by myriads and myriads of these creatures does not exceed the hum of a single wasp. The slightest zephyr disperses them; and if in their progress they chance to be over your head, if you walk 
slowly on, they will accompany you, and regulate their motions by yours." All the male, and a great number of the female ants, become the prey of birds or fish, or are destroyed in various ways; but a few females remain, some of which become the founders of new colonies, while others return to their original nests, when it is said that they are seized forcibly by some of the working ants, who tear off their wings, and keep them prisoners till they are ready to lay their eggs. During the time that the female ants are in this state of durance, the working ants, though hanging pertinaciously to each leg, to prevent their going out, at the same time attend upon them with the greatest care, feeding them regularly, and conducting them where the temperature is suitable for them; but never quitting them for a single moment. As soon as the female begins to lay her eggs, the working ants which are in attendance on her carry them off, and deposit them in proper places for them to be hatched. Eacl female lays four or five thousand eggs in the course of a year; so that when a single female founds a colony, she is very soon enabled to people it. When a female has founded a colony, the working ants begin to pay a homage to her very similar to that which bees render to their queen.

Of all the stratagems employed by insects, perhaps the most curious are those of the Ant-lion (Myrmeleon, fig. 123). This insect in

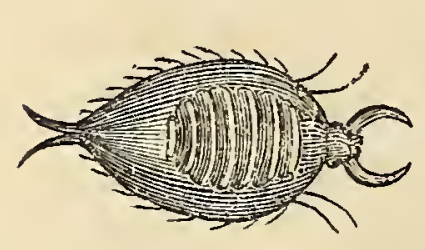
the larva state bears considerable resemblance to the wood-louse; and, as Messrs. Kirby and Spence observe, "if we looked only at its external conformation and habits, we should be apt to conclude it

fig. 123.

The Ant-lion. one of the most helpless animals in the creation. Its sole food is the juices of other insects, particularly ants; but, at the first view, it seems impossible that it should ever secure a single meal. Not only is its pace slow, but it can walk in no other direction than backwards; you may judge, therefore, what would be such a hunter's chance of seizing an active ant. Nor would a stationary posture be more favourable; for its grim aspect would infallibly impress upon all wanderers the prudence of keeping at a respectful distance." In this helpless condition, instinct teaches the ant-lion to accomplish by artifice what it would otherwise have been quite unequal to. The female generally lays her eggs in a loose, sandy soil; so that, as soon as the larva is hatched, it finds itself in the situation most suitable to it. Its first effort is to trace in the sand a circle; and this being done with wonderful exactness, it proceeds to excavate the cavity by throwing out the sand. "Placing itself in the inside of the circle which it has traced, it thrusts 
the hind part of its body under the sand, and with one of its fore-legs serving as a shorel, it charges its flat and square head with a load, which it immediately throws over the outside of the circle, with a jerk strong enough to carry it to the distance of sereral inches." Walking backwards, and constantly repeating this process, it soon arrives at the part of the circle from which it set out. It then traces another furrow in the same manner, and then others, till it has excarated a conical hole rather more than two inches deep, about three inches wide at the top, and contracting to a point at the bottom. In the course of its labours the ant-lion frequently meets with small stones, which it places on its head one at a time, and jerks off orer the margin of the pit. If, however, the stone is too large, it contrives with great difficulty to get it on its back; and, keeping it in a "steady position by an alternate movement of the segments which compose that part, it carefully walks up the ascent with its burden, and deposits it on the outside of the margin. When, as occasionally happens, the stone is round, the labour becomes more difficult and painful; and a spectator, watching the motions of the ant-lion, feels an inexpressible interest in its behalf. He sees it, with vast exertion, elevate the stone, and begin its arduous retrograde ascent; at every morement the burden totters to one side or the other; the adroit porter lifts up the segments of its back to balance it, and has already nearly reached the top of the pit, when a stumble or a jolt mocks all its efforts, and the stone tumbles headlong to the bottom. Mortified, but not despairing, the ant-lion returns to the charge, again replaces the stone on its back, again ascencls the side, and artfully avails itself, for a road, of the channel formed by the falling stone, against the sides of which it can support its load." In this manner it frequently tries without success, renewing its efforts again and again, till at last it either succeeds or abandons the hole in despair. When all is finished, the ant-lion buries itself in the sand at the bottom of its pit, only leaving exposed its two large horn-like forceps, with which it seizes its prey. No sooner does an ant or any other insect approach the edge of the cleverly-contrived slope, than the sand gives way, and the unfortunate insect rolling to the bottom, is instantly seized; and if not sufficiently powerful to make any resistance, it is as instantly killed, and its body, after it has been sucked dry, is tossed, by a jerk of the head of the ant-lion, beyond the immediate boundary of the carity. Sometimes, however, it happens that a large and rigorous-winged insect-such as a wasp, a bee, or a beetle-tumbles headforemost into the pit; and when this is the case, a tremendous battle ensues, and "the result at last is, that either the ant-lion is 
dragged out of its den and stung to death, or dropped upon the ground and left a prey to birds, or that the winged insect is maimed, disabled, drawn into the sand, and slain. If an insect incapable of flight, or from its situation unable to use its wings, but of larger size than the Myrmeleon deems it prudent at once to seize upon, chances to fall into the snare, it is overwhelmed in its attempts to reascend by repeated showers of sand, which its enemy directs upon it with unerring aim." The showers of sand are thrown up by the head of the insect; and it is astonishing the quantity it conveys each time, and the force and precision with which it hurls its ammunition on the foe.

\section{THE STINGS OF INSECTS.}

The sharp and penetrating instruments wherewith the tails of wasps, bees, hornets, and some other insects are armed, are weapons given to them for self-defence, and differ in every respect from the probosces of flies, gnats, \&c., which are intended as instruments whereby they are enabled to procure their sucking food, using them from necessity only. Touch or squeeze a bee or a wasp, it instantly darts forth its sting; but no provocation can cause a gnat or a flea to bite; on the contrary, when disturbed or hurt, it draws in its proboscis, and hastens away, only to use it when undisturbed and in safety.

Some of the wasp tribe are very peculiar in their habits, and are active agents in the economy of nature. The solitary, or mason wasps, curiously construct their nests, forming cells, in which they most carefully rear their young. The social wasps, like the bees, live in communities, and have nearly the same divisions of labour and regulations for the government of their colony. The structure and mechanical contrivance of their stings can only be seen under the microscope. The sting itself is enclosed in a horny case or sheath, which ends in a pointed extremity; a slit opens and permits two barobed darts to be protruded, which penetrate the flesh deeply; and from the peculiar arrangement of their serrated edges, prevent their immediate withdrawal; while, by the muscular effort required for this purpose, a small sack or bag near the root is pressed upon, and its irritating contents squeezed into the wound; after the fluid is injected, the insect possesses the power of contracting the barbed points; it then withdraws the sting from the flesh. In fig. 124 the sting of the wasp is shown, with its attachments and muscular arrangements; and it can be seen that the sting itself is most wonderfully adapted to become an instrument of a very effective and deadly construction. The parts seen near it are 
brushes, evidently placed there for the purpose of cleaning or wiping it.

The proboscis or trunk of the honey-bee next demands our attention; this it uses, with its accessories, to collect the honey for its food

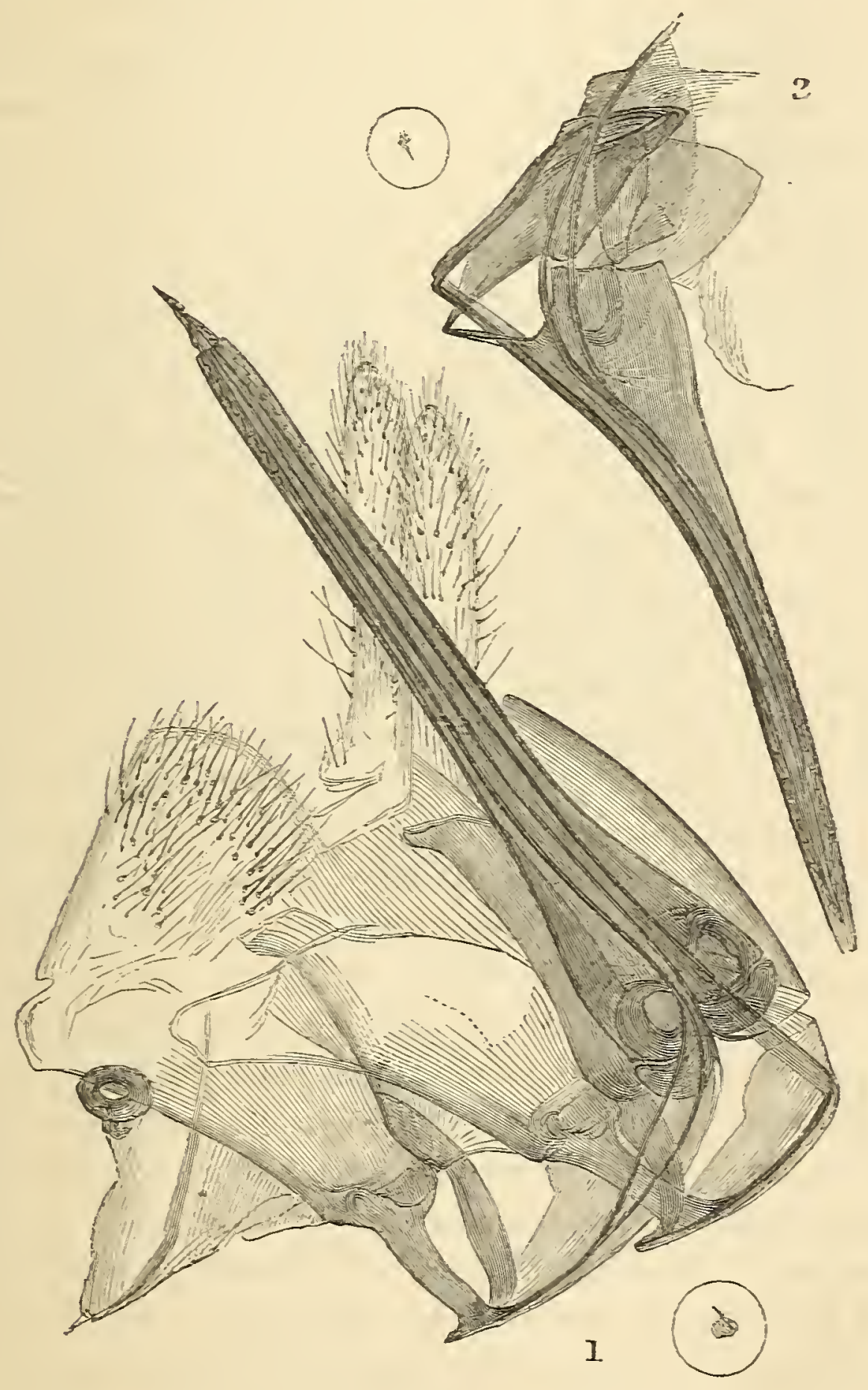

fig. 124.

1. Sting of Wasp. 2. Sting of Bee. The small circle encloses the object of the natural size.

when roring about from flower to flower. The proboscis itself (fig. $12.5)$ is rery curiously divided; the divisions are elegant and regular; beset with triangular hairs; and being numerous, appear at first sight as a number of different articulations. The two exterior lancets are 
spear-shaped, and of a membranaceous or horny substance set on one side with short hairs, and having their interior hollow; at the base of each is a hinge-articulation, permitting considerable motion in several directions; evidently used by the little busy insect for the purpose of distending the internal parts of flowers, and thus facilitating the introduction of the proboscis. The two shorter feelers are closely connected to the proboscis, and terminate in three jointed articulations. Swammerdam thought these were used as fingers in assisting the removal of obstructions; but it is more probable that they are used by the insect for the purpose of storing and removing the bee-bread to the pocketreceptacle in the legs. The lower part of the proboscis is so formed that it may be considerably enlarged at its base, and thus made to contain a larger quantity of the collected juice of flowers; while, at the same time, in this cavity the nectar is soon transformed into pure honey by some peculiar chemical process of its own; the proboscis tapers off to a little nipple-like extremity. At its base may be seen two shorter and stronger mandibles, serving the little insects for tools in constructing their cells; from between these is protruded a long and narrow tongue or lance; the whole most ingeniously connected to the head by a cartilaginous structure, with a series of muscles and ligaments. The proboscis being cylindrical, extracts the juice of the flower in a somewhat similar way to that of the butterfly; when loaded with honey, the next care is to fill the very ingenious pockets situated in its hind-legs (one of which is shown at No. 2) with bee-bread; when these little pockets are filled with as much pollen as the bee can conveniently carry, it flies back to the hive with its valuable load, where the bee is speedily assisted to unload by its fellow-workers; and the pollen is at once kneaded and packed close in the cells provided for its preservation. The quantity of this collected in one day by a single hive during favourable weather is said to be at least a pound; this seems to constitute the food of the working-bees in the hive. The wax is another secretion exuding from the skin of the insect, and is found in little pouches in the under-part of the body; it is not collected and brought home ready for use, as has been generally supposed. The waxen walls of the cells are, when completed, strengthened by a varnish, called propolis, collected from the buds of the poplar and other trees, and besmeared over them by the wonderful apparatus we have shown in the engraving. If a bee is attentively observed when it has placed itself upon a flower, the activity and promptitude with which it uses this apparatus is truly surprising. It lengthens it, applies it to the bottom of the petals, then shortens it, bending and turning it in all 




fig. 125.

1. Honey-bee's tongue. 2. Leg, showing pocket for carrying the Bee-bread. The small circles enclose the objects of their natural size.

possible directions, for the purpose of exploring the interior, and removing the whole of the pollen. In the words of Brooke :

"The dainty suckle and the fragrant thyme,

By chemical reduction they sublime;

Their sweets with bland attempering suction strain,

And curious through their neat alembics drain;

Imbib'd recluse, the pure secretions glide,

And vital warmth concocts th' ambrosial tide." 


\section{ARACHNIDA OR SPIDER TRIBES.}

Spiders are animals which, although not strictly belonging to the insect tribes, are generally described with them, and certainly afford the microscopist much matter for observation; they are articulated animals, and possess the same power of renewing a lost limb as the crustacea. The diadem spider (Epeira diadema) is one of the largest of British spiders; it is a garden spider, and is easily recognised by the beautiful little gem-like marks on its body and legs. Spiders abound on every shrub; and when we consider that the spider is destitute of a distinct head, without horns, one half of its body attached to the other by a very slender connection, and so soft as not

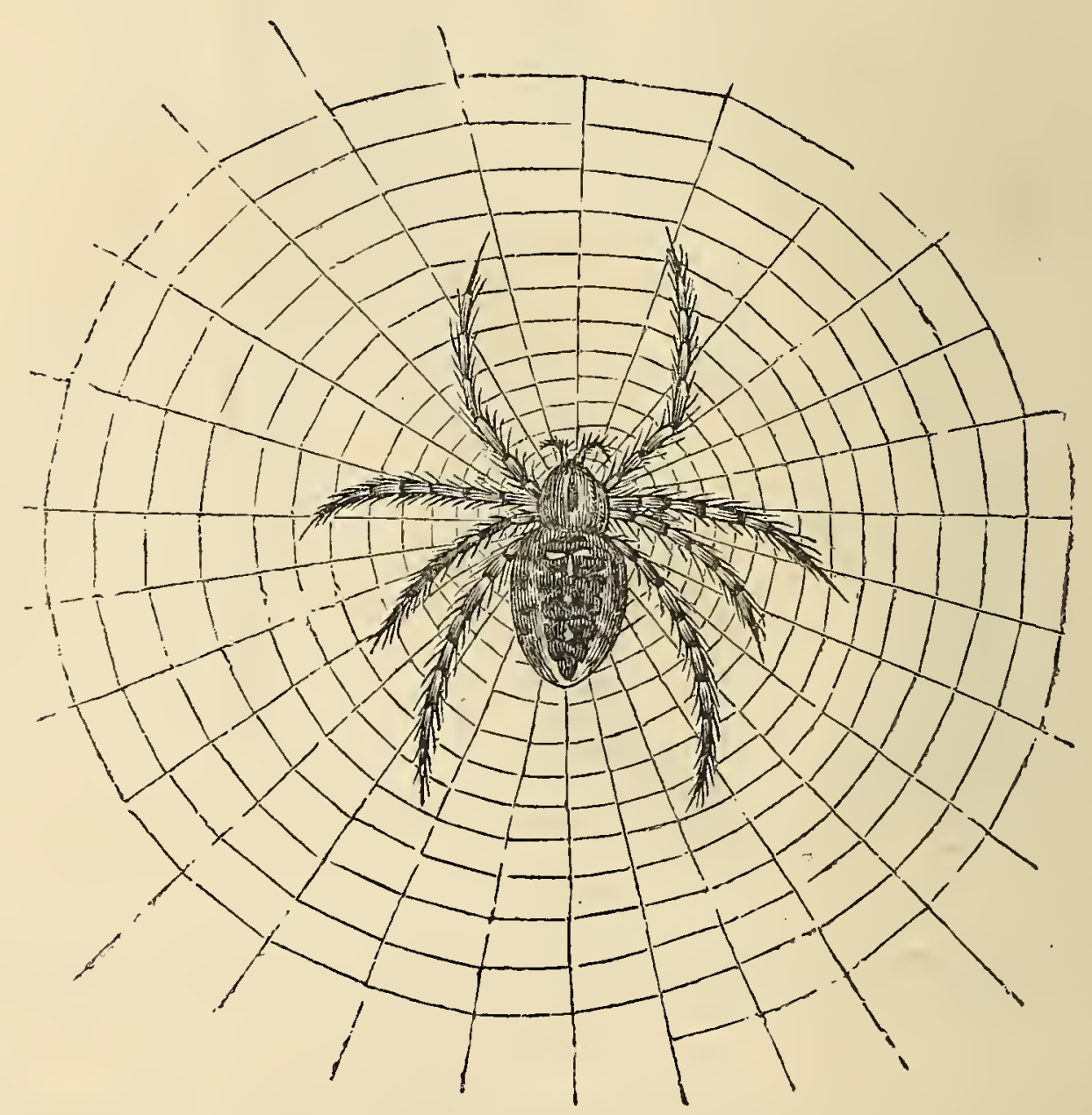

fig. 126. The Garden-spider.

to bear the least pressure,-its limbs so slightly attached to its body that they fall off at a very slight touch,--it appears ill adapted either to escape from danger which threatens it on all sides, or to supply itself with food: the economy of such an insect deserves notice.

The several small appendages, represented in fig. 127, it is important not to confound with each other. Of these the two longest, No. 1, having 
articulated processes, seem to be feelers; the others, being the organs by which their silky threads are emitted, are four in number. Their structure is very remarkable; the surface of each of the spinnarets is pierced by an infinite number of minute holes, as seen in No. 2, from each of which there escape as many little drops of a liquid, which, drying the moment they come in contact with the air, form so many delicate threads. Immediately after the filaments have passed out of the pores, they unite first together, and then with those of the next, to form one common thread; so that the thread of the spider is composed of a large number of minute filaments, perhaps many thousands, of such extreme tenuity, that the eye cannot detect them until they are twisted together into the working thread. In the two pairs of spinnarets

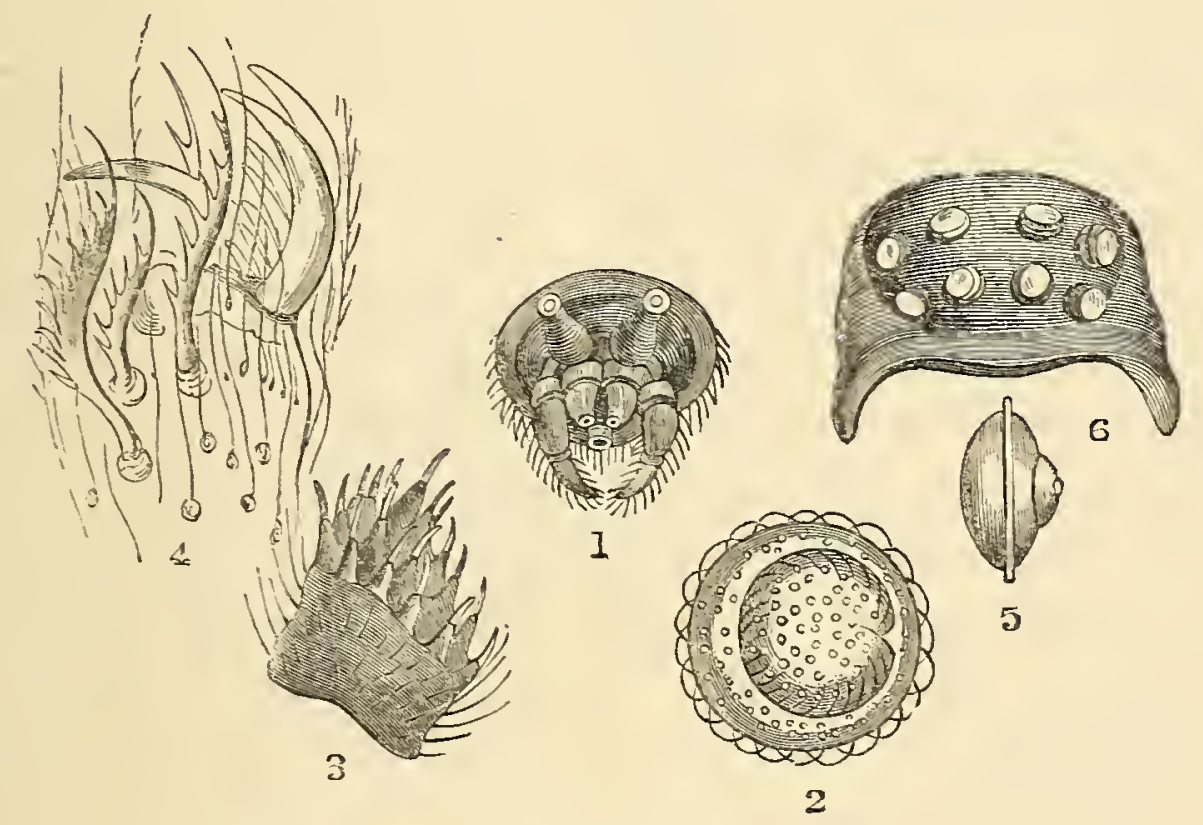

fig. 127 .

1. Spinnarets of the spider. 2. Extreme end of the upper pair of spinnarets 3. End of under pair of spinnarets. 4. Foot of the spider. 5. Side riew of eye of spiders. 6. The eight eyes are shown.

different anatomical structure is to be detected; the pair above, which are a little longer than the lower, show a multitude of small perforations, the edges of which do not project, and which therefore resemble a sieve. The other shorter pair have projecting tubes, independent of the perforations which also exist (No.3). The tubes are hollow, and perforated at their extremities; and it is supposed that the agglutinating threads issue from these tubes, while those emitted from the perforations do not possess that property. It may be observed, by throwing' a little dust on a circular spider's web, that it adheres to the threads which are spirally disposed, but not to those that radiate from 
the centre to the circumference; the latter are also stronger than the others. "The top-line of this web appears to be first spun, either by attaching a thread to a neighbouring tree, and then carrying it along until it is of sufficient strength, when it is attached to some adjacent object to which the spider has crawled; or by throwing out a floating line whilst the spider remains stationary, the action of the air carrying this line on until it becomes attached to some object, when, in either case, it is doubled and redoubled until it is of sufficient strength to bear the weight of the intended fabric, together with the spider itself. The other outer threads of the frame-work are then added, and then cross lines carried from one point of the web to another exactly opposite, forming a complete series of spokes or radii, which the insect then attaches together by a spiral series of transverse bars of a more glutinous thread." The rapidity with which these webs are constructed is astonishing, as is also the accuracy with which the webs are formed. There are many different kinds of spiders; but nearly all of them envelope their eggs in a covering of silk, forming a round ball, which the spider takes care to hang up in some sheltered place till the spring: The mode in which the ball is formed is very curious: the mother spider "uses her own body as a gauge to measure her work, in the same way as a bird uses its body to gauge the size and form of its nest. The spider first spreads a thin coating of silk as a foundation, taking care to have this circular by turning round its body during the process. It then, in the same manner, spins a raised border round this till it takes the form of a cup, and, at this stage of the work, it begins to lay its egg's in the cup, not only filling it with these up to the brim, but piling them up above it into a rounded heap, as high as the cup is deep. Here, then, is a cup full of eggs, the under half covered and protected by the silken sides of the cup, but the upper still bare and exposed to the air and the cold. It is now the spider's task to cover these, and the process is similar to the preceding, that is, she weaves a thick web of silk all round them, and, instead of a cup-shaped nest like some birds, the whole egg's are enclosed in a ball much larger than the body of the spider that constructed it."

The feet of the spider, one of which is represented at No. 4, are curiously constructed. Each foot, when magnified, is seen to be armed with strong, horny claws, furnished with bent teeth on the under-surface, which gradually diminish towards the extremity of the claw. By this apparatus the spider is enabled to regulate the issue of its rope from the spinnarets, and also to suspend itself with the greatest ease by the larger central claw. Some have, in addition, a remarkable comb 


\section{PLATE X.}

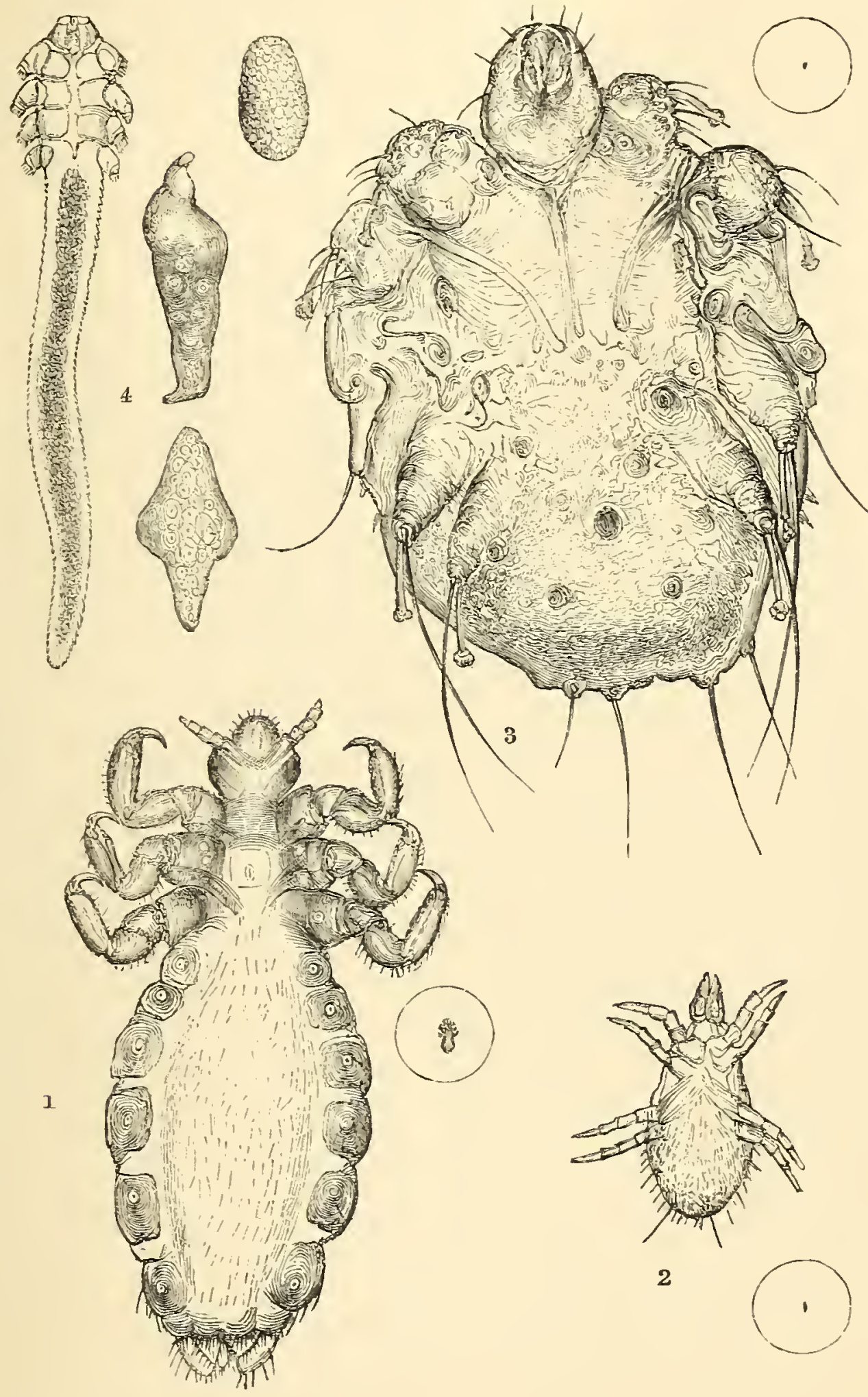

\section{PARASITES.}

1. Louse HuMar; magnified ว̃0 diameters.

2. Acarus from Cheese, Cheese-Mite; magnified 50 diameters.

3. Acarus Scabifi, Itch-Insect; magnified 350 dianeters.

4. Extozoos Foliculorux, or Grub from IIuman Skin, in its varions stages of existence, from the Egg upwards; magnified 250 diameters.

(THE SMALL CIRCLES EXCLOSE THE OBJECTS OF THE XITURAL SIZE.) 

ing claw, for the purpose of separating certain fibrous bands that enter into the composition of its delicate web.

\section{PARASITICAL INSECTS.}

We now pass on to a tribe of insects of a less-inviting form and character, and which at first sight may appear not so agreeable for examination as the preceding; nevertheless, their most extraordinary habits, together with their strange mode of life and numerous other peculiarities, offer many points of interest to the microscopic observer.

Parasites or ticks infest the skin, lurk among the hair and feathers of quadrupeds and birds, and even of other insect races, whence they draw an abundant supply of support for their singular mode of existence. Every species of animal in creation appears to have a peculiar race of parasites of its own assigned to it; some even have several different kinds; so that this despicable group is richer in species than both the great classes of quadrupeds and birds put together. Mr. Henry Denny has figured and described a greater number of parasite insects in his Monographic Anoplurom than any previous observer. He says, "that the opinion entertained of each animal having its peculiar parasite, is not entirely borne out by facts; nevertheless, that those infesting the quadruped will not be foưnd in the bird, being almost always confined to animals of the same species, or of similar habits. For instance, the Docophorus icteroicles is to be found on nearly every species of duck. The Neimus obscurus infests several species of sandpipers, godwits, \&c. ; the Neimus rufus, the hawks and falcons; the Docophorus lari, all the gulls. In quadrupeds it is rather more doubtful, as they are frequently transferred by association; for instance, the Trichodectes scalcuris has been found upon both the ox and the ass, feeding in the same stall. The Hermatopinus piliferus, infesting dogs, have been found in swarms upon the ferret. The Pecticulus, besides being found on man, is also found on the Quadnumana, Rodentia, Carnivora, Pachydermata, and the Ruminantia. We include in this group acari; though from their having eight legs-parasites have only six-they are not generally admitted to belong to the same class of insect life."

Commencing our description with those infesting and annoying mankind, and seemingly sent on purpose to compel us to be more careful and cleanly in our person and habitation, - the most curious, as well as the most common, of the tribe, is the Hlea. In our observation of this insect we must at once be struck with its surprising agility and its great strength, enabling it to take such extraordinary leaps; 
what muscular vigour and elasticity of fibre must be possessed by it! In proportion to its bulk, the strength of the horse or the elephant is as nothing in comparison with it! The neck of the flea is long, the body

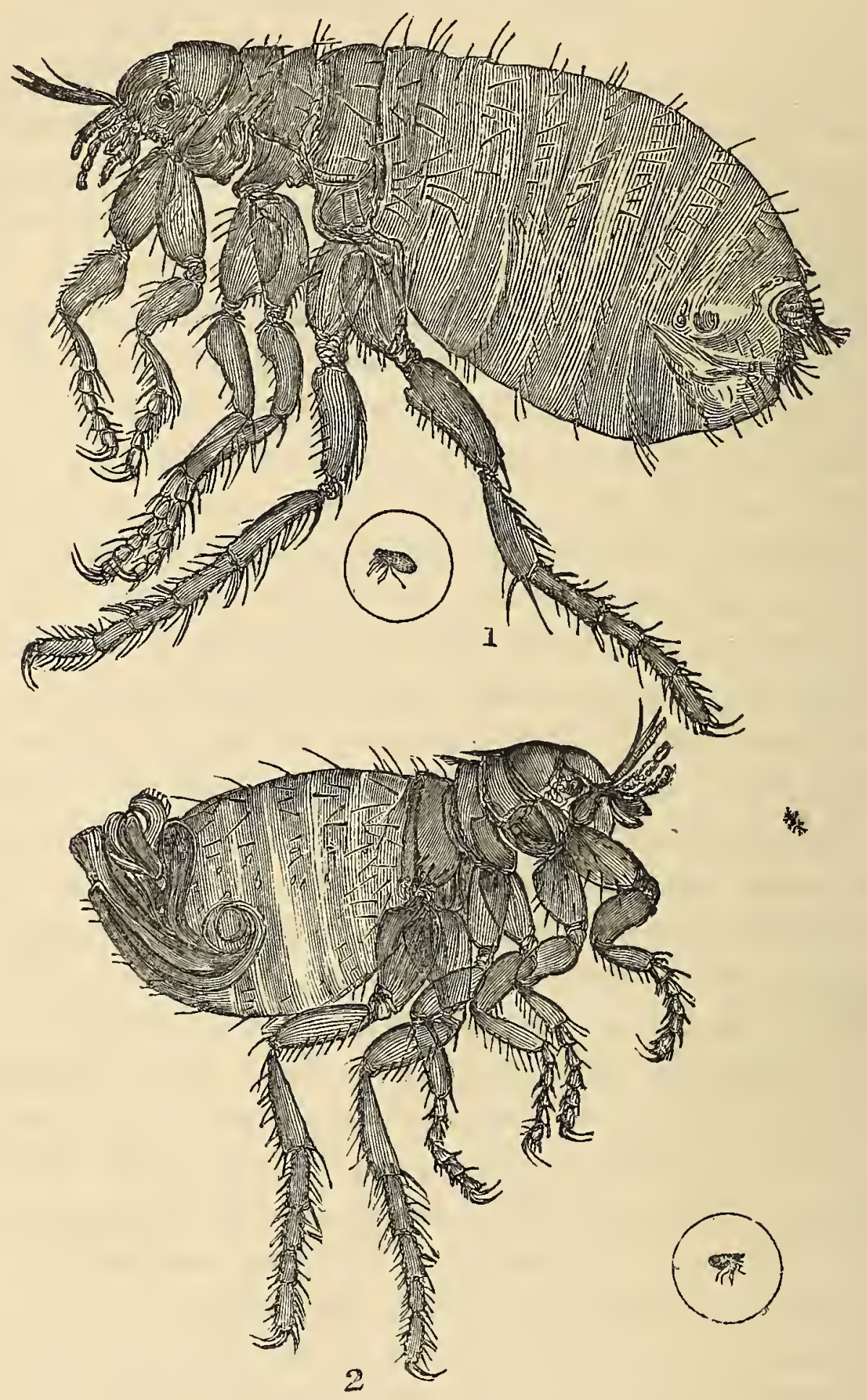

fig. 128.

1. Female Flea. 2. Male Flea. The small circles enclose the little animals of the natural size.

covered over with scales, the edges of which are set with short spikes or hairs; from its head project a pair of antennæ or horns, its proboscis, and a pair of sharp lancets, with which it penetrates the skin. On 
each side of the head a large sharp eye is placed. It has six manyjointed powerful legs, terminating in two sharp-hooked claws; they are so constructed that it can fold them up as it were, and when it leaps, it springs them out; so that its whole strength is excited at the same moment. The two lancets or piercers are enclosed in a sheath, which renders them difficult to be seen; after they have pierced the flesh, the proboscis is used to draw up the blood. The female flea, fig. 128, lays a great number of eggs, sticking them together with a glutinous matter; those of fleas infesting the dog or cat are made fast to the roots of the hairs; in four days' time the eggs are hatched, and a small white worm or grub is seen crawling about, and feeding most actively. No. 4, fig. 129, is a magnitied view of one; it is covered with short hairs, which prevent its being easily dislodged. After remaining in this state about nine or ten days, it assumes the pupa form, which it retains for four days; and in nine days more it becomes a perfect flea. The head of the flea found in the cat (No. 3, fig. 129) is seen to be very different in its form from that of the human flea (fig. 128). Its jaws are supplied with formidable hooklets, and from the first and second joints behind the head project short and strong spines; these are, no doubt, for the purpose of enabling it to maintain a firmer and better hold on the animal. The bed-bug is another intruder on the peace and comfort of mankind, provided as it is with piercing instruments of the most uncomfortable character; the pretty appearance of the eggs has been before alluded to, and represented in fig. 80, page 114 .

The Louse (Plate X. No. 1). Whenever wretchedness, disease, and hunger seize upon mankind, this horrid parasite seldom fails to appear in the train of such calamities, and to increase in proportion as neglect of personal cleanliness engenders loathsome disease. When examined under the microscope, our disgust of it is in no way diminished. In the head may be distinguished two large eyes, near to which are situated the antennæ; the fore part of the head is long, and somewhat tapering off to form a snout, which serves as a sheath to the proboscis and the instrument of torture with which it pierces the flesh and draws the blood. To the fore part of its body six legs are affixed, having each five joints, terminated by two unequal hooks; these, with other parts of the body, covered with short hairs. Around the outer edge of the body may be seen small circular dots, the breathing apertures, with which all this class of animals are provided, rendering them very tenacious of life, and difficult to kill. There is another louse, rather differing in its characteristics from this, formed about the body of the very poor and dirty, called the body or crab-louse. Leeuwenhoek carried his re- 
searches on the habits of these species further than most investigators, even allowing his zeal to overcome his disgust for such creatures as the louse. In describing its mode of taking food, \&c. he observes: "In

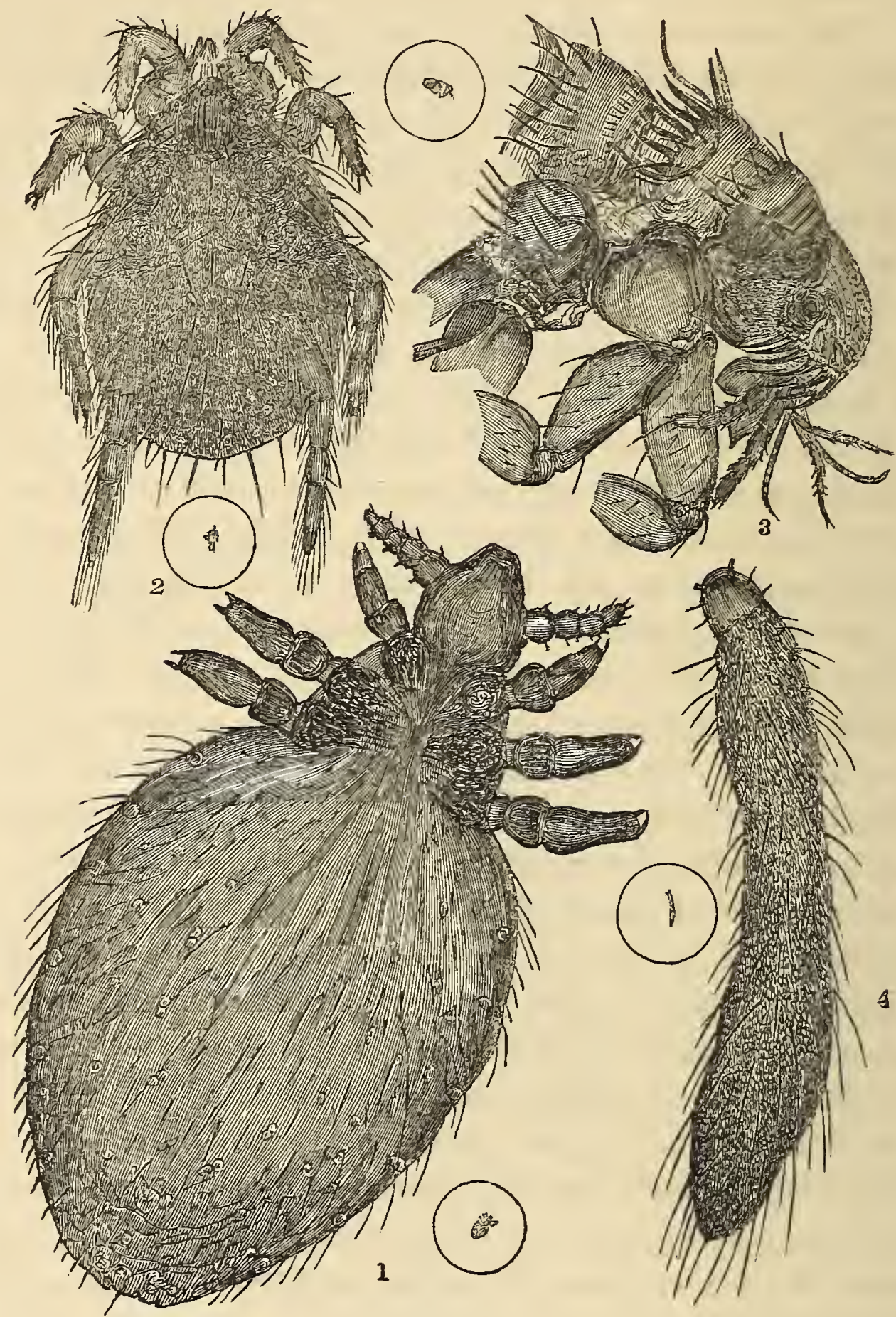

fig. 129.

1. Dog's Parasite. 2, Rat acarus. 3. Head of Cat Flea. 4. Larva or grub of Flea.

The natural size of the object is shown within the small circles near each.

my experiments, although I had at one time several on my hand drawing blood, yet I very rarely felt any pain from their punctures; which is not to be wondered at, when we consider the excessive slenderness of the piercer; for, upon comparing this with a hair taken from the back of my hand, I judged, from the most accurate computation I 
could form by the microscope, that the hair was seven hundred times the size of this incredibly slender piercer, which consequently by its punctures must excite little or no pain, unless it happens to touch a nerve. Hence I have been induced to think that the pain or uneasiness those persons suffer who are infested by these creatures, is not so much produced from the piercer as from a real sting, which the male louse carries in the hind part of his body, and uses as a weapon of defence." He found from experiments made to ascertain the increase of these vermin, that from two females he obtained, in eight weeks, the incredible number of 10,000 eggs.

The Cheese-mite (Plate X. No. 2) has a peculiar elongation of its snout, forming strong, cutting, dart-shaped mandibles. Some naturalists have made this characteristic the basis of a subdivision, grouping them into suctorial and masticatory acari. All indeed present a great diversity of form, size, and colour; and in the cheese-mite we observe the peculiar adaptation of form and apparatus necessary for cutting its way into or through the food on which it is destined to exist. Mites multiply very rapidly; they are hatched from eggs in about eight days, and if deprived of food will kill and eat each other very greedily. They infest almost the whole of our dried articles of food.

The Itch-insect (Plate X. No. 3, magnified 350 diameters). Dr. Bononio discovered the true character of the very troublesome disease known as the itch. Upon examining one of the pustules, or little bladders, from between the fingers, with the points of very fine needles, under the microscope, he perceived a small animal, very nimble in its motion, covered with short hairs, having a formidable head with a pair of strong mandibles or cutting-jaws, and eight legs, from the extremities of which are appended remarkable feet, each provided with $\mathbf{a}$ sucker, by means of which it no doubt sucks or draws its way beneath the skin, having first cut out a small section with its mandibles; here the insects form a nest, lay eggs, and multiply rapidly, being most difficult to dislodge.

To find the itch-insect, the operator must examine carefully the parts surrounding each pustule, and he will see a red line or spot communicating with it; this part, and not the pustule, must be probed with a fine-pointed instrument; the operator must not be disappointed by repeated failures. As it is often very difficult to detect the haunts of the insect, an eye-magnifier will greatly assist.

Dr. Bourguignon has of late bestowed much time in studying the habits of this troublesome parasite. To arrive at this knowledge, the 
Doctor had recourse to a peculiar kind of movable microscope, which enabled him to observe it under the skin of the diseased person. The microscope is composed of the frame of an ordinary instrument, the optical and essential parts of which have been raised from the socket that supported it, and articulated to a movable knee at the extremity of a lever. The rays of light from a lamp or candle are brought to a brilliant focus by means of the condensing or bull's-eye lens; which focus is directed upon the chosen point of observation.

He then saw that the feet were armed with suckers, which enabled it to fasten itself in the furrows under the skin, aided by the small bristles; and being likewise covered by the same in various parts of the body, it more firmly fixes itself there; and with its terrible mandibles it accomplishes its destructive mission. It has no eyes; but in the moment of danger it quickly draws in its head and feet, somewhat resembling the tortoise; its march is precisely that of the tortoise. It usually lays sixteen eggs, which are carefully deposited in furrows under the skin, and ranged in pairs; these are hatched in about ten days.

No. 4; Plate X., the Entozoa folliculorum, is another very remarkable insect found beneath the skin of man: it may be obtained, says Mr. Quekett, "from some spot where the sebaceous follicles, or fat glands, are very abundant; such as the forehead, the side of the nose, and the angles between the nose and lip; if the part where a little black spot or a pustule is seen be squeezed rather hard, the oily matter accumulated will be forced out in the globular form and various stages of growth, as represented in the drawing; if this be laid on a glassslide, and a small quantity of oil be added to it, so as to separate the harder portions, the little insect in all probability will be floated out; after the addition of more oil, it may be taken away from the oily matter by means of a fine-pointed sable pencil-brush, and transferred to a clean slide, where it may be covered over with thin glass, and mounted in the usual way. The Entozoa are more abundant in the skins of some persons; but where black spots are seen about the face, they may generally be found.

The term Entozoa is applied to this class of insects, found within the body; and that of Epizoa to those found on the exterior of the body.

Another troublesome anirnal of this class infests the extremities, called the Guinea-worm; it is represented in fig. 130. The Guineaworm (Filaria medinensis) is an extremely common parasite of the human frame in some parts of the world; it is chiefly to be met with in the tropics; the proper habitation of the worm is the torrid zone, although it is not confined to that zone; and from its great frequency 
in Guinea it derives its name. The entrance of the worm into the body is apparently unattended with any observable pain ; and the per-

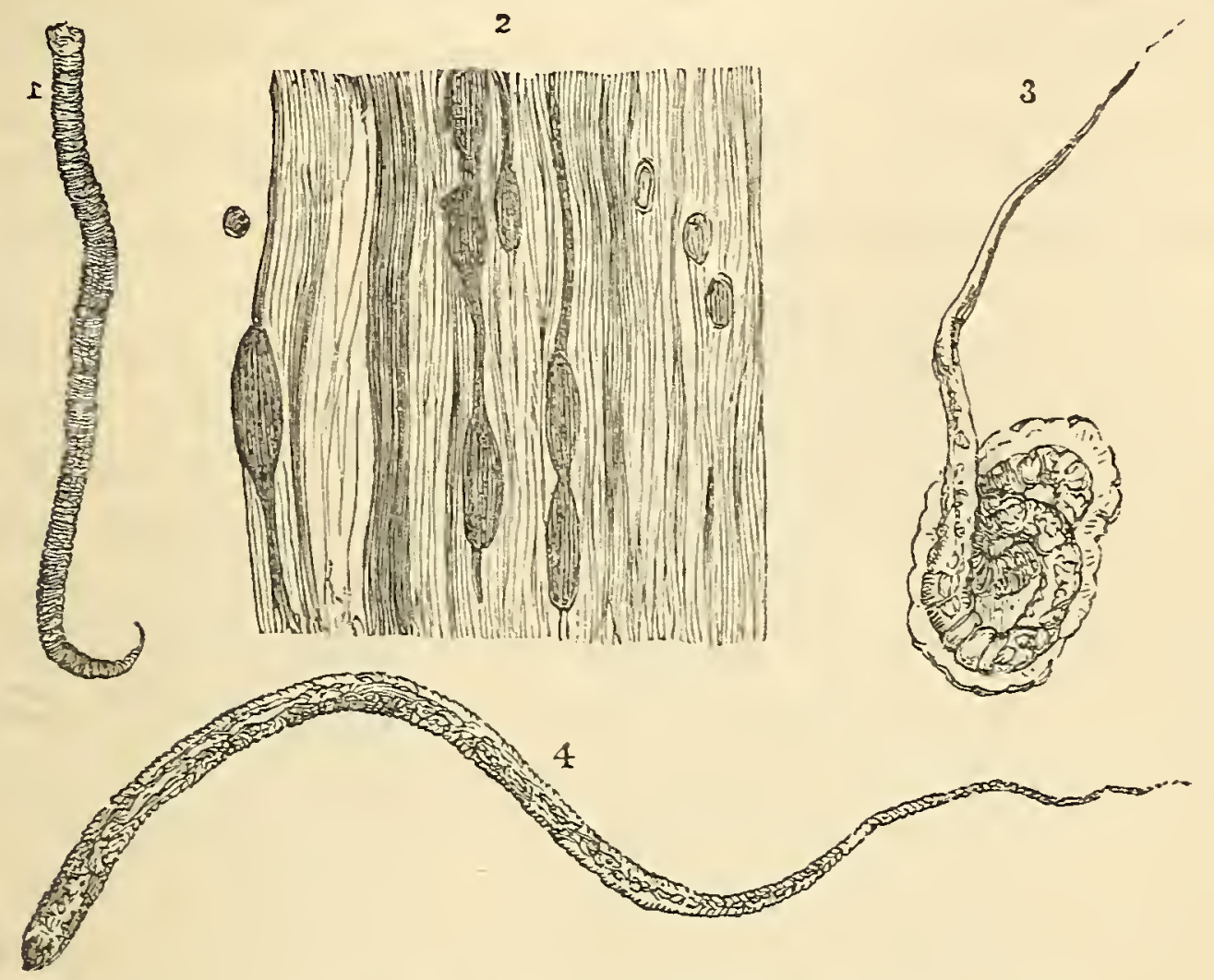

fig. 130. The Guinea Worm, taken from the leg of a Negro.

1. Represents the form of the worm when first taken from one of the sacs seen at fig. 2. 3. The young worm rolled up. 4. The worm extended.

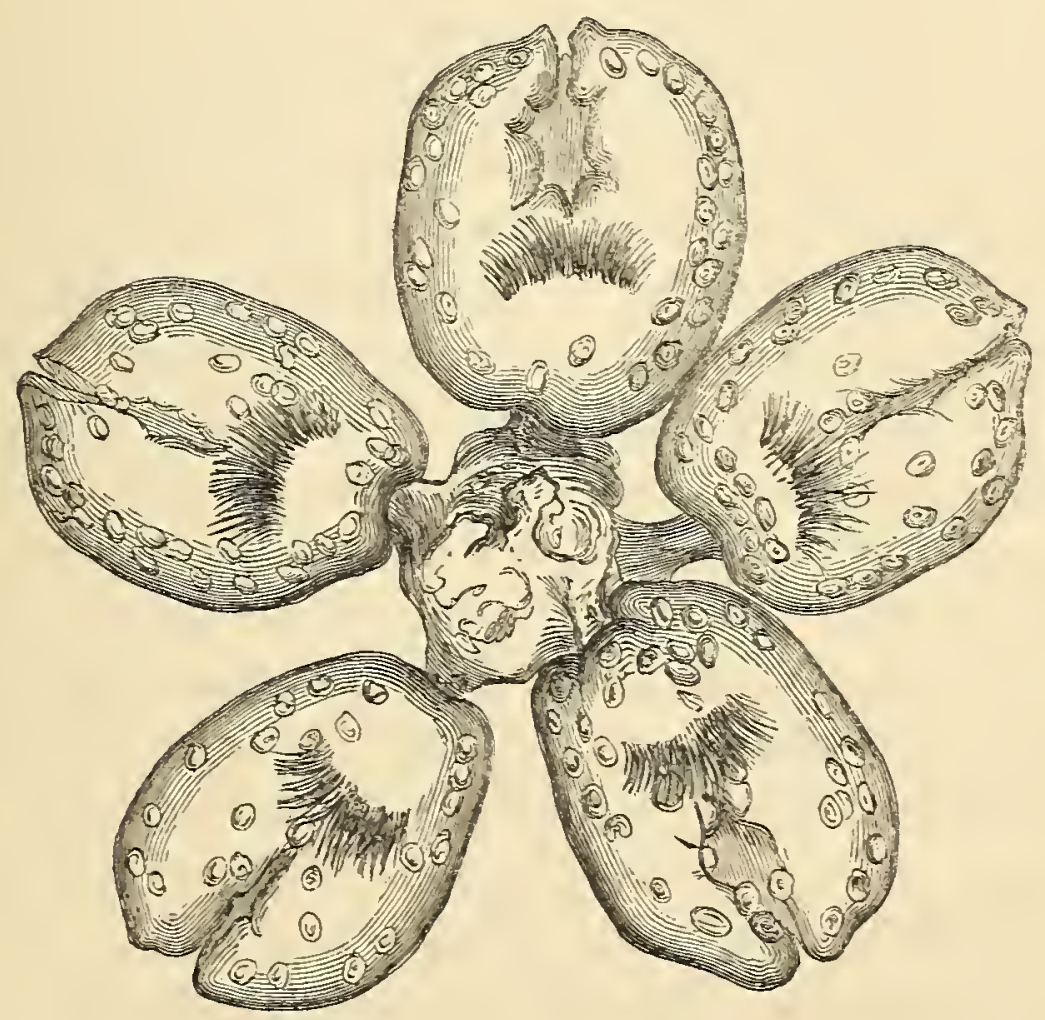

fig. 131. Echinococci found in the human liver, magnified 250 diameters. 
son affected is unconscious of its presence until the period when it is ready to make its exit. In the first instance it lies dormant under the skin for several months, as shown in No. 2, enclosed in an oblong sac. In this embryo or egg-like state it produces little or no inconvenience, It here commences a rapid growth, estimated by Mr. Busk at about an inch in a week, until it often reaches the enormous length of six feet. When the full growth is attained, the worm perforates the skin; and in so doing creates excessive pain, inflammation, and suppuration in the part, and presents his head at the surface through a small pustule apparently containing matter only. The whole worm, when mature and fresh, is of a transparent milky-white colour, and presents an indistinct brownish, wavy, longitudinal streak; it is round, and about one-twelfth of an inch in diameter; it is tough and elastic to a remarkable degree, and may therefore with care be withdrawn, by a cylindrical or screw motion, entire from its hiding-place. The muscles are frequently attacked by one of this class of parasites; it then constitutes a well-known disease among pork-dealers, and is called by them mecusly-pork.

The Echinococcus is one of the class Epizoa, a minute animalcule found in hydatid cysts in the interior of the body of man and other animals. Fig. 131 represents a bunch taken from the liver of a boy who died in Charing-cross Hospital from a rupture of the liver, occasioned by the wheel of an omnibus passing over him. The simple cysts containing these animalculæ are always situated in cavities in the interior. These cavities may be situated in any part of the tissues or organs of the body; they are more frequently found in the solid viscera, and especially in diseased livers or lungs of the lower animals.

Mr. Busk, who has examined several of these cysts, says: "When a large hydatid cyst,-- for instance, in the liver of the sheep,- - very shortly after the death of the animal, is carefully opened by a very small puncture, so as to prevent at first the too-rapid exit of the fluid, and consequent collapse of the sac, its internal surface will be found covered with minute granulations resembling grains of sand. These bodies are not equally distributed over the cyst, but are more thickly situated in some parts than in others. They are detached with the greatest facility and on the slightest motion of the cyst, and are rarely found adherent after a few days' delay. When detached, they subside rapidly in the fluid, and consequently will then be usually found collected in the lowest part of the cyst, and frequently entangled in fragments of its innermost thin membrane. When some of these granulations are placed between glass under the microscope, and viewed with 
a power of 250 diameters, each will be found composed of individual Echinococci, more or less closely packed together, and enveloped almost entirely in a delicate thin membrane. Upon further pressure being employed, it will be seen, after the rupture of the enveloping

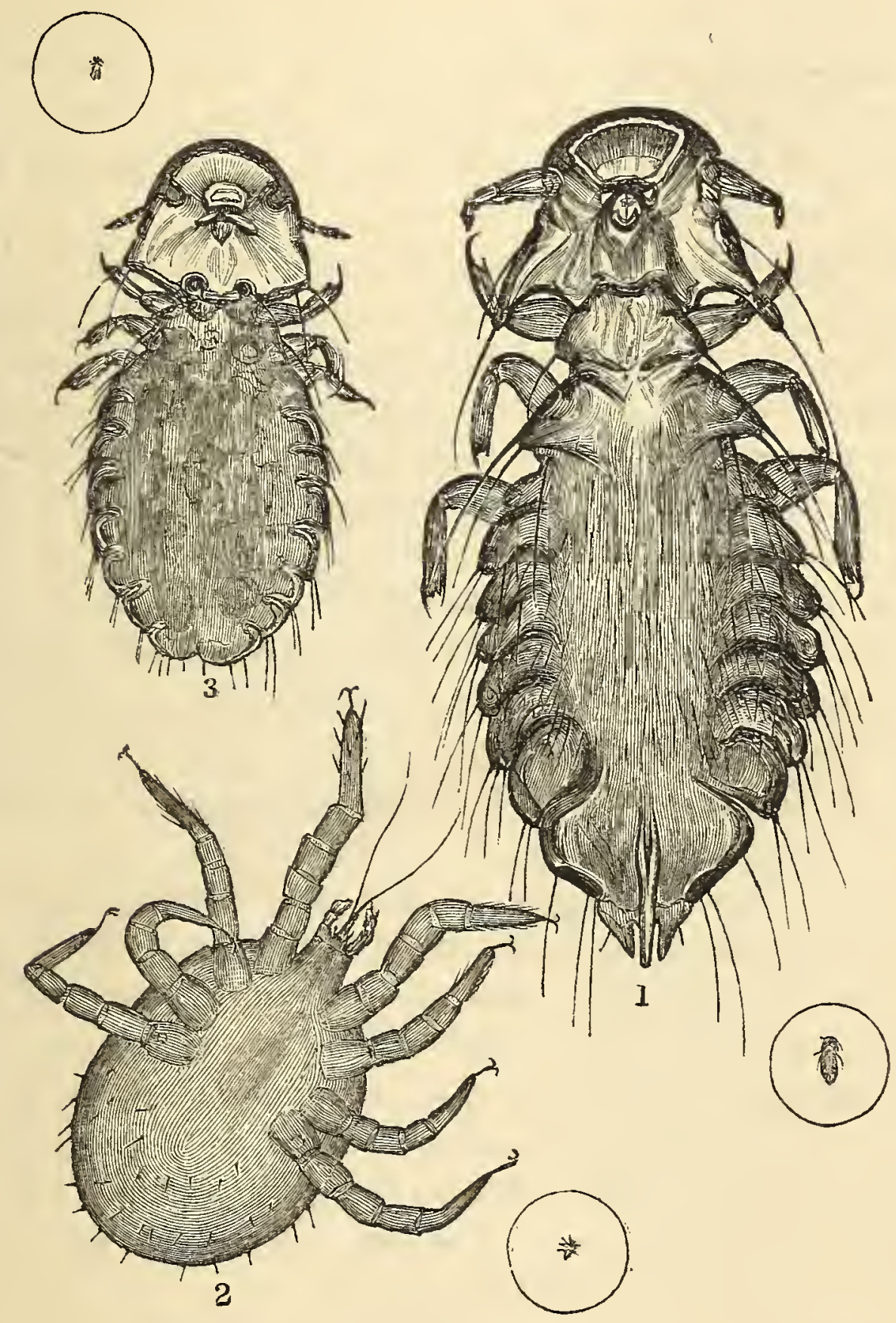

fig. 132 .

1. Parasite of Turkey. 2. Acarus of common Fowl: its under surface. 3. Parasite of Pheasant. The small circles enclose each object of the natural size.

membrane, that the Echinococci composing the granulations are all attached to a common central mass by short pedicles; which, as well as the central mass, appear to be composed of a substance more coarsely granular by far than that of which the laminæ of the cyst are formed. 
This granular matter is prolonged beyond the mass of Echinococci into a short pedicle, common to the whole, and by which the granulation is attached to the interior of the hydatid cyst, as represented in fig. 131.

"In specimens preserved in spirits, Echinococci of all imaginable forms and appearances are to be met with,-_differences owing to decomposition or to mechanical injury; and in many cases no trace of them can be found except the hooklets or spines, which, like the fossil remains of animals in geology, remain as certain indications of their source, and not unfrequently afford the only proof we can obtain of the true nature of the hydatid.

"In almost all the aggregations of Echinococci, besides the perfect specimens, there are to be seen one or more of a different appearance and of various shapes-round, clavate, or oval-like the others, attached to the common mass by a pedicle: they are composed of granular matter, denser apparently, and of a deeper colour, than that of which the bodies of the perfect animals are composed, and increasing in density towards the free extremity.

"Some of this class of animals have been found in the chambers of the eye and brain. It is indeed difficult to conceive how these living things find their way into such situations, and apparently cannot live out of them. There is no doubt that the origin of the Epizoa is favoured by all causes which tend to disturb the equality between the secerning and absorbent systems."

Dr. J. Leidy has established the fact, that cryptogamic vegetables exist, as a normal condition, in the interior of several species of healthy animals. He describes three new genera of entophytes-Enterobrus, Cladophytus, and Arthranitus-all being confervoid or mycodermatoid. All are found growing from the mucous membrane of the small intestines, and commencement of the large intestines of Julus marginatus (Say); and from Entozoa inhabiting these cavities in the small animal. They were uniformly found in 116 examinations of animals of this species, made immediately after death.

In one instance, an acarus three lines long had no less than twentyeight individuals of Enterobrus, averaging a line in length, besides a quantity of two other genera, growing upon it; and yet moved about in so lively a manner, that it did not appear the least incommoded by its load of vegetation.

The important point in these observations is, that they show that cryptogamic vegetables may exist in the internal organs, and upon Entozoa inhabiting these organs, without disturbing the health, and even as an ordinary and normal condition. 
We have cryptogamia producing diseases of the skin, in a variety of very troublesome forms, many of them difficult of cure: but whether in these instances they are the cause or effect of the morbid state, is not decided. Dr. Leidy regards the microscopic forms known as Vibrio

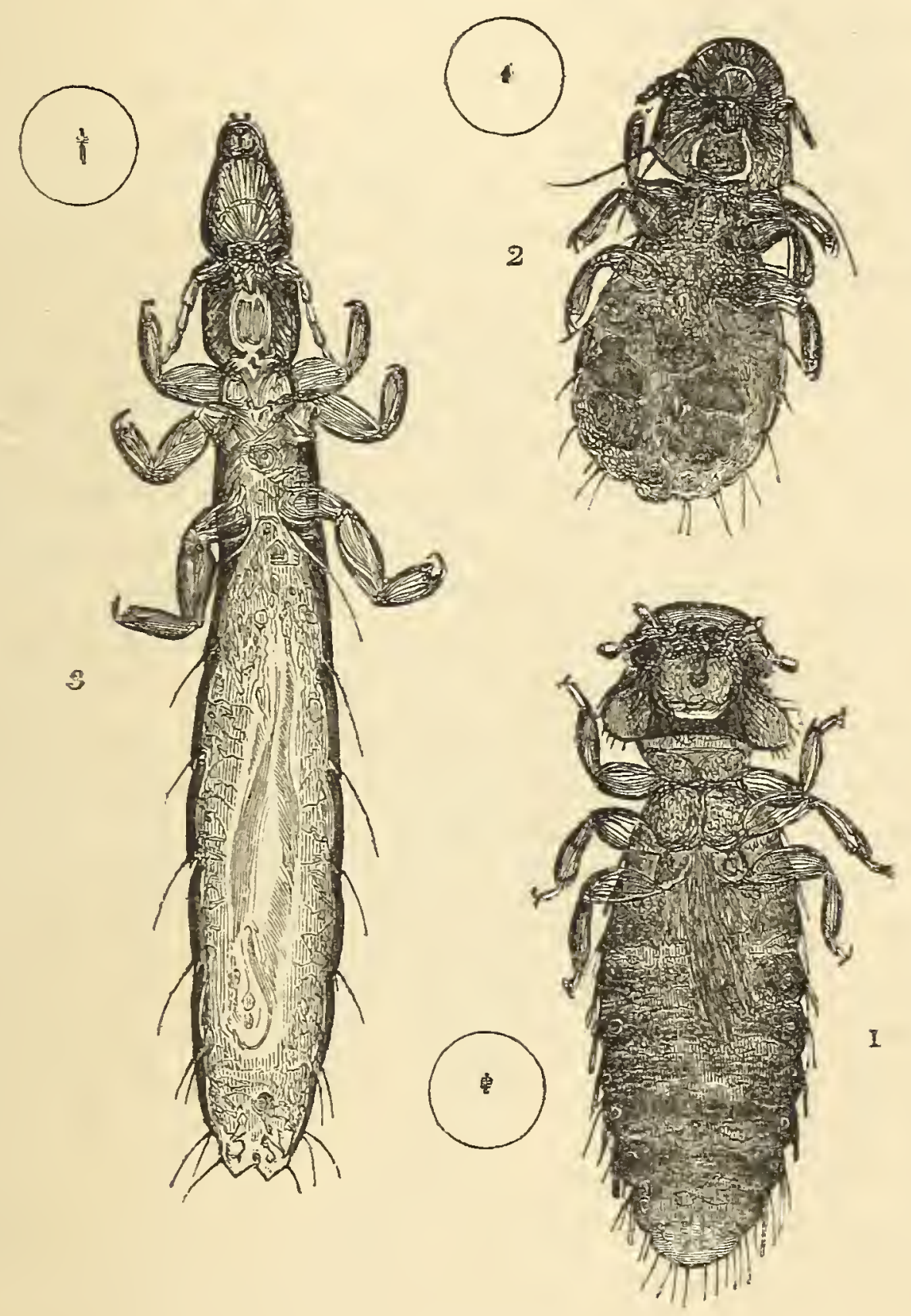

fig. 133.

1. Parasite of Eagle. 2. Parasite of Vulture. 3. Parasite of Pigeon. The small circles enclose the objects of the natural size.

as vegetables; and states that he has made the discovery of several new species : he likewise notices the existence of Gregarina in the ventriculus of Julus marginatus. Gregarina has recently attracted much attention, from its being supposed to be an animal consisting only of 
two cells, and said by Siebold to be destitute of an alimentary canal. Dr. Leidy describes a papilla surmounting the superior cell, with traces of an external communication from the cavities of the cells. He regards it as the larva of the Entozoon.

Dr. Burnett of New York devoted much attention to these curious creatures : we therefore append his interesting account of "Parasites and their relations to other animals," as bearing, first, on the distinct creation of types of animals; and secondly, on the local creation of these types wherever they are found. "In the general fauna of the earth, the fact that totally distinct genera and species exist, exposed to the same external influences, is a strong argument against their being the results of modifications of a single type, and in favour of their having been created as we now see them. The same is true as regards these parasites; we have different species, and even different genera, upon a single animal, all exposed to the same external changes.

"If genera and species are mere modifications of a primitive family type, we should expect to find a uniformity in the special character of parasites of all the species of the genera of that family. For instance, if the Sciuridea are but modifications of a primitive type (the squirrel), we should expect to find certain parasites common to all, with a uniform specific character, with widely-separated genera; which, as far as his experience goes, is not true; for though in many cases certain species of parasites are common to the whole family, yet there is an evident tendency for each species of the higher animals to have its own peculiar species of parasites. Though we can easily imagine that the same species may be found in animals and birds of the same family, with similar habits and associating together, we cannot understand that the same species of parasites should be found in widely-different families, of entirely dissimilar habits; yet such is the fact: and it is not reconcilable with the hypothesis of a successive production of types by a series of changes of their structure; on the contrary, it would go to show that the existing specific types were as such created.

"As to the local creation of genera and species, we know that the existence of the world's animals has not that commonness which might be supposed; they have relations of a local nature, connected with a remarkable diversity of forms. At any rate, this fact is certain, that each particular region has a marked tendency to have its own peculiar fauna. Climate has undoubtedly a great influence on the character of a fauna; we see in the same zone; separated by impassable barriers, a tendency to similar animal productions; though there is, at the same time, sufficient diversity to exclude the idea of a common origin: dif- 
ferent countries have analogous, not identical species. Geological data, the history of the surface of the earth, and our fast-increasing knowledge of the intimate relations of animals to the circumstances in which they live, all lead to the conclusion of their local creation in their pre-

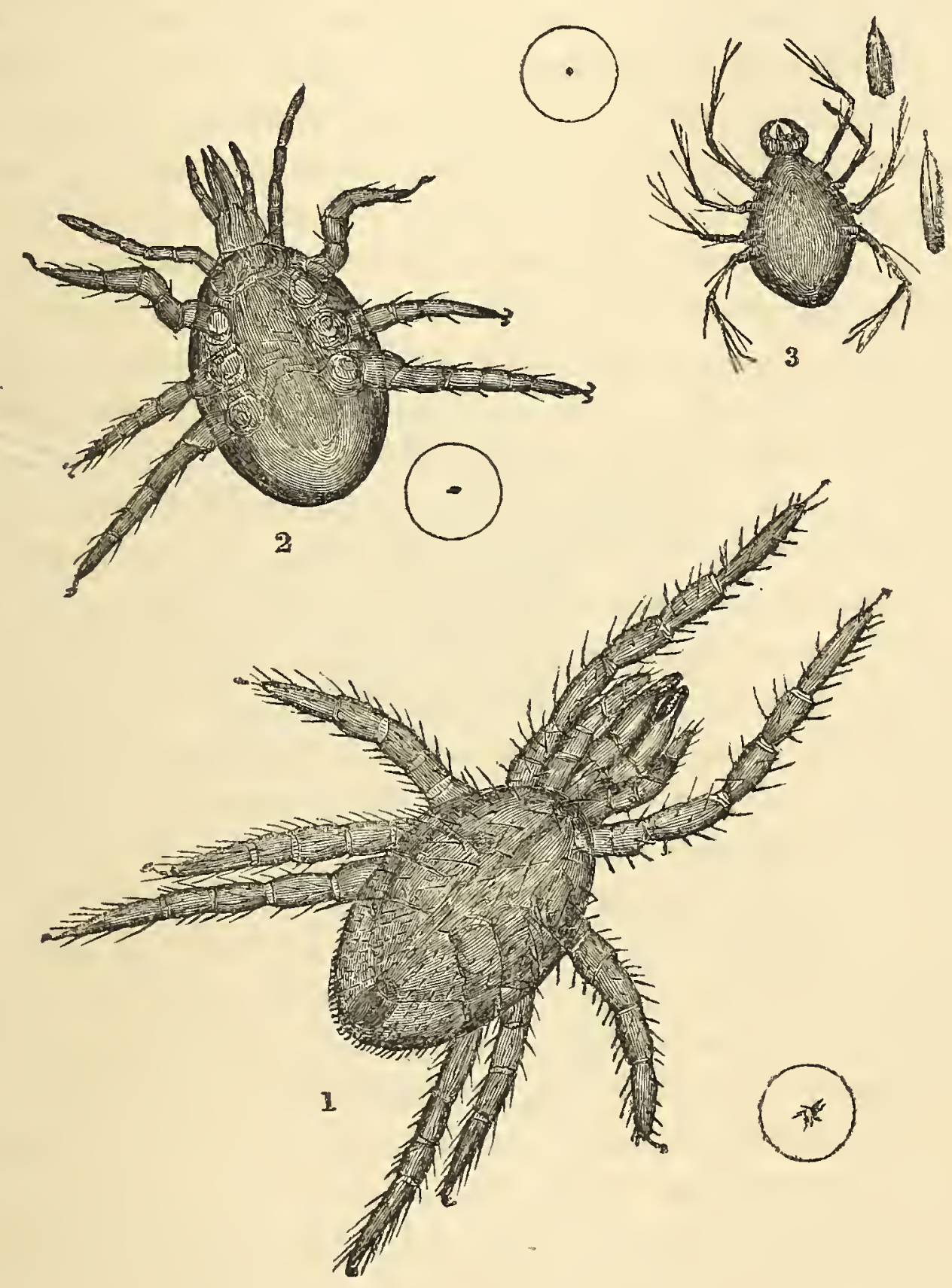

fig. 134.

1. Acarus of Beetle. 2. Acarus of Fly. 3. Acarus of Cloths-Moth. The small circles enclose the objects of the natural size.

sent, habitats. The relations of parasites afford considerable proofs against the hypothesis, that the differences of terrestrial condition have caused the differences in animals. We could not suppose that the pediculi living on European birds would be different from those of the analogous species of American birds; if they arose from a common 
stock, their parasites ought to agree as to species. This is not the fact: not only do the parasites of our animals, compared with analogous species in Europe, present differences greater than those of the animals on which they live (their species being more distinct than those of the higher animals); but even our species of birds or mammals having no representative on the other continent, have their own parasites as distinct as themselves. Those animals which, by their own power of locomotion or human means, are common to both continents, have parasites identical in character, as far as observation goes.

"The pediculi of our (American) cow, horse, dog, \&c. do not differ from those of the same animals in Europe; the same is true in some birds. The legitimate inference from these facts is, that the analogous species of animals of the different continents were created as such, and therefore have their proper parasites, and did not emanate from parent stocks. Man himself is, fortunately, annoyed but by few parasites; his ubiquity renders the conditions of his existence different from those of other animals. Many of the higher mammals man has gathered around him in domestic life; and as they go wherever he goes, the facts relating to them can have no great weight.. The pediculi of the Quadrumana, or monkeys, seem to be different from those of man: in fact, the species found on man are not found on monkeys, except when their presence may be accounted for by accident; and those of the monkeys are found only on them."

Dr. Burnett has also established to his satisfaction the following facts : "1. That although there are single species of parasites peculiar to particular animals, there are others which are found on different species of the same genus; as is the case in the parasites living on birds of the genus Larus (gulls), and the diurnal birds of prey. 2. The parasites of the human body confine themselves strictly to particular regions; when they are found elsewhere, it is the result of accident. Thus, the Pediculi capitis live in the head; $P$. vestimenti upon the surface of the body; the $P$. tabescentium on the bodies of those dying of marasmus; and the $P$. inguinalis about the groins, armpits, mouth, and eyes." From an examination of the structure of these animals, Dr. Burnett is of opinion that they should be placed in an order by themselves, closely allied to the insecta; the mandibulate parasites occupying the highest, and the haustellate the lowest position in the order: thus confirming the observations made by Mr. Denny, before referred to.

There is a remarkable species of acarus described by Dr. Robins, found spinning a white silky web on the base of the sparrow's thigh, or 
on the fore-part of its body: on raising this delicate web, you perceive that it is filled with minute eggs, from which the young issue, being in due time hatched by the warmith of the body it is destined to annoy. Another, curiously enough, selects the pulmonic orifice of the snail: when the animal dilates this orifice for the purpose of allowing the air to penetrate its respiratory cavity, the female acarus slips through it, lays her eggs in the folds of the mucous membrane, where they are gradually developed; issuing forth from the egg, they select some portion of the snail's body upon which to feed and perfect their growth.

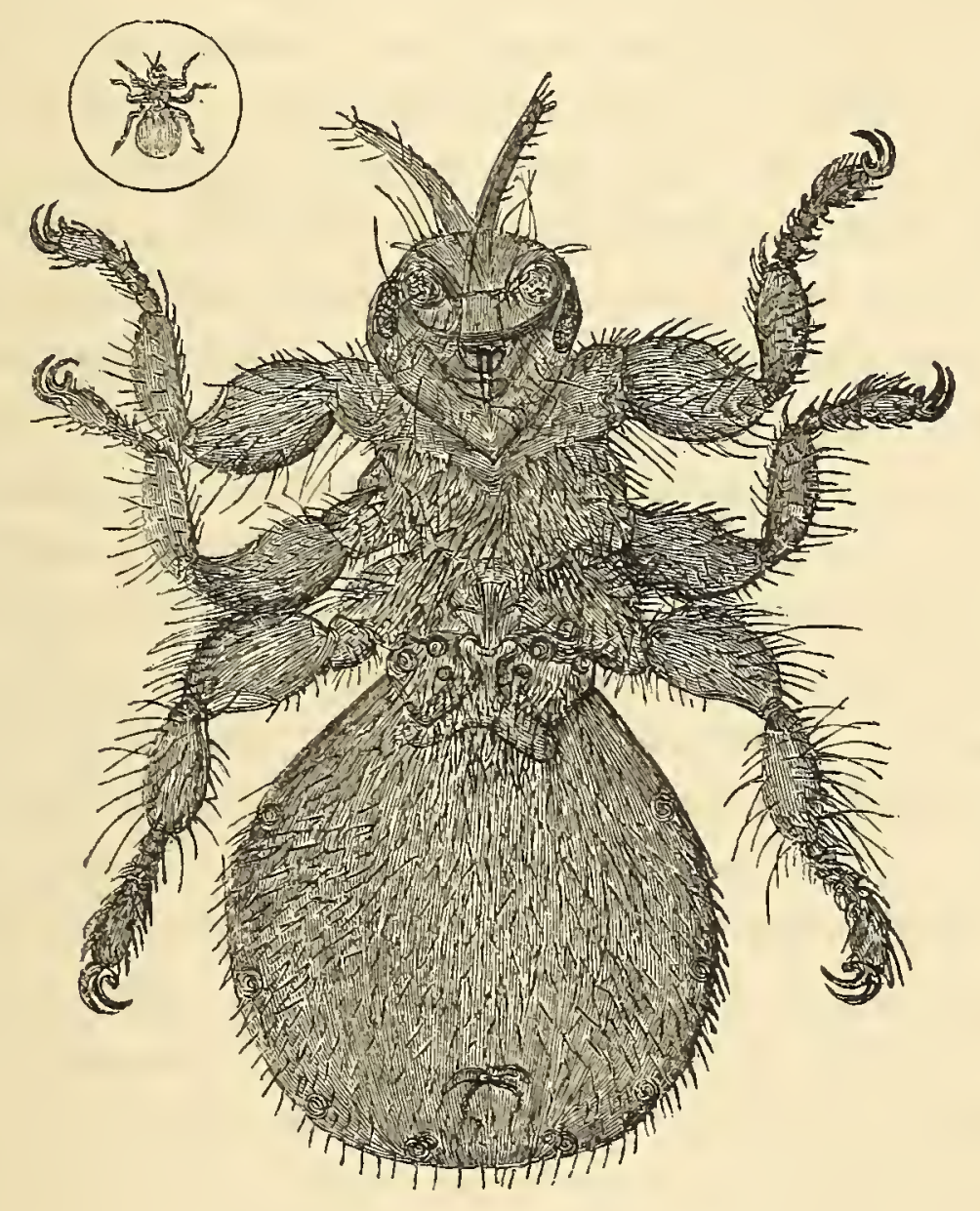

fig. 135 .

The Sheep-tick, a parasite. The small circle shows one of life-size.

The water-snail, Lymnerus, is infested with a parasite of the family Cercaricedce, which attaches itself by a series of hooklets and bristles to such parts of the body and mantle as give a secure lodgment to it; they look like little tufts of thread hanging from the sides of the animal, without its having the power to dislodge them.*

* A very interesting account of the parasite tribes is given in Rhedi's "Treatise de Generatione Insector'um," and in H. Denny's "Monographia Anoplurorum Britanniæ," Bohn, London, 1842. 
We have before referred to Swammerdam's careful dissections under the microscope. In no department of nature did he bestow more care than in his many examinations of insects. He first killed them by immersion in spirits of wine and water, or in spirits of turpentine; preserving them for some time in the same fluid, to give firmness, and render his dissections more easy. When he had divided the insect transversely with his fine scissors, he particularly noted the relative position of the various parts, and then proceeded to remove the viscera very cautiously with fine-pointed instruments, carefully washing away all the fat and other matters with fine camels'-hair pencils; or, by putting the whole into water, and then shaking them gently, he separated the air-vessels or trachæa in a perfect state from the other parts. At other times he made use of a very fine syringe to inject water into and thoroughly cleanse them; after which they were distended by blowing in air, and hung up to dry. $\mathrm{He}$ is said to have often succeeded by immersing insects in balsam. Again, that he frequently made punctures in some insects with a fine needle, and after squeezing out all their moisture through the holes made in this manner, he filled them with air by means of very slender glass tubes, then dried them in the shade, and afterwards anointed them with oil of spike in which a little resin had been dissolved: those prepared in this way retained their forms and kept well for years. Swammerdam discovered that the fat of all insects was perfectly soluble in spirit of turpentine; after steeping them in it, he washed them well in water, and was thus enabled to show the viscera plainly. He frequently spent whole days in thus cleansing a single caterpillar of its fat, in order to discover the construction of its heart. His singular mode of stripping off the skin of the caterpillar, just as it was on the point of spinning its cone, was effected by taking hold of its thread and letting it drop into scaldingwater, and then suddenly withdrawing it, the skin easily peels off; after this, it was put into distilled vinegar and spirit of wine mixed in equal proportions: this gave a firmness to the parts; and the exuvia or skin readily separated, when the pupa was seen to be enclosed by it, with the butterfly again in the pupa.

Parasites may be quickly killed by immersing them in spirits of wine or spirits of turpentine; after a time take them out, dry them; and if transparent, they may be at once mounted in glycerine or Goadby's solution. If opaque, they had better be mounted in Canada balsam. (Also see general directions for mounting objects.)

Insects furnish so many objects for examination, that we can only enumerate a few of the most interesting of them: 
ANTENNE.

Cockchafer.

Cockroach.

Mole Cricket.

Cyrambyx.

Aphis.

Atropus (Death-watch).

Bee (Leaf-cutting).

Beetle (Asparagus).

,, (Diamond), \&c.

EGGS.

Various Moths.

Flea.

Fly.

Bed-bug.

Water-beetle, \&c.

ELATRA.

Beetle.

Cicada.

Dermestes.

Flame Beetle.

Dytiscus.

Rose Beetle.

Golden Beetle.

Diamond Beetle, \&c.

(All these are rery beautiful when viewed as opaque objects.)

The eyes and feet of insects form a diversity of objects.
HATRS.

Indian Bee.

Stag Beetle.

Wing of Fly.

Moths.

Caterpillars, \&c.

LARTA.

Ephemera.

Gnat.

Hydrophilus.

Nepa.

Tortoise Beetle.

Boat Fly, \&c.

Ant.

Spider.

Lady-bird (Coccinella).

Lacquey Moth, \&c.

Gadfly.

FROBOSCIS.

Harvest Tick.

Hornet.

Hydrometra, \&c.

\section{SCALES}

From the rarious Moths and Butterflies, skins of Spiders, internal organs, tongues, tracheæ, wings, \&ce.

\section{INSECT CHANGES.}

The metamorphoses of the insect race offer some of the most curious and wonderful of nature's phenomena for contemplation. "We see," says an old author, "some of these creatures crawl for a time as helpless worms upon the earth, like ourselves; they then retire into a covering, which answers the end of a coffin or a sepulchre, wherein they are invisibly transformed, and come forth in glorious array, with wings and painted plumes, more like the inhabitants of the heavens than such worms as they were in their former state. This transformation is so striking and pleasant an emblem of the present, intermediate, and glorified state of man, that people of the most remote antiquity, when they buried their dead, embalmed and enclosed them in an artificial covering, so figured and painted as to resemble the caterpillar in the intermediate state; and as Joseph was the first we read of that 
was embalmed in Egypt, where this custom prevailed, it was probably of Hebrew original."

Faint and imperfect symbol though it be, yet it may perchance offer a glimpse of the metamorphosis awaiting our own frail bodies. Between the highest and lowest degree of corporeal and spiritual perfection, that there are many intermediate degrees, the result of which is one universal chain of being, no one can for a moment gainsay.

"The great class of insects which furnishes four-fifths of the existing species of the animal kingdom, has two chief divisions. In the one, the Ametabola, we have an imperfect, in the other, the Metabola, a perfect metamorphosis: that is, in the former there is no quiescent pupa state, and the metamorphosis is accompanied by no striking change of form; in the latter there is an inactive pupa that takes no nourishment, and so great a change of form, that only by watching the progress of the metamorphosis can we recognise the pupa and the image as being the same animal. The Metabola correspond, as it were, to the flowering plants; the Ametabola to the Cryptogamia. It is well worthy of remark, that among plants the Cryptogamia, and among insects the Ametabolous, first appeared on our earth. The most ancient forests, composed of tree-ferns, club-mosses, and equiseta, were inhabited by Locustce and Blattce, the first insects. There have not yet been found," says Professor Heer, in his History of Insects, "in the carboniferous and tribassic rocks any traces: of insects that can be with certainty referred to any of the other insect orders."

And with regard to the scarcity of insects in the earlier periods of the earth's history, he says: "Nor need we wonder, if we consider that at present also our Lycopodia and Equiseta harbour no insects, and the Filices very few. The hosts of insects that now live on the flowers and their honey, on the fruits and seeds, could not at that time have been in existence, the vegetable world being then destitute of flowers and fruits; inasmuch as in the tertiary period the land was almost entirely occupied with woody plants and forests, and offered but few herbaceous flawering plants from which the butterflies and bees could derive their nourishment."

As heat is the principal agent in giving impulse and vigour to organic life, it will be found that insects undergo as great a change under the increasing temperature of the earth and atmosphere, on approaching: the equator, as is well known to take place in vegetables and the larger animals. Their species are continually varying, even under the same parallel of latitude; so that countries similar to each other in soil, temperature, and all other circumstances which might be supposed to 
have an influence on animals, present the most striking differences in their insect productions. One thing has been especially pointed out by Latreille, that the countries most fruitful in insects are those in which vegetation is richest and most speedily renewed.

The importance of insects in a commercial point of view is scarcely ever glanced at. In Great Britain alone not less a sum than 50,000l. is paid annually for the dried carcasses of the tiny insect, the Cochineal; an insect found on the Opuntic, a species of cactus, with large, flat, roundish, leaf-like stalks, which produce the flowers and fruit, without the tree bearing any leaves properly so called. These flat, leaflike stalks are extremely succulent; and on them appears a white woolly substance, resembling what is commonly known as blight on appletrees: this woolly substance is the covering of the female Cochineal, the insect used for the dye. When fully grown, these insects are collected in

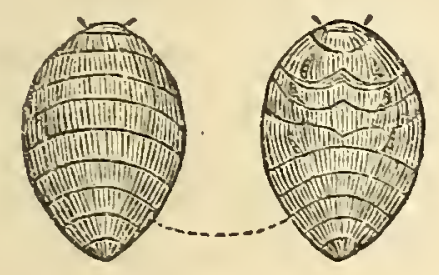

The Female of the Cochineal Insect (Coccus cacti).

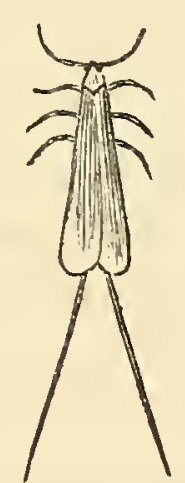

The Male of the Cochineal Insect.

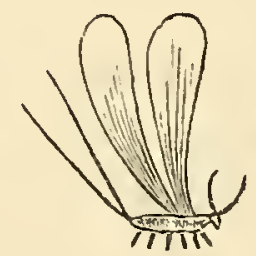

fig. 136 .

Mexico, and other countries where the plant grows in great abundance, by women, who brush them off with the tail of a squirrel or a deer. They are then killed by dipping them in boiling water, or exposure to heat in ovens or the sun, and are then ready for sale. It is the female insect only from which the dye is taken; the male insect has wings, as seen in fig. 136 .

Another Indian insect, the Coccus lacca, furnishes the well-known substance lac, a substance of much utility in the arts, as entering into the composition of varnish, sealing-wax, \&c.: it is a glutinous and semi-transparent exudation which envelopes the whole body of the insect, after it has fixed itself to the branch of a tree; and in this it resembles the frothy matter, before alluded to, with which some of the aphides cover themselves. Of the secretions peculiar to insects, silk may well be regarded as the most valuable, since it has become nearly as essential to our own purposes as it is to the economy of the pro- 
ducers of it. The vessels which secrete it consist of two tubes; these unite at the extremity, and open into a small perforated filiform organ, placed between the palpi on the under lip; this is termed the spinnaret, and the size of its aperture determines the thickness of the thread. The fluid, before it comes in contact with the air, is viscous and transparent in the young larva, but thick and opaque in the mature ones. It is found, by chemical analysis, to be chiefly composed of a gummy matter, a small portion of another substance resembling wax, and a little colouring matter. It may be placed in boiling water without undergoing any change; the strongest acids are required to dissolve it; and it has never yet been imitated artificially. More than 1,500,000 of human beings derive their sole support from the culture and manufacture of silk; and upwards of $200,000 l$. may be said to be annually circulated by the silk-worm. Then we have large sums of money changing hands from the labours of the useful little bee; tons weight of honey and wax are yearly consumed; England pays more than 50,000l. for foreign honey and wax, besides her own produce. A great variety of scents, which from their agreeable odour are much used in perfumery, are also the produce of insects. The Spanish Fly is absolutely indispensable in the treatment of certain forms of disease; and that invaluable agent, Formic-acid, or Chloroform, was first discovered in, and produced from, the Formic-ant, from which it derives its name. Then there are the Gall-nuts produced by a small fly, so useful in dyeing, ink-making, \&c. Gallic-acid is also obtained from the grain-weevil, Calandra granaria; and what is known as Bombic-acid from the silk-worm.

"Much more extensive and important than any of the foregoing, but, as less palpable, even more disregarded, are the general uses of insect existence. Disease engendered of corruption, in substances animal and vegetable, would defy all the precautions of man, unless these were aided by scavenger insects, those myriads of flies and carrion-beetles, whose perpetual labours, even in our tempered climate-but infinitely more so in warmer regions-are essentially important to cleanliness and health.

"A use of this nature, and one performed perhaps to an extent we little think of, is the purification of standing waters by the innumerable insects which usually inhabit them. We have witnessed ample proof of the efficacy in this respect of gnat larvæ, when keeping them to observe their transformations. Water, swarming with these 'lives of buoyancy,' has been perfectly sweet at the end of ten days; while that from the same pond, containing only vegetable matter, has become speedily offensive. 
"We have already pointed out the utility of insects in affording ever-new subjects of interesting inquiry. And let those who will, look scorn upon our pursuit; but few are more adapted to improve the mind. In its minute details, it is well calculated to give habits of observation and of accurate perception; while, as a whole, the study of this department of nature, so intimately linked with others above and below it, has no common tendency to lift our thoughts to the great Creative Source of Being; to Him who has not designed the minutest part of the minutest object, without reference to some use connected with the whole."*

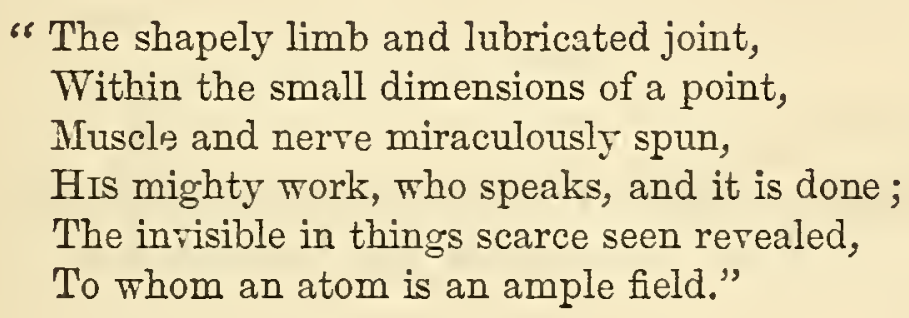

* Episodes of Insect Life. 


\section{CHAPTER III.}

ANIMAL STRUCTURE.

PHYSIOLOGY. HISTOLOGY. CELL THEORY. GROWTH OF TISSUES. SPECIAL TISSUES. SKIN, TEETH, BONE, ETC.

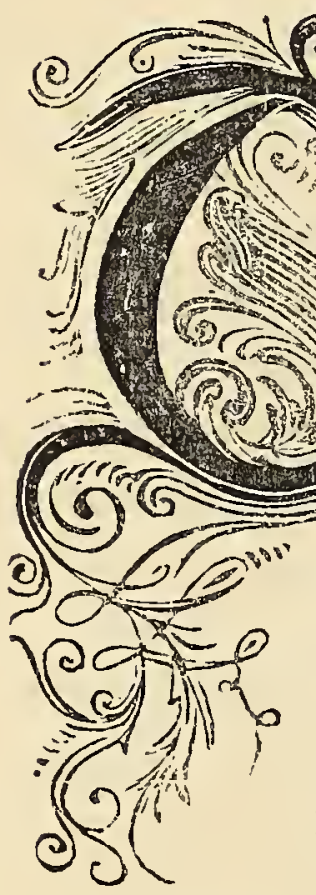

HE most complicated state in which matter exists, is where, under the influence of life, it forms bodies with a curious internal structure of tubes and cavities, in which fluids are moving and producing incessant internal change," says the philosophic Dr. Arnott. "These are called organised bodies, because of the various organs which they contain; and they form two remarkable classes: the individuals of one of which are fixed to the soil, and are called vegetables, - of these we shall consider the structure at a future stage; and the individuals of the other are endowed with power of locomotion, and are called animals: it is some of the peculiarities and minute structure of this latter class that we are now about to examine. The phenomena of growth, decay, death, sensation, self-motion, and many others, belong to life; but, from occurring all in material structures, which subsist in obedience to the laws of physics and chemistry, the life is truly a superstructure on the other two, and cannot be studied independently of them. Indeed, the greater part of the phenomena of life are merely chemical and physical phenomena, modified by an additional principle. The phenomena of life, from thus involving generally the agency of all the sets of laws, are by far the most complex of any; and the discovery or detection of the peculiar laws of life, although they are as fixed as the laws of chemistry or physics, has been very slow, and is as yet far from being completed."

The study of the Science of Life, or the building up of the living structure, is termed Physiology; and that part of it more particularly relating to the minute structure of the organs of animals has been 
termed Histology.* It is generally divided into animal and vegetable life, or, as Bichat terms it, organic life and relative life. In organised beings, the way in which nature works out her most secret processes is by far too minute for observation by unassisted vision; even with the aid of the improved microscope, only a small portion has, up to this time, been revealed to us. To point out in detail the discoveries made through the employment of this instrument, as regards physiology, would be to give a history of modern biological science; for there is no department in this study which is not more or less grounded upon the facts and teachings of the microscope.

To the casual observer, the brain and nerves appear to be composed of fibres. The microscope, however, reveals to us, as was first pointed out by Ehrenberg, that these supposed fibres do not exist, or rather that they all consist of numerous tubes, the walls of which are distinct, and contain a fluid which may be seen to flow from their broken extremities on pressure. In looking at a muscle, it appears to be made up of fine longitudinal fibres only. The microscope tells us that each of these supposed fine fibres is composed of numerous smaller ones, and that these are crossed by lines, which have received the name of transverse striæ; that muscular contraction, the cause of motion in animals, is produced by the relaxation or approximation of these transverse striæ.

The microscope has shown us that a distinct net-work of vessels lies between the arteries and veins, partaking of the properties of neither, and possessed of others peculiar to themselves. These have been denominated intermediary vessels by Berres, and serve to connect the arterial with the venous system.

On regarding with the naked eye the different glands, in which the secretions are formed, how complex they appear, how various in conformation! The microscope teaches us that they are all formed on one type; that the ultimate element of every gland is a simple sacculated membrane to which the blood-vessels have access; and that all glands are formed from the greater or less number, or different arrangement only of the primary structure.

Our notions respecting the skin were vague until the microscope discovered its real anatomy, and showed us the existence and relations of the papillæ, of the sudorific organs and their ducts, the inhalent muscular apparatus, and so on. All our knowledge of epidermic structures, such as hair, horn, feather, \&c., the real structure of cartilage, 
bone, tooth, tendon, cellular tissue, and, in a word, of all the solid textures, has been revealed to us by the same agency; so that it may be truly said, that all our real knowledge of structural anatomy, and all our acquaintance with the true composition of every organ in the body, have been arrived at by means of the microscope, and could never have been known without it.

In addition to this, and what is of greater importance, after having studied the healthy structure of the body, most beneficial aid is afforded in the diagnosis of diseases, which in many cases were overlooked or undistinguishable without the assistance of this instrument. It is on this account constantly resorted to by the medical profession for the benefit of their fellow-creatures.

We cannot, with our limited space, do more than take a microscopic view indeed of a few of the fundamental tissues entering into the formative process of the animal economy; taking for our starting-point the cell, which we recognise as "the common centre alike of animal and vegetable organism." "To Schwann and Schleider are we indebted," says Mr. Huxley, "for this service to the biological sciences. At a time when the researches of innumerable guideless investigators, called into existence by the tempting facilities afforded by the improvement of microscopes, threatened to swamp science in minutiæ, and to render the noble calling of the physiologist identical with that of the putterup of preparations, they stepped forward with the cell-theory as a colligation of the facts. To the investigator they afforded a clear basis and starting-point for his inquiries; for the student they grouped together immense masses of details in a clear and perspicuous manner; and if not absolutely true, it was the truest thing that had been done in biology* for half a century."

Dr. Carpenter's definition of a cell is, " a closed vesicle, or minute bag, formed by a membrane in which no definite structure can be discerned, and having a cavity which may contain matter of variable consistence. In such a cell every organised fabric, however complex, originates. The vast tree, almost a forest in itself; the zoophyte, in which we discover the lowest indications of animality; and the feeling, thinking, intelligent man,-each springs from a germ that differs in no obvious particular from the permanent condition of one of those lowly beings."

"The history of the animal cell, in its simplest form, is precisely

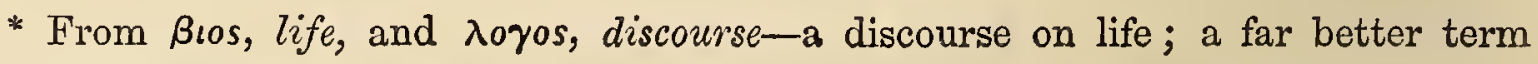
than physiology. 
that of the regetable cell of the lowest kind. It lives for itself and by itself, and is dependent upon nothing but a due supply of nutriment and a proper temperature for the continuance of its growth, and for the due performance of its functions, until its term of life is expired. It originates from a reproductive granule, previously formed by some other cell; this granule attaches to itself, assimilates, and organises the particles of the nutrient fluid in its neighbourhood; and converts some of them into the substance of the cell-wall, whilst it draws others into the cavity of the cell. In this manner. the cell gradually increases in size; and whilst it is itself approaching the term of its life, it usually makes preparation for its renewal by the derelopment of reproductive granules in its interior, as may be seen in Plate XI. No. 1a, which may become the germs of new cells, when set free by the rupture of the cellwall. There is an important difference, however, in the endowments of the animal and regetable cell. The latter can in general obtain its nutriment, and the materials for its secretion, by itself combining inorganic elements into organic compounds. The former, howerer, is totally destitute of this power: it can produce no organic compound; and we have yet to learn how far its power of converting one compound into another may extend; its chief endowment seems to be that of attracting or drawing to itself some of the various substances which are contained in the nutritive fluid in relation with it."* In general, the cells of animal tissues are furnished with a nucleus (central dot). This would appear to denote the ending and tying in of the orid body, somewhat similar to the drawing in of the string to close up the mouth of a small bag; or it may be the aggregation of minute molecules around the original granular germ. This nucleus appears to be the chief instrument in the future functions going on in the cell; the cell membrane only enclosing and limiting the contents of the cell. These constituents may be of a nature to give solidity and permanence to the texture: thus the cells of the skin are strengthened by a deposit of horny matter ; those of the shell by the deposit of carbonate of lime ; those of bones and teeth by a mixture of mineral and earthy matter. The nucleus seems always to perform a more important part when it has a distinct existence; in many cases the multiplication can be clearly perceived to take place by the division of the nucleus into one or more portions, as we have seen in the ova of the Lymneus, each giving origin to a new part or cell. This seems to be the case in the ordinary production of cartilage-cells; for, on examining a section of

* Dr. Carpenter's Principles of Human Physiology. 
cartilage that is undergoing rapid extension, we find groups of cells in all respects corresponding with those of the simple cellular plants (see Plate XI.* No 8).

And thus we shall find that all the tissues most actively engaged in the maintenance of the vital functions of the human body are per-

\section{* Description of Plate XI.-Antmal Structure.}

No. 1. a, Simple isolated cells containing reproductive granules; $b$, mucous membrane of stomach, showing cells, with the open mouths of tubes at the bottom of each, magnified 50 diameters.

No. 2. a, Diagram of a portion of the involuted mucous membrane, showing the continuation of its elements in the follicles and villi, with a nerve entering its submucous tissue. The upper surface of one villus is seen covered with cylindrical epithelium; the other is denuded, and with the dark line of basement membrane only running around it. $b$, epithelium scales, separated and magnified 200 diameters ; in the centre of each is a nucleus, with a smaller spot in its interior, called the nucleoli. $c$, pavement epithelium scales, from the mucous membrane of the bronchial or air tubes of the lung, showing nuclei, with a double nucleoli in some. $d$ represents another form of epithelium, termed the vibratile or ciliated; the nuclei are visible, with cilia at their upper or free surfaces, magnified 250 diameters.

No. 3. $a$ is one of the tubular follicles from a pig's stomach, cut obliquely to display the upper part of its cavity, and the cylindrical epithelium forming its walls, with a few of them detached, to show their true form; and at the lower part, the nucleated extremities of the cylinders of epithelium are seen. This is altogether a better view than that from the human stomach, No.2. $b$ shows a section of the lymphatics, and capillary blood-vessels, distributed beneath the mucous surfaces; the lymphatics take their origin from the radiated cells.

No. 4. Cells of adipose tissue, or fat, magnified 100 diameters.

No. 5. A single fat cell separated, and magnified 250 diameters.

No. 6. A capillary of blood-vessels distributed in the fat tissue.

No. 7. Section of the tendo-Achillis as it joins the cartilage; showing the stellate cells of tendon gradually coalescing to form the round or oval cells of the cartilage.

No. 8. A vertical section of cartilage, with clusters of cells arranged in columns previous to their conversion into bone, which is seen condensed at the upper surface. The greater opacity of this portion is owing to the increase of osseous fibres, the opacity of the cell contents, and the multiplication of the oil-globules; the dark intercellular spaces become filled up with vessels.

No. 9. A small transverse section of the same, showing the gradual change of the cartilage-cells at $a$ into the true bone-cells, termed lacunce, at $b$, with their characteristic canaliculi.

No. 10 is a stellate nerve-corpuscle, with tubular processes issuing from them : at $a$ it is filled with corpuscles containing black pigment, above which is a corpuscle in the nucleus of which is seen nucleoli; at $b$ is a corpuscle enclosed within its sheath, and filled with granular matter : taken from the root of a spinal nerve.

No. 11 shows the continuity of muscle; the upper portion, with connective tissue ; the lower portion: from the tongue of lamb.

No. 12. Branched muscle, ending in stellate connective cells, from the upper-lip of the rat.

No. 13. Black pigment cells from the human eye, lining the internal surface of the eye, magnified 150 diameters. 
PLATE XI.

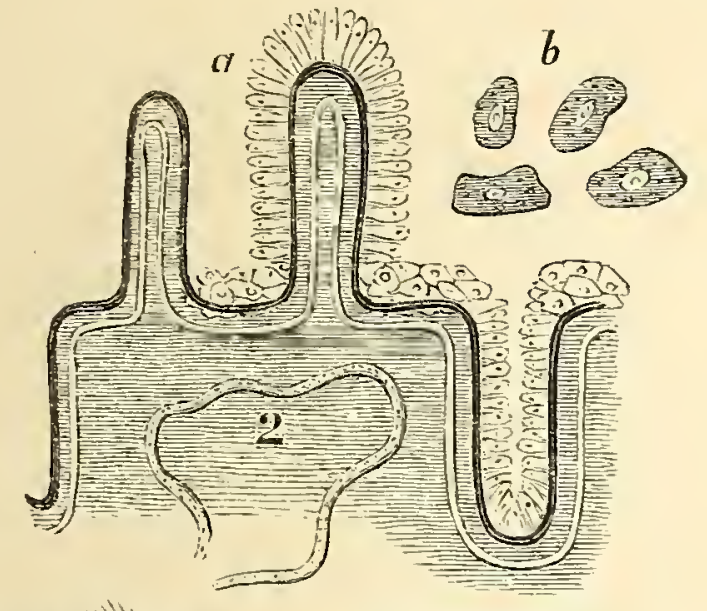

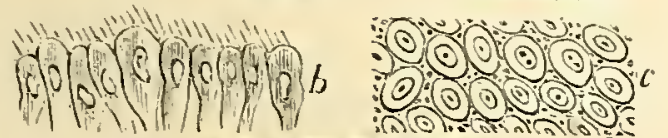
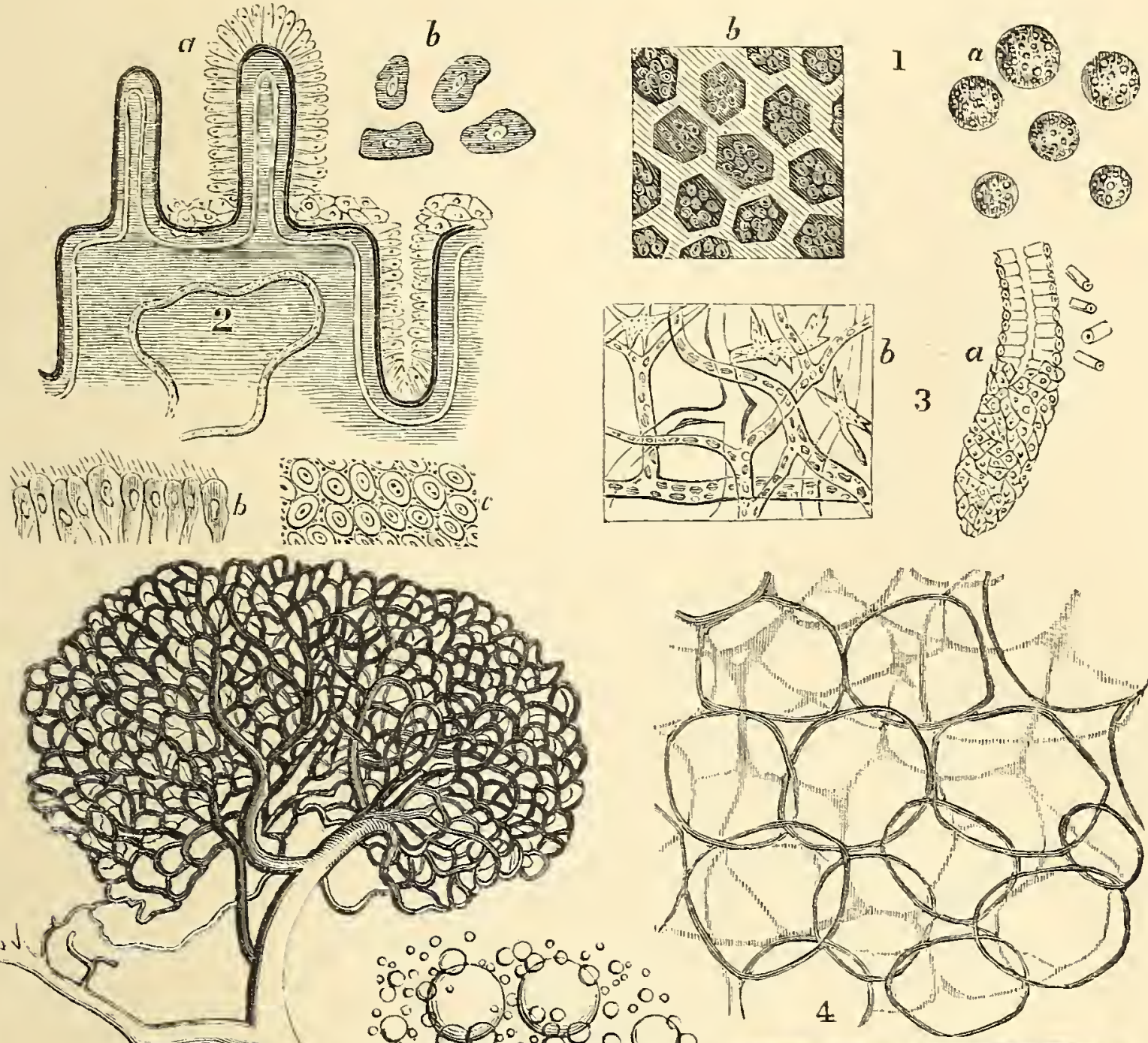

col go

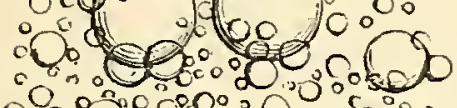

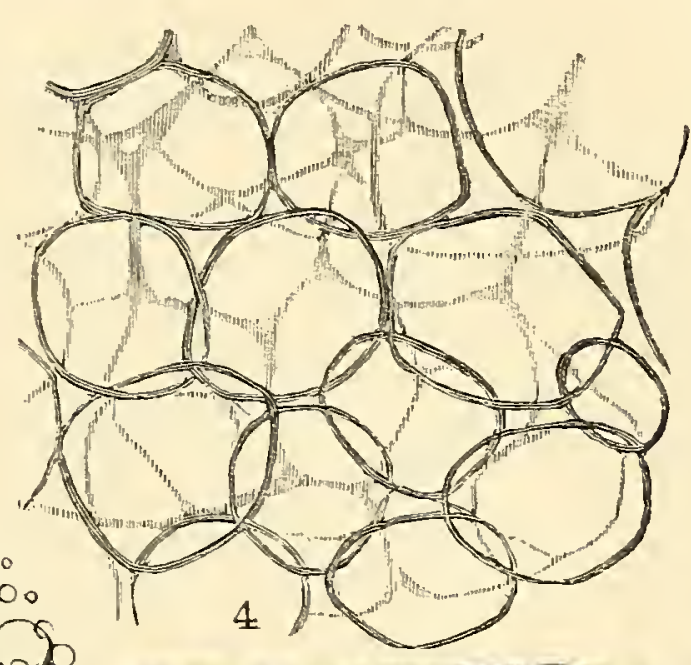

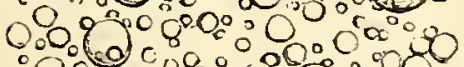

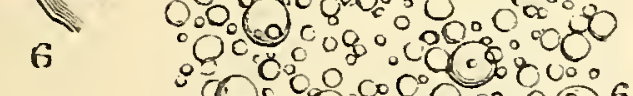

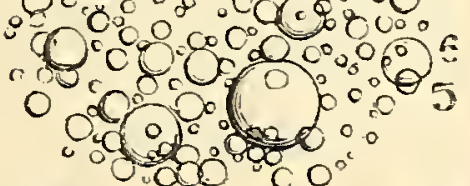

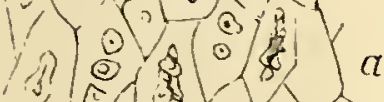

V 80 ,

cond

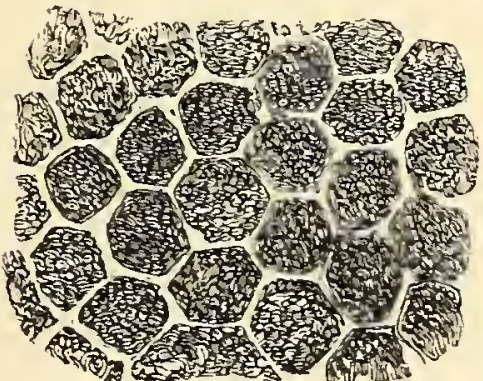

13
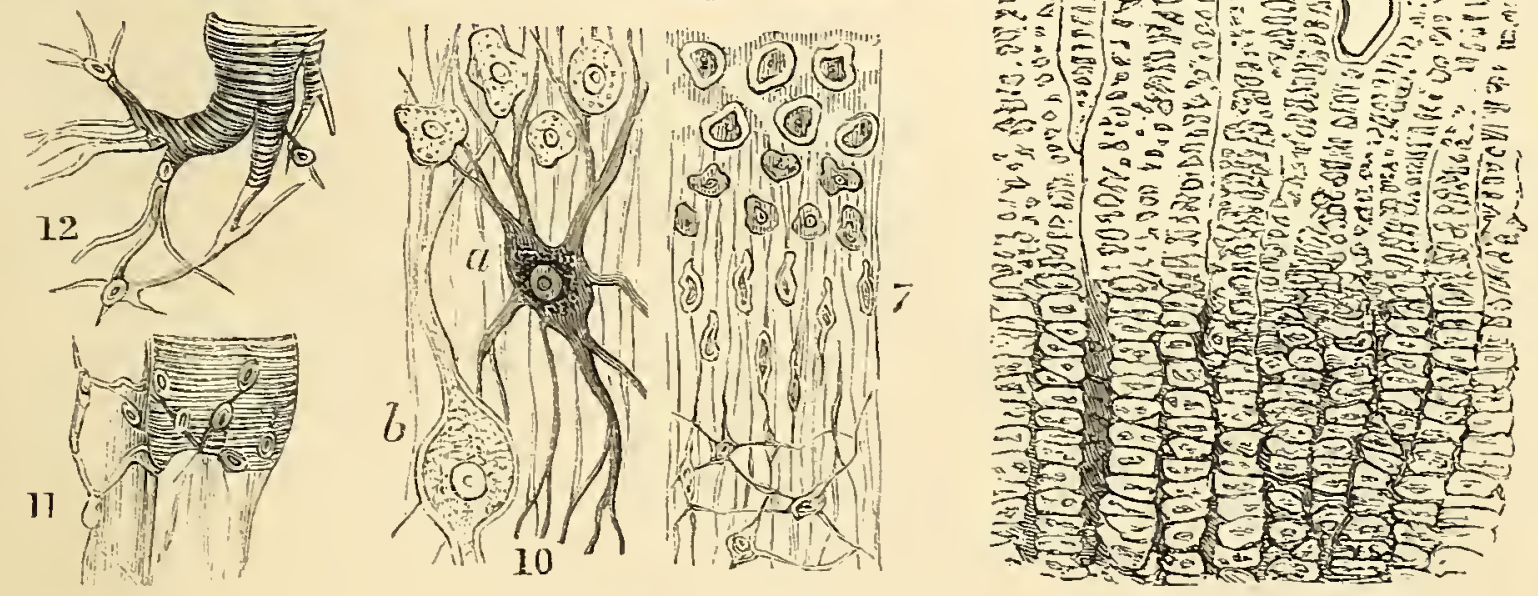

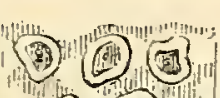

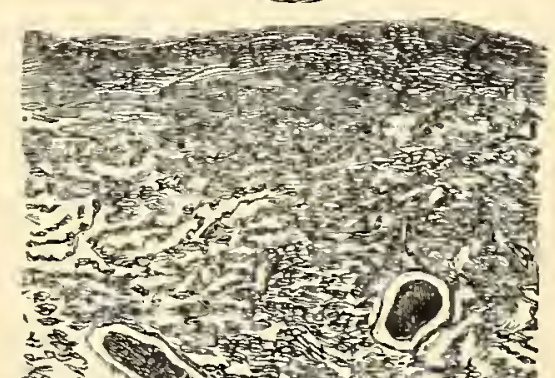

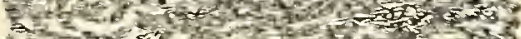

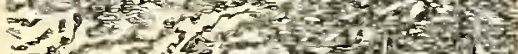
7n 3 .

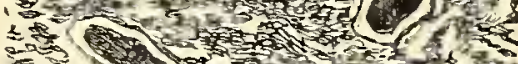
군

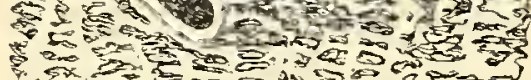
政 5

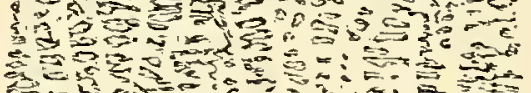

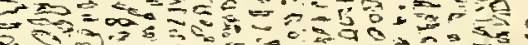

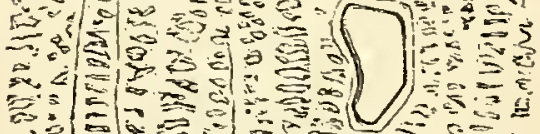

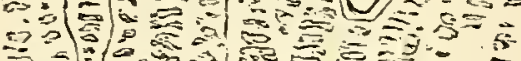
30 -

ANIMAL STRUCTURES. 

formed by these cells or globules, varying in size from infinite minuteness to forms visible to the unassisted eye ; that one system of cells secretes the bile, another the fat, another the nerve matter, and so forth. But how these special products are formed by cells apparently of similar organisation, we know not. Whether the special endowment belonging to the system of cells of a particular organ depends on the intimate structure of the wall or tissue of such cells, and this structure be so attenuated and infinitesimal as to elude our observation; or whether it results from the transmission of some peculiar modification of that mysterious vital force we term nervous influence, are questions to which no satisfactory reply can be given.

Cells and their nuclei undergo various metamorphoses; from being originally oval, spheroidal, or polygonal, they may become elongated, and assume the spindle or fusiform shape seen in woody fibres. Or they may shoot out prolongations in a radiating or stellate form, so that their shape becomes altogether irregular (Plate XI.), as in bone-cells, No.9, or nerve matter, No.10. They may, by the breaking up of the cellwalls, coalesce with other cells, end to end, and thus form a tube, as in No. 3 b, the lymphatics; and these may again become connected with others, so as to form a capillary network for the circulation of the blood, as in No.6. Or the tube may form a simple straight fibre; and the nuclei of its component cells may originate a new deposit, either as the fibrous portion of nervous tissue, or in the form of an aggregation of new cells, as seen in the more perfect kind of muscular fibre, No. 11.

Again, there are some tissues in which no other cell agency can be traced than that concerned in the preparation of the plastic material. This is doubtless the case in the formation of a very delicate structureless membrane known under the name of Basement or Primary Membrane, which is found to enter into the composition of most of the tissues of the body. "And this fact," says Dr. Carpenter, "most seriously affects the unicellular theory of Schwann, as here no vestige of cell-structure can be seen; and it would rather appear to resemble that of which the walls of the cells are themselves constituted. There are forms of this Basement Membrane which can be seen to consist of flattened polygonal cells, closely adherent by their edges, every one havingits own granular nucleus. And it seems to be from these granular germs, sometimes scattered through the membrane, and in other instances collected in certain spots, that the epithelium cells of the skin and other parts take their origin; and if this be the case, we must regard the basement membrane as a transitional rather than as a permanent structure, continually disintegrating, and yielding up its con- 
tained cell-germs on its free surface, and as constantly being renewed by the blood beneath. For the epidermic structures appear to constitute an exception to the general rule, that the tissues reproduce themselves, since they are cast off without leaving their germs behind them ; and the cells which replace them must be derived from new germs, more directly supplied from the blood than is elsewhere the case." The epithelium cells are represented in Plate XI. Nos. 1, 2, and 3.

"It would seem doubtful also, with regard to the simple fibrous tissues, whether they are generated by a metamorphosis of cells, in the same manner as the osseous, muscular, and nervous ; or whether they are not produced, like the basement membrane, by the consolidation of a plastic fluid which has been elaborated by cells. In the case of the other tissues, it would seem probable that, in the very act of the dissolution of the parent structure, the germs of the new structures destined to replace it are set free, as happens in the reproduction of the simple cellular plants."

T. H. Huxley, Esq., F.R.S., in reviewing the unicellular theory of Schleiden and Schwann," says, that he believes it "to be based upon erroneous conceptions of structure, leading to errors in physiology. Animal physiologists go on using Schwann's nomenclature, furgetting that the whole doctrine of the vegetable cell, from which he drew that nomenclature, has been completely upset by Von Mohl and others; and at present, beyond the mere fact of a common vesicularity at one period of their existence, one would be led, on opening successively two works on animal and vegetable structure, rather to predicate their total discrepancy than any uniformity between them."

The views entertained by Mr. Huxley are so entirely original, scientific, and logical, that we have been induced to bring that portion of them which bears on the subject-matter before us to the notice of our readers. He starts with the inquiry of "What are cells?"

"What is the meaning of the unquestionable fact, that the first indication of vitality, in the higher organisms at any rate, is the assumption of the cellular structure?

"In answering these questions, we would first draw attention to the definition of the nature of development in general, first clearly enunciated by Von Baer. 'The history of development,' he says, 'is the history of a gradually increasing differentiation of that which was at first homogeneous.' The yelk is homogeneous ; the blastoderma is a portion of it which becomes different from the rest, as the result of

* British and Foreign Medico-Chirurgical Review, October 1853. 
the operation of the laws of growth ; the blastoderma, again, comparatively homogeneous, becomes differentiated into two or more layers; the layers, originally identical throughout, set up different actions in their various parts, and are differentiated into dorsal and visceral plates, chorda dorsalis, and bodies of vertebræ, \&c.......

"Now, why should we not extend this view to histology, which is only ultimate morphology? As the whole animal is the result of the differentiation of a structureless yelk, so is every tissue the result of the differentiation of a structureless blastema; the first step in that differentiation being the separation of the blastema into endoplast and periplast, or the formation of what is called a 'nucleated cell.' Then, just as in the development of the embryo, when the blastodermic membrane is once formed, new organs are not developed in other parts of the yelk, but proceed wholly from the differentiation of the blastoderm, - so, histologically, the 'nucleated cell,' the periplast with the endoplast, once formed, further development takes place by their growth and differentiation into new endoplasts and periplasts. The further change into a special tissue, of course, succeeds and results from this primary differentiation. . . . . . The cellular structure precedes the special structure ; but is the latter, therefore, the result of a 'cell-force,' of whose existence there is, on other grounds, no evidence whatever? For us, the primarily cellular structure of plants and animals is simply a fact in the history of their histological development.

"We would therefore submit the following sketch of a general theory of the structure of piants and animals :

"Vitality-the faculty, that is, of exhibiting definite cycles of change in form and composition-is a property inherent in certain kinds of matter.

"There is a condition of all kinds of living matter in which it is an amorphous germ-that is, in which its external form depends merely on ordinary physical laws, and in which it possesses no internal structure. Now, according to the nature of certain previous conditions, the character of the changes undergone-of the different states necessarily exhibited - or, in other words, the successive differentiations of the amorphous mass, will be different.

"Conceived as a whole, from their commencement to their termination, they constitute the individuality of the living being; and the passage of the living being through these states is called its development. Development, therefore, and life, are, strictly speaking, one thing; though we are accustomed to limit the former to the progressive half of life merely, and to speak of the retrogressive half as decay, con- 
sidering an imaginary resting-point between the two as the adult or perfect state.

"The individuality of a living thing, then, or a single life, is a continuous development; and development is the continual differentiation, the constant cyclical change of that which was at first morphologically and chemically indifferent and homogeneous. The morphological differentiation may be of two kinds : in the lowest animals and plants-the so-called unicellular organisms-it may be said to be external; the changes of form being essentially confined to the outward shape of the germ, and being unaccompanied by the development of any internal structure.

"But in all other animals and plants, an internal morphological differentiation precedes or accompanies the external ; and the homogeneous germ becomes separated into a certain central portion, which we have called the endoplast, and a peripheral portion, the periplast. Inasmuch as the separate existence of the former necessarily implies a cavity in which it lies, the germ in this state constitutes a vesicle with a central particle, or a 'nucleated cell.'

"There is no evidence whatever that the molecular forces of the living matter (the vis essentialis of Wolff, or the 'vital forces' of the moderns) are by this act of differentiation localised in the endoplast, to. the exclusion of the periplast, or vice versê. Neither is there any evidence that any attraction or other influence is exercised by the one over the other; the changes which each subsequently undergoes, though they are in harmony, having no causal connection with one another, but each proceeding, as it would seem, in accordance with the general determining laws of the organism.

"The endoplast grows and divides; but, except in a few more or less doubtful cases, it would seem to undergo no other morphological change. It frequently disappears altogether; but as a rule, it undergoes neither chemical nor morphological metamorphosis. So far from being the centre of activity of the vital actions, it would appear much rather to be the less important histological element.

"The periplast, on the other hand, which has hitherto passed under the names of cell-wall, contents, and intercellular substance, is the subject of all the most important metamorphic processes, whether morphological or chemical, in the animal and in the plant. By its differentiation every variety of tissue is produced; and this differentiation is the result not of any metabolic action of the endoplast, which has frequently disappeared before the metamorphosis begins, but of intimate molecular changes in its substance, which take place under 
the guidance of the vis essentiatis, or, to use a strictly positive phrase, occur in a definite order, we know not why.

"The metamorphoses of the periplastic substance are twofold-chemical and structural. The former may be of the nature either of conversion,-change of cellulose into xylogen, intercellular substance, \&c. of the indifferent tissue of embryos into collagen, chondrin, \&c.; or of deposit, as of silica in plants, of calcareous salts in animals.

"The structural metamorphoses, again, are of two kinds: vacuolation, or the formation of cavities; as in the intercellular passages of plants, the first vascular canals of animals; and fibrillation, or the development of a tendency to break up in certain definite lines rather than in others, a peculiar modification of the cohesive forces of the tissue, such as we have in connective tissue, in muscle, and in the 'secondary deposits' of the vegetable cell.

"Now to illustrate and explain these views, let us return to the regetable and animal tissues, and trace out the modifications of these, which are identical with all young tissues, into some of the typical adult forms.

"Take a sphagnum leaf; the point must be older than the base. At the base of the leaf we find nothing but minute endoplasts, each resembling the other, imbedded in a homogeneous periplastic substance; as we trace these upwards, we find that some of the endoplasts increase in size more rapidly than the others, and eventually totally disappear, leaving only the endoplastic cavity or 'cell' which contained them. In the surrounding cells the endoplasts are very obvious as granular primordial utricles. After the disappearance of the endoplasts, changes commence in the periplastic substance or wall of the cell, more or less circular or spiral thickenings taking place in it, so as to form the well-known fibrecell of the sphagnum leaf (see a section figured in Vegetable Tissues); and at the same time, a process of resorption occurs in particular parts of the wall, so that round apertures are formed. Nothing can be more instructive than this case, the leaf being composed of a single layer of delicate and transparent cells, so that there are no interfering difficulties of observation; and we see demonstrated, in the most striking manner, that the endoplast or primordial utricle has nothing to do with the metamorphoses wbich occur in the periplastic substance. The disappearance of the primordial utricle in cells which are undergoing thickening, was, in truth, long ago pointed out by Von Mohl; but neither he nor any of his successors seem to have noticed how completely this fact does away with that activity of the primordial utricle, and passivity of the cell-wall, which they all assume. We have here, in fact, 
the cell-wall commencing and carrying through its morphological changes after the primordial utricle has completely disappeared; and we see that the so-called secondary deposit in this case is a morphological differentiation of the periplast, which, at the same time, exhibits its peculiar powers by setting up a resorption of its substance at another point. Here, however, we have no marked chemical differentiation; for an instance of which we may turn to the collenchyma of the beet-root. In the fully-formed beet-root we have no less than three compounds disposed around each cell cavity. . . . . . . . We may cail one of these portions cell-membrane, and another intercellular substance; but they are, assuredly, all nothing but differentiated portions of one and the same periplast.

"Woody tissues present preeisely the same phenomena; the inner layers of the periplastic substance having, very generally, a different composition from the outer.

"Morphologically we have already noticed the lamination of the periplastic substance; and we may mention its fibrillation,--a process which takes place almost invariably in the inner layers of the periplasts, and to which the well-known spirality of the so-called secondary deposits must be referred: but a more important process for our present purpose is what we have called Vacuolation,- the development of cavities in the periplastic substance independent of the endoplast, and which, to distinguish them from the cells, may conveniently be termed vacuolce. In the youngest vegetable tissues there are no such cavities, the periplastic substance forming a continuous solid whole; and it is by this vacuolation, which occurs as the part grows older, that all the intercellular passages are formed, and that many cells obtain that spurious anatomical independence to which we have adverted above. The exaggerated development of the vacuolæ in the pith of the rush converts the periplastic substance, with its proper endoplastic cavities, into regular stellate cells. (Plate XV. No. 20.)

"Sufficient has been said to illustrate the differentiation of the primitive vegetable structure into its most complex forms. If we turn to the animal tissues, we shall find the same simple principles amply sufficient to account for all their varieties.

"In the plant there are but two histological elements-the periplastic substance and the endoplasts; cell-wall and intercellular substance being merely names for differentiated portions of the former, cell-contents representing a part of the latter. In the animal, on the other hand, if we are to put faith in the present nomenclature, we find cell-wall, intercellular substance, and cell-contents forming 
primitive elements of the tissues, and entering into their composition as such: there have been no small dispites whether the collagenous portion of connective tissue is intercellular substance or cellwall, the elastic element being pretty generally admitted to be developed from distinct cells. Again, it appears to be usual to consider the fibrillæ of striped muscle as modified cell-contents, while the sarcolemma represents the cell-walls. We confess ourselves quite unable to find any guiding principle for this nomenclature, unless it be that the toughest structure surrounding a 'nucleus' is to be taken as cell-wall, anything soft inside it being' contents, and anything external to it intercellular substance; which is hardly a caricature of the vagueness which pervades histological works upon this subject. This results, we think, from the attempts to determine the homology of the parts of the tissues having been made from the examination of their embryonic conditions, where it is often very obscure, and hardly to be made out. It is another matter if we adopt the 'principle of continuity' of Reichert,-a method of investigation which has been much neglected. This principle is simply, that whatever histological elements pass into one another by insensible gradations are homologous and of the same nature; and it is so clear and easy of application, that we can but wonder at its hitherto limited use.

"We now proceed to analyse the nature of the constituents of some of the most characteristic tissues in this way, starting from that of embryonic cartilage.

"Connective tissue occurs in two forms, which, however, pass into one another by infinite gradations, - the solid and the areolated: of the former, we may take a tendon as an example; of the latter, the loose areolar tissue which is found in forming the inner layer of the skin and mucous membranes. Plate XI. No. 7 represents the junction between the tendo-Achillis and the cartilage of the os calcis in a young kitten. At the top part we have pure cartilage, the endoplasts lying within cavities whose walls present more or less defined contours. At the middle part the cavities are somewhat elongated; and a faint striation is obvious in the upper portion of the periplastic substance, which becomes stronger and stronger as we proceed lower down, until it ends in an apparent fibrillation. A chemical change has at the same time taken place, so that in this portion the striated part of the periplast is swollen up more or less by acetic acid, the walls of the cavities remaining unaffected, and thence becoming more distinct; while the top portion, the cartilaginous, the whole periplast, was nearly equally insensible to this re-agent. The next portion, on the contrary, nearest the tendon, and passing into it, 
is completely tendinous in its structure. The periplast exhibits strong fibrillation, and is very sensitive to acetic acid; while not only the walls of the cavities, but the intermediate periplast, in certain directions, which radiate irregularly from them, have changed into a substance which resists acetic acid even more than before, and is in fact elastic tissue. Compare this process with that stated to occur in the collenchyma of the beet-root; and we have the fibrillation of the outer portion of the periplast round each endoplast, and its conversion into collagen, answering to the lamination of the intercellular substance, and its conversion into a vegetable gelatinous matter, whilst the elastic corresponds with the cellulose inner wall.

"The testimony of numerous observers agrees that cartilage is converted into connective tissue in the way described. Professor Kölliker, who unwillingly admits the fact, suggests, nevertheless, that such con. nective tissue as this is not true connective tissue, inasmuch as it presents differences in its mode of development, the collagenous element in the latter being always developed from cells.

"Now we might be inclined to ask, if the substance of the tendoAchillis is not connective tissue, what is? But it is better to attack Kölliker's stronghold, the areolated gelatinous connective tissue, which is, as he justly observes, the early form of foctal connective tissue generally.

"If the outer layer of the corium of the skin, or the submucous gelatinous tissue in the enamel organ, be teased out with needles, we shall obtain various stellate or ramified bodies, containing endoplasts, which Kölliker calls cells; and which, as he states, do assuredly pass into and become bundles of fibrillated and connective tissue. But is this really a different mode of development from that already described? We think not. Indeed, if that portion of this young gelatinous connective tissue which lies immediately adjacent to the epidermis or epithelium be examined, it will be found to present a structure in all respects similar to very young cartilage; that is, there is a homogeneous matrix, in which the endoplasts are dispersed. (Plate XI. No. 7.) If this be traced inwards, it will be found that the endoplasts become more widely separated from one another; and that the matrix in places between them is softened and altered, while in their immediate neighbourhood, and in the direction of irregular lines stretching from them, it is unaltered. This is, in fact, the first stage of that process which we have called vacuolation. In this condition the intermediate softened spots still retain sufficient consistence not to flow out of a section; but yielding, as it does, in these localities much more readily than in others, it is 
easy enough to tear out the firmer portion in the shape of 'cells,' which are fusiform, irregular, or stellate; and the whole tissue has therefore been described by Reichert, Virchow, and Schwann, as consisting of cells connected by an 'intercellular substance.' Both 'cell-walls' and 'intercellular substance,' however, are portions of the same periplast, and together correspond with the matrix of the cartilage. When, therefore in the course of further development, the 'intercellular substance' becomes quite fluid and so disappears, the outer portion of these cells being converted into fibrillated collagenous tissue, and the inner into elastic substance, we have, notwithstanding the apparently great difference, in reality exactly the same mode of metamorphosis of the same elements as in the preceding instance. Connective tissue, therefore, we may say, consists in its earliest state of a homogeneous periplast inclosing endoplasts. The endoplasts may elongate to some extent, but eventually become lost, and cease, more or less completely, to be distinguishable elements of the tissue. The periplast may undergo three distinct varieties of chemical differentiation, that is, into the gelatinous 'intercellular substance,' the collagenous ' cell-wall,' and the elastic ' cell-wall;' and two varieties of morphological differentiation, vacuolation and fibrillation; and the mode in which these changes take place gives rise to the notion that the perfect tissue is composed of elements chemically and mechanically distinct.

"The proper understanding of the nature and mode of development of the component parts of connective tissue is, we believe, of the first importance in comprehending the other tissues. If we clearly bear in mind, in the first place, that the periplast is capable of undergoing modifications quite independently of the endoplasts; and secondly, that in consequence of their modification elements may become optically, mechanically, or chemically separable from a perfect tissue, which were not discoverable in its young form, and never had any separate existence,-many of the great difficulties and perplexities of the cell-theory will disappear. Thus, for instance, with regard to the structure of bone (Plate XI. No. 9), there can be no doubt that the 'nuclei' of the corpuscles are endoplasts, and that the calcified matrix is the periplast. This calcified matrix has, however, in adult bone, very often a very regular structure, being composed of definite particles. To account for these, Messr's. Tomes and De Mrorgan, in their raluable essay on ossification, in the Philosophical Transactions, 1853, suppose that certain 'osteal cells' exist and become ossified. We have no intention bere of entering upon the question of the existence of these ' osteal cells' as a matter of fact, but we may remark, that they are 
by no means necessary, as the appearance might arise from a differentiation of the periplast into definite particles, corresponding with that which gives to connective tissue its definite and fibrillated aspect. So with regard to the vexed question, whether the lacunæ have separate parietes or not, how readily comprehensible the opposite results at which different observers have arrived become, if we consider that their demonstrability or otherwise results simply from the nature and amount of the chemical difference which has been established in the periplast in the immediate neighbourhood of the endoplast, with regard to that in the rest of the periplast. In Plate XI. No. 9 (see also description of bone) we have a piece of bone exhibiting every variety of lacunæ, from those without distinct walls, to those which constitute regular stellate 'bone-corpuscles.' Finally, in bone, the formation of the 'Haversian-spaces' of Tomes and De Morgan is a process of vacuolation, strictly comparable to that which we have described as giving rise to the areolated connective tissue. Cancellated bone is, in fact, areolated osseous tissue. Once having comprehended the fact, that the periplast is the metamorphic element of the tissues, and that the endoplast has no influence nor importance in histological metamorphosis, there ceases to be any difficulty in understanding and admitting the development of the tubules of the dentine and the prisms of the enamel, without the intervention of endoplasts. These are but extreme and.obvious cases, in which nature has separated for us two histological elements and two processes, which are elsewhere confounded together.

"One of the most complicated of tissues is striped muscle; yet the true homology of its elements seems to us to become intelligible enough upon these principles. Dr. Hyde Salter has pointed out, that in the tongue the muscles pass directly into the bundles of the submucous connective tissue which serve as their tendons. We have figured such a transition at Plate XI. No. 11: the tendon, the lower part of which may be seen passing insensibly into the striped muscle, the glandular sarcous elements of the latter appearing, as it were, to be deposited in the substance of the tendon (just as the calcareous particles are deposited in bone), at first leaving the tissue about the walls of the cavities of the endoplasts, and that in some other directions, unaltered. These portions, which would have represented the elastic element in ordinary connective tissue, disappear in the centre of the muscular bundle, and the endoplasts are immediately surrounded by muscle; just as, in many specimens of bone (see figs. of bone), the lacunæ have no distinguishable walls. On the other hand, 
at the surface of the bundle the representative of the elastic element remains, and often becomes as much developed as the sarcolemma. There is no question here of muscle resulting from the contents of fused cells, \&c. It is obviously and readily seen to be nothing but a metamorphosis of the periplastic substance, in all respects comparable to that which occurs in ossification, or in the development of tendon. In this case we might expect, that as there is an areolar form of connective tissue, so we should find some similar arrangement of muscle; and such may indeed be seen very beautifully in the termination of the branched muscles, as they are called. In Plate XI. No. 12, the termination of such a muscle, from the lip of a rat, is shown; and the stellate 'cells' of areolated connective tissue are seen passing into the divided extremities of the muscular bundle, becoming gradually striated as they do so. In the muscle it is obvious enough, that whatever homology there may be between the stellate 'cells' and the nuscular bundles with which they are continuous, there is no functional analogy, the stellate bodies having no contractile faculty. But the nervous tubule is developed in essentially the same manner as a muscular fasciculus, the only difference being, that fatty matters take the place of syntonin. Now it commonly happens that the nervetubules terminate in stellate bodies (Plate XI. No. 10) of a precisely similar nature; and these, in this case, are supposed to possess important nervous functions, and go by the name of ' ganglionic cells.' From what has been said, however, it is clear that these may be genetically, and not functionally, connected with the nervous tubules; and that so far from being the essential element of the nervous centres and expansions, it is possible that the 'ganglionic cells' have as little nervous function as the stellate cells in the lip of the rat have contractile function. It thus would appear, that life, so far as it is manifested by structure, is for us nothing but a succession of certain morphological and chemical phenomena in a definite cycle, of whose cause or causes we know nothing; and that, in virtue of their invariable passage through these successive states, living beings have a development, a knowledge of which is necessary to any complete understanding of them."

And therefore Mr. Huxley does not consider the "nucleus" of the animal cell the homologue of that in the plant, but of its primordical utricle only; that the cells are not machines by which alone further development can take place, even with Dr. Carpenter's restriction, to be considered as "instrumental" to that development, but simply "indications of it." "Wolff sought to establish the doctrine, that the vital pheno- 
mena are not necessarily preceded by organisation, nor in any way the result or effect of formed parts, but that the faculty of manifesting them resides in the matter of which living bodies are composed; as such are termed vital or molecular forces."

By what force the determinate arrangement of atoms into cells or cell-contents is effected, is a subject of which nothing is positively known, or probably ever will be. The term vital or molecular forces should be received, then, only as a general expression for the power by which the vital phenomena are produced. Physiologists must not, at present, attempt to define this power; whether it resides in a single organ or in the whole organism,-whether it be a property of some particular kind of matter, or something superadded to common matter. Tiedeman and De Blainville have demonstrated life, as far as we know of it, " to be a power of reaction modified in every part according to its texture, and called into operation in each texture by appropriate stimuli. The property to which is referred the formative power of living beings, is, however, no simple property, such as the attraction of mechanical science, or the affinity of chemistry. These manifest themselves in acts so simple and almost uniform, that the hypothesis which assumes the existences of such properties supplies at once the language in which their laws of action may be enunciated. But in the simplest exercise of living formative power, even in that which accomplishes the formation of a cell, there is evidence of the operation of many forces. Mechanical force is shown in the determination of the position, shape, and relations of the cell; chemical force in the determination of the composition of its walls and contents; and with these, as if directing them, that vital force, different from them and from all other known physical forces, is in operation, by virtue of which the cell acquires properties that characterise the species or organs to which it is attached, and becomes capable of taking part in vital processes, even in such processes as those in which itself originated."

Dr. T. Williams declares both the philosopher and chemist alike at fault in their explanations of this vital force: "that the power which moulds a cell, configurates a fibre, or shapes the tubularity of a vessel, transcends the conceptions of the pure chemist. It cannot be experimentally catechised; it cannot be imitated by any artificial arrange.ment. Even in the attempt to explain the form of organised solids, all mathematical and mechanical conceptions utterly fail. Wherefore the discordal shape of the animal blood-cell? why the elliptical of the fish? This is the vital force; chemistry compounds the materials, vitality chisels them into form. Chemistry unites the elementary atoms into composite 
groups; vitality utilises these compound groups, and no other, in the fabrication of the solids. The floating cells are the only parts of the fluids which in this sense exhibit the evidence of vital force. Vitality gives to these products a special arrangement; but no other principles could be shaped, even by the vital force, into corpuscles. It requires conditions of action, as well as every other force. There is, therefore, some mysterious relation between the shape and the substance, between the material and the form."

"It will be sufficient to remind the thoughtful querist," says Coleridge, "that herein consists the essential difference, the contradistinction of an organ from a machine: that not only the characteristic shape is evolved from the invisible central power, but the material mass itself is acquired by assimilation. The germinal power of the plant transmutes the fixed air and the elementary base of water into grass or leaves; and on these the organific principle in the ox or the elephant exercises an alchemy still more stupendous. As the unseen agency weaves its magic eddies, the foliage becomes indifferently the bone and its marrow, the pulpy brain or the solid ivory. That what you see is blood, is flesh, is itself the work; or, shall I say, the translucence of the invisible energy which soon surrenders or abandons them to inferior powers (for there is no pause nor chasm in the activities of nature), which repeat a similar metamorphosis according to their kind? These are not fancies, conjectures, or even hypotheses, but facts; to deny which is impossible, not to reflect on which is ignominious."

"Sleep is a recreance to body; but when was mind asleep?

Even in a swoon it dreameth, though all be forgotten afterward.

The muscles seek relaxing, and the irritable nerres ask peace;

But life is a constant force, spirit an inquietable impetus :

For death is merely absent life, as darkness absent light:

Not eren a suspension; for the life hath sailed away, steering gladly somewhere. And I look to recognise again, through the beautiful mask of their perfection, The dear familiar faces I have somewhile loved on earth.

Yea, there is a fitness and a beauty in ascribing immortality to mind,

That its energies and lofty aspirations may have scope for indefinite expansion."*

\section{THE ELEMENTARY TISSUES.}

The division of the elementary tissues into sub-divisions, or a proper classification and arrangement, is due, in the first instance, to Professor Schwann. The classification now generally adopted is that given by Messrs. Todd and Bowman, in their Physiological Anatomy, which we here append, with Professor Quekett's modifications.

\footnotetext{
* Proverbial Philosophy.
} 


\section{A Classification of the Animal Tissues.}

1. Simple membrane: employed alone in the formation of compound membranes .

2. Fibrous tissues

3. Cellular tissues

4. Sclerous or hard tissues

5. Compound membranes; composed of simple membrane, and a layer of cells of various forms (epithelum or epidermis), or of areolar tissue and epithelum

6. Compound tissues; $a$, those com. posed of tubes of homogeneous membrane, containing a peculiar substance

$b$, those composed of white fibrous tissues and cartilage
Examples: Walls of cells, capsule of lens of the eye, sarcolemma of muscle, $\& c$.

Examples: White and yellow fibrous tissue, areolar tissue, elastic tissue, \&c.

Examples: Cartilage, fat, pigment, grey nervous matter, \&c.

Examples: Rudimentary skeleton of invertebrata, bone, teeth, \&c.

Examples: Mucous membrane, skin, true or secreting glands, serous and synovial membranes.

Examples: Muscle, nerve.

Example: Fibro-cartilage.

\section{STMPLE MEMBRANE.}

When examined under the highest powers of the microscope, it is perfectly transparent and structureless; nevertheless, it is nourished by blood-vessels which run over its outer surface, but do not penetrate to its interior. Its office in the animal economy appears to be, to serve as a covering to the more delicate parts, and to form the cellwalls of the blood-globules, lymph, fat, \&c. In Plate XI. No. 4, a portion of fat is shown to consist of a series of cells, with walls of simple or structureless membrane; at No. 5, the contents of a single fat-cell is seen under a quarter-inch object-glass; and at $\mathrm{No}$. 6, same plate, capillaries of vessels distributed to the fat-globules running round and about the cells, but never penetrating their walls. The capsule of the lens of the eye is a membrane the most perfectly structureless, however highly magnified. The human blood-globule (fig. 130, No. 3) is enclosed in this membrane, forming a double-convex disc; and these are very easily discernible from other blood-discs : for instance, compare them with the ovid form of the white corpuscule of those in the bloodvessel of the frog, No. 2; the same difference in appearance will be seen to exist when compared with those from birds and other animals. Beneath the vessel of the frog a layer of hexagonal epithelium scales is seen, with their nucleated centres. 


\section{PLATE XII.}
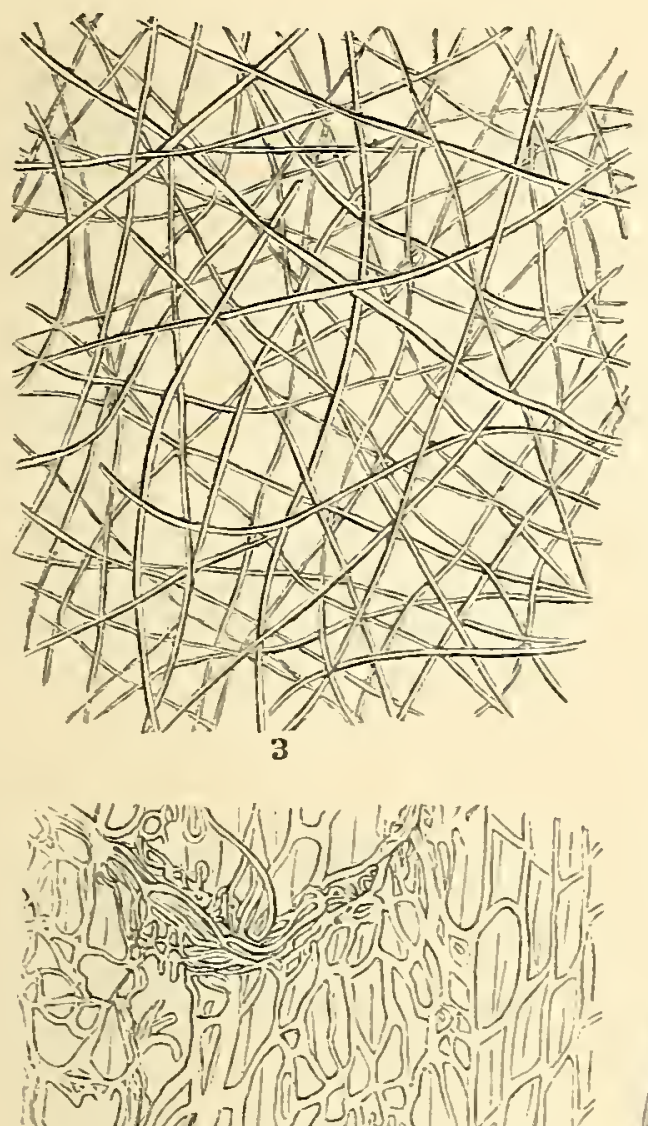

जoly

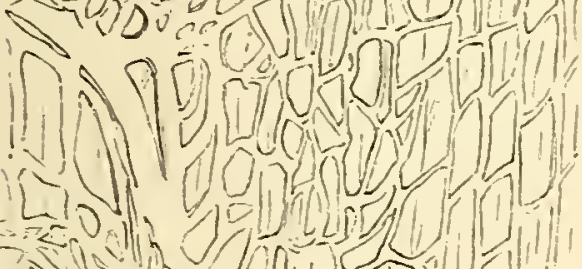

4
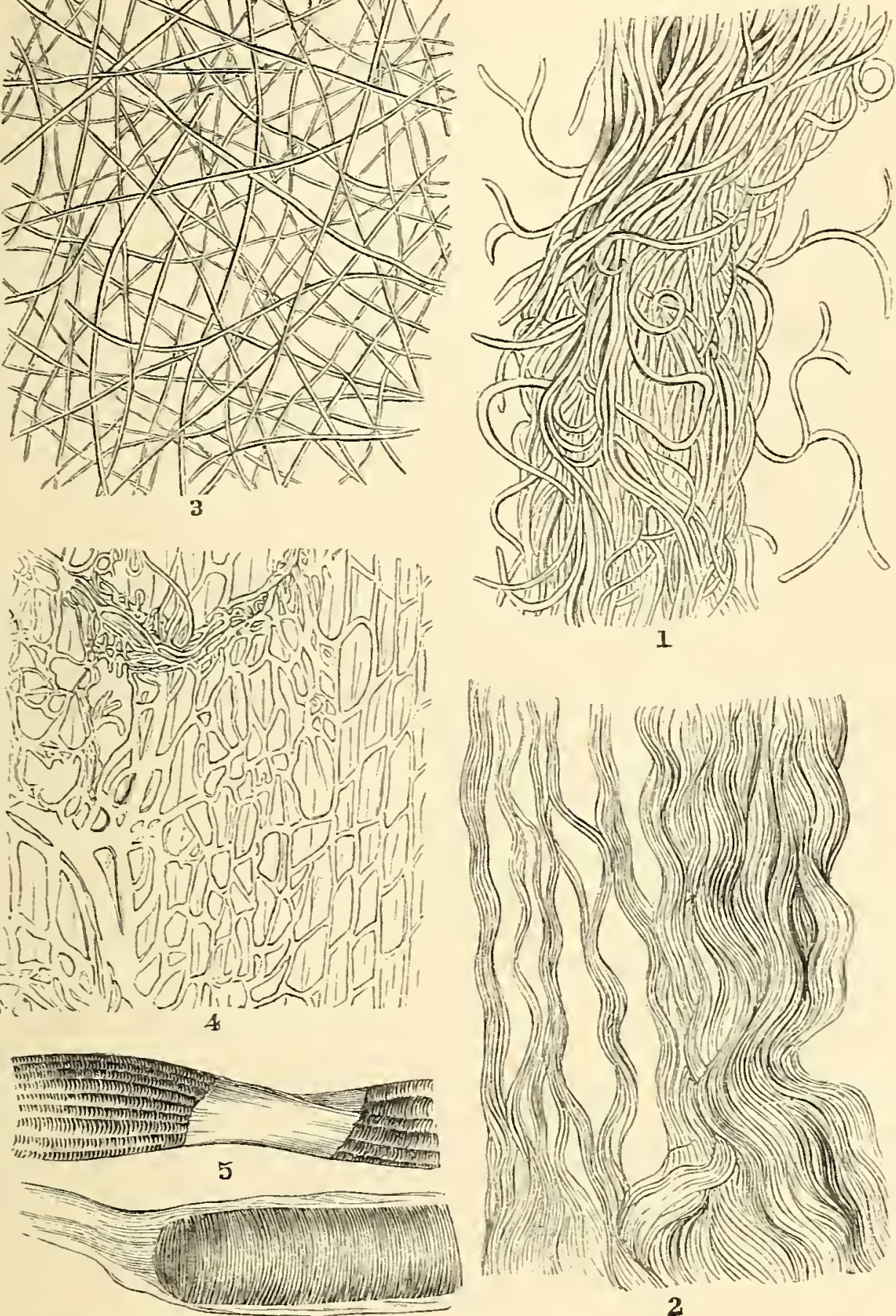

1
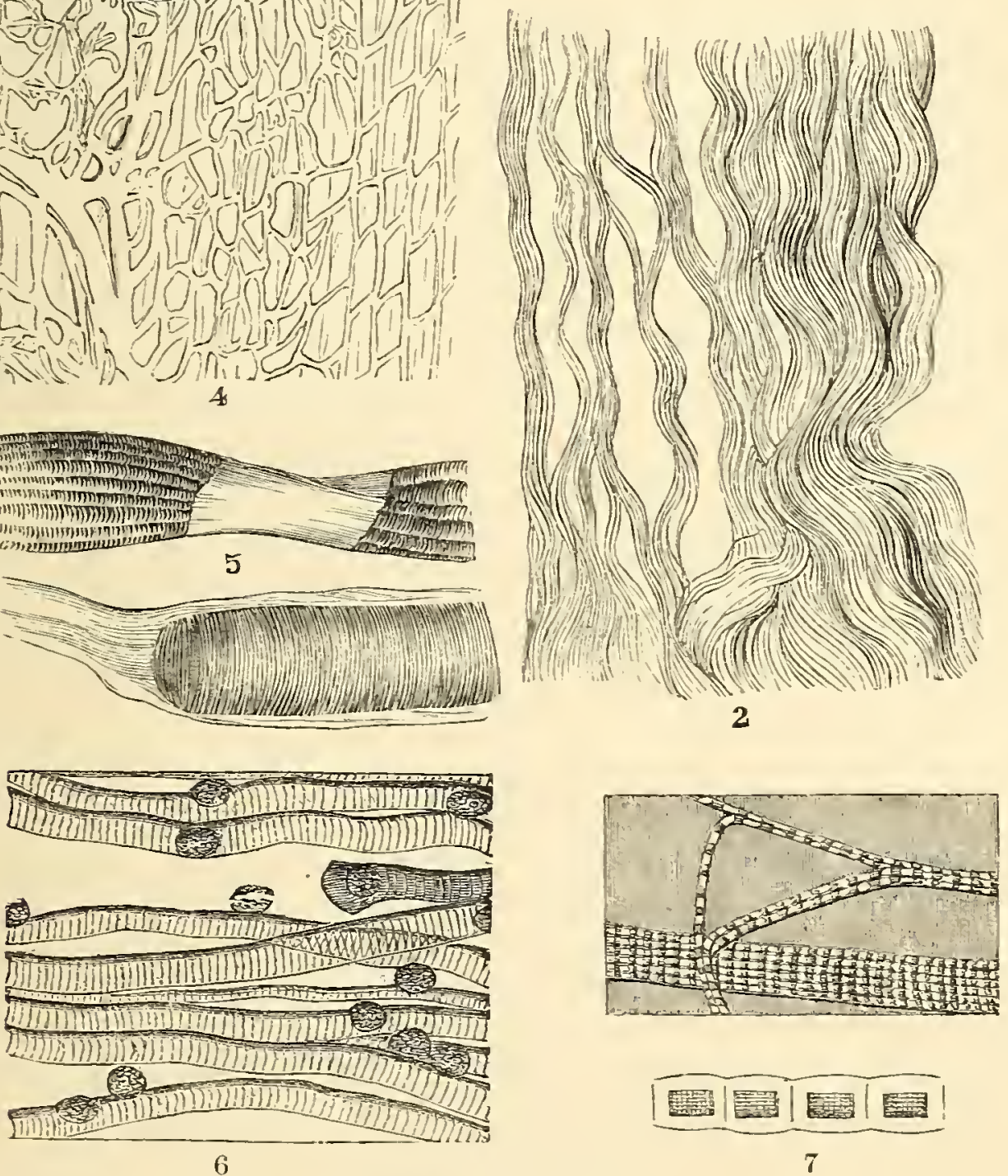

ANIMIAL TISSUES

1. Yellow-fibrous, or elastic tissue, taken near a livament. 2 . White-fibrous, or non-elastic tissue 3. Fibrous tissue, lining the interior of the egg-shell, the lime haring been remored by diluted hydrochloric acid. 4. White-fibrous tissue connecting the Tendons. 5. Bands of muscular fibre, connected together by structureless membrane, magnified 50 diameters. 6. Muscular fibre, broken up into irregular and distinct bands with a few blood-globules distributed about, magnified $₫ 50$ diameters. 7. Muscular fibre or a fasiculus of muscle taken from a young pig, magnificd 600 diameters. 

Mr. Gulliver accurately measured the blood-discs; and he found "that in the families of the Carnivora there is such a well-marked diversity in the size of the corpuscles, that the fact may be used as a help to classification: between the $\operatorname{dog}$ and the wolf the difference is not greater than that which exists among the varieties of the dog, whilst the discs of the fox are much smaller. The large size of the blood-discs in reptiles, especially in the Batrachia, has been of great service to the physiologist, by enabling him to ascertain many particulars regarding their structure which could not have been otherwise
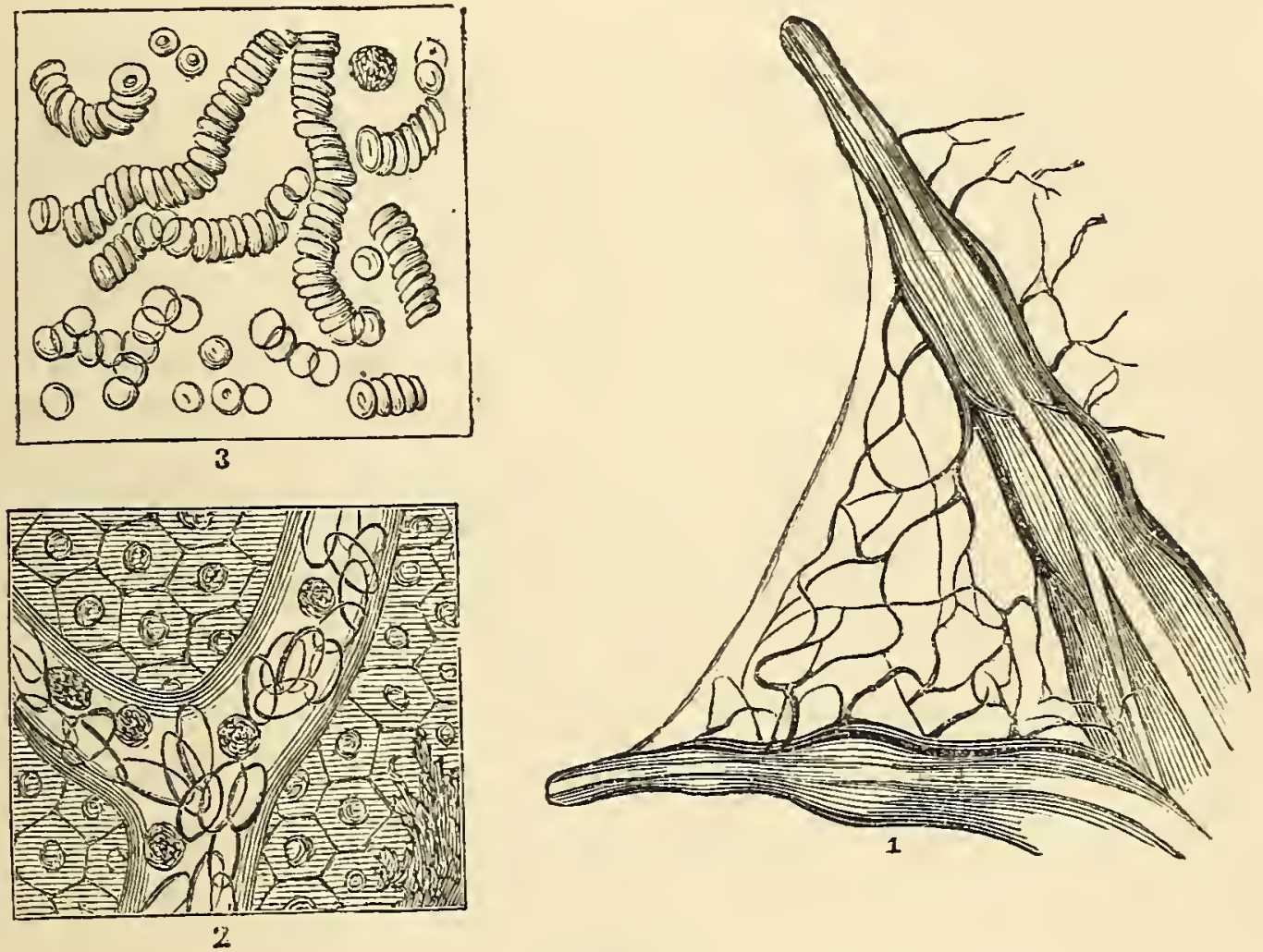

\section{fig. 137 .}

1. A portion of the web from a frog's foot, spead out and magnified to show the distribution of the blood-vessels. 2. A portion of same highly magnified, showing the ovid form of the blood-discs in the vessels. 3. Human blood-discs, showing the peculiar way in which they run together when fresh drawn.

determined with certainty. Among other facilities which this occasions, is that of procuring their separation from the other constituents of the blood; for they are too large to pass through the pores of ordinary filtering-paper, and are therefore retained upon it after the fluid part of the blood has flowed through."

It is not in the blood alone that cells floating in a fluid are found; but the chyle and lymph are colourless corpuscles flowing along their especially-adapted ducts and tubes, carrying the nutritive particles 
gathered from the food to the blood-vessels, for the reparation of the framework or growth that is so incessantly going on in the animal. In Plate XI. No. $3 b$, is seen the arrangement of the chyliferous tubes with their corpuscles, likewise enclosed in a structureless membrane.

\section{CRYSTALLISATION OF THE BLOOD.}

A new and very interesting subject has lately been observed-the production from the blood, in certain circumstances, of red "albuminous crystals," which, though formed of animal matter, and sometimes, in all probability, during life, have yet forms as regular as any inorganic crystals.

"It has been litherto held to be the rule, that crystallisation is peculiarly a property of inorganic chemistry, and that crystalline forms are met with in the animal economy only when the material giving rise to them has lost all claim to be considered as an integral part of the living tissues. The discovery of the crystallising power of the albuminous elements of red blood (hcematoidin of Virchow) has, however, made it probable that there are exceptions to this general law.

"Various authors, as Sir E. Home, Scherer, \&c., had described reddish crystals in blood which had been effused into tissues or organs; but Virchow was the first who paid particular attention to their actual nature, as different from saline or earthy crystals. He considers that the formation of these red crystals is the terminal step of the transformation of the colouring matter of the blood, which, first diffused, passes into the granular, then into the crystalline form; the crystals being yellowish or red, of a rhomboidal form, occasionally as large as the ordinary triple phosphate crystals in the urine, and forming spontaneously in extravasated blood, in the course of from a fortnight to three weeks after its escape from the vessels. Kölliker has advanced another step in this investigation, having detected the crystals in the interior of unbroken blood-corpuscles. In the blood of a dog's liver he found a great number of blood-globules, containing from one to five rodlets of a dark-yellow colour; and in unchanged corpuscles from the splenic blood the gradual formation of one or two of the crystals could be followed. Dr. Funke introduces the matter under yet a new aspect; he has succeeded in forming the crystals from the blood. If we add water to a drop of blood spread out upon the object-glass of the microscope, when the drop has just begun to dry up, the edges of the heaps of blood-corpuscles are seen to undergo a sudden change : a few corpuscles disappear, others receive dark thick edges, become angu- 
lar and elongated, and are extended into small well-defined rodlets. In this manner an enormous quantity of crystals are formed, which are too small to enable us to determine their shape; they rapidly move lengthways, the entire field of vision being gradually covered with a dense network of acicular crystals crossing one another in every direction, other crystals presenting the form of rhombic plates."

Dr. Garrod also discovered, that by a slow evaporation of portions of the serum of blood taken from patients labouring under rheumatism and gout, he could obtain strings of crystals of uric acid or urate of soda; indicating a certain state of disease, which may prove of great value to the medical man.

At No. 1, fig. 137, the foot of the frog is stretched out, to show the distribution of the blood-vessels in the web: the two sets of vessels - the arteries and veins-may be very readily made out when kept steadily on the stage of the microscope, as previously directed; the rhythm and valvular action of the latter may be observed, although they are much better seen in the wing of the long-eared bat, as pointed out by Mr. Wharton Jones.

The circulation in the foot of the frog and the tail of the newt is, for the most part, the capillary circulation. The ramifications of the minute arteries form a continuous network, from which the small branches of the veins take their rise. The point at which the arteries terminate and the minute veins commence cannot be exactly defined: the transition is gradual ; but the intermediate network is so far peculiar, that the small vessels which compose it maintain nearly the same size throughout; they do not diminish in diameter in one direction, like arteries and veins; hence the term capillary, from capillus, a hair. (See fig. 138.) The size of the capillaries is proportioned in all animals to that of the blood-corpuscles; thus, amongst the Reptilia, where the blood-corpuscles are the largest, the capillaries are also the largest: but it does not follow that they should be always of the same size in all the tissues of one and the same animal; for if we examine and carefully measure in the human subject their sizes in different tissues, we shall find that they vary greatly even in individual tissues; and, at a rough estimate, examples may occur as large as a thousandth, whilst others are so small as the four or five thousandth of an inch. They should be measured, if possible, in their natural state; when injected, their size is slightly increased; but when dried, they diminish so considerably, that in some specimens vessels imperfectly filled with injection have been known to shrink from the three to the twenty thousandth of an inch. 
Capillaries are, with very few exceptions, always supported by an areolar network, which serves not only as an investment to them, but connects them intimately with the tissues they are destined to supply.

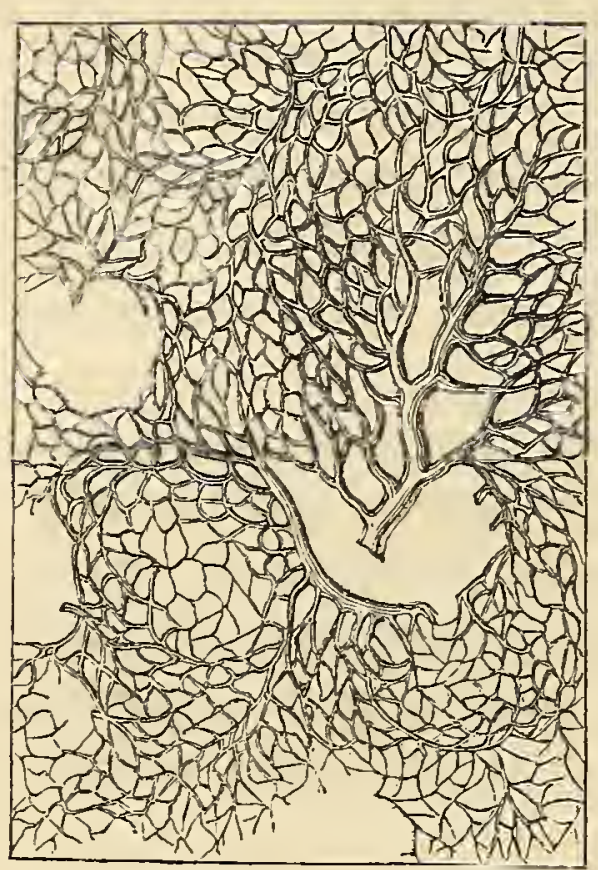

1

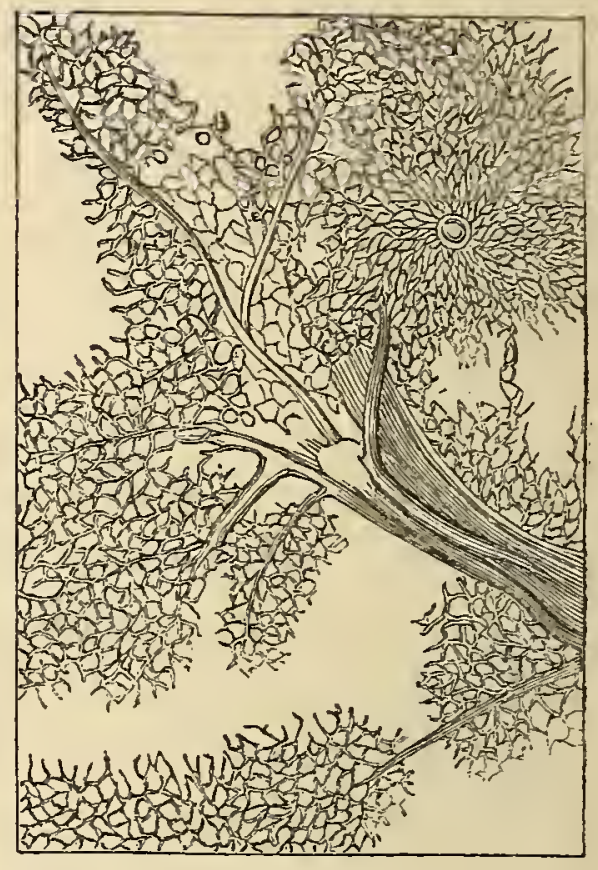

2

fig. 138.

No. 1 represents the fine network of air-tubes for supplying the lungs with air. No. 2, the network of capillaries for conveying the blood to the lungs.

There is a possibility, in first examinations, of mistaking or confounding capillaries with nerves, especially if the part under observation should have been left for some time in the strong preserving or alkaline solutions in the act of cleansing. A weak solution of caustic soda, and also another of acetic acid, are both made use of: the first is more generally available for the purpose of discovering nerves; the latter in tracing out vessels, structure of the papillæ, the unstriped muscle, \&c.; inasmuch as it renders their nuclei more obvious, while soda would make them less so. It is very useful sometimes to use these re-agents alternately; and the rule is, to apply them to the object only while under the microscope, so as to watch their gradual operation.

\section{FIBROUS TISSUE, ELASTIC AND NON-ELASTIC.}

In the higher animals this white and yellow fibrous tissue performs extensive and important duties. The yellow is elastic and of great strength, and consists of bundles of fibres which are highly elastic, 
represented in Plate XII. No. 2. The white, No. 1, though nonelastic, is of great strength, and of a shining silvery appearance.

"The two kinds of fibrous tissue differ from each other in many respects, but chiefly in their ultimate structure, their physical properties, and their colour: both are largely employed in the animal economy, but principally in those parts subservient to the organs of locomotion.

"The white fibrous tissue is (when perfectly cleared of the areolar) of a silvery lustre, and is composed of bundles of fibres running for the most part in a parallel direction; but if there be more than one plane of fibres, they often cross or interlace with each other: in some specimens it is difficult to make out the fibres distinctly, except in certain lights; and in these cases it appears that this tissue may be composed of a longitudinally striated membrane, which may be now and then split up into fibres.

"The white fibrous tissue is principally employed in the formation of ligaments and tendons, - a purpose for which it is admirably fitted on account of its inelasticity; it also is concerned in the formation of fibrous membranes, viz. the pericardium, dura mater, periosteum, perichondrium, the sclerotic coat of the eye, and all the different fasciæ. It is sparingly supplied with blood-vessels and nerves: the former always run in the areolar tissue, connecting the bundles of fibres together; but in the generality of the fibrous tissues the blood-vessels are not well seen, except in the dura mater and in the periosteum.

"The yellow fibrous tissue is highly elastic; it consists of bundles of fibres covered with, and connected together by, areolar tissue: the fibres are of a yellow colour, in some cases round, in others flattened; they are not always parallel, but frequently bifurcate and anastomose with other neighbouring fibres. It is always rather difficult to separate the fibres from each other; and when they are separated, the elasticity of each individual fibre is shown by its tendency to curl up at the end. The fibres in the human subject vary in diameter from the 1-5000tin to 1-10,000th of an inch. The acetic acid of ordinary strength does not act on the yellow fibrous tissue; nor after maceration in water or spirit for a very long time does its elasticity diminish. Very long boiling is said to extract from it a minute quantity of a substance allied to gelatine; but no nuclei or trace of cells can be seen in it when acetic acid is added: these, however, are readily shown when the white fibrous element is treated with this acid."**

* Professor Quekett, Nicroscopical Society's Transactions. 
The areolar tissue is one entering very largely into the construction of the animal body, its principal use being that of connecting or binding the tissues together; it may be said to be a modification of the two tissues just described: it exists more plentifully beneath the skin than elsewhere, and looks like a delicate web of white fibres. It is represented in Plate XII. Nos. 4 and 5, as crossing and recrossing of bundles of tissue.

\section{IIUSCULAR FIBRE.}

Muscular fibre is held together by a very delicate tubular sheath, nearly resembling the simple structureless membrane. It cannot always be discerned; but when the two ends are drawn asunder, it will be perceived to rise up in wrinkles; or the fragments of the torn muscle will be seen to be connected by the untorn membrane, as at No. 5, Plate XII. This membrane is termed Myolemma. It will be best seen by subjecting a piece of muscle to the action of fluids, such as diluted acetic or citric acid, or alkalies; which occasion it to swell, and it is then of easy separation. It has no share in the contraction of the muscle itself, which is made up of a series of bundles of highly elastic fibres : this may be seen upon reference to the greatly-magnified portions of one of these separated bundles at No. 6 ; and the ultimate structure of a fibre is shown under a magnifying power of 600 diameters at No. 7.

Here we may pause to observe the very remarkable similarity between this animal muscular fibre, and that of a portion of Conferve, when seen under a high magnifying power.

Dr. Barry has been for some time engaged in the examination of muscular fibre; and, from repeated observations, he believes it to be made up of a twin-spiral,- - that the flattened particles represented in No. 7, Plate XII. are in a state resembling rings, or those of the blooddises in fig. 137 ; each having its pellucid centre or nucleolus, which, when the outer part assumes the spiral form, is left behind; a line of such nucleoli being the foundation of future growth.

We cannot think Dr. Barry perfectly correct in his description; although it Las been truly observed by M. Wichura, "as a very remarkable phenomenon, that the circularly or heliacally acting forces of nature follow an unchanging, definite, lateral direction in their course. The planets describe heliacal lines winding to the right in space, by virtue of their circulation from west to east; since this is combined with the advance in company with the sun, towards a point in the 
northern hemisphere. In the department of physics we meet with allied phenomena in the circular polarisation of light, and in the course of electro-magnetic spirals. Organic life exhibits similar laws in the circulation of the blood, in all cases starting from the left side of the animal's body; and in the heliacal windings of the shells of molluscs, which follow a direction determinate for every species. But plants, above all, give evidence of a wonderful obedience to such laws, in the direction of the spiral vessels, the heliacally winding trunks of trees, winding stems and leaves; and probably, also, in the circulation of their saps."* In the lowest forms of animal life, and in that of insects, the spiral form will be seen to be most extensively distributed throughout; as may be observed by a reference to our several woodcuts.

We have another form of muscular fibre known as the non-striated, or involuntary. It consists of a series of tubes presenting a flattened appearance, without the transverse striæ so characteristic of the former; but elongated nuclei are developed immediately upon the application of a little diluted acetic acid. Wharton Jones, F.R.S., first demonstrated this structure, in his lectures at Charing Cross Hospital about 1843: he was led to infer from appearances in very young fibre, "that the striped muscular fibre is originally composed of similar elements to the unstriped, or plain muscular tissue; which, in the process of development, became enclosed in a sarcolemma (simple membrane) common to many of them: the fibres then split into smaller fibres (fibrillce). Thus accounting for the nuclei of striped muscular fibre; which, according to his views, are the persistent nuclei of the primitive muscular fibre-cells."

The non-striated fibre is beautifully seen in connection with the skin surrounding the hair, a few fibres of which are separately shown at No. 3, Plate XIII. Professor Kölliker originally described these muscles of the skin, of which there appear to be one or two in connection with each hair-follicle: that they arise from the more superficial parts of the outer skin, and may then be seen to pass down to the root of the hair, close behind the fat gland embracing it.

It is, indeed, remarkable that the skin, where covered with hair, should alone be provided with these muscular fibres; the effect of the contraction of which must be to thrust up the hair-follicles, and depress the intermediate portions of the skin, and thus produce that peculiar state of the surface well known as goose-skin, a condition of the skin before unaccounted for.

* M. Wichura on the "Winding of Leaves of Plants." 
In Plate XIII., No. 1, we have represented a vertical section of the skin under a $\frac{1}{4}$-inch object-glass.

The skin is the seat of two processes in particular; one of which is destined to free the blood of a large quantity of fluid, and the other to draw off a considerable amount of solid matter. To effect these processes, we meet with two distinct classes of glandulæ in its substance: the sudoriferous, or sweat glands; and the sebaceous, or oil glands. They are both formed, however, upon the same simple plan, and can frequently be distinguished only by the nature of their secreted product.

The sudoriferous or perspiratory glands form small oval or globular masses, situated just beneath the cutis, in almost every part of the surface of the body. Each is formed by the convolution of a single tube, which thence runs towards the surface, as the efferent duct, making numerous spiral turns in its passage through the skin, and penetrating the epidermis rather obliquely; so that its orifice is covered by a sort of little valve of scarf-skin, which is lifted up as the fluid issues from it. The convoluted knot of which the gland consists is copiously supplied with blood-vessels. On the palm of the hand, the sole of the foot, and the extremities of the fingers, the apertures of the perspiratory ducts are visible to the naked eye; being situated at regular distances along the little ridges of sensory papillæ, and giving to the latter the appearance of being crossed by transverse lines. On the sensitive layer they make a similar impression, dividing the ridges into little square allotments; each allotment being separated from that which precedes and follows it by the shaft of a perspiratory tube, as may be seen in the drawing. Mr. Erasmus Wilson says: "To arrive at something like an estimate of the value of the perspiratory system, in relation to the rest of the organism, I counted the perspiratory pores on the palm of the hand, and found 3528 in a square inch. Now, each of these pores being the aperture of a little tube of about a quarter of an inch long, it follows that in a square inch of skin on the palm of the hand there exists a length of tube equal to 882 inches, or $73 \frac{1}{2}$ feet. Surely such an amount of drainage as 73 feet in every square inch of skin-assuming this to be the average for the whole body-is something wonderful ; and the thought naturally intrudes itself, What if this drainage were obstructed? Could we need a stronger argument for enforcing the necessity of attention to the skin ?"*

The oil-glands of the skin are similar in structure to the perspira-

* Wilson on the Management of the Skin. 


\section{PLATE XIII.}

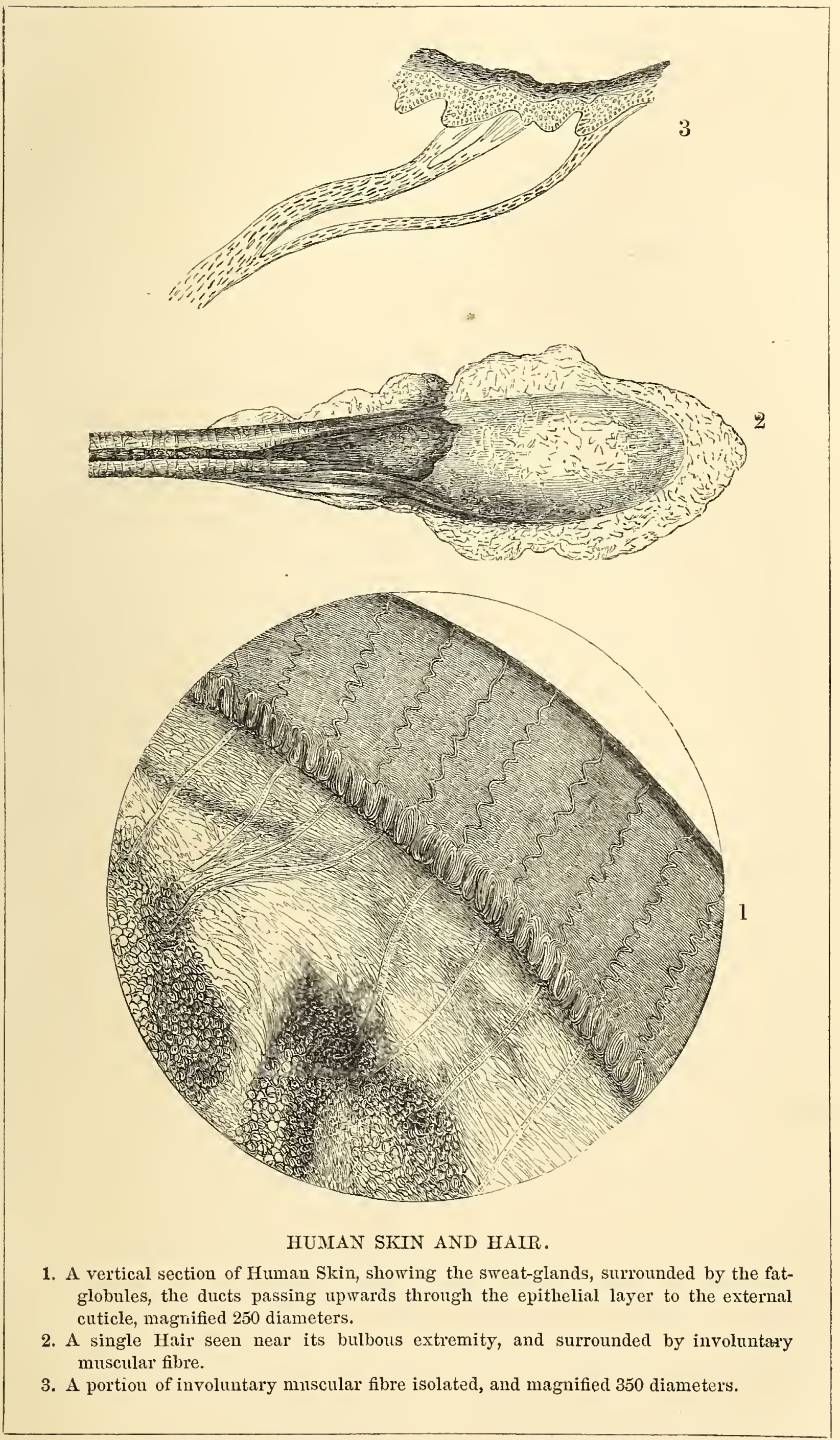



tory ducts, being composed of three layers, derived respectively from the scarf-skin, which lines their interior; the sensitive skin, which is the medium of distribution of their vessels and nerres; and the corium, with its fibres, which gives them strength and support. Like the perspiratory tubes, they are in some situations spiral; but this is not a constant feature; more frequently they pass directly to their destination; and they are also larger, as shown in the drawing, proceeding from the oil or fat resicle situated at its lower extremity. They are freely distributed to some parts, whilst in other's they are entirely absent: in a few situations they are worthy of particular notice; as in the eyelids, where they possess great elegance of distribution and form, and open by minute pores along the edges of the lids; in the earpassages, where they produce that amber-coloured substance known as the wax of the ears; and in the scalp, where they resemble small clusters of grapes, and open in pairs into the sheath of the hair, supplying it with a pomatum of Nature's own preparing.

The contents of the glands and oil-cells are derived from the blood flowing through the capillaries contained in the walls of the tubes and glands: when the cells are fully formed, that is, distended, they yield up their contents, and collapse into scales, termed epithelium-scales; the fluid matters being distributed to the skin as they pass along to the surface, constituting the unctuous element of the skin. The cells themselves are expelled from the tubes in company with their fluid products, and cast off in the form of minute flattened scales, like those of the scarf-skin, generally recognised as dandriff. The whole of the layer, through which the sweat-spiral proceeds to the surface, is almost wholly composed of these nucleated cells or scales in different stages of growth; those nearest to the surface being the oldest, are constantly removed by wear and tear, or by washing.

Sometimes, however, the contents of the cells are too dry and dense for this mode of escape; they then collect and obstruct the tube, causitg it to inflame and swell, when a small round spot of impacted matter is formed; and if this be squeezed between the fing'er-nails, a small mass of unctuous matter issues forth; or the maggot known as the Entozoon folliculorum, before referred to and represented in Plate X. No. 4.

These scales form the outer corering to the membranes of the body generally: we scrape them from the tongue and teeth as fur, in the forms represented in Plate XI., Nos. 1 and 2, called the tesselated or parement epithelium.

The cylindrical, or ciliated epithelium (Plate XI. No. $3 a$ ), is more 
especially found to be a covering to the surface of the mucous membranes of the stomach, intestines, and many other parts of the body; intended, no doubt, by its continual motion, to produce a current of the fluids; at the same time assisting in the absorption of the nourishing juices. On the other hand, they secrete and throw out the fluid known as mucus; which appears to be expressly formed for the protection of the internal membranes from the irritating particles of food, or other matters passing over them. The cilia seen on the epithelium are in appearance the same as those surrounding the volvox, the vorticella, and all other animals.

\section{H A I R.}

Of the endless variety of beautiful objects disclosed to us by means of the microscope, the hairs of different animals are not the least interesting; and of these none excel in beauty of structure those of the Bat tribe. According to physiologists, hair is developed in highly vascular sacs, or follicles, situated in the cutis or true skin. A hair commences at the bottom of the follicle, by a swelling termed the root or bulb; this is softer than the rest of the hair, is hollow, and contains within it the pulp, which is probably a vascular prolongation of the bottom of the follicle: each hair is formed by the aggregation of successive cytoblasts or new cells, and is gradually protruded from the follicle, both by the elongation of its constituent cells, and by the addition of new layers of these to its base; the apex and shaft of hair being formed before the bulb, just as the crown of a tooth is before its fang. The cytoblasts are round and loose at the base of the hair, but are more compressed and elongated in the shaft; and by this rectilinear arrangement the hair assumes a fibrous character (see human hair, Plate XIII. No. 2). Of sixteen species of the Bat tribe, the hairs of which have been examined by Professor Quekett, all were analogous in structure to Fig. 139, No. 1; and the curious surfaces which these hairs present were in reality owing to the development of scales on their exterior." By submitting hairs to a scraping process, minute scalelike bodies, tolerably constant as regards their size and figure, can be procured; so that Bat's hair may be said to consist of a shaft inverted with scales, which are developed to a greater or less degree, and vary in the mode of their arrangement in different species of the animal; that part of the hair nearest the bulb is nearly free from scales, but as we proceed toward the apex the scaly character becomes evident. Many of the scales are not unlike in shape those from the wings of 
butterflies, but are much more minute, and exhibit no trace of striæ on their surfaces; but those taken from dark-coloured hairs have colouring-matter deposited on them in small patches. In some cases they appear to terminate in a pointed process, like the quill observed in butterflies' scales; and in others the free margin is serrated. By scraping, many of them will be detached separately; but in some few cases as many as four or five will be found joined together: in the larger hairs the cellular structure of the interior, as well as the fibrous character of the shaft, can be well seen when the scales have been removed."

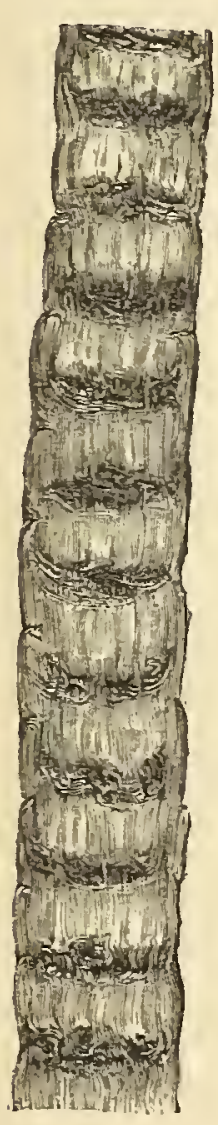

3

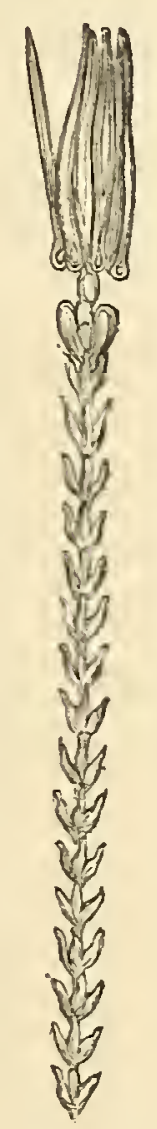

2

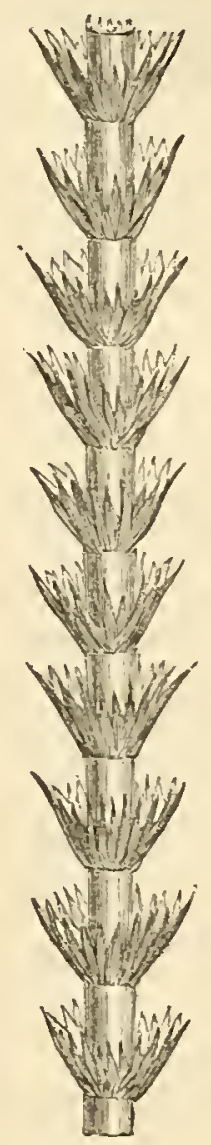

1

fig. 139.

1. Hair from the Indian Bat, magnified 500 diameters. 2. Hair from the Dermestes, magnified 250 diameters. 3. Hair from the Mouse, magnified 250 diameters.

The hair owes the greater part of its colour to what are termed pigment-cells: as these decay, and become gradually divested of the colouring-matter, they appear whitened without it, or turn grey. These hexagonal cells also give colour to the skin of the negro, and are seen situated immediately beneath the transparent coat. A small section is given in Fig. 140, No. 1: the vacant spaces denote the situation of the root of a hair. We have these black or pigment cells in other situations 
at No. 2 : they are represented taken from the back part of the eye of a sheep, placed there for the purpose of absorbing the rays of the light.
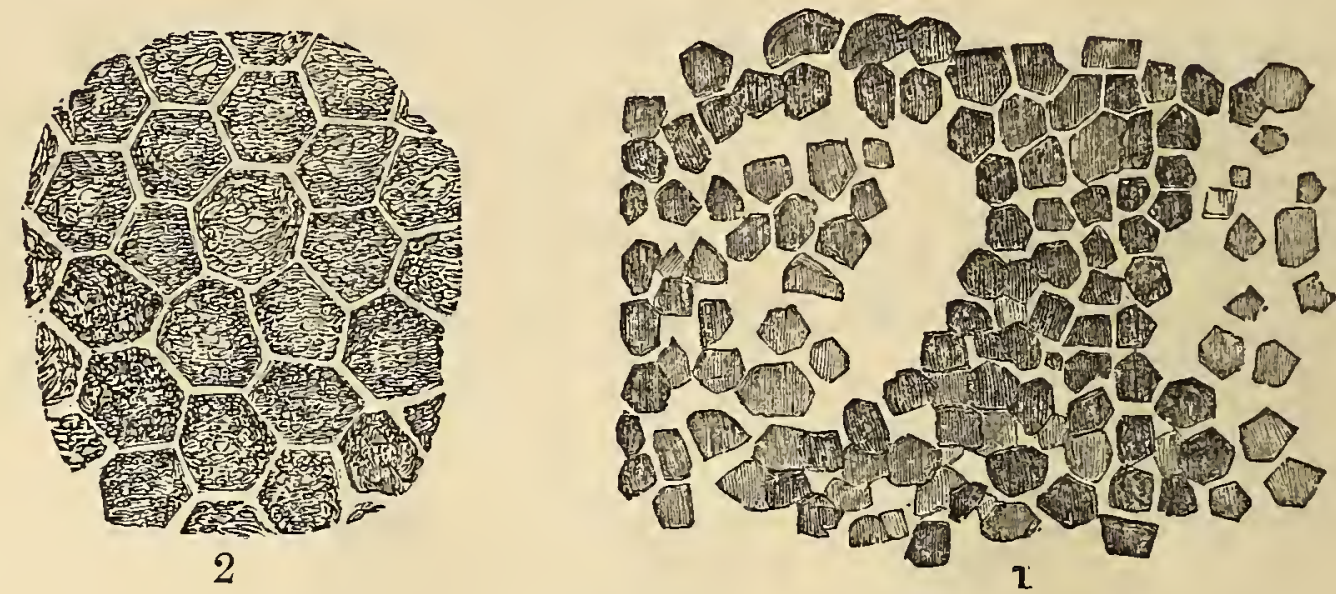

fig. 14?

NERVES.

The structure of these important fibres, upon which our sensations, perceptions, and indeed our whole existence depend, must be glanced at. Under the microscope, nerves will be seen to be minute fibres or tubules, full of nervous matter, arranged in bundles, connected by intervening fibro-cellular tissue, in which blood-vessels ramify. A layer of the same, or of strong fibrous tissue, also surrounds the whole nerve, and forms a sheath for it. The slight pressure of the thin glass, when placed on the nerve-fibre, causes nearly the whole of the contents to flow out in the form of a granular material; it therefore becomes requisite to exercise more care in the breaking up of structure, to view these tubules, which should be immersed in a very weak solution of spirit and water. As the nerves approach the brain or spinal cord, they gradually become smaller, and do not measure more than from $\frac{1}{10000}$ to $\frac{1}{14000}$ of an inch in diameter. Whilst the structural appearances and constitution of the brain and nervous system have a certain general similarity, there is yet an obvious divisibility of the tissues into two distinct kinds, the grey and the white matter; a divisibility which applies alike to the encephalon, the spinal cord, and the nerves. The difference in these nervous substances is not an affair of colour only; it refers also to their intimate structure and organisation: the white matter is made up of bundles of tubular fibres; whilst the grey is composed of aggregated cells, and is often denominated the vesicular neurine. To collections of this vesicular substance the term 'ganglion' is 
very generally applied; because the knots of nervous matter, which were formerly supposed to give origin to the nerves, and which are distributed so largely throughout the body, are vesicular in their composition. And thus the identity in structural constitution has led to the employment of the word ganglion as a common term; although the ganglionic or spheroidal form is not at all essential, as was at one time supposed, to the constitution of what is now called ganglionic substance. Physiological and pathological researches have rendered it more than probable that the vesicular and the fibrous substances have universally separate and distinct offices in the animal economy; the ganglionic structures being the source of functional change, and the fibrous matter being simply for the conduction of impressions originating in the former. This theory, in the promulgation of which $\mathrm{Mr}$. Solly shares probably in the most eminent degree, is now received very generally as scientific truth. The nerve-corpuscles and stellate nervefibres are represented in Plate XI. No. 10.

All the sensory ganglia, it may here be noticed, besides their instrumentality in inducing the simpler forms of consciousness, react upon the muscular system, when stimulated from without; and that, too, in apparent independence of thought or volition. The movements thus arising Dr. Carpenter very aptly designates consensual: they are seen when the dazzled eye withdraws instinctively from the light; or when the startle follows upon a loud and unexpected sound.

\section{CARTILAGE.}

Cartilage is usually described as a highly elastic and flexible substance, of a whitish-grey colour, sometimes approaching a jellow; forming the first framework of the earlier stages of existence, and the entire skeleton in some kinds of fishes, as the skate, lamprey, \&c. It is nourished without coming into direct contact with the blood-vessels, and is therefore said to be non-vascular, deriving nourishment by imbibition from the surrounding blood-vessels. In like manner does the frond of the alga draw into its interior the requisite nourishment for its growth from the surrounding fluid. When examined microscopically, the simplest form of cartilage is found to resemble in a striking mauner the cellular tissue of vegetables; it consists of an aggregation of cells of a spherical or oval figure, capable in some cases of being separated from each other, but every cell having a nucleus, with a nucleolus in its interior. In figs. 141 and 142 we have representations of some varieties of this structure. In the more highly advanced scale of 
animals a strong fibrous capsule or sheath surrounds the cartilage-cells, and some of the fibres dip in amongst the cells, and bind them firmly together. In those of the Ray and Shark kind, where the entire skele-

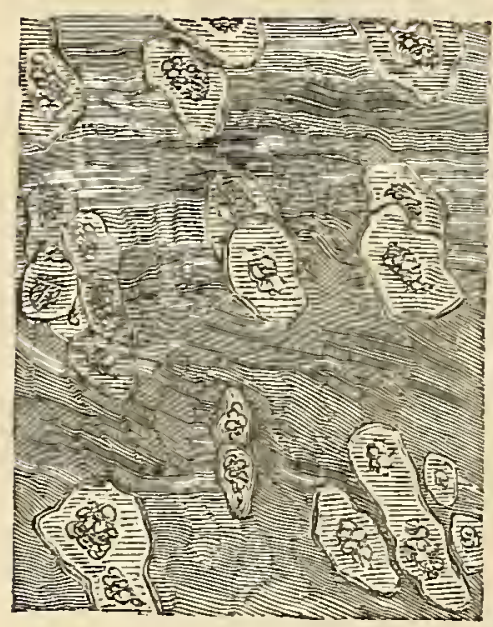

3

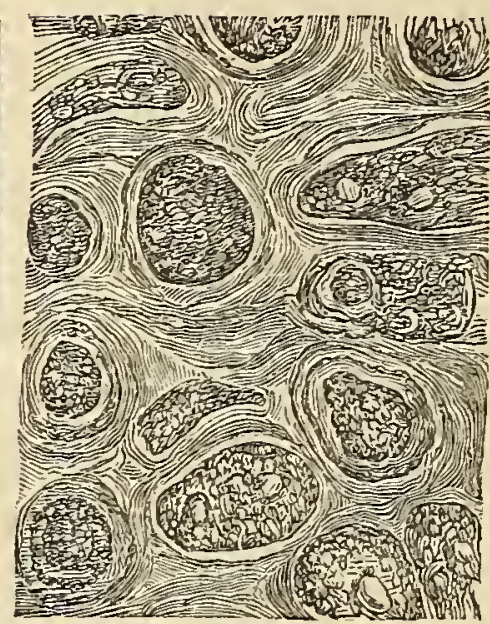

2

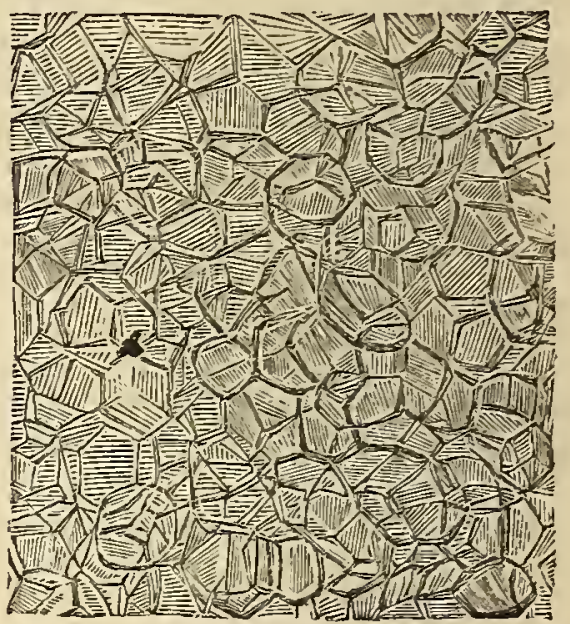

1

fig. 141 .

1. Cartilage from ear of mouse, resembling a section of regetable tissue, with several superimposed layers. 2. Cartilage from rabbit's ear, showing large cells imbedded in a fibrous matrix. 3. Cartilage from human ribs, with cells in groups, and each having a granular nucleus: magnified 200 diameters.

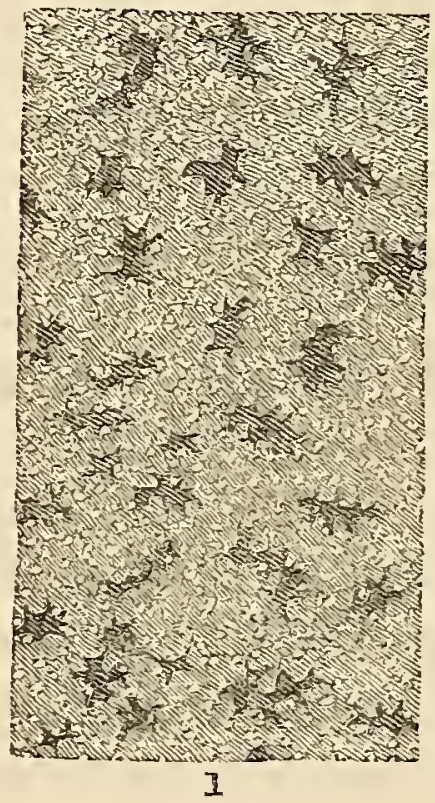

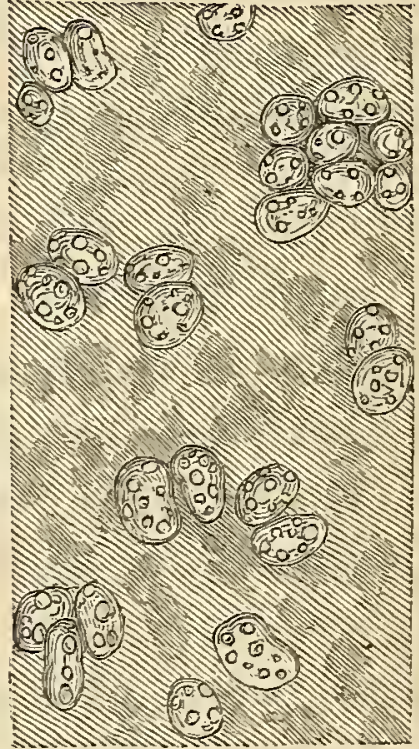

2

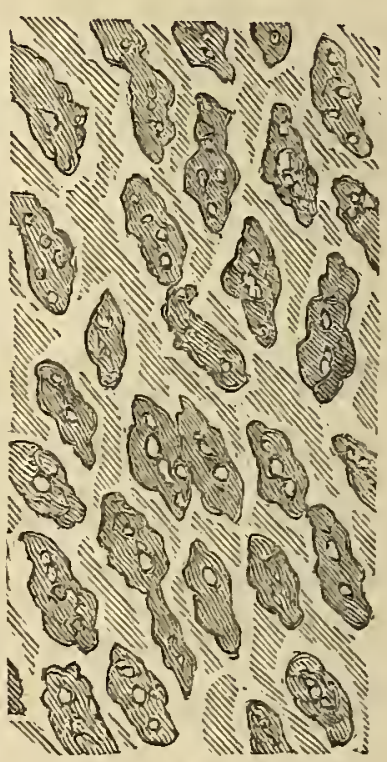

3

fig. 142.

1. Cartilage from the cuttle-fish, showing a peculiar form of cell. 2. Cartilage from the head of skate, with nucleated cells in clusters, and nucleoli in their interior. 3. Cartilage from the frog, and cells having nucleoli : magnified 250 diameters.

ton is cartilaginous, the cell is imbedded in a matrix, which may be strictly termed intercellular. The cells may be frequently or entirely isolated, as seen in the section from the ear of a mouse (Fig. 141, No. 1), 
and it then rarely or ever becomes converted into bone. In the higher animals it is generally invested by a fine and delicate membrane, termed the perichondrium, which brings the vessels into closer contact with the cartilage; and when in close contact with the extremities of bones, it is covered by a fringed membrane having a large number of vessels terminating in it for the purpose of supplying a lubricating fluid to the ends of the bones: this is the synovial membrane, a very beautiful structure when injected and viewed under the microscope.

In the early stages of existence, the framework, a very large proportion of it, is composed of cartilage, which, by the gradual addition of earthy matter, becomes consolidated into bone. The mode of development, and the change from one to the other, is represented in the section, Plate XI. No. 8 ; it will there be seen that the calcareous matter is deposited in straight lines, which stretch from the ossified surface into the substance of the matrix of the cartilage, and the amount of calcareous matter in which gradually diminishes as we recede from the ossified part. If the deposit has taken place to any great extent, the calcareous matter becomes crowded and consolidated; as the process advances, the bone thickens. A series of grooves, of a stellate figure, No. 9, are found upon its surface, which are gradually converted into closed canals for the passage of the blood-vessels.

\section{CONSOLIDATED TISSUES.}

These structures are formed by some chemical combination with the gelatine of the fibres; and the cells of the cartilage becoming consolidated by mineral deposits, the gradual transition that we have before referred to is the result. We have observed precisely the same change occurring in the calcified areolar structure of the skeleton coverings of the Echinodermata, and other Crustaceæ. Another example is presented to us in the mineral matter deposited in the cavities of cells in the shells of the mollusca, which we are enabled to separate, a consistent membrane being left after the carbonate of lime is dissolved out by an acid. We have the same arrangement occurring in the enamel of the tooth, the consolidating material being the phosphate in place of the carbonate of lime. A section of a molar-tooth is shown in fig. 143, the dark marginal line representing the enamel; the dotted border the dentine traversed by tubuli; and the radiating crystalline portion the true bone, made up of a series of minute chambers, with a central aperture for the blood-vessel, and for the purpose of conveying the nutritive fluid to the tooth. This bone, or cementum, of the tooth, is com- 
posed of a mass of earthy matter and cartilage, with bone corpuscies; and dentine is stated by Professor Owen to be an ossification of the pulp of the tooth.

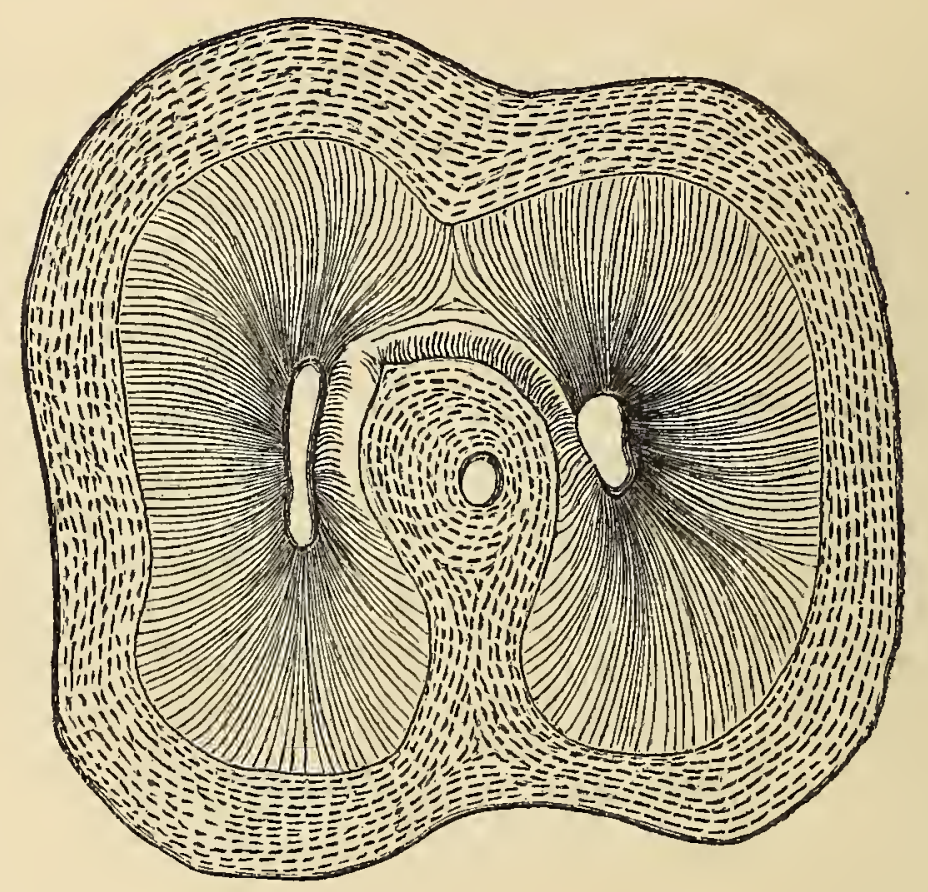

fig. 143.

A transverse section of a human molar or double tooth, magnified 50 diameters.

Czermak discovered that the curious appearances of globular conglomerate formations in the substance of dentine, are entirely dependent on its mode of calcification; and he attributes the contour lines to the same cause. Contour markings vary in intensity and number; they are most abundant in the root, and most marked in the crown. Vertical sections exhibit them the best. In preparing the specimen, first make the section accurately, then decalcify it by submersion in dilute muriatic acid. It should then be dried and mounted in Canada balsam, with continued heat, so as to allow the specimen to soak in the fluid resin for some time before it cools. It;is the white opacity of the extremity of the contour markings that produces the appearance of rings on a tooth-fang.

"The tooth-substance appears," says Czermak, "on its inner surface, not as a symmetrical whole, but consisting of balls of various diameter, which are fused together into a mass with one another in different degrees, and on which the denterial tubes, in contact with the germ cavity, are terminated. By reflected light one perceives this stalactite-like condition of the inner surface of the tooth-substance very distinctly, by means of the varied illumination of the globular elevations, and by the shadows which they cast. Here one has evidently to do 
with a stage of development of the tooth-substance; for the older the tooth is, the less striking in general are these conditions, and the more even is the surface of the wall of the germ-cavity. In very old teeth considerable unevennesses again make their appearance; these, however, are not globular, but have a cicatrised, distorted appearance. It is best to make the preparation from a tooth of which the root is not perfectly completed. With such preparations one is readily convinced that the ground-substance of the last-formed layer of the tooth-substance appears, at least partly, in the form of balls, which are fused among one another, and with the balls of the penultimate layers; and one also perceives that in general their diameter becomes less and less, somewhat in the form of a point, towards the periphery of the toothsubstance. The majority of these balls are pierced through by one or more tubes, crosswise, from within outwards. Very frequently, however, they appear homogeneous, and contain no tubes." To obtain specimens, procure a tooth of which the fang is half grown; then introduce the point of a penknife into its open extremity, and scraping the inner surface, small portions may be detached which exhibit the globules admirably.

"Another method of obtaining specimens which further illustrate the internal surface of the dentine is the following: In rubbing down a section of a tooth, as the operator approaches the pulp-cavity, the last fibre of dentine frequently bulges into the unresisting cavity; and, instead of grinding up into particles, comes away in a little sheet, a little film of dentine parallel with the pulp-cavity's surface, the innermost layer, and the one last formed. This should be carefully preserved and mounted. On viewing such a specimen by transmitted light, one sees the globules scattered about-some isolated, others more or less confluent; and between them a pale, rather indefinite structure, uniting the whole into a sheet.

"But the most instructive specimens are to be obtained from the very thin cap of dentine found upon the fotal pulp. The thin edge should be cut off, and examined on the inner surface; it should be moist, and never allowed to get dry. In such specimens the globules are very apparent; but, as Czermak observes, they do not appear supericial, but in the substance of the dentine. It is here, in the moist specimens, that the focus reaches the globules; and, consequently, there is no superficial stalactite, like bulgings of globules; it is only in dry specimens that that is seen. Now, if such a specimen be sieeped in dilute muriatic acid, so as to remove all the earthy materials, the globules instantly vanish, and the dentine where they were seen assumes the same 
aspect as that where they were not seen. No other change is produced. The existence of the globules, therefore, seems dependent upon the presence of earthy material. This suggested to Czermak the idea that the organic material of dentine is, during the calcifying process, impregnated with earthy salts in globular forms; and that, by a deeper degree of calcific impregnation, the whole tissue is imbued with the hardening element, and the globules are fused. Such a doctrine is capable of explaining all the circumstances of the case ; and we have only to imagine an arrest of calcification at the globular stage, over the surface of the pulp as it exists at any one time, to explain all the phenomena of the contour markings." *

\section{BONE.}

Professor Owen has said, "If the microscope be essential to the full and true interpretation of the vegetable remains of a former world,

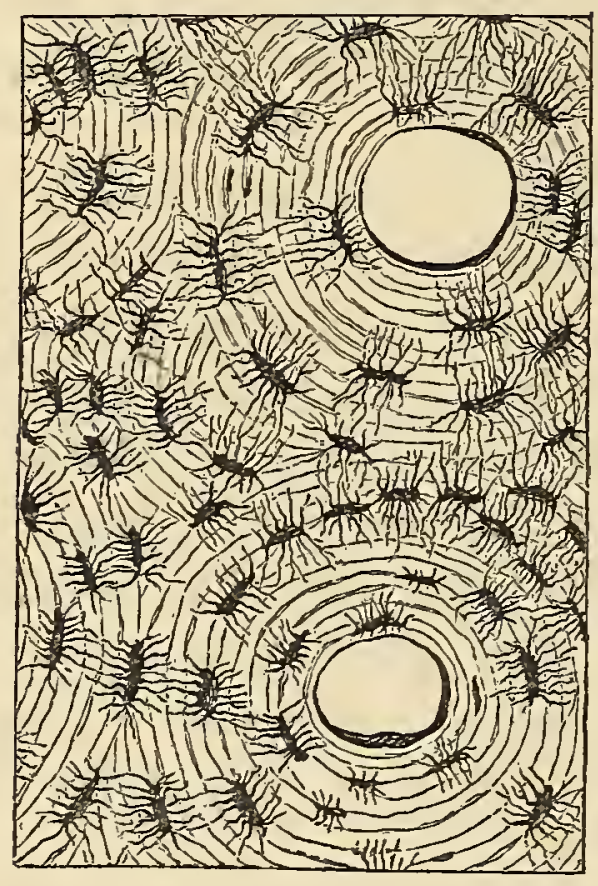

1

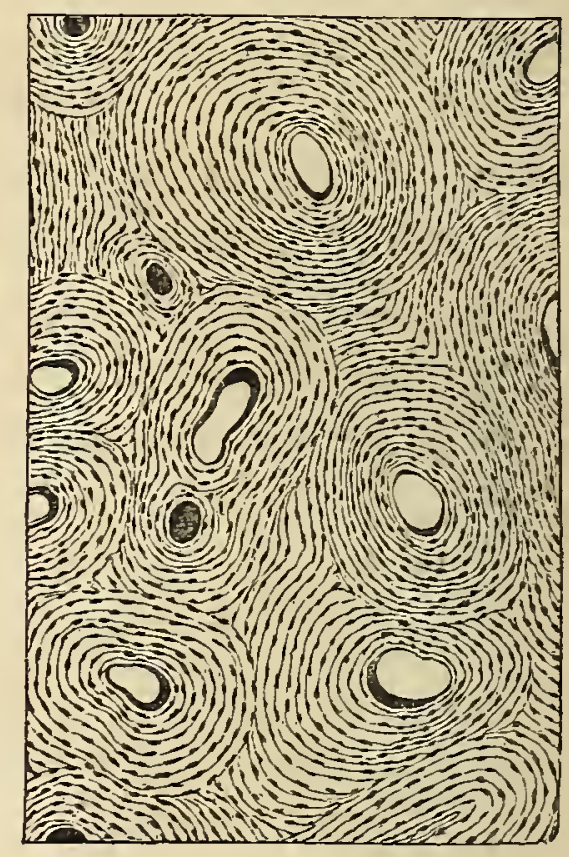

2

fig. 144.

1. A transverse section of the human clavicle, or collar-bone, magnified 95 diameters ; which exhibits the Haversian canals, the concentric laminæ, and the concentric arrangement of bone-cells around them. Some of the Haversian canals are white, others black : the latter are filled with a deposit of opaque matter, used in the grinding and polishing the section. When viewed under a lower power, they appear to be only a series of small black dots, as shown in No. 2 .

it is not less indispensable to the investigator of the fossilised parts of animals. It has sometimes happened that a few scattered teeth have

* James A. Salter, M.B., Quarterly Journal of Microscopical Science, July 1853. 
been the only indications of animal life throughout an extensive stratum; and when these teeth happened not to be characterised by any well-marked peculiarity of external form, there remained no other test by which their nature could be ascertained than that of the microscopic examination of their intimate tissue. By the microscope alone could the existence of Keuper-reptiles in the lower sandstones of the new red system, in Warwickshire, have been placed beyond a doubt.

"By the microscope, the supposed monarch of the Saurian tribesthe so-called Basilosaurus - has been deposed, and removed from the head of the reptilium to the bottom of the mammiferous class. The microscope has degraded the Saurocephalus from the class of reptiles to that of fishes. It has settled the doubts entertained by some of the highest authorities in palæontology as to the true affinities of the gigantic Megatherium; and by demonstrating the identity of its dental structure with that of the Sloth, has yielded us an unerring indication of the true nature of its food."

Fronr an excellent paper on the "Intimate Structure of Bone," by Professor Quekett,* we extract the following; and from it may be seen the useful results to be obtained by the aid of the microscope:

"Every bone may be said to consist of two parts, a hard and a soft part: the hard is composed of carbonate and phosphate of lime, and of carbonate and phosphate of magnesia, deposited in a cartilaginous or other matrix; whilst the soft consists of that matrix, and of the periosteum which invests the outer surface of the bone, and of the medullary membrane which lines its interior or medullary cavity, and is continued into the minutest pores. If we take for examination a long bone of one of the extremities of the human subject, or of any mammalian animal, we shall find that it consists of a body or shaft and two extremities : if a vertical section of such a bone be made, we shall also find that the middle of the shaft contains a central cavity, termed the medullary cavity, which extends as a canal throughout the whole of it, or else is entirely or partially filled up with a cellular bony structure, which cells are termed cancelli, and the structure a cancellated structure. On a more careful examination of the bony substance, or shaft, we shall find it to be slightly porous, or rather occupied, both on its external and internal surfaces, by a series of very minute canals, which, from their having been first described by our countryman Clopton Havers, are termed to this day the Haversian canals, and serve for the transmission of blood-vessels into the interior of the bone. Further than this we cannot proceed without optical assist-

\footnotetext{
* Microscopical Society's Transactions, 1816.
} 
ance; but if now a thin transverse section of the same bone be made, and be examined by the microscope with a power of 200 linear, we shall see the Haversian canals very plainly, and around them a series of concentric bony laminæ, from three to ten or twelve in number. If the section should consist of the entire circle of the shaft, we shall notice, besides the concentric laminæ round the Haversian canals, two other series of laminæ, the one around the outer margin of the section, the other round the inner or medullary cavity. Between the laminx is

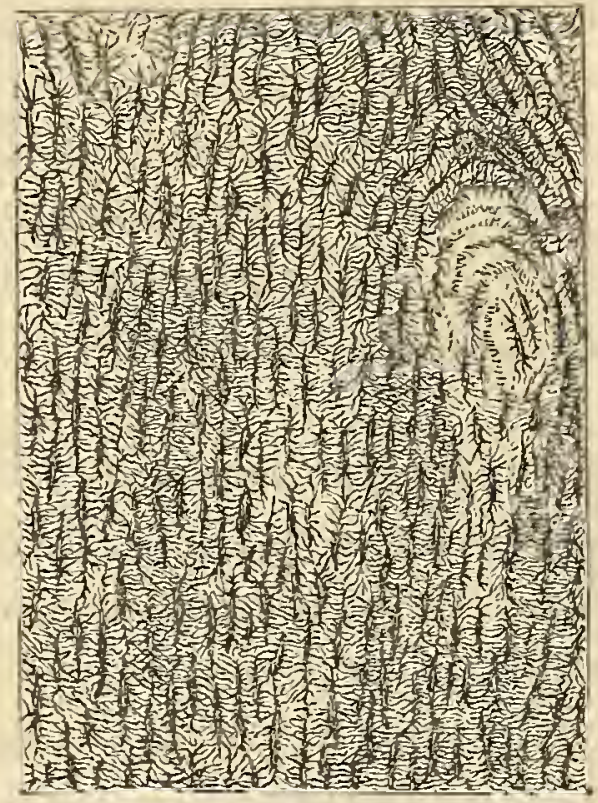

I

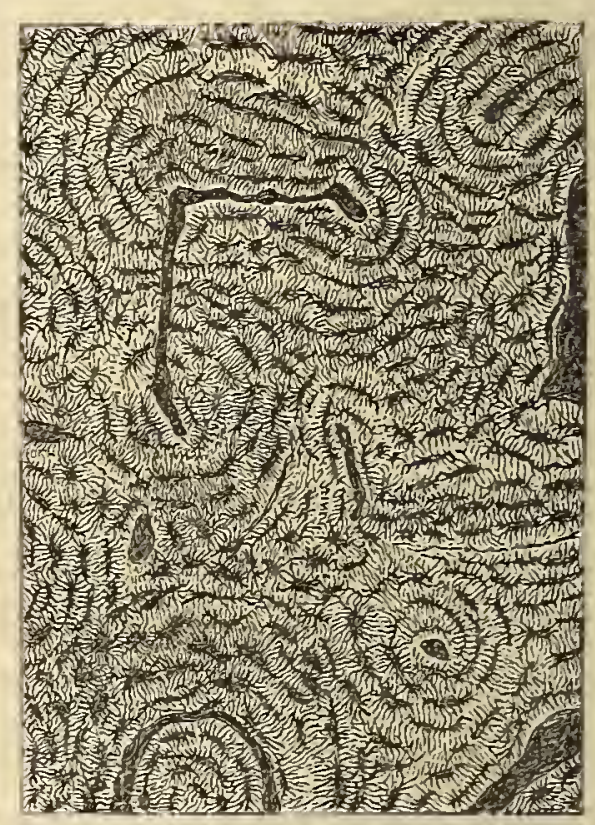

2

fig. 145.

1. A transverse section of the humerus, or fore-arm bone, of a Turtle (Chelonia mydas). It exhibits traces of Haversian canals, with a slight tendency to a concentric arrangement of bone-cells around them. The bone-cells are large and very numerous, but occur for the most part in parallel rows.

2. A transverse section of the femur, or leg-bone, of an Ostrich, magnified 95 diameters. When contrasted with the preceding figure, it will be noticed that the Haversian canals are much smaller and much more numerous, and many of them run in a transverse direction.

situated a concentric arrangement of spider-like looking bodies, which have, by different authors, received the names of osseous corpuscles, lacunæ, or bone-cells, according as to whether they were ascertained to be solid or hollow : these bone-cells have little tubes or canals radiating from them, which are termed canaliculi by-some authors, and tubes and pores by others: those bone-cells which are nearest the Haversian canals have the canaliculi of that side radiating towards and opening into the Haversian canals ; whilst the canaliculi of the opposite side communicate with those of the layer of canaliculi more external to them; and those in the outer row have most of their canaliculi given 
off from that side of the bone-cell which is nearest its own Haversian canal: hence arises the transparent white line which often may be noticed as surrounding each concentric system of laminæ and bonecells : in some cases, however, part of the bone-cells of the external row anastomose with another series of bone-cells, which are situated between the concentric laminæ. The average length of the lacunæ or bone-cells in the human subject is the 1-2000th of an inch; they are of an oval figure, and somewhat flattened on their opposite surfaces, and

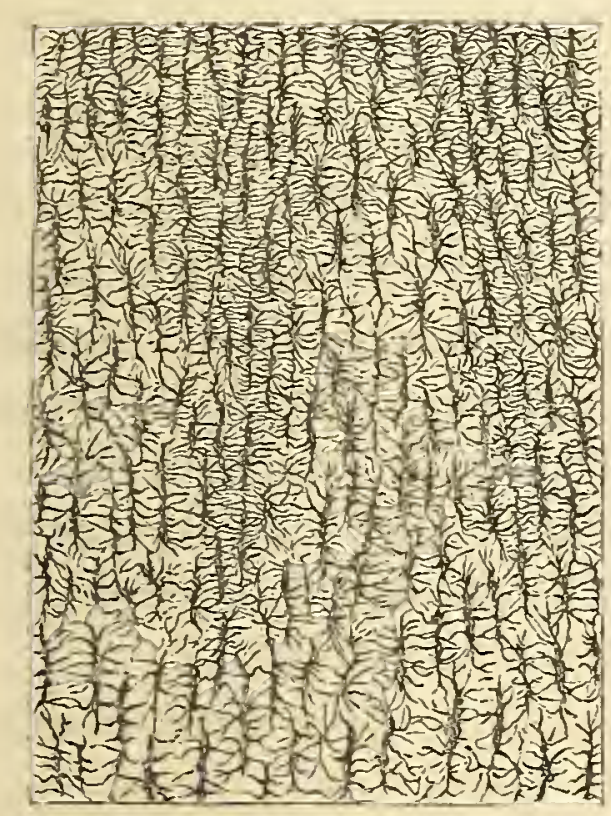

1

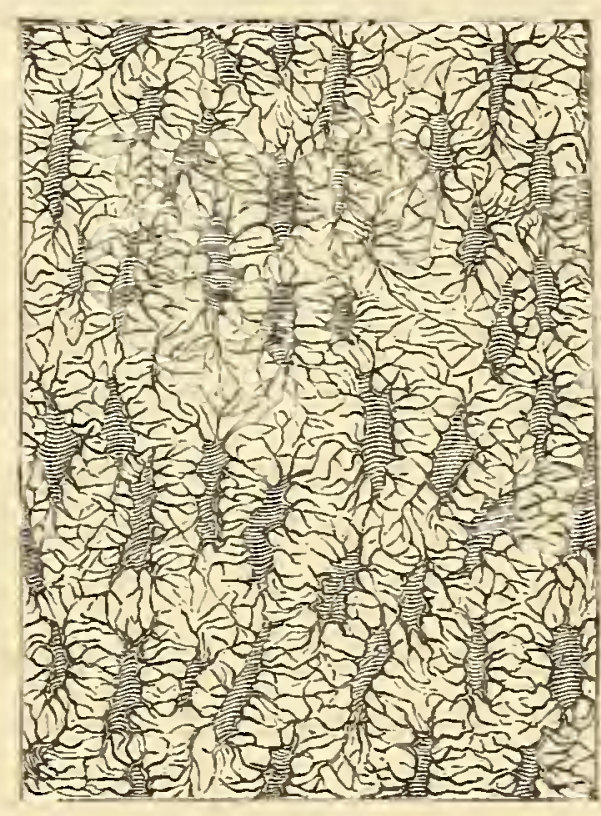

2

fig. 146 .

1. A horizontal section of the lower jaw-bone of a Conger eel, which exhibits a single plane of bone-cells arranged in parallel lines. There are no Haversian canals present; and when this specimen is contrasted with that of fig. 145, No. 1, it will be noticed that the canaliculi given off from each of the bone-cells of this fish are rery few in number in comparison with that of the reptile.

2. A portion of the cranium of a Siren (Siren lacertina), which is remarkable for the large size of the bone-cells and of the canaliculi, they being larger in this animal than in any other yet examined. As in the preceding specimen, no Haversian canals are present.

are usually about one-third greater in thickness than they are in breadth; hence, as will be presently shown, it will become necessary to know in what direction a specimen is cut, in order to judge of their comparative size. The older anatomists supposed them, from their opacity, to be little solid māsses of bone; but if the section be treated with spirits of turpentine coloured with alkanet-root, or if it have been soaked in very liquid Canada balsam for any great length of time, it can then be unequivocally demonstrated that both these substances will gain entrance into the bone-cells through the canaliculi. The bone- 
cells, when viewed by transmitted light, for the most part appear perfectly opaque; and they will appear the more opaque the nearer the section of them approaches to a transverse one : for when the cells are cut through their short diameter, they are often of such a depth that the rays of light interfere with each other in their passage through them, and darkness results ; whereas if the section be made in the longdiameter of the cells, they will appear transparent. When viewed as an opaque object, with a dark ground at the back and condensed light, the bone-cells and canaliculi will appear quite white; and the intercellular substance, which was transparent when viewed by transmitted light, is now perfectly dark.

"Thus much may be said to compose the hard part of the bone; we must now turn our attention to the soft part. This, as has been before stated, consists of the periesteum, which invests the outer, and of the medullary membrane, which invests the inner surface, lines the $\mathrm{Ha}$ versian canals, and is continued from them, through the canaliculi, into the interior of the bone-cells; and of the cartilaginous or other matrix, which forms the investment of the minute ossific granules. The earthy matter of the bone may be readily shown by macerating the section for a short time in a dilute solution of caustic potash.

"The animal matter may be procured by using dilute hydrochloric acid instead of caustic potash, when the earthy matter will be removed, and the section will exhibit nearly the same form as when the earthy constituent was present; and when viewed microscopically, it will be noticed that all the parts characterising the section previous to its maceration in the acid will be still visible, but not so distinct as when both constituents were in combination. When, however, the animal matter is removed, the bone will not exhibit the cells and the canaliculi, but will be opaque and very brittle, and exhibit nothing but the Haversian canals and a granular structure.

"If we consider what has been already mentioned as entering into the composition of a bone, viz. the medullary cavity, the Haversian canals, the canaliculi, and the bone-cells, we shall find that every part thus described has been more or less hollow; where, then, is the true bony substance? This is no other than the small granules of ossific matter which are situated between the canaliculi of the bone-cells, each granule having an investment of soft animal matter, by which the whole mass of granules is kept in firm apposition.

"The parts, then, which a transverse or a longitudinal section of a long bone of a mammalian animal will exhibit will be the Haversian canals, the concentric bony laminæ, the bone-cells and their canaliculi; 
although all these parts, except the bony laminæ, may be seen in all mammalian bones. Whether long or otherwise, they are, nevertheless, so differently arranged in the flat bones, such as those of the skull, and in the irregular bones, such as the vertebræ, as to require a short description at this stage of our inquiry.

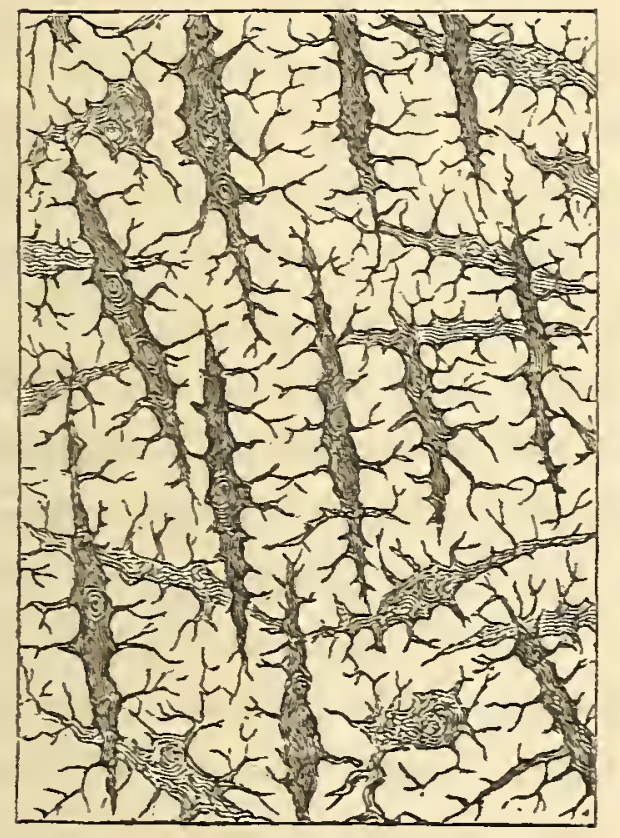

1

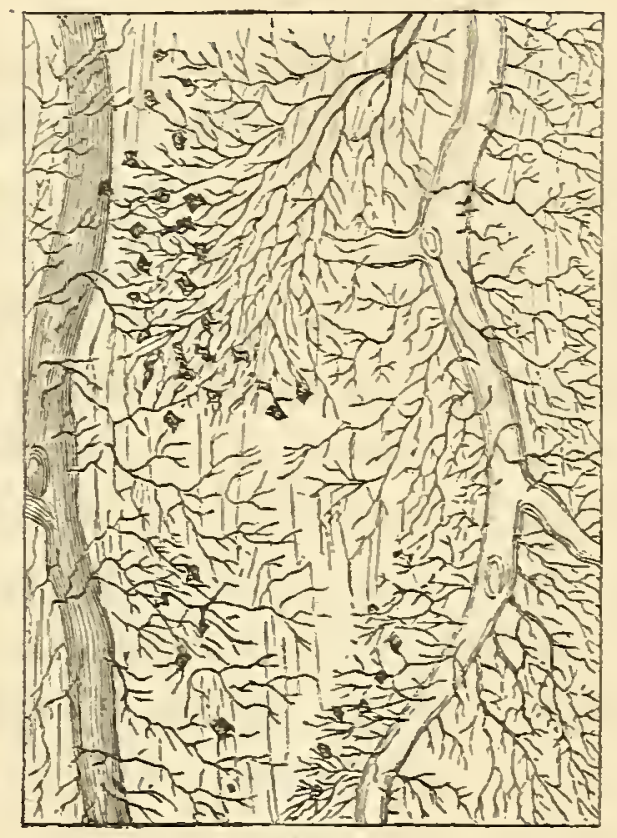

2

147.

1. A small portion of bone, taken from the exterior of the shaft of the humerus of a Pterodactyle, which exhibits the elongated bone-cells characteristic of the orders of Reptilia.

2. A horizontal section of a scale, or flattened spine, from the skin of a Trygon, or Sting Ray, which exhibits large Harersian canals, with uumerous wary parallel tubes, like those of dentine, communicating with them. It will be noticed that this specimien shows, besides these rary tubes, numerous bone-cells, whose canaliculi communicate with the tubes, as in many specimens of dentine.

"The bones of the cranium are in all cases composed of two thin layers of compact texture, which inclose another layer of variable thickness, which is cellular or cancellated. The two outer layers are called tables, - the one being the outer, the other the inner table; and the middle or cancellated layer is termed the diploe : in this last the principal blood-vessels ramify. The outer table of the skull is less dense than the inner; the latter, from its brittleness, is termed by anatomists the vitreous table. When a vertical section of a bone of the skull is made so as to include the three layers above mentioned, bone-cells may be seen in all, but each of the three layers will differ in structure : the middle or cancellated structure will be found to resemble the cancellated structure in the long bones, viz. thin plates of bone, 
with one layer of bone-cells without Haversian canals; the outer layer will exhibit Haversian canals of large size, with bone-cells of large size, and a slightly laminated arrangement; but the inner or vitreous layer will be found to resemble the densest bone, as the outer part of the shaft of a long bone for instance, and will exhibit both smaller Haversian canals, and more numerous bone-cells of ordinary shape around them.

"A transverse section of the long bone of a bird, when contrasted with that of a mammal, will exhibit the following peculiarities: the Haversian canals are much more abundant, and much smaller; and they often run in a direction at right angles to that of the shaft, by which means the concentric laminated arrangement is in some cases lost; the direction of the canals often follows the curve of the bone; the bone-cells also are much smaller and much more numerous; but the number of canaliculi given off from each of the cells is much less than from those of mammals : the arerage length of a bone-cell of the ostrich is 1-2000th of an inch, the breadth 1-6000th.

"In the Reptilia, the bones may be either hollow, cancellated, or solid; and, generally speaking, whichever form prevails, the bone may be said to be very compact and heavy, but the specific gravity not so great as that of birds or mammals.

"The short bones of most of the Chelonian reptiles are solid, but the long bones of the extremities are either hollow or cancellated; the ribs of the serpent tribe are hollow, the medullary cavity performing the office of an Haversian canal; the bone-cells are accordingly arranged in concentric circles around the canal. The vertebræ of these animals are solid; and the bone, like that of some of the birds, is remarkable for its density and its whiteness. When a transverse section is taken from one of the long bones, and contrasted with that of a mammal or bird, we shall notice at once the difference which the reptile presents : there are very few, if any, Haversian canals, and these of large size; and at one view, in the section, we shall find the canals and the bone-cells arranged both vertically and longitudinally: the bone-cells are most remarkable for the great size to which they attain; in the turtle they are 1-375th of an inch in length : the canaliculi, too, are extremely numerous, and are of a size proportionate to that of the bone-cell.

"In fishes we have a greater variation in the minute structure of the skeleton than in either of the three classes already noticed; and there are certain remarkable peculiarities in the bones of fishes which are so characteristic, that a bone of one of these creatures can never be confounded with that of any animal of a higher class, when once the true 
structure has been satisfactorily understood. We lose now those character's which were evident in mammals and birds, and often, to a certain degree, in reptiles, viz. the concentric laminated arrangement of the bony matter, with a corresponding concentric arrangement of bone-cells around the Haversian canals; and we shall find that the true Haversian canals may be even absent in some fishes, their place being supplied by numerous layers of bone-cells. Of all the varieties of structure in the bones of fishes, by far the greater number exhibit nothing more than a series of ramifying tubes, like those of teeth; others exhibit Haversian canals, with numerous fine tubes or canaliculi, like ivory tubes, connected with them; others consist of Haversian canals, with fine tubes and bone-cells; whilst a rare form, found only as yet in the sword of the Swordfish (Istiophorus), exhibits Haversian canals and a concentric laminated arrangement of the bone, but no bone-cells. The Haversian canals, when they are present, are of large size, and very numerous, and then the bone-cells are, generally speaking, either absent or but few in number ; their place being occupied by tubes or canaliculi, which are often of larger size. The bone-cells are remarkable for their graduate figure, and for the canaliculi which are derived from them being few in number ; they are readily seen to anastomose freely with the canaliculi given of from neighbouring cells; and if the specimen under examination be a thin layer of bone, such as the scale of an osseous fish, from the cells lying nearly all in one plane, the anastomose of the canaliculi will be rendered beautifully distinct. In the majority of the bones of fishes the cells are not present, their place being occupied (as has been before stated) by enlarged canaliculi or tubes; but in all the hard bones, the enlarged tubes and the cells together, or else the cells alone will be found. In the hard scales of many of the osseous fishes, such as the Lepidosteus and Callicthys, and in the spines of the Siluridce, the bone-cells are beautifully seen; in the true bony scales comprising the exo-skeleton of the cartilaginous fishes, the bone-cells are to be seen in great numbers. In the spines of some of the Ray family may be noticed a peculiar structure: the Haversian canals are large and very numerous, and communicating with each canal are an infinite number of wavy tubes, which are connected with the canals in the same manner as the dentinal tubes of the teeth are connected with the pulp-cavity; and if such a specimen was placed by the side of a section of the tooth of some of the Shark tribe, the discrimination of one from the other would be no easy matter. In the spine of a Ray the analogy between bone and the ivory of the teeth is made more evi- 
dent; for in this fish we have tubes, like those of ivory, anastomosing with the canaliculi of bone-cells.

"Having said thus much on the minute structure of the bone composing the skeleton in the four vertebrated classes, let us proceed at once to the application of the facts which have been laid down; and let us, for example, suppose that a fragment of bone of an extinct animal be the subject of investigation. It has been stated, that the bone-cells in mammalia are tolerably uniform in size; and if we take 1-2000th of an inch as a standard, the bone-cells of birds will fall below that standard: but the bone-cells of reptiles are very much larger than either of the two preceding; and those of fishes are so entirely different from all three, both in size and shape, that they are not for a moment to be mistaken for one or the other, so that the determination of a minute yet characteristic fragment of tishes' bone is a task easily performed. If the portion of bone should not exhibit bone-cells, but present either one or other of the characters mentioned in a preceding paragraph, the task of discrimination will be as easy as when the bonecells exist. We have now the mammal, the bird, and the reptile to deal with : in consequence of the very great size of the cells and their canaliculi in the reptile, a portion of bone of one of these animals can readily be distinguished from that of a bird or a mammal; the only difficulty lies between these two last: but notwithstanding that on a cursory glance the bone of a bird appears very like that of a mammal, there are certain points in their minute structure in which they differ; and one of these points is in the difference in size of their bone-cells. To determine accurately, therefore, between the two, we must, if the section be a transverse one, also note the comparative sizes of the Haversian canals, and the tortuosity of their course; for the diameter of the canal bears a certain proportion to the size of the bone-cells, and after some little practice the eye will readily detect the difference. The fragments necessary for the purpose of examination are to be selected with some little care; and on the whole, a small chip (or two) from the exterior of the shaft of a long bone is sufficient : but as many fossil bones are coated with a layer of earthy deposit on their external surface, it will be requisite to get beneath this deposit, as it very seldom happens that the bone-cells are visible in it; but by a fragment from about the middle of the laminæ of the shaft the characteristic bonecells can at once be recognised. But in the comparison of the bone of a mammal with that of a bird, from the peculiarity in the arrangement of the Haversian canals in the latter class, it is highly important 
always to bear in mind that the specimens used for comparison should be cut in one and the same direction; for as it has been stated that the bone-cells, on which we are to rely for our determination, are always longest in the direction of the shaft of the bone, it would follow, that if one section were transverse and the other longitudinal, there might be a vast difference in the measurement of the bone-cells, in consequeace of their long diameter being seen in the one case, and their short diameter in the other; hence the caution of having all the sections made in one direction. In all doubtful cases, the better plan is to examine a number of fragments, both transverse and longitudinal, taken from the same bone, and to form an opinion from the shape of bone-cell which most commonly prevails."

We have thus examined a few of the more important structures of the animal economy. Imperfect in a detailed point of view though that examination be, we trust it may be found to smooth the way, or in some degree assist the investigations of the student to a better and more general survey of the whole fabric: such a survey will not be unattended with difficulties and disappointments; but it will, nevertheless, fully reward him for any amount of labour he may bestow. Some excellent hints were thrown out by Dr. Bennett in his "Lectures on the Study of Clinical Medicine." " On the proper mode of investigating animal structure," he says, "the principal physical characters to be regarded in microscopic examinations are the following:

"1. Shape. Accurate observation of the shape of bodies is very necessary, as many are distinguished by this physical property. Thus the human blood-globules presenting a round bi-concave disc, are in this respect different from the oval corpuscles of the camelidx, of birds, reptiles, and fishes. The distinction between round and globular is very requisite. Human blood-corpuscles are round and flat; but they become globular on the addition of water. Minute structures seen under the microscope may also be likened to the shape of wellknown objects, such as that of a pear, balloon, kidney, heart, \&c.

"2. Colour. The colour of structures varies greatly, and often differs under the microscope from what was previously conceived regarding them. Thus the coloured corpuscles of the blood, though commonly called red, are, in fact, yellow. Nany objects present different colours, according to the mode of illumination; that is, as the light is reflected from or transmitted through their substance, as in the case of certain scales of insects, feathers of birds, \&c. Colour is often produced, modified, or lost, by re-agents; as when iodine comes in contact with 
starch-granules, when nitric acid is added to chlorophyle, or chlorinewater to the pigment-cells of the choroid, and so on.

"3. Edge or border. This may present peculiarities worthy of notice. Thus it may be dark and abrupt on the field of the microscope, so fine as to be scarcely visible; or it may be smooth, irregular, serrated, beaded, \&c.

"4. Size. The size of the minute bodies, fibres, or tubes, which are found in the various textures of animals, can only be determined with exactitude by actual measurement. It will be observed, for the most part, that these minute structures vary in diameter; so that when their medium size cannot be determined, the variations in size from the smaller to the larger should be stated. Human blood-globules in a state of health have a pretty general medium size; and these may consequently be taken as a standard with advantage, and bodies may be described as being two, three, or more times larger than this structure.

"5. Transparency. This physical property varies greatly in the ultimate elements of numerous textures. Some corpuscles are quite diaphanous; others are more or less opaque. The opacity may depend upon corrugation or irregularities on the external surface, or upon contents of different kinds. Some bodies are so opaque as to prevent the transmission of the rays of light; in this case they look black when seen by transmitted light, though white if viewed by reflected light: others, such as fatty particles and oil-globules, refract the rays of light strongly, and present a peculiar luminous appearance.

"6. Surface. Many textures, especially laminated ones, present a different structure on the surface from that which exists below. If then, in the demonstration, these have not been separated, the focal point must be changed by means of the fine adjustment. In this way the capillaries in the web of the frog's foot may be seen to be covered with an epidermic layer, and the cuticle of certain minute fungi or infusoria to possess peculiar markings. Not unfrequently the fracture of such structures enables us, on examining the broken edge, to distinguish the difference in structure between the surface and the deeper layers of the tissue under examination.

"7. Contents. The contents of those structures which consist of envelopes, as cells, or of various kinds of tubes, are very important. These may consist of included cells or nuclei, granules of different kinds, pigment matter, or crystals : occasionally their contents present definite moving currents, as in the cells of some vegetables; or trembling rota- 
tory molecular movements, as in the ordinary globules of saliva in the mouth.

" 8. Effects of re-agents. These are most important in determining the structure and chemical composition of numerous tissues. Thus water generally causes cell-formations to swell out from endosmosis; while syrup, gum-water, and concentrated saline solutions cause them to collapse from exosmosis. Acetic acid possesses the valuable property of dissolving coagulated albumen, and in consequence renders the whole class of albuminous tissues more transparent. Thus it operates on cell-walls, causing them either to dissolve, or become so thin as to display their contents more clearly. Ether, on the other hand, and the alkalies, operate on fatty compounds, causing their solution and disappearance. The mineral acids dissolve most of the mineral constituents that are met with; so that in this way we are enabled to tell with tolerable certainty, at all events, the group of chemical compounds to which any particular structure may be referred."

If it be desired to examine the vital fluid, blood, the smallest drop, caused by the prick of a fine needle, should be placed on a strip of glass; which must then be waved backwards and forwards, that the blood may dry as quickly as possible; by this means the corpuscles or blood-dises will retain their form: if the preservation of the specimen is wished, a thin glass cover should be placed over it, and cemented down in the way directed at page 72 .

To the advanced observer, the examination of the mucous membrane will afford some instruction. If the specimen be small, it should be pinned on a piece of cork, and well washed by means of a small syringe. If the investing epithelium be required for examination, a portion may be detached from the surface by a small knife, placed on a glass slide, and viewed as a transparent object with a power of 250 diameters. The villi are best seen upon injected specimens.

\section{TO VIEW THE CIRCULATION OF BLOOD IN THE FROG.}

The part most commonly employed for this purpose is the transparent web of the hind foot; and in order to secure the animal, and keep its web open, various contrivances have been had recourse to. The older microscopists, Baker, Adams, and others, were in the habit of tying the frog to a frame of brass with some fine cord: in the present day the entire body of the animal, with the exception of the foot to be examined, is secured in a linen bag; and this is fastened to a plate of brass, termed the frog-plate, as shown at $a a a$ in fig. 148: this should 
be contrived so as to be held firmly by some part of the stage of the microscope, and permit of its being moved about with it. Although the shape of the plate differs with every maker, the mode of using it, nevertheless, is nearly the same in all. A linen bag should be provided, about three or four inches in length, and two and a half inches broad, as shown at $b b$, having a piece of tape, $c c$, sewn to each side, about midway between the mouth and the bottom; the mouth itself should be capable of being closed by a drawing-in string, $d d$. Into this bag the frog is placed, and only the leg which is about to be examined kept out of the mouth; the string $d d$ is then to be drawn so tight around the small part of the leg, as to prevent the foot from being pulled into the bag, but not to stop the circulation; three short pieces of thread,

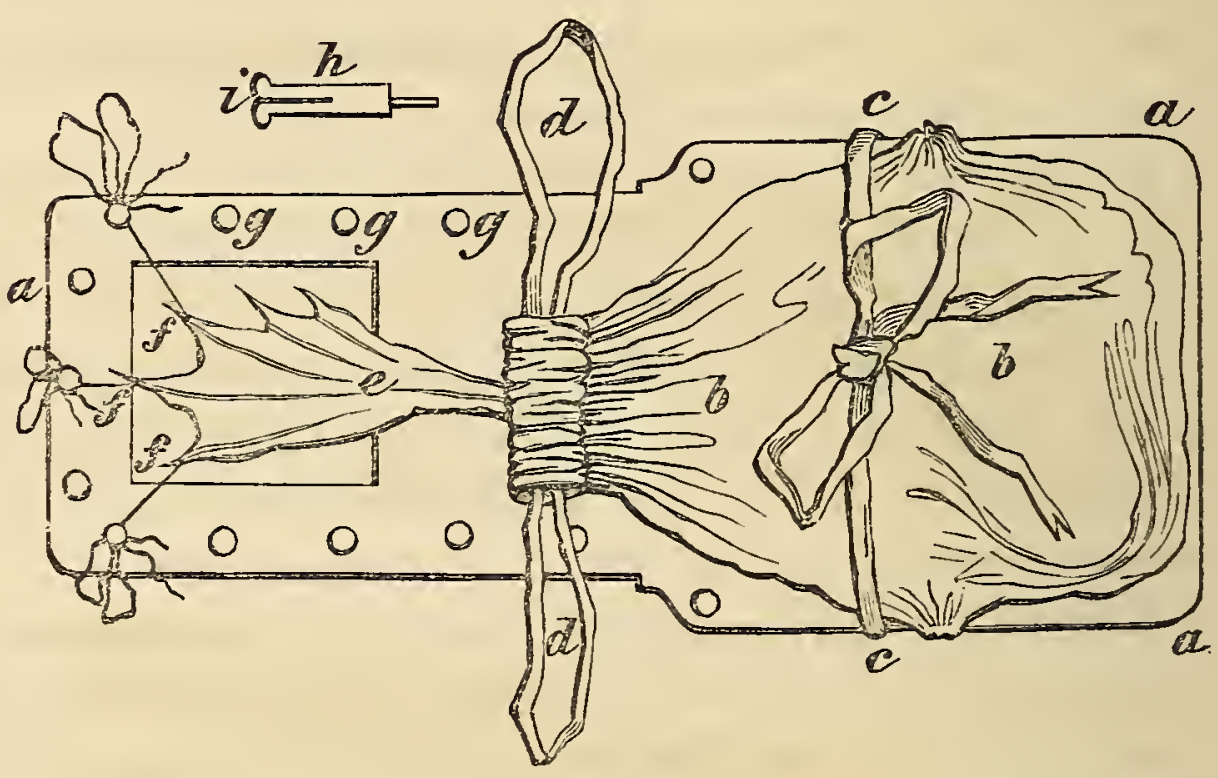

fig. 1 .

$f f f$, are now to be passed around the three principal toes; and the bag with the frog is to be fastened to the plate $a a$ by means of the tapes $c c$. When this is accomplished, the threads $f f f$ are to be passed either through some of the holes in the edge of the plate, three of which are shown at $g g g$, in order to keep the web open; or what answers better is a series of pegs of the shape represented by $h$, each having a slit $i$ extending more than half-way down it; the threads are wound round these two or three times, and then the end is secured by putting it into the slit $i$. The plate is now ready to be adapted to the stage of the microscope : the square hole over which the foot is placed must be brought over the aperture in the stage through which the light passes to the object-glass, so that the web may be strongly illuminated by the mirror. The power required to view the circulation will be the 
one-inch or half-inch object-glass; a higher power will be needed to show the rhythm of the veins.

In the common newt, as well as the frog, the circulation may be viewed : the tail of the young animal being much used for this purpose, and showing other interesting points of structure, we therefore give a short account of this interesting little creature.

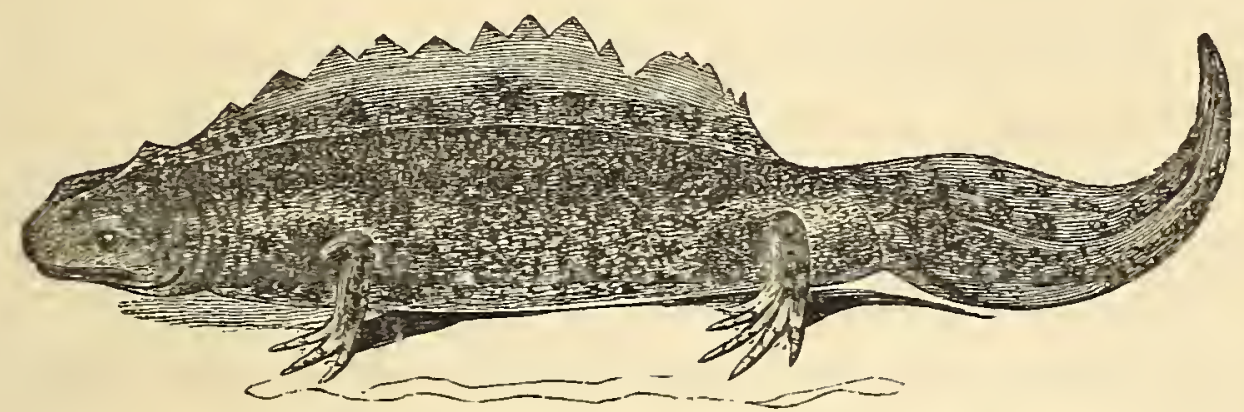

fig. 149. The common warty newt: male.

The warty newt is in a state of great activity early in spring. It is common in ponds and large ditches, where it feeds upon the tadpole of the common frog. The male and female newt are nearly the same in appearance during winter ; but in spring a beautifully-cut crest rises from the back of the male, which is highly ornamental.

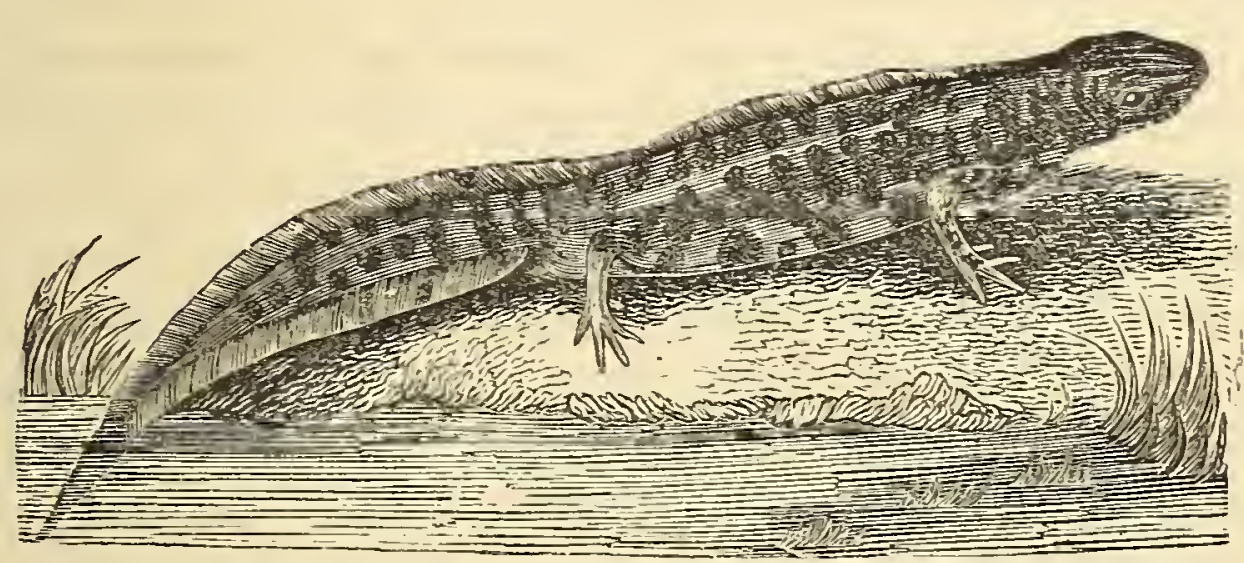

fig. 150. The common smooth icwt: male.

The manner in which the eggs are deposited is very interesting. "The female, selecting the leaf of some aquatic plant, sits, as it were, upon its edge ; and folding it by means of her two hinder feet, deposits a single egg in the duplicature of the folded part of the leaf, which is thereby glued most securely together, and the egg is thus effectually protected from injury." As soon as the female has in this way deposited an egg, she seeks another leaf, on which she deposits another 
egg in the same manner; and in this way she proceeds till she has deposited as many eggs as she requires. The egg is very slightly tinged with buff, and is surrounded by a substance resembling the white of a common egg, in which it keeps continually whirling round. It now goes through nine changes from the egg till it becomes a perfect animal; and for a considerable time it remains in a tadpole state, almost like the common frog.

The smooth newt is found in considerable numbers in almost every ditch and pond, especially where the water is tolerably clear; and it affords food not only to several kinds of fish, but to the warty newt, which is much larger than itself. Its own food consists of gnats and other small insects, and also of the Planorbis and other British molluscous animals, which it devours when they are quite young.

They are readily found in many of the ponds around London. The watercress-pickers meet with them in large numbers.

\section{FISH.}

It has often been observed, that we are surrounded by wonders which we do not notice because they are of daily occurrence, but which excite the greatest surprise when they are pointed out to us. The - truth of this observation is forcibly exemplified as regards fish. We see them every day exposed for sale on stalls, and we eat them frequently at our tables, without once cousidering by what a curious and delicate organisation these creatures are enabled to see and breathe in an element that carries death to us and to quadrupeds. The sight of fishes appears to be remarkably strong, as it is by sight chiefly that they discover their prey. Hence a fish is easily deceived by an artificial fly, or the imitation of a frog or other small aquatic or amphibious animal; which, if it were guided by the smell, or any other sense than the sight, could not happen. The mode in which fishes breathe is, however, the most curious. They have no lungs; but, instead of them, they have gills, carefully covered with a lid and a flap, both of which the fish can open or keep closed at pleasure. The gills are composed of arches bordered by a kind of fringe, which, when examined through the microscope, is seen to be covered with a velvetlike membrane, "over which myriads of wonderfully minute bloodvessels are spread, like a delicate net-work. There are commonly four of these fringed arches : they are movable, and allow the currents of water driven down by the action of the mouth to flow freely through them, so as to lave every fibril. It is absolutely necessary that this 
should be the case, since the gills lose their power of acting as soon as they become dry; and hence a fish cannot live long after it is taken out of the water. As there is danger, however, of the food taken by the fish being carried through the gills by the stream of water constantly flowing through them, the minor curve of the arch formed by the gills is studded with spines, which prevent any thing but air or water passing through them.

A knowledge of the form and structure of scales of fishes, like that of teeth, has been shown by M. Agassiz to afford an unerring indication of the particular class to which the fish may belong: in the examination of fossil remains, the application of this knowledge has been attended with extraordinary results. As a class of objects for the microscope, the scales of fishes are exceedingly curious and beautiful, especially when mounted in fluid or Canada balsam, and viewed by polarised light. Many are best seen as opaque objects, and are then mounted dry between glasses. M. Agassiz divided the scales into four orders, which he named Placoid, Ganoid, Ctenoid, and Cycloid; in the first two the scales are more or less coated with enamel, in the others they are of a horny nature. To the Placoid order belong the skates, dogfish, and sharks;-cartilaginous fishes, having skins covered with small prickly or flattened spines. To the Ganoid belong the sturgeon, lepidosteus, and hussar-fish; the fish of this order are more generally found in a fossil state, and their scales are of a bony structure. To the Ctenoid belong the pike, perch, pope, busse, \&c.; their scales are notched like the teeth of a comb. To the Cycloid belong the salmon, herring, eel, carp, and the majority of our edible fishes; their scales are circular and laminated. The scales of the eel will be found of an oval figure, and, with many others, are very beautiful objects when viewed by polarised light. 


\section{CHAPTER IV.}

VEGETABLE STRUCTURE-VITAL AND CHEMICAL CHARACTERISTICS-THE VEGETABLE CELL-FUNGI-ALGE-MOSSES-VOLVOX-DESMIDACE压 -STRUCTURE OF PLANTS-PREPARATION FOR MICROSCOPIC EXAMINATION-REFLECTIONS-CONCLUSION. *

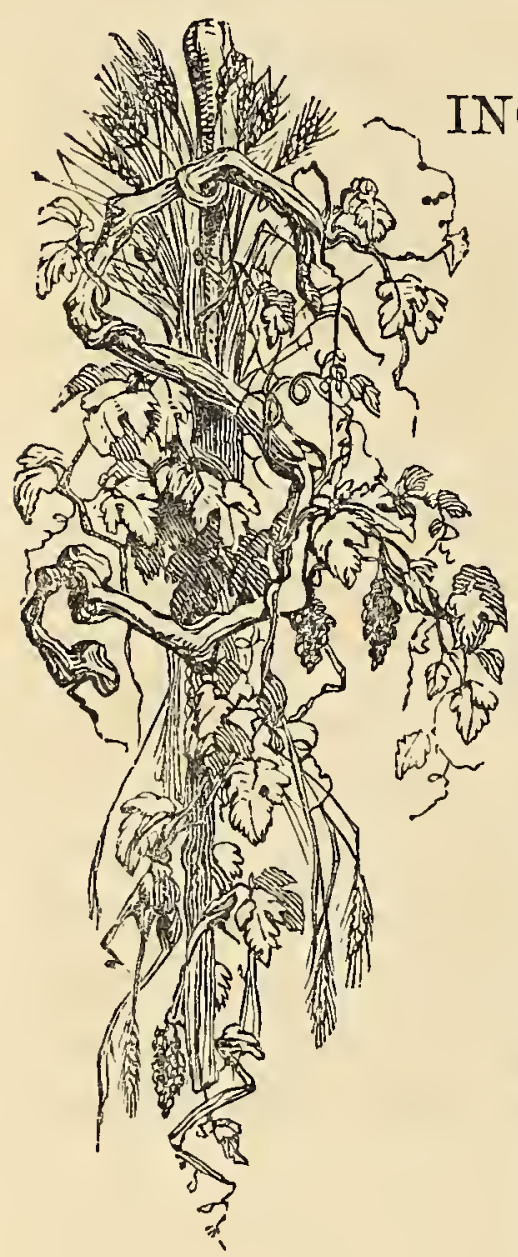

NCE the introduction of the achromatic microscope, we have obtained nearly the whole of the valuable information which we now possess relative to the minute structure of vegetables. Before that time, although some progress had been made in vegetable physiology, yet the means of distinguishing one structure from another, with their several external characters, comprehended the amount of our botanical knowledge. "The vegetation which every where adorns the surface of the globe, from the moss that covers the weather-worn stone, to the cedar that crowns the mountain, is replete with matter for reflection. Not a tree that lifts its branches aloft, not a flower or leaf that expands beneath the sunlight, but has something of habit, of structure, or of form, to arrest the attention." The microscopist sees proof of a higher

* Description of Plate XIV.-Desmidacea.

\begin{tabular}{|c|c|c|c|c|c|c|}
\hline u m oblongum & $\begin{array}{r}\text { No. } \\
. \quad 1\end{array}$ & Closterium & & & & 20, 25, 31, 35 \\
\hline licrasterias rotata & 2 & Penium & 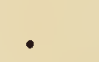 & & & . 21 \\
\hline , denticulata & 6 & Staurastrum & & 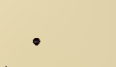 & & $22,23,32$ \\
\hline Desmidium quadrangulatum & $.3,16$ & Pediastrum & birac & diatun & & - 24 \\
\hline Didymoprium Grevillii & - 4 & , & pect & icum & . & 29 \\
\hline Didymoprium Borreri & 5 & Volvox glob & oata & - & - & 26 \\
\hline Sphærozosma vertebratum & 7 & Penium Jent & neri & & & 27,30 \\
\hline Xanthidium & $3,14,17,19$ & Aptogonum & desn & aidiun & & 28 \\
\hline Staurastrum tumidum & 15,18 & Spirotænia & . & . & . & 34 \\
\hline Cosmarium Ralfsii & 11,12 & Docidium & . & . & & 33 \\
\hline
\end{tabular}


PLATE XIV.

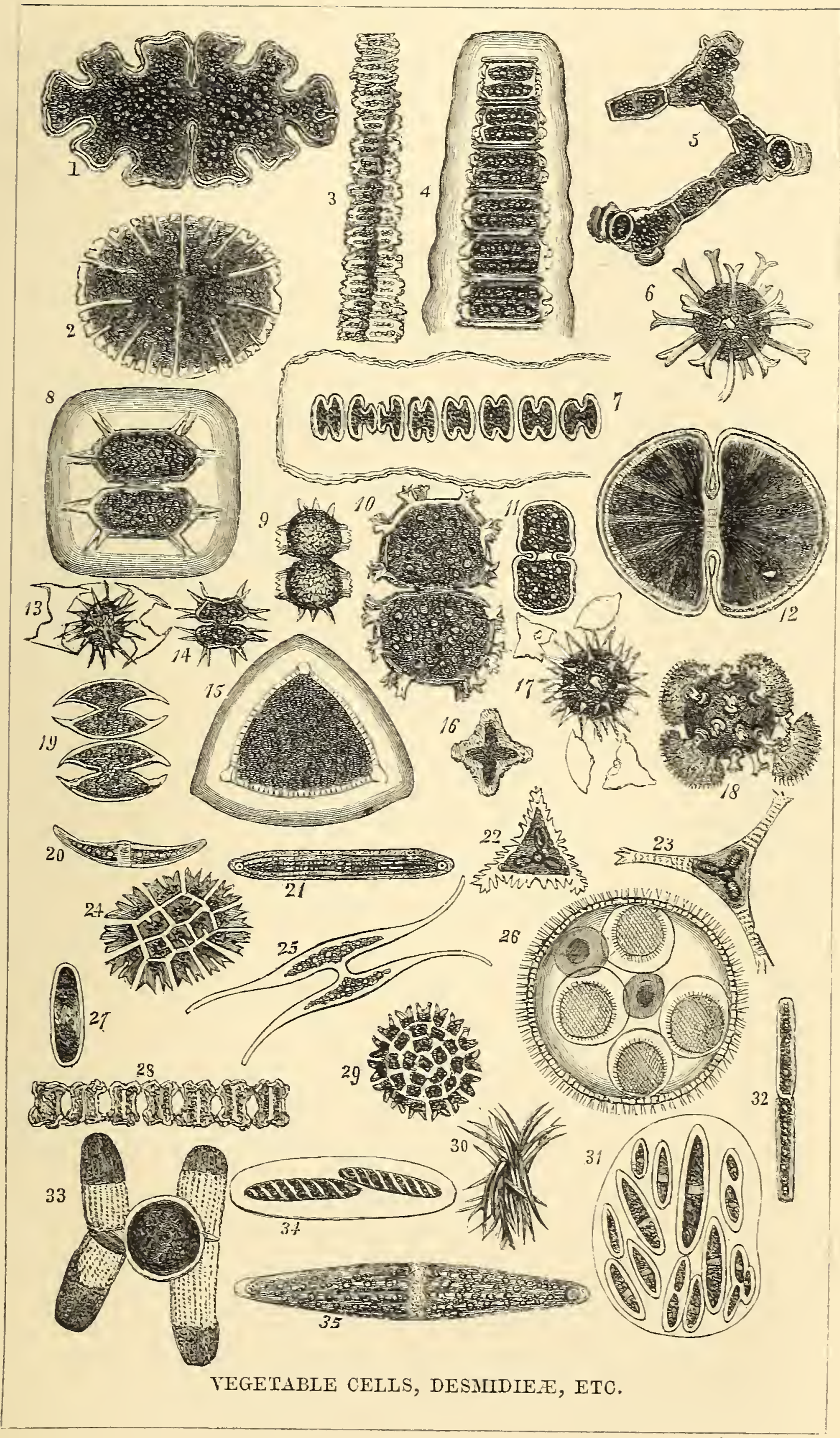



life in plants than he before conceived; and he becomes convinced, after examining the functions which their organs are destined to perform, that animals and plants are only separate links in the great chain of organic nature.

Plants are organised beings; that is, individuals composed of a number of essential and mutually dependent parts : in common, therefore, with animals, they possess a principle which is in continued action; and which operates in such a manner, that the individual parts which it forms in the body are adapted to the designs of the whole. Or, in more intelligible language, plants are living bodies. Like animals, they are the offspring of other beings similar to themselves; they grow, are endowed with excitability, have their periods of infancy, addult age, decay, and death. Their affinity to animals is much closer than is commonly supposed. The vital or creative power exists already in the germ, in plants as well as in animals; and by its influence the essential parts of the future plant are formed. It might be supposed that the lateral generation of plants - namely, that renewal of the indiridual. which is the result of budding or gemmation,-is sufficient to distinguish them from animals: but this opinion is erroneous; as we find that the formation of germs or buds is common in animals belonging to the class polypiferc. In the hydra, for example, we perceive the germs developed as small oroid elevations upon the cylindrical body of the animal; and when examined in this state, they are, like the first formation of the buds in plants, mere masses of cells; but as their growth proceeds these cells undergo a special arrangement, so as to produce the different tissues of the body, and acquire the proper form of the polype: on the same principle the bud in the plant is gradually developed, until it terminates in the perfect branch.

Plants, like animals, possess excitability, or the faculty of being acted upon by external stimuli, impelling them to the exertion of their regetative powers.

Light acts on plants, directing the growth of the stem, vigour, and colour; the direction of the branches, position of leaves, the opening. and shutting of flowers. Heat influences the protrusion of buds, and. other stimulants affect the regetable irritability; as an instance of this, cut plants, when fading, rerive if placed in water impregnated with certain chemicals.

Besides the physical and physialogical distinctions generally pointed out as marking the line between animals and plants, chemistry furnishes many others. Thus, one of the great functions of a plant is to decompose water, and assimilate its components to the vegetable tissues; 
whilst it is a property of animal life constantly to re-form it from its elements. The oxygen derived from the atmosphere, by whatever means it is introduced into the animal system, is expended in the production of carbonic acid and water, both of which are thrown off as excretions. It is true that water is exhaled in great quantities from the surfaces of plants; but it is that fluid which has been taken into the system of the plant, and has not undergone decomposition; it is, therefore, not actually found in the body of the vegetable, as it is in that of the animal. During the process of vegetation proteine is formed from the constituents of water, with carbonic acid and ammonia; proteine is formed in the animal body, and enters largely into the blood and muscle.

There is the closest affinity in the chemical nature of the products between plants and animals. Vegetable albumen is identical in composition with that in blood and in eggs; caseine does not materially differ in milk and the juices of some plants: we have many other equally striking characteristics, which modern chemical investigations have unfolded. Plants in some characteristics differ most strikingly, in being almost destitute of voluntary sensation and motion: here we would not have sensibility confounded with irritability, a principle which plants, in common with animals, possess. The simplest forms of animal life manifest both sensation and volition; even those that are fixed to rocks and other bodies presenting a ramified and vegetative form : for instance, the compound polypes; each individual polype displays both sensation and voluntary motion. It is, nevertheless, difficult to attribute satisfactorily the movements of some plants to irritability alone. Thus we find plants, in an apartment with light admitted on one side, not only turn the upper surface of their leaves to the light, but bend their stems and branches towards it. Many other instances might be cited; but none of them, excepting the movements of the Oscillatoria, more closely resemble volition. Plants, again, differ from animals in having no nervous system. All animals, without distinction, have a nervoxs system. "Ehrenberg described and distinctly traced "vessels and nerves in the rotatoria and some infusoria."

Another great distinction is connected with the function of digestion, which the simplest form of animals possess: those even which turn inside out, the hydra, \&c. have an internal cavity, into which their food is taken at intervals; but vegetables are nourished from the surface, and by continual imbibition.

It has been supposed, because the sap rises in plants, and in the interior of some internodia and cells of some simple plants a rotatory 
motion of fluid can be perceived, plants, like animals, have a circulation of fluids. This opinion is at least disputable, the sap of plants ascending only once, for that which is termed the descending sap of the plant is the proper juice prepared in the leaf; and the fact of the currents being observed in opposite directions in the leaf of Chilidonium majus, is no proof of the existence of a circulation. But, it may be asked, is the motion in the chara or the cells of the Valisneria spiralis, or in the hairs of the radicle fibres of frog's-bit, any proof of a circulation? It is certainly a proof of the motion of a fluid in the cells of a plant, effected in a manner not understood, and is very different from a general circulation of the sap; which is the only answer that can be made to such an inquiry: and the true circulation in animals is derived from an internal impelling power, and not from external influences.

A more distinctive character is obtained in the products of the respiratory function in plants. Respiration is performed by the entire surface in most animals, as it is by all plants; but the products are different. In plants, the process consists chiefly in the conversion of carbonic acid and water into vegetable matter; hence oxygen is exhaled from the leaves, and carbonic acid absorbed by them from the atmosphere; and it is by the decomposition of that acid in the leaf, that the greater part of the oxygen is restored to the air. And although plants exhale carbonic acid during the night, and in the shade, yet the quantity is small; and plants are, in reference to their respiration, a balance in the opposite scale to animals; they remove from the air the carbonic acid exhaled from the lungs and spiraculæ of animals, and re-supply the oxygen requisite for their respiration. Without the vegetable tribes, the atmosphere would soon cease to be fitted for the present race of animals; without the carbonic acid formed by animal respiration, plants would lose the greater part of their nutriment; and by their reciprocal action the atmosphere is preserved very nearly unchanged. Therefore the great distinctive feature between the two may be said to be essentially that pointed out hy Dr. Lankaster, in the nature of the distinctive character of the gases inhaled and exhaled by animals and by plants. With this brief survey we may conclude our comparative view of plants and animals by stating, that whilst they are endued with many properties and functions common to both, they possess others sufficiently distinctive, which prevent them from being: regarded as parts of the same link in the great chain of vital existence.

To pass on to a more intimate examination of the various structures entering into a plant, at this part of our inquiry, it will be seen 
that we have objects of the deepest interest presented to our notice; and strikingly differing as we find plants and animals in some essentials, we shall here, at our starting-point, find them gradually coalescing, until they meet in a common granule - "that of the simple and individual cell."

Mülder, in describing this starting-point of life, says: "The cell is a concave globule. This concave globule is an individual; in the most simple form in which it can possibly exist (in the lowest moulds), it possesses all the powers of the molecules united into one whole, and thus reduced to a state of equilibrium. This state depends not only on the nature of the substances and of their elements, carbon, hydrogen, oxygen, and nitrogen, but also on their form. The state of equilibrium, therefore, could not exist, unless this concave globular form existed. Moreover, this hollow globule possesses the whole of these forces in a state of mutual combination, co-operating for one end; this being a peculiarity which also apparently depends on the globular form. Since these two ideas are founded on pure observation, we may steadfastly adhere to them; and therefore correctly infer that in organic nature, besides all the peculiarities existing in the carbon, hydrogen, oxygen, and nitrogen, we must suppose, as a chief consequence of this, a tendency to form membraneous, concave, spherical little bodies, in which, because of this form, new peculiar properties manifest themselves, which cannot be brought out by other forms. Thus, by matter and form, by form and matter, all that we observe in nature is, to a great extent, determined. This general conclusion is drawn from the innumerable phenomena we perceive in the organic world; phenomena which vary, whether, on the one hand, the materials differ, while the forms are the same; or, on the other, the materials are the same, while the forms differ. If, therefore, the vegetable kingdom consisted of one common cellular substance,-this being different, however, only as to the form, either in various tribes, or species, or organs of plants, the effects of the same chemical body, of the same cellular substance, must, of necessity, be different for each different form. This has, in fact, been found to be true. These little individuals, these little cells, become other individuals when different in form, or when connected together in a different manner, though they consist almost of the same substances. The very smallest difference in the nature of the substances they consist of, or with which they are in contact, can infinitely influence that difference of form; and thus the material products of different forms are as innumerable, and as frequently modified, as the different forms produced through their difference in substance are innu- 
merable and frequently modified. Finally, if the form and substance are constant, the products of the cells must be also constant; if either the form or the substance of the cells differs, these products must be different.

"It is only right, therefore, that they who study the doctrine of life should set the highest value upon the knowledge of forms, and should not rest satisfied with merely knowing the component parts, or with enumerating a series of chemical substances which appear on the analysis of an organic body, even if it were possible to get only natural products by an artificial analysis."

Cells from which plants are formed are very small delicate closed sacs, partaking of many forms, and enclosed in a perfectly transparent membrane, so excessively thin that it is with difficulty detected, unless iodine or some colouring-matter be previously added. Dead and old cells form an exception, as they become thickened, and the broken surfaces are then readily detected. At one time the cells were said to be developed by an extrication of gaseous matter among mucus; but the double walls which separate cells are irreconcilable with such an origin. Mr. Thwaites regards the original wall of the cell as a mere shell, having quite a subordinate office to perform in
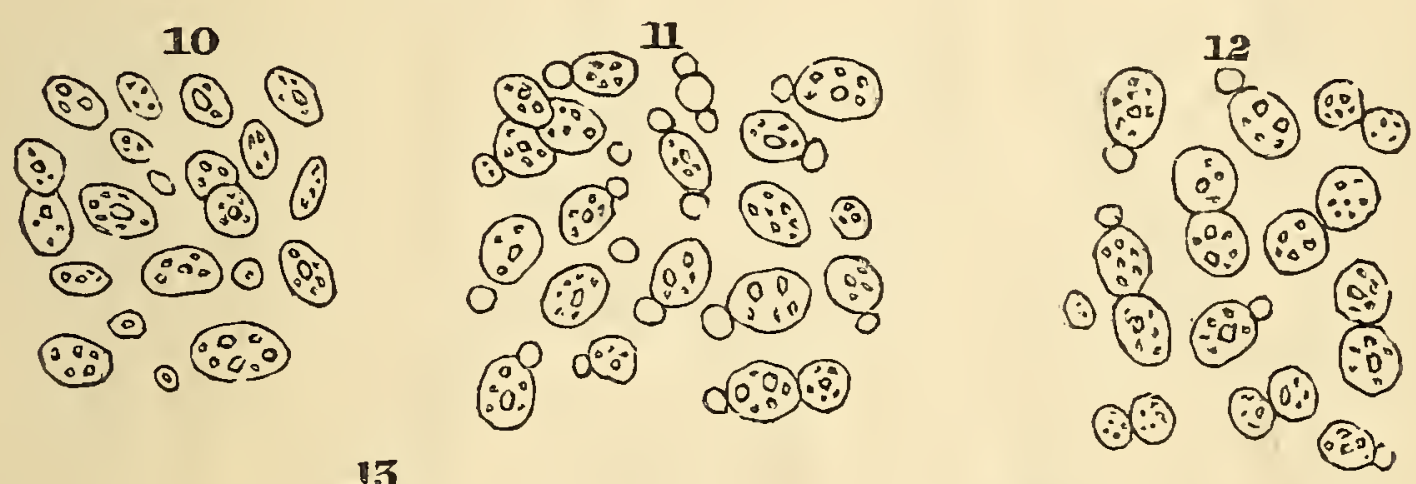

13
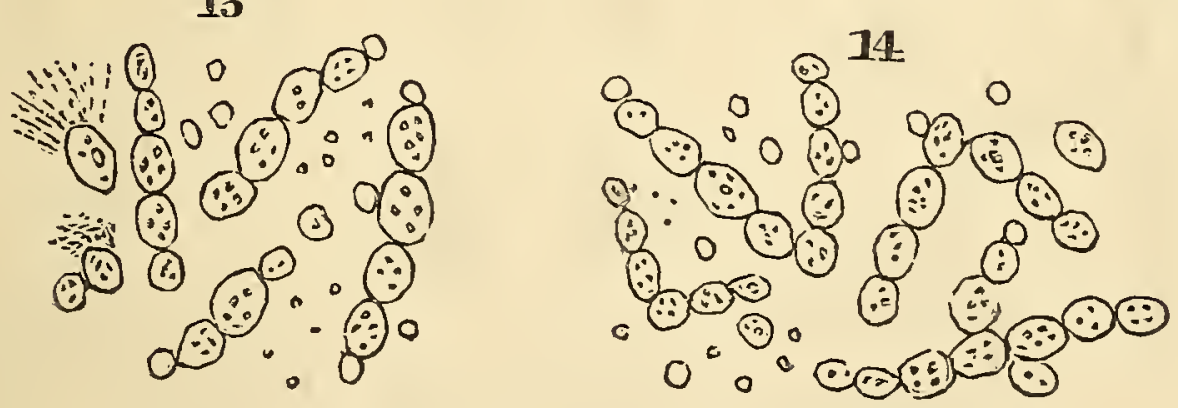

fig. 151. The growth of the Yeast-plant.

the growth of plants; and he ascribes all the vital powers of growth to the cytoblast and colouring-matter of the central nucleolus. $\mathrm{He}$ supposes the cell-membrane to arise from the action of electrical currents upon mucus, and that fissiparous division is caused by the presence of two centres of electrical force, each giving rise to a set of 
currents, and producing two cell-membranes instead of the original one. For further information on this very interesting subject, see Henfrey's translation of Mohl's Vegetable Cell.

The first and most curious exemplification of the simple cell is the fungi known as the Yeast Plant: it consists of two parts, the cell-walls, composed of a matter termed cellulose, and the contents of the cells, resembling fat or oil, a protenic substance. The notion that yeast was an organised being, in fact a living plant, was at first strongly opposed by even Berzelius and Liebig; but by the microscope they have been convinced both of its organisation and vitality. The scientific name by which it is known is Fermentum cervisice, or Torula cervisia, ; it consists of globular or ovoidal transparent nucleated cells, represented in
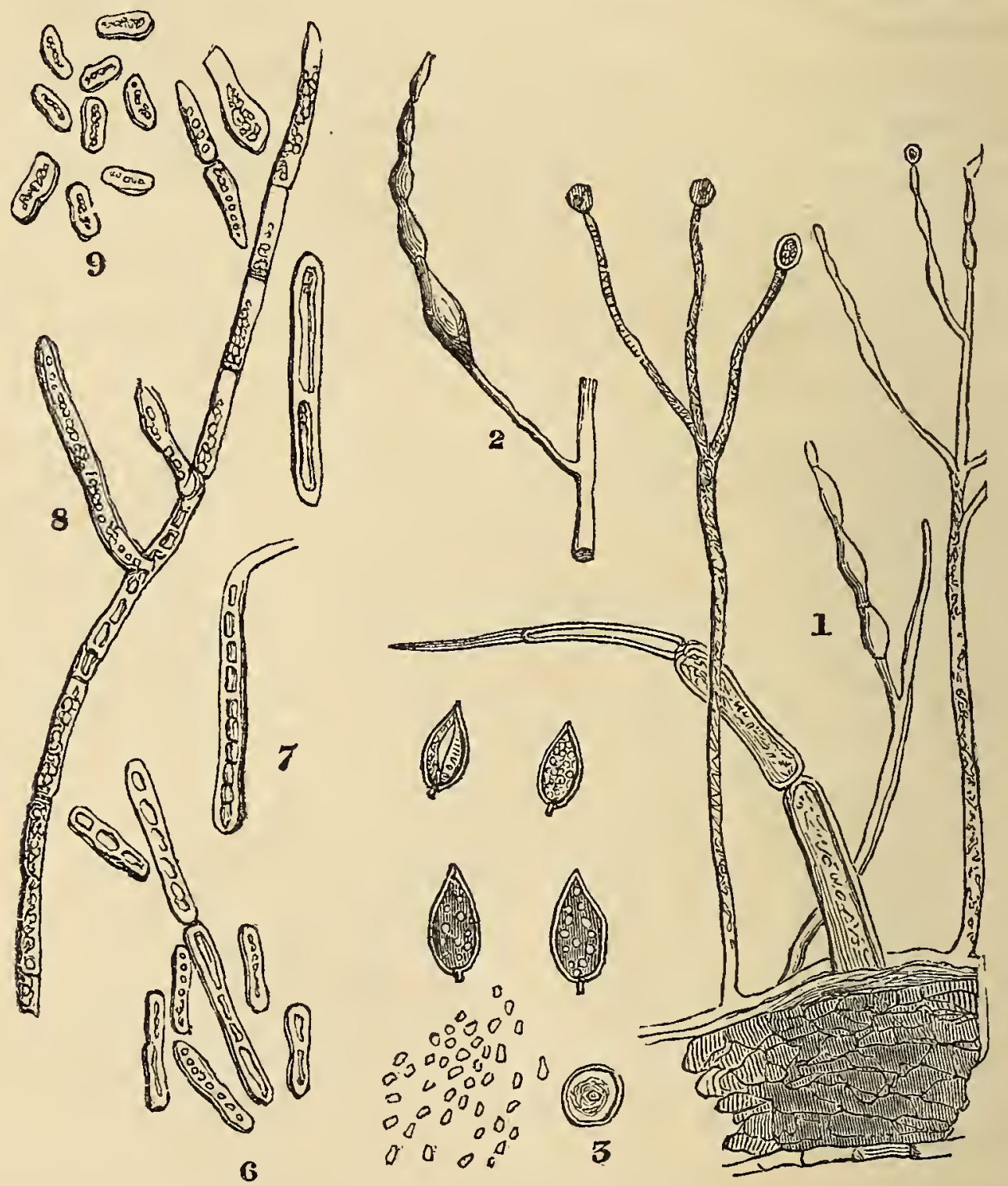

fig. 152. Fungoid disease of Tomata.

1. A section of the Tomata, showing the sporangiæ growing from the spawn or root (mycelium). 2. Showing the budding from the upper part of a branch. 3. Vertical and lateral view of sporangix, with their granular contents turned out. 6, 7, and 8 show different stages of growth of Mycoderma cervisiæ. 9. Torulæ diabetica. 
the accompanying fig, 151, and showing its stages of growth, as first observed by Turpin, who carefully watched the changes after mixing it with some newly-made beer. The fresh yeast has the appearance seen at No. 10 ; one hour after it had been added to the wort, germination commenced, and produced two buds or cells, as at No. 11. In three hours they were doubled, and attained the size of the maternal cell. In eight hours the plants began to ramify, and some to explode, emitting a fine powder ; and in three days jointed filaments with lateral branches were produced, as in No. 14.

Yeast cells occasionally form in the human body under certain states of disease, principally occurring in the urine of patients; hence the cell has been named Tomlce diabetica: for the sake of comparison a few of these cells highly magnified are represented at No. 9, fig. 152 . Mr. Busk met with a peculiar disease of the stomach in some patients under his care, vomiting another form of this remarkable fungi, named by Professor Goodsir Saccina ventriculi; this presented under the microscope an appearance very nearly resembling the Gonium pectorate, a peculiar infusorial animalcule shown in fig. 85. The Mycoderma cervisice of Desmazièrs is another stage of growth of the same plant deposited in the porter-vats; its various stages are shown in fig. 152, Nos. 6,7 , and at 8 ; the perfect plant is seen, with its granular contents in the stem.

One of the most remarkable of this tribe has been committing great devastation among our grape crops during the past two years. A section of the grape, magnified 75 diameters, is seen in fig. 153; the fungi or mildew is growing from a section of the skin of the grape.

"The grapes," says Mr. Harris, "when blighted, are covered with what appears to be a white powder, like lime, a little darkened with brown or yellow. These fungi send forth laterally, in all directions, thread-like filaments, which become so completely interwoven with one another as entirely to cover and enclose the skin of the grape in a compact and firm net-work, and on each is seen the egg-shaped capsule or seed-pod. The pips and juice go on swelling; and at times, when struck late in the season, the grapes become partially ripe and coloured; but very soon, expansion from within going on, checked by this net-work the grape bursts; but as it cannot burst as it would do were it unconfined, the edges of the part where it bursts turn inward in place of outward, and the pips are exposed to view as the teeth of a man when the lips are drawn back. If the grapes are struck in an early stage of their growth, they dry up, fall off, and become very offensive, but do not split." 
Fungoid diseases among our growing crops attracted but little attention until the mischief produced by them became serious; and the microscope has enabled us to determine the vegetable destroyer in its variety of forms: thus, we have our corn-crops withering under the blighting influence of the Uredos and Puceinias, our vines, \&c. under that of the Oidium, our esculents by the Botrytis infestans (potatoblight): the same disease infected the tomata, as represented in fig. 152 .

The microscope has revealed to us that many of the skin-diseases attacking the human frame are but other forms of the same growth of parasitic plants, either cryptogamic, or a low form of the fungi, represented by filaments at first simple, then ramified, and

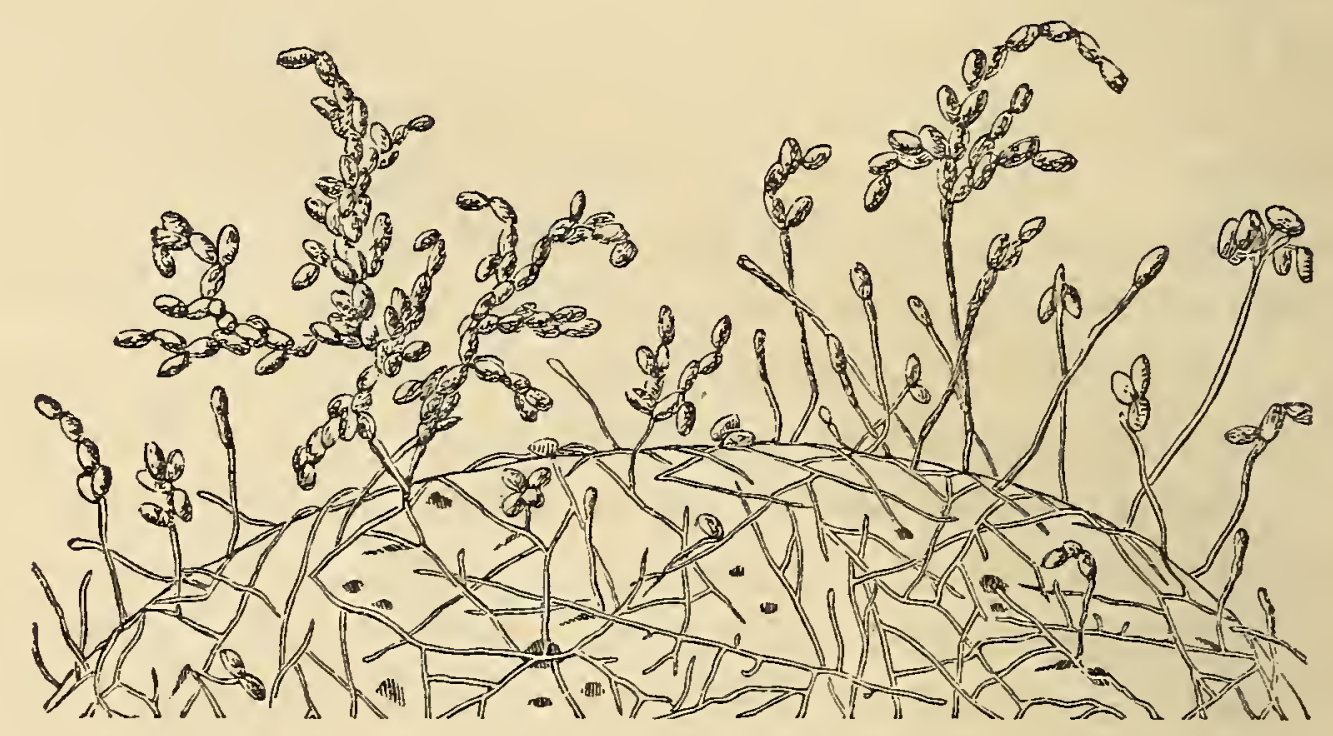

fig. 153. Section of a grape.

formed by a single elongated cell, or several cells placed end to end, as in those of the yeast. The disease known as ringworm, infesting the heads of children, is one out of forty-eight different species of this fungi. The conditions of growth of this low form of vegetable life on the human body is the same as in all other cases. Whenever the healthy chemical processes of nutrition are impaired, and the incessant changes between the solids and the fluids slacken, then the skin may furnish a proper soil for the fungi to take root in, should it come in contact with the sporules. That dreadful disease known as cancer will no doubt ultimately prove a vegetable growth, or conversion of the nutritive cell into that of a fungoid vegetable cell. The general history of civilisation teaches us that skin diseases were very prevalent, and that leper-houses were ordinary institutions in all the countries of Europe, as long as the lower classes continued impoverished, ill-clothed, ill-fed, and badly lodged. 
Animals, birds, insects, and fishes alike suffer from the ravages of the fungi. One of the most prevalent of these observed among our domestic pets is the fungi growing over the upper surface of the goldfish; death is almost certain when this white fungoid disease once commences its ravages. Great devastation is at times committed amongst silkworms by fungi, just as they are about to enter the chrysalis state.

We may range also by the side of these the fungi known as mushrooms, toadstools, puff-balls; and also a large number of microscopic plants forming those appearances which are referred to generally under the terms of mouldiness, mildew, blight, smut, dry-rot, \&c. It is well known that fruit-preserves are very liable to be attacked by the common bead-mould; which no care employed in completely closing the mouths of the jars can prevent. We may remark, however, that they are much less liable to suffer in this way, if not left open for a night before they are tied down; it is therefore probable that the germs of the mould sow themselves before the jar is covered. Some particular kinds of cheese also derive their flavour from the quantity of fungous growth which spreads through the mass whilst it is yet soft.

The leathery boletus is merely an enormous aggregation of the vegetable mould-plant or mucor; and their powers of reproduction are so great, that extensive tracts of snow are suddenly reddened by the gory-dew, Protococcus nivalis (red-snow) of the northern regions. That the red-snow plant consists of a cellular or filamentous tissue, may be easily ascertained by means of a microscope of even moderate powers; and one of a higher power demonstrates that the filaments are nothing more than cells drawn out. Sometimes, as in the genus Uredo, the cells are spheroidal, having little connection with each other; each cell containing propagating matter, and all separating from each other in the form of a fine powder when ripe. In plants of a more advanced organisation, as the genus Monilia, the constituent cells are connected in series which preserve their spherical, and also contain their own reproductive matter; while, in such plants as Aspergillas, the cells partly combine into threads forming a stem, and partly preserve their spheroidal form for fructification.

It is probable, however, that in all fungi, and certain that in most of them, the first development of the plant consists in what we here call a filamentous matter, which radiates from the centre formed by the space, or seeds; and that all the cellular spheroidal appearances are subsequently developed, more especially with a view to the dispersion of the species. That very curious fungus called in Scotland siller cups (Nidularia campanulata), fig. 154, consists of a curious leathery 
cup, in which are a number of small thecæ, which contain the sporules; and each plant looks like a bird's nest with several eggs in it. It generally grows on a twig, or a bit of rotten wood, and one has been found growing on a wooden tally, fixed in a pot containing a green-

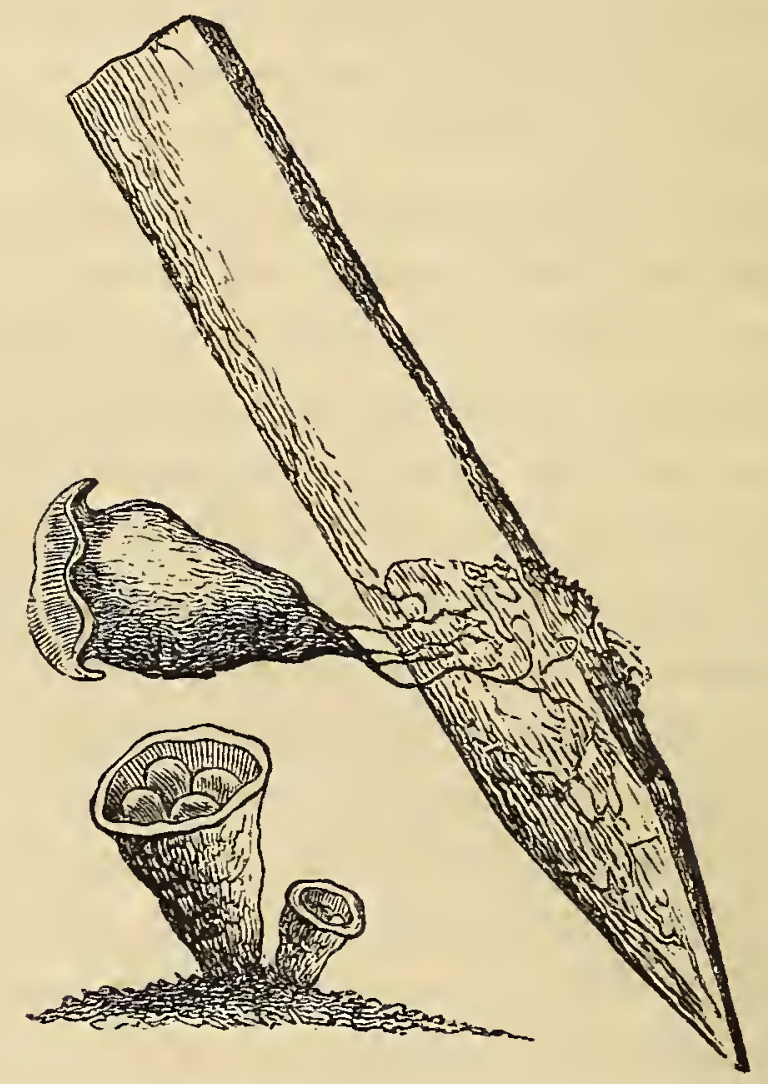

fig. 154. Siller Cups : Nidularia campanulata.

house plant. Seyeral kinds of Agaricus have blue stems, others orange, yellow, and green, with caps of various colours, some of which are scarlet or crimson, and others have beautiful shades of purple or violet.

CONFERY

The jointed algæ or confervæ are chiefly inhabitants of fresh water. They present the appearance of thread-like tubes, having joints differing in length, and the manner in which their contents are arranged. An endless variety of these plants may be found in clear ditches and running streams. They multiply by means of little granules contained in their tubes, which are gradually enclosed in tube after tube added to the end of the previous one. Among these confervæ, the most remarkable are the Zognema and Oscillatoria, both of which evince certain degrees of approach to the animal kingdom. The species of the latter genus form dark green and purple slimy patches in damp places, or in water, and are exceedingly remarkable for the power they possess of 
moving spontaneously. When in an active state their tubes are seen to unite and twist about, just as if they were vegetable worms; but they grow like plants, and their manner of increase is also vegetable." Disjointed algæ are extremely curious; they are characterised by their original or final spontaneous separation into distinct fragments, which have a common origin, but an individual life. They multiply by spontaneous division. It is upon the stems of other plants immersed in water, or floating in pools or ditches, that these curious productions are met with; in their habits they are so paradoxical, that it cannot surprise us that naturalists were so long undecided as to whether they were not really minute animals. In the algæ, the seeds are imbedded either in the frond itself or in some especial receptacle; the fronds develop cellules, in which the reproductive nucleolus is enclosed, acquiring a deeper colour. They become finally separated. The higher kinds of algæ inhabit sea-water only; and they then assume the forms of more perfect plants, forming vast submarine forests of the most luxuriant vegetation. A few species are represented in our frontispiece : many kinds furnish a wholesome and palatable food for man : the Laver of this country, the Carrageen, or Irish Moss, with others, belong to this group; and from them are formed the edible birds'-nests, which are considered so great a delicacy by the Chinese. The valuable medicinal substance, Iodine, is also produced from algæ.

The mosses are another low form of vegetable life; and Linnæus called them servi,-_servants or workmen,-as they seem to labour to produce vegetation in newly-formed countries, where soil is not yet

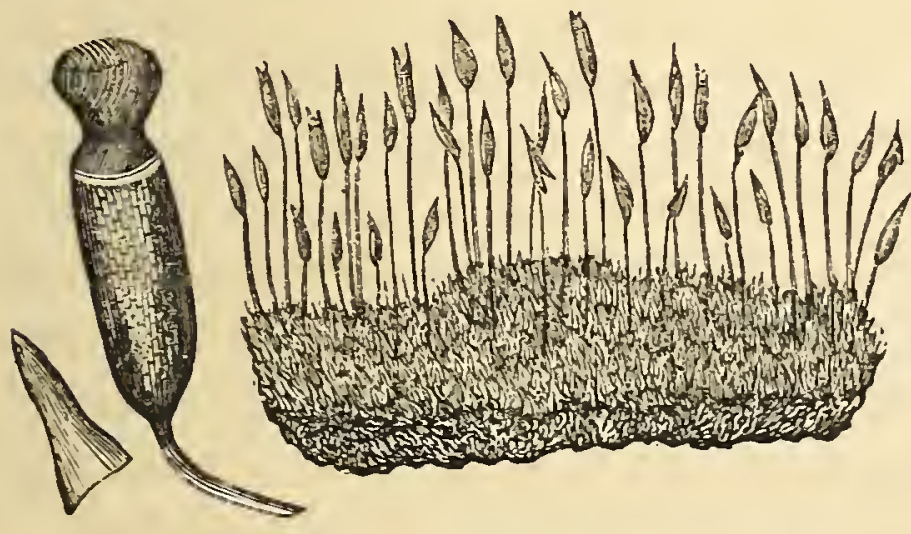

fig. 155. Screw Moss.

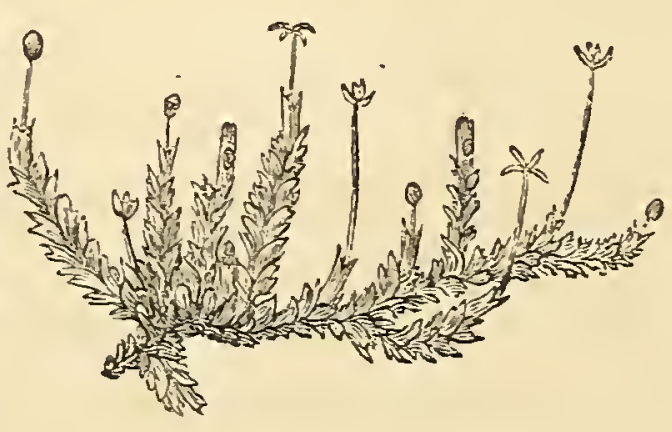

fig. 156. Scale Moss.

formed. They also fill and consolidate bogs, and form rich mould for the growth of larger plants, which they protect from the winter's cold. The common or wall screw-moss (Tortula muralis), fig. 155, which grows almost every where on old walls and other brick-work, 
if examined closely, will be found to have springing from its base numerous very slender stems, each of which terminates in a dark brown case, which is, in fact, its fruit. As the fruit ripens, a little cap which covers it like an extinguisher, rises gradually, and is at last thrown off; and when the lid of the fruit, which is also conical, falls off, a curious tuft of twisted hairs appears, forming a kind of fringe; and it is from these twisted hairs that the plant takes its popular name of screw-moss. If a patch of the moss is gathered when in this state, and the green part at the

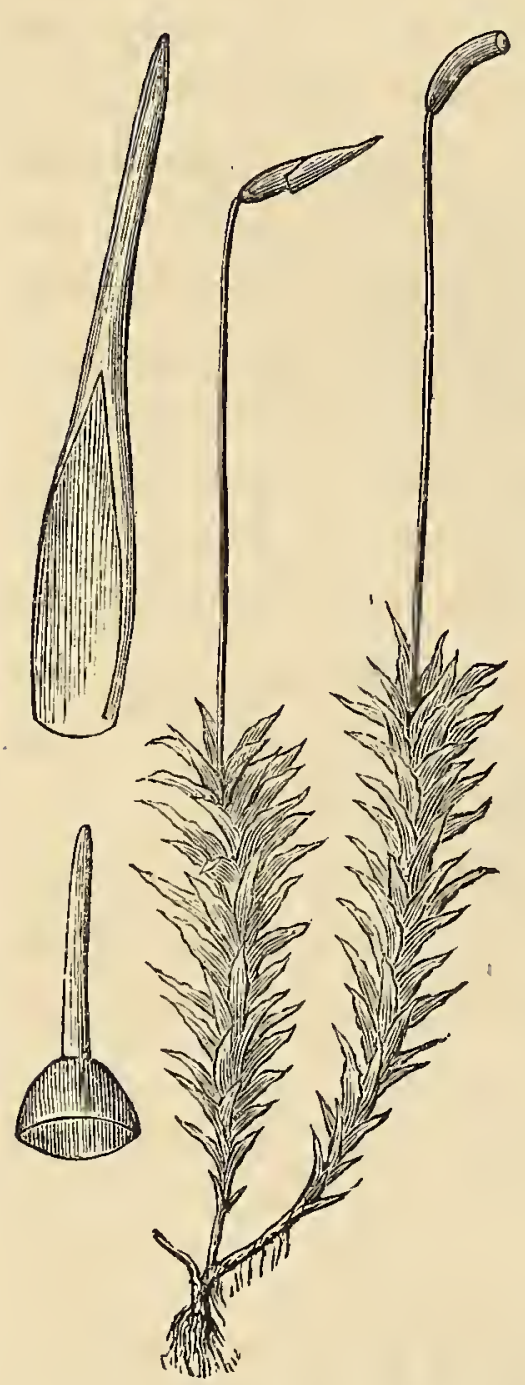

fig. 157. Undulated Hair Moss. base is put into water, the threads of the fringe will uncoil and disentangle themselves in a most curious and beautiful manner, and thus afford an opening to the seeds, which are exceedingly small, and are contained within a thin bag, attached to the central column of the case. It may here be mentioned, that all mosses and lichens are more easiiy detached from the rocks and walls on which they grow in frosty weather than at any other season, and consequently they are best studied in winter. One of the commonest kinds of scalemoss, fig. 156 (Jungermannia bidentata), grows in patches, in moist, shady situations, near the roots of trees, upon commons, and on hedgebanks. The seed-vessels are little oval bodies, which, if gathered when unexpanded, and brought into a warm room, burst under the eye with violence the moment a drop of water is applied to them, the valves of the vessel taking the shape of a cross, and the seeds distending in a cloud of brown dust. If this dust be examined by the microscope, a num. ber of curious little chains, looking something like the spring of a watch, will be found among it, their use being to scatter the seeds; and if the seed-vessel be examined while in the act of bursting, these little springs will be found twisting and writhing about like a nest of serpents. The undulated hair moss (Polytrichum undulatum), fig. 157, is found on moist shady banks, and in woods and thickets. The seed-vessel has a curious shaggy cap; but in its construction it is very similar to that of the screw-moss, except that the fringe round its opening is not twisted. 
We now proceed to an examination of a more complex form of cell, one that has attracted the attention of all microscopists from its earliest discovery to the present day. It was for a very long time classed with the lower orders of animal life; and there it remained for the micro-chemical investigators of the present time to settle the perplexing question by assigning it truly a place amongst vegetables. The cell referred to is known as the Volvox globata or revolving-cell, represented in Plate XIV. No. 26, also in fig. 85, Nos. 4, 5, and 6, where its near resemblance to the lower form of animal life is strikingly shown. These revolving globular bodies are found of various sizes, some so large as to be discoverable by the naked eye.

Leeuwenhöek first perceived the motion of what he termed globes, " not more than the 30th of an inch in diameter, through water ; and judged them to be animated." These globes are studded with innumerable minute green spots at their surface, each of which is a cell about the 3500 th part of an inch in size, with a vivid nucleus having many ever-active cilia that bristle over their spherical home, and are bound to each other by bands forming a beautiful network. Within this globe, busy active nature is at work to carefully provide a continuance of the species; and from six to twenty little bright green spheres have been found encased in the larger transparent shell. As one of these arrives at maturity, the parent shell enlarges, then bursts asunder to launch forth its offspring into its watery world. Both the older and younger spheres possess openings through which the waters freely flow, affording food and air to the little hives of organised beings.

Professor Williamson carefully examined the Volvox globata, and proved that it belongs not to the animal but to the vegetable kingdom. He has shown that the increase of the cells is carried on in a manner precisely analogous to that of the algæ; that between the outer integument and the primordial cell-wall of each cell, a hyaline membrane is secreted, causing the outer integument to expand; and as the primordial cell-wall is attached to it at various points, it causes the internal colouring-matter, or endochrome, to assume a stellate form (see fig. 85, No.6), the points of one cell being in contact with those of the neighbouring cell : these points forming at a subsequent period the lines of communication between the green spots generally seen on the fullgrown volvox. Cilia might be distinctly seen on the outer edge of the adult volvox; by compressing and rupturing one, they could even be counted. Mr. Busk has been able to satisfy himself, by the addition of the chemical test iodine, of the presence of a very minute quantity 
of starch in the interior of the volvox, which must be considered as conclusive of their vegetable character. A singular provision is made in the structure of the gemmules, consisting of a slender elastic filament, by which it is attached to the parent cell-wall; at times it appears to thrust itself out, as if in search of food; it is then seen quickly to recover its former nestling-place by contracting the tether.

"Wonderful as it may appear, we have here an example of all the functions of vegetable life,_namely, absorption, assimilation, exhalation, secretion, reproduction, \&c. - effected by a single cell. This is ever continued in the highest and most complicated orders of vegetable life, in which there is a variety of organs adapted for the performance of different offices, the functions of which are effected by the agency of cells, obtaining materials of formation and support from the ordinary chemical agents around them. Thus the more man gains a knowledge of the wonders displayed in these minute objects of creation, the more is the mind humbled and inspired with reverence for the First Great Cause."*

\section{THE DESMIDACE $Æ . \quad$ PLATE XIV.}

The disputed question of the animal or vegetable nature of these cells has received much valuable elucidation from Mr. Ralfs, who has given to the world the results of his laborious researches in lis excellent work on The British Desmidiex, published in 1848. The conclusions of this painstaking author have been generally accepted by men of science. Removing, as he has done, to the vegetable kingdom, a great many objects which Ehrenberg and others had classed as Infusoria, he has added to the botanist's stores a fruitful source of delightful study. The interest which has so long attached to this topic will warrant us in devoting some space to its consideration, and we avail ourselves for that purpose of some extracts from Mr. Ralfs' able work, with a recommendation to those of our readers who would wish to familiarise themselves more completely with this peculiar species, to consult the pages of the work itself.

"The Desmidiece are grass-green in colour, surrounded by a transparent structureless membrane, a few only having their integuments coloured; they are all inhabitants of fresh water. Their most obvious peculiarities are the beauty and variety of their forms and their external markings and appendages; but their most distinctive character is their evident division into two cr more segments. Each cell or joint

- Dr. Mantell's Wonders of Geology. 
in the Desmidiex generally consists of two symmetrical valves or segments; and the suture or line of junction is in general well marked.

"In the Desmidieæ the multiplication of the cells by repeated transverse division is full of interest; both on account of the remarkable manner in which it takes place, and because it unfolds the nature of the process in other families, and furnishes a valuable addition to our knowledge of their structure and physiology.

"The compressed and deeply-constricted cells of Euastrum offer" most favourable opportunities for ascertaining the manner of their division; for although the frond is: really a: single cell, yet this cell in all its stages appears like two, the segments being always distinct, even from the commencement. As the connecting portion is so small, and necessarily produces the new segments, which cannot arise from a broader base than its opening; these are at first very minute, though they rapidly increase in size. The segments are separated by the elongation of the connecting tube, which is converted into two roundish hyaline lobules. These lobules increase in size, acquire colour, and gradually put on the appearance of the old portions. Of course, as they increase, the original segments are pushed further asunder, and at length are disconnected; each taking with it a new segment to supply the place of that from which it has separated:

"It is curious to trace the progressive development of the new. portions. At first they are devoid of colour, and have much the appearance of condensed gelatine; but as they increase in size, the internal fluid acquires a green tint, which is at first very faint, but soon becomes darker; at length it assumes a granular state. At the same time the new segments increase in size, and obtain their normal figure; the covering in some species shows the presence of puncta or granules; and lastly, in Xanthidium and Staurastrum the spines and processes: make their appearance; beginning as mere tubercles, and then lengthening until they attain their perfect form and size; but complete separation frequently occurs before the whole process is completed. This singular process is repeated: again and again, so that the older segments are united successively, as it were, with many generations. In Sphærozosma the same changes take place, and are just as evident; but the cells continue linked together, and a filamentis formed, which elongates more and more rapidly as the joints increase in number. This continued multiplication by division has its limits:- the segments: gradually enlarge whilst they divide, and at length the plant ceases to grow; the division of the cells is no longer repeated; the internal matter changes in appearance, increases in density, and contains starch- 
granules, which soon 'become numerous; the reproductive granules are perfected, and the individual perishes.

"When the cells approach maturity, molecular movements may be at times noticed in their contents, precisely similar to what has been deseribed by Agardh and others as occurring in the Confervæ. This movement has been aptly termed a swarming.

"All the Desmidiex are gelatinous. In some the mucus is condensed into a distinct and well-defined hyaline sheath or covering, as in Didymoprium Grevillii and Staurastrum tumidum; in others it is more attenuated, and the fact that it forms a covering is discerned only by its preventing the contact of the coloured cells. In general its quantity is merely sufficient to hold the fronds together in a kind of filmy cloud, which is dispersed by the slightest touch. When they are left expesed by the evaporation of the water, this mucus becomes denser, and is apparently secreted in larger quantities to protect them from the effects of drought. I have shown," continues Mr. Ralfs, "that the cell in the Desmidieæ consists of two valves united by a central suture, and that during its division the new-formed portions are interposed between these valves. The Desmidiex are membranous; or should a few species contain silica, it is not present in sufficient quantity to interfere with their flexibility. They rarely have acute angles, and are seldom if ever rectangular. They are often deeply incised or 'lobed, warted or spinous. The internal matter is of a herbaceous-green colour, and starch-vesicles abound in the mature cell. They couple and form either orbicular or quadrate seed-like bodies, and are remarkable for the resistance which they oppose to decomposition. Meyen states he has 'distinctly seen that the large and small granules contained starch, and were sometimes even entirely composed of it ;' and that 'in the month of May he had observed many specimens of Closterium in which the whole interior substance was granulated, and all the grains gave with iodine a beautiful blue colour, as is the case with starch." "*

Did we trust solely to the eye, we should indeed be very liable to pronounce these variable and beautiful forms to far more closely re-

* The test for starch can be easily applied, and so remove any doubt that may exist. It is only necessary to bear in mind that unless granular matter be seen in the interior of the cell, starch cannot be present. A small quantity of diluted tincture of iodine may be applied, removing the free iodine by the aid of heat, occasionally adding a little water to facilitate its removal. This also will assist in the removal of the brownish stain which at first obscures the characteristic purple tint; and then, by applying the highest power of the microscope, the peculiar colour of the purple iodide of starch can in general be easily perceived. 
semble animals than vegetables in their appearance. All favours this supposition. Their symmetrical division into parts, the exquisite disc form, finely cut and toothed Micrasterias, the lobed Euastrum, the Cosmarium glittering as it were with gems, the Xanthidium armed with spines, the scimitar-shaped Closterium embellished with striæ, the Desmidium resembling a tape-worm, and the strangely insect-like Staurastrum, sometimes furnished with arms, as if for the purpose of seizing its prey;-all these characteristics appear to a superficial observer to belong rather to the lowest forms of animal than vegetable life. Another indication may be adduced by rendering apparent their power of motion, as Dr. Bailey did, taking a portion of mud covered with Closteria, placing it in water exposed to light; after a time, it will be seen tinat if the Closteria are buried in the mud, they will work their way to the surface, and cover it with a green stratum: this is no doubt owing to the stimulus of the light exerted upon all matter, although at first appearing very like a voluntary effort. Another is afforded by their retiring beneath the surface when the pools dry up. Mr. Ralfs states that he has taken advantage of this circumstance to obtain specimens less mingled with foreign matter than they would otherwise have been. If a species be much mixed with mud, he fills a saucer with the earth, makes it into a paste with water, then spreads a thick layer upon calico; in a short time, as it dries, the stratum may be scraped off the calico with a knife. But whatever motive power the Desmidiex may be supposed to possess, it is evidently only in common with that of the Algæ and various other species of this class.

The slow forward and backward movement which has been observed in several Desmidieæ and Diatomaceæ is explained by Nägeli in the following way: "The cells have no special organs for these movements; but as, in consequence of their nutritive processes, they take in and give out fluid matters, the cells necessarily move when the attraction and emission of the fluids are unequally distributed on parts of the surface, and are so active as to overcome the resistance of the water. This motion, consequently, is observed more particularly in those cells which, in consequence of their taper forms, easily pass through the water: these cells, moreover, move only in the direction of their long axis. If one half of a spindle-shaped or ellipsoidal cell chiefly or exclusively admits material, the other half, on the contrary, giving it out, the cell moves towards the side where the admission takes place. But as in these cells both sides are physiologically and morphologically similar, so it is, that first the one, and then the other half 
receives or emits; and consequently the cell moves sometimes in one, sometimes in the other direction. In this way possibly may be explained all those motions which are so readily noticed in the Bacillariæ. Only a complete misconception of these plant-motions could have induced Ehrenberg to seek for motile organs in these organisms: According to him, it would appear, for instance, that the Navicularia can project an undivided motile organ, like the foot of a snail, from one of the central openings of the shield. This pedal organ is said to be closely applied to the shield, but to admit of its being extended as far as the two extremities. Many careful observers have looked for this organ, but without success.

"The mode of finding and taking the Desmidiece, \&c.-As the difficulty of obtaining specimens of these plants is very great, it will materially assist the efforts of the microscopist to learn the method adopted by Mr. Ralfs, Mr. Jenner, and Mr. Thwaites. In the water the filamentous species resemble the Zygnemata; but their green colour is generally paler and more opaque. When they are much diffused in the water, take a piece of linen about the size of a pocket-handkerchief, lay it on the ground in the form of a bag, and then, by the aid of a tin box, scoop up the water and strain it through the bag, repeating the process as often as may be required. The larger species of Euastrum, Micrasterias, Closterium, \&c., are generally situated at the bottom of the pool, either spread out as a thin gelatinous stratum, or collected into finger-like tufts. If the finger be gently passed beneath them, they will rise to the surface in little masses, and with care may be removed and strained through the linen as above described. At first. nothing appears on the linen except a mere stain or a little dirt, but by repeated fillings-up and strainings a considerable quantity will be obtained. If not very gelatinous, the water passes freely through the linen, from which the specimen can be scraped with a knife, and transferred to a smaller piece; but in many species the fluid at length does not admit of being strained off without the employment of such force as would cause the fronds also to pass through; and in this case it should be poured into bottles until they are quite full. But many species of Staurastrum, Pediastrum, \&c. usually form a greenish or dirty cloud upon the stems and leaves of the filiform aquatic plants; and to collect them requires more care than is necessary in the former instances. In this state the slightest touch will break up the whole mass, and disperse it through the water. I would recommend the following method as the best adapted for securing them:-Let the hand be passed very gently into the water and beneath the cloud, the palm. 
'upwards and the fingers apart, so that the leaves or stem of the inverted plant may lie between them, and as near the palm as possible; then close the fingers, and keeping the hand in the same position but concave, draw it cautiously towards the surface; when, if the plant has been allowed to slip easily and equably through the fingers, the Desmidieæ in this way brushed off will be found lying in the palm. The greatest difficulty is in withdrawing the hand from the surface of the water, and probably but little will be retained at first; practice, however, will soon render the operation easy and successful. The contents of the hand should be at once transferred either to a bottle, or in case much water has been taken up, into the box, which must be close at hand; and when this is full, it can be emptied on the linen as before. But in this case the linen should be pressed gently, and a portion only of the water expelled, the remainder being poured into the bottle, and the process repeated as often as necessary.

"Sporangia are collected more frequently by the last than the preceding methods. When carried home, the bottles will apparently contain only foul water; but if it remain undisturbed for a few hours the Desmidieæ will sink to the bottom, and most of the water may then be poured off. If a little filtered rain-water be added occasionally to replace what has been drawn off, and the bottle be exposed to the light of the sun, the Desmidieæ will remain unaltered for a long time.

"Respecting the uses of the Desmidieæ little is known. Doubtless, in common with other aquatic vegetables, they tend to preserve the purity of the water in which they live; and Mr. Williamson has ascertained that to a great extent they furnish food for the bivalved
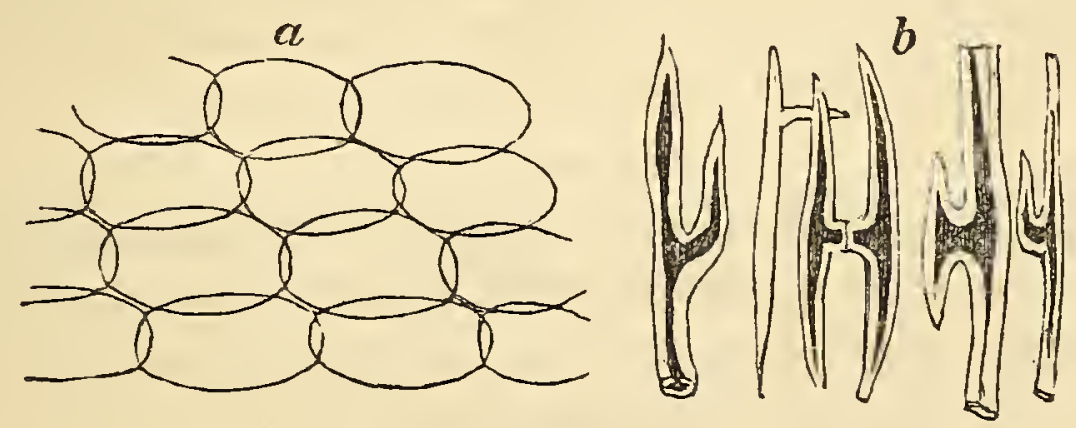

fig. 158 .

a, elementary cells. $\delta$, branched cellular tissue.

molluses which inhabit fresh waters. The Desmidieæ prefer an open country. They abound on moors and in exposed places, but are rarely found in shady woods or in deep ditches. To search for them in turbid waters is useless; such situations are the haunts of animals, not 
the habitats of the Desmidiex; and the waters in which the latter are present are always clear to the very bottom."

Proceeding onward in our course of inquiry, we find the cell passing to higher forms, and the transparent membranous cell-wall becoming thickened; spontaneous fissure then takes place; and thus is formed a series of connected cells variously modified and arranged, according to the conditions under which they are developed, and the functions which they are destined to exercise. The typical form, as

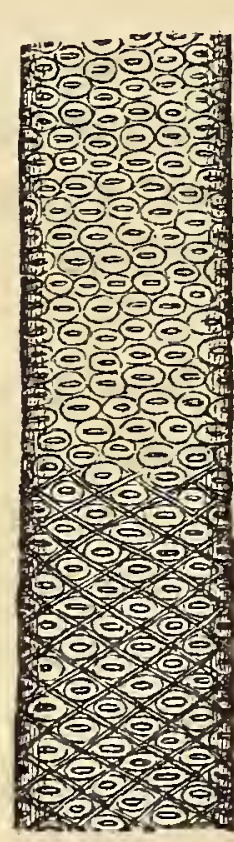

5

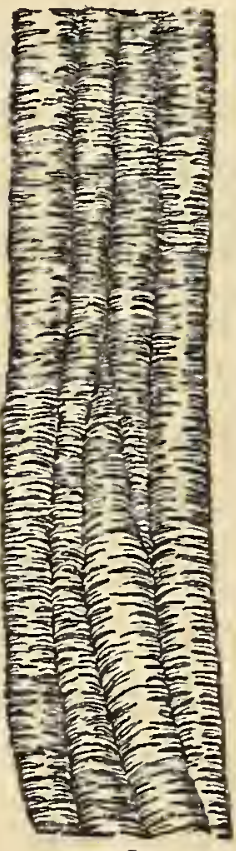

4

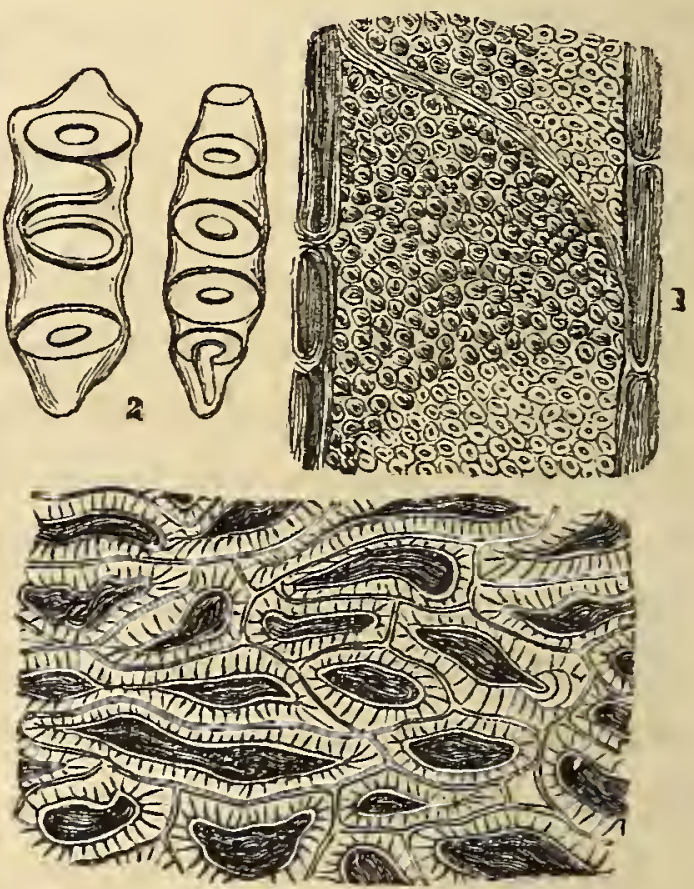

3

fig. 159.

1. Vertical section of root of Alder, with outer wall. 2. Spiral vessels from the Opun. tia vulgaris. 3. Section of nut (croylus avellanus), showing cells with small radiating pores. 4. An interior cast of siliceous portion of the spiral tubes in the Opuntia. 5. Vertical section from the Elm; in the lower portion small spiral fibres are seen between the pores.

we have before observed, of the vegetable cell is spheroidal ; but when developed under pressure within walls, or denser tissues, they take other shapes, as the oblong, lobed, square, prismatical, cylindrical, fusiform, muriform, compressed, stellate, filamentous, \&c. : it is then termed the Parenchym; and the cells woven together are called cellular tissue. In pulpy fruits the cells may be easily separated one from the other : a thin transverse section from a strawberry is represented in No. 15, Plate XV. : within the cells are smaller cells, commonly known as the pulp. Fig. 158, $a$, is the elementary form of oval cells or vesicles, proceeding on to the formation of branched cellular tissue, $b$. Remarkable specimens of the filamentous tissue may be seen in No. 19, 
Plate XV., the fungiform elongated cells from the Mushroom; only another and more closely connected growth of the mucedinous fungi, or, as it is commonly called, the spawn of the mushroom.

In No. 20, Plate XV., is seen the stellate tissue cut from the stem of a rush; and here we have the formative net-work dividing into ducts for the purpose of conveying the juices to the leaves of the plant. These ducts undergo other transformations; the cell itself may become gradually changed into a spiral continuous tube or duct, as seen in No. 21, Plate XV.; these are sometimes formed by the breaking

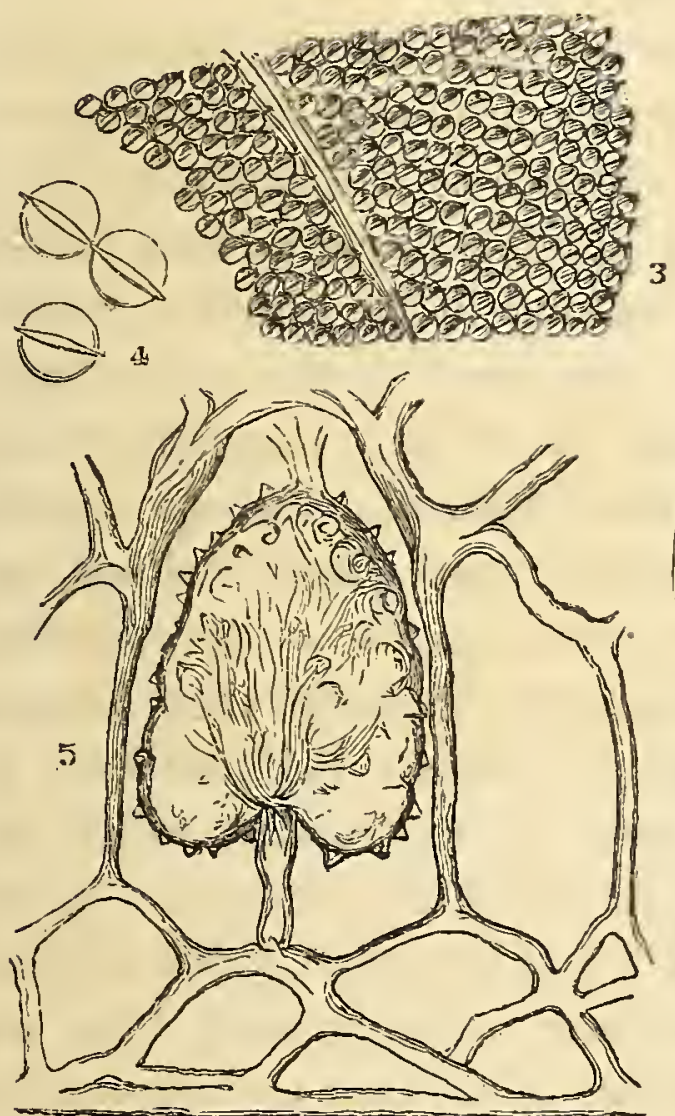

fig. 160 .

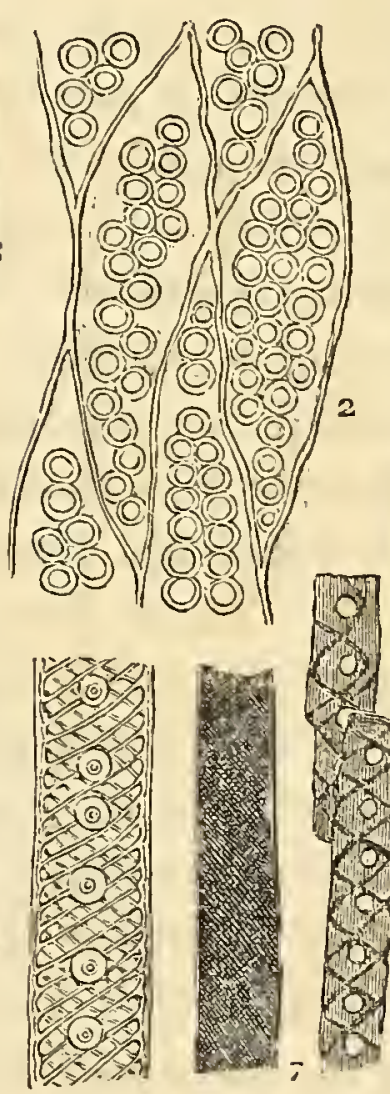

G

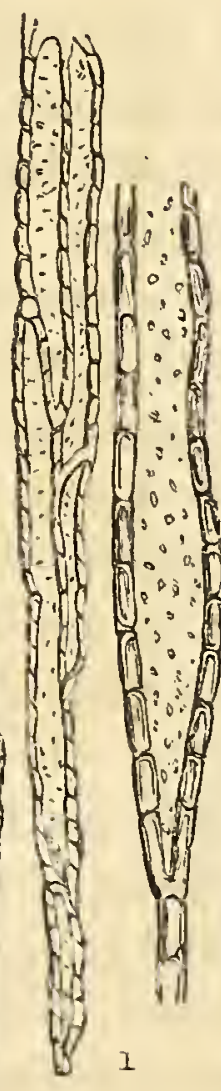

1

1. Woody fibre from the root of the Elder, with smail pores: 2. Woody fibre of fossil wood, with large pores. 3. A section of stem of Clematis; with pores. At 4 the pores are highly magnified, to show the way in which the line passes round them. 5. A vertical section of a leaf of the India-rubber tree, exhibiting a central gland. 6. Woody fibre, bordered with pores and spiral fibres. 7. Portions of fossil wood found in coal.

down of the partitions shown in the previous figure - in the centre we have a compound spiral duct, very much resembling the trachea of the silkworm. Another important change occurring in the original cell is that of its conversion into woody fibre, so largely entering into the 
texture of vegetables. These fibres are elongated cells of a spindle shape, or tubes drawn out to a point at each end, whose internal cavities become gradually filled up, as represented occurring at $b$, fig. 158 . The spiral thread may be either free, or adhering to the side of the tube. In the first case it forms the so-called sperm-animalculce of the antheridix of mosses; its elastic and hygrometric properties give it the appearance of motion: seen in fig. 159, No. 2. Nägeli believes this arises from the terminal enlargement of such fibres being a free nucleolus; and he also assumed that the motile threads, or vibratory cilia, found on the spores of some of the algæ, are of an analogous nature, the spore being a nucleolus.

In fig. 160, No. 1, we find a modification of the cellular tissue, having its sides marked by pits or dots, produced in consequence of the cell contents being unequally deposited over the inside of the cell-wall or membrane. The office of this peculiar tissue is said to be for the purpose of conveying fluids with rapidity in the direction of the woody tissues that surround it, or from the lower to the upper extremities, and is usually called the porosity of wood. Common woody fibre (Pluerenchyma) has its sides free from definite markings. In the coniferous plants, the tubes are furnished with circular dises, as shown at No. 6, fig. 160. These discs are thought to be contrivances to enable the tubules of the woody tissue to discharge their contents from one to the other, or into the cellular spaces. Such as have aromatic secretions are furnished with glands, a circumstance which has led to the division of woody tissue into simple and glandular. A large central gland is seen in a section of a leaf from the Ficus elastica, Indiarubber tree, No. 5, fig. 160. Professor Quekett says, "the nature of the pores or discs in the coniferce has been long a subject for controversy; it is now certain that the bordered pores are not peculiar to one fibre, but are formed between two, contiguous to each other, and always exist in greatest numbers on those sides of the woody fibres parallel to the medullary rays. They are hollow; their shape bi-convex, Nos. 3, 4; and in their centre is a small circular or oval spot (No. 6, fig. 160, and Nos. 1 and 5, fig. 159): the latter may occur singly, or be crossed by another at right angles, which gives the appearance of a cross. No. 2, fig. 160, is a vertical section of fossil wood, remarkable for having three or four rows of weody tissue, occupied by large pores without central markings."

At No. 7, fig. 155, we have represented fragments of Durham coal, composed almost entirely of woody cells, in which are two flat- 
tened spiral bands interlacing each other at regular distances, and having small angular spaces between them.*

Plants are likewise furnished with lactiferous ducts or tissue, the proper vessels of the old writers. These ducts convey a peculiar fluid,

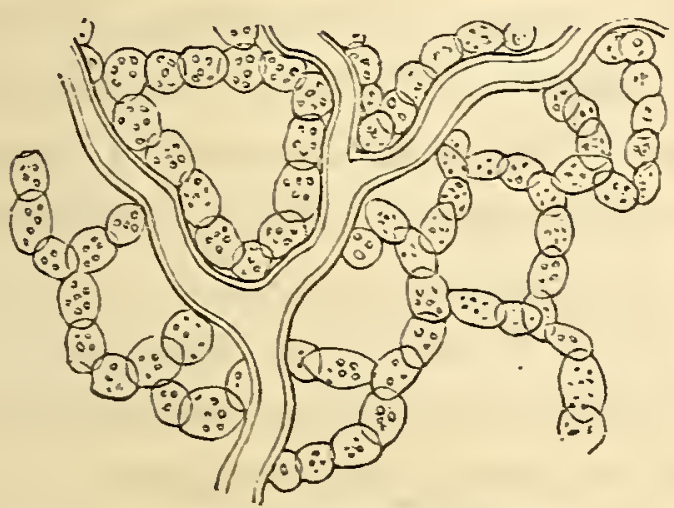

Lactiferous tissue.

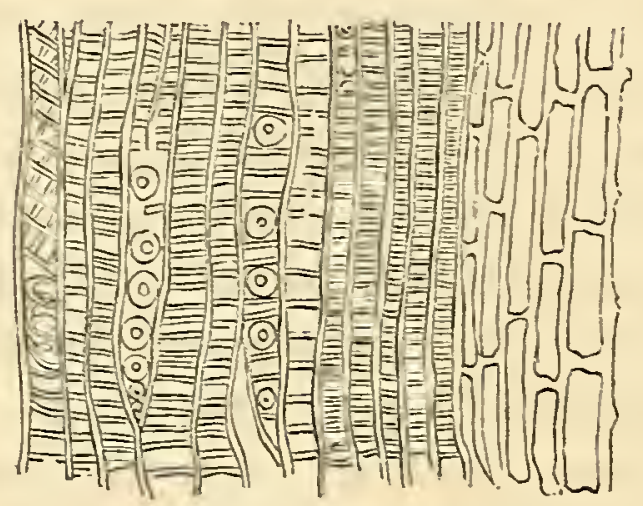

Reticulated ducts.

fig. 161.

called latex, usually turbid, and coloured red, white, or yellow; often, however, colourless. It is supposed they carry latex to all the newlyformed organs which are nourished by it. The fluid becomes darker after being mounted for specimens to be viewed under the microscope.

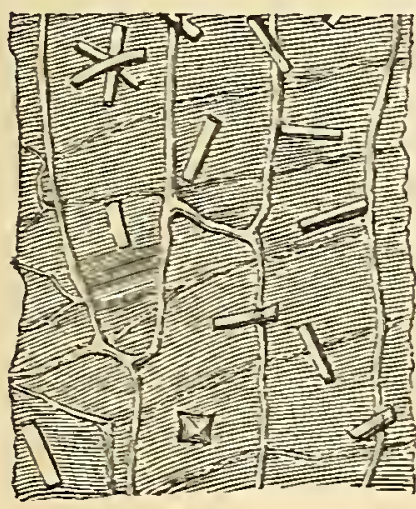

1.

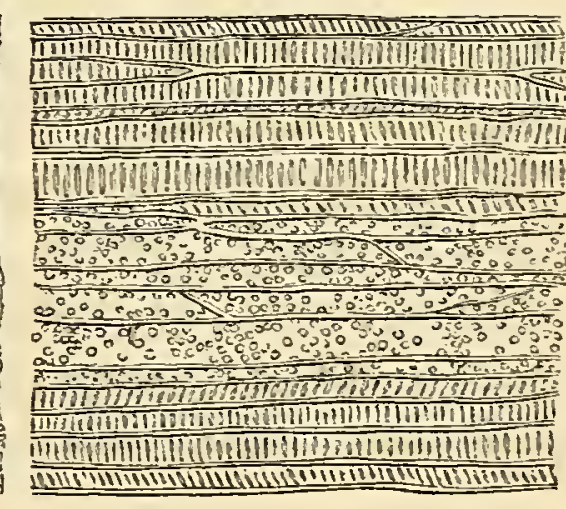

2.

1. Section from the outer layer of the bulb of an Onion, showing a crystal of oxalate of lime, with raphides. 2. A rertical section of root of a Fern.

This tissue is remarkable from its resemblance to the earliest aggregation of cells, the yeast-plant, and therefore has some claim to being considered the stage of development preceding that of the reticulated ducts seen near it in fig. 161. In the section from the India-rubber

* In the January number, 1854, of the Microscopical Journal will be found a vert interesting account of a microscopical examination of coal and its allied species by Professor Quekett. 
tree, fig. 160, a net-work of these lactiferous tubes may be found filled with a brownish or granular matter; that in fig. 161 is an enlarged view of this tissue from the liber of an Exogen, near the root.

Amongst the cell-contents of some plants we have a beautiful form of crystals, known as Raphides: the term is derived from paфıs, a needle; the crystals, when first noticed, were of this shape. They are composed of the phosphate and oxalate of lime; their use in the economy of the plant is not known. "Whether the result of chemical affinity or of a vital process, cannot be decided; but it is certain that they can be produced artificially in the tissue of plants."

The French philosopher, Geoffroy St. Hilaire, has been endeavouring to prove that crystals are the possible transition of the inorganic to organic matter. "Crystals have naturally been supposed to conceal the first beginnings of the phase named organic, because in crystals we first meet with determinate form as a constituent element. The matter named inorganic has no determinate form; but a crystal is matter arranged in a particular and essential form. The differences, however, between the highest form of crystal and the lowest form of organic life known-a simple reproductive cell-are so manifold and striking, that the attempt to make crystals the bridge over which inorganic matter passes into the organic is almost universally regarded as futile."*

These crystals may be always found in a section from the outer coat of the onion, fig. 162 ; and in fig. 163, No. 4, we have a cell filled with the stellate form of crystals, from the Garden Rhubarb.

Professor Quekett says: "If we examine a portion of the layers of an onion or of a squill, or take a thin section of the stem or root of the garden rhubarb, we shall observe many cells in which either bundles of needle-shaped crystals or masses of a stellate form occur.

"Raphides were first noticed by Malpighi in Opuntia, and were subsequently described by Jurine and Raspail. According to the latter observer, the needle-shaped or acicular are composed of phosphate, and the stellate of oxalate of lime. There are others having lime as a basis, combined with tartaric, malic, or citric acid. These are easily destroyed by acetic acid; they are also very soluble in many of the fluids employed in the conservation of objects ; some of them are as large as the 1-40th of an inch, others are as small as the 1-1000th. They occur in all parts of the plant; in the stem, bark, leaves, stipules, sepals, petals, fruit, root, and even in the pollen, with

* Mr. Lowes, Leader, Aug. 1853. 
few exceptions. They are always situated in the interior of cells, and not, as has been stated by Raspail and others, in the intercellular passages." Some of the containing cells become much elongated; but still the cell-wall can be readily traced. In some species of Aloe, as, for instance, Aloe verrucosa, with the naked eye you will be able to discern small silky filaments. When these are magnified they are found to be bundles of the acicular form of raphides.

"In portions of the cuticle of the medicinal squill-Scilla maritimaseveral large cells may be observed, full of bundles of needle-shaped crystal. These cells, however, do not lie in the same plane as the smaller ones belonging to the cuticle. In the cuticle of an Onion every
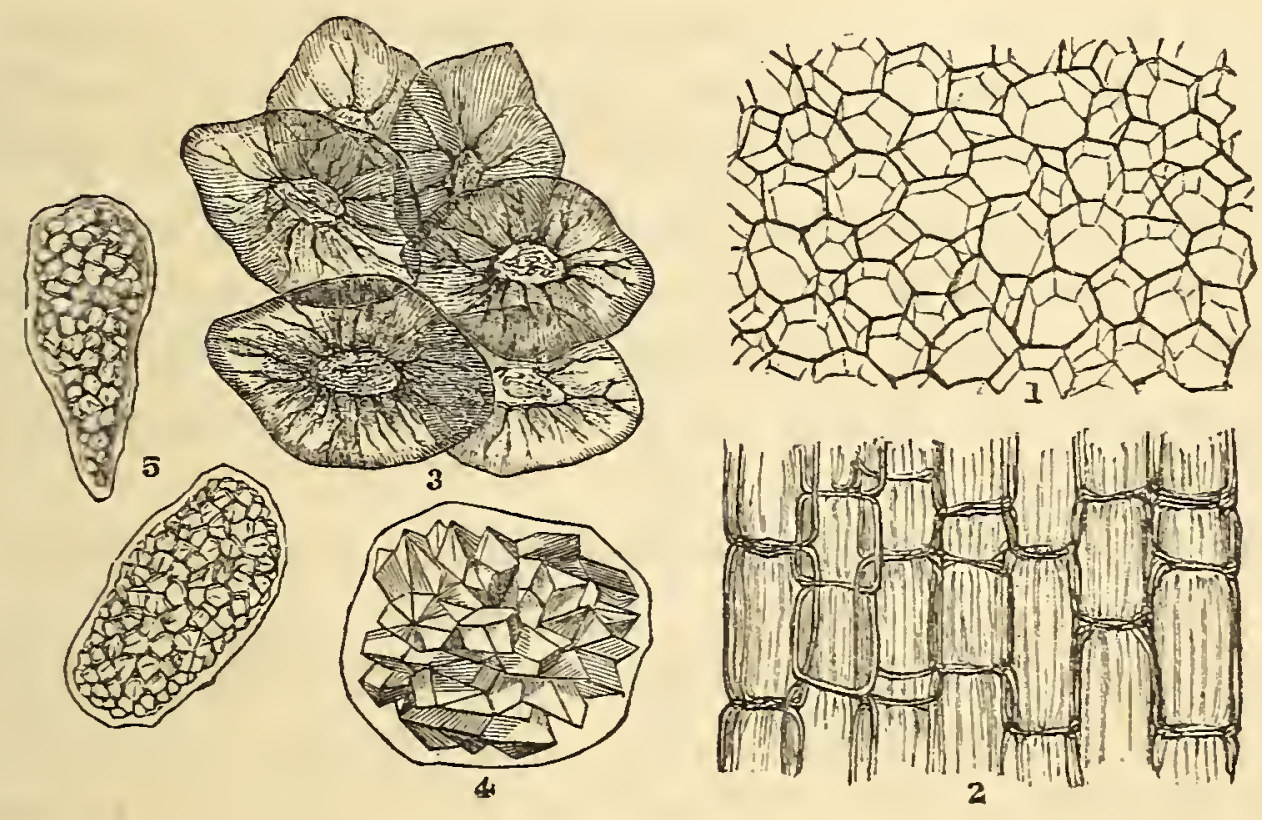

fig. 163.

1. Transverse section of stem of Equisetum, showing the hexagonal shape of cells. 2. A rertical section, showing the elongated cell. 3. Cells of the Pear, showing Sclerogen or gritty tissue. 4. Cells of garden Rhubarb, filled with raphides. 5. Cells from same, filled with starch-grains.

cell is occupied either by an octahedral or a prismatic crystal of oxalate of lime: in some specimens the octahedral form predominates; but in others from the same plant the crystals may be principally prismatic, and are arranged as if they were beginning to assume a stellate form. Some plants, as many of the cactus tribe, are made up almost entirely of raphides. In some instances every cell of the cuticle contains a stellate mass of crystals; in others the whole interior is full of them, renclering the plant so exceedingly brittle, that the least touch will

* As an exception, many years ago they were discorered in the interior of the spiral ressels in the stem of the grape-rine; but with some botanists this would not be considered as an exceptional case, the ressels being regarded as elongated cells. 
occasion a fracture; so much so, that some specimens of Cactus senilis. said to be one thousand years old, which were sent a few years since to Kew from South America, were obliged to be packed in cotton, with all the care of the most delicate jewellery, to preserve them during the transport.

"Raphides, of peculiar figure, are common in the bark of many trees. In the hiccory (Carya alba) may be observed masses of flattened prisms having both extremities pointed. In vertical sections of the stem of Elocagnus angustifolia, numerous raphides of large size may be seen in the pith. Raphides are also found in the bark of the apple-tree, and in the testa of the seeds of the elm; each cell contains two or more very minute crystals.

"It is at present not known what office raphides perform in the economy of the plant. Some have gone so far as to state, that they are deposits to be applied to the mineral part or skeleton of the plant; but the fact of their being insoluble in vegetable acids would prove this view of their use to be erroneous. The more rational supposition is, that they are generally accidental deposits formed by the union of vegetable acids with lime or other base existing in the plant or taken up from the soil."

In fig. 164 we have other representations of the crystalline structure of plants, in sections taken from wheat, grass, and the leaf of Deutzia scabia. This insoluble material is called silica, and is abundantly distributed throughout certain orders of plants, forming a skeleton after the soft vegetable matters have been destroyed: masses of it, having the appearance of irregularly-formed blackened glass, may be always found after the burning of hay or straw; it is caused by the fusion of the silica contained in the cuticle combining with the potash in the vegetable tissue, thus forming a silicate of potash (glass). To display this siliceous matter, it is necessary to dissolve the tissue by boiling it in nitric acid for several hours; the organic portion is thus destroyed, and the siliceous matter remains, a perfect cast of the original structure.

In the Graminacece, especially the canes; in the Equisetum hyenale, or Dutch rush; in the husk of the rice, wheat, and other grains, silica may be abundantly found. The Pharus cristatus, an exotic grass, fig. 164, No. 2, we have beautifully-arranged masses of silica with raphides. The leaves of the Deutzia, No. 3, are remarkable for their stellate hairs, developed from the cuticle of both their upper and under surfaces; forming most interesting and attractive objects when examined under the microscope, either by polarised or condensed light. 
The most generally-distributed and conspicuous of the cell-contents is Starch; at the same time it is one of great value and interest, performing a similar office in the economy of plants as that of fat in animals. It occurs in all plants at some period of their existence, and is the chief and great mark of distinction between the regetable and animal kingdoms. Its presence is detected by testing with iodine, by
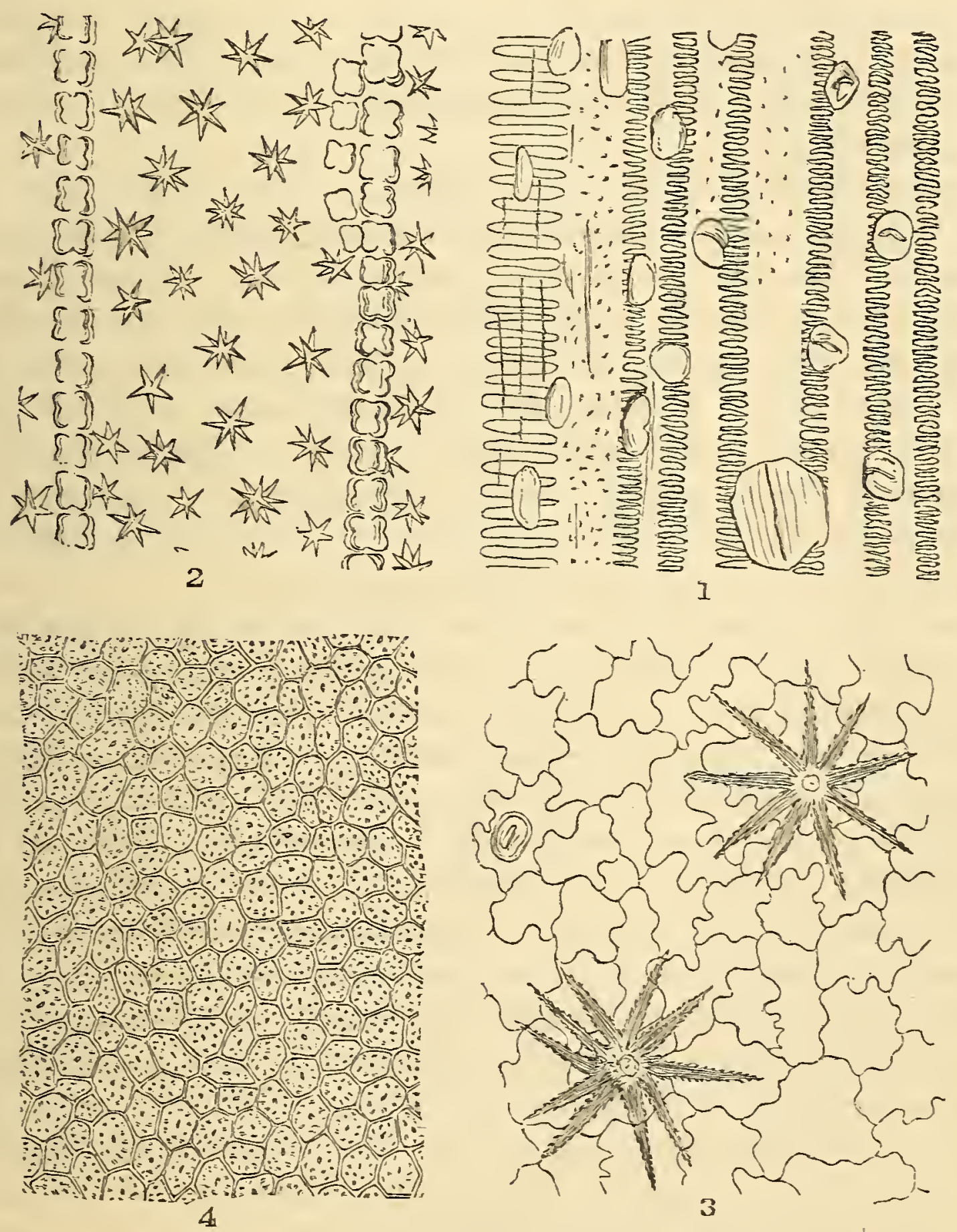

fig. 164.

1. Portion of the husk of Wheat, showing siliceous crystals. 2. Siliceous cuticle of blade of grass (Pharus cristatus). 3. Siliceous cuticle from under-surface of leaf of Deutzia scabia. 4. Section of a Cane; the cell-walls of silica, and internal pores filled with granular matter. 
means of which it is stained of a characteristic blue or violet colour. Being insoluble in cold water, it may therefore be readily washed away from the other matters contained in the cellular parts of fullgrown plants : it is often found in small granular masses in the interior of cells, as shown at No. 10, Plate XV. from the garden rhubarb. Starch-grains are variable in size : the Tous-les-mois, No. 5, are very large; in the potato, No. 14, are smaller; and in rice, No. 6, they are very small indeed. Nearly all present the appearance of concentric irregular circles; each granule having at one of its ovid extremities a circular spot termed the hilum, around which a large number of curved lines are arranged.

Leeuwenhoek, to whom we are indebted for the earliest notice of starch-granules, enters with considerable minuteness into a description of those of several plants - such as wheat, barley, rye, oats, peas, beans, kidney-beans, buckwheat, maize, and rice; and very distinctly describes experiments made by him in order to investigate the structure of the starch-granule. Dr. Reissek regards the granule as a perfect cell, from the phenomena presented during its decay or dissolution, when left for some time in water. Schleiden and others, after examining its expansion and alteration under the influence of heat and of sulphuric acid, consider it to be of a solid homogeneous nature.

Mr. Busk, having devoted considerable attention to the subject, agrees with M. Martin in believing the primary form of the starch-granule to be "a spherical or ovate vesicle, the appearance of which under the microscope, when submitted to the action of strong sulphuric acid, conveys the idea of an unfolding of plaits or rugæ, which have, as it were, been tucked in towards the centre of the starch-grain."* The mode of applying the concentrated sulphuric acid is thus described by Mr. Busk:- "A small quantity of the starch to be examined is placed upon a slip of glass, and covered with five or six drops of water, in which it is well stirred about; then with the point of a slender glass rod the smallest possible quantity of solution of iodine is applied, which is to be quickly and well mixed with the starch and water; as much of the latter as may be must be allowed to drain off, leaving the moistened starch behind, or a portion of it is to be removed by an inclination of the glass; the starch is then to be covered with a piece of thin glass. The object must be placed on the field of the microscope, and the $\frac{1}{4}$-inch object-glass brought to a focus close to the upper edge of the thin glass. With a slender glass rod a small drop

* G. Busk, F.R.S., on the Structure of the Starch-granule, Quarterly Journal of Microsconical Science, April 1853. 


\section{PLATE XV.}

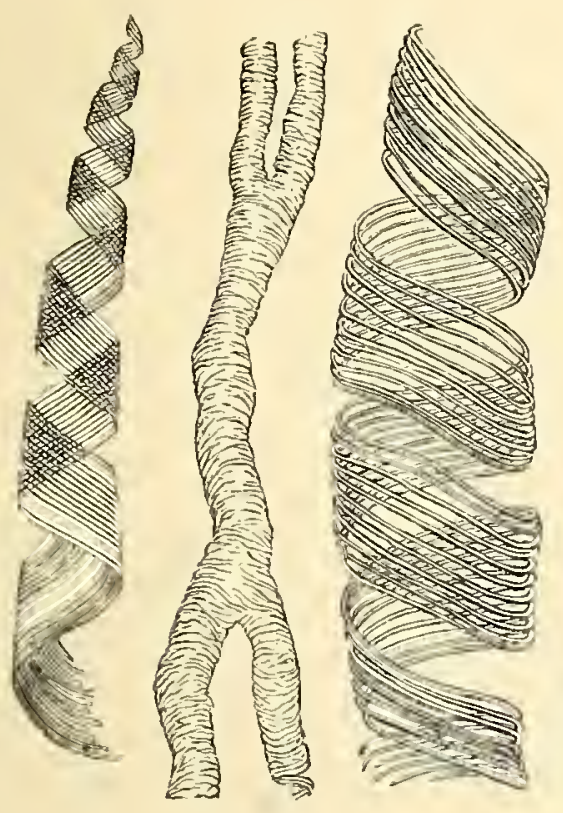

21
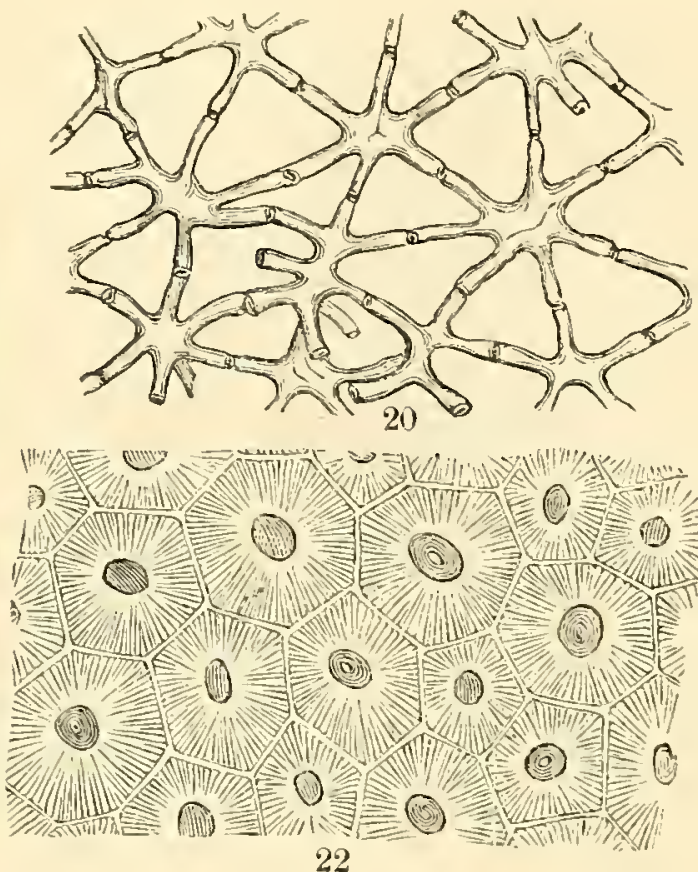

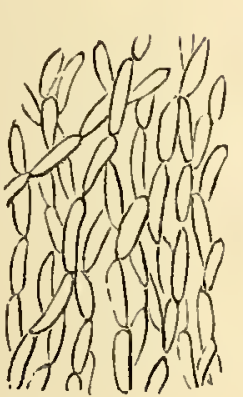

19
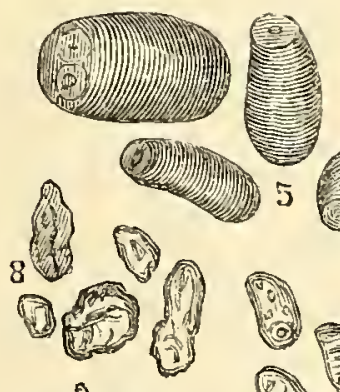

a

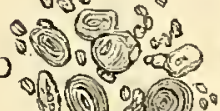
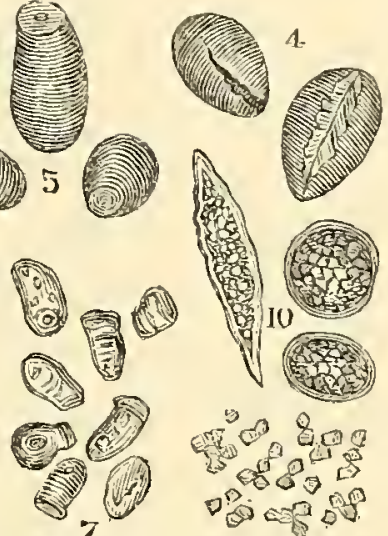

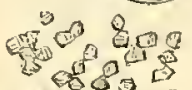

perag of

22

18

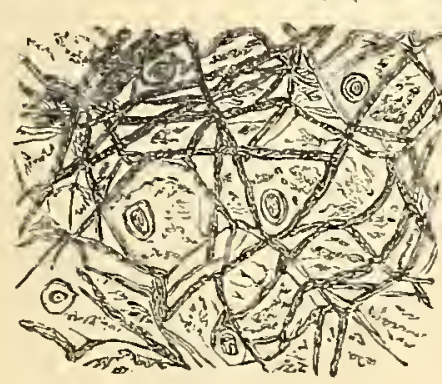

16

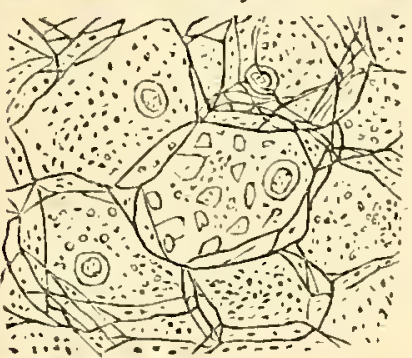

17

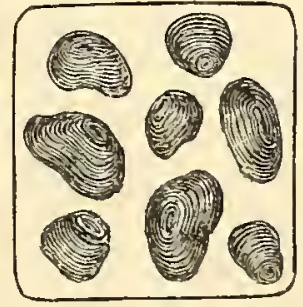

14
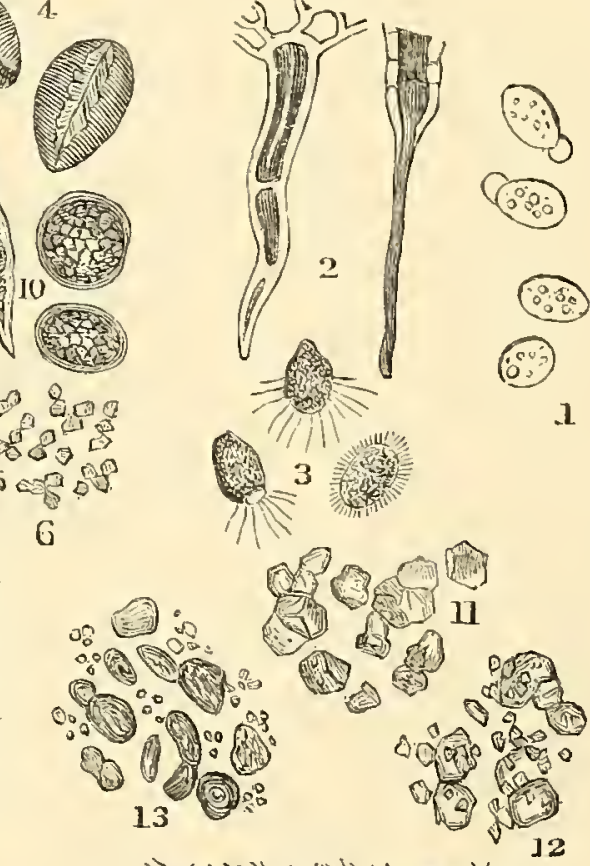

$\therefore 0$
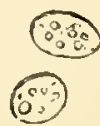

1

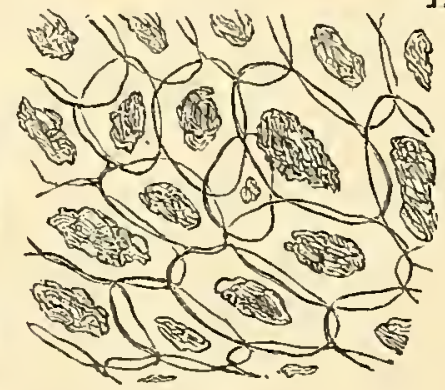

15

VEGETABLE STRUCTURES.

1. Cells of Torula Cerevisia, or Yeast Plant. 2. Hairs of Urtica Dioica, or Stinging-Nettle. 3. Cilinted spores of Confervæ. 4. Starch-grains broken by applying heat. 5. Starch from Tous-les-mois. 6. Starch from Rice. 7. Starch from Sago. 8. Imitation Sago Starch. 9. Wheat Starch. 10. Rhubarb Starch contained in their cells. 11. Maize Starch. 12. Oat Starch. 13. Barley Starch. 14. Potato Starch. 16. Section of Potato attacked by fungoid discasc. 17. Scction of Potato, showirg total absence of Starch grains. 18. Section of Potato, cells filled with heal thy Starch grains. 15. Section of a ripe Strawberry, cells oroid, and containing a brown nueleus. 19. Mrushroom Spawn, elongated cells. 20. Simple stellate tissuc from the stem of a Rush. 21. Spiral vessels from the Leck and Rhubarb. 22. Radiating cells or pores from the outer shell of the Ivory-Nut. 

of strong sulphuric acid is to be carefully placed immediately upon, or rather above the edge of the cover, care being taken that it does not run over it. The acid quickly insinuates itself between the glasses, and its course may be traced by the rapid change in the appearance of the starch-granules with which it comes in contact. The course of the acid is to be followed by moving the object gently upwards; and when, from its diffusion, the re-agent begins to act more slowly, the peculiar changes in the starch-granules may be readily witnessed. Whilst pressing or moving the glasses, the starch disc becomes torn, and is distinctly seen, especially in the blue-eoloured ones, to consist of two layers, an upper and lower one; and the collapsed vesicular bodies of an extremely fine but strong and elastic membrane." Mr. Busk believes the hilum to be a central opening into the interior of the ovate vesicle.

Instead of the sulphuric acid, the iodised solution of chloride of zinc proposed by Schultz (see p. 84) may be very advantageously employed. Nitric acid communicates to wheat-starch a fine orangeyellow colour; and recently-prepared tincture of guaiacum forms a blue colour with the starch of good wheat-flour.

Pure wheat-flour is almost entirely dissolved in a strong solution of potash, containing twelve per cent of the alkali; but mineral substances used for the purpose of adulteration remain undissolved.

Wheat-flour is frequently adulterated with various substances; and in the detection of these adulterations, the microscope, together with a slight knowledge of the action of chemical re-agents, lends important assistance. It enables us to judge of the size, shape, and markings on the starch-grains, and thereby to distinguish the granules of one meal from that of another. In some cases the microscopic examination is best aided by the application of a solution of potash. Thus we may readily detect the mixture of wheat-flour with either potato-starch, the meal of the pea, or bean, by the addition of a little water to a small quantity of the fiour; and then by adding a few drops of a solution of potash (made of the strength one part liquid potasi to three parts of water), the granules of the potato-starch immediately swell up, and acquire three or four times their natural size, while those of the wheatstarch are scarcely affected by it: if it be adulterated with pea or bean meal, the hexagonal tissue of the seed is at the same time rendered very obvious uncter the microscope. Polarised light may be used as an additional aid in this detection. Wheat-starch presents to our view a beautiful black cross proceeding from the central hilum; whereas the starch of the oat shows nothing of the kind. 
The diseases of wheat and corn are most readily detected under the microscope; these will be seen to be produced by a parasitic fungi, as well as animalculæ: we need scarcely add, that all are more or less dangerous if used as articles of food when found in this state.

Before leaving the subject of starch, allusion may be made to the prevalent and destructive epidemic among potatoes, which is a disease of the tuber, not of the haulm or leaves.

"Examined in an early stage, such potatoes are found to be composed of cells of the usual size; but they contain little or no starch: this will be seen upon reference to Nos. 16 and 17, Plate XV. Hence it may be inferred that the natural nutriment of the plant being deficient, the haulm dies, the cells of the tuber soon turn black and decompose, and fungi are developed as in most other decaying vegetable substances.

"This will undoubtedly explain the most prominent symptom of the potato-disease, the tendency to decomposition; and is a point in which the microscope confirms the result of chemical experiment: for it has been found that the diseased potatoes contain a larger proportion of water than those that are healthy. A want of organising power is evidently the cause of this deficiency of starch; but we fear the microscope will never tell us in what the want of this organising force consists."*

Writing of the potato-disease, Mr. Herepath says: "I find, in almost every instance, that the epidermis of the stalk below the surface of the ground is more or less in a state of decay, often disintegrated and completely rotten; the leaves and branches accord with the state of that part of the stalk below the ground. The tuber beneath the outer skin is first spotted brown, like a bruised apple; these spots extend and penetrate towards the centre, quite changing the nature of the potato. Those near the surface are most injured; in some cases the lowest on the root are not at all affected, while the upper ones are useless. I should therefore expect that the longer the crop remains in the land, the greater the injury will be. It seems, from the microscopic appearances, that the starch escapes injury for a long time after the skin and cellular parts are gone; and as the whole of the nutritive powers of the potato reside in the starch, I should recommend that wherever the disease has shown itself to any extent, the crop should be dug whether ripe or not, and the starch extracted by the following simple process:- after washing the roots, let them be rasped fine and thrown into a large tub or other vessel; pour a considerable quantity

* Professor Quekett's Histology of Vegetables. 
of water, and well agitate and rub the pulp with the hands: all the starch or fecula will, from its great weight, fall to the bottom, while the skin and fibrous matter will be carried away by the water; wash the starch with one or two more waters, allowing it to fall after each washing; spread it upon cloths in a warm room to dry: in this way about twenty or twenty-one pounds will be obtained from every hundred pounds of potatoes, and it contains as much nourishment as the original roots : it will keep any length of time, and might be used with flour to make bread, pies, puddings, \&c., as well as farinaceous spoonmeat."

A few plants are provided, like insects; with weapons of a defensive character. Those in the Urtica dioica, commonly called Stingingnettle, are elongated hairs, developed from the cuticle, usually of a conical figure, and containing an irritating fluid; in some of them circulation is visible: when examined under the microscope, with a power of 100 diameters, they present the appearance seen at No. 2, Plate XV. At No. 3, same plate, we have represented the very interesting ciliated spores from Confervoc.

The next of the cell-contents falling under our notice is that to which the leaves owe their green colour, and is known as Chloro$p h y l$. It is of a resinous, nature, containing nitrogen formed under. the action of light, and not otherwise. In the autumn its colour changes to red or yellow. It is soluble in spirit of wine or ether, but not in water; and is usually contained in cells lying below the surface. The astonishing circulation of the fluid-contents of vegetable cells may be examined at the same time as the Chlorophyl granules, by selecting for the purpose the transparent water-plants Chara, Nitella, and Vallisneria; or the hairs of Groundsel and Tradescantia.

It is necessary to take a thin section or strip from the flattened leaf of Vallisneria, which will exhibit a series of oblong cells, as seen in fig. 165, No. 4. Among the granules a few of a larger and more transparent character than the rest may be seen, having the nucleolus: of the cell within them.

Charc vulgaris is the plant in which the important fact of vegetable circulation was discovered, and in which, from the extreme simplicity. of its structure, much more is possibly observable, and with lower magnifying powers than other plants require. Fig. 165, No. 1, is a portion of the plant of the natural size. Every knot may produce roots; but it is remarkable that they always proceed from the upper surface of the knot, and then turn downwards; so that it is not pecu- 
liar that the first roots also should rise upwards with the plant, and come out of the seed-skin, and then turn downwards.

The stems and arms are tubular, and entirely covered with smaller tubes; the circulation can mostly be observed in these: see No. 2. Any ordinary cutting to obtain sections would squeeze the tube flat, and spoil it and the lining; it is, therefore, better to avoid this, by laying the Chara on smooth wood, just covered with water; then, with a sharp knife, make suddenly a number of quick cuts across it, and so obtain the various sections required. Wet a slip of glass, and turn the wood over, so as just to touch the water, and the sections will fall from the wood on to the glass, and thus be ready for the microscope.

Mr. Varley gives the following directions for cultivating these plants :

"The Chara tribe is most abundant in still waters or ponds that never become quite.dry.; if found in running .water, it is mostly met with out of the current, in holes or side bays, where the stream has little effect, and never on any prominence exposed to the current. If the Chara could bear a current, its fruit would mostly be carried on and be deposited in whorls.; but it sends out from its various joints very long roots into the water, and these would by agitation be destroyed, and then the plant decays; for although it may grow long before roots are formed, yet when they are produced their destruction involves the death of the plant. In order, therefore, to preserve Chara, every care must be taken to imitate the stillness of the water by never shaking or sudidenly turning the vessel. It is also important that the Chara should be disturbed as little as possible ; and if requisite, it must be done in the most gentle manner, as, for instance, in cutting off a specimen, or causing it to descend in order to keep the summit of the plant below the surface of the water.

"Imitate the freshness of the water by having an extent of the surface, which it is requisite to skim frequently, or suffer to overfiow by the addition of more water. These precautions being attended to, a clear bright surface is kept. It is also desirable to change a small portion of the water; but this should be done without agitation. The best vessels for cultivating this plant in are either wide pans holding three or four gallons, or glass jars a foot or more high ; into these the Chara may be placed, either with clean water alone, or a little earth may be sprinkled over it, so as to keep it at the bottom, or the bottom may be covered one inch with closely-pressed mould, in order that the water may be put in without disturbing it. On this lay the Chara, with a little earth over the lower ends to fix it. By causing the water 
to overflow is the readiest way to skim the surface, though dipping out gently will do ; but in all cases of pouring in water, hold something, such as a saucer or flat piece of wood, to receive the pouring, and make it spread, instead of allowing it to descend at onre on the surface. Pans in the open air nearly full of water will be kept in order by the wind and rain, only taking care to supply the deficiency (the effect of evaporation), and to change some of the water if it is consi-

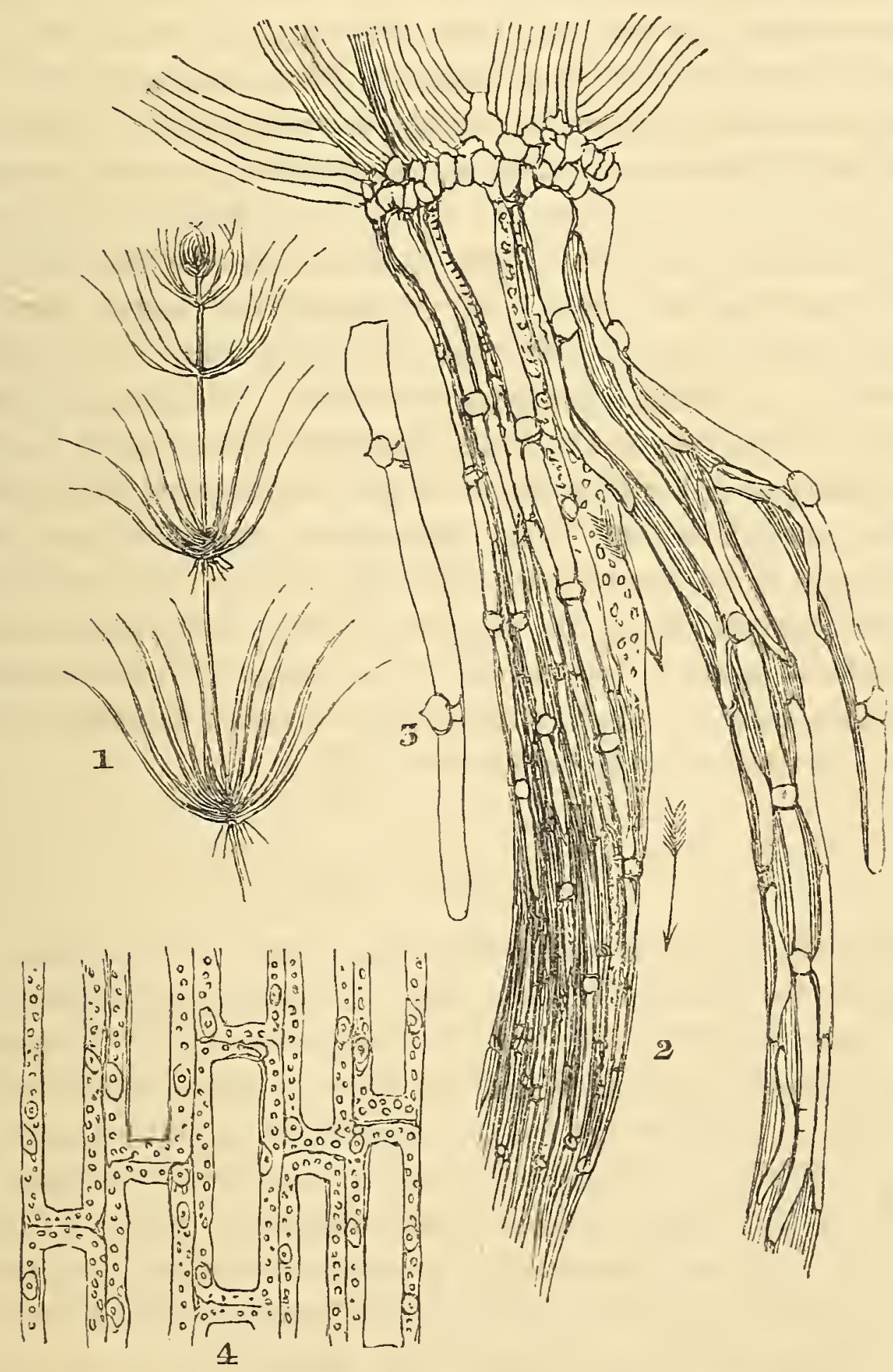

fig. 165 .

1. Branch of Chara vulgaris. 2. Magnified view: the arrows indicate the course of the granules in the tubes. 3. A limb of ditto, showing the budding at joints. 4. Portion of a leaf of Vallisneria spiralis, with cells and granules. 
dered necessary. The vessels kept indoors have a film which is always forming on the water, and which requires to be frequently removed.

"Imitate the equal temperature of its native holes, by sinking the pan a little within the earth; but during frosty weather keep the pan indoors, and at the lower part of the house, as this situation is generally the most uniform in its temperature.

"The Chara will live in any temperature above freezing, and grows quicker as the warmth increases; but above the earth, as outside of a first-floor window, it will not bear the daily difference between the mid-day sun and the cold of sun-rising. The glass jars I keep within the house as nearly uniform in warmth as convenient. Similar care is requisite for Vallisneria ; but the warmest and most equal temperature is better suited to this plant. It should be planted in the middle of the jar in about two inches deep of mould, which has been closely pressed; over this place two or three handfuls of leaves, then gently fill the jar with water. When the water requires to be changed, a small portion is sufficient to change at a time. It appears to thrive in proportion to the frequency of the changing of the water, taking care that the water added rather increases the temperature than lowers it.

"The natural habitat of the Frog-bit is on the surface of ponds and ditches; in the autumn its seeds fall, and become buried in the mud at the bottom during the winter ; in the spring these plants rise to the surface, produce flowers, and grow to their full size during summer. Chara may be found in many places around London, the Isle of Dogs, and in ditches near the Thames bank."

\section{VASCULAR TISSUE.}

This tissue in plants is somewhat analogous to the vascular system of animals; for this reason, and also for that of its tubular appearance, it was called by the older botanists trachece. It consists of rounded, square, columnar, elongated tubes or cells, with membranous walls, having a spiral fibre within. In some cases many fibres are seen running in the same direction, forming a band, when they are termed compound-spiral. Spiral fibres are represented in Plate XV. No. 21. Under this head other membranous tubes are included, in which the arrangement of the fibre has been considerably modified in its deposition. Elongated tubes or ducts, with porous walls, come under the head of vascular tissue ; they somewhat differ from the spiral varieties, inasmuch as they cannot be unrolled without breaking. It is a curious fact, that mostly the spiral coils from right to left; and it has been 
suggested that the direction of the fibre may determine that in which the plant coils round an upright pole. The Hop has left-handed spirals, and is a left-handed climber, which would therefore appear to support this theory. The nature of the fibre, and the development of the tissue, has been frequently a subject of dispute with botanists. The late Mr. Edwin Quekett gave much attention to this subject; and in an excellent paper published in the Microscopical Society's Transactions, 1840, gives the results of his observations. He says :

"In order to watch the development of the membranous tube of a vessel, no better example can be chosen than the young flower-stalk of the long-leek (Allium porrum) in the state in which this regetable is usually sent to market; it is then most frequently found to be about an inch or more in length, and from a quarter to half an inch in diameter. This organ occurs very low down amidst the sheathing bases of the leaves; and from having to lengthen to two or three feet, and containing large vessels, forms a very fit subject for ascertaining the early appearances of the vascular tissue.

"To examine the development of ressels, it is necessary to be very careful in making dissections of the recent part; and it will be found useful to macerate the specimen for a time in boiling water, which will render the tissues more easily separable. When the examination is directed in search of the larger vessels, it will be found that at this early stage they present merely the form of very elongated cells, arranged in distinct lines, amongst which some vessels, especially the annular, will be found matured, even before the cytoblasts have disappeared from the cells of the surrounding tissue.

"As development proceeds, the vessels rapidly increase in length, till they arrive at perfection. No increase in diameter is perceptible after their first formation. At this period, in the living plant the young ressels appear full of fluid, which is apparently, as remarked by Schleiden, of a thick character, and which he has designated regetable jelly; which, by boiling, or by the addition of alcohol, the contents, or at least the albuminous portion, becomes coagulated. From this circumstance, erery cell appears to enclose another in a shrivelled condition; this state is sometimes so far extended, that a thick granular cord is all that can be seen of the contents. When the granules have arranged themselves throughout the entire length of the tube, those which were first deposited, and had then some slightly visible space between them, become reinforced by others, or nourished by the contents of the ressel; so that space becomes obliterated, the fibre assuming a thread-like shape with defined borders, and sufficiently large to allow of the transmission 
of white light. When this action has progressed throughout the entire vessel, the transparency is restored, and the entire mass of granules disappear. The vessel having arrived at maturity, the liquid contents are absorbed, as happens in the cells of the pith; the vessel is then empty. Probably its being seen in these different states, at one time full and at others empty, may account for the discrepancies existing among botanists as to the functions these vessels perform. The period of growth at which the laying down of fibre commences, determines the
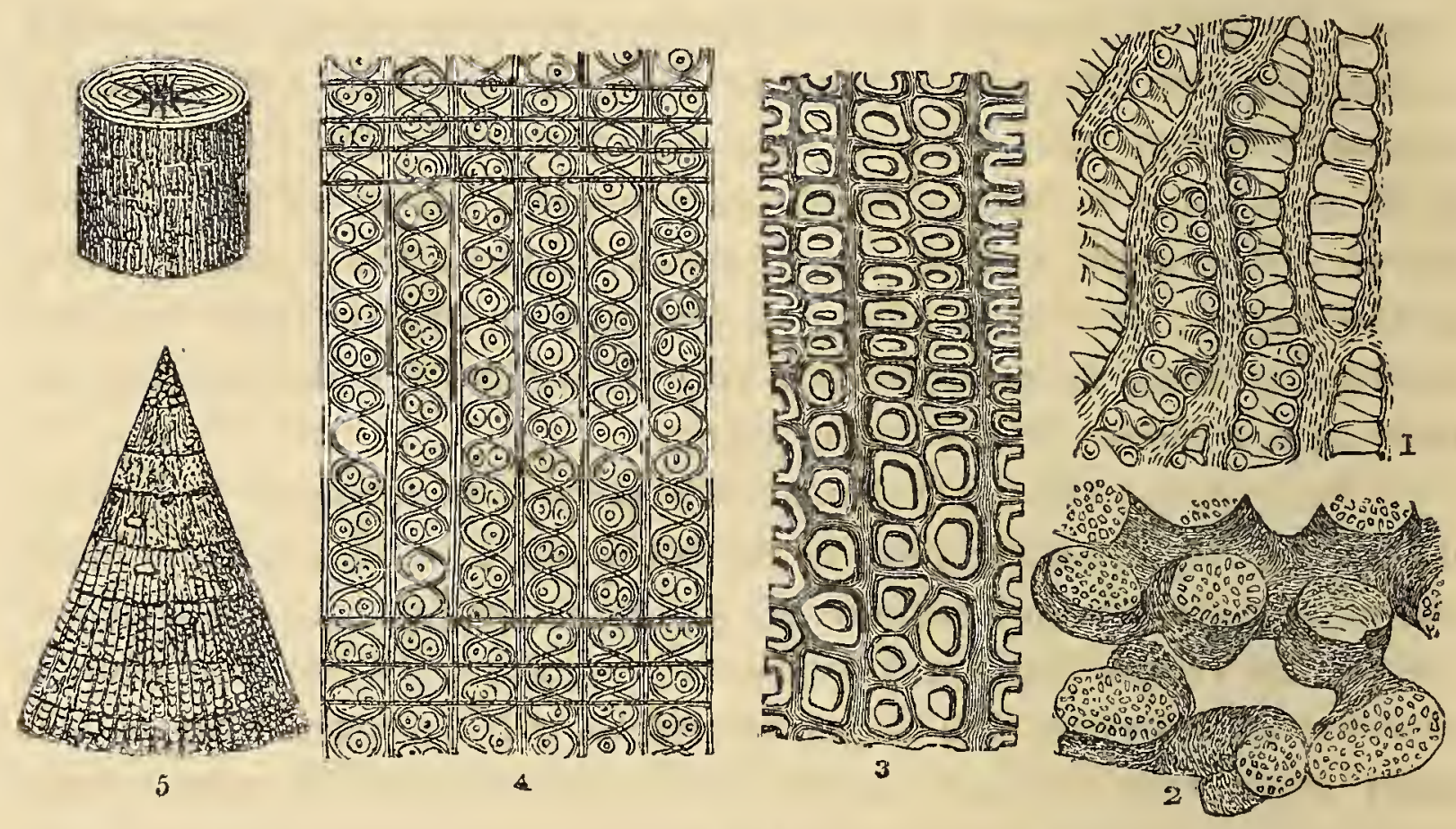

fig. 166.

1. A portion of the leaf of Sphagnum, showing ducts, vascular tissue, and spiral fibre, in the interior of its cells. 2. Porous cells obtained from the testa of Gourdseed, communicating with each other, and resembling ducts. 3. A transverse section of Taxus baccata (Yew'), showing the woody fibre. 4. Vertical section of the same, exhibiting pores and spiral fibres. 5. A section from the stem of a coniferous plant, with a transverse cutting magnified, and showing the zones of annual growth, termed the medullary rays.

distance between the several coils; for instance, when it is first formed the coils are quite close, scarcely any perceptible trace of membrane existing between them. In the annular vessel, the development of the cell and the adherence of the granules to each other are conducted in the same manner; the deposit showing a tendency towards the spiral direction, by the presence of a spire connecting two rings, or by a ring being developed in the middle of a spiral fibre. The annular vessel is the first observed in the youngest parts of plants, and when found alone indicates a low degree of organisation; as shown by its occurrence in Sphagnum, Equisetum, and Lycopodium, which plants, 
in the ascending scale of regetation, are almost the first that possess vascular tissue.

"It will be found that spiral fibre occurring with rings marks a higher step in the scale of organising power, the true spiral more so, and the reticulated and clotted mark the highest; this being the order in which these several vessels are placed in herbaceous exogens proceeding from within outwards, the differences of structure of the several vessels being indices of the vital energy of the plant at the several periods of its development. In those vessels in which the annular or spiral character of the fibre is departed from, some curious modifications of the above process are to be observed, as in the reticulated vessels met with in the common balsam (Balsamina hortensis). The primary formation of fibre in these vessels is marked by the tendency of the granules to take a spiral course, when it happens that some one of the granules becomes enlarged by the deposition of new matter around it. This becomes a point originating another fibre or branch, which becomes dereloped by the successive attraction of granules into bead-like strings, taking a contrary direction to the original fibre, forming a cross-bar, or ramifying, thereby causing the appearance by which the vessel is recognised.

"In the exogrenic vessel, the development of fibre proceeds in the same manner as in the last example; but the vessels will be seen to be dotted with a central mark, usually of a red colour, which, when viewed under high power, may be thought to resemble a minute garnet set in the centre of each dot. This red colour is owing to the dot being somewhat hollowed or cupped, and the centre only thin membrane. These vessels are best seen in the young shoots of the Willow. In the endogenic ressel the connecting branches are given off beneath each other, so that the dots which are rounded are arranged in longitudinal rows; and in the acrogenic or scalariform, which vessels are generally angular, and present distinct facets, the branches come off in the same line, corresponding generally to the angles of the ressel; but the spaces left between are linear instead of round."

Mr. Quekett affirms, in opposition to the views entertained by Mirbel, Richard, and Bischoff: "The dots left in these several vessels are not holes, neither do they consist of broken-up fibre, but are the membranous tube, unsupported by internal deposit; and on account of the extreme tenuity of the tissue, and the minute space between the fibres, the light in its transmission becomes decomposed, and appears of a greenish-red hue. The structure of the dot is best seen by examining the broken edge of any such vessels, when it will be found that the 
fracture has.been caused by the vessel giving way from one dot to another, so that the torn edge of the membrane can be observed in each dot."

\section{THE PREPARATION OF VEGETABLE TISSUES.}

The preparation and preservation of vegetable tissues is a matter of much importance to the miscroscopist; we therefore believe that we shall be doing good service to the student by adding a few general directions for this purpose.

Vegetable tissues are readily prepared for the microscope by making thin sections, either by maceration, by tearing, or by dissection.

The spiral and other vessels of plants require to be dissected out under a simple magnifying-glass. Take, for instance, a piece of asparagus, and separate with the needle-points the vessels, which must be finished under the magnifying-glass, in a single drop of distilled water. When prepared, keep in spirits of wine and water until mounted.

Vascular tissue is separated by maceration and dissection. The cuticle or external covering of plants assumes various attractive forms, and is best seen in the pelargonium, oleander, \&c. : it may be mounted dry, or in Canada balsam.

Cellular tissue is best seen in sections of the pith of elder, pulp of peach, pear, \&c. The petals of flowers are mostly composed of cellular tissue, and their brilliant colours arise from the fluid contained within the cells. In the petal of the anagallis, or scarlet chickweed, the spiral vessels diverging from the base, and the singular cellules which fringe the edge, are very interesting. The petal of the geranium is one of the most beautiful objects for microscopic examination. The usual way of preparing it is by immersing the leaf in sulphuric ether for a few seconds, allowing the fluid to evaporate, and then putting it up dry. Dr. Inman of Liverpool suggests the following method: first peel off the epidermis from the petal, which may be readily done by making an incision through it at the end of the leaf, and then tearing it forwards by the forceps. This is then arranged on a slip of glass and allowed to dry; when dry, it adheres to the glass. Place on it a little Canada balsam diluted with turpentine, and boil it for an instant over the spirit-lamp; this blisters it, but does not remove the colour. Cover then with a thin slip, to preserve it. Many cells will be found showing the mamilla very distinctly, and the hairs surrounding its base, each being slightly curved and pointed towards the apex of the mamilla. It is these hairs and the mamilla which give the velvety appearance to the petal. 
Fibro-cellular tissue is found readily in Sphagnum or bog-moss, as also in the elegant creeper Cobæa scandens. In some orchidaceous plants the leares are almost entirely composed of it. A modification of this form of tissue is found in the testa of some seeds, as in those of Salvia, Collomia grandiflora, \&c.

Ferns, with their curious and interesting sporules, when ripe burst, and are dispersed to a distance; so that they should be gathered before they come to maturity, and mounted as opaque objects. The development of ferns may be observed by placing the seeds in moistened flannel, and keeping them at a warm temperature. At first a single cellule is produced, then a second; after this the first divides into two, and then the others; by which a lateral increase takes place. It must be observed, that ferns do not form buds like other plants; but that their leares, or fronds as they are properly called, when they first appear, are rolled up in a circular form, and gradually unfoll, as in fig. 167. Ferns hare no

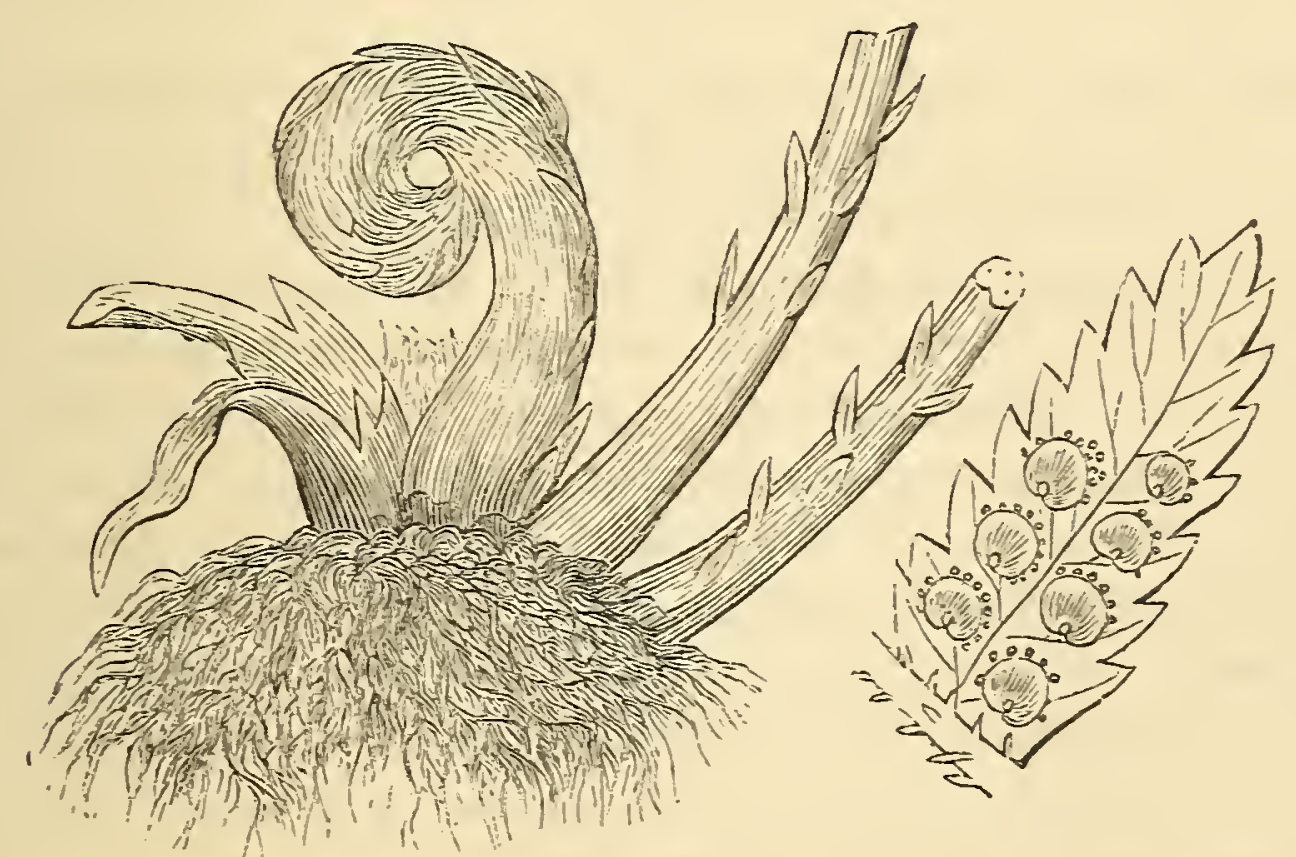

fig. 167. Male Fern.

visible flowers; and their seeds are produced in clusters, called sori, on the backs of the leares. Each sorus contains numerous thecæ, and each theca encloses almost innumerable spores or seeds. There are numerous kinds of fern, all remarkable for some interesting peculiarity, but which from want of space we cannot here enumerate.

The siliceous cuticle from the stems of grasses exhibits the beautiful arrangement of silica so constant in exogenous plants, and all of which are rery interesting objects for polarised light: Equisetum, wheat, barley, oat, or rye straw, Malacca cane, bamboo. Hairs are 
found principally upon the under-surface of leaves; they are best seen when viewed as opaque objects. Good specimens may be obtained from leaves of Deutzia, Anchusa tinctoria, Borago officinalis, Dolichos pruriens. Pollen should be viewed by refiected light, with a magnifying power of 100 diameters. That of the Passion-flower is curious, and if immersed in dilute sulphuric acid opens and disperses the grains. The pollen of the Datura Stramonium, if placed on a slide, and a few drops of dilute acid added, will send forth a tube of some length; the granular matter in the pollen may be seen to pass along the tube until it is emptied. For these observations a power of 200 diameters is required. Remarkable forms of pollen will be found in the following plants: Anagallis arvensis, Fuchsia globosa, Convolvolus, Jasmine, Lychnis, Penstemon, Polygonum orientale.

The following are amongst the most characteristic examples of woody fibre: flax, hemp, China-grass, section of pine, yew, date-palm, cedar-wood, cork, oak, mahogany, root of gooseberry, sycamore, furze, apple, vine.

Thin sections of charred wood sometimes show structures not seen in any other way. In fossil woods, after making thin sections, it is necessary to grind them on a lapidary's wheel, and afterwards polish them. In the ashes of coal a variety of vegetable structures may be discovered; these must be rendered transparent by immersion in Canada balsam. Stones and shells of nuts are prepared in the same way; or we may grind them between a piece of cork and fine emerypowder on box-wood, as we do sections of bone. In the cotton-plant, the hairs are attached to and envelop the seeds; their fibres are readily distinguished from those of linen, wool, \&c.: being tubular fibres of cellular tissue, these tubes, from the thinness of their sides, collapse, and appear like flat ribbons or bands, woven or interlaced together; which is a reason for cotton being preferred to linen (flax) in making lint for surgical purposes.

Sections of woods, if cut from hard woods, containing gum, resin, ic., should be soaked in essential oil, alcohol, or ether, before mounting as transparent objects. A razor may be fixed to the bench for the purpose of cutting these fine sections, or a fine plane will answer very well. The best instrument is the one used by Mr. Topping, fig. 168. $a b$ is a flat piece of mahogany, seven inches long and four wide, to the under-surface of which is attached, at right angles, a piece $g$ of same size as $a b$. $d$ represents a flat plate of brass, four inches long and three wide, screwed to the upper-surface of $a b$; to the middle of this plate is attached a tube of the same metal $e i$, 
three inches long and half-an-inch in diameter, and provided at its lower end with a screw $f$, working in a nut, and having a disc $k$ exactly adapted to the bore of the tube; this disc is connected with the upper-end of the screw, and is moved up or down by it. $c$ is another

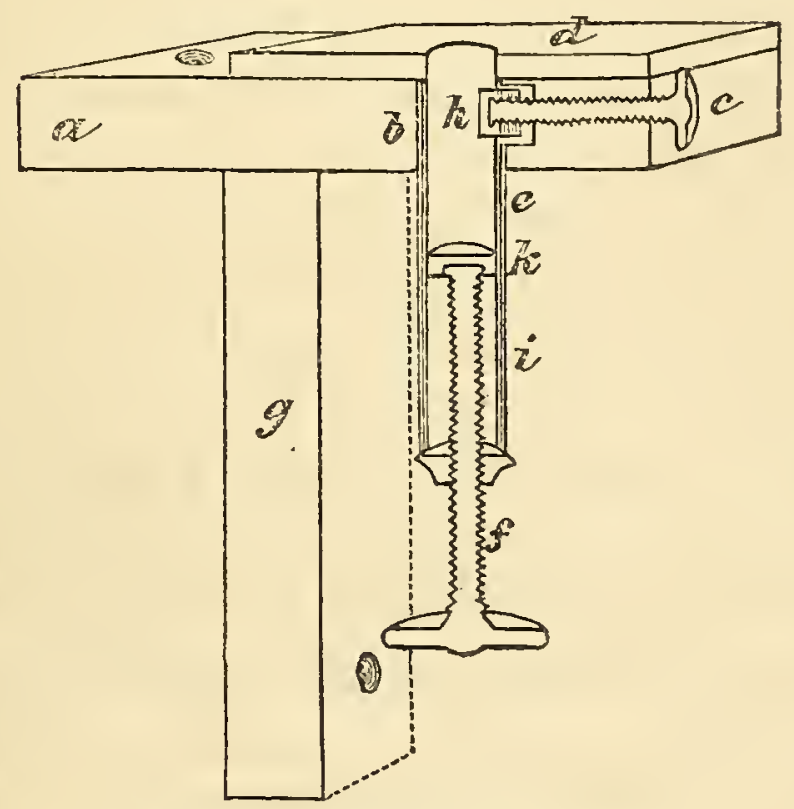

fig. 168. Cutting Machine.

screw connected with a curved piece of brass $h$, which is capable of being carried to the opposite side of the tube by it. The piece of wood about to be cut is put into the tube $e$, and is raised or depressed. by the screw $f$, whilst, before cutting, the curved piece of metal $h_{6}$ should be firmly pressed against it by the screw $c$. This instrument is to be fastened to the edge of a bench or table, where it may be always kept ready for use. The knife to be employed may be one constructed for the purpose; or a razor ground flat on one side will be found to answer very well.

"Method of making Sections. - If the wood be green, it should be cut to the required length, and be immersed for a few days in strong alcohol, to get rid of all resinous matters. When this is accomplished, it may be soaked in water for a week or ten days; it will then be ready for cutting. If the wood be dry, it should be first soaked in water and afterwards immersed in spirit, and before cutting placed in water again, as in the case of the green wood. If the machine to be employed be such as described, the wood (if sufficiently large) should be cut so as to fit tightly into the square hole, and be driven into it by a wooden mallet; if, on the contrary, it be round, and at the same time too small for the hole, wedges of deal or other soft wood may be employed to fix it firmly: these will have the advantage of affording support, and if 
necessary, may be cut with the specimen, from which they may afterwards be easily separated. The process of cutting consists in raising the wood by the micrometer screw, so that the thinnest possible slice may be taken off by the knife; after a few thick slices have been removed to make the surface level, a small quantity of water or spirit may be placed upon it; the screw is then to be turned one or more divisions, and the knife passed over the wood until a slice is removed; this, if well wetted, will not curl up, but will adhere to the knife, from which it may be removed by pressing blotting-paper upon it, or by sliding it off upon a piece of glass by means of a wetted finger. The plan generally adopted is to have a vessel of water by the side of the machine, and to place every section in it: those that are thin can then be easily separated from the thick by their floating more readily in the water; and all that are good, and not immediately wanted, may be put away in bottles with spirit and water, and preserved for future examination. If the entire structure of any exogenous wood is required to be examined, the sections must be made in at least three different ways; these may be termed the transverse, the longitudinal, and the oblique, or, as they are sometimes called, the horizontal (seen at No. 5, fig. 167), vertical, and tangental: each of these will exhibit different appearances, as may be seen upou reference to fig. 164 . $b$ is a vertical sec-

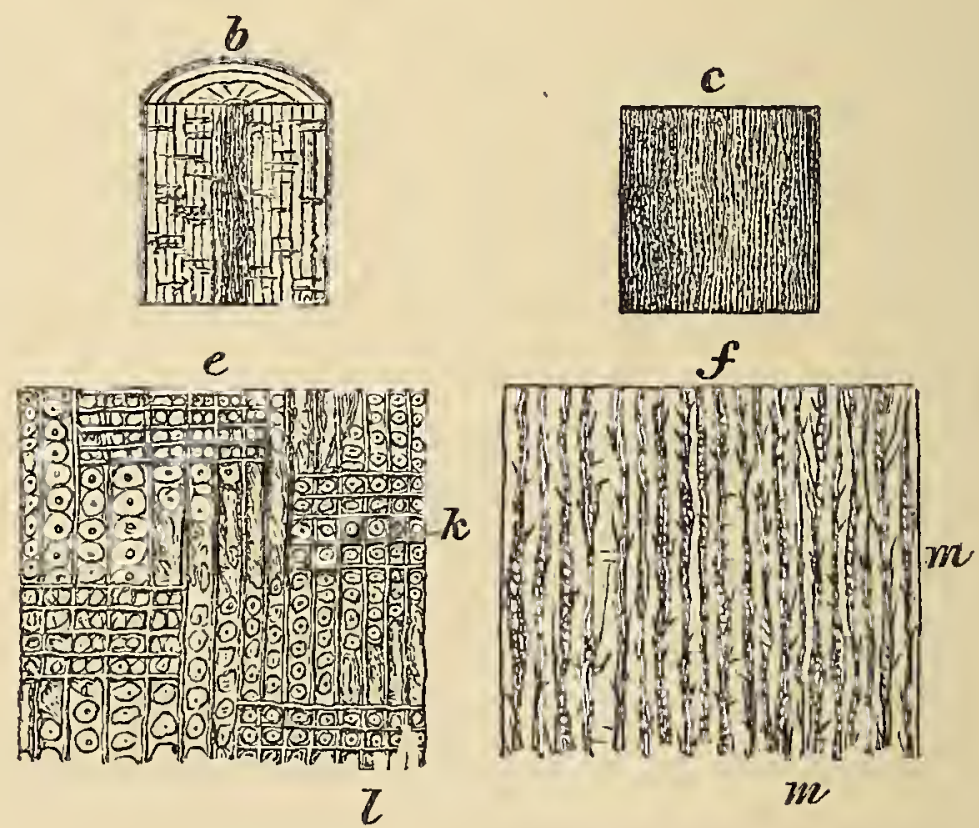

fig. 169.

tion through the pith of a coniferous plant: this exhibits the medullary rays, which are known to the cabinet-maker as the silver grain; and at $e$ is a magnified view of a part of the same : the woody fibres are seen 
with their dots $l$, and the horizontal lines $k$ indicating the medullary rays cut lengthwise; whilst at $c$ is a tangental section, and $f$ a portion of the same magnified: the openings of the medullary rays $m m$, and the woody fibres with vertical slices of the dots, are to be seen. Very instructive preparations may be made by cutting oblique sections of the stem, especially when large vessels are present, as then the internal structure of the walls of some of them may oftentimes be examined. The diagram above given refers only to sections of a pine; all exogenous stems, however, will exhibit three different appearances, according to the direction in which the cut is made; but in order to arrive at a true understanding of the arrangement of the woody and vascular bundles in endogens, horizontal and vertical sections only will be required. Many specimens of wood that are very hard and brittle may be much softened by boiling in water; and as the cutting-machine will answer other structures besides wood, it may here be stated, that all horny tissues may also be considerably softened by boiling, and can then be cut very easily." *

\section{CONCLUDING REMARKS.}

When using the microscope, a thinking and reflective mind will naturally be led to a consideration of matter, - a subject at once interesting, suggestive, and momentous. Our ideas of matter, space, and duration are merely comparative, derived from ourselves and from objects around us, and restricted within certain limited bounds; beyond which if we endeavour to extend them, they become vague and indistinct. The beginnings and endings, the exceeding greatness or the very littleness of things, are to us, in a certain sense, a source of perplexity and confusion. About thirty years since it was a generally received and favourite theory, that all organised bodies were made up of globules, which were incapable of being resolved into any other kind of structure, and of which the diameter was estimated at about 1-8000th of an inch. But the great improvements that have been effected in the microscope, and the general advance and extension of knowledge respecting the ultimate constitution of organised structures, have dissipated this view and exposed its erroneous nature, by showing that as far as our means of observation can carry us, there is no body, however minute, which is not capable of being resolved into still smaller particles.

"Let a man,"-Addison has remarked in the Spectator, with great

* Quekett on the Microscope. 
force and felicity of language,- " try to conceive the different bulk of an animal which is twenty, from another which is a hundred times less than a mite,-or to compare in his thoughts the length of a thousand diameters of the earth with that of a million,-_and he will quickly find that he has no different measures in his mind adjusted to such extraordinary degrees of grandeur or minuteness. The understanding, indeed, opens an infinite space on every side of us; but the imagination, after a few faint efforts, is immediately at a stand, and finds itself swallowed up in the immensity of the void that surrounds it. Our reason can pursue a particle of matter through an infinite variety of divisions ; but the fancy soon loses sight of it, and feels in itself a kind of chasm that wants to be filled with matter of a more sensible bulk. We can neither widen nor contract the faculty to the dimensions of either extreme. The object is too big for our capacity when we would comprehend the circumference of a world, and dwindles to nothing when we endeavour after the idea of an atom."

"The telescope has suggested to me," says the eloquent Dr. Chalmers, "that beyond and above all that is visible to men, there may be fields of creation which sweep immeasurably along, and carry the impress of the Almighty's hand to the remotest scenes of the universe :the microscope suggests to me, that within and beneath all that minuteness which the aided eye of man has been able to explore, there may yet be regions of invisibles; and that, could we draw aside the mysterious curtain that veils it from our senses, we might there see a theatre of as many wonders as astronomy has unfolded, a universe within the eompass of a point so small as to elude all the powers of the microscope; but where the wonder-working God finds room for the exercise of all His attributes, where $\mathrm{He}$ can raise another mechanism of worlds, and fill and animate them with all the evidences of His glory."

The gradations in the chain of universal being, through all its varying links, from the worm or the vegetable up to man himself, were long since pointed out by that diligent and scientific inquirer Soane Jenyns. His knowledge on the subject was, indeed, far inferior to that which we possess at the present day; nevertheless, his remarks have an abiding interest.

"The manner," he writes, "by which the consummate wisdom of the Divine Artificer has formed this gradation, so extensive in the whole and so imperceptible in its parts, is this:- He constantly unites the highest degree of the qualities of each inferior order to the lowest degree of the samie qualities belonging to the order next above it; by 
which means, like the colours of a skilful painter, they are so blended together and shaded off into each other, that no line of distinction is any where to be seen. Thus, for instance, solidity, extension, and gravity-the qualities of mere matter-being united with the lowest degree of regetation, compose a stone; from whence this regetative power, ascending through an infinite variety of flowers, plants, asd trees, to its greatest perfection in the sensitive plant, joins there the lowest degree of animal life in the shell-fish which adheres to the rock; and it is difficult to distinguish which possesses the greatest share, as the one shows it only by shrinking from the finger, and the other by opening to receive the water which surrounds it. In the same manner, this animal life rises from this low beginning in the shell-fish, through innumerable species of insects, fishes, birds, and beasts, to the confines of reason, where, in the dog, the monkey, and chimpanzee, it unites so closely with the lowest degree of that quality in man, that they cannot easily be distinguished from each other. From this lowest degree in the Hottentot, reason, with the assistance of learning and science, advances through the various stages of human understanding, which rise above each other, till in a Bacon or a Newton it attains the summit."

"When we consider," says the learned and philosophic Locke, "the infinite power and wisdom of the Maker, we have reason to think that it is suitable to the magnificent harmony of the universe, and the great design and infinite goodness of the Architect, that the species of creatures should also, by gentle degrees, ascend upward from us towards His infinite perfection, as we see they gradually descend from us downwards. Which, if it be probable, we have reason then to be persuaded that there are far more species of creatures above us than there are beneath; we being, in degree of perfection, much more remote from the infinite being of God than we are from the lowest state of being, or that which approaches nearest to nothing."

Coleridge pursues the same idea in the following beautiful passage of his Table-talk:

"Every rank of creatures, as it ascends in the scale of creation, leaves death behind it or under it. The metal at its height of being seems a mute prophecy of the coming regetation, into a mimic semblance of which it crystallises. The blossom and flower, the acmé of regetable life, divides into correspoudent organs with reciprocal functions, and by instinctive motions and approximations seems impatient of that fixure by which it is differenced in kind from the flower-shaped Psyche that flutters with free wing abore it. And wonderfully in the insect realm doth the irritability, the proper seat of instinct, while yet the nas- 
cent sensibility is subordinate thereto,-most wonderfully, I say, doth the muscular life in the insect, and the musculo-arterial in the bird, imitate and typically rehearse the adaptive understanding, yea and the moral affections and charities of man. Let us carry ourselves back in spirit to the mysterious week, the teeming work-days of the Creator, as they rose in vision before the eye of the inspired historian ' of the generations of the heavens and earth, in the days that the Lord God made the earth and the heavens.' And who that hath watched his ways with an understanding heart, could, as the vision evolving stifl advanced towards him, contemplate the filial and loyal bee; the home-building, wedded, and divorceless swallow ; and, above all, the manifoldly intelligent ant tribes, with their commonwealth and confederacies, their warriors and miners, the husband-folk, that fold in their tiny flocks on the honied leaf, and the virgin sisters with the holy instincts of maternal love, detached and in selfless purity, - and not say to himself, Behold the shadow of approaching Humanity, the sun rising behind in the kindling morn of creation! Thus all lower natures find their highest good in semblances and seekings of that which is higher and better. All things strive to ascend, and ascend in their striving. And shall man alone stoop? Shall his pursuits and desires, the reflections of his inward life, be like the reflected image of a tree on the edge of a pool, that grows downward, and seeks a mock-heaven in the unstable element beneath it, in neighbourhood with the slim water-weeds and oozy bottom-grass that are yet better than itself and more noble, in as far as substances that appear as shadows are preferable to shadows mistaken for substance? No! it must be a higher good to make you happy. While you labour for any thing below your proper humanity, you seek a happy life in the region of death."

These are lofty and philosophical truths, which must impress every mind, and awaken a responsive echo in every heart. Now, in the wide and diversified field of investigation which the marvels and beauties of nature offer, we find that to every created thing a certain measure of space is assigned, and that its powers of observation are circumscribed and limited; although, undoubtedly, some move and put forth their energies in a sphere of greater extent and importance than others, according to their allotted position and varied wants in the scale of existence. But all are co-relative and mutually dependent in the one grand and harmonious scheme of universal creation. This earth is the spot appointed for man to dwell and act upon-the scene of his life's drama-the theatre for the exercise of his faculties. $\mathrm{He}$ stands foremost of all sentient creatures here below, and links instinct 
and reason together. True, the sphere of his bodily action is comparatively narrow and restricted; but his mind is untrammelled in its sphere, and can soar and penetrate into regions far beyond the bounds of matter. Formed for the enjoyment of intellectual pleasures, his happiness arises from his knowledge; and his knowledge increases in proportion as he discovers and contemplates the variety, order, beauty, and perfection of the works of Nature. Whatever, therefore, can assist him in extending his observations, is to be valued as in the same degree conducive to bis happiness. "What we know at present, even of things the most near and familiar to us, is so little in comparison of what we know not, that there remains an illimitable scope for our inquiries and discoveries; and every step we take serves to enlarge our capacities, and give us still more noble and just ideas of the power, wisdom, and goodness of God. This marvellous universe is so full of wonders, so teems with objects of latent beauty, that perhaps eternity alone will open up and develop sufficient opportunities to enable us to survey and admire and appreciate them all."

Professor Hunt, in an able passage of his Poetry of Science, thoughtfully and eloquently says: "All thing's visible around us are aggregations of atoms. From particles of dust which, under the microscope, could scarcely be distinguished one from the other, are all the varied forms of nature created. This grain of dust, this particle of sand, has strange properties and powers. Science has discovered some; but still more truths are hidden within the irregular molecule of matter which we now survey, than even philosophy dares dream of. * * * It gathers around it other particles, they cling together, and each acting: upon every other one, and all of them arranging themselves around the little centre according to some law, a beautiful crystal results, - the geometric perfection of its form being the admiration of all. It exerts some other powers, and-atom cohering to atom, obeying the influences of many external radiant forces-undergoes inexplicable changes; and the same dust which we find forming the diamond, aggregates into the lordly tree; blends to produce the graceful, scented, and richlypainted flower; and combines to yield the luxury of fruit. It quickens with yet undiscovered energies, it moves with life; dust and vital force combine; blood and bone, nerve and muscle, result from the combination. Forces which we cannot by the utnost refinements of our philosophy detect, direct the whole; and from the same dust which formed the rock and grew in the tree, is produced a living and a breathing thing, capable of receiving a divine illumination, of bearing in its new state the gladness and glory of a soul." 
But when the soul shall become divested of flesh, the pleasures of sense can be no more; and if, by a continued habit, any longings after them shall hang about it, such must create a proportionate degree of uneasiness and disquietude; as they can never possibly be gratified. But if its principal delight has been in the contemplation of the beauties of creation and the adoration of the Almighty Author, it soars when disembodied into the celestial regions, duly prepared for the full enjoyment of intellectual happiness.

"And lives the man, whose universal eye

Has swept at once th" unbounded scheme of things ;

Mark'd their dependence so, and firm accord,

As with unfaltering accent to conclude

That this availeth nought? Has any seen

The mighty chain of beings, lessening down

From infinite perfection to the brink

Of dreary nothing, desolate abyss !

From which astonish'd thought recoiling, turns?

Till then, alone let zealous praise ascend,

And hymns of holy wonder, to that Power,

Whose wisdom shines as lovely on our minds,

As on our smiling eyes his servant sun."-THOMson. 


\section{N D E X.}

ACAlephe, 250.

Acarus of beetle, 325 .

— of cloth moth, 325 .

of fowl, 321.

of fly, 325 .

of rat, 316 .

scabiei, 317.

Achnanthes, 232.

Achromatic illuminator, 54 . object-glasses, 33 .

Actiniadæ, 190.

Actinia gemmacea, 194.

- maculata, 194.

- mesembryanthemum, 193.

rubra, 193.

Tuediæ, 195.

Actinocyclus, 235.

Adams' microscope, 8.

Alcyonidæ, 159.

Alcyonellæ, 212.

Trembley on, 212.

Alcyonium, spiculæ of, 218.

Algæ, 396.

Air-pump for removing air-bubbles, 74 .

Amici's microscopes, 10.

Annelidæ, 251.

Anguinaria, 173.

Animalculæ, cilia of, 122. collecting, 65 .
Animalculæ, marine, 129.

— polygastria, 125.

_- rotifera, 142 .

Animal cell, 326.

- connective tissue, 345 .

elementary tissue, 351.

— life and structure, Huxley on, 349.

- cell, history of, 336 .

— membrane, 339.

— simple membrane, 352.

-— structural metamorphoses, 343.

- structure, 334 .

_ structure, mode of investigating, 379.

Anthea, 195.

Ants, 303.

Ant-lion, 304.

Arachnoidiscus, 236.

Aristophanes, microscope known to, 2.

Artists, designs for, 235.

Astatia, varieties of, 136 .

Asteroidæ, 162.

\section{Bacillaria, 137.}

Baker on the microscope, 8 .

Barry, Dr., on spiral fibre, 358.

Bee's tongue, leg, \&c. 309.

Beetles, description of, 290.

Bennett, Dr., on investigating animal structure, 379. 
Berg-mehl, 151.

Binocularity, applied to microscope, 113.

Binocular microscope, Wenham's, 113.

Blood corpuscles, 353.

- crystallisation of, 354 .

Bone, birds', 376 .

—_ fishes', 376 .

human, 370.

reptiles, 376 .

Bonnani's microscope, 7.

Boys, Mr. Thos., on mounting objects, 74.

Brachionæ, 145.

Brewster's, Sir David, on viewing objects, 51 .

Brookes', Mr., on viewing opaque objects, 60 .

Bryozoæ, 198.

- Bowerbankia,'200.

Burnett, Dr., on parasites, 324.

Busk, Mr.; on Anguinaria spatulata, 173.

— on HydaLids, '320.

— on starch granules, 414.

Butterflies and moths, 294.

\section{Camera-lucida, 93.}

- Quekett, Professor, on the use of the, 94 .

Campanularia, 190.

— volubilis, 189.

Campylodiscus clypeus, 235.

Capillaries, 356.

Carpenter, Dr., on the animal cell, 336.

Carter, Mr., surgeon, on foraminifera, 245.

Cartilage, 365.

Caryophilliæ, 178.

Celaporidæ, 175.

Cells of glass for dissections, 69 .

Cellulariæ, 176.

Cellular tissue of plants, 406 .

Cements, 73.

Cementing pencil, 73.

Chara vulgaris, 418.

Cheese-mite, 317.

Circulation of blood in frog, how to view, 381.

Clionæ, 210.
Cocconeis, 235.

Cocconema Böeckii, 232.

Cochineal insect, 331 .

Cockchafer, 293.

Coleridge's reflections on life, 351 .

- on scale of creation, 431.

Collecting objects, 89 .

- animalculæ, 65 . stick, Mr. Williamson's, 65.

Collodion for multiplying objects, 78 .

Confervæ, 396.

Consolidated tissues, 367 .

Corallidæ, 163.

Coral reefs, Capt. Basil Hall on, 167.

Dr. Macculloch on, 166.

Coryne-stauridia, 181.

Coscinodiscus, 235.

Crane-fly, 275.

Crisiadæ, 169.

Crustaceæ, 253.

Crystals of snow, 111 .

- urinary salts, 107.

Cutting machine, 427.

Czermak upon tooth substance, 368 .

Deane, Mr., on mounting objects, 75 .

Death-watch beetle, 291.

Delabarre's microscope, 8.

Denny, Mr., on parasites, 313 .

Desmidaceæ, 400.

Deutzia scabia, 412.

Diatomaceæ, 231.

Rev. W. Smith on collecting and preserving, 237.

Diaphragm, description of, 53.

Dipping-tube for animalculæ, 63.

Disc circular, 71.

Dissecting knives and needles, 68 .

Divini's microscope, 6 .

Dragon-fly, description of, 216.

Echinococci, 319.

Echinidæ, 247.

Egg of bed-bug, 114.

Eggs of insects, 299.

Enchelis, 134

Entozoa foliculorum, 318. 
Epizoa, 318.

Escharæ, 204.

Eye-pieces, value of, 43 .

Eyes of insects, 27.

FeEt of insects, 280.

Ferns, 425.

- sections of, 426 .

Fibre, muscular, 358.

Fibrous tissue, 356.

Fishes' scales, 384.

Flea, 314.

- cat's, 316.

- larva of, 316.

Flosculariæ, 148.

Gosse's description of, 150.

Stephanoceros, 148.

Flustræ, 201.

— carbasea, 202.

- chartacea, 202.

foliacea, 202.

Lamouraux on, 201.

Foraminifera, 245.

Forceps for holding objects, 63 . Varley's, 64.

Frauenhofer's glasses, 8.

Frog-plate, 382.

— circulation, 381.

Fungoid growths, 394.

GALLIONELLA sulcata, 233.

Garrod's, Dr., crystals in blood, 355.

Gas-lamp, Highley's, 61.

Gasteropoda, 261.

Glycerine for mounting objects, 76 .

Gnat, description of, 28\%.

Goadby's fluids, 70.

Gorgoniæ, 169.

- spiculæ of, 219.

Gorham's, Mr., holder, 73.

Gosse, Mr., on marine animalculæ, I 29.

Grant, Dr., on Flustra, 201.

Grape blight, 393.

- section of, 394 .

Guinea worm, 318.
HAIR, 363.

Harris, Mr., on the grape-blight, 393.

Herapath on the uses of polarised light, 10 .

iodo-quinine, 104.

Highley's photographic camera for microscope objects, 118.

- gas-lamp, 61.

Hill's treatise on the Microscope, 8 .

History of animalculæ, 122.

Holland's eye-piece, 25.

Hooke's microscope, 6.

Huyghenian eye-piece, 37.

Huxley, T. H. on the unicellular theory, 340.

- general theory of vitality, 341.

Hydatids, 320.

Hydra, 154.

ICELAND spar, 98.

Improvements in microscope, 8.

Infusoria, history of, 121, 122.

- description of, 126.

Bakel's account of, 127 .

Infusorial animalculæ, 125.

Injecting, mode of, 79 .

Insects, changes of, 329 .

- commercial importance of, 331.

- dissection of, by Swammerdam, 328.

- distribution of, 330 .

eggs, 299.

_ feet, 280.

habits, 272 .

itch, 317 .

- mouths, 282.

_- parts of, for examination, 329.

__ probosces, $30 \%$.

- stings, 306.

—_tongues, 273, 383.

—u uses of, 332. wings, 285.

Isis hippuris, 169.

Isthmia enervis, 234.

JACKson's, Mr., micrometer, 39. 
Jenyns, S., on the gradation of beings, 430.

Johnston, Dr., on sponges, 209.

Jones, Wharton, Mr., on non-striated muscular fibre, 359.

- on the circulation in frog's foot, 355.

KING, Mr., on polarised light, 103.

Knives, Quekett's and Valentin's, 70.

Kolliker on the muscles of the skin, 359 .

Lankester, Dr., on sponges, 211. on the distinction between plants and animals, 211.

Laomedia, 189.

Leech, medicinal, 252.

Leeuwenhoek's microscope, 4.

Lenses, achromatic; 21.

- chromatic aberration of, 21.

Coddington, 26.

concavo-convex, 19 .

condensing, 59.

different forms of, 14.

double convex, 21.

- meniscus, 20.

method of tracing progress of rays through, 14.

- periscopic, 26.

plano-convex, 18.

- spherical aberration of, 17.

— refraction of light through, 13.

- Stanhope, 27.

Lieberkhun's microscope, 3.

- specula, 60 .

Life, theory of, 341.

- Wolff's doctrine of, 349.

— Williams, Dr. 'T., on, 350.

Limniades, 215.

Lister's lenses, 10.

Louse, 315.

Lucernaria, 197.

- campanularia, 198.

Lymnea stagnalis, 265.

MÀdReporid 178.
Marine animal plants, 159.

— vivaria in the Zoological Gardens, 206.

Materials for injecting, 79 .

Membranipora pilosa, 176 .

Micrometers, 38.

Microscope, Baker on the, 8.

—- Baker's student's, 49.

- Bonnani's, 7.

compound, 29.

Huyghenian eye-piece of, 36 .

Lieberkhun's, 3.

simple, 27.

- simple, for dissections, 27.

-_ Ross's, body of, 35.

— Ross's small compound, 45.

— large compound, 46.

Mollusca, 255.

Monads, 131.

Mosses, 369.

Moths and butterflies, 294.

Mülder on the vegetable cell, 390 .

Muscular fibre, 358.

Naviculer, 137.

— Quekett on, 234.

Nerves, 364.

Newt, 383.

Nicols' prism, 99.

Nitella, 417.

Noctiluca miliaris, 130.

Notamia, 171.

Notommata aurita, 146.

OBJECT-GLASSES, 33.

Objects, Shadbolt on collecting, 89 .

—_ mounting and preserving, 72. Mr. Boys on mounting, 74 .

On collecting salt-water specimens, 269 .

Owen, Professor, on animalculæ, 152.

- on microscopic investigation, 370 .

Parabolic reflectors, 57.

Parasites, 313.

— of cat, $3 \mathrm{l} 6$.

of $\mathrm{dog}, 316$.

of eagle, 323 . 
Parasite of fowl, 321.

of pheasant, 321.

of pigeon, 323 .

of sheep, 327 .

of turkey, 327.

of vulture, 323 .

Parkes', Dr., caution on using reagents, 86 .

Pedicula, 326.

Pearls, 256.

- artificial, 257.

Pennatulidæ, 182.

Photography applied to microscope, 117 .

Pigment cells, 363.

Plants, chorophyl in, 417.

_ cellular tissue of, 406 .

— crystals in, 410.

- histological elements of, 344 .

- lactiferous tissue of, 409 .

- preparation of tissues, 424 .

- raphides in, 410 .

- sections of, 422 .

- siliceous cuticle of, 413 .

- starch in, 414.

- starch of potato, 416 .

- structure of, 387 .

- vascular tissue of, 420 .

- circulation in, 418.

— vital characteristics, 389.

Polarisation of light as applied to the microscope, 96.

Polygastria of Ehrenberg, 125.

Polygastric animalculæ, 130.

Polypifera, 153.

Porifera, 208.

Potato, disease of, 416.

Preservation of algæ, 86 .

Z zoophytes, 226.

Proteus, the, 134.

Queкeтt, Edwin, on the vascular tissue of plants, 421.

- Professor, on advantages of polarised light, 112.

— on ciliary movement, 123.

— on raphides in plants, 411 .

- on the structure of bone, 371 .
Ralfs', Mr, on Desmideæ, 404.

Read, Rev. J. B., on animals found in chalk, 241.

Reflector, parabolic, 57.

Reflector, Shadbolt's, Mr., 59.

Retipora, $17 \%$.

Ross, Mr., on illumination, 52.

Rotifera, 142.

Salter, Dr., on dentine, 369.

Sarcina ventriculi, 393.

Saw-fly, 300.

Schachit's, Dr., preservative fluid for microscopic uses, 83.

Scissors for dissecting, 70.

Section cutting-machine, 427.

Selenite, 103.

Serpula, 252.

Sertularia filicula, $22 \%$.

Sertularidæ, 185.

Shadbolt, Mr., on collecting objects, 89 .

Siller-cups, 396.

Single microscope, 3.

Skeletons of zoophytes, 217.

Skin, fungoid diseases of, 394 .

- section of, 360.

Smith's and Beck's troughs, 67.

Smith, James, Mr., on management of light in viewing objects, 51 .

Snow, crystals of, 111.

- red, 395.

Sphagnum, leaf of, 422 .

Spider tribes, 310.

Sponges, 208.

Starch in plants, 414.

- tests for, 402 .

Stentors, 140.

Stinging-nettle, leaves of, 417.

Swammerdam's dissection of insects, 328 .

TEALE on actiniæ, 195.

Tissues, consolidated, 367. - elastic and non-elastic, 356 .

Tomata, diseased, 392.

Tooth, section of, 368 .

Topping's fluid for mounting, 88 .

- test-objects, 298. 
Torula cervisiæ, 392. diabetica, 393.

Tourmaline, 101.

Trembley on animalculæ, 135.

Troughs for animalculæ, 67 .

Tubiporidæ, 179.

WASPS' tongue, \&c., 275.

Water microscope, 5 .

Wenham's, Mr., binocular microscope, 113.

illuminator, 56.

Wheat-flour, adulterations of, 415 . portions of husk, 413.

Wichura, M., on the winding of leaves, 358.

Wollaston's and Coddington's lenses, 26.

Wonders of microscope, 1.20.

Wood, cutting sections of, 426.

Woodward, Mr., on polarised light, 101.

VALJISNERIA, 417.
Valkeria, 206.

Varley, Mr., on the culture of waterplants, 418.

Vegetables, structure of, 386.

- cellular tissue of, 406 .

- vascular tissue in, 420.

- preparation of tissues, 424 .

Vesicularidæ, 20.5.

Vibrio spirilla, 135.

Virgularia mirabilis, 183.

Volvox globata, 399.

Vorticellæ, 139.

XANTHIDIE, 239.

Yeast-PLANT, 391.

Zoological Society's marine vivaria, 206.

Zygoceros rhombus, 236.

THE END. 


\title{
LIST OF BOOKS
}

PUBLISHED AT THE

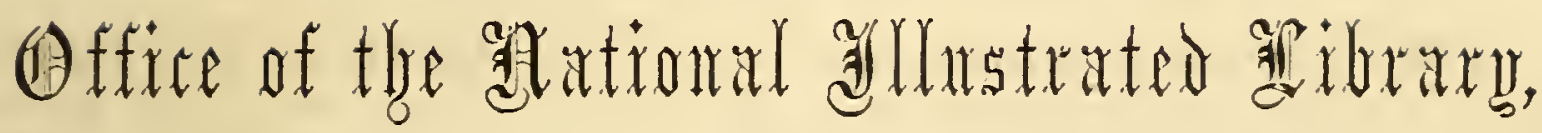

\author{
MILFORD HOUSE, STRAND;
}

ALSO BY

W. S. ORR \& CO., AMEN CORNER, PATERNOSTER ROW, LONDON.

Fourth Edition.

WEBSTER'S DICTIONARY

OF THE

ENGLISH LANGUAGE;

Exhibiting the Origin, Orthography, Pronuneiation, and Definition of Words ; comprising, also, a Synopsis of Words variously pronounced by different Orthoepists; and Walker's Key to the Classical Pronunciation of Greek, Latin, and Scripture Proper Names. A New Edition, revised and enlarged, by C. A. Goodrich, Professor in Yale College. With the addition of a Vocabulary of Modern Geographical Names, and their Prollunciation. The new words that have been added amount to several thousands, and the Dictionary now contains 27,000 words more than "Todd's Edition of Johnson." The work is handsomely printed upon a fine paper, in a elear readable type, in double columus.

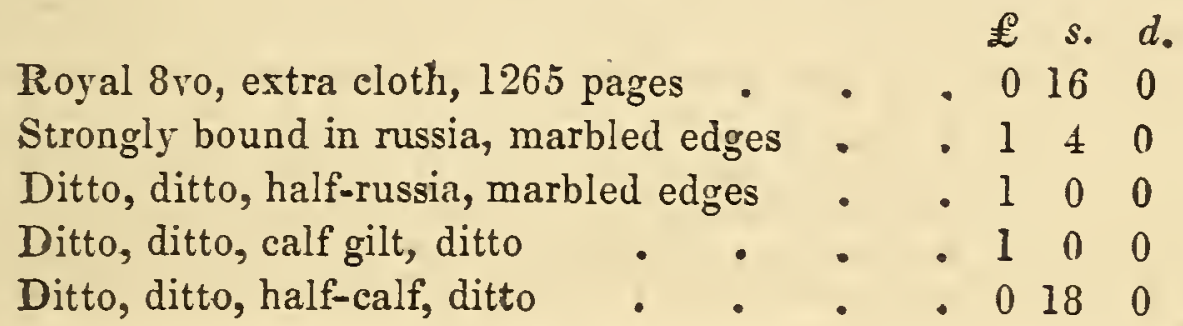

Testimonials.

"We can have no hesitation in giving it as our decided opinion, that this is the most elaborate and successful undertaking of the kind which has ever appeared."-Times.

"The present edition is in a handsome portable form-imperial octavo; it is clearly and correctly printed, upon moderately thick paper; and it is sold at a price proportioned rather to the wants of the public than to its intrinsic cost-so cheap is it compared with other similar publications."-Observer.

"A mine of philological wealth."-Wm. Russell, Esq.

"Best Dictionary of the English Language."-Morning Chronicle.

"A noble mollument of erudition."-Literary Gazelte.

"Most complete, accurate, and reliable Dictionary of the Language."-Thirty Members of United States Senate.

"It is acknowledged, both in this country and in America, to be the most copious and most excellent at present in circulation."-Editor of the London Imperial Dictionary, 1849.

"We rejoice that it bids fair to become the standard Dictionary to be used by the numerous millions of people who are to inhabit the United States."-Signed by 104 Members of Congress.

"Washington, Jan. $31,1850$.

"I possess many Dictionaries, and of most of the learned and cultivated languages, ancient and modern; but I never feel that I am entirely armed and equipped in this respect, without Dr. Webster at command."-Daniel Webster.

"This third edition of Webster's unrivalled work gires us an opportunity of recording our judgment in its favour, and in harmony with authorities who have described it as the standard of our language. Dr. Johnson places the writer of dictionaries among the unhappy mortals who are exposed to censure, without the hope of praise; one whom mankind have considered not as the pupil but as the slave of science, the prisoner of literature, and its humble drudge where toils are not cheered by a smile from learning and genius as they press forward to conquest and glory. If this be the rule, Dr. Webster is the exception. There is, we suppose, no scholar or writer in this country who does not give the greatest honour to the man whose lengthened labours, aided by his very able associates, have compiled this vast treasure of learning. Great indeed was the work of Dr. Johnson, but it has necessarily ceased to be a dictionary of a language which has been growing and enriching itself ever since he laid down his pen, and of which period we have the benefit in the 27,000 words more than are contained in Todd's Johnson. The book is essential to every one who wishes to know the words that are now in our tongue, their definition given with severe simplicity and beauty, their correct pronunciation, and their
classification in synonymes."-Leeds Times. 


\title{
ILLUSTRATED EDUCATIONAL WORKS.
}

\author{
Two Hundredth Thousand, price $1 s$. , or post free, $1 s .6 d$.
}

\section{The Illustrated London Spelling-Book; illustrated by upwards} of One Hundred and Seventy beautiful Woodcuts of Objects and Scenes described; forming by far the most alluring Introduction to Learning ever published. Coloured, price $2 s$. 144 pages.

“ 'The Illustrated Lond on Spelling-Book' contains no fewer than one hundred and seventy woodcuts, well executed; and, what is still more important, well chosen as to subject, of a cheerful and practical character, instead of the fantastic, nay cabalistic forms which disfigured our earliest edncational books. Even the alpliabet subjects will be suggestive to the little learner, and such as will induce him to ask questions relating to tliem : this is the moment for imparting information with effect; for it is never so strongly impressed upon the mind as when given the moment it is asked for. At the same time, the progressive plan of the book is closely watched; the child is not frightened by impossibilities at its tender age; but, both in the spelling and reading, the progress of the scliolar is consulted by the gradual increase in the length of the words. The reading-lessons are pretty little narratives, mostly original, and of just such incidents of amusement and instruction as are most likely to attract the attention of a child; they are cheerful throughout, although ' the good seed' is not forgotten to be thrown in the path of clildhood - a priceless feature in its first lessons, in spite of the secular cant and coldness of the day. Among the pictures, subjects of natural history predominate; and there cannot be a readier means of leading children to understand the beauty of earth and all that therein is, than by well-drawn figures of striking objects in the kingdoms of nature."-Illustrated London News.

\section{Fifty-first Thousand, price $2 s$.}

\section{The Illustrated London Reading-Book ; containing a Series of}

Lessons on the most amusing and instructive subjects, selected with great care from the best English Authors in prose and verse. The whole beautifully illustrated with above Two Hundred and Fifty Engravings.

" 'The Illustrated London Reading-Book' is the next stage in the series. It contains some hundred and fifty lessons, mos:ly selected from standard authors. The subjects are not of the old "Tom and Harry' class, or of a hackneyed character; but of actual living interest, in many instances describing wonders which are at the moment arising around us. Historiettes are sprinkled throughout the book. Its leading recommendation is the vivacity as well as variety of its contents : they are, to quote a common and often misused phrase, 'highly graphic ;' the evellts and incidents have a certain picturesqueness of character which must prove highly attractive to all growths; and both teaclier and learner may profit in their progress, though in a different ratio. The engravings are of ligher pretensions than those of the Spelling-book; some are from pictures by eminent living painters; and not unfrequently they take the reader to remote corners of the earth, as well as familiarise him with the wonders of lis own country. Appended is a vocabulary of words used in the volume, and rendered necessary by the somewhat advanced nature of:certain of the information conveyed in the descriptive lessons."-Illustrated London News.

Thirty-first Thousand, price $2 s$.

\section{The Illustrated London Instructor; being a Companion to} the Reading-Book ; consisting of Extracts from English Classical Authors, from the earliest periods of English literature to the present day. With One Hundred and Twenty fine Engravings.

"' 'The Illustrated London Instructor,' the third volume of the series, has a still higher aim than its predecessors-the teaching of the art of elocution, by 'selections from the best ancient and modern authors in every branch of English composition, most fitted for tlie purpose of eliciting and strengthening the powers of reading and speaking.' The contents are less discursive than those of the 'Read. ing-book;' and the 'Instructor,' as its name implies, is more directly educational. The plan commences with an Essay on Elocution and Composition, thougll the author does not fail to enforce the importance of 'the oral example of a competent teacher-without which, all books professing to give instruction in elocution are comparatively of little value.' The selections, about one hundred in number, consist of Moral and Miscellaneous Essays: Historical and Biographical Readings ; Aucient Eloquence; Natural History; and Dramatic and Poetic Readings. The masterpieces of English literature by the elder authors have supplied the staple of the volume; although there is an almost equa] proportion of graceful compositions by living writers. These impart much novelty, whilst they do not impair the soundness of the papers, many of which treat of the higher branches of study. The Illustrations are tasteful, various, and appropriate, and are, perhaps, of more artistic design than the Engravings of the 'Reading-book.' "-Illustrated London News.

\section{Second Edition.}

The Illustrated London Geography. By Joseph Guy, jun., of Magdalen Hall, Oxford, Author of numerous popular Educational Works. Demy 8vo, with about One Hundred Engravings of Cities, Customs, and Wonders of the World, \&c. The Drawings are made with great care from truthful sources. Nine 
Maps have been engrared by a patent process expressly for this work, and are corrected to the present period. Cloth, $2 s$.; coloured plates, $3 s$.

“The present volume is beautifully 'got up' and, being carefully compiled, presents a complete epitome of geographical knowledge."-Tait's Magazine.

"A cliss-book for young learners. The salient features of the geography of the sereral countries, with brief notices of their government and religion - of historical incidents, natural productions, physical characteristics, \&c.-are given in a concise style that is likely to make an impression on the mind of the pupil, the effect being aided by maps and numerous engravings."-Brislol Mercury.

"The Government press has produced no such elementary works as those of Ingram, Cooke, and Co., and other publishers-no such spelling-books, reading-books, and treatises on Geography, Optics, and Astronomy."-Morning Advertiser, September $28 t h, 1853$.

\section{The Illustrated London Astronomy, for the Use of Schools} and Students. By J.R. Hrxp, F.R.A.S. (of Mr. Bishop's Observatory, Regent's Park). With numerous illustrative Drawings and Diagrams. Demy .8ro, cloth, $2 s$.

"Mr. Hind has certainly managed to simplify his sublime subject, and gire a great amount of information in a few words."-Art Journal.

"Mr. J. R. Hind is so well known in connection with planetary researches, that his appearance in an educational library is as surprising as it is gratifying. "-Sherborne and Dorchester Journal.

\section{The Elements of Natural Philosophy (double volume). By} JABEZ HogG, M.R.C.S. Illustrated by uprards of 300 Woodcuts. Demy 8vo, cloth, $4 s$.

"Remarkable for its completeness, the number of admirable jllustrations, and its cheapness. It will be extensively useful to schools and private students."-Daily Neus.

"It is completed in a stỵle exceedingly creditable both to its editor and puolishers; and we know of no work we can so strongly recommend as an interesting and useful introduction to the physical sciences."-Medical Times and Gazette.

\section{The Mllustrated London Architectural, Engineering, and $\mathrm{Me}$ -} chanical Drawing-Book. By R. S. Burs. With numerous Engravings. Demy 8vo, cloth, $2 s$.

"This book should be given to every youth, for amusement as well as for instruction."-Taunfon Journal.

\section{Electric Science: its History, Phenomena, and Applications.} By F. BAKEwell, Author of "Evidences of Christianity," \&c. Copiously illustrated. Demy 8 ro, cloth, $2 s$.

"So much attention is drawn to this subject; its powers, though coeral with the creation, have been only so recently applied to facilitate communication; and we look to it so confidently for farther assistance, - that there is no compendium of recent date of such value to ourselres or to our children: the explanations are singularly clear, even to the non-scientific; and the rolume, dealing as it does in miracles of facts, possesses all the interest of a novel."-Art Journal.

\section{John Barnett's New Edition of Wade's Handbook to the}

PIANOFORTE : comprising an easy Rudimental Introduction to the Study of that Instrument, and Music in general; the Art of Fingering according to the Modes of the best Masters, exemplified in rarious Exercises, Scales, \&c., in all the Mrajor and Minor Kers; and interspersed by Relaxations from Study, consisting of Popular Melodies and Romances, and Selections from the Pianoforte Compositions of the most celebrated Masters ; also a short and easy introduction to Harmony or Counterpoint, and a new Yocabulary of Terms. Demy 4to, neatly bound in cloth, with Engrarings and Diagrams. Price $6 s$.

"As a manual, the book before us is decidedly commendable; and we have great pleasure in proffering it to the attention of the pupil and student, who wiil find in it much matter worthy their most careful and serious perusal."-M M sical World.

\section{The Illustrated New Testament (Authorised Version); with} upmards of One Hundred and Twenty Engravings, beautifully bound in cloth, embossed and gilt sides, gilt edges. The Illustrations are from drawings executed by eminent Artists expressly for this edition; with Notes historical, explanatory, and descriptive ; and embellished by a novel Panoranic Picture of the Holy Land, and a View of Lower Egypt. 4to, cloth gilt, $5 s$. 


\section{The Illustrated Practical Geometry. Edited by Robert Scott}

\section{BurN, Editor of "The Illustrated London Drawing-Book." Demy 8vo, cloth, $2 s$.} (Second Edition.)

"Suited to the youthful mind, and calculated to assist instructors, filled as it is with really good diagrams and drawings, elucidatory of the text."-Globe.

\section{The First Six Books of Euclid, with numerous Exercises.} Printed on a New Plan, with accurately executed Diagrams. Demy 8vo, cloth, $2 s$.

The present work, embracing the First Six Books of the Elements of Euclid, or, in other words, the foundation of Plane Geometry, is intended as a companion volume to that issued with it on Practical Geometry; the one embracing the theory, the other the application.

The text adopted is that of the distinguished mathematician, Dr. Simson. In very few instances has any change been made, and that only when it seened to be positively necessary. Several attempts have been made, by altering the text and the arrangement, to reduce the demonstrations of Euclid to what may be considered a more popular form, but these have been attended with little success; and a text like that of Simson, clear, direct, and unencumbered, like absolute truth itself, will always be adopted by scholar and student.

It has been thought unnecessary to extend this volume beyond the first six books of the Elements, or to enlarge it by notes, as it will be followed by other works carrying the subject forward. A number of Exercises on each book have been given, the solutions of which will be published separately.

First Lessons in Arithmetic, on a New Plan. By Hugo Reid, late Principal of the People's College, Nottingham, and Author of numerous Educational Works. Demy 8vo, cloth, $2 s$.

"The First Lessons in Arithmetic are calculated to awaken the dullest intellect."-Stamford Merc.

"The book is capitally adapted for the use of schools and for private tuition; and is remarkable for the beauty of its typography."-Derby Mercury.

Mechanics and Mechanism. By Robert Scott Burn. With about 250 Illustrations. Demy 8vo, cloth, 2s. (Second Edition.)

"One of the best-considered and most judiciously-illustrated elementary treatises on mechanics and mechanism which we have met with. The illustrations, diagrams, and explanations are skilfully introduced, and happily apposite; numerous, and beautifully executed. As a hand-book for the instruction of youth, it would be difficalt to surpass it."-Derby Mercury.

\section{The Illustrated London Drawing-Book: comprising a Com-} plete Introduction to Drawing and Perspective; with Instructions for Etching on Copper or Steel, \&c. \&c. Illustrated with above Three Hundred Subjects for Study in every branch of Art. By Robert Scotr Burn. Demy 8vo, cloth, 2s. (Second Edition.)

"Here is a work which has long been a desideratum with the English people. It is the completest thing of the kind which has ever appeared."-Tait's Magazine.

"s. This is a very capital instruction-book, embodying a complete course of lessons in drawing, from the first elements of outline-sketching up to the more elaborate rules of the art."-Bristol Mercury.

A New and greatly improved Elementary Grammar of the ENGLISH LANGUAGE, uniform with the "Illustrated London Spelling-Book." Strongly bound in cloth, $1 s$.

\section{Handbook of Foliage and Foreground Plants. By George} Barnard, Esq., Author of "Sketches of Switzerland," "Drawing Book of Trees," \&c. Post 8vo, cloth, $6 s$.

This book is completely illustrated with Sixty progressive Plates, filled with various studies of the best style of lithography, and will be found peculiarly valuable as a text-book for Students.

In preparation, and will shortly be published, Works on Geology, Algebra, the Steam-Engine, \&c. \&c. 


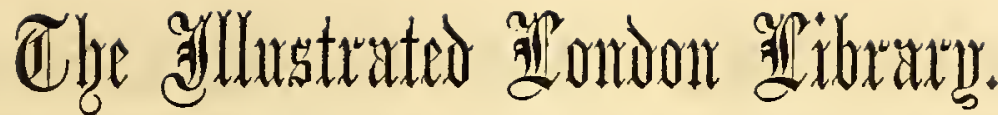

Demy 8vo, cloth, 6s. per Volume; calf, marbled edges, 10s. 6d.; morocco extra, gilt edges, $12 s$.

Second Edition, revised.

I.

NINEVEH AND ITS PALACES:

The Discoveries of Botta and Layard applied to the Elucidation of Holy Writ. By Joseph Bononi. Illustrated with above Two-Hundred Engravings.

II.

OUR IRON ROADS :

Their History, Construction, and Social Influences. By Frederick S. Williams. With numerous Illustrations and Diagrams.

Second Edition, revised.

III.

THE THREE COLONIES OF AUSTRALIA:

New South Wales, Victoria, and South Australia: their Pastures, Copper Mines, and Gold Fields. By SAmuel Sidver, Author of "The Australian Handbook," \&c., with numerous authentic Engravings.

\section{IV. \& V.}

AN ILLUSTRATED LIFE OF FIELD-MARSHAL THE DUKE OF WELLINGTON,

THE COMMANDER-IN-CHIEE OF THE BRITISH ARMY.

By J. H. Stocqueler, Esq., Author of "The British Officer," "The Handbook of British India," \&c. \&c. 'Two Volumes.

Household Gods of the Cilicians.

VI.

\section{LARES AND PENATES:}

Or, Cilicia and its Governors. Being a short Historical Account of that Province, from the Earliest Times to the Fresent Day. Together with a Description of some Household Gods of the Ancient Cilicians, broken up by them on their Conversion to Christianity, and first discovered and brought to this country by the Author, WILliaM BURCKHARDT BARKER, M.R.A.S., many years resident at Tarsus in an official capacity. Edited by William Francis Ainsworth, F.R.G.S., F.G.S.

VII.

\section{ENGLISH FORESTS AND FOREST-TREES:}

Being an Account, Legendary, Historical, and Descriptive, of the Forests and Trees of England, and the associations and events connected with them. Profusely illustrated.

VIII.

\section{THE THREE PRESIDENCIES OF INDIA:}

Their Rise, Progress, and Present Condition. A complete Review of the British Indian Possessions, from the Earliest Period to the year 1853. By JoHN CAPPER, Esq., F.R.A.S. Profusely illustrated.

IX.

ALGERIA :

The Topography and History, Political, Social, and Natural, of French Africa. By John Reynell Morell. Profusely illustrated. 


\section{THE NATIONAL ILLUSTRATED LIBRARY.}

\section{In Monthly Volumes, containing about 300 Pages and 50 Engravings, Crown 8vo,}

handsomely bound in cloth, gilt.

Per Volume

Calf, marbled edges, or morocco, gilt edges :

Calf, do. do., 2 vols. in 1

Morocco, gilt, do. do.

$\begin{array}{rr}\text { s. } & d . \\ 2 & 6 \\ 7 & 6 \\ 9 & 0 \\ 10 & 6\end{array}$

WORKS ALREADY PUBLISHED.

I. to IV. Boswell's Life of Dr. Johnson. Complete in Four Volumes. Fourth Edition.

v. 'The Illustrated Book of English Songs. Third Edition.

vi. 'The Mormons, or Latter-Day Saints. Third Edition.

VII. The Orbs of Heaven; or, the Planetary and Stellar Worlds. Fourth Edition.

VIII. Pictures of Travels in the South of France. From the French of ALexander DuMas. Second Edition.

IX. \& XIII. Huc's 'Travels in Tartary, Thibet, and China, in 1844, '5, and '6. Unabridged Edition. Two Voluues.

x. A Woman's Journey round the World. Unabridged. From the German of IDA PfElfFer. Third Edition.

xr. \& xII. Memoirs of Extraordinary Popular Delusions. By Chardes Mackay, LL.D. Second Edition.

XIV. Beswell's Journal of a Tour to the Hebrides. Companion Volume to "Boswell's Life of Johnson." By R. CaRRUThers, EsQ., of Inverness. Second Edition.

$x v$. Narrative of a Residence at the Capital of the Kingdom of Siam. By Frederick Arthur Neale.

XVI. The Illustrated Book of Scottish Songs. From the Sixteenth to the Nineteenth Century. Second Edition.

xVII. Picturesque Sketches of London, Past and Present. By Thomas Mrluer.

XviII. Madame Pfeiffer's Visit to Iceland and the Scandinavian North. Second Edition.

xIx. The Israel of the Alps: a History of the Persecutions of the Waldenses. By the Rev. Dr. Alexrs Muston.

xx. Madame Ida Pteiffer's Visit to the Holy Land, Egypt, and Italy. Second Edicion.

XXI. \& XXII. A Narrative of the United States' Exploring Expedition. Two Volumes.

XXIII. \& XXIV. 'The Iliad of Homer. Translated into English Verse by ALEXANDER Pope. A New Edition. Two Volumes. In morocco extra, for School Prizes, Two Volumes, $15 s$. ; or Two Volumes in One, 10s. $6 \mathrm{~d}$.; ditto, calf, marbled edges, $9 s$.

*** This Edition of Homer's Iliad contains the Classical Compositions of Flaxman, beautifully drawn by T. D. Scott, E、q.. and engraved in the most careful manner by J. L. Williams, Esq.

xxy. 'The Odyssey of Homer, with Flaxman's Illusirations, \&c. One Volume. Edited by the Rev. THEODuRE Acors BuckL Ex.

xxvi. 'The Complete Angler of Izaak Walton and Charles Cotion. New Edition, superbly illustrated with Fifty Engravings of Fishes, Fishiny Tackle, Flies, and Portraits and Landscapes. Edited by "EPHemera," of "Bell's Life in London."

* This Edition of the Complete Angler con. tains 100 pages of Notes; both explanatory of the Text, and adapting to modern experience and practice its obsolete instructions.
Xxvir. Extraordinary Men : their Boyhood and Early Life. Illustrated with Fifty Engravings of Portraits, Birth-places, \&c. \&c.

XxviII. The Pilgrim's Progress from this World to that which is to come. By JoHN Bunyan. A New Edition, with a Memoir by J. M. HARE. The Allegory illustrated with Thirty Outline Drawings, and the Biographical Sketch with interesting relics and recollections of the Author.

** To insure the completeness of the present Edition, it has been carefully reprinted VERBATIM, without abridgment or interpolation, from the genuine old Editions of John Bunyan.

xxix. Wanderings in Spain. From the French of Gautier. Copiously Illustrated by Maceuold.

xxx. The Life of Alexander Pope, with Extracts from his Correspondence. By $\mathbf{R}$. Carruthers, Esq., of Inverness.

xxxI. The Life, Public and Domestic, of the Right Hon. Edmund Burke. By Perer BURKE, Esq., of the Inner Temple and the Northern Circuit. Profusely Illustrated with Portraits, Scenes of Events, and Landscape Views, relating to the great Orator and the other noted persons of his time and career.

XXXII. The Life and Works of Alexander Pope, edited by R. CARRUTHERS, Esq., of Inverness (to be completed in Four Volumes). Volume II., containing the Rape of the Lock, Windsor Forest, Imitations of Chaucer, Essay on Criticism, Vertumnus and Fomona, Abelard and Heloise, Epistles, (ides, \&c. \&c. Illustrated with Incidents, Localities, and Portraits, by J. Portch and T. D. Scotr.

xxxIII. The Poetical Works of Alexander Pope. Edited by R. CARRUTIERs, Esq., of Inverness. Vol. III., containing the Dunciad, the Essay on Man, \&c. Completely Illustrated.

xxIv. The Natural History and Antiquities of Selborne. By the Rev. Grimert White, M.A. Edited, with Notes and Additions, by Sir William J A RiNe, Bart., F.R.S.E., F.L.S., M.W.S. Fully Illustrated.

"No one at the present day needs to be told of the merits of one of the rilost charming books on local history in the language. The merits of this edition consist in the fact of its oeing accompanied by copious notes of Sir William Jardine, and illustrated by seventy engravings, comprising subjects of natural history, and views of Selborne, its vicinity and antiquities. It is an appropriate holiday-book, and certainly one of the cheapest ever produced."-Daily News.

xxxy. 'The Life and Poetical Works of Alexander Pope, edited by R. CARRuthers, Esq., of Inverness. Volume IV., containing Satires, Miscellaneous Pieces, \&c. Illustrated with Incidents, Localities, and Portraits, by J. Portch and T. D. Scort.

xxxvi. Johnson's Lives of the Poets. Completed by William Hazlitr. 4 Volumes. Illustrated with Portraits, Incidents, and Localities, by J. GILBERT, T. D. ScotT, and other Artists.

Will be shortly published, in Two Volumes, the HISTORY of RUSSIA. By J. Duncan, Esq. 


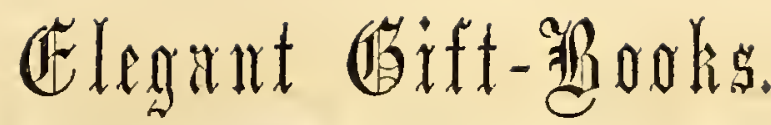

\section{Magnificent Edition of UNCLE TOM's CABIN. \\ Uncle Tom's Cabin; or, Life among the Lowly.}

By Harriet Beecher Srowe. Now ready, in a handsome form (demy 8vo), printed on the best paper by Messrs. Bradburr and Evars, elegantly bound in cloth gilt, illustrated with One Hundred and Twelve Drawings by GEorge Thomas, engraved by W. L. Thомas, and Fifty Ornamental Initial Letters and Tailpieces by T. R. MACQUOID. Price 12s., gilt edges, forming a most superb gift -book.

*** Every effort has been made to render this edition the best that has yet appeared, with respect alike to typography, binding, and artistic illustration. The Drawings are, however, its chief feature. A protracted residence in the United States of America has afforded Mr. Geonge Thomas peculiar opportunities of making himself familiar with the physiognomy of the Negro race, and the characteristics of Life in the Southern States; thus his Sketches are of a character entirely different from any that have appeared in the earlier editions of Mrs. STowe's world-renowned Tale.

\section{The Life of Martin Luther, the German Reformer,}

In Fifty Pictures; to which is added a Sketch of the Rise and Progress of the Reformation in Germany. Super-royal 8 vo, beautifully illustrated with Fifty Plates in the highest style of German Art, handsomely bound in cloth, gilt, price $12 s$.

\section{The Coinage of the British Empire,}

From the earliest Period to the present Time, with a Chapter on the proposed System of Decimal Coinage. By H. Noel Humphrers, Author of "The Origin and Progress of the Art of Writing," \&c. Completely illustrated by fac-similes of the British and Colonial Coins, represented by a Chromolithographic process in their respective metals, gold, silver, and copper. 4to, elegantly bound in cloth extra, gilt edges, $1 l$. 1s.; or bound in imitation of the ancient carved book-covers, $1 l .5 s$.

\section{The Origin and Frogress of the Art of Writing.}

Being a Complete History of the Art in all the stages of its development, from the simple pictorial writing of the early Chinese and Mexicans, and the cuneiform inscription of the Assyrians, to the different styles of European MSS. from the 6 th to the 1 lith century, and the progress of ordinary writing from the invention of printing to the present time. By H. NOEL HuMPhreYs, Author of the "Illuminated Books of the Middle Ages," "The Art of Missal Painting," "Ancient Coins and Medals," \&c. \&c. Illustrated by numerous coloured Plates, executed in a highlywrought style of Chromolithography, from examples of the painted writing of the Egyptians and Mexicans, and from beautiful specimens of the illuminated writings of the Mediæval and more recent periods of Modern History, including fac-similes from the Magna Charta, Domesday Book, \&c., followed by a series of fac-simiies from private letters, works in MS., signatures, \&c. \&c., and a great variety of autographs, chronologically arranged. 4 to, handsomely bound in cloth, $1 l .1 s$. The same handsomely bound in antique style, $16.5 s$.

New Edition, with upwards of Fifty Illustrations by JoHN GILBERT, engraved in the first style of art by DALZIEL Brothers.

\section{The Salamandrine; or, Love and Immortality.}

\section{By Charles Mackay, LL.D., Author of "Egeria," "Legends of the Isles," \&c. \&c.}

\section{A Holiday Book for Christmas and the New Year.}

Amusement for Winter Evenings, containing Fairy Legends and Tales, Christmas Carols and Music, Poetry, Pictures of Christmas Sports, Manners and Customs; \&c. \&c., specially adapted to the Season. Profusely and superbly illustrated, in elegant binding. This splendid book contains above Two Hundred and Fifty Engravings of the highest order ; it will prove at all times a delightful Table Book for the Drawing-Room. The volume is the size of the Illustrated London News, printed on a fine thick paper made expressly for it. It contains instruction and amusement for all ages. But few copies remain, and it will not be reprinted. Folio, in cloth, or in emblematical binding, $1 l .1 s$.

\section{The Parables of Friedrich Adolph Krummacher.}

Translated from the Eighth German Edition (the last revised by the Author). Embellished with Forty beautiful Drawings by J. R. Clayton, Esq., engraved by Dalziel Brothers. Small 4to, beautifully printed on the finest paper, and tastefully hound in cloth, gilt edges, $6 s$. These Parables, by the Author of "Elijah the Tishbite," must be welcome to all readers, inculcating, as they do, in an alluring form, the deepest truths of Christianity. As a book for Sabbath reading, it is highly commended. and is in universal use throughout Germany. 


\section{THE UNIVERSAL LIBRARY}

\section{OF THE BEST WORKS OF THE BEST AUTHORS OF ALL NATIONS, IN ALL DEPARTMENTS OF LITERATURE.}

\section{Beautifully and uniformly printed in Royal Octavo, with two or more first-class Illustrations to each Number, and a handsome cover.}

\section{THE FOLLOWING WORKS HAVE ALREADY APPEARED:-}

Second Edition.

I. Scott's Lady of the Lake, and Lay of the Last Minstrel. 1s.

II. Izaak Walton's Lives of Donne, Wotton, Herbert, Hooker, and Sanderson. $1 s$.

III. Anson's Voyage round the World. $1 s$.

Iv. Goldsmith's Vicar of Wakefield, and Saintine's Picciola. 1s.

v. Alison's Essays on Taste. $1 s$.

vi. Sterne's Tristram Shandy. pages.) $1 s .6 d$.

(168

VII. Fables of La Fontaine. Translated from the Frencl by E. Wright. 1s.

VIII. Sedgwick's Home, Paul and VirVirginia, the Indian Cottage, and the Exiles of Siberia. With Three Engravings, (120 pages.) $1 s$.

Ix. Uncle Tom's Cabin. New edition; with Portrait and Memcir of Mrs. Harriet BEEChER Stowe; a Digest of the Slave Laws of the Southern States of America; and Statistics of American Slavery since the Declaration of Independence. (180 pages.) 1s. $6 d$.

x. Kœmpfer's Account of Japan. 1s.

xI. Translations of Goethe's Faust, and the Tragedies of Schiller. With Four En. gravings. (170 pages.) 1s. $6 d$.

XII. Stephiens' Travels in Egypt, Arabia, and the Foly Land. 1s.

XIII. Emerson's Essays and Orations. $1 s$.

XIV. Milton's complete Poetical Works. 1s. $6 d$.

xv. Bremer's Mora, H. Family, Bondmaid, Axel and Anna. With Six Engravings. 1s. $6 d$.

xvr. Stephens' Travels in Turkey, Greece, Russia, and Poland. With Frontispiece and Vignette. $1 s$.
XVII. Goldsmith's Citizen of the World. With Frontispiece and Vignette. 1s.

XvirI. Michelet's Life of Martin Luther. With Engravings and Vignette. $1 s$.

XIX. Memoirs of the Life of Colonel Hutchinson. By Lucy Hutchrsson. With Frontispiece and Vignette. 1s.

xx. The Life of Charles XII. King of Sweden, by Voltaine; and the Life of Lord Herbert of Cherbury. With Frontispiece and Vignette. $1 s$.

xxr. Essays, Civil and Moral, by Francis Bacon; and Locre on the Reasonableness of Christianity. With Four Illustrations. 1s.

xxII. The Neighbours, and Minor Tales. By Frederika Bremer. With Frontispiece and Vignette by C. KEAME. 1s. $6 d$.

xxirI. Ritson's Robin Hood, with Title and Frontispiece. 1s.

xxIv. The Mutiny of the Bounty. With rontispiece and Vignette by E. DuNCAN. $1 s$.

xxv. The Canterbury Tales of Geoffrey Chaucer. Edited, with a Glossary, \&c., by Thomas WRIGHT, Esq., M.A., F.S.A., \&c. Double Part (230 pages), with Frontispiece and Vignette. $2 s$.

XXVI. Goldsmith's Essays. With Frontispiece and Vignette. $1 s$.

Xxvir. The Poetical Works of Robert Burns. With Frontispiece and Vignette. $1 s$.

XXVIII. Goldsmith's Plays and Poems. Illustrated with Frontispiece and Vignette. $1 s$.

Xxıx. The Life of Peter Wilkins, a Cornishman. By a Passenger in the "Hector." With Frontispiece and Vignette. $1 s$.

xxx. Crabbe's Tales. With Frontispiece and Vignette. $1 s$.

Poetry.-Volume I., containing Scott's Lay of the Last Minstrel, Lady of the Lake; La Fontaine's Fables; Goethe's Faust; Schiller's Piccolomini, and Wallenstein's Death; Milton's Poetical Works. Royal 8vo, handsomely bound in cloth gilt.

Fiction.--Volume I., containing Goldsmith's Vicar of 'Wakefield; Saintine's Picciola; Sedgwick's Home, Paul and Virginia, the Indian Cottage, and the Exiles of Siberia; Uncle Tom's Cabin; Bremer's Mora, H. Family, Bondmaid, Axel and Anna.

Voyages and Travels.-Volume I., containing Anson's Voyage round the World; Stephens' Travels in Egypt, Arabia, and the Holy Land; Stephens' Travels in Turkey, Greece, Russia, and Poland; Kempfer's Account of Japan; Bligh's Voyage in the Bounty to the South Seas. With Twelve Illustrations by Gilbert, DuncaN, and others. Royal 8vo, cloth (600 pages). 


$\$ 10$ 
i)

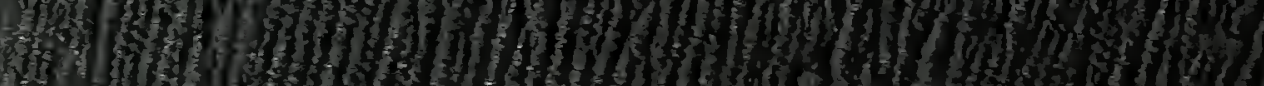

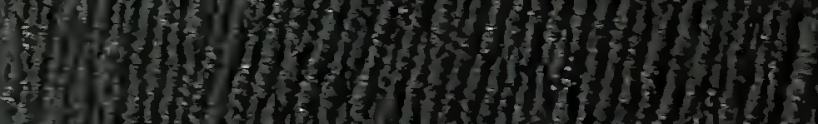

*1.

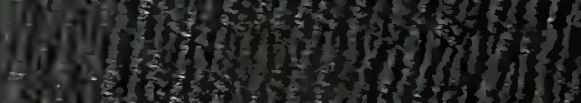

(i:

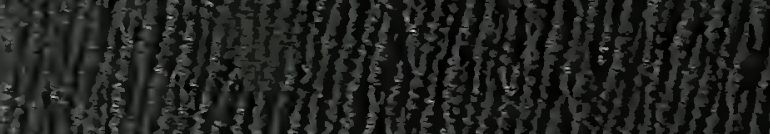

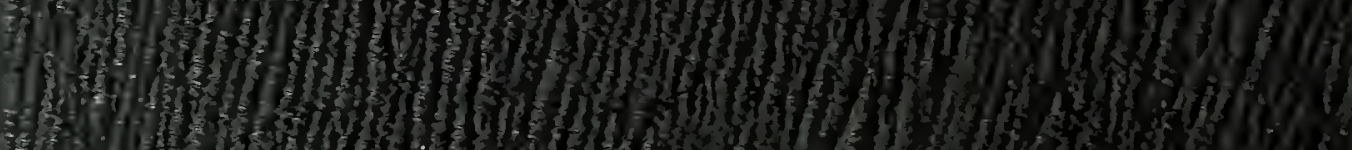

a

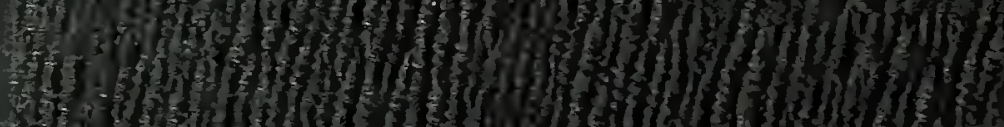

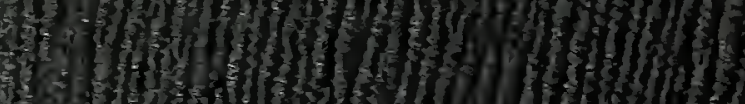

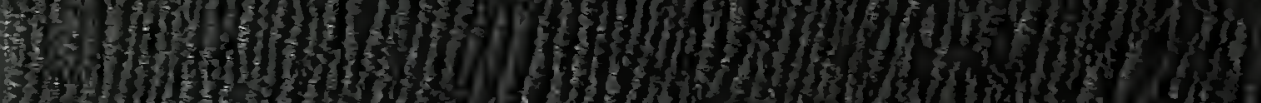

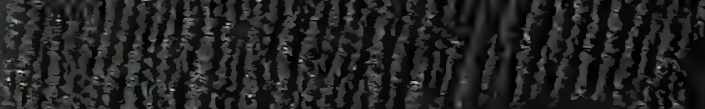

W.

(3)

HII)

7hth

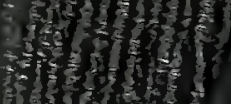

6)

W

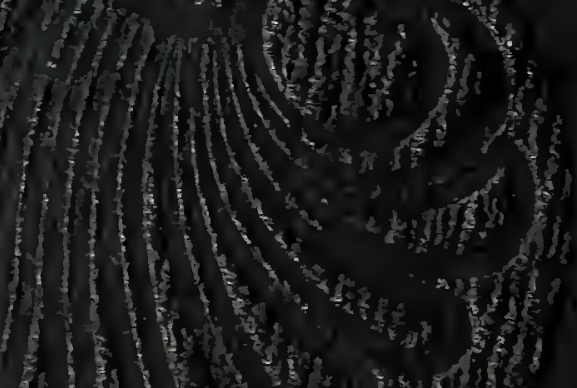
8)

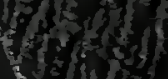

